\title{
Multi-Frequency Recirculating Planar Magnetrons
}

\author{
by
}

Geoffrey Bruce Greening
A dissertation submitted in partial fulfillment
of the requirements for the degree of
Doctor of Philosophy
(Nuclear Engineering and Radiological Sciences)
in the University of Michigan
2017

Doctoral Committee:

Professor Ronald M. Gilgenbach, Co-Chair

Professor Yue Ying Lau, Co-Chair

Professor Brian E. Gilchrist

Assistant Research Scientist Nicholas M. Jordan 
Geoffrey Bruce Greening geofgree@umich.edu

ORCID ID: 0000-0002-2765-6347

(C) Geoffrey Bruce Greening 2017 


\section{ACKNOWLEDGEMENTS}

I'd like to begin by thanking my advisors and dissertation committee co-chairs, Professors Ronald Gilgenbach and Y. Y. Lau. Ron has been a source of insight and advice for both scientific and professional challenges. My many conversations with him have proved to be an invaluable part of my education at the University of Michigan, and his early suggestion that I interview for an internship with L-3 Communications Electron Devices ultimately led to my decision to pursue a career in vacuum electronics. I will be forever grateful for his support during my time as a graduate student. Similarly, the many hours I've spent in Y. Y.'s office have been profoundly enjoyable, both for his explanations of physics and his many excellent and entertaining stories. Without a doubt, Y. Y. is the finest lecturer I've encountered at Michigan, and it has been a privilege and a pleasure to attend his courses.

I'd also like to thank the other members of my dissertation committee, Professor Brian Gilchrist and Dr. Nick Jordan. Professor Gilchrist has kindly agreed to be a member of several committees for students of Professor Gilgenbach, and I am grateful that he agreed once more to offer his time. Dr. Nick Jordan has patiently tolerated my many interruptions of his lunches and my incessant discussions of the daily challenges we faced in the laboratory. I've thoroughly enjoyed my time working with Nick, and I cannot imagine anyone being a better fit to manage the lab during my time as a student.

Without the help of the other members of the lab, this research would have been impossible. In particular, I'd like to thank my fellow microwave students, Steve Exelby and Drew Packard, who were instrumental in assisting me with the data collection process. Dave Simon, Sonal Patel, Adam Steiner, and Dave Yager have also provided a fresh perspective on some of the challenges encountered in this project, and I am grateful to them for sharing their knowledge. To my good friend Matt Weis, I'd like to say thanks for making the undergraduate and graduate experience more enjoyable. Special thanks are also owed to Mark Perreault, who fabricated several components used in this experiment. Thanks also go to Rob Sattler for his assistance with component fabrication, and Frank Hegeler for supplying the velvet electron emitters used for this project.

My ability to complete this dissertation was the result of advice and instruction from many excellent mentors over the course of many years. Each has contributed to my educa- 
tion. Some of those I'd like to acknowledge (in no particular order) include: Gene Overton, Doug Norris, Ben Lampe, Shernaz Minwalla, Phil MacKethan, Jim Bogard, Mike Worthington, Chris Wheeland, Mike Boyle, Peter Mardahl, Matt Franzi, Ed Cruz, Brad Hoff, and Dave French.

Finally, I'd like to thank my parents for their encouragement and support. The project did not always progress smoothly, and having someone patiently listen to my troubles helped me to think through the process of overcoming whatever obstacles arose. Mom kindly dedicated a substantial amount of time proofreading my draft to ensure my sentences did not get obnoxiously out of control. The process of writing a dissertation is indeed a long one, and I thank my Dad for setting an example that perseverance can yield eventual success.

This work was funded by the Office of Naval Research under grant numbers N0001413-1-0566 and N00014-16-1-2353, and L-3 Communications Electron Devices. Additional support was provided by the Directed Energy Professional Society through the Directed Energy Professional Society Graduate Scholarship. 


\section{TABLE OF CONTENTS}

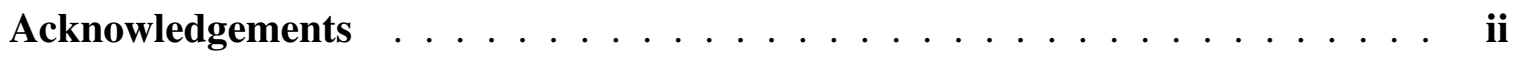

List of Figures $\ldots \ldots \ldots \ldots \ldots \ldots \ldots$ vii

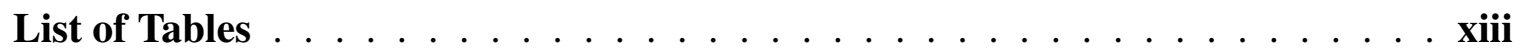

List of Appendices . . . . . . . . . . . . . . . . . . . . xv

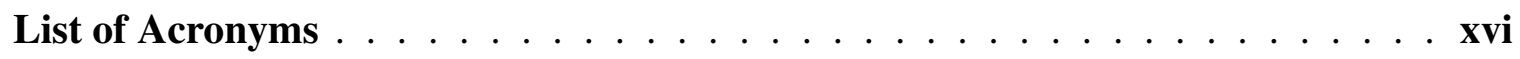

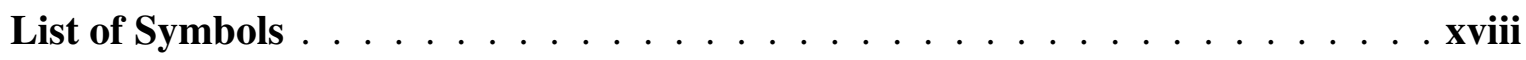

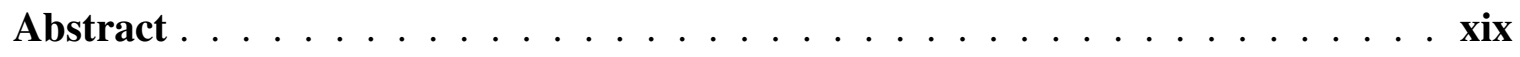

\section{Chapter}

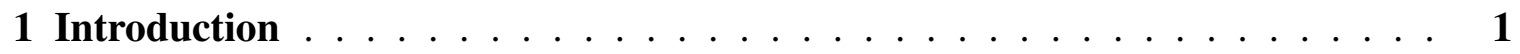

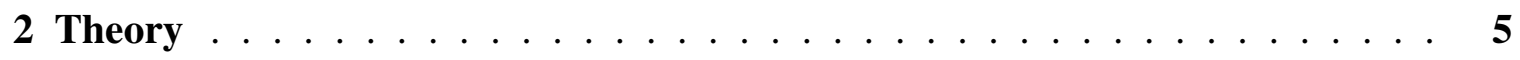

2.1 Planar Cavity Arrays . . . . . . . . . . . . . . . . . . 5

2.2 Electron Dynamics . . . . . . . . . . . . . . . . . . . . 14

2.3 Locking of Harmonic Frequencies . . . . . . . . . . . . . . 21

3 Design of the MFRPM . . . . . . . . . . . . . . . . . . 26

3.1 Design Criteria and Constraints . . . . . . . . . . . . . . . 26

3.2 Analytical Basis . . . . . . . . . . . . . . . . . . 29

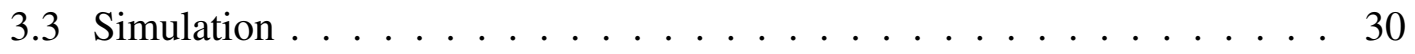

3.3.1 Cold Tube Modeling . . . . . . . . . . . . . . . 30

3.3.2 Hot Tube Modeling . . . . . . . . . . . . . . . 35

4 Experimental Configurations . . . . . . . . . . . . . . . . . 40

4.1 Magnetron Hardware . . . . . . . . . . . . . . . . . . . . . . 41

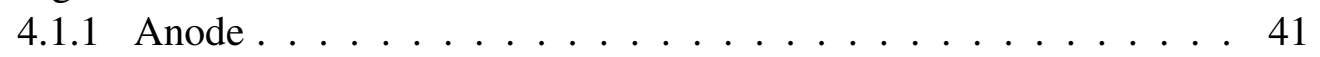

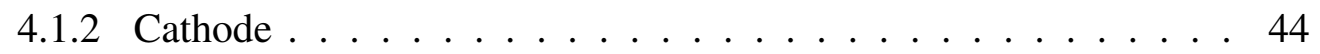

4.1 .3 Microwave Extractor . . . . . . . . . . . . . 45

4.2 Electromagnet . . . . . . . . . . . . . . . . . . . . . . . . . . . . 48

4.2.1 The Nonuniform Magnetic Field . . . . . . . . . . . . . . . . 49

4.2.2 The Uniform Magnetic Field . . . . . . . . . . . . . . 50 
4.3 Melba-C Pulsed Power Driver . . . . . . . . . . . . . . . . . 51

4.4 Triggering Sequence $\ldots \ldots \ldots \ldots \ldots$

4.5 Diagnostics . . . . . . . . . . . . . . . . . . 54

4.5.1 Pulsed Power Diagnostics $\ldots \ldots \ldots$. . . . . . . . . . . 54

4.5.2 Microwave Diagnostics . . . . . . . . . . . . . . 56

4.6 Pulse-Shortening Imaging Configuration . . . . . . . . . . . . . . . 59

5 Experimental Results for the Standard Load . . . . . . . . . . . . . . . 61

5.1 Summary of Findings . . . . . . . . . . . . . . . . . . . 61

5.1.1 Isolated LBO with Standard Load and Uniform Magnetic Field . 62

5.1.2 Isolated SBO with Standard Load and Uniform Magnetic Field . 62

5.1.3 MFRPM with Standard Load and Nonuniform Magnetic Field . . 63

5.1.4 MFRPM with Standard Load and Uniform Magnetic Field . . . . 64

5.1.5 Comparison of Metrics . . . . . . . . . . . . . . 65

5.2 Operation Metrics . . . . . . . . . . . . . . . . . . . 67

5.2 .1 Standard Metrics . . . . . . . . . . . . . . . . . . . 67

5.2.2 Additional Analysis . . . . . . . . . . . . . . . . . 71

5.3 Isolated LBO with Standard Load and Uniform Magnetic Field . . . . . 75

5.3.1 Standard Metrics . . . . . . . . . . . . . . . . . . . . . 75

5.3.2 Additional Analysis . . . . . . . . . . . . . . . 80

5.3.3 Comparison with Simulation . . . . . . . . . . . . . 82

5.4 Isolated SBO with Standard Load and Uniform Magnetic Field . . . . . 84

5.4 .1 Standard Metrics . . . . . . . . . . . . . . . . . . . . . . . . 84

5.4 .2 Additional Analysis . . . . . . . . . . . . . . . . 89

5.4.3 Comparison with Simulation . . . . . . . . . . . . . . 91

5.5 MFRPM with Standard Load and Nonuniform Magnetic Field . . . . . 94

5.5.1 Standard Metrics . . . . . . . . . . . . . . . . . . . . 95

5.5 .2 Additional Analysis . . . . . . . . . . . . . . 102

5.6 MFRPM with Standard Load and Uniform Magnetic Field . . . . . . 106

5.6 .1 Standard Metrics . . . . . . . . . . . . . . . . . 106

5.6 .2 Additional Analysis . . . . . . . . . . . . . 113

5.6 .3 Comparison with Theory . . . . . . . . . . . . . . . . . 121

5.6.4 Comparison with Simulation . . . . . . . . . . . . . . . . 124

5.7 Impact of Magnetic Field Optimization . . . . . . . . . . . . . 127

5.8 Pulse-Shortening Analysis _ . . . . . . . . . . . . . . . . . 128

6 Experimental Results for the Taper Load . . . . . . . . . . . . . . . 132

6.1 Summary of Findings . . . . . . . . . . . . . . . . . . . 132

6.1.1 MFRPM with Taper Load and Nonuniform Magnetic Field . . . . 132

6.1.2 MFRPM with Taper Load and Uniform Magnetic Field . . . . . . 135

6.1.3 Comparison of Metrics . . . . . . . . . . . . . . 136

6.2 Operation Metrics . . . . . . . . . . . . . . . . . 137

6.2 .1 Standard Metrics . . . . . . . . . . . . . . . . . . . 138

6.2 .2 Additional Analysis . . . . . . . . . . . . . . . . . 138

6.3 MFRPM with Taper Load and Nonuniform Magnetic Field . . . . . . 139 
6.3.1 Standard Metrics . . . . . . . . . . . . . . . . . . . . . . 139

6.3.2 Additional Analysis . . . . . . . . . . . . . . . . . . 147

6.4 MFRPM with Taper Load and Uniform Magnetic Field . . . . . . . . . 155

6.4.1 Standard Metrics . . . . . . . . . . . . . . . 156

6.4 .2 Additional Analysis . . . . . . . . . . . . . . . . . 164

6.5 Impact of Magnetic Field Optimization . . . . . . . . . . . . . . . 170

7 Summary and Conclusions . . . . . . . . . . . . . . . . 172

7.1 Summary . . . . . . . . . . . . . . . . . . 172

7.2 Conclusions . . . . . . . . . . . . . . . . . . . . . . 174

7.3 Suggested Future Work . . . . . . . . . . . . . . . . . . 174

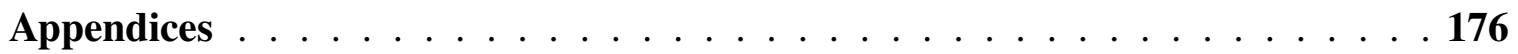

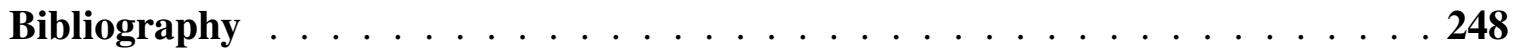




\section{LIST OF FIGURES}

1.1 Comparison between a conventional cylindrical cavity magnetron (top center), a recirculating planar magnetron (lower left), and a multi-frequency recirculating planar magnetron (lower right). . . . . . . . . . . . 3

2.1 A unit cell of a planar cavity array. . . . . . . . . . . . . . . . 6

2.2 Electromagnetic field in the unit cell for a $180^{\circ}$ phase advance. . . . . . . . . . 7

2.3 Different electromagnetic modes for an 8-cavity cylindrical magnetron. Different modes are identified by their respective phase advance per vane. Image sourced from [7] with corrections applied to (d). . . . . . . . . . . 8

2.4 Dispersion diagram showing a beam mode and the dispersion relation for the LBO, which includes the fundamental and higher-order circuit mode with space harmonics. . . . . . . . . . . . . . . . . 11

2.5 Particle orbits and analytic models for a planar magnetron diode. . . . . . . . . 14

2.6 Hartree diagram illustrating the Hull cutoff and threshold voltages for the LBO $5 \pi / 6$-mode and $\pi$-mode. . . . . . . . . . . . . . . . . . 17

2.7 The $\pi$-mode crossed-field interaction leading to phase focusing of the electrons. 19

2.8 Qualitative illustration of the fundamental energy transfer mechanism in crossedfield devices. . . . . . . . . . . . . . . . . . . . . . . 20

2.9 Circuit model for the SBO subject to an external driver, which models the second harmonic content of the LBO. . . . . . . . . . . . . . . . . . . 22

3.1 Basic components of the MFRPM. . . . . . . . . . . . . 27

3.2 Top sectional view of the microwave extractor showing the distance between the outer conductor of the coaxial transmission lines and the cathode endcap. . 29

3.3 HFSS model of the experimental MFRPM configuration. . . . . . . . . . . . . 32

3.4 Electric field geometry for various anode modes of interest in the MFRPM. . . 33

3.5 Electric field geometry for the parasitic chamber mode. . . . . . . . . . . . 34

3.6 MAGIC simulation geometry for the MFRPM. . . . . . . . . . . 35

3.7 MAGIC isolated oscillator configurations. . . . . . . . . . . . 36

3.8 MAGIC simulation illustrating particles and fields during $\pi$-mode operation by the LBO and SBO. The simulation used full emission and voltage correction. . 38

4.1 Top sectional diagram of the MELBA-C vacuum chamber and MFRPM components. The anode support structure is not shown. . . . . . . . . . . . . 40

4.2 Photographs of MFRPM components. . . . . . . . . . . . . . . . 41

4.3 Photographs of two magnetron anode configurations. . . . . . . . . . . . . 42 
4.4 Photographs of RF and DC gaskets used to improve electrical contacts. . . . . 43

4.5 The MCC-2 cathode. . . . . . . . . . . . . . . . . . . . . 44

4.6 Photographs of the microwave power extraction assembly. . . . . . . . . . 46

4.7 Cold test results obtained using a network analyzer. . . . . . . . . . . . . . 47

4.8 Magnetic field profiles within the MFRPM. . . . . . . . . . . . . . . 50

4.9 Photographs of the MELBA-C Marx generator. . . . . . . . . . . . . . 52

4.10 Diagram illustrating the trigger sequence times and approximate timescales during a shot. In the diagram, $M$ is the trigger delay between the electromagnet and MelBA-C, and $C_{d}$ is the command crowbar time. . . . . . . . 53

4.11 Microwave diagnostic configurations. . . . . . . . . . . . . 57

4.12 Photographs of the pulse-shortening imaging configuration. . . . . . . . . . 60

5.1 Overlay of multiple time-shifted shots compensating for jitter in the MELBA-C start time. . . . . . . . . . . . . . . . . . . 70

5.2 TFA plots of the RF voltage from the LBO on shot 13597 using different resolutions and RIKs. . . . . . . . . . . . . . . . . . . . . . . . . 72

5.3 Sample of the RF voltage signal from the LBO on shot 14610 before and after splining and filtering. . . . . . . . . . . . . . . . 74

5.4 Isolated LBO: Peak microwave output powers near $1 \mathrm{GHz}$ vs. magnetic field. . 75

5.5 Isolated LBO: Frequency metrics. . . . . . . . . . . . . . . . 76

5.6 Isolated LBO: Electrical characteristics vs. magnetic field. . . . . . . . . . . . 77

5.7 Isolated LBO: Energy conversion metrics vs. magnetic field. . . . . . . . . . . 78

5.8 Microwave start and peak power times. . . . . . . . . . . . . . . . 79

5.9 Isolated LBO: Power and dominant frequency vs. magnetic field. . . . . . . . 80

5.10 Isolated LBO: Analysis of shot 14924. . . . . . . . . . . . . . . . 81

5.11 Isolated LBO: Comparison between simulation and experiment. . . . . . . . 83

5.12 Isolated SBO: Peak microwave output powers near $2 \mathrm{GHz}$ vs. magnetic field. . 84

5.13 Isolated SBO: Frequency metrics. . . . . . . . . . . . . . . . 85

5.14 Isolated SBO: Electrical characteristics vs. magnetic field. . . . . . . . . . . 86

5.15 Isolated SBO: Energy conversion metrics vs. magnetic field. . . . . . . . . . 87

5.16 Microwave start and peak power times. . . . . . . . . . . . . . 88

5.17 Isolated SBO: Analysis of shot 14970 . . . . . . . . . . . . . . . . . 89

5.18 Isolated SBO: Analysis of shot 14971. . . . . . . . . . . . . . . . . 90

5.19 Isolated SBO: Comparison between simulation and experiment. . . . . . . . . 93

5.20 Current at peak power vs. series shot number. . . . . . . . . . . . . . . . 94

5.21 Standard Load and Nonuniform Magnetic Field: Peak microwave output powers for both oscillators vs. magnetic field. . . . . . . . . . . . . . . 95

5.22 Standard Load and Nonuniform Magnetic Field: Dominant and competing frequencies of both oscillators vs. magnetic field. . . . . . . . . . . . . 96

5.23 Standard Load and Nonuniform Magnetic Field: Dominant and competing frequency histograms for both oscillators. . . . . . . . . . . . . . . 97

5.24 Standard Load and Nonuniform Magnetic Field: Peak oscillator powers vs. dominant operating frequencies. . . . . . . . . . . . . . . . . . 98

5.25 Standard Load and Nonuniform Magnetic Field: Electrical characteristics vs. magnetic field. . . . . . . . . . . . . . . . . . . 99 
5.26 Standard Load and Nonuniform Magnetic Field: Energy conversion metrics vs. magnetic field. . . . . . . . . . . . . . . . . . . . 100

5.27 Standard Load and Nonuniform Magnetic Field: Temporal metrics vs. magnetic field. . . . . . . . . . . . . . . . . . . . . . . . . 101

5.28 Standard Load and Nonuniform Magnetic Field: LBO dominant frequency and $0.5 \times$ SBO competing frequency vs. magnetic field. . . . . . . . . . 102

5.29 Standard Load and Nonuniform Magnetic Field: Analysis of the LBO on shot 13630. . . . . . . . . . . . . . . . . . . . . . . . . 104

5.30 Standard Load and Nonuniform Magnetic Field: Analysis of the SBO on shot 13626. . . . . . . . . . . . . . . . . . . . . 105

5.31 Standard Load and Uniform Magnetic Field: Peak output powers for both oscillators vs. magnetic field. . . . . . . . . . . . . . . . . . 106

5.32 Standard Load and Uniform Magnetic Field: Dominant and competing frequencies of both oscillators vs. magnetic field. . . . . . . . . . . . . 107

5.33 Standard Load and Uniform Magnetic Field: Dominant and competing frequency histograms for both oscillators. . . . . . . . . . . . . . 108

5.34 Standard Load and Uniform Magnetic Field: Peak oscillator powers vs. dominant operating frequencies. . . . . . . . . . . . . . . . . 108

5.35 Standard Load and Uniform Magnetic Field: Electrical characteristics vs. magnetic field. . . . . . . . . . . . . . . . . . . . . . . . . . . . . 109

5.36 Standard Load and Uniform Magnetic Field: Energy conversion metrics vs. magnetic field. . . . . . . . . . . . . . . . . . . . 111

5.37 Standard Load and Uniform Magnetic Field: Temporal metrics vs. magnetic field. . . . . . . . . . . . . . . . . . . . . . . . 112

5.38 Standard Load and Uniform Magnetic Field: LBO dominant frequency and $0.5 \times$ SBO dominant frequency vs. magnetic field. . . . . . . . . . . . 113

5.39 Standard Load and Uniform Magnetic Field: Analysis of shot 14588. . . . . . 116

5.40 Standard Load and Uniform Magnetic Field: Mean phase differences $\overline{\Delta \phi}$ vs. magnetic field for a variety of phase shifts applied to the LBO RF voltage signal.118

5.41 Standard Load and Uniform Magnetic Field: Phase analysis of shots having temporal windows $\geq 5 \mathrm{~ns}$ during which each oscillator produced at least $67 \%$ of their respective peak powers. . . . . . . . . . . . . . . . 119

5.42 Comparison between operation metrics and the analytic locking condition. . . 123

5.43 Standard Load and Uniform Magnetic Field: Individual oscillator performance comparison between simulation and experiment. . . . . . . . . . . 125

5.44 Standard Load and Uniform Magnetic Field: Overall MFRPM performance comparison between simulation and experiment. . . . . . . . . . . . 126

5.45 Comparison between experimental pulse-shortening images and simulated RF electric fields for the SBO $\pi$-mode. . . . . . . . . . . . . . . . . . . . . 129

5.46 Microwave amplitudes and corresponding colorized fast framing camera images during shot 15104 . Images were provided by Dr. Nick Jordan. . . . . . . 131

6.1 Taper Load and Nonuniform Magnetic Field: Peak microwave output powers for both oscillators vs. magnetic field. . . . . . . . . . . . . . . . . . . . 139 
6.2 Taper Load and Nonuniform Magnetic Field: Dominant and competing frequencies of both oscillators vs. magnetic field. . . . . . . . . . . . . . 141

6.3 Taper Load and Nonuniform Magnetic Field: Dominant and competing frequency histograms for both oscillators. . . . . . . . . . . . . . . . . . . 142

6.4 Taper Load and Nonuniform Magnetic Field: Peak oscillator powers vs. dominant operating frequencies. . . . . . . . . . . . . . . . . . . . 143

6.5 Taper Load and Nonuniform Magnetic Field: Electrical characteristics vs. magnetic field. . . . . . . . . . . . . . . . . . . . . . . . 144

6.6 Taper Load and Nonuniform Magnetic Field: Energy conversion metrics vs. magnetic field. . . . . . . . . . . . . . . . . . . . . 145

6.7 Taper Load and Nonuniform Magnetic Field: Temporal metrics vs. magnetic field. . . . . . . . . . . . . . . . . . . . . . . . 146

6.8 Taper Load and Nonuniform Magnetic Field: Effects of the waveguide taper load on LBO operation. . . . . . . . . . . . . . . . . . . . . . . 148

6.9 Taper Load and Nonuniform Magnetic Field: First comparison set of dominant and competing frequencies vs. magnetic field. . . . . . . . . . . . . . . 149

6.10 Taper Load and Nonuniform Magnetic Field: Second comparison set of dominant and competing frequencies vs. magnetic field. . . . . . . . . . 150

6.11 Taper Load and Nonuniform Magnetic Field: Peak LBO output powers at harmonic frequencies vs. magnetic field. . . . . . . . . . . . . . . 153

6.12 Taper Load and Nonuniform Magnetic Field: Dominant and competing LBO harmonic frequency histograms. . . . . . . . . . . . . . . 153

6.13 Taper Load and Nonuniform Magnetic Field: Peak LBO harmonic powers vs. dominant operating frequencies. . . . . . . . . . . . . . . . . . . 154

6.14 Taper Load and Uniform Magnetic Field: Peak output powers for both oscillators vs. magnetic field. . . . . . . . . . . . . . . . . . . 156

6.15 Taper Load and Uniform Magnetic Field: Dominant and competing frequencies of both oscillators vs. magnetic field. . . . . . . . . . . . . . 157

6.16 Taper Load and Uniform Magnetic Field: Dominant and competing frequency histograms for both oscillators. . . . . . . . . . . . . . . . . . . 159

6.17 Taper Load and Uniform Magnetic Field: Peak oscillator powers vs. dominant operating frequencies. . . . . . . . . . . . . . . . 160

6.18 Taper Load and Uniform Magnetic Field: Electrical characteristics vs. magnetic field. . . . . . . . . . . . . . . . . . . . . . . . 161

6.19 Taper Load and Uniform Magnetic Field: Energy conversion metrics vs. magnetic field. . . . . . . . . . . . . . . . . . . . . . . 162

6.20 Taper Load and Uniform Magnetic Field: Temporal metrics vs. magnetic field. 163

6.21 Taper Load and Uniform Magnetic Field: Peak LBO output powers at harmonic frequencies vs. magnetic field. . . . . . . . . . . . . . . . . 164

6.22 Taper Load and Uniform Magnetic Field: Dominant and competing LBO harmonic frequency histograms. . . . . . . . . . . . . . . . 165

6.23 Taper Load and Uniform Magnetic Field: Peak LBO harmonic powers vs. dominant operating frequencies. . . . . . . . . . . . . . . 166

6.24 Taper Load and Uniform Magnetic Field: First comparison set of dominant and competing frequencies vs. magnetic field. . . . . . . . . . . . . 167 
6.25 Taper Load and Uniform Magnetic Field: Second comparison set of dominant and competing frequencies vs. magnetic field. . . . . . . . . . . . . . 169

B.1 SBO frequency vs. number of cells per SBO cavity. . . . . . . . . . . . . 179

C.1 Left portion of the LTspice MelBA-C circuit model. . . . . . . . . . . . . . 185

C.2 Middle portion of the LTspice MELBA-C circuit model. . . . . . . . . . . . 185

C.3 Right portion of the LTspice MelBA-C circuit model. . . . . . . . . . . . 185

C.4 Comparison of LTspice voltage traces with experimental MelBA-C shots. . . 186

C.5 Comparison of delayed ( $90 \mathrm{~ns}$ ) and rapid (10 ns) time to closure of all switches. The delayed simulation is identical to that shown in Fig. C.4b. . . . . . . . 187

C.6 Sample shots illustrating ideal Melba-C Marx erection. . . . . . . . . . . . 188

C.7 Sample shots illustrating typical and poor MELBA-C Marx erection. . . . . . . 189

D.1 Block diagram illustrating the LBSD microwave detector calibration configuration. . . . . . . . . . . . . . . . . . . . . . 190

D.2 Sample plot illustrating the calibration points obtained for LBSD HM01 used for MELBA-C shots. The diode output was terminated at $50 \Omega \ldots \ldots$. . . . 191

D.3 Photographs illustrating the configurations used to calibrate the directional couplers. . . . . . . . . . . . . . . . . . . . . . 193

D.4 Frequency-dependent attenuation of waveguide directional couplers. . . . . . 194

D.5 Examples of waveguide load matches. . . . . . . . . . . . . . . . 195

D.6 Sample plot illustrating the attenuation of cables A+B . . . . . . . . . . 195

D.7 Plot illustrating the calibration points obtained for the MELBA-C voltage monitor. . . . . . . . . . . . . . . . . . . . . . . . 197

D.8 Calibration configuration for the Rogowski coil current diagnostic. . . . . . . 198

D.9 Photograph of the rod cathode with a velvet electron emitter used for calibrating the Melba-C Rogowski coils. . . . . . . . . . . . . . . . . . . . . 199

D.10 Calibrated currents for two MELBA-C shots using a rod cathode and stainless steel current collector. . . . . . . . . . . . . . . . . . . . . 200

D.11 Circuit diagram showing the paths of the current detected by the ENTC Rogowski coil. . . . . . . . . . . . . . . . . . . . . . 201

D.12 Calibration of the magnetic field. . . . . . . . . . . . . . . . . 204

E.1 Identification of the optimal Marx trigger delay time. . . . . . . . . . . . 206

F.1 Taper Load and Nonuniform Magnetic Field: First comparison set of dominant and competing frequencies vs. magnetic field. . . . . . . . . . . . . 209

F.2 Taper Load and Nonuniform Magnetic Field: Second comparison set of dominant and competing frequencies vs. magnetic field. . . . . . . . . . . 210

F.3 Taper Load and Nonuniform Magnetic Field: Third comparison set of dominant and competing frequencies vs. magnetic field. . . . . . . . . . . . . 211

G.1 Taper Load and Uniform Magnetic Field: First comparison set of dominant and competing frequencies vs. magnetic field. . . . . . . . . . . . . 213

G.2 Taper Load and Uniform Magnetic Field: Second comparison set of dominant and competing frequencies vs. magnetic field. . . . . . . . . . . . 214 
G.3 Taper Load and Uniform Magnetic Field: Third comparison set of dominant and competing frequencies vs. magnetic field. . . . . . . . . . . 215

H.1 Crowbar switch geometry. . . . . . . . . . . . . . . . . . . . 217

I.1 The SBO anode. . . . . . . . . . . . . . . . . . . . 221

I.2 The SBO microwave extraction antenna. . . . . . . . . . . . . . . . . 222

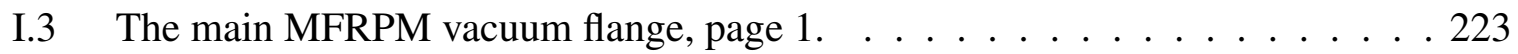

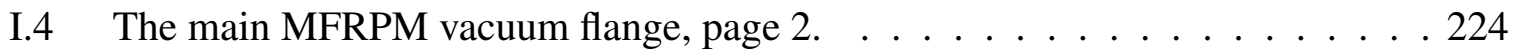

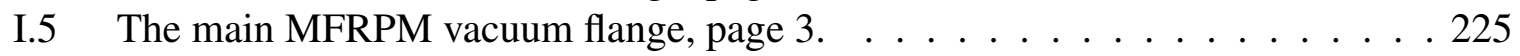

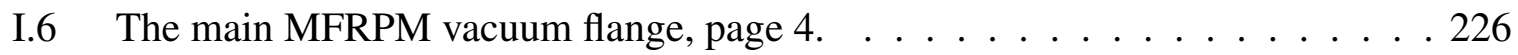

I.7 The main MFRPM vacuum flange, page 5. . . . . . . . . . . . . . . . 222

I.8 The main MFRPM vacuum flange, page $6 . \ldots \ldots \ldots$

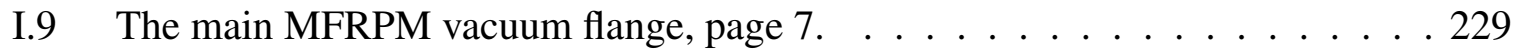

I.10 The main MFRPM vacuum flange, page $8 \ldots \ldots \ldots . \ldots \ldots$

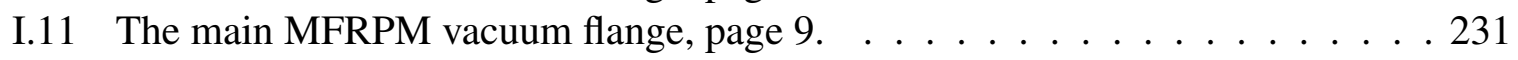

I.12 The WR-650 microwave window vacuum flange, page $1 \ldots \ldots$. . . . . . . 232

I.13 The WR-650 microwave window vacuum flange, page 2 . . . . . . . . . . 233

I.14 The WR-650 microwave window vacuum flange, page 3 . . . . . . . . . . 234

I.15 The WR-650 microwave window vacuum flange, page 4 . . . . . . . . . . 235

I.16 The WR-650 microwave window vacuum flange, page 5 . . . . . . . . . . 236

I.17 The Lexan vacuum flange insulator. . . . . . . . . . . . . . . . . . 237

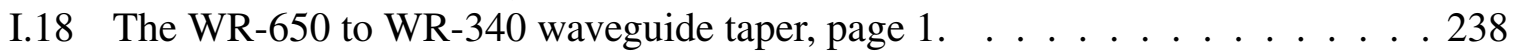

I.19 The WR-650 to WR-340 waveguide taper, page 2. . . . . . . . . . . . . . . 239

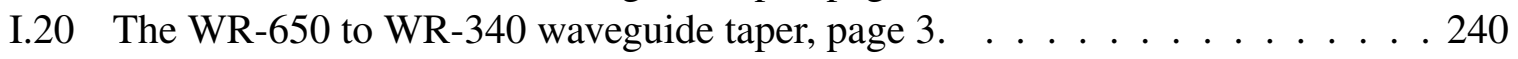

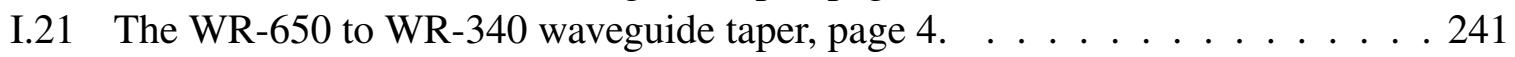

I.22 The WR-650 to WR-340 waveguide taper, page $5 . \ldots \ldots \ldots \ldots$

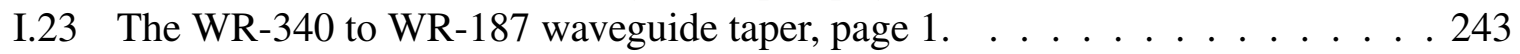

I.24 The WR-340 to WR-187 waveguide taper, page 2. . . . . . . . . . . . . . . 244

I.25 The WR-340 to WR-187 waveguide taper, page $3 . \quad \ldots \ldots \ldots$. . . . . . . 245

I.26 The WR-340 to WR-187 waveguide taper, page $4 . \ldots \ldots \ldots$

I.27 The WR-340 to WR-187 waveguide taper, page $5 . \ldots \ldots \ldots \ldots$ 


\section{LIST OF TABLES}

3.1 Summarized cold-tube simulation results obtained using HFSS for the modes of interest in the MFRPM. . . . . . . . . . . . . . . . . . . . 31

3.2 Summary of the MAGIC simulation sets of magnetic field parameter scans. . . 37

4.1 Summarized cold test results for the $\mathrm{LBO}$ and SBO $\ldots \ldots \ldots$

5.1 Isolated LBO: Summarized performance metrics near $0.16 \mathrm{~T}$. . . . . . . . 62

5.2 Isolated LBO: Summarized performance metrics for the $5 \pi / 6$ mode near $B=$

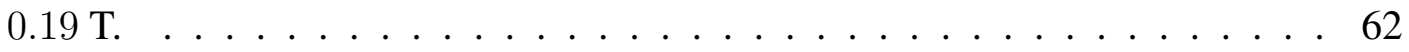

5.3 Isolated LBO: Summarized performance metrics for the region of optimal operation in $B=0.22-0.25 \mathrm{~T}$. . . . . . . . . . . . . . . . . 62

5.4 Isolated SBO: Summarized performance metrics for the region of optimal operation near $B=0.16$ T. . . . . . . . . . . . . . . . . . . . 63

5.5 Standard Load and Nonuniform Magnetic Field: Summarized performance metrics for the region of optimal operation in $B=0.21-0.23 \mathrm{~T}$. . . . . . . . 64

5.6 Standard Load and Nonuniform Magnetic Field: Summarized performance metrics in $B=0.14-0.17 \mathrm{~T}$, wherein the SBO operated in the $7 \pi / 8$-mode. . . 64

5.7 Standard Load and Nonuniform Magnetic Field: Summarized performance metrics for the LBO parasitic chamber mode. . . . . . . . . . . . . . . . . 64

5.8 Standard Load and Uniform Magnetic Field: Summarized performance metrics in $B=0.16-0.18 \mathrm{~T}$ wherein consistent frequency-locking occurred. . . . . 65

5.9 Standard Load and Uniform Magnetic Field: Phase metrics in $B=0.16-$ $0.17 \mathrm{~T}$ encompassing peak power production, wherein consistent frequencylocking and preferential selection of a relative phase difference occurred. . . . 65

5.10 Standard Load: Comparison of metrics for individual oscillators. . . . . . . . . 66

5.11 Standard Load: Comparison of MFRPM metrics. . . . . . . . . . . . . . 66

6.1 Taper Load and Nonuniform Magnetic Field: Summarized performance metrics near 0.17 T. . . . . . . . . . . . . . . . . . . . . . . 133

6.2 Taper Load and Nonuniform Magnetic Field: Summarized performance metrics near $0.17 \mathrm{~T}$ for the LBO harmonics. . . . . . . . . . . . . . . . . 134

6.3 Taper Load and Nonuniform Magnetic Field: Summarized performance metrics in the region of optimal operation in $B=0.22-0.23 \mathrm{~T}$. . . . . . . . . . 134

6.4 Taper Load and Nonuniform Magnetic Field: Summarized performance metrics for the LBO harmonics in the region of optimal operation in $B=0.22$ $0.23 \mathrm{~T}$. 
6.5 Taper Load and Nonuniform Magnetic Field: Summarized performance metrics for the SBO $6 \pi / 8$-mode in $B=0.13-0.17$ T . . . . . . . . . . . . 134

6.6 Taper Load and Uniform Magnetic Field: Summarized performance metrics near 0.17 T. . . . . . . . . . . . . . . . . . . . . . . . . 135

6.7 Taper Load and Uniform Magnetic Field: Summarized performance metrics near $0.17 \mathrm{~T}$ for the LBO harmonics. . . . . . . . . . . . . . . . . . . 135

6.8 Taper Load: Comparison of metrics for individual oscillators. . . . . . . . . 136

6.9 Taper Load: Comparison of MFRPM metrics. . . . . . . . . . . . . . . . . . 137

6.10 Taper Load: Comparison of metrics for the LBO frequency harmonics. . . . 137

B.1 Comparison of parallel computing configurations. . . . . . . . . . 182

D.1 Calibration parameters for all diodes. . . . . . . . . . . . . . . 192

D.2 Calibration parameters for all cables. . . . . . . . . . . . . . 196

D.3 Calibrated attenuation for other RF components at frequencies of interest. . . 196

D.4 Magnetic field calibration functions $f(x)$ for the different experimental configurations, where $f(x)$ is the magnetic field in Tesla, and $x$ is the peak voltage from the electromagnet Pearson coil. . . . . . . . . . . . . . . . 205 


\section{LIST OF APPENDICES}

A HFSS Simulation Implementation . . . . . . . . . . . . . . . . . 176

B MAgIC Simulation Implementation $\ldots \ldots$. . . . . . . . . . . . . . .

C Simulation of the MELBA-C Marx Generator $\ldots \ldots \ldots$

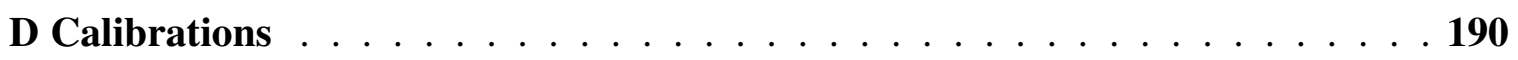

E Magnetic Field Optimization . . . . . . . . . . . . . . . . . . 206

F MFRPM with Taper Load and Unoptimized Magnetic Field: Plots of Frequencies vs. Magnetic Field . . . . . . . . . . . . . . . . . . . 208

G MFRPM with Taper Load and Optimized Magnetic Field: Plots of Frequencies vs. Magnetic Field . . . . . . . . . . . . . . . . . . . . . . 212

H Crowbar Switch Reconfiguration . . . . . . . . . . . . . . . 216

I Hardware Production Drawings . . . . . . . . . . . . . . . 220 


\section{LIST OF ACRONYMS}

$\begin{array}{ll}\text { AK gap } & \text { Anode-Cathode Gap } \\ \text { CV } & \text { Constant Voltage } \\ \text { CW } & \text { Continuous Wave } \\ \text { DV } & \text { Dynamic Voltage } \\ \text { ENDL } & \text { Endloss Current } \\ \text { ENTC } & \text { Entrance Current } \\ \text { FE } & \text { Full Emission } \\ \text { FFT } & \text { Fast Fourier Transform } \\ \text { HFSS } & \text { High Frequency Structural Simulator developed by Ansoft } \\ \text { HPM } & \text { High-Power Microwaves } \\ \text { LBO } & \text { L-Band Oscillator } \\ \text { LBSD } & \text { Low-Barrier Schottky Diode } \\ \text { LE } & \text { Limited Emission } \\ \text { LTspice } & \text { Simulation Program with Integrated Circuit Emphasis developed by } \\ \text { PMT } & \text { Linear Technology Corporation } \\ \text { MCC } & \text { Mode-Control Cathode } \\ \text { MEC } & \text { Magnetron Entrance Current } \\ \text { MELBA-C } & \text { Michigan Electron Long Beam Accelerator with Ceramic Insulator } \\ \text { MFPM } & \text { Multi-Frequency Recirculating Planar Magnetron } \\ \text { PML } & \text { Particle-In-Cell } \\ \text { PMotly Matched Layer } \\ \text { PMliplier Tube }\end{array}$




$\begin{array}{ll}\text { RF } & \text { Radio Frequency } \\ \text { RIK } & \text { Reduced Interference Kernel } \\ \text { RPM } & \text { Recirculating Planar Magnetron } \\ \text { RPM-12A } & \text { Recirculating Planar Magnetron with Aluminum Anode } \\ \text { RPM-12B } & \text { Recirculating Planar Magnetron with Plastic Anode and Copper } \\ \text { RPM-12C } & \text { Recirculating Planar Magnetron with Plastic Anode and Copper Spray } \\ \text { SBO } & \text { S-Band Oscillator } \\ \text { SWS } & \text { Slow-Wave Structure } \\ \text { TFA } & \text { Time-Frequency Analysis } \\ \text { TIG } & \text { Trigger Isolation Gap } \\ \text { TRL } & \text { Thru-Reflect-Line } \\ \text { TWTA } & \text { Traveling Wave Tube Amplifier } \\ \text { UM } & \text { University of Michigan } \\ \text { VOLM } & \text { Voltage Monitor } \\ \text { VSWR } & \text { Voltage Standing-Wave Ratio }\end{array}$




\section{LIST OF SYMBOLS}

\begin{tabular}{|c|c|}
\hline$b$ & Anode-Cathode Gap Spacing \\
\hline$B$ & Magnetic Field \\
\hline$B_{\mathrm{BH}}$ & Buneman-Hartree Threshold Magnetic Field \\
\hline$\beta$ & Propagation Constant \\
\hline$\beta_{p}$ & $c$-Normalized RF Phase Velocity \\
\hline$c$ & Speed of Light in Vacuum \\
\hline$\Delta \phi$ & Relative Phase Difference between the LBO and SBO \\
\hline$\overline{\Delta \phi}$ & Mean Phase Difference (Representative Phase Difference of a Shot) \\
\hline$|e|$ & Elementary Charge \\
\hline$E$ & Electric Field \\
\hline$f$ & Temporal Frequency \\
\hline$h$ & Cavity Depth \\
\hline$\kappa$ & Experimental Frequency-Locking Parameter \\
\hline$L$ & Circuit Pitch \\
\hline$\lambda_{g}$ & Guide Wavelength \\
\hline$m_{0}$ & Electron Rest Mass \\
\hline$\omega$ & Angular Frequency \\
\hline$Q$ & Quality Factor \\
\hline$\sigma_{\Delta \phi}$ & Phase Variation (Standard Deviation of $\Delta \phi$ ) \\
\hline$V$ & Voltage \\
\hline$v_{d}$ & Guiding Center Drift Velocity \\
\hline$v_{p}$ & RF Phase Velocity \\
\hline$w$ & Cavity Width \\
\hline
\end{tabular}




\begin{abstract}
The cavity magnetron is generally accepted as the standard for compactness and high microwave power with applications in industry, science, and defense, with the latter including counter-electronics. In this application, magnetrons are limited because they are narrow-band devices. To expand the range of frequencies that can be produced using a single magnetron, a novel multi-frequency variant of the Recirculating Planar Magnetron (RPM) was designed, fabricated, and experimentally demonstrated. This multi-frequency RPM (MFRPM) was the first high-power magnetron capable of generating multiple microwave frequencies simultaneously and demonstrated the first known instance of harmonic frequency-locking in a magnetron.

The MFRPM design consisted of two planar cavity arrays coupled by cylindrical electron recirculation bends. The two arrays formed a $1 \mathrm{GHz}$ L-Band Oscillator (LBO) and a $2 \mathrm{GHz}$ S-Band Oscillator (SBO). Experiments were conducted using a 0.1-0.3 T axial magnetic field produced using a pulsed pair of Helmholtz coils and a $-300 \mathrm{kV}, 200-400 \mathrm{~ns}$, 1-5 kA pulse applied to a Mode-Control Cathode (MCC) using the MELBA-C Marx generator. Six experimental configurations were tested using three anodes (the isolated LBO, the isolated SBO, and the MFRPM), two microwave loads (a standard, matched load, and a waveguide taper load used to characterize the LBO frequency harmonics), and two axial magnetic fields (uniform and nonuniform). Using these configurations, an in-depth characterization of MFRPM operation determined: 1) the identity of the observed electromagnetic modes, and the degree of mode competition, 2) the frequencies, powers, and other electrical characteristics associated with those modes and the LBO frequency harmonics, 3) the magnetic fields corresponding to optimal operation, 4) the operational impact of a nonuniform axial magnetic field, and 5) the origin and performance characteristics of a novel harmonic frequency-locked state observed in the MFRPM.

The uniform magnetic field consistently yielded better performance relative to the nonuniform magnetic field. In the harmonic frequency-locked state at $0.17 \mathrm{~T}$ with the uniform magnetic field, the MFRPM LBO produced $32 \pm 3 \mathrm{MW}$ at $0.984 \pm 0.001 \mathrm{GHz}$, and the SBO produced $13 \pm 2 \mathrm{MW}$ at $1.970 \pm 0.002 \mathrm{GHz}$. Relative to the other operating states, the locked state was remarkably consistent. In $B=0.16-0.17 \mathrm{~T}$, the phase drift during a
\end{abstract}


typical locked shot was $8 \pm 4^{\circ}$, and the lock duration was $14 \pm 3$ ns. The average phase difference between the oscillators was $93 \pm 17^{\circ}$. The locking appeared to be Adler-like, where the LBO was the driving oscillator and the SBO was the driven oscillator. Changes in the relative phase difference between the oscillators correlated with changes in the magnetic field, suggesting the coupling occurred through the second harmonic content of the LBO-modulated electron beam as it propagated from the LBO to the SBO. A comparison of the experimental results for this locked state with a new theory for harmonic locking was inconclusive.

Using the uniform magnetic field at $0.17 \mathrm{~T}$, the LBO second harmonic power was $178 \pm$ $60 \mathrm{~kW}$ at $1.962 \pm 0.013 \mathrm{GHz}$. The LBO fourth harmonic power was $5 \pm 1 \mathrm{~kW}$ at $3.916 \pm$ $0.018 \mathrm{GHz}$. In general, LBO harmonic powers increased when the fundamental circuit modes were operating at reduced power with considerable mode competition. Harmonic powers were also as much as $150 \%$ higher using the nonuniform magnetic field relative to the uniform magnetic field. 


\section{CHAPTER 1}

\section{Introduction}

High-Power Microwaves (HPM) have a number of applications in the fields of industry, science, and defense. Industrial examples include bulk cooking of foods, product testing for electromagnetic compatibility and vulnerability, and Radio Frequency (RF) sources for medical accelerators [1-3]. Scientific applications include RF sources for particle accelerators and fusion plasma heating [4]. Defensive applications include military radar systems, defeating improvised explosive devices, and counter-electronics [5,6]. This dissertation focused on the development of a variant of the cavity magnetron [7], which addresses some challenges in the field of counter-electronics. The cavity magnetron is generally accepted as the standard for compactness and high microwave power [8], and recent developments in magnetron sources have the potential to generate microwave powers of hundreds of $\mathrm{MW}$ at efficiencies near $70 \%[9,10]$, with some experimental success [11]. At lower efficiencies, the magnetron is a proven HPM source capable of generating GW microwave power levels $[12,13]$. However, the cavity magnetron has some limitations in counter-electronics applications because it is a narrow-band device. Considerable research has therefore been devoted to improving the range of accessible frequencies that can be produced using a single magnetron. The following methods discussed in this introduction were summarized in [14].

One method of producing different frequencies involves operating the magnetron in different resonant electromagnetic modes by adjusting the magnetron operating voltage and axial magnetic field [15]. This approach requires flexibility in the range of voltages and/or magnetic fields that can be applied to the magnetron, which introduces complexity to the power supply and/or electromagnet, and many magnetrons use permanent magnets instead of electromagnets. The method is also predicated on a Slow-Wave Structure (SWS) (an integral component in magnetron design) having: 1) very different frequencies with 2) similar phase velocities that are all 3) well-matched to the microwave extractor. The second condition is particularly difficult to achieve in magnetron design, and is typically infeasible at relativistic voltages due to the incorporation of features prone to arcing, such as anode 
straps [16]. Operating the magnetron at very different frequencies (and therefore very different phase velocities) demands that the electron $\vec{E} \times \vec{B}$ drift velocity be very different for the two modes, which usually compromises efficiency (due to having a fast phase velocity) or output power (due to having an excessively slow phase velocity). Mode-hopping in relativistic devices is possible due to cathode plasma expansion [17], although this is generally undesirable due to the lack of temporal control, and does not produce simultaneous emission at the two frequencies. In addition, the neighboring modes (between which hopping has been observed) tend to be relatively close in frequency and phase velocity, although this depends on the dispersion relation of a given SWS.

Another approach used to produce different frequencies involves the use of mechanical plungers to modify the resonant frequency of a given mode $[6,7,18]$. While this method is effective, it is complicated due to the mechanical-vacuum interface, ultimately limited in the range of available tunability (e.g., the aforementioned reference quotes $33 \%$ tunability), and cannot produce different frequencies simultaneously.

The last approach involves the incorporation of two different SWSs in a single HPM source. Such a concept has been presented in the literature for other devices, but a dualfrequency magnetron has not yet been investigated. Some examples of dual-frequency concepts include magnetically-insulated line oscillators [19,20], klystron-like concepts [21], backward-wave oscillators [22], and traveling-wave tubes [23].

In this dissertation, the dual SWS approach is employed using the novel Recirculating Planar Magnetron (RPM) [24] geometry to design, fabricate, and experimentally demonstrate a prototype Multi-Frequency Recirculating Planar Magnetron (MFRPM) composed of an L-Band Oscillator (LBO) and an S-Band Oscillator (SBO) [14]. Figure 1.1 shows examples of a conventional cylindrical cavity magnetron, RPM, and MFRPM. The RPM has several potential advantages over conventional cylindrical cavity magnetrons, and has considerable flexibility in its design [25]. Most notable is the large cathode area. In order to generate HPM, magnetron cathodes operate using explosive electron emission in a space charge limited regime [26-29], so the currents available to conventional cylindrical magnetrons are limited by the comparatively small cathode area. The large cathode area therefore makes the RPM an especially promising HPM source. The inverted cylindrical magnetron variant (where the cathode resides on the outside, and the anode on the inside) solves the small-area cathode problem with the added advantage of enhanced electron bunching in the circular bends because of the negative mass instability [30-32], but suffers from heat dissipation issues from the smaller anode area, and introduces considerable practical challenges for microwave extraction from the anode [33-35]. Other RPM advantages include a favorable scaling of the magnetic field volume with cavity number 


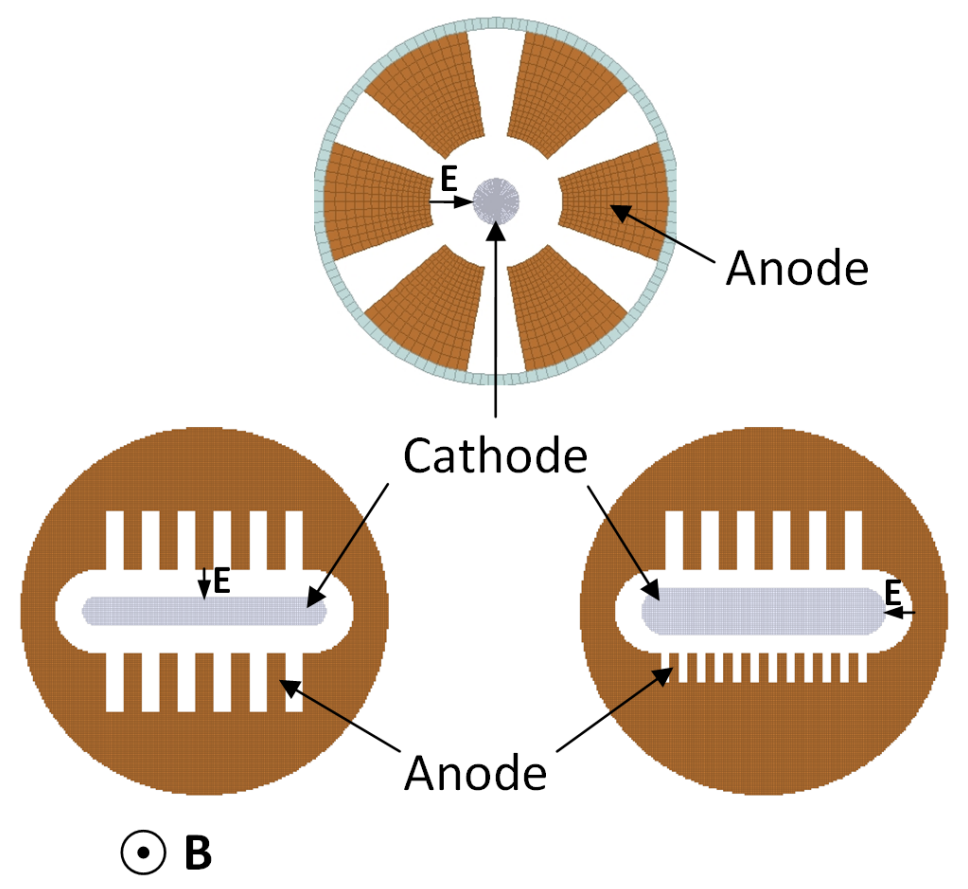

Figure 1.1: Comparison between a conventional cylindrical cavity magnetron (top center), a recirculating planar magnetron (lower left), and a multi-frequency recirculating planar magnetron (lower right).

(as $N$ ), a large anode volume that facilitates heat dissipation, and a decoupled relation between the cavity number and Anode-Cathode Gap (AK gap) spacing. The power generated by an RPM scales linearly with cavity number if power is extracted from all cavities [36], whereas the scaling in cylindrical devices is more complicated. The RPM combines these favorable features of a planar magnetron with the high efficiency afforded by full electron beam recirculation.

Different geometric variations of the magnetron have been the focus of research at the University of Michigan (UM) for several years. For example, the effect of conventional, planar, and inverted magnetron configurations on start-up has been theoretically investigated [32, 37, 38]. Experimental research at UM has largely focused on the first RPM prototype, termed the RPM-12A $[10,39,40]$. The RPM-12A consisted of two planar cavity arrays of six cavities, and demonstrated $150 \mathrm{MW}$ microwave powers near $1 \mathrm{GHz}$. In addition to the aluminum RPM-12A, additively-manufactured variants (the RPM-12B and RPM-12C) have also been experimentally demonstrated [41]. These variants were geometrically identical to the RPM-12A, and experiments yielded comparable operation to the RPM-12A. The RPM-12B was fabricated using a copper electroplating process applied to 3D-printed plastic anodes coated in conducting epoxy, and the RPM-12C was fabricated 
using a thermal-sprayed process applied to a similar plastic anode. For this dissertation, the most relevant prior work includes the development of a cathode design to passively promote locked operation of the individual planar cavity arrays in the RPM-12A [10,42], which was successfully demonstrated experimentally [40]. Locking between the structures occurred through a slotted cathode that allowed feedthrough of the electromagnetic fields (and currents) between the oscillators to improve the coupling between them. The MFRPM was designed to investigate whether similar locking would occur between frequency harmonics.

Along with the design, fabrication, and demonstration of the MFRPM prototype, the scope of this dissertation also includes an in-depth characterization of operation using six experimental configurations. The configurations tested three anodes (the isolated LBO, the isolated SBO, and the MFRPM), two microwave loads (the standard load and the waveguide taper load), and two axial magnetic fields (uniform and nonuniform). The characterization determined: 1) the identity of the observed fundamental operating electromagnetic modes, and the degree of mode competition, 2) the frequencies, powers, and other electrical characteristics associated with those modes and the LBO frequency harmonics, 3) the magnetic fields corresponding to optimal operation, 4) the operational impact of a nonuniform axial magnetic field, and 5) the origin and performance characteristics of a novel harmonic frequency-locked operating state observed in the MFRPM. A related study was also performed to image plasma-related pulse-shortening in the MFRPM oscillators. While preliminary, the imaging results appeared to provide valuable insight that might explain some of the MFRPM operation described herein.

This dissertation is organized into seven chapters. Chapter 2 provides an introduction to the theory of planar cavity arrays and beam dynamics needed to design the MFRPM, as well as a theory recently proposed by Y. Y. Lau on harmonic frequency-locked operation [43]. Chapter 3 covers the design of the MFRPM by describing the necessary criteria, practical constraints, application of the theory, and simulations conducted using two commercial codes. Chapter 4 describes the experimental hardware and configurations. Chapters 5 and 6 report the results obtained using the standard load and waveguide taper load, respectively. Both results chapters begin with a summary of the most important findings followed by an in-depth analysis and discussion of the data. The dissertation concludes with Chapter 7, which presents a summary of the work, notable conclusions, and suggested future work. 


\section{CHAPTER 2}

\section{Theory}

This chapter provides an introduction to the theory and analytic models used to design the Multi-Frequency Recirculating Planar Magnetron (MFRPM) and interpret the experimental results. The discussion is organized into three sections. The first covers the theory of planar cavity arrays, which form the oscillators that interact with the electron beam to generate microwaves. The planar cavity array theory provides a powerful tool for understanding magnetron operation, and the interpretation is discussed in detail. The second section describes the electron dynamics, which includes the condition for synchronism between the electrons and the microwaves, as well as the intrinsically efficient crossed-field interaction. The chapter concludes with a new theory to predict the conditions under which frequency-locked operation may occur between two oscillators having frequencies $f_{1}$ and $f_{2}=2 \times f_{1}$.

\subsection{Planar Cavity Arrays}

The MFRPM generates microwaves by exciting a resonant mode on a Slow-Wave Structure (SWS), which is a circuit on which an electromagnetic wave propagates at a speed less than the speed of light in vacuum. The MFRPM resonant circuit is a planar cavity array, which is a set of coupled unit cells like the one illustrated in Fig. 2.1. Each cell includes the cathode, anode, and anode-cathode gap (AK gap), and the figure shows the labeling conventions used for the dimensions of each part. In a magnetron, the cavities also function as the anode, which collects the electron current from the cathode.

Each unit cell is a resonant microwave cavity, and can be imagined as a simple $L_{i}$-C oscillator with some additional capacitance between the SWS vanes and the cathode [7]. Here, $L_{i}$ refers to an inductance, which should not be confused with the circuit pitch $L$ in Fig. 2.1a. Figure 2.1b shows the lossless equivalent circuit imposed on the unit cell structure. Thus, at their simplest, the planar cavity arrays are a set of coupled $L_{i}-C$ oscillators. 


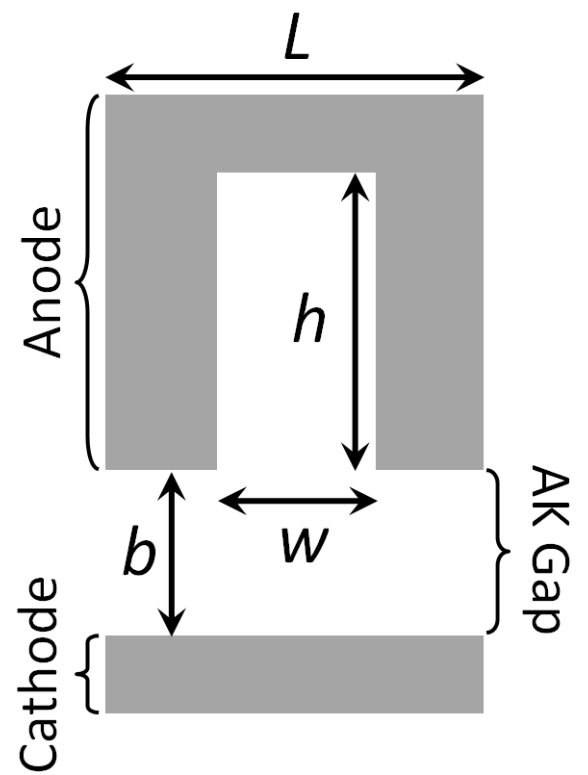

(a) Dimensions of a unit cell.

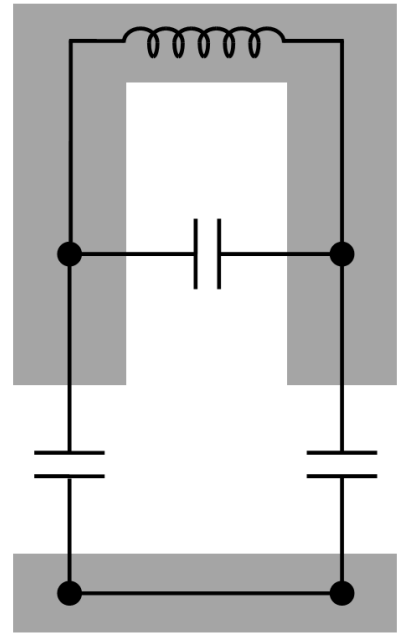

(b) Equivalent circuit of a unit cell.

Figure 2.1: A unit cell of a planar cavity array.

The circuit model in Fig. 2.1b also provides an intuitive explanation of the beamloading phenomenon observed in magnetrons, which is also called frequency-pushing [6]. Beam-loading refers to a reduction in the resonant frequency as the current is increased. The explanation lies in the frequency dependence of the cell, which is approximately given by $1 / \sqrt{L_{i} C}$. The Beam Dynamics section will show that the presence of the electron beam in the AK gap usually makes the AK gap distance $b$ look electrically smaller, although there are exceptions. Since capacitance scales inversely with distance, the presence of the electron beam increases the capacitance of the circuit and therefore lowers the resonant frequency.

The basic structure of the electric and magnetic fields in a single microwave cavity is shown in Fig. 2.2, which was generated with High Frequency Structural Simulator (HFSS) [44] (a commercial simulation code) using a methodology described in Appendix A. In the fundamental circuit mode, an oscillating voltage potential exists across the cavity, which leads to the horizontal electric field varying from zero in the back of the cavity to a maximum between the vane tips. The cavity depth $h$ is therefore approximately a quarter of the wavelength. Note that the horizontal component of the fringing cavity electric field decays in magnitude across the AK gap from the maximum at the vane tips to zero at the cathode surface, and that wider cavities (having larger $w$ ) have electric fields that decay more slowly with distance from the cavity compared to narrower cavities. In the fundamental mode, the current flows from one vane tip, around the cavity, to the other vane 


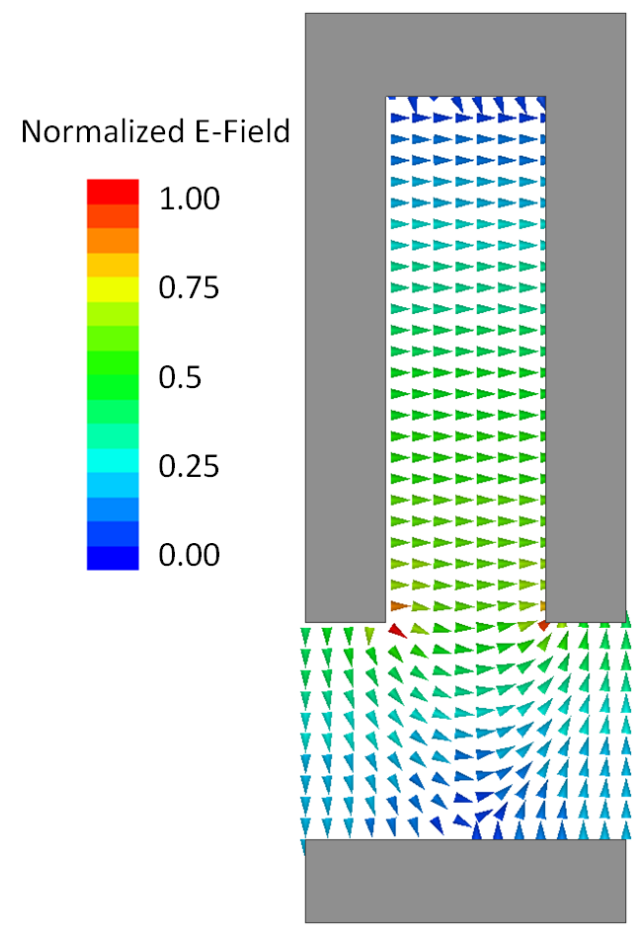

(a) Electric field in the fundamental mode.

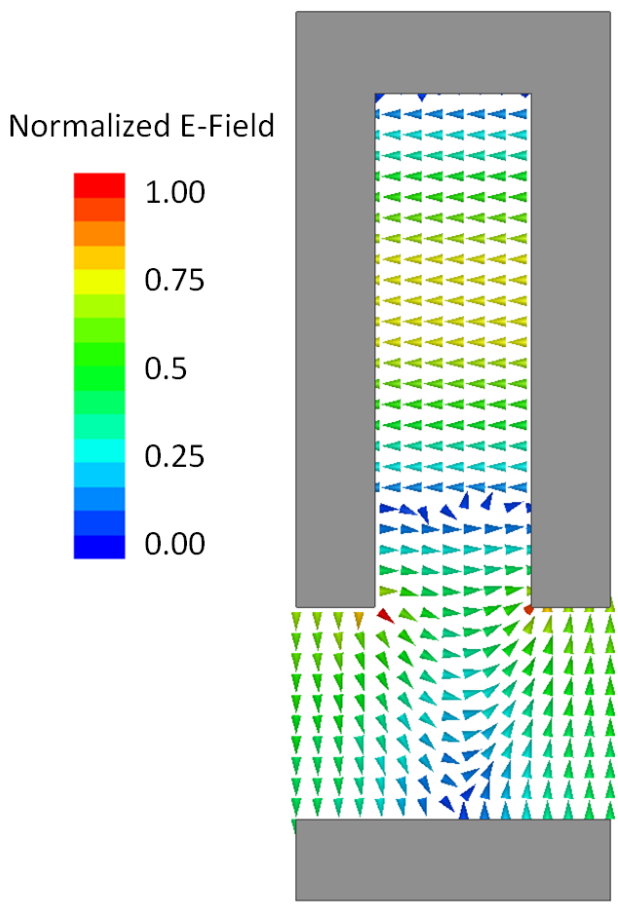

(c) Electric field in the higher order mode.

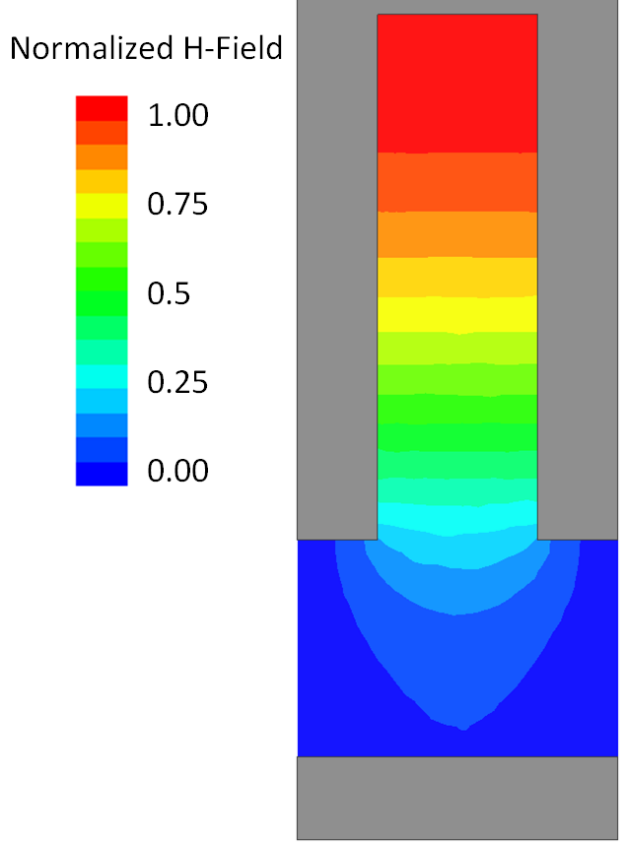

(b) Magnetic field in the fundamental mode (orthogonal to the paper).

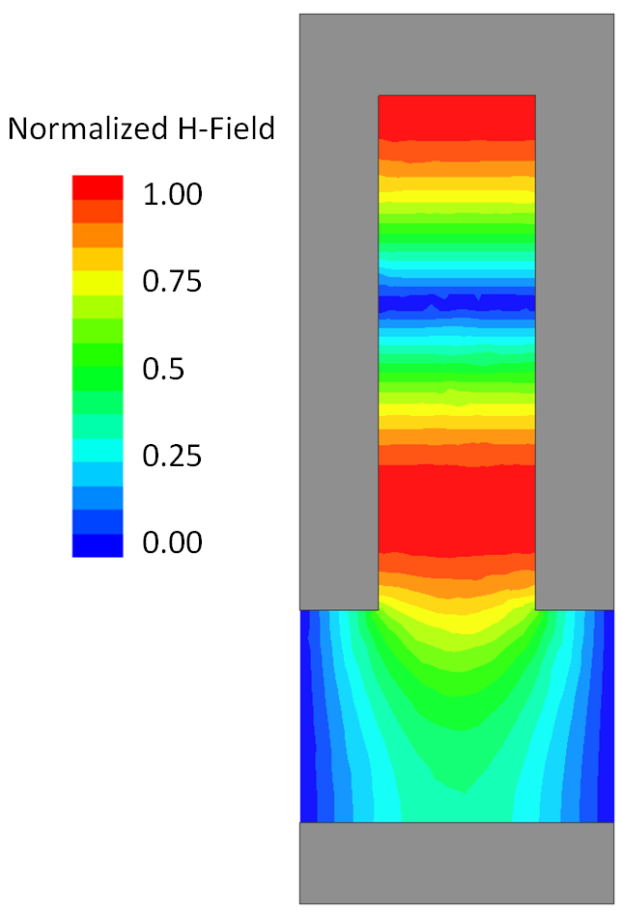

(d) Magnetic field in the higher order mode (orthogonal to the paper).

Figure 2.2: Electromagnetic field in the unit cell for a $180^{\circ}$ phase advance. 


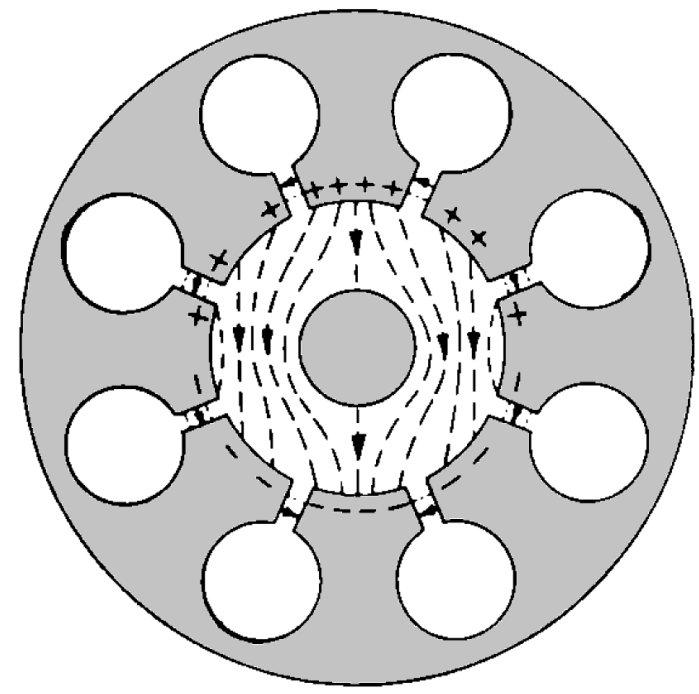

(a) $\pi / 4$-mode electric field.

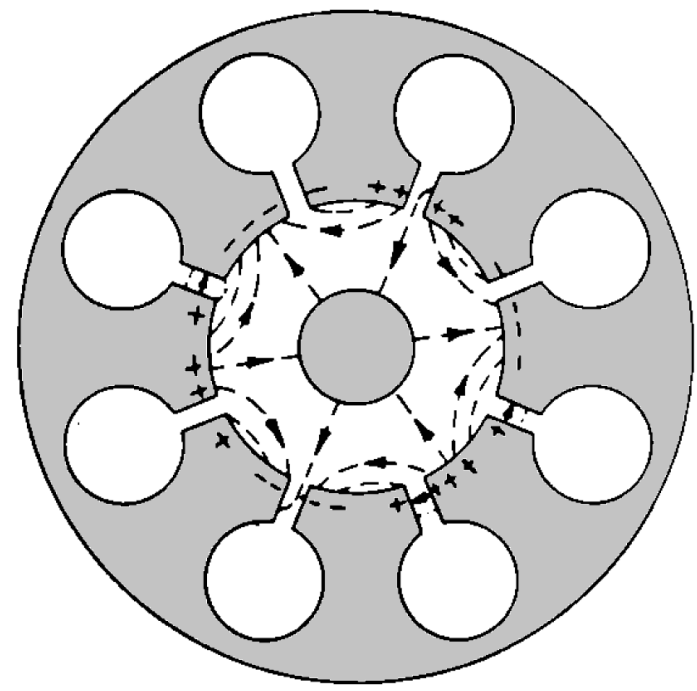

(c) $3 \pi / 4$-mode electric field.

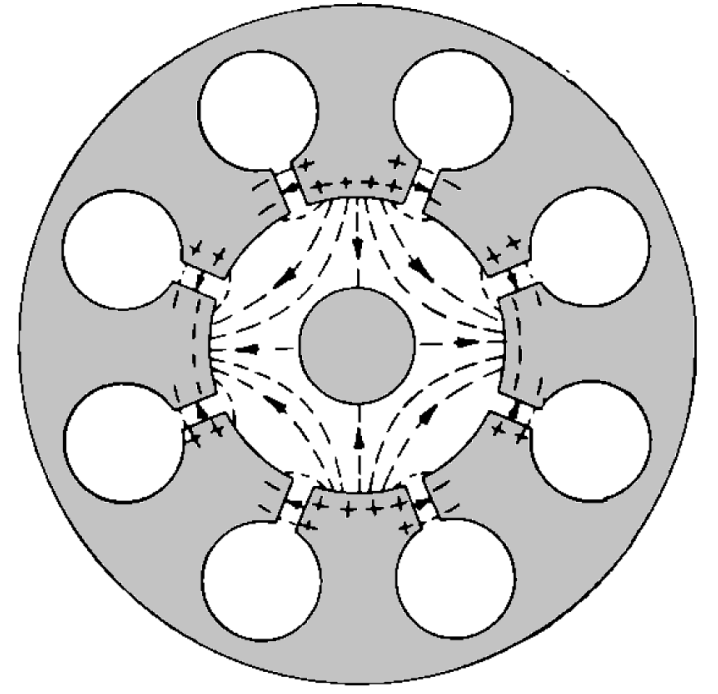

(b) $\pi / 2$-mode electric field..

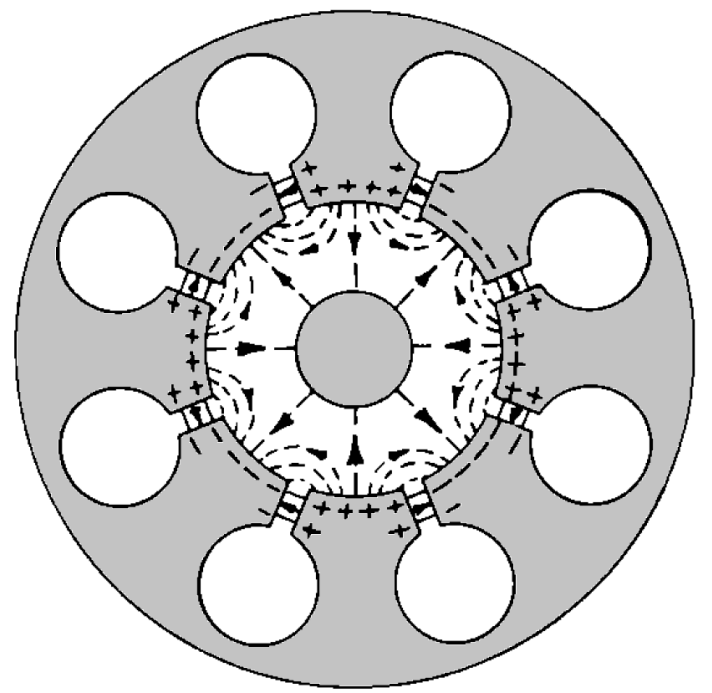

(d) $\pi$-mode electric field.

Figure 2.3: Different electromagnetic modes for an 8-cavity cylindrical magnetron. Different modes are identified by their respective phase advance per vane. Image sourced from [7] with corrections applied to (d). 
tip, as illustrated by the magnetic field in Fig. 2.2b.

The microwave cavities also support additional electromagnetic field geometries at higher frequencies, which are called the higher-order circuit modes. Figures $2.2 \mathrm{c}$ and $2.2 \mathrm{~d}$ show the electric and magnetic fields for the next circuit mode. These higher modes can be imagined as squeezing additional field half-wavelengths in the cavity along with the original quarter-wavelength. In all modes, the electric field magnitude is always zero at the back of the cavity, and maximized between the vane tips. Nearly all magnetrons are designed to operate solely in the fundamental circuit mode. However, because the MFRPM was designed to operate at $f_{1}$ and $f_{2}=2 \times f_{1}$, the low-frequency structure (the L-Band Oscillator, or LBO) was theoretically susceptible to interference from the high-frequency structure (the S-Band Oscillator, or SBO), which could promote LBO operation in a higher-order circuit mode.

In addition to the higher-order circuit modes, other modes of operation are possible when the individual microwave oscillators are coupled together as an array of cavities. Figure 2.3 illustrates the electric fields for different electromagnetic modes in an 8-cavity cylindrical magnetron [7]. When multiple cavities are coupled together, the different modes correspond to different RF phase advances per vane, and are named accordingly (e.g., a mode having a phase advance of $\pi$ radians per vane is called the $\pi$-mode). For cylindrical magnetrons, which are re-entrant because the SWS is cylindrically continuous and uninterrupted, the number of fundamental resonant modes $n$ is related to the number of cavities $N$ by $n=N / 2$ (ignoring the distinction between forward- and backward-wave modes, which are explained shortly). In a planar structure, which is not re-entrant, the number of fundamental resonant modes is equal to the number of cavities (again, ignoring the aforementioned distinction).

For the desired crossed-field interaction to occur, magnetrons must be designed to synchronize the phase velocity of electromagnetic waves propagating on the SWS circuit with the mean velocity of the electron beam, which is roughly the $\vec{E} \times \vec{B}$ drift velocity. Thus, to design the MFRPM, the dimensions of a planar cavity array must be adjusted to achieve a specified wave propagation velocity and frequency. The phase velocity of the RF is given by $v_{p}=\omega / \beta$, where $\omega$ is the angular velocity, and $\beta$ is the propagation constant in the direction of the $\vec{E} \times \vec{B}$ drift velocity. The wavelength of the RF wave on the SWS is called the guide wavelength $\lambda_{g}$, which is related to the propagation constant by $\beta=2 \pi / \lambda_{g}$. From Fig. 2.3, different modes clearly have different guide wavelengths. The design of the SWS therefore requires knowledge of the relation between the geometric dimensions, the angular frequency $\omega$, and the propagation constant $\beta$ for the different electromagnetic modes.

Plots of $\omega$ vs. $\beta$ are called dispersion diagrams because they illustrate the dispersion 
relation $\omega(\beta)$, where the angular frequency $\omega$ is a function of the propagation constant $\beta$. Points on the diagram falling on the dispersion relation curve for a SWS correspond to values of $\omega$ and $\beta$ that can propagate on the circuit. If an electron beam has a given velocity $v$ projected in the direction of the circuit phase velocity, the zero-intercept line having a slope equal to $v$ is called the beam mode, represented by the straight line $\omega=\beta v$ in the $\omega$ - $\beta$ plane. Since the RF phase velocity of the circuit mode is $v_{p}=\omega / \beta$, an interception between the cold circuit dispersion relation curve and the beam mode therefore represents some $\omega$ and $\beta$ corresponding to a mode that: 1) can exist on the SWS, and 2) propagates with the same phase velocity as the velocity of the electron beam. At these intercept locations, the synchronous crossed-field interaction can occur, which is described later in the Electron Dynamics section.

A dispersion relation can be analytically derived for an infinitely long, two-dimensional array of planar unit cells like the one illustrated by Fig. 2.1a on page 6, which approximates the structures in the MFRPM (see Fig. 1.1). The derivation applies Floquet's theorem to the periodic structure and makes some assumptions about the form of the electric and magnetic fields in the cavities [45]. Notably, in the derivation, the electric and magnetic field for a propagating mode on the SWS are expanded as a summation of space harmonics, as shown by Eq. 2.1 for the electric field. Each harmonic has a different propagation constant $\beta_{n}$ and phase velocity $v_{p n}$, which are shown by Eqs. 2.2 and 2.3.

$$
\begin{gathered}
\vec{E}(x, y, z, t)=\sum_{n=-\infty}^{\infty} \vec{E}_{n}(x, y) e^{j \omega t-j \beta_{n} z}, \\
\beta_{n}=\beta_{0}+\frac{2 \pi n}{L}, n=0, \pm 1, \pm 2, \ldots, \\
v_{p n}=\frac{\omega}{\beta_{n}} .
\end{gathered}
$$

The propagation constant $\beta_{0}$, which corresponds to $n=0$, is called the fundamental mode, and all other values of $n$ are called the $n^{\text {th }}$ space harmonic. This leads to a crucial, nuanced interpretation of SWS electromagnetic modes: each individual electromagnetic mode is the superposition of an infinite number of space harmonics, and each space harmonic is characterized by a unique phase velocity. Therefore, of all the space harmonics that constitute any single electromagnetic mode, only one harmonic can be synchronous with an electron beam characterized by a single, constant velocity. An infinite number of terms in Eq. 2.1 is necessary to represent a discontinuous function (e.g., the electric field at a vane tip) in a Fourier representation. 


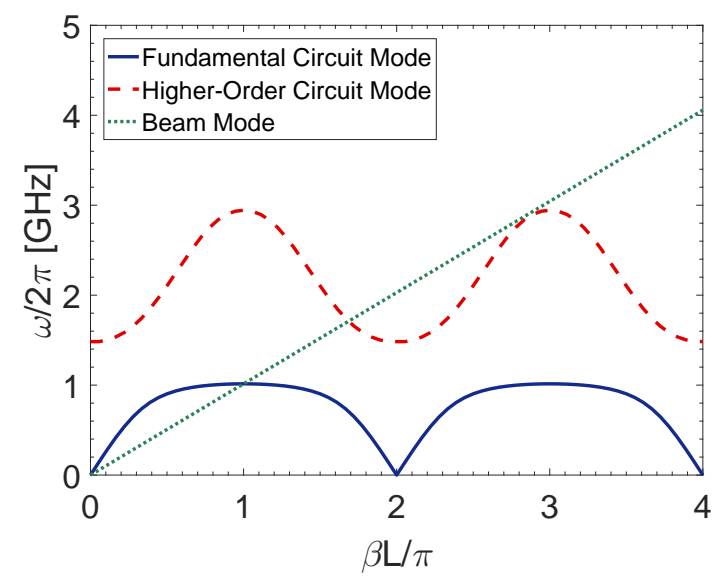

Figure 2.4: Dispersion diagram showing a beam mode and the dispersion relation for the LBO, which includes the fundamental and higher-order circuit mode with space harmonics.

After applying the boundary conditions on the electric and magnetic fields, one obtains an expression $D\left(\omega, \beta_{0}\right)=0$, which is the dispersion relation. An excellent, detailed derivation is written in full in [45] for infinitely thin vane widths, and the interpretations and explanations in this chapter are largely from that work. An analysis of these cavity arrays, also called vane type lines, has also been the subject of many other works [46-50]. The model in [45] was modified by Franzi et al. to account for nonzero vane widths and the slotted cathode used in the single-frequency RPM-12A magnetron, which is derived in detail in $[10,42]$, and will not be repeated here. For infinitely long, two-dimensional, planar cavity arrays having nonzero vane widths (see Fig. 2.7 on page 19 for a drawing), the cold-tube dispersion relation reads

$$
\frac{\cot (\omega h / c)}{(\omega h / c)}=\left(\frac{w}{L}\right) \sum_{n=-\infty}^{\infty} \operatorname{sinc}^{2}\left(\frac{\beta_{n} w}{2}\right) \frac{\operatorname{coth}\left(\gamma_{n} b\right)}{\gamma_{n} h} .
$$

Equation 2.4 uses the geometric parameters from Fig. 2.1a on page 6, where $h$ is the cavity depth, $w$ is the cavity width, $L$ is the circuit pitch, $b$ is the AK gap spacing, $\omega$ is the angular frequency, $c$ is the speed of light, $\beta_{n}=\beta_{0}+2 n \pi / L$ and $\gamma_{n}=\sqrt{\beta_{n}^{2}-(\omega / c)^{2}}$ for $n=0, \pm 1, \pm 2, \ldots$, and $\beta_{0}$ is the propagation constant of an electromagnetic mode.

Figure 2.4 shows an example of a dispersion relation generated from Eq. 2.4 using the dimensions of the LBO $(h=6.3 \mathrm{~cm}, w=1.92 \mathrm{~cm}, L=3.84 \mathrm{~cm}, b=2.6 \mathrm{~cm})$. The dispersion diagram is a very powerful tool for understanding MFRPM operation, and several important features of the plot are noteworthy and will be explained in detail. The horizontal axis corresponds to the space harmonic propagation constant $\beta_{n}$, which is customarily labeled as $\beta$ [45]. For convenience, the vertical axis is in $\mathrm{Hz}$, and the horizontal axis is 
in $\beta L / \pi$ so that the $\pi$-mode corresponds to $\beta L / \pi=1$. The fundamental space harmonic ( $n=0$ in Eq. 2.2) corresponds to $0<\beta L / \pi<2$ in Fig. 2.4. As will be described in the Beam Dynamics section, the $\pi$-mode is typically the desired mode of operation, and corresponds to a phase advance of $180^{\circ}$, or $\pi$ radians, per vane (or, equivalently, per circuit pitch).

Figure 2.4 includes the fundamental circuit mode having fields like those of Figs. 2.2a and $2.2 \mathrm{~b}$ (see page 7), the first higher-order circuit mode having fields like those of Figs. 2.2c and $2.2 \mathrm{~d}$, and a line representing the beam mode characterized by a single, constant velocity. Each point on the horizontal axis corresponds to a different space harmonic of a resonant electromagnetic mode. Recall that each resonant mode is described as having a different phase advance per vane, which was illustrated by Fig. 2.3 on page 8 . The terminology identifying the modes in those figures refers to the phase advance between one cavity and its neighbor, which equals the value of $\beta_{0} L$ in radians. For example, $\beta_{0} L=\pi$ for the $\pi$-mode. Thus, $\beta_{n}=\beta_{0}$ in the region $\beta L / \pi \in[0,2]$, which is the region of the dispersion diagram illustrating the dispersion relation for the fundamental space harmonics. The periodicity of this region arises because each electromagnetic mode is described by the superposition of the different space harmonics, each of which is plotted on the dispersion diagram. For example, the $\pi$-mode consists of the superposition of the space harmonics residing at points $(1,1),(3,1),(5,1), \ldots$ in Fig. 2.4 .

The space harmonics are important because they explain why a magnetron can operate at a much slower beam velocity than would be expected based only on the synchronism condition (discussed in the next section) for the fundamental space harmonic. For example, an electron beam that is synchronous with the $\beta L / \pi=3$ mode is said to be synchronous with the $3 \pi$-mode, even though the electromagnetic fields are the same as the $\pi$-mode. The fields are the same because, as previously stated, the electromagnetic mode of interest (the $\pi$-mode) is the superposition of all the space harmonics. An electron beam that is synchronous with the $3 \pi$-mode is simply another way of stating that the beam is synchronous with the first higher space harmonic of the $\pi$-mode. Physically, the electron beam transits from one cavity to the next at one-third of the normal $\pi$-mode velocity, thereby arriving when the cavity has the same RF phase as if it were traveling at the normal $\pi$-mode velocity, meaning synchronous operation is possible despite the slower electron beam velocity [6]. This could be important because, in the Brillouin flow that prevails in crossed-field devices, the electron velocity may range from zero on the cathode to some peak value at the top of the Brillouin flow. In Fig. 2.4, the plotted beam mode is synchronous with only one space harmonic of the $\pi$-mode of the fundamental circuit mode (namely, the fundamental $\pi$-mode space harmonic corresponding to $n=0$, where $\beta_{n}=\beta_{0}$ ). Visually, the synchronism con- 
dition is satisfied because of the interception between the dispersion relation and the beam mode at the point $(1,1)$.

Nearly all crossed-field devices are designed to operate assuming an electron beam that is synchronous with a fundamental space harmonic of the fundamental circuit mode. The fundamental space harmonics, which reside in $\beta L / \pi \in[0,2]$, are symmetric about $\beta L / \pi=1$. This symmetry corresponds to frequency-degenerate pairs of modes, where the same frequency corresponds to two propagation constants identifying two modes with different phase and group velocities. The portion of the curve having positive slope (and therefore positive group velocity) corresponds to forward-wave modes, whereas the portion of the curve having a negative slope (and therefore negative group velocity) corresponds to backward-wave modes. Most magnetrons are designed to operate in the $\pi$-mode, which has zero group velocity and exists on the structure as a standing wave, whereas amplifiers are designed to operate near a $\pi / 2$ or $3 \pi / 2$ phase advance per vane corresponding to a forwardor backward-wave mode, respectively $[49,51]$. Amplifiers are designed to operate away from the band edges, where group velocities are zero, in order to avoid excitation of band edge oscillations [52]. These principles also apply to other microwave vacuum electronic devices; backward-wave oscillations are important in the design of traveling-wave tubes, for instance $[45,53]$. Near the $\pi$-mode, the fundamental circuit mode is nearly flat, so vane type lines are considered very dispersive. A dispersive structure is problematic because it is highly susceptible to mode competition, which arises due to the small frequency separation between adjacent modes. This can lead to simultaneous excitation of the adjacent modes and extreme subsequent beating in the generated microwave signal.

Since the analytic curves were derived under the assumption of an infinitely long planar cavity array, the curves are continuous. However, because a physical magnetron has a finite number of cavities, the dispersion relation would have individual points on the plot corresponding to the resonances. The reason for the discrete values stems from the need to satisfy the boundary conditions of a non-infinite structure [7]. As an example, for a 6-cavity planar structure like the LBO, the forward-wave fundamental space harmonic of the fundamental circuit mode has resonances that reside at points $(1 / 6,0.42),(2 / 6,0.74)$, $(3 / 6,0.90),(4 / 6,0.98),(5 / 6,1.01)$, and $(6 / 6,1.01)$ on Fig. 2.4 (page 11).

The last noteworthy point illustrated by Fig. 2.4 is that, in addition to the fundamental circuit mode, the beam mode also intercepts the higher-order circuit mode at a point $(f, \beta L / \pi)=(1.67,1.7)$. Thus, the electron beam having a velocity that is synchronous with the $1 \mathrm{GHz} \pi$-mode fundamental space harmonic is theoretically able to also excite the $1.7 \mathrm{GHz}$ resonance on the higher-order circuit mode via a backward-wave interaction. The beam is said to be synchronous with the higher-order $5 \pi / 3$-mode in addition to the 
$\pi$-mode.

\subsection{Electron Dynamics}

Electron motion in crossed-field devices is a complicated subject. Prior to the advent of computer modeling, several analytic models were developed using a variety of approximations to study magnetron operation [7,47-49]. These models are cumbersome and very complicated, or are based on many ad-hoc assumptions. Due to advances in numerical modeling and computational power, computer simulation tools have largely replaced these models in modern magnetron design. However, analytic descriptions of electron dynamics in smoothbore structures endures as an excellent illustration of electron behavior in crossed-field devices, and some of the comparatively simple analytic expressions remain indispensable for generating candidate magnetron designs prior to refinement and optimization using computer simulations. The most notable are the Hull cutoff assumption and the Buneman-Hartree condition, which are addressed in this section.

The resonant structures for the MFRPM are planar, so some understanding of electron behavior can be gained by studying a smoothbore planar magnetron diode like that shown in Fig. 2.5. Simon [38] has explicitly shown that the smoothbore magnetron was an adequate description for the equilibrium state. Still, two models dominate the literature: a

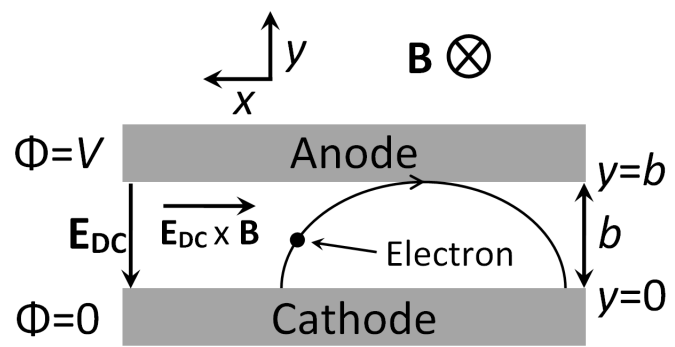

(a) Electric and magnetic field configuration with a single electron orbit at Hull cutoff. Vector quantities are represented by boldface letters.

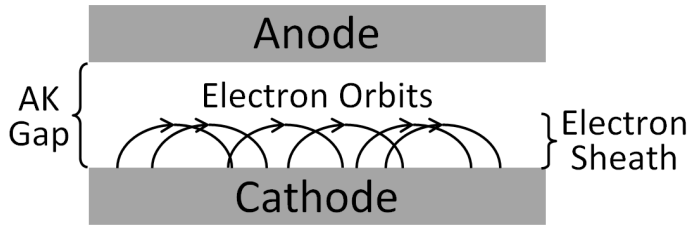

(b) Illustration of the sheath formed from many cycloidal single-particle orbits.

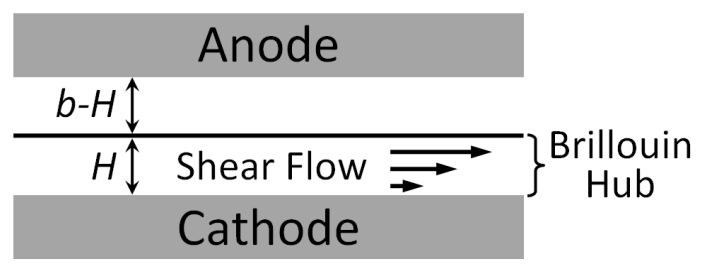

(c) The Brillouin flow model.

Figure 2.5: Particle orbits and analytic models for a planar magnetron diode. 
multistream model based on individual cycloidal particle orbits, and the Brillouin model that idealizes the electron motion as a laminar flow [54]. Full derivations of the following equations in planar and cylindrical smoothbore magnetrons have been published in many textbooks $[6,45,47,54]$ and theses $[10,38,55,56]$, and will not be repeated here. The following discussion instead focuses on the relevance of the results as they apply to the design of the MFRPM and the interpretation of the experimental results.

In Fig. 2.5, the cathode is the electron source, and is held at zero electric potential $\Phi(y=0)=0$ with respect to the anode potential $\Phi(y=b)=V$, which produces the constant electric field $\vec{E}_{\mathrm{dc}}$. An axial magnetic field $\vec{B}$ (into the page) is also imposed on the structure. Crossed-field devices are named for this orthogonality between the electric and magnetic field.

An electron emitted from the cathode is accelerated by the DC electric field and deflected by the magnetic field. If the magnetic field is sufficiently high, the electron will graze the anode before its inertia carries it back to the cathode, as illustrated by Fig. 2.5a. This magnetic field is called the Hull cutoff $\left(B_{\mathrm{HC}}\right)$, and it represents the critical field for magnetic insulation. Provided that $B>B_{\mathrm{HC}}$, no current will flow across the diode (in a strictly one-dimensional model). Equation 2.5 shows the non-relativistic expression for $B_{\mathrm{HC}}$.

$$
\begin{gathered}
B_{\mathrm{HC}}=\sqrt{\frac{2 m_{0} V}{|e| b^{2}}}, \\
V_{\mathrm{HC}}=\frac{1}{2} \frac{|e|}{m_{0}} B^{2} b^{2} .
\end{gathered}
$$

For a fixed magnetic field, there exists a critical voltage for magnetic insulation below which no current will flow. The non-relativistic expression for the Hull cutoff voltage $V_{\mathrm{HC}}$ is shown by Eq. 2.6. In these expressions, $m_{0}$ is the electron mass, $e$ is the elementary charge, and the other quantities remain consistent with their previous definitions. Equations 2.5 and 2.6 are valid even when a DC space charge is present in the gap (see, e.g., [57]).

Figure $2.5 \mathrm{~b}$ illustrates the cycloidal orbits of several electrons at a magnetic field $B>$ $B_{\mathrm{HC}}$. The drift velocity is given by $\left|v_{D}\right|=\left|\vec{E}_{\mathrm{dc}} \times \vec{B}\right| / B^{2}=E_{\mathrm{dc}} / B \propto V / B$ [58]. With a large electron population, an electron sheath takes shape. In the Brillouin flow model depicted in Fig. 2.5c, the sheath is treated as a shear flow, and is also commonly called the Brillouin hub. Electrons in the hub undergo rectilinear motion with a velocity equal to the $\vec{E} \times \vec{B}$ drift, where $\vec{E}$ now includes both the DC electric field and the self electric field of the hub electrons. Thus, describing the electron "beam" as having a single velocity is a significant simplification, and should be kept in mind when discussing virtually any 
aspect of crossed-field device operation. Between the cycloidal flow model (Fig. 2.5b) and the Brillouin flow model (Fig. 2.5c), the Brillouin flow is the preferred state according to extensive simulations [38,59-61].

Equation 2.7 gives the expression for the Brillouin hub height, which depends on both the voltage and the magnetic field. When the operating voltage $V$ is small relative to $V_{\mathrm{HC}}$, the hub height increases linearly with voltage and decreases as $1 / B^{2}$ as $B$ is increased [54]. Thus, lower magnetic fields bring the edge of the electron hub closer to the anode and increase the electron drift velocities.

$$
H=b\left[1-\sqrt{1-\frac{V}{V_{\mathrm{HC}}}}\right] .
$$

If an RF wave is traveling on the anode, electrons must match its phase velocity to undergo the desired crossed-field interaction, which will be described shortly. This requirement is called the synchronism condition, and it provides the critical relation between the voltage, magnetic field, AK gap size, and RF phase velocity. This synchronism condition is known as the Buneman-Hartree condition [7,57]. While the synchronism condition can be derived using both a single-particle formulation and the Brillouin model, a recent study suggested the latter model showed better agreement with experiments [57]. Another reinforced this result by showing improved agreement for inverted magnetrons [38]. Nonetheless, the synchronism condition derived using the single-particle formulation, which is called the Buneman-Hartree condition, continues to endure in the magnetron literature due to its convenience and simplicity. In the planar geometry of interest in this dissertation, the synchronism condition is the same for both the single-particle and Brillouin flow models [57]. Equation 2.8 shows this expression, which also gives the theoretical minimum voltage required for interaction between the electron beam and the RF wave on a SWS. It should be noted that, in the Brillouin flow formulation, the shear flow layer that is synchronous with the RF is the top layer of the electron hub, which lies closest to the SWS.

$$
V_{\mathrm{BH}}=b B v_{p}-\frac{m_{0}}{2|e|} v_{p}^{2} .
$$

Most derivations of the synchronism condition in a planar geometry do not account for relativistic effects. If these are taken into account, the modified Hull cutoff voltage, Hull cutoff magnetic field, Buneman-Hartree voltage, and Buneman-Hartree magnetic field become Eqs. 2.9-2.12, where $\beta_{p}$ is the $c$-normalized RF phase velocity $\left(\beta_{p}=v_{p} / c=\right.$ $\omega / \beta c$ ), where $c$ is the speed of light, and the other parameters are the same as previously 


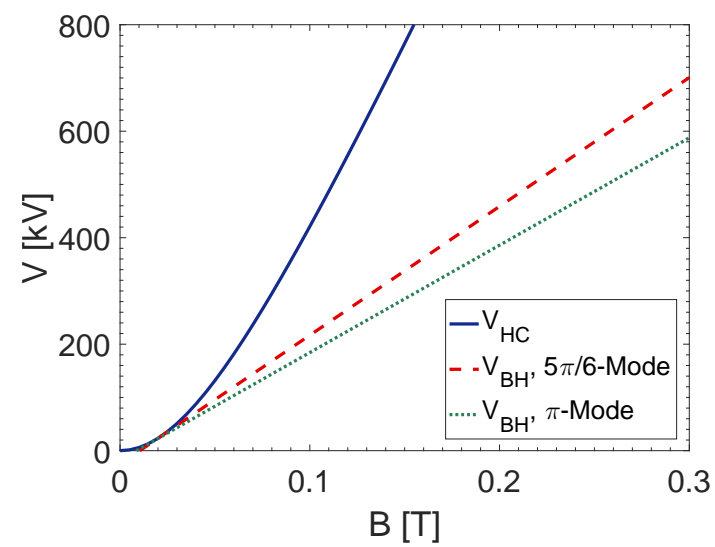

Figure 2.6: Hartree diagram illustrating the Hull cutoff and threshold voltages for the LBO $5 \pi / 6$-mode and $\pi$-mode.

defined.

$$
\begin{gathered}
V_{\mathrm{HC}}=\sqrt{(B b c)^{2}+\left(\frac{m_{0} c^{2}}{|e|}\right)^{2}}-\frac{m_{0} c^{2}}{|e|}, \\
B_{\mathrm{HC}}=\left(\frac{m_{0} c}{|e| b}\right) \sqrt{\frac{2|e| V}{m_{0} c^{2}}+\left(\frac{|e| V}{m_{0} c^{2}}\right)^{2}}, \\
V_{\mathrm{BH}}=b B \beta_{p} c-\left(1-\sqrt{1-\beta_{p}^{2}}\right) \frac{m_{0} c^{2}}{|e|}, \\
B_{\mathrm{BH}}=\left(\frac{1}{b \beta_{p} c}\right)\left(V+\frac{m_{0} c^{2}}{|e|}\left(1-\sqrt{1-\beta_{p}^{2}}\right)\right) .
\end{gathered}
$$

Equations 2.9-2.12 are derived from the single-particle orbit theory, and are valid for cylindrical geometry as well, in which case $b$ is replaced by $\left(r_{a}^{2}-r_{c}^{2}\right) / 2 r_{a}$, where $r_{a}$ is the anode radius and $r_{c}$ is the cathode radius [57]. For this dissertation, the operating voltage of the magnetron is approximately constant, so Eq. 2.12 is the most relevant for comparison with experimental results. The experimental parameters (described in Chapter 4) are: $b=$ $2.6 \mathrm{~cm}, \beta_{p}=0.26$ for the $\pi$-mode, and $V=300 \mathrm{kV}$. From Eq. 2.12, the Buneman-Hartree condition for synchronism predicts a magnetic field $B_{\mathrm{BH}}=0.16 \mathrm{~T}$. Thus, near this magnetic field, both MFRPM oscillators should operate in the $\pi$-mode.

Figure 2.6 shows a Hartree diagram (which customarily includes the Hull cutoff), which shows the threshold voltages for various modes. In the plot, the LBO threshold voltages for the $5 \pi / 6$-mode and $\pi$-mode are shown. The theory predicts that operation in a given mode can only occur if the voltage $V \geq V_{\mathrm{BH}}$, so the region above a given threshold volt- 
age curve is theoretically capable of oscillation in the mode corresponding to the curve. The region above the Hull cutoff curve is purely conducting because the magnetic field is too low to maintain insulation, so magnetrons must operate below the Hull cutoff and theoretically above the Hartree threshold. Since these curves were produced using several approximations, exact quantitative agreement with experiments is rare, but the qualitative predictions are usually accurate. Using the LBO operating at $300 \mathrm{kV}$ as an example, the $5 \pi / 6$-mode should be observed at lower magnetic fields than the $\pi$-mode because the $5 \pi / 6$ mode threshold magnetic field ( $B=0.13 \mathrm{~T}$ ) is lower than the $\pi$-mode threshold magnetic field ( $B=0.16 \mathrm{~T})$. Similar reasoning applies to the SBO as well, where the threshold magnetic field for the $6 \pi / 8$-mode is lower than the $7 \pi / 8$-mode, which is lower than the $\pi$-mode threshold. This interpretation assumes the most synchronous mode will be the dominant mode of oscillation, although theoretically, any mode for which $V \geq V_{\mathrm{BH}}$ is satisfied is capable of oscillation. For instance, at $B=0.12 \mathrm{~T}$ and $V=300 \mathrm{kV}$, both the $5 \pi / 6$-mode and $\pi$-mode are possible modes of operation, but the beam velocity is closer to the $5 \pi / 6$ mode threshold than the $\pi$-mode threshold; the $5 \pi / 6$-mode would be the expected mode of operation.

Figure 2.7 illustrates the crossed-field interaction that leads to the phase focusing of the electrons, which is an effect that is intrinsic to crossed-field devices [54,62]. It is the phase focusing effect that leads to the very high efficiencies of magnetrons and crossedfield amplifiers. Figure 2.7a shows the RF electric field of a planar cavity array operating in the $\pi$-mode. In the moving frame of the electrons, which propagate to the right due to the $\vec{E}_{\mathrm{DC}} \times \vec{B}$ drift velocity from the voltage imposed between the anode and cathode, the RF electric fields appear to be approximately constant because the electron velocity is matched to the RF phase velocity. In this moving frame, the effect of the RF fields on electron motion can be understood by determining how the single-particle drift velocity is modified from the usual $\vec{E}_{\mathrm{DC}} \times \vec{B}$ drift velocity in the Brillouin hub. Since the RF electric field is superposed on the DC electric field, the drift velocity component due to the RF can be determined by finding $\vec{E}_{\mathrm{RF}} \times \vec{B}$. Figure $2.7 \mathrm{~b}$ shows these drift velocity components in different locations at the same snapshot in time as Fig. 2.7a. The net effect on electron motion is shown in Fig. 2.7c. As the electrons propagate rightward in the figures due to the DC electric field drift component, the RF drift component draws only those electrons that are in a favorable phase to the anode. Electrons in an unfavorable phase are returned to the cathode or migrated to the favorable phase regions, thus producing a void of electrons in the unfavorable phase. The result is the formation of electron spokes, which are a form of beam modulation. One notable consequence of the moving space charge of the modulated electron beam is the induction of currents on the anode having harmonic frequency content. 


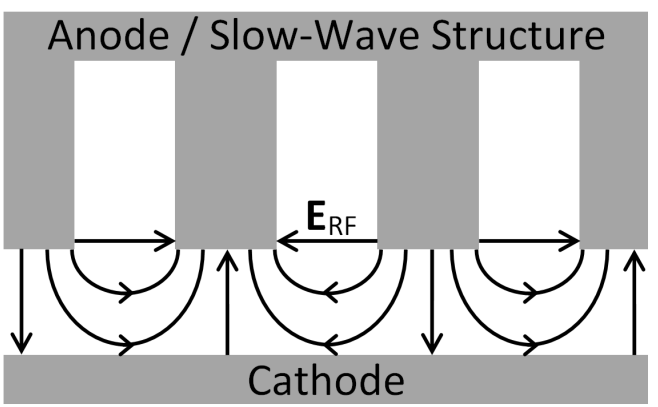

(a) Cavity $\pi$-mode RF electric field.
$B \otimes$

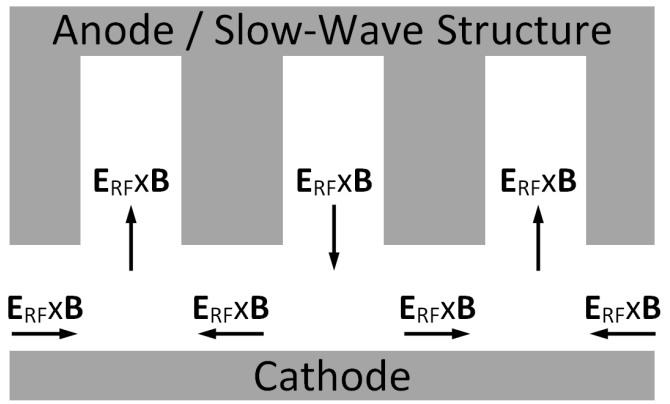

(b) $\mathrm{RF} \vec{E} \times \vec{B}$ electron drift velocity vectors.

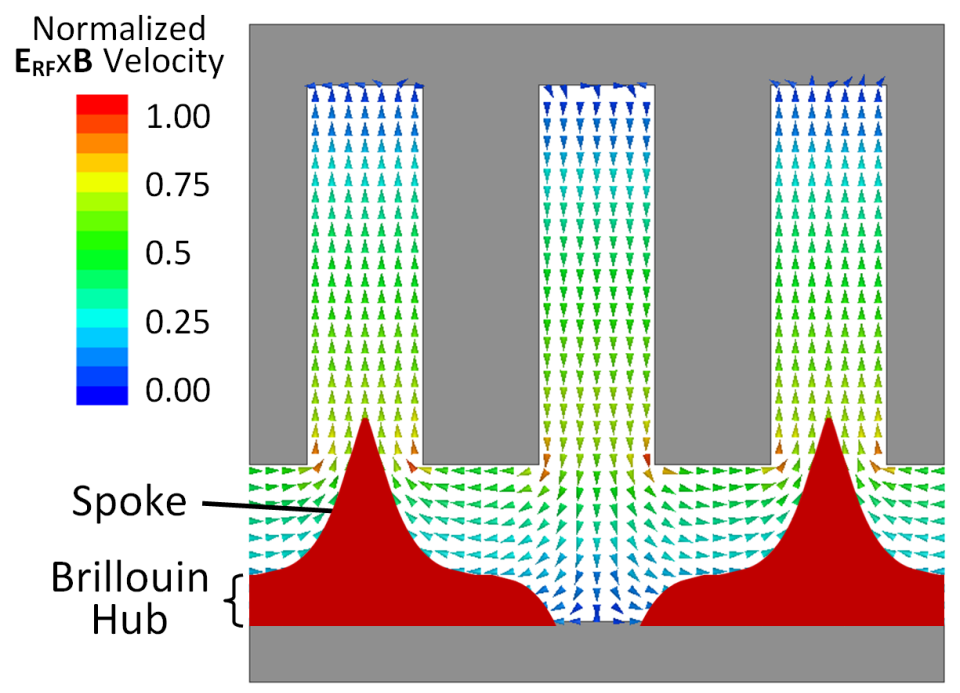

(c) RF $\vec{E} \times \vec{B}$ drift velocities and phase focusing of the electrons leading to spoke formation.

Figure 2.7: The $\pi$-mode crossed-field interaction leading to phase focusing of the electrons.

Later in the chapter, $\S 2.3$ explores whether the second harmonic of the LBO, which has a frequency near $2 \mathrm{GHz}$, can injection-lock the SBO, which also has a frequency near $2 \mathrm{GHz}$. The harmonic content of electron beams has been the focus of study in many microwave vacuum electron devices, such as klystrons [63] and TWTAs [64,65]. Another consequence is the gradual reduction in magnetron efficiency as the current density is increased due to the energy invested in focusing the electrons by overcoming their electrostatic repulsion. This is part of the reason that relativistic magnetrons, which operate at extremely high currents, have lower efficiencies than conventional magnetrons [66].

Due to phase focusing, in-phase electrons migrating to the anode propagate with a drift velocity that is approximately constant and equal to the RF phase velocity (neglecting the drift velocity contribution of the RF electric field to the overall speed). From an energy standpoint, the kinetic energy invested in the electrons to maintain synchronism with the 


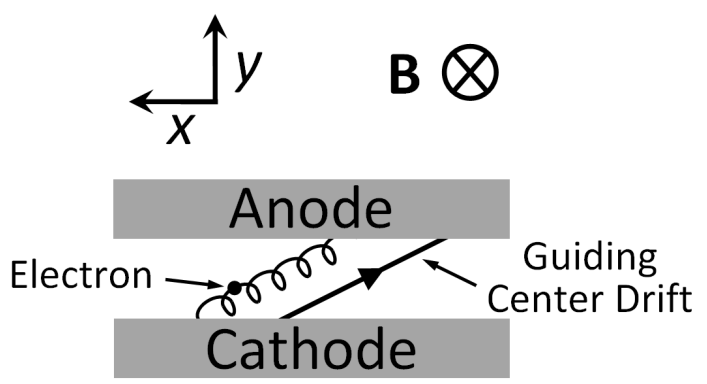

(a) Electron motion toward the anode.

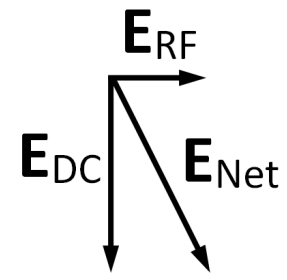

Electric Field

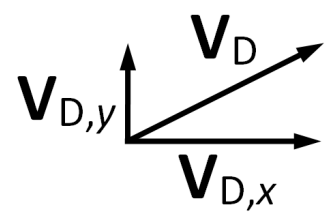

Drift Velocity

(b) Electric field and guiding center velocity vectors and components.

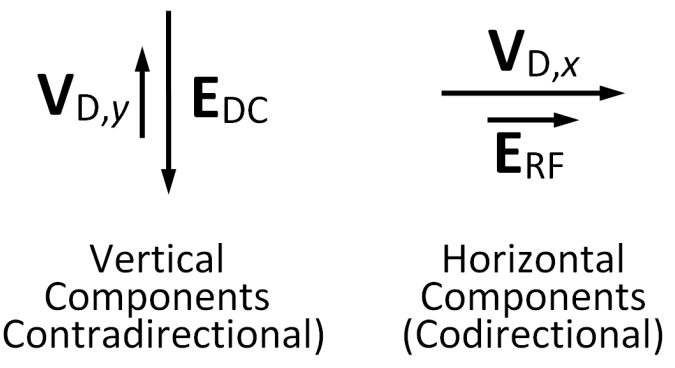

(c) Comparison of vector components.

Figure 2.8: Qualitative illustration of the fundamental energy transfer mechanism in crossed-field devices.

$\mathrm{RF}$ wave is unavailable for transfer to microwave energy, so some fraction of the AK gap potential is intrinsically lost in the crossed-field interaction. This potential is called the synchronous voltage $V_{0}$, and is found from Eq. 2.13. Thus, the remaining potential represents the theoretical maximum efficiency of the magnetron, which is given by $\eta_{e}=1-V_{0} / V$, where $V$ is the AK gap voltage $[6,48]$. For many magnetrons, the theoretical maximum efficiency exceeds $95 \%$. In actuality, electrons typically impact the anode with far greater energy than $e V_{0}$, and there are many other practical constraints that limit magnetron efficiency.

$$
e V_{0}=\frac{1}{2} m_{0} v_{p}^{2}
$$

Fundamentally, for the crossed-field interaction to transfer energy to the RF electric field, the electrons must do work on the field by undergoing motion against it, where some component of the RF electric force on the electrons must oppose their velocity. Figure 2.8a shows the net electric field vector and net electron drift velocity vector for the favorable phase. Vector lengths assume the RF electric field amplitude is half that of the DC electric field amplitude, which is consistent with most simulation results for the MFRPM. These vectors are broken into vertical and horizontal components. Figure $2.8 \mathrm{c}$ shows that, in the 
favorable phase, the vertical drift velocity direction is opposite that of the vertical electric field component. Because the vertical electric field component arises from the voltage across the diode, the DC voltage does work on the electron as it moves toward the anode. The horizontal drift velocity direction is the same as RF electric field, so the electron does work on the RF as it moves toward the anode, which is the fundamental origin of the energy transfer to the microwaves. However, as previously described, the average electron velocity remains approximately constant. Thus, it is the guiding center motion arising from the single-particle cycloidal drifts in the favorable phase that leads to the transfer of energy from the DC voltage potential to the RF field, which is an energy transfer mechanism that is unique to crossed-field devices. This explanation provides a qualitative description; however, a rigorous theory for energy transfer between the RF electric field and the kinetic and potential energy of the electrons remains an open problem for crossed-field devices. In all other microwave vacuum electronic devices, electrons are first accelerated to high velocity before doing work on microwave fields, thereby converting kinetic energy to microwave energy [54].

\subsection{Locking of Harmonic Frequencies}

As described in Chapter 3, the MFRPM was designed using two planar cavity arrays having operating frequencies near $1 \mathrm{GHz}$ (the $\mathrm{LBO}$ ) and $2 \mathrm{GHz}$ (the SBO). However, due to differences in their coupling to the microwave extractor and the variable effects of beamloading, it was unlikely that their operating frequencies would be related exactly by a factor of 2. However, the spoke current in the LBO may contain a second harmonic component near $2 \mathrm{GHz}$. The spoke transits the cylindrical bend and enters the SBO, where the $2 \mathrm{GHz}$ harmonic content of the current might be sufficient to frequency-lock the $2 \mathrm{GHz}$ SBO. In an attempt to understand the conditions under which such a locking might occur between the oscillators, this section presents a new theory adapted by Y. Y. Lau [43] from a previous magnetron injection-locking model developed by Pengvanich et al. for magnetrons of the same frequency, which has been experimentally verified under various conditions [67-71].

The new model treats the $2 \mathrm{GHz} \mathrm{SBO}$ as an oscillator driven at a frequency harmonic of the LBO. This assumption was based on many experimental observations discussed at length in Chapter 5. Briefly, the key experimental results leading to this model include 1) the substantially higher powers observed from the $\mathrm{LBO}$ relative to the SBO, and 2) the relatively minor change in the LBO operating frequency between the unlocked and locked states, whereas the SBO operating frequency changed considerably. Because this behavior appears to be analogous to Adler master-slave injection locking [72], the second harmonic 


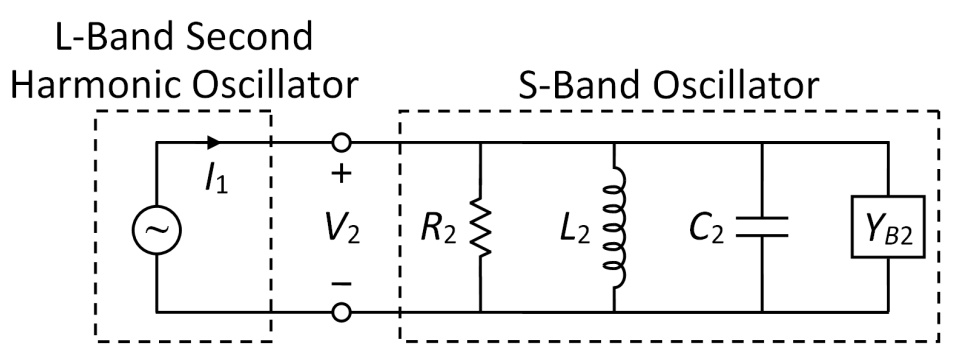

Figure 2.9: Circuit model for the SBO subject to an external driver, which models the second harmonic content of the LBO.

of the LBO is treated as the master oscillator and the SBO as the slave oscillator.

Figure 2.9 shows the basic circuit model used to represent the SBO, which consists of three components. The first is the $R-L-C$ oscillator, which adds a parallel loss element to the lossless circuit equivalent for a microwave cavity shown in Fig. 2.1b (page 6) earlier in the chapter. In that figure, resistive losses would naturally be incorporated using a series resistance. However, it is desirable to transform the series resistance to a parallel shunt resistance because 1) it is analytically more convenient, and 2) using the shunt resistance $R$, the ratio $R / Q$, also called the characteristic impedance, can be used to describe the microwave cavity independent of losses because the ratio is a figure of merit that depends only on the cavity geometry $[6,73]$. Thus, the parallel $R-L-C$ circuit can be used to represent the single-mode cold-tube magnetron characteristics, and is a standard approximation for magnetrons in circuit models [74]. Because the model only includes a single $R-L-C$ component, the theory focuses on only one mode of oscillation and ignores much of the multi-mode complexity described earlier.

The second component is the beam-loaded admittance $Y_{B 2}$. This quantity accounts for the hot-tube magnetron characteristics, which includes the magnetron gain mechanism, the DC- and RF-electron interactions, and the effects of beam-loading [48, 67, 69, 74, 75]. Empirical expressions for the conductance and susceptance are used to define the beamloaded admittance [74].

The third model component is the driving signal from the LBO. In most experimental applications of this model, the driving signal was injected to the magnetron oscillator via the magnetron's power extractor, which provides direct coupling to the oscillator cavities. However, in the MFRPM, the coupling between the oscillators occurs within the magnetron, and is thought to occur through a combination of the modulated electron beam recirculating between the oscillators and feedthrough of the electromagnetic fields through a slotted cathode. The simple driving term in the circuit model therefore represents perhaps the most significant simplification of the experimental configuration. 
Referring to Fig. 2.9, current conservation on the circuit yields Eq. 2.14, where the left-hand side represents the SBO and the right-hand side is the LBO driving term,

$$
\frac{V_{2}}{R_{2}}+\frac{V_{2}}{j \omega L_{2}}+j \omega C_{2} V_{2}+Y_{B 2} V_{2}=I_{1}
$$

where $I_{1}$ represents the second harmonic current from the LBO. When the external LBO driver is removed $\left(I_{1}=0\right)$ and no SBO electron beam is present $\left(Y_{B 2}=0\right)$, the cold-tube characteristics for the $R-L-C$ oscillator model are related by Eq. 2.15, where $\omega_{02}$ is the natural, free-running angular frequency of the SBO mode, $Q_{02}$ is the cold quality factor, and $Z_{2}$ is the characteristic impedance.

$$
\text { Cold-Tube }\left\{\begin{aligned}
\omega_{02} & =\sqrt{\frac{1}{L_{2} C_{2}}} \\
Q_{02} & =\omega_{02} R_{2} C_{2} \\
Z_{2} & =\sqrt{\frac{L_{2}}{C_{2}}}=\frac{R_{2}}{Q_{02}}
\end{aligned}\right.
$$

When the external LBO driver is removed and the beam-loaded admittance $Y_{B 2}$ is added (Fig. 2.9), the hot-tube SBO characteristics become the hot free-running frequency $\omega_{2}$, hot $Q_{2}$, and free-running voltage amplitude $V_{02}$. The circuit equation (2.14) is modified. Rearranging, it reads,

$$
(j \omega)^{2} V_{2}+\left(\frac{1}{R_{2} C_{2}}\right)\left(1+R_{2} Y_{B 2}\right)(j \omega) V_{2}+\frac{1}{L_{2} C_{2}} V_{2}=j \omega I_{1} .
$$

Equation 2.16 is then rewritten using the time-domain representation. The relations $1 / R_{2} C_{2}=\omega_{2} / Q_{2}$ and $\omega_{2}^{2}=1 / L_{2} C_{2}$ are used for substitutions, and the beam-loaded admittance is replaced through simplifications of the empirical conductance and susceptance [69]. If $\omega_{1}$ is the free-running frequency of the LBO, and $V_{01}$ is the free-running complex amplitude, the second harmonic complex amplitude and phase must be proportional to $V_{01}^{2} e^{j 2 \omega_{1} t}$. In a frequency-locked state, the SBO locks to twice the frequency of the LBO, so $\omega_{2} \simeq 2 \omega_{1}$ and the driving term on the right-hand side is assumed to be proportional to the LBO second harmonic. Collecting constants using a complex constant $k$, Eq. 2.16 becomes Eq. 2.17, which is the governing equation for master-slave oscillators with a harmonic frequency driving term.

$$
\frac{d^{2} V_{2}}{d t^{2}}+\frac{\omega_{2}}{Q_{2}}\left[1-\frac{\left|V_{2}\right|}{V_{02}}\right] \frac{d V_{2}}{d t}+\omega_{2}^{2} V_{2}=k V_{01}^{2} e^{j 2 \omega_{1} t}
$$

Equation 2.17 explicitly models the locking of the free-running $\mathrm{SBO}\left(V_{2}\right)$ by the second 
harmonic current of the LBO [43].

Under zero-drive conditions, where the right-hand side of Eq. 2.17 is zero, a solution is $V_{2}(t)=V_{02} e^{j \omega_{2} t}$, as should be the case for the free-running SBO. As in Adler's theory of locking between oscillators in a master-slave configuration, a solution should take the form of Eq. 2.18, where $|A|$ and $\phi$ are real constants.

$$
V_{2}(t)=|A| e^{j\left(2 \omega_{1} t\right)+j \phi}
$$

Equation 2.18 is constructed so the SBO voltage $V_{2}(t)$ oscillates exactly at a frequency $2 \omega_{1}$ and has a constant phase $\phi$ with respect to the LBO second harmonic drive, which is represented by the right-hand side of Eq. 2.17. Without a loss of generality, the quantity $k V_{01}^{2}$ is assumed to be real and positive. Substituting Eq. 2.18 into Eq. 2.17,

$$
\left\{\left(j 2 \omega_{1}\right)^{2}+\frac{\omega_{2}}{Q_{2}}\left[1-\frac{|A|}{V_{02}}\right] j 2 \omega_{1}+\omega_{2}^{2}\right\}|A| e^{j 2 \omega_{1} t+j \phi}=k V_{01}^{2} e^{j 2 \omega_{1} t}
$$

Rearranging,

$$
\begin{aligned}
-\left(2 \omega_{1}\right)^{2}+\frac{\omega_{2}}{Q_{2}}\left[1-\frac{|A|}{V_{02}}\right] j 2 \omega_{1}+\omega_{2}^{2} & =\frac{k V_{01}^{2}}{|A|} e^{-j \phi} \\
& =\frac{k V_{01}^{2}}{|A|}(\cos \phi-j \sin \phi) .
\end{aligned}
$$

Equating the real parts of Eq. 2.20,

$$
\begin{aligned}
& -\left(2 \omega_{1}\right)^{2}+\omega_{2}^{2}=\frac{k V_{01}^{2}}{|A|} \cos \phi \\
& \Rightarrow\left(\omega_{2}-2 \omega_{1}\right)=\frac{k V_{01}^{2}}{|A|\left(\omega_{2}+2 \omega_{1}\right)} \cos \phi \simeq \frac{k V_{01}^{2} \cos \phi}{2 \omega_{2} V_{02}},
\end{aligned}
$$

since $\omega_{2} \simeq 2 \omega_{1}$ and $|A| \simeq V_{02}$. Equation 2.21 has a real solution for $\phi$ if and only if

$$
\left|\omega_{2}-2 \omega_{1}\right|<\frac{k V_{01}^{2}}{2 \omega_{2} V_{02}} .
$$

The locking condition in Eq. 2.22 is similar in form to Adler's condition for injection locking [72]. Since $V_{01}^{2} \propto P_{1}$, where $P_{1}$ is the power of the free-running LBO at frequency $\omega_{1} / 2 \pi$, and $V_{02}^{2} \propto P_{2}$, where $P_{2}$ is the power of the free-running SBO at frequency $\omega_{2} / 2 \pi$, 
the locking condition may be rewritten as [43]

$$
\begin{array}{r}
\left|\omega_{2}-2 \omega_{1}\right|<C \frac{P_{1}}{\sqrt{P_{2}}} \\
\Rightarrow\left|\omega_{2}-2 \omega_{1}\right| \frac{\sqrt{P_{2}}}{P_{1}}=\kappa<C .
\end{array}
$$

Equation 2.23 says that the left-hand side of the inequality, denoted by $\kappa$, must be less than some critical constant for locking to occur. Thus, for a range of experimental conditions, the locking parameter $\kappa$ can be evaluated for comparison to this analytic model. By varying the value of the locking parameter using the powers and/or frequencies, an experiment would be expected to identify a specific value for $\kappa$ above which locking was not observed, and below which locking was observed. This value represents the threshold constant $C$ in the theory. 


\section{CHAPTER 3}

\section{Design of the MFRPM}

This chapter describes the design of the Multi-Frequency Recirculating Planar Magnetron (MFRPM), and is organized into three sections. The first section provides an overview of the design criteria and practical constraints introduced by the laboratory equipment and the use of existing components. The second section explains the application of the theory from Chapter 2 to produce anode geometries meeting the aforementioned criteria. The third section discusses cold-tube and hot-tube simulations of the MFRPM, which were used to refine the theory-based design and provided a framework to interpret and understand the experimental results reported in Chapters 5 and 6.

\subsection{Design Criteria and Constraints}

The MFRPM project sought to fabricate a magnetron that demonstrated simultaneous HPM generation at two different frequencies [14]. The first Recirculating Planar Magnetron (RPM) prototype, termed the RPM-12A, was designed for operation at a single frequency near $1 \mathrm{GHz}[10]$. Based on the understanding gained from RPM-12A experiments and simulations $[39,40,42]$, the decision was made to reuse the existing $1 \mathrm{GHz}$ planar cavity array as one of the two oscillators in the MFRPM. A schematic drawing of the MFRPM is shown in Fig. 3.1. The cylindrical electron recirculation bends, which coupled the two planar cavity arrays, were also slated for reuse in the MFRPM. Motivated by the frequency-locked operation in the RPM-12A [40] promoted by the ModeControl Cathode (MCC) [42], and by extensive previous research into magnetron phaselocking $[13,67-70,75,76]$, the frequency of the second oscillator was chosen to be $2 \mathrm{GHz}$, which is the second harmonic of the repurposed RPM-12A structure. By choosing a harmonic of the $1 \mathrm{GHz}$ structure, it was hoped that a similar locking phenomenon would be observed in the MFRPM. Thus, the MFRPM design consisted of a planar cavity array at $1 \mathrm{GHz}$, termed the L-Band Oscillator (LBO), and another planar cavity array at $2 \mathrm{GHz}$, 


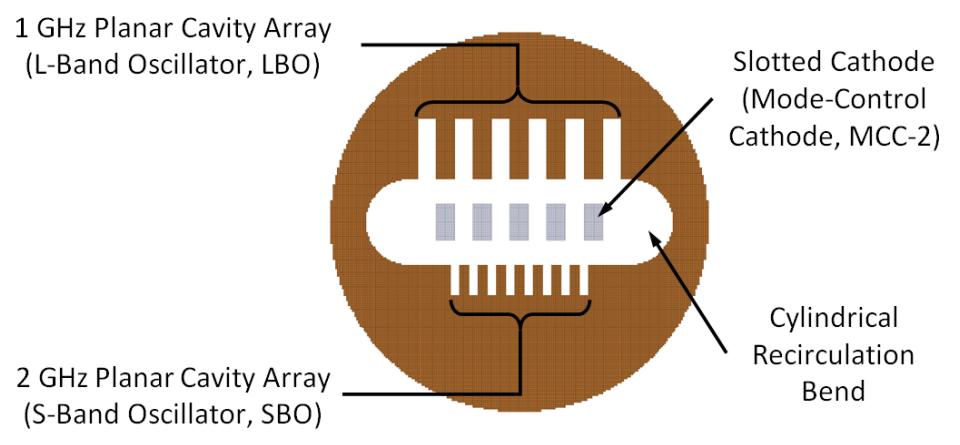

Figure 3.1: Basic components of the MFRPM.

termed the S-Band Oscillator (SBO), which are connected by two cylindrical recirculation bends (Fig. 3.1).

A full discussion of the anode dimensions and photographs of the hardware (Fig. 4.2) can be found on page 41 of Chapter 4 . The axial length of the RPM-12A anode was $11 \mathrm{~cm}$, which was less than half the $30 \mathrm{~cm}$ free-space $1 \mathrm{GHz}$ wavelength in order to impede axial mode formation. However, in order to reuse the RPM-12A components in the MFRPM, the SBO also needed to be $11 \mathrm{~cm}$ long. Magnetron design typically calls for anode lengths that are less than a full free-space wavelength to avoid mode competition [3]; the original RPM-12A design was therefore very conservative. For the SBO, the $11 \mathrm{~cm}$ length remains less than the $15 \mathrm{~cm} 2 \mathrm{GHz}$ free-space wavelength, so axial mode formation was not expected. Simulations (discussed later in this chapter) also suggested the risk of axial mode competition was minimal. Thus, the original anode length was preserved to avoid modifying the existing hardware.

As described in greater detail in Chapter 4, the pulsed-power source used to drive the magnetron had a fixed output voltage approximately equal to $-300 \mathrm{kV}$, and operates well using 100-150 $\Omega$ loads. In order to achieve approximately uniform electron emission from both sides of the cathode, and to avoid having one oscillator pulse-shorten faster than the other due to diode plasma closure, a uniform Anode-Cathode Gap (AK gap) distance was chosen for both planar cavity arrays (Fig. 3.1). The magnetic field is the same for both structures, so identical AK gaps produce identical electron beam drift velocities past the oscillators. This fixed electron beam velocity therefore constrained the RF phase velocity of the SBO to be the same as the LBO, which was 0.26 c. Like the LBO, the SBO was also designed to operate in the $\pi$-mode.

The previous success of the second MCC variant, which was termed the MCC-2 and used with the RPM-12A, motivated its reuse with the MFRPM. However, several modifications were made to control the amount of emitted current and meet the pulsed-power 
source specifications. These modifications are described later in Chapter 4. The MCC-2 design constrained the AK gap to $2.6 \mathrm{~cm}$, which was approximately 1.35 and 2.7 times the cavity widths of the LBO and SBO, respectively. The fixed operating voltage, RF phase velocity, and AK gap constrained the axial magnetic field to approximately $0.16 \mathrm{~T}$ based on the relativistic Buneman-Hartree condition discussed in Chapter 2.

The theory chapter remarked that the decay of the cavity fringing fields in the AK gap depended on the cavity widths. The strength of the interaction between the cavity fields and the electron beam depends on the AK gap size relative to the cavity width, among other factors. Simulations suggested that the SBO operated well despite the narrower SBO cavities. The MCC-2 was therefore suitable for use with the MFRPM.

As outlined in the previous theory chapter, the risk of mode competition in the highly dispersive planar cavity arrays increases with the number of cavities. Thus, the number of SBO cavities was kept to a minimum. If the SBO locks to the LBO via a similar mechanism as was observed in the RPM-12A, the SBO circuit length should be equal to the cathode width to allow feedthrough of the RF fields between the oscillators. By choosing the MCC-2 as the cathode, the number of cavities was functionally fixed because the choice of frequency and phase velocity fixed the circuit pitch. If the pitch and circuit length are fixed, the number of cavities is therefore also fixed. Figure 3.1 illustrates this idea. The eight SBO cavities are just long enough to span all the slots of the MCC-2. While additional cavities could be added to the SBO, there would likely be no benefit due to the greater susceptibility to mode competition and the absence of all-cavity power extraction. To constrain the investigated parameter space (and to remain consistent with the original RPM-12A design), the SBO vane widths and cavity widths were equal.

In addition to the LBO and RPM-12A recirculation bends, several other experimental components were reused for the MFRPM experiment. Most notable were the electromagnet coils and the vacuum chamber, which placed significant constraints on the feasibility of any microwave power extraction assembly. The geometry of an RPM makes it favorable for a coaxial, all-cavity extraction technique [10] based on an all-cavity extractor used in cylindrical magnetrons $[77,78]$. However, due to the vacuum chamber and electromagnet coils, this promising extraction approach was impossible given the choice of operating frequencies and the reuse of existing RPM components. Thus, a simple axial extraction technique, which was used for the RPM-12A [40], was modified for use with the MFRPM. The approach was motivated by the same method used in household microwave oven magnetrons, which uses an antenna connected directly to a vane to couple microwaves into a coaxial transmission line [79].

Figure 3.2 shows a top sectional view of the MFRPM, the extraction antennas, and the 


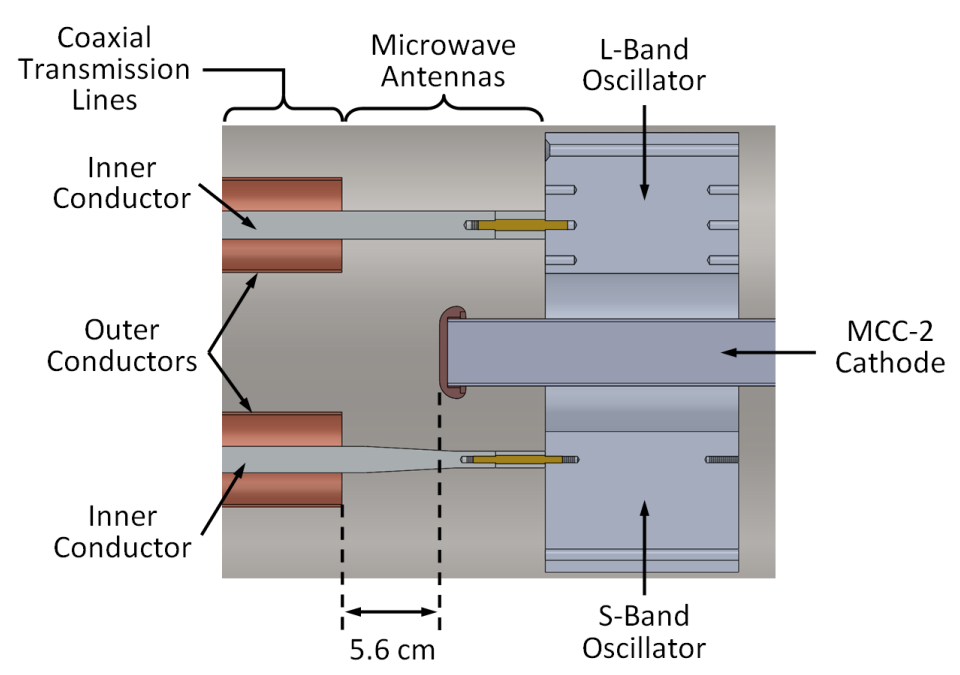

Figure 3.2: Top sectional view of the microwave extractor showing the distance between the outer conductor of the coaxial transmission lines and the cathode endcap.

coaxial transmission lines. In the $1 \mathrm{GHz}$ RPM-12A, the separation length between the outer conductor of the coaxial lines and the oscillators was optimized using the commercial simulation code HFSS to provide strong coupling of the $\pi$-mode to the extractor. The design converged on a distance approximately equal to $\lambda / 2$, where $\lambda$ is the wavelength. The optimization was effective as evidenced by the powers and efficiencies demonstrated in experiment [40]. Obtaining similarly good coupling between the SBO and the extractor would require lengthening the outer conductor of the coaxial line to reduce the distance from the SBO. However, Fig. 3.2 shows that this would bring the outer conductor unacceptably close to the cathode endcap by reducing the original $5.6 \mathrm{~cm}$ separation, which would substantially increase the risk of an arc forming between the components during the voltage pulse. Thus, the separation was kept the same, which approximately corresponds to a full wavelength at the $2 \mathrm{GHz}$ SBO frequency. This greater distance resulted in weaker coupling between the SBO and the extractor, and therefore the microwave power extracted from the SBO was expected to be lower than the extracted LBO power.

\subsection{Analytical Basis}

Design of the SBO cavities was straightforward based on the constraints outlined in the previous section and the theory presented in Chapter 2. To summarize, the constrained design parameters were an operating voltage $V=300 \mathrm{kV}$, an RF phase velocity $v_{p}=0.26 c$ in the $\pi$-mode, an AK gap distance $b=2.6 \mathrm{~cm}$, vane widths equal to the cavity widths $w$, 
and an operating frequency of $2 \mathrm{GHz}$ in the $\pi$-mode.

The RF phase velocity is related to the guide wavelength by $v_{p}=\omega / \beta=f \lambda_{g}$. In the $\pi$-mode, the phase advance per vane corresponds to $180^{\circ}$, so the $\pi$-mode guide wavelength $\lambda_{g}=2 L$, where $L$ is the circuit pitch, and is related to the cavity width by $L=2 w$. Hence, $L=v_{p} / 2 f$ and $w=v_{p} / 4 f$.

Using $\omega=2 \pi \times 2 \mathrm{GHz}, w=0.96 \mathrm{~cm}$, and $L=1.92 \mathrm{~cm}$, the dispersion relation given by Eq. 2.4 in $\S 2.1$ of Chapter 2 (page 11) can be solved to determine the cavity depth $h$. For the SBO, $h$ was determined to be $3.18 \mathrm{~cm}$ after further refinement using simulations. The cavity depth was the final geometric parameter needed to fabricate the anode block. Figure I. 1 in the appendix on page 221 shows the hardware production drawing used for fabrication.

To determine the threshold magnetic field for $\pi$-mode operation, the AK gap $b=$ $2.6 \mathrm{~cm}, c$-normalized phase velocity $\beta_{p}=0.26$, and operating voltage $V=300 \mathrm{kV}$ were used with Eq. 2.12 for the Buneman-Hartree condition in $\S 2.2$ of the theory chapter (page 17). For both the LBO and SBO, the threshold magnetic field was $B=0.16 \mathrm{~T}$. This concludes the determination of all the geometric and electrical parameters needed to create the MFRPM prototype.

\subsection{Simulation}

To understand the complex operation of the MFRPM, two types of simulations were used to model hot- and cold-tube operation, which refers to the presence or absence of the electron beam. Cold-tube operation was modeled using the High Frequency Structural Simulator (HFSS) finite-element, frequency-domain code [44], and hot-tube operation was modeled using the MAGIC finite-difference, time-domain, Particle-In-Cell (PIC) code [80].

\subsubsection{Cold Tube Modeling}

The purpose of the cold-tube simulations was to identify the frequencies of the fundamental anode modes and their respective field geometries, and to understand how they coupled to the microwave extractor. The details regarding simulation implementation can be found in Appendix A. Table 3.1 summarizes the findings.

Figure 3.3 shows the geometry used to model the MFRPM experimental configuration. Several assumptions and simplifications were used in the simulation, although the geometry of the anode, cathode, and microwave extractor were nearly identical to the components used in the experiment. Microwaves coupled into the coaxial transmission lines 
Table 3.1: Summarized cold-tube simulation results obtained using HFSS for the modes of interest in the MFRPM.

\begin{tabular}{clc}
\hline \hline ANODE & \multicolumn{1}{c}{ MODE } & HFSS [GHz] \\
\hline LBO & $5 \pi / 6$-Mode & 0.977 \\
LBO & $\pi$-Mode & 0.996 \\
LBO & Chamber Mode & 1.058 \\
SBO & $6 \pi / 8$-Mode & 1.965 \\
SBO & $7 \pi / 8$-Mode & 1.996 \\
SBO & $\pi$-Mode & 2.017 \\
\hline \hline
\end{tabular}

were assumed to be completely absorbed, which was implemented in the simulations using a Perfectly Matched Layer (PML) absorber. In reality, some power is reflected back to the coaxial transmission line from the coax-to-waveguide mode converters and the waveguide loads. From the earlier work reported in [10], the frequencies of interest for both oscillators were well within the pass-band of the couplers. The Voltage Standing-Wave Ratio (VSWR) was $<1.5$ in all cases. As shown by the waveguide load calibrations in Appendix D.2.2, the waveguide load match for both oscillators corresponded to a VSWR $<2$, which was sufficient for the experiment, but higher than the typical 1.2 VSWR standard for many magnetrons. Thus, while the simulated termination of the coax lines did not exactly match the experiment, the assumption was nonetheless reasonably representative of the configuration. Similarly, the MELBA-C side of the simulation was also modeled as perfectly absorbing using PML. This simplification may be significant because the coaxial transmission line leading to the Marx generator is complicated by a series of stepped voltage rings insulated by a ceramic. The line is terminated by a metal plate. However, despite these complications, the line was assumed to be perfectly absorbing.

Several features of the electric field geometries are notable. Figure 3.4 illustrates the electric fields for the different modes using a zoomed view of the anode to emphasize the different phase advances per vane and the relative field amplitudes in the different locations of the planar cavity arrays. Broadly, there are two mode types, termed even and odd modes.

The odd modes, such as the LBO $5 \pi / 6$-mode and SBO $7 \pi / 8$-mode, have field nulls located in the center of the planar cavity array. For example, the LBO $5 \pi / 6$-mode in Fig. 3.4a has an average phase advance per vane of $5 \pi / 6$ radians. In the structure, the mode has $\pi$ mode-like fields on the ends and a segment of zero phase advance in the center, which can be seen by the electric fields pointing in the same direction in the cavities adjacent to the center vane. A similar mode pattern is observed for the SBO $7 \pi / 8$-mode in Fig. 3.4d. For the odd modes, the power density is highest away from the center vane. The combination of zero phase advance and low field amplitudes at the center vane leads to very weak cou- 


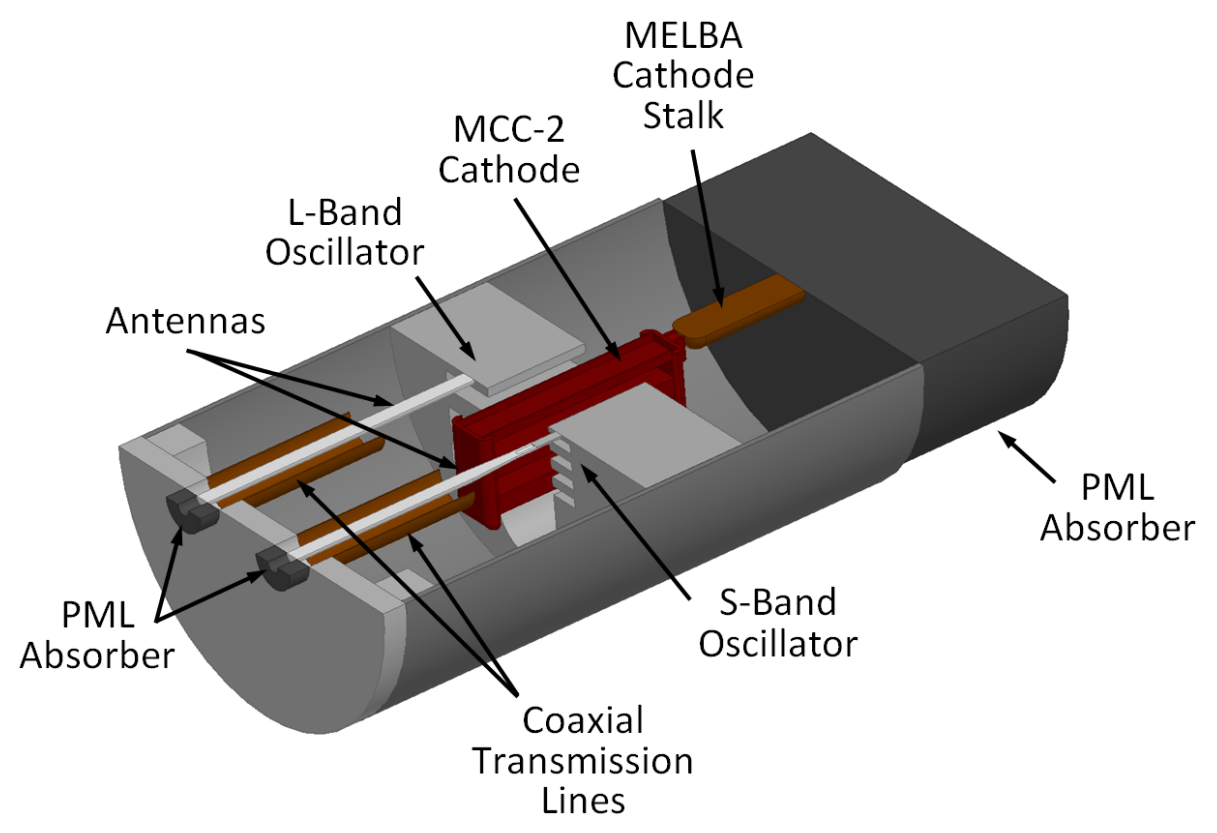

Figure 3.3: HFSS model of the experimental MFRPM configuration.

pling of these modes to the microwave extractor. Thus, magnetron operation in the odd modes was not expected to yield high microwave powers in the extraction waveguide. The different locations of the electric field amplitude maxima were also useful for mode identification using the results of the plasma-related pulse-shortening imaging study discussed in Chapter 5.

By contrast, the $\pi$-mode for both the LBO and SBO have field maxima at the center vane, which is shown by Figs. $3.4 \mathrm{~b}$ and $3.4 \mathrm{e}$. These modes couple effectively to the microwave extractor. The SBO $6 \pi / 8$-mode in Fig. 3.4c also couples to the microwave extractor, although the mode has field amplitude maxima near the ends of the cavity array. Note that the $6 \pi / 8$-mode has a phase advance per vane of $6 \pi / 8$ on average, and that the mode has $\pi$-mode-like fields in the center and ends, and has segments of zero phase advance at the vanes adjacent to the center vane.

HFSS also identified an unexpected mode at $1.058 \mathrm{GHz}$ that could couple to the electron beam. The field structure is similar to a cylindrical $\mathrm{TE}_{01}$ mode in the front of the vacuum chamber between the MFRPM anode and the front vacuum flange, as shown by Figs. 3.5a and 3.5b. The spacing between the anode and flange approximately corresponds to a half-wavelength at the solved frequency. The mode may be capable of coupling to the electron beam because the electric field geometry is $\pi$-mode-like on the ends of the LBO, as shown by Fig. 3.5c. Furthermore, the fields are strong in these locations. However, a zero phase advance at the center extraction vane suggests that, like the odd modes, this 


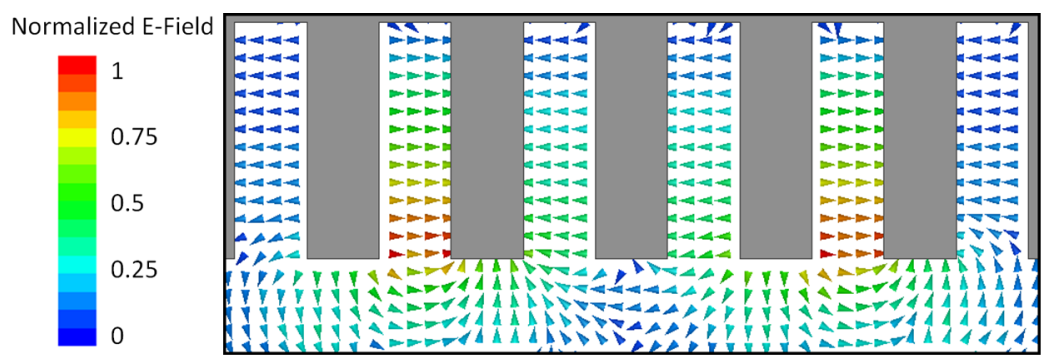

(a) LBO $5 \pi / 6$-mode.

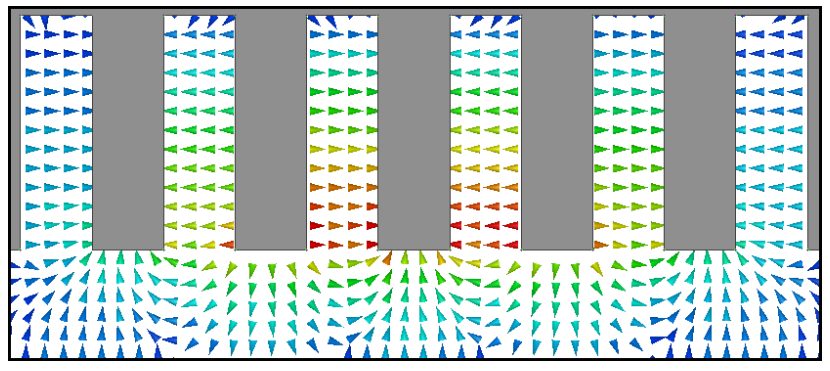

(b) LBO $\pi$-mode.

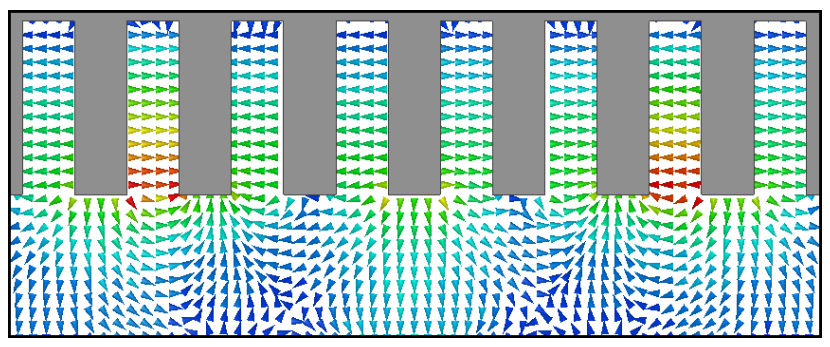

(c) SBO $6 \pi / 8$-mode.

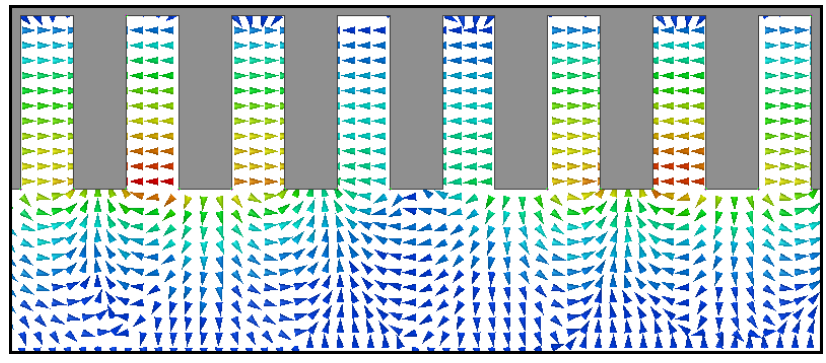

(d) $\operatorname{SBO} 7 \pi / 8$-mode.

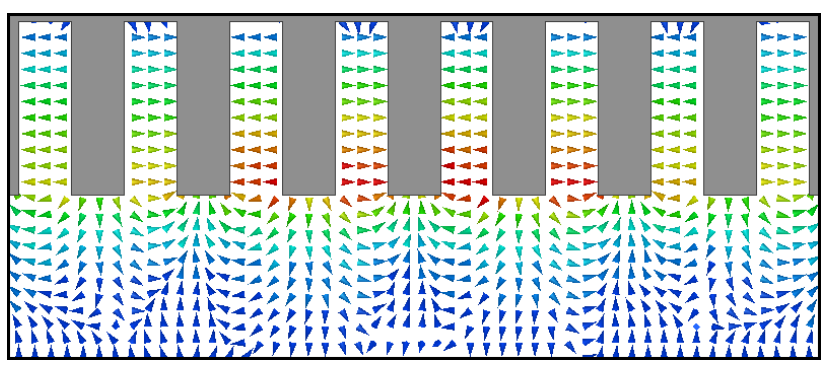

(e) $\mathrm{SBO} \pi$-mode.

Figure 3.4: Electric field geometry for various anode modes of interest in the MFRPM. 


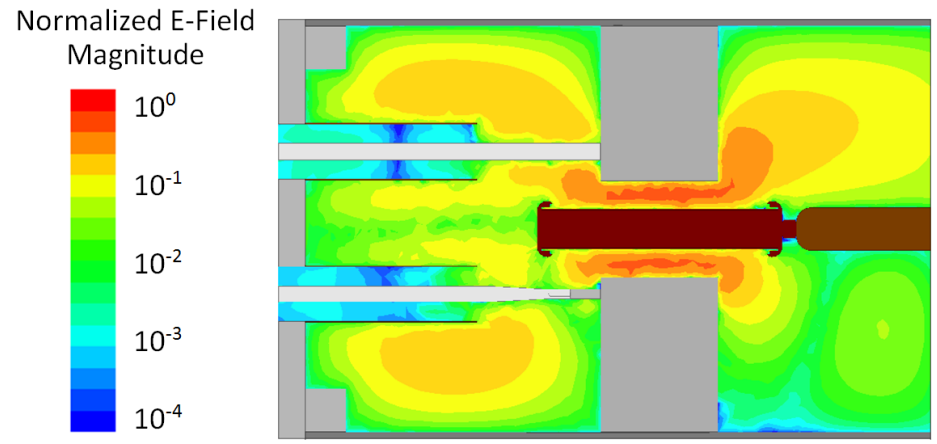

(a) Top sectional view of the parasitic chamber mode. The front chamber length approximately corresponds to a half-wavelength.

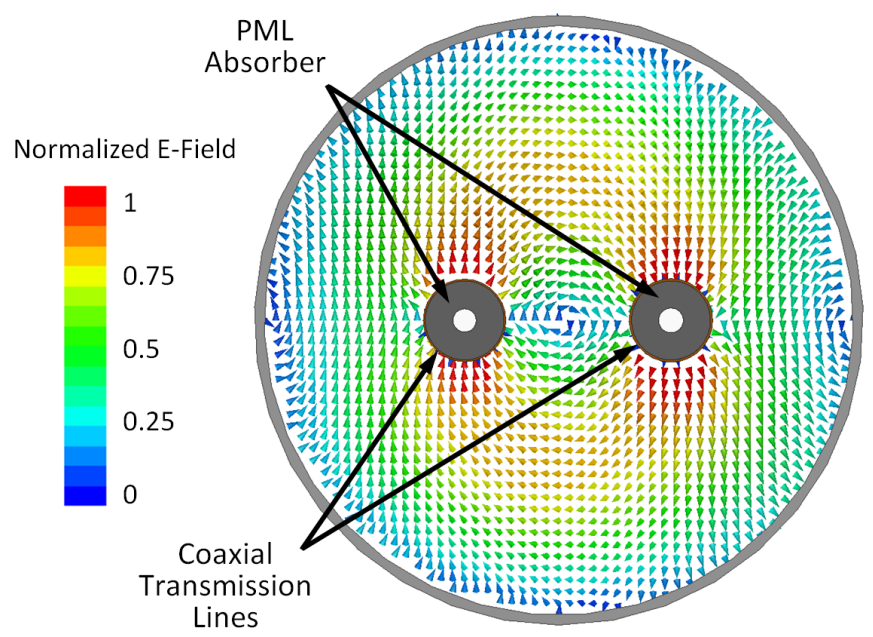

(b) Front sectional view showing the $\mathrm{TE}_{01}$-like electric field. The section resides approximately halfway between the anode and the front vacuum chamber flange.

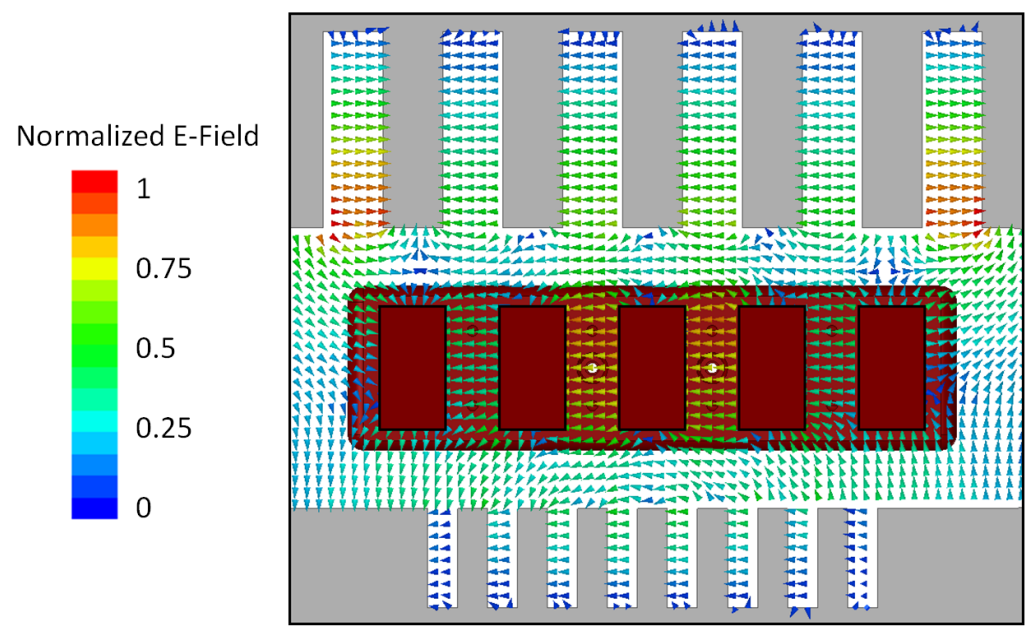

(c) Anode electric field vectors for the parasitic chamber mode.

Figure 3.5: Electric field geometry for the parasitic chamber mode. 
parasitic chamber mode was not expected to couple strongly to the microwave extractor.

\subsubsection{Hot Tube Modeling}

The purpose of the hot-tube simulations was to: 1) identify the beam-loaded frequencies of the fundamental anode modes, 2) estimate the extracted microwave powers for the different modes, 3 ) assess how the presence of the electron beam affects the fields on the anodes, 4) assess the proclivity for mode competition, and 5) assess how the aforementioned parameters were affected by changes in the axial magnetic field. Implementation of PIC simulations required careful consideration of many factors, eight of which are described in detail in Appendix B.

Three geometries were modeled: 1) the MFRPM, 2) the isolated LBO, and 3) the isolated SBO. Figure 3.6 illustrates the geometry for the MFRPM configuration. Like the HFSS simulation configuration, the MFRPM MAGIC model reproduced the experimental geometry as accurately as possible within the limits of computational feasibility. The same assumptions regarding absorbing boundaries discussed in the previous HFSS section also

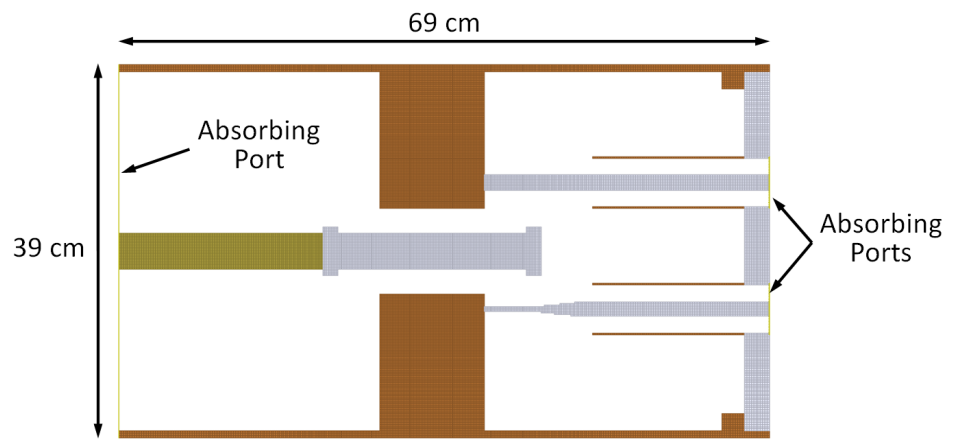

(a) Top sectional view.

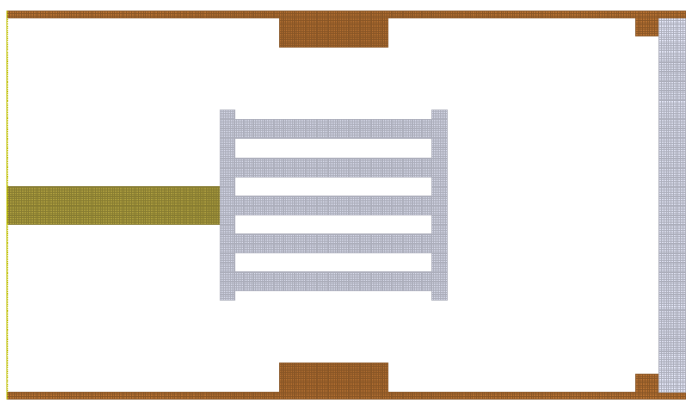

(b) Side sectional view.

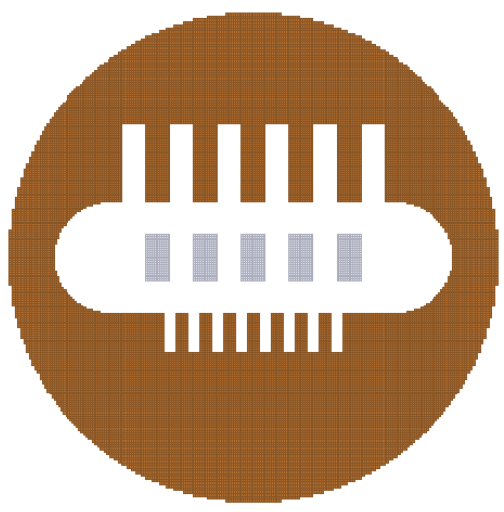

(c) Anode sectional view.

Figure 3.6: MAGIC simulation geometry for the MFRPM. 


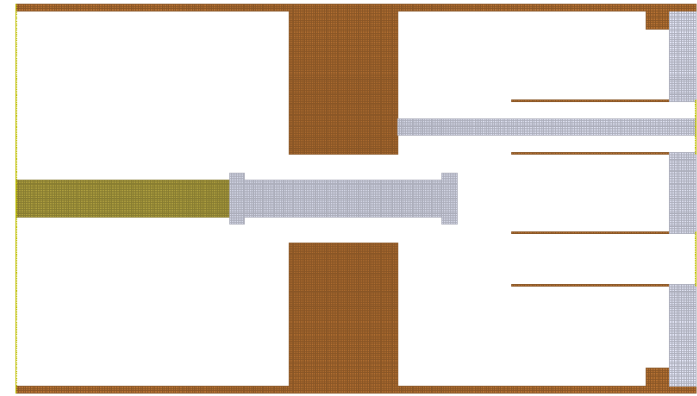

(a) Top sectional view of the MAGIC isolated LBO configuration.

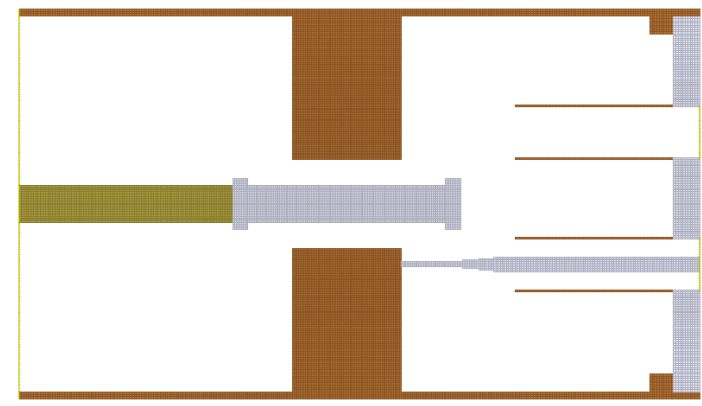

(c) Top sectional view of the MAGIC isolated SBO configuration.

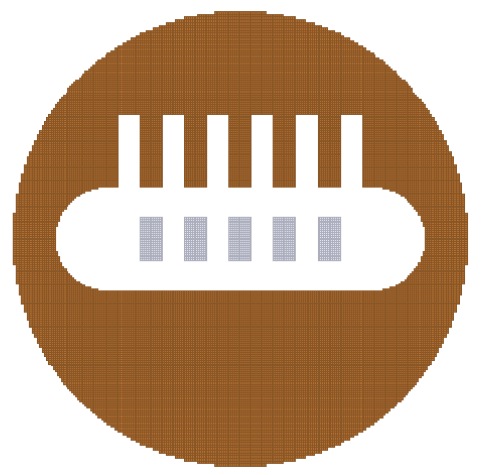

(b) Anode sectional view of the MAGIC isolated LBO configuration.

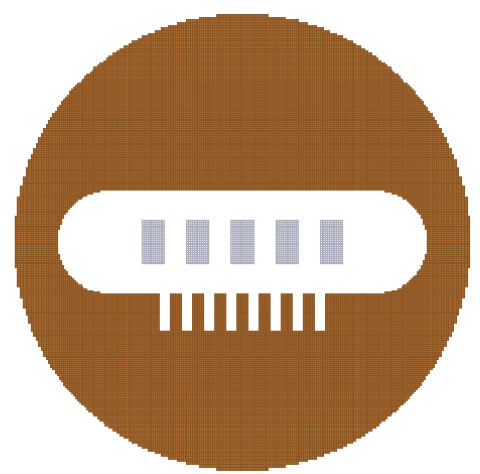

(d) Anode sectional view of the MAGIC isolated SBO configuration.

Figure 3.7: MAGIC isolated oscillator configurations.

apply to the MAGIC model. Both the microwave extraction ports and the MELBA-C coaxial transmission line were modeled as absorbing ports, as shown by Fig. 3.6a. In order to estimate the endloss current that might occur in the experiment, the cathode endcaps were not enlarged in the simulation, which is sometimes done to ease computational requirements by achieving faster magnetron start-up and by reducing the particle population. A comparison of Figs. 3.6a and 3.6b with photographs of the cathode in Chapter 4 shows that the MAGIC model is a reasonably close approximation to the experimental hardware.

Figure 3.7 shows the models for the isolated anode configurations. Like the MFRPM model, these configurations mirror those described in the experimental configuration chapter. Note the inclusion of the outer conductor of the coaxial transmission line corresponding to the removed oscillator. As with the experiment, the outer conductors were not removed in order to minimize changes to the geometry when testing the oscillators in their isolated configurations.

While Appendix B describes the details of simulation implementation, several impor- 
Table 3.2: Summary of the MAGIC simulation sets of magnetic field parameter scans.

\begin{tabular}{ccc}
\hline \hline ANODE & DC EXCITATION & EMISSION \\
\hline LBO & Constant & Full \\
SBO & Constant & Full \\
MFRPM & Constant & Full \\
MFRPM & Dynamic & Full \\
MFRPM & Dynamic & Limited \\
\hline \hline
\end{tabular}

tant factors should be mentioned. A total of five simulation sets were performed, where each set was composed of 10 simulations conducted at magnetic fields ranging from approximately $0.12 \mathrm{~T}$ to $0.21 \mathrm{~T}$. For all simulations, the axial magnetic field was assumed to be uniform. Two DC voltage excitation models were used: a Constant Voltage (CV) model, and a Dynamic Voltage (DV) model. The CV model maintained a constant diode voltage despite changes in the magnetron impedance, and the DV model attempted to accurately model the changes in the diode voltage resulting from changes in the load impedance. Additionally, two electron emission models were used: Limited Emission (LE), which emitted from $10 \%$ of the cathode area (which was consistent with the experiment), and Full Emission (FE), which used $100 \%$ of the cathode area. The five simulation sets are summarized by Table 3.2 .

Due to computational limitations, the simulation sets were not fully converged because the mesh was too coarse. However, as described in the appendix, the effects of unconverged simulations were determined and factored into the interpretation of the results. The most significant effect was an overestimation of the degree of beam-loading on the operating frequencies.

Figure 3.8 shows an example of some results from a simulation of the MFRPM using the $\mathrm{CV}$ and FE models. The voltage, Entrance Current (ENTC) and Endloss Current (ENDL), and the extracted microwave powers are shown by Fig. 3.8a. The voltage rise-time was approximately equal to the $150 \mathrm{~ns}$ MELBA-C rise-time, and the simulation was run until steady-state was achieved. Notably, the endloss current was approximately $50 \%$ of the total current. Figure $3.8 \mathrm{~d}$ indicates that the loss occurs from the cylindrical recirculation bends due to the short cathode endcaps, which do not cover the bends.

The coupling of the electric fields to the coaxial transmission lines is shown by Fig. 3.8b, which reinforces the earlier discussion of the full, $2 \mathrm{GHz}$ wavelength separation between the outer conductor of the coaxial transmission line and the SBO, and the half, $1 \mathrm{GHz}$ wavelength separation between the coaxial outer conductor and the LBO. Even when operating well in the $\pi$-mode, the SBO does not generate comparable extracted microwave powers relative to the LBO. For both oscillators, the odd modes were barely detectable at 


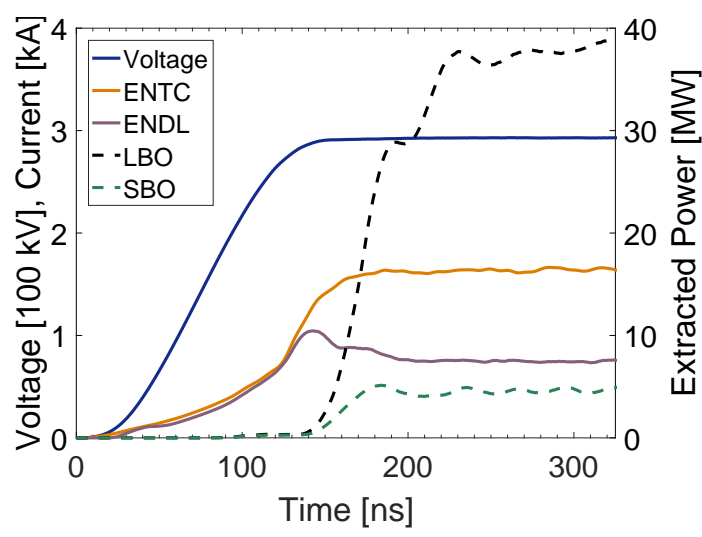

(a) Simulation voltage, currents, and extracted microwave powers.

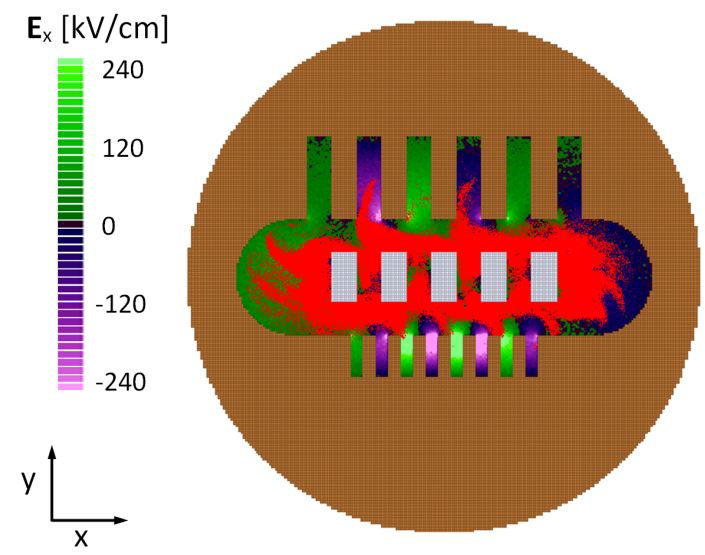

(c) Anode sectional view showing electron spoke formation and preservation of some beam modulation around the cylindrical recirculation bends.

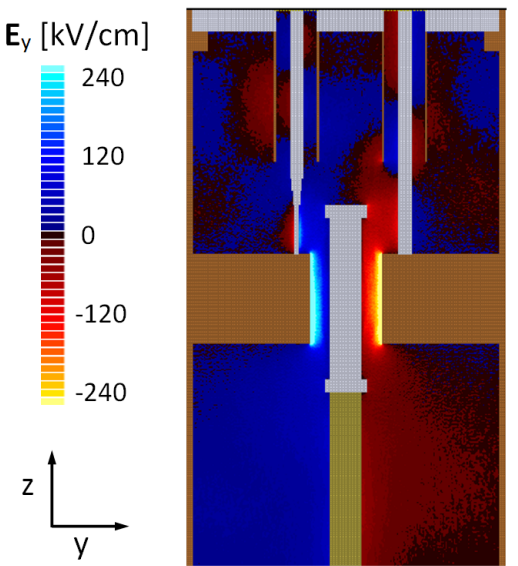

(b) Illustration of electric fields and the coupling from the anode to the microwave extractor. Left (right) oscillator is the SBO (LBO).

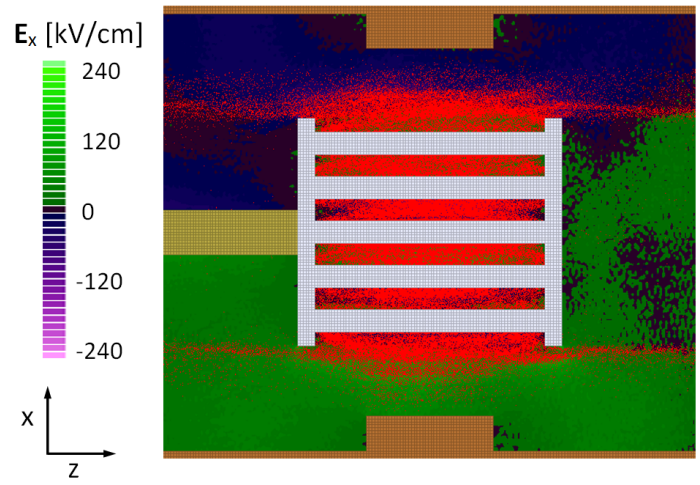

(d) Chamber sectional side view showing electron endloss current due to absence of endcaps covering the cylindrical recirculation bends.

Figure 3.8: MAGIC simulation illustrating particles and fields during $\pi$-mode operation by the LBO and SBO. The simulation used full emission and voltage correction.

the extractor output, even when the modes were extremely dominant. Thus, under conditions where the even and odd modes are competing, the experiment was expected to detect the even modes as the dominant frequencies. At no time did the LBO operate purely in the parasitic chamber mode, although the extracted microwave signal sometimes contained a minor frequency component corresponding to the mode.

Perfect $\pi$-mode operation is shown by Fig. 3.8c, which illustrates the $180^{\circ}$ phase advance per vane in both oscillators and the formation of electron spokes. Also notable is the preservation of some beam modulation around the cylindrical recirculation bends. Finally, while the effect is less pronounced in this particular simulation, it was very common to 
observe the first few cavities on each oscillator seen by the electron beam (in Fig. 3.8c, the upper right LBO cavities and lower left SBO cavities) oscillating at very low amplitude, suggesting those cavities are partially disrupted by the recirculated, modulated electron beam. This observation may be supported by the pulse-shortening study discussed in Chapter 5. Finally, due in part to the coarse mesh (which affected the frequencies) and the high $Q$-factor of the SBO, no evidence of harmonic frequency-locked operation between the LBO and SBO was found in these simulations.

A full discussion of these simulation results is left to $\S \S 5.3 .3,5.4 .3$, and 5.6.4 in the experimental results chapters, where they are compared to the experimental observations and interpreted to explain the operation of the magnetron in the laboratory. 


\section{CHAPTER 4}

\section{Experimental Configurations}

This chapter describes the experimental configuration components used for this dissertation: the magnetron hardware, the electromagnet, the pulsed power driver, the triggering sequence, and the experiment diagnostics. Figure 4.1 illustrates the main components of the experimental configuration. Diagnostics are addressed with details concerning the postprocessing methods used to obtain the results presented in Chapters 5 and 6. This chapter concludes with a description of the pulse-shortening imaging configuration, which was notably different from the configuration used to collect the majority of the results presented in this dissertation.

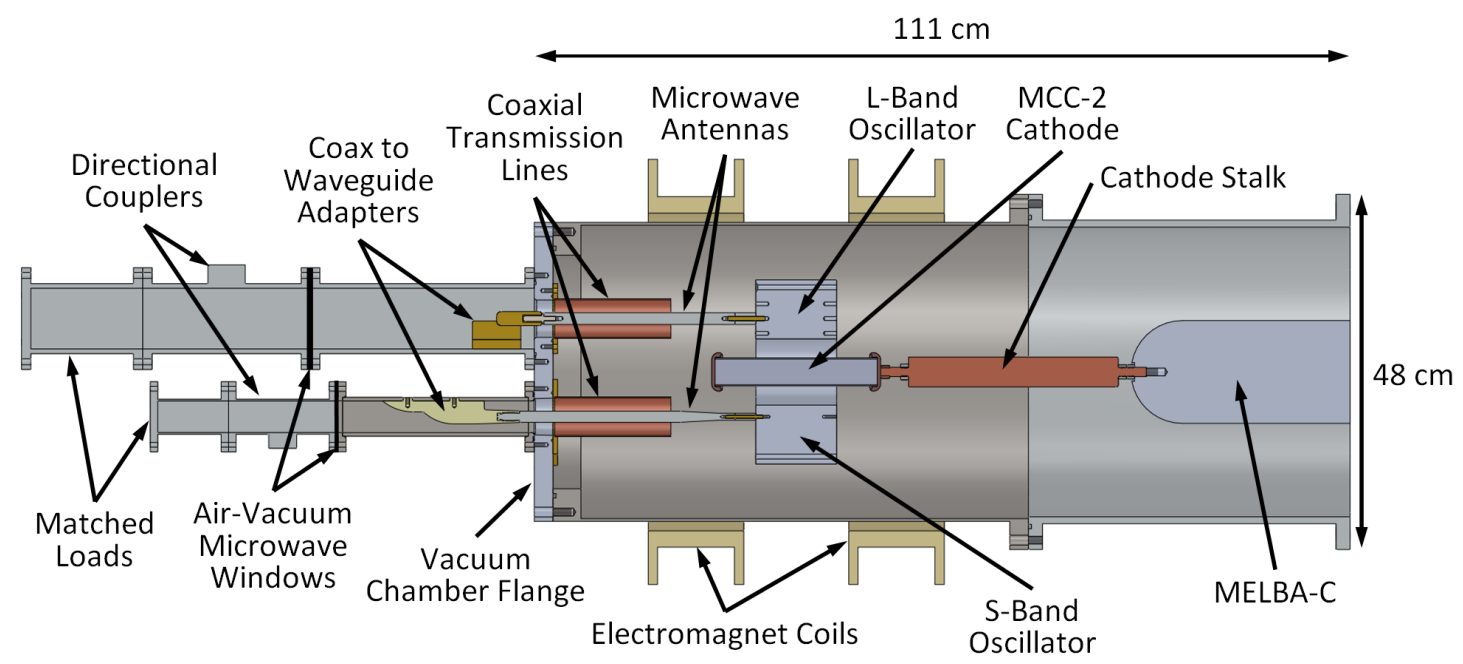

Figure 4.1: Top sectional diagram of the MELBA-C vacuum chamber and MFRPM components. The anode support structure is not shown. 


\subsection{Magnetron Hardware}

Discussion of the magnetron hardware is separated into the three primary components involved in magnetron design: the anode, the cathode, and the microwave extractor.

\subsubsection{Anode}

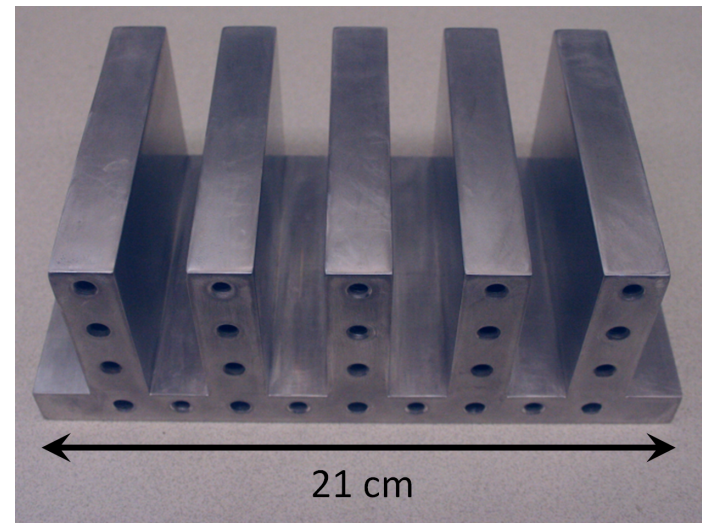

(a) Photograph of the LBO.

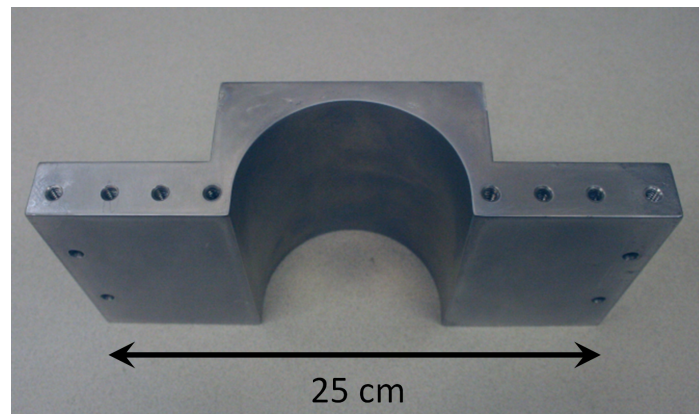

(c) Photograph of a cylindrical bend.

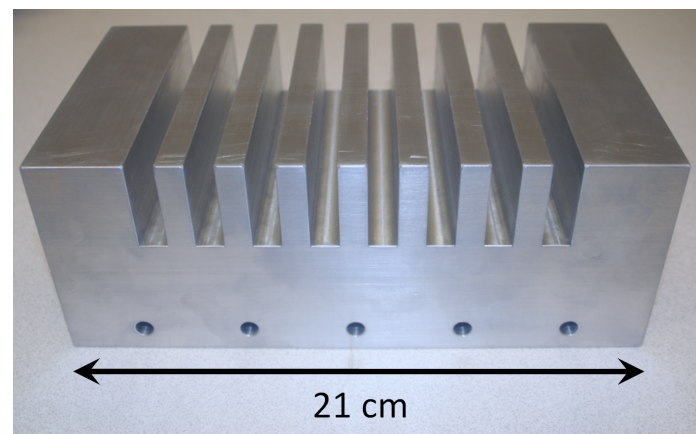

(b) Photograph of the SBO.

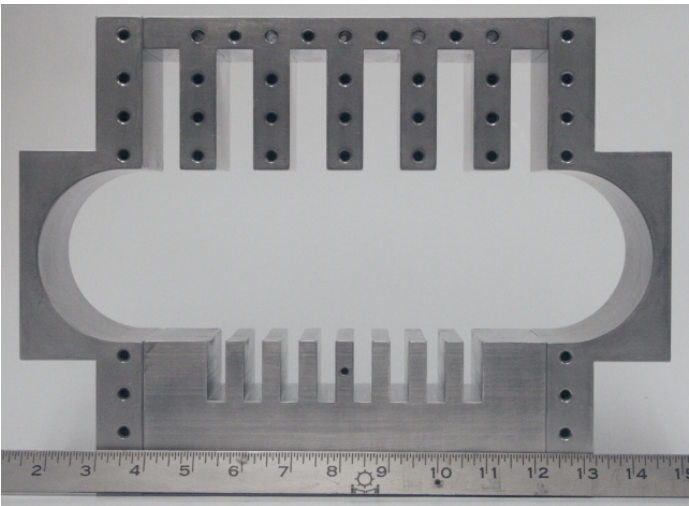

(d) Photograph of the assembled MFRPM anode. Ruler is in inches.

Figure 4.2: Photographs of MFRPM components.

The Multi-Frequency Recirculating Planar Magnetron (MFRPM) anode consists of two Slow-Wave Structures (SWSs) coupled by two cylindrical electron beam recirculation bends. Photographs of the anode sections are shown in Fig. 4.2. All components were fabricated from blocks of 6061 aluminum alloy and had a uniform axial length of $11 \mathrm{~cm}$. The recirculation bends, which are shown in Fig. 4.2c, were originally designed for the RPM-12A, and were suitable for use in the MFRPM.

Two SWSs were used: the L-Band Oscillator (LBO) and the S-Band Oscillator (SBO). 


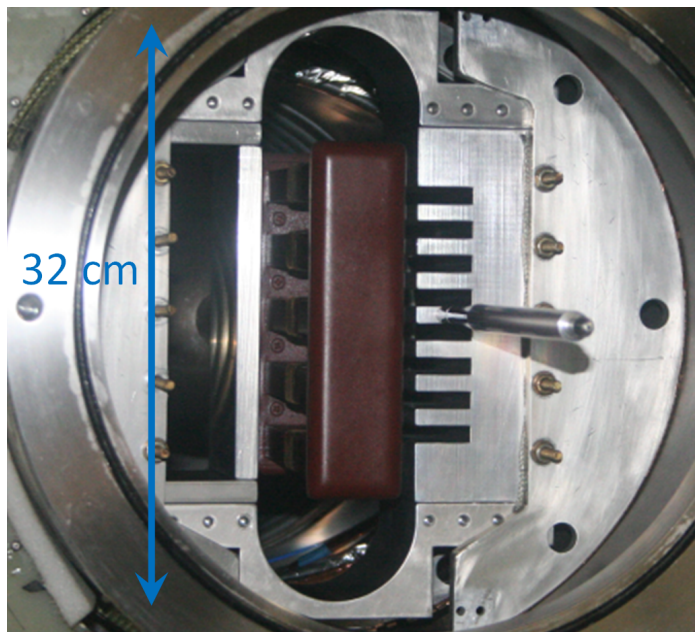

(a) Photograph of the isolated SBO configuration in the MELBA-C vacuum chamber.

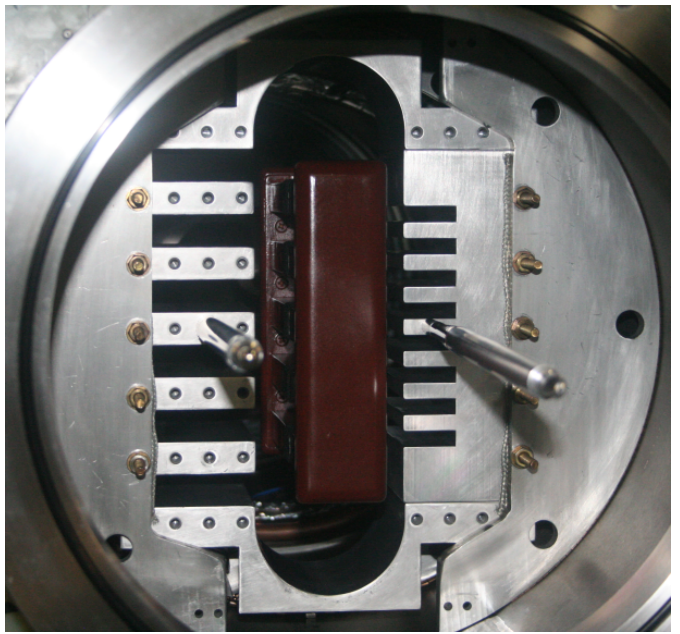

(b) Photograph of the MFRPM configuration in the MELBA-C vacuum chamber.

Figure 4.3: Photographs of two magnetron anode configurations.

The LBO, shown in Fig. 4.2a, was originally designed for the RPM-12A, and detailed hardware drawings can be found in [10]. The LBO consisted of six uniform cavities having a cavity depth and width equal to $6.3 \mathrm{~cm}$ and $1.92 \mathrm{~cm}$, respectively. Vane widths were also $1.92 \mathrm{~cm}$. These dimensions produced resonators having an unloaded $\pi$-mode frequency and guide wavelength of $1.01 \mathrm{GHz}$ and $7.68 \mathrm{~cm}$, respectively, which corresponded to an RF phase velocity of $0.26 c$.

The SBO is shown in Fig. 4.2b, and consisted of eight uniform cavities having a cavity depth and width equal to $3.18 \mathrm{~cm}$ and $0.96 \mathrm{~cm}$, respectively. Vane widths were equal to the $0.96 \mathrm{~cm}$ cavity width. The resonators were designed to have an unloaded $\pi$-mode of $2.02 \mathrm{GHz}$ and a guide wavelength of $3.84 \mathrm{~cm}$, which also corresponded to an RF phase velocity of $0.26 c$. Chapter 3 described the SBO design process in greater detail. The fully assembled MFRPM anode is shown in Fig. 4.2d.

Three anode configurations were used in this dissertation: the isolated LBO, the isolated SBO, and the MFRPM. In the isolated configurations, one of the SWSs was replaced with a smooth electron drift section. An example is shown in Fig. 4.3a, which shows the smooth drift region in place of the LBO. The full MFRPM configuration is shown for comparison in Fig. 4.3b. For all anode configurations, a cylindrical support structure was used to fix the anode in place within the vacuum chamber. A good electrical connection between the support structure and the vacuum chamber was established using copper finger stock. The support structure can be seen in both photographs shown by Fig. 4.3.

Evidence of arc damage between the support plate for the outer conductor of the mi- 


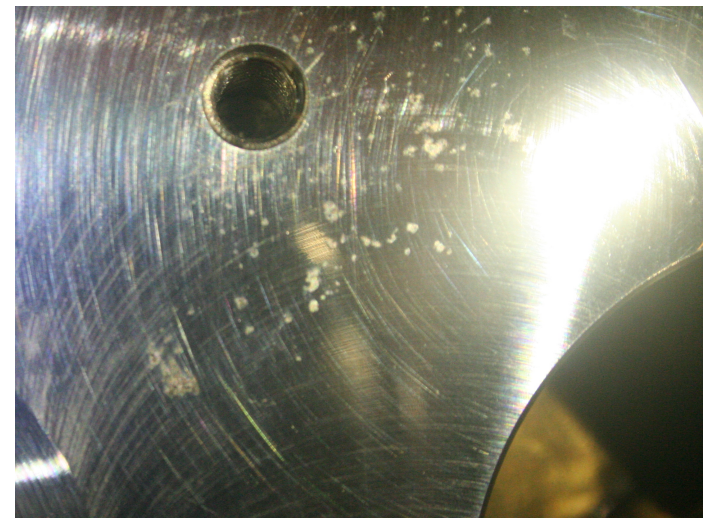

(a) Arc damage between the coaxial outer conductor support plate and vacuum chamber endplate.

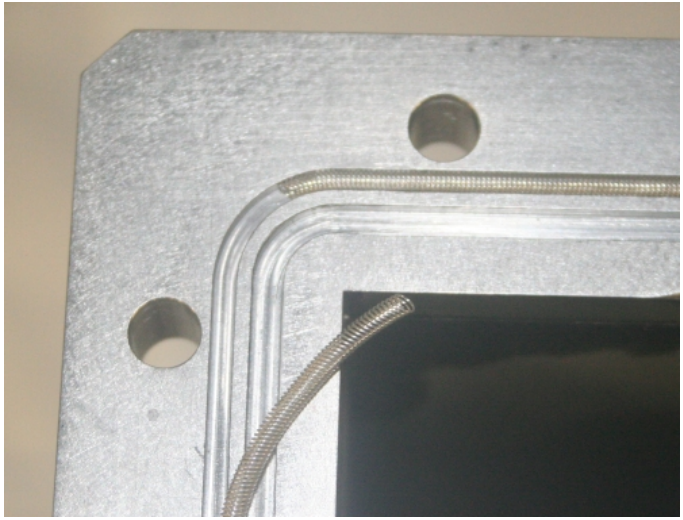

(b) Bal-Seal coil in a waveguide flange.

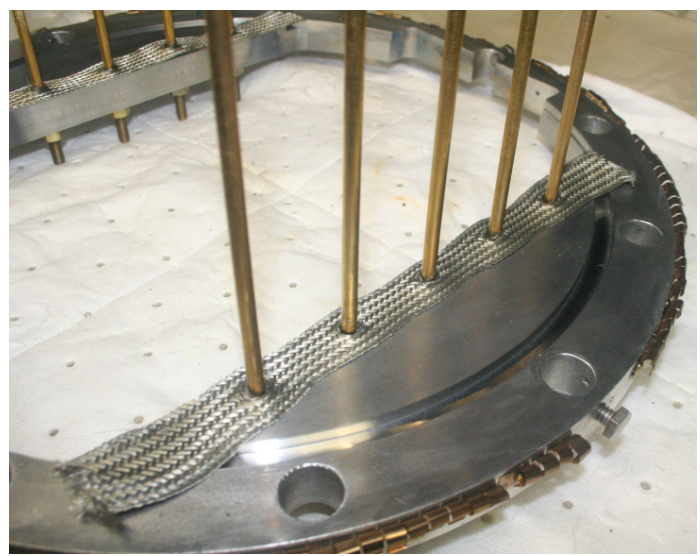

(c) Conducting braid on the MFRPM support structure.

Figure 4.4: Photographs of RF and DC gaskets used to improve electrical contacts.

crowave extractor coaxial transmission lines and the vacuum chamber end plate was found during earlier RPM-12A experiments. Figure 4.4a shows a photograph of some of the arc marks. Additional damage was noted between the RPM-12A anode and the support structure. To address these issues, Bal Seal RF gaskets were used between critical RF components to improve their electrical connection and reduce contact resistance $[81,82]$. Poor $\mathrm{RF}$ and DC contacts are a known source of disruption in vacuum electronics [83]. Figure $4.4 \mathrm{~b}$ shows an example of Bal Seal coil on a waveguide flange. Ground braid was used in an attempt to improve the electrical connection between the magnetron anode and the grounded support structure. The braid and support structure are shown in Fig. 4.4c. 


\subsubsection{Cathode}

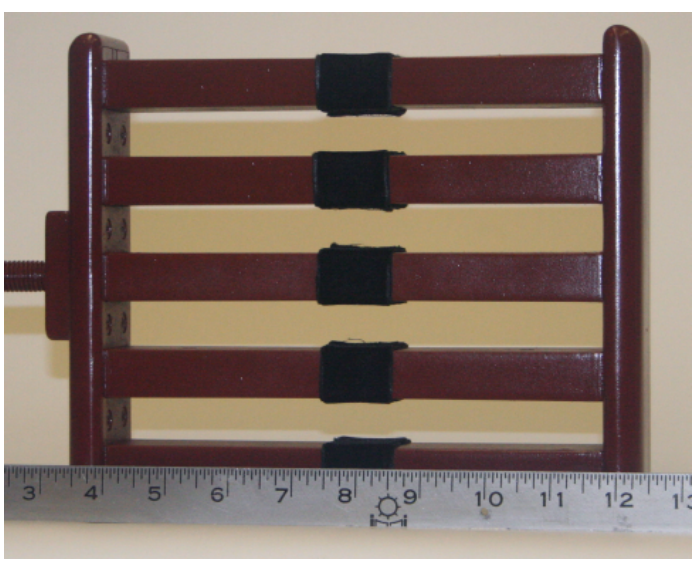

(a) Photograph of the MCC-2 cathode. The ruler is in inches.

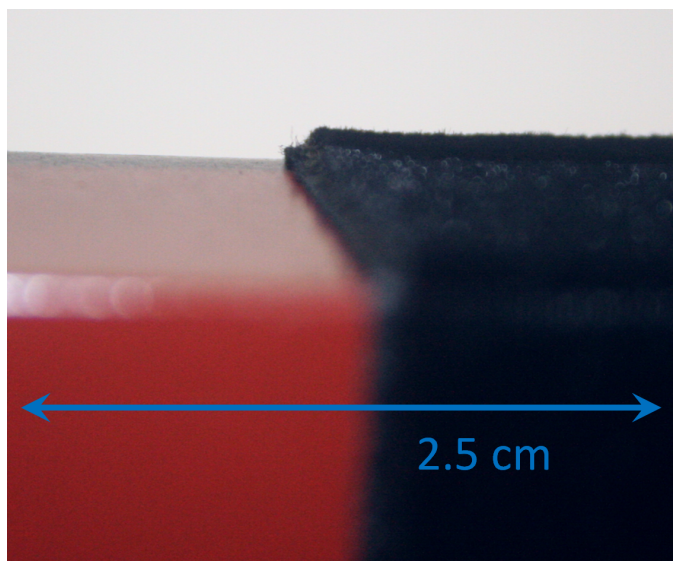

(b) Photograph of the heat-treated edge of the velvet electron emitter.

Figure 4.5: The MCC-2 cathode.

The cathode used for this dissertation was a periodically slotted structure termed a Mode-Control Cathode (MCC), which was originally developed for the RPM-12A to promote phase-locked operation [40]. The material was 6061 aluminum alloy. While geometrically similar to a transparent cathode [84], an MCC is unique in that it functions as an EM coupler [42], an emission-primed electron source [56,85,86], and introduces beneficial perturbations to the DC electric field [87]. Since the cathode was the second MCC prototype developed for use with an RPM, the cathode was termed the MCC-2. A photograph is shown by Fig. 4.5a. The cathode featured endcaps to reduce endloss currents [88-90], although the endcap design was conservative to reduce the risk of arcing between the endcaps and microwave extraction antennas.

The cathode consisted of five rectangular bars that were $1.92 \mathrm{~cm}$ wide and $3.8 \mathrm{~cm}$ thick, which resulted in a $2.6 \mathrm{~cm}$ AK gap in the magnetron. The separation between the bars was $1.92 \mathrm{~cm}$, which resulted in an overall width of approximately $17 \mathrm{~cm}$. The bar widths and gaps were designed to be the same as the vane and cavity widths of the LBO. The axial length of the MCC-2 was $23 \mathrm{~cm}$, which was $12 \mathrm{~cm}$ longer than the $11 \mathrm{~cm}$ axial length of the magnetron anode.

Five coats of Glyptal insulating enamel were necessary to reduce excessive current emission from the bare aluminum surface. Aggressive electron emission control was critical to match the electrical impedance of the magnetron to the specifications for the MELBA-C Marx generator. The electron emitter was velvet fabric provided by Frank Hegeler at the Naval Research Laboratory. Velvet has been extensively studied as an electron emitter for 
explosive-emission cathodes operating in a space-charge-limited regime [91-95]. The velvet was attached to the bare aluminum surface using conducting silver epoxy adhesive. In order to reduce excessive current emission from the velvet edges, the fibers were heated to $220^{\circ} \mathrm{C}$ to produce a smooth edge free of protruding material. To reduce the likelihood of undesirable emission from the triple point between the velvet, metal, and vacuum, the interface edges were hidden beneath the velvet by overlapping approximately $3 \mathrm{~mm}$ of velvet over the Glyptal-painted surface [55]. A previous study confirmed that velvet functions well as an electron emitter only if a good electrical interface exists between the velvet and metal [96]. Figure $4.5 \mathrm{~b}$ shows a close-up photograph of an emitter.

\subsubsection{Microwave Extractor}

The microwave power extractor was motivated by the same technique used in common household microwave oven magnetrons [79]. A diagram of the extractor components was shown earlier in this chapter in Fig. 4.1 on page 40, and photographs of extraction assembly components are shown by Fig. 4.6. Microwave power was coupled from the centermost vane on each SWS to a simple rod antenna (Fig. 4.6a). At a distance from the magnetron that was approximately equal to half of a $1 \mathrm{GHz}$ wavelength (or a full $2 \mathrm{GHz}$ wavelength), the antennas entered copper tubes to form coaxial transmission lines (Fig. 4.6b). Microwaves propagated down the lines in the TEM mode toward the coax-to-waveguide adapters for the LBO and SBO shown in Figs. 4.6c and 4.6d, respectively. The adapters then converted the coaxial TEM mode to the waveguide $\mathrm{TE}_{10}$ mode, which continued to propagate down the output waveguides shown in Fig. 4.6e. The output waveguides were vacuum-sealed using microwave-transparent Lexan windows that formed the barrier between air and vacuum. The components beyond the microwave windows vary depending on the configuration, and are discussed in greater detail in the Microwave Diagnostics section later in this chapter. Hardware production drawings for the fabricated components can be found in Appendix I.

To improve the RF electrical contact between the antenna components, copper wool was compressed between the joints and trimmed using a razor blade to eliminate any protruding metal. The distance between the magnetron and the copper tubes forming the coaxial transmission lines was originally optimized for use with the RPM-12A, which resulted in acceptable coupling between the LBO and the extractor. However, for similar coupling between the SBO and the extractor, the distance between the tubes and the SBO would need to be approximately half the original distance used for the LBO, which would bring the copper tube edges unacceptably close to the cathode endcaps, thereby introducing a 


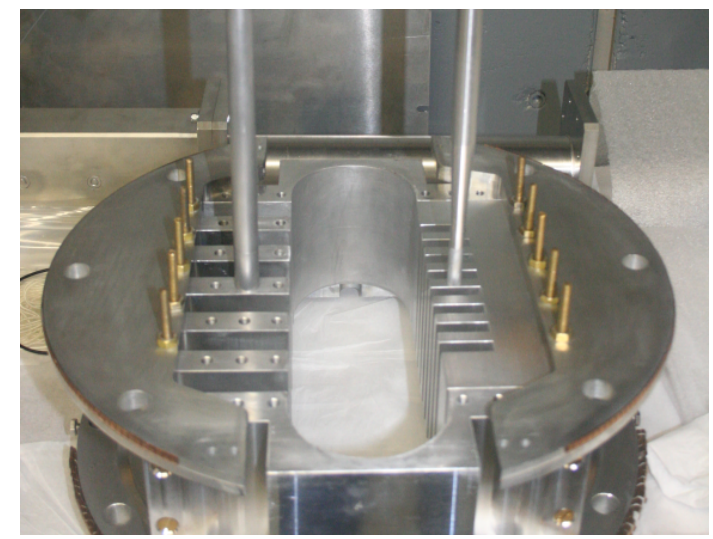

(a) MFRPM with attached microwave antennas.

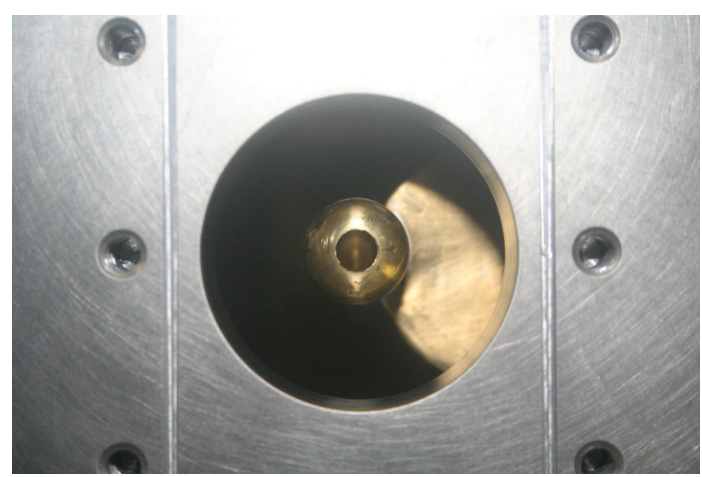

(c) LBO coax-to-waveguide adapter.

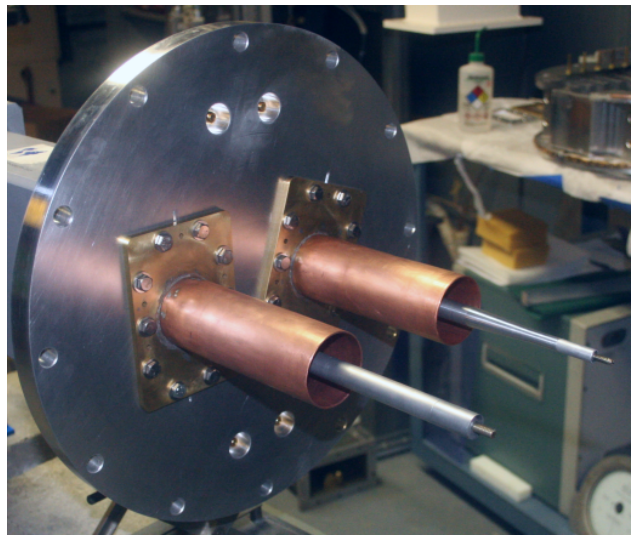

(b) Microwave antenna transition to coaxial transmission lines.

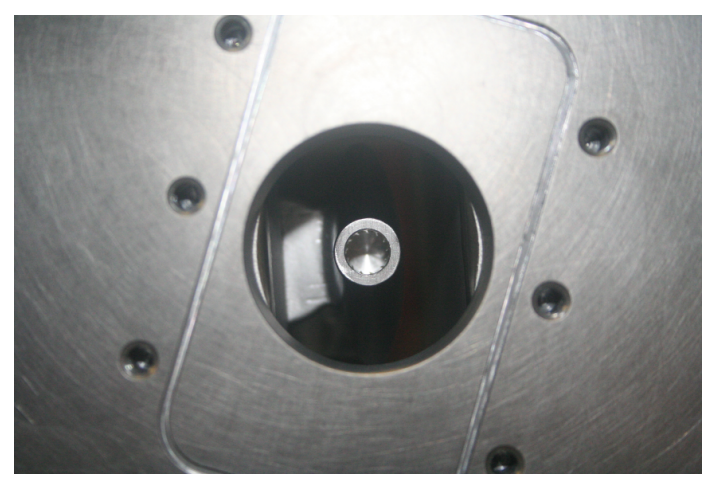

(d) SBO coax-to-waveguide adapter.

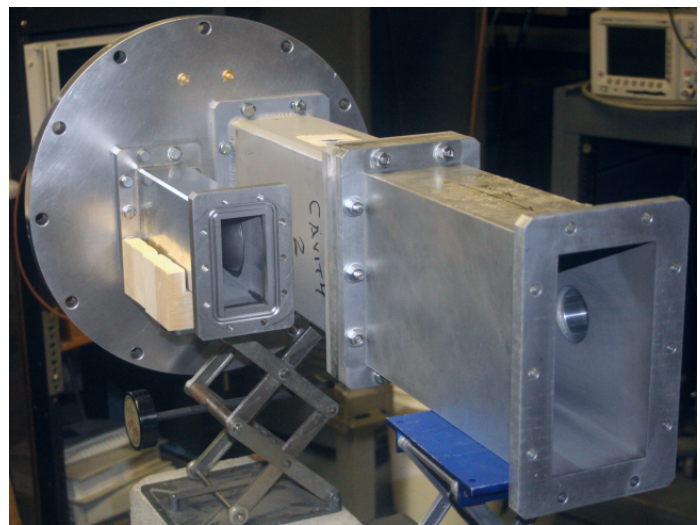

(e) Output waveguides attached to the vacuum chamber flange.

Figure 4.6: Photographs of the microwave power extraction assembly. 


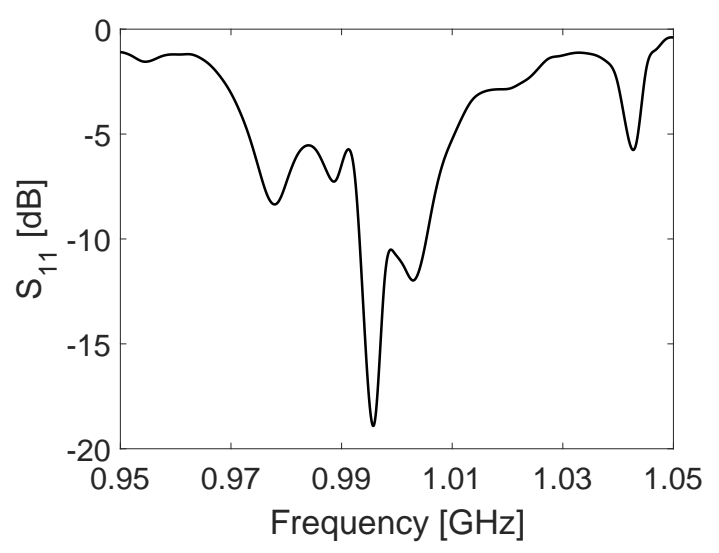

(a) Cold test results for the LBO.

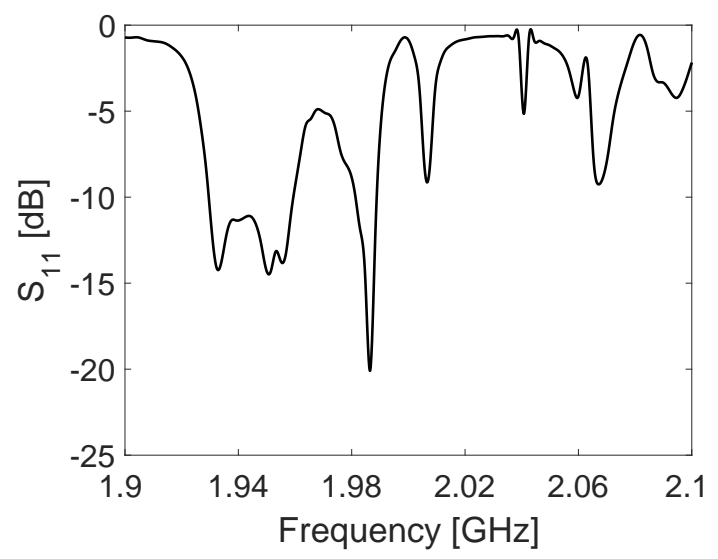

(c) Cold test results for the $\mathrm{LBO}$ second harmonic.

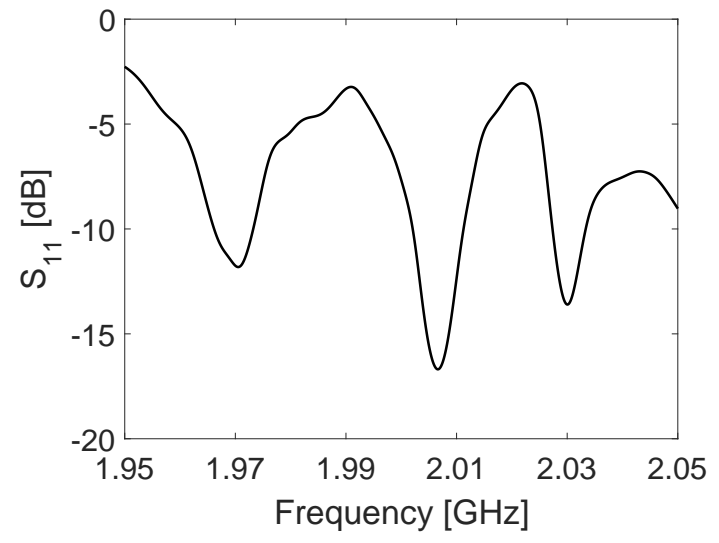

(b) Cold test results for the SBO.

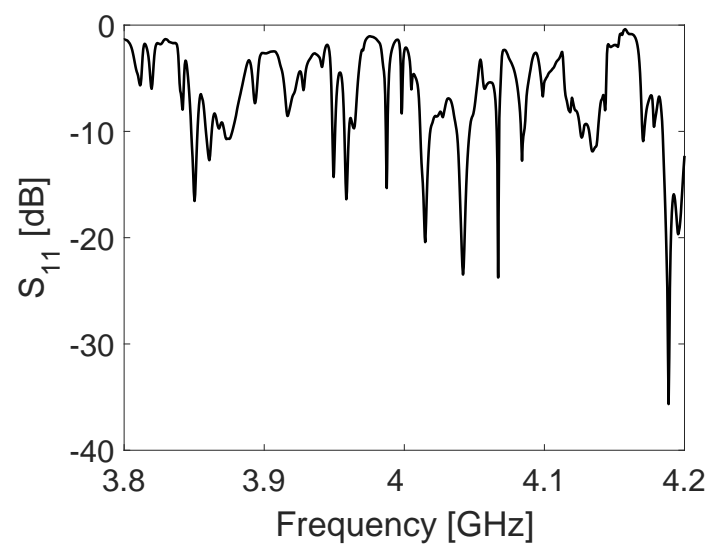

(d) Cold test results for the LBO fourth harmonic.

Figure 4.7: Cold test results obtained using a network analyzer.

significant arc risk. As a consequence of this extraction design, the coupling between the SBO and the extractor was expected to be weaker relative to the LBO. A more thorough discussion can be found in Chapter 3.

Cold tests are shown in Fig. 4.7. The results were obtained using an HP 8722D vector network analyzer. Since the resonant frequencies of the modes are reduced by as much as a few percent during hot operation due to beam-loading and the magnetic field profile, the "hot" quality factors of the resonances cannot be reliably estimated from the cold tests alone. However, cold tests can confirm the presence of expected resonances, as well as reveal the existence of undesired modes.

Figure 4.7a shows the results for the LBO. There were five apparent frequencies of interest: $0.978 \mathrm{GHz}, 0.989 \mathrm{GHz}, 0.996 \mathrm{GHz}, 1.003 \mathrm{GHz}$, and $1.043 \mathrm{GHz}$. Comparison with the HFSS results from $\S 3.3 .1$ in the design chapter suggested the $0.978 \mathrm{GHz}$ and $0.996 \mathrm{GHz}$ frequencies corresponded to the $5 \pi / 6$-mode and $\pi$-mode, respectively, and that the $0.989 \mathrm{GHz}$ and $1.003 \mathrm{GHz}$ frequencies corresponded to modes that were supported by 
Table 4.1: Summarized cold test results for the LBO and SBO.

\begin{tabular}{clcc}
\hline \hline ANODE & \multicolumn{1}{c}{ MODE } & HFSS [GHz] & EXPERIMENT [GHz] \\
\hline LBO & $5 \pi / 6$-Mode & 0.977 & 0.978 \\
LBO & $\pi$-Mode & 0.996 & 0.996 \\
LBO & Chamber Mode & 1.058 & 1.043 \\
SBO & $6 \pi / 8$-Mode & 1.965 & 1.971 \\
SBO & $7 \pi / 8$-Mode & 1.996 & 2.007 \\
SBO & $\pi$-Mode & 2.017 & 2.030 \\
\hline \hline
\end{tabular}

the anode, but were unlikely to couple to the electron beam through the crossed-field interaction due to the geometry of the fields. The $1.043 \mathrm{GHz}$ mode corresponded to a parasitic chamber mode previously discussed in Chapter 3, which may be capable of coupling to the electron beam.

Figure $4.7 \mathrm{~b}$ shows the results for the SBO. Comparison with HFSS suggested the three modes of interest at $1.970 \mathrm{GHz}, 2.007 \mathrm{GHz}$, and $2.030 \mathrm{GHz}$ corresponded to the $6 \pi / 8$ mode, $7 \pi / 8$-mode, and $\pi$-mode, respectively. Table 4.1 summarizes the comparison between HFSS and experimental cold tests.

Figures $4.7 \mathrm{c}$ and $4.7 \mathrm{~d}$ show the cold test results for the LBO frequency harmonics using the waveguide taper configuration described later in this chapter. Both figures revealed the existence of numerous resonances, a finding that was consistent with HFSS eigenmode solver results. However, precisely which modes could couple to the electron beam was not known because the quantitative agreement with HFSS was insufficiently strong, and the degree of beam loading on the resonances was unknown. The relations between the hot test LBO harmonic and fundamental frequencies are determined in Chapter 6.

\subsection{Electromagnet}

The axial magnetic field was produced using two pulsed electromagnets in a pseudoHelmholtz configuration. An ideal Helmholtz configuration calls for the coil separation to be equal to the radii of identical coils. In the experiment, the coil radii were $22 \mathrm{~cm}$, and the coil separation was $27 \mathrm{~cm}$. The non-ideal separation was a consequence of a legacy configuration used for the A6 magnetron, which needed the greater distance to fit the extraction waveguide between the coils. Since the configuration was not modified for use with the RPM-12A, the non-ideal separation was maintained for this dissertation in order to compare the performance of the MFRPM directly to the results from RPM-12A experiments. As discussed in the following sections, direct measurement of the magnetic field confirmed that the most important consideration affecting the magnetic field was the diffusion time 
through the vacuum chamber and anode structures.

The coils were provided by the Air Force Research Laboratory, and consisted of 115 turns of $0.0381 \mathrm{~cm} \times 7.62 \mathrm{~cm}$ copper sheet [56]. The inductance per coil was approximately $200 \mu \mathrm{H}$. The driving configuration used seven capacitors rated at $200 \mu \mathrm{F}$, and were charged to a maximum of $5 \mathrm{kV}$ to produce magnetic fields as high as $0.27 \mathrm{~T}$ in the MFRPM AK gap.

Determination of the magnetic field involved the application of a calibration relating the electromagnet current to measurements obtained using a Hall probe. The calibration details can be found in Appendix D.5. The electromagnet current was measured using a Model $301 X$ Pearson current transformer terminated at $50 \Omega$ at a Textronix 3052 oscilloscope. The signal was digitally processed to remove any DC voltage offset and a small movingwindow average filter was applied to reduce noise. The peak voltage was then fed to the calibration function to determine the representative magnetic field in the MFRPM AK gap at the time of the MELBA-C voltage pulse. Changes in the Marx trigger delay time or the components in the vacuum chamber, such as the anode or cathode, require the generation of a new calibration function using the procedure in Appendix D.5.

Some control can be exerted over the magnetic field profile in the magnetron AK gap by varying the trigger delay between the electromagnet and the MELBA-C Marx generator. For this dissertation, two magnetic field profiles were used: a nonuniform magnetic field, and a field that was optimized for uniformity throughout the MFRPM.

\subsubsection{The Nonuniform Magnetic Field}

The nonuniform magnetic field was produced using a $9.7 \mathrm{~ms}$ Marx trigger delay after the triggering of the electromagnet. The $9.7 \mathrm{~ms}$ delay was originally used for the A6 magnetron and the RPM-12A. As a legacy delay, initial MFRPM experiments preserved the $9.7 \mathrm{~ms}$ timing for performance comparisons. Further study of the magnetic field led to an investigation of the impact of different magnetic field profiles on MFRPM operation that are addressed in Chapters 5 and 6.

The axial magnetic field profile along the planar cavity arrays was measured using a Hall probe and the calibration procedure outlined in Appendix D.5. Figure 4.8a shows the probed locations, which correspond to the horizontal axis values in Figs. $4.8 \mathrm{~b}$ and $4.8 \mathrm{c}$. The variation in the magnitude of the nonuniform axial magnetic field along the planar cavity arrays was $40 \%$, with the maximum falling in the center of the cavity arrays, and the minimum falling in the cylindrical bends. Plotted magnetic field values for configurations using the nonuniform magnetic field used a calibration that averaged the field values along 


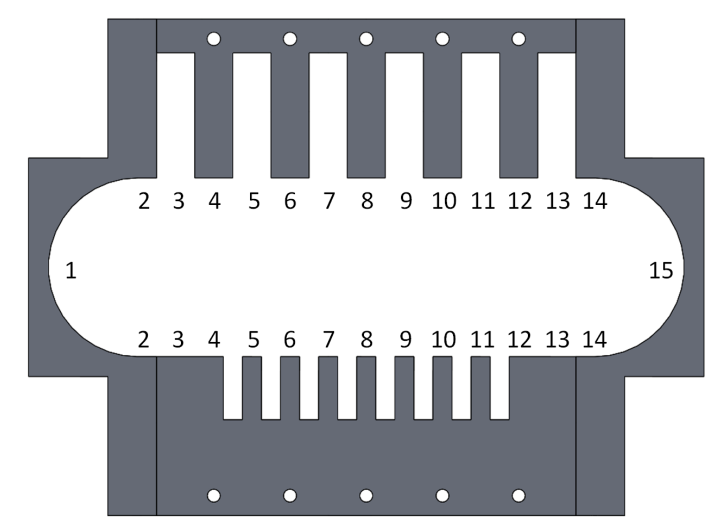

(a) Hall probe sampling positions used to measure the MFRPM magnetic field profile.

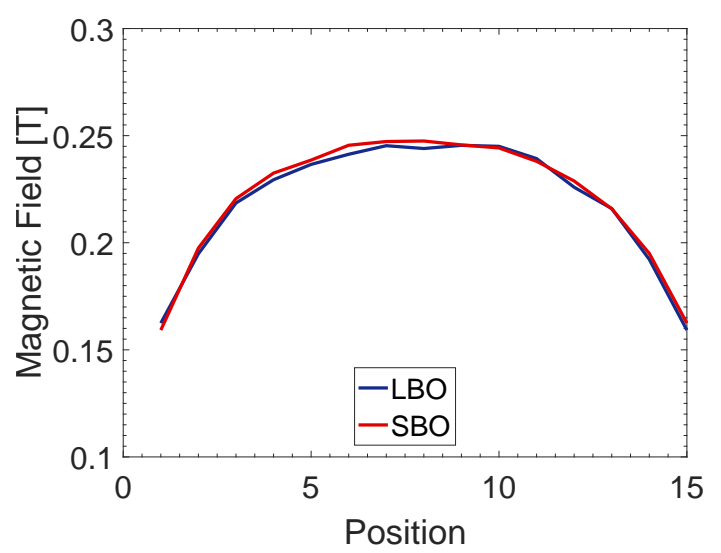

(b) The nonuniform magnetic field profile. Plotted values are from a $5 \mathrm{kV}$ capacitor bank charge.

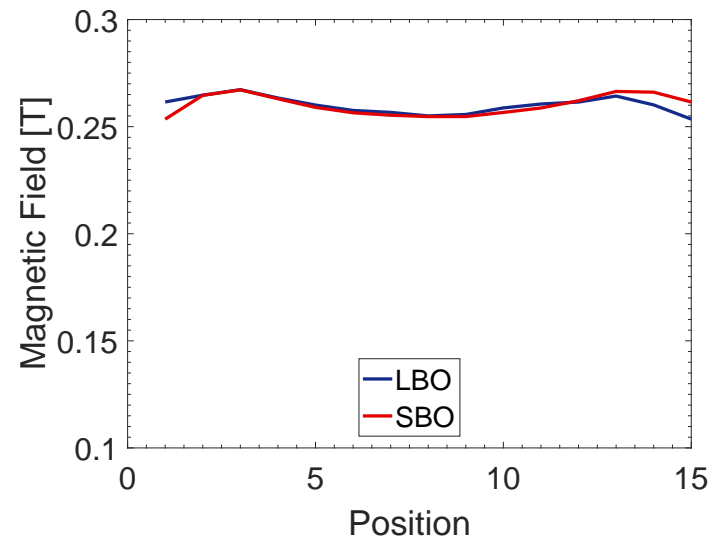

(c) The uniform magnetic field profile. Plotted values are from a $5 \mathrm{kV}$ capacitor bank charge.

Figure 4.8: Magnetic field profiles within the MFRPM.

the array. Despite the asymmetry introduced by the different planar cavity arrays, the magnetic field variation along the LBO and SBO was nearly identical.

Additional measurements of the axial variation of the field found minor variation relative to the middle of the magnetron due to differences in the magnetic field diffusion time, depending on the location. In all cases, the magnetic field was higher axially outside of the magnetron, and lower in the middle. The variation was less than 5\%, and was highest in the cylindrical bends.

\subsubsection{The Uniform Magnetic Field}

The uniform magnetic field was produced using a $13.7 \mathrm{~ms}$ Marx trigger delay. The procedure to determine the optimal Marx trigger delay time is described in detail in Appendix E. Figure 4.8c confirms that the field optimization dramatically improved the uniformity relative to the original nonuniform magnetic field. No discernible differences in 
the field profile along the cavity arrays were found between the LBO and SBO, and unlike the nonuniform magnetic field, nearly zero axial variation was observed.

\subsection{Melba-C Pulsed Power Driver}

The Michigan Electron Long Beam Accelerator with Ceramic Insulator (MELBA-C) is a pulsed Marx generator composed of a main Marx circuit and an Abramyan voltage compensation circuit [97-99]. It consists of 16 capacitors rated for $\pm 100 \mathrm{kV}$ and $1 \mu \mathrm{F}$, and is capable of delivering a $-1 \mathrm{MV}, 150 \mathrm{~ns}$ rise-time, flat-top pulse at $10 \mathrm{kA}$ for $1 \mu$ s to a load with collapsing impedance. The main Marx circuit comprises seven stages, with 2 capacitors per stage, and the Abramyan circuit comprises a single stage consisting of 2 capacitors. With the ceramic insulator, the MELBA-C vacuum chamber can achieve vacuum pressures in the range $10^{-7}-10^{-6}$ torr. With a pulse repetition rate functionally limited to 0.5 shots per minute, MELBA-C is not considered a rep-rated pulsed-power source. Figure 4.9 shows photographs of the Marx generator.

Operation of the Marx generator involves charging the capacitors in parallel, followed by rapidly and simultaneously closing the set of spark-gap switches to discharge the capacitors in series so their voltages add. To protect the load from complete diode closure, the remaining Marx charge is quickly and safely shunted to ground after a preset time by closing an additional switch on a crowbar circuit.

Without a voltage compensation circuit, the draining charge from the capacitors and the collapsing impedance of the load would lead to unacceptable voltage droop during the pulse. The Abramyan circuit addresses this issue by superposing a low-frequency oscillation on the Marx output voltage. During the first half-period of the oscillation, the ringing Abramyan circuit subtracts from the output voltage, and during the second half-period, it adds to the output voltage. The MELBA-C design has a tuned Abramyan circuit frequency that is suitable for achieving $1 \mu$ s pulses within flat-top voltage specifications. In general, optimal pulse shapes are achieved using 100-150 $\Omega$ load impedances. Figures C.1-C.3 in Appendix $\mathrm{C}$ on page 185 show a detailed MELBA-C circuit diagram created using LTspice.

For this dissertation, MELBA-C was configured to output approximately $-300 \mathrm{kV}$. Optimally matched loads should therefore be designed to draw 2-3 kA. By operating the Marx generator at a reduced voltage, the lifetime of the components has been substantially prolonged. MELBA-C has surpassed its 10,000 shot specification, with the current shot total exceeding 15,000. Conversion to the lower operating voltage necessitated modification of the crowbar switch, which is described in Appendix H. In general, due to the rapid onset of magnetron microwave generation and severe microwave pulse-shortening, MELBA-C volt- 


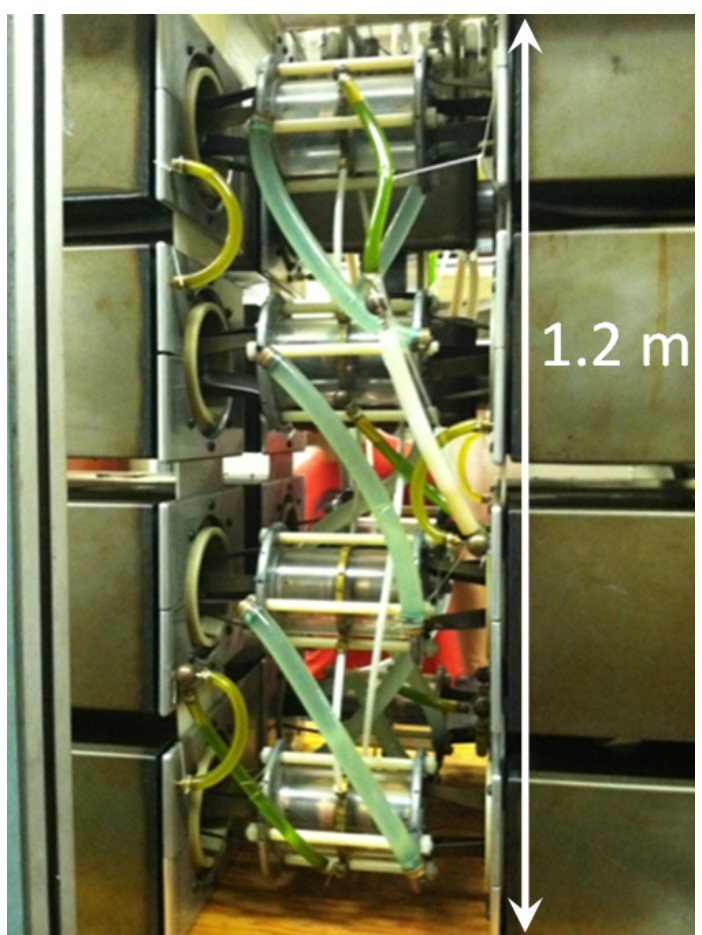

(a) Melba-C side view showing switches and $\mathrm{CuSO}_{4}$ resistors.

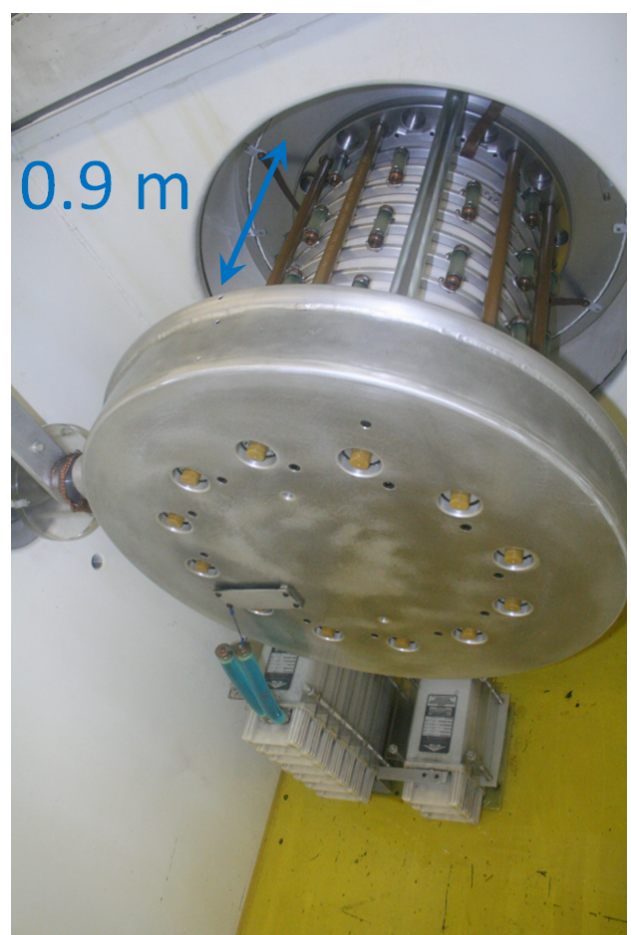

(b) MELBA-C ceramic insulator, filter capacitors, and $\mathrm{CuSO}_{4}$ resistors.

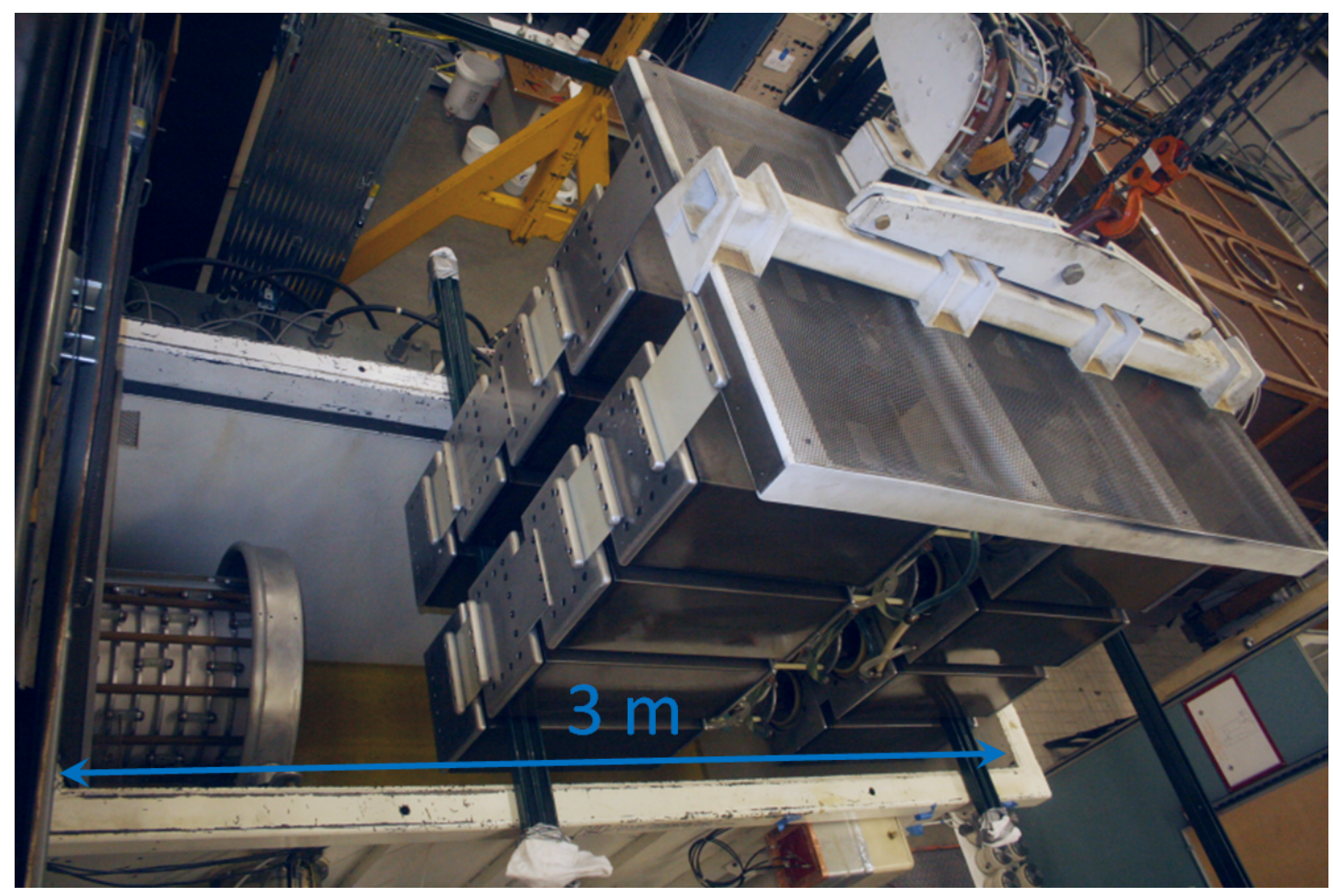

(c) Top view of MelBA-C suspended over the oil tank and ceramic insulator.

Figure 4.9: Photographs of the MELBA-C Marx generator. 
age pulse durations in this dissertation were typically limited to 200-400 ns. Appendix C contains extensive additional discussion of MELBA-C LTspice simulations, new switch diagnostics, and comparisons with experimental results.

\subsection{Triggering Sequence}

Figure 4.10 shows a diagram illustrating the trigger sequence used for this dissertation. The trigger sequence was controlled using a BNC 575 pulse generator. At the beginning of a shot, the first trigger pulse is sent to close the electromagnet ignitron switches, causing current to begin flowing in the electromagnet coils. The first pulse also initiates data collection on a Tektronix 3052 oscilloscope used to monitor the electromagnet current. The magnetic field diffuses into the magnetron in approximately $10 \mathrm{~ms}$ (the time depends on the choice of anode). At $500 \mathrm{~ns}$ prior to achieving the desired magnetic field profile (whether uniform or nonuniform), another trigger pulse is sent to initiate a MELBA-C pulse. This trigger pulse also initiates data collection on two Tektronix 3054, one Agilent 54855A, and two Tektronix TDS 2014 oscilloscopes used to monitor the performance of the Marx generator and the magnetron. The $500 \mathrm{~ns}$ offset accounts for the time between the MELBA-C trigger pulse and the time at which a rise in MELBA-C voltage is first observed. The early scope trigger time relative to the start of the MELBA-C pulse was necessary to record a portion of noise-free data on each channel to determine and remove any DC offset during digital post-processing. The time to reach peak voltage is approximately $150 \mathrm{~ns}$, and the

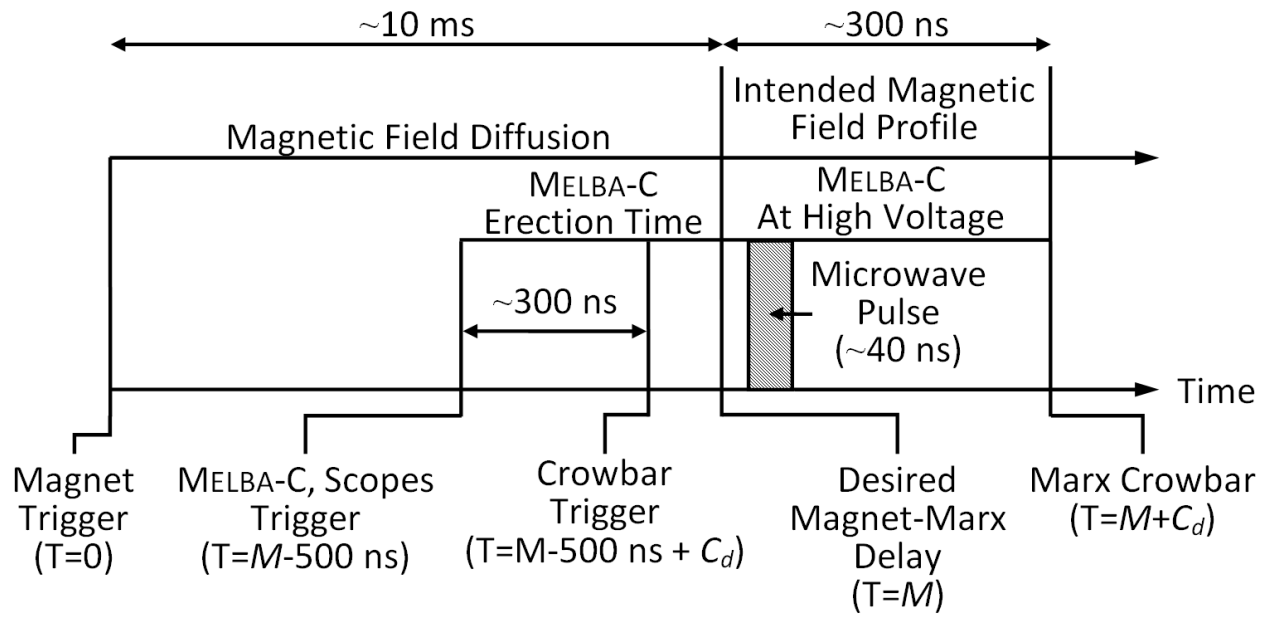

Figure 4.10: Diagram illustrating the trigger sequence times and approximate timescales during a shot. In the diagram, $M$ is the trigger delay between the electromagnet and MELBA-C, and $C_{d}$ is the command crowbar time. 
magnetron typically begins producing microwaves during the voltage rise. Thus, the beginning of MELBA-C high voltage coincides with the desired magnetic field obtained at a time $M$ after triggering the electromagnet. Since both the MELBA-C rise-time and typical microwave pulse widths ( $\simeq 40 \mathrm{~ns}$ ) are short relative to $M$, the magnetic field during magnetron operation is functionally unchanged. The Marx generator crowbar switch is then closed at a time $C_{d}$ after the Marx erects. The crowbar trigger is sent early because it also takes approximately $500 \mathrm{~ns}$ until the voltage is observed to fall. The crowbar delay time $C_{d}$ therefore represents the desired voltage pulse duration, and was typically 300-400 ns.

\subsection{Diagnostics}

Several diagnostics were used for this series of experiments. These broadly fall in two categories: pulsed power diagnostics, and microwave diagnostics.

\subsubsection{Pulsed Power Diagnostics}

The pulsed power diagnostics monitored the performance of the pulsed power systems, which include the electromagnet and the MELBA-C Marx generator.

\subsubsection{Current Measurement}

As previously discussed in the electromagnet section, knowledge of the peak electromagnet current was required to determine the magnetic field in the magnetron using a calibration function. A Pearson current transformer (model 301X, $0.01 \mathrm{~V} / \mathrm{A}$, with $50 \Omega$ termination) was used to measure the current. This diagnostic did not require calibration because it is considered a standard in the pulsed power and microwave component community [100].

Measurement of the MELBA-C Marx generator current was accomplished using a Rogowski coil. Multiple coils were installed axially upstream from the magnetron. Relative to Fig. 4.1 on page 40 at the beginning of this chapter, the coils were located on the far-right side beyond the figure border. All coils were calibrated using a Pearson current transformer, as documented in Appendix D.4. However, only one coil was determined to be reliable during magnetron operation, which is termed the Entrance Current (ENTC). The ENTC coil is embedded in the MELBA-C flange in the oil tank, and is therefore completely shielded from the electron beam.

A detailed and thorough description of Rogowski coil operation can be found in [101]. Briefly, a Rogowski coil consists of a series of wire loops, and each loop describes a small 
area bordered by the loop. From Faraday's law, a time-changing magnetic flux through the loop area will produce a voltage between the terminals of the loop, so it follows that the loop voltage $V_{\text {loop }}$ is proportional to the time rate of change in the magnetic flux $d \Phi / d t$. Using Kirchoff's voltage law, and accounting for the resistance of the load between the terminals of a Rogowski coil and the inductance of the series of loops, it follows that the total magnetic flux $\Phi_{t}$, coil voltage $V_{c}$, and coil current $I_{c}$ are related by

$$
V_{c}=\frac{d \Phi_{t}}{d t}=I_{c} R+L \frac{d I_{c}}{d t} .
$$

To eliminate cable reflections, the Rogowski coil load was $50 \Omega$. Omitting the details, the coil response is well-approximated by the relation $V_{c}=I_{c} R=d \Phi_{t} / d t$ provided that $d I_{c} / d t \ll I_{c} /(L / R)$, meaning a coil with a sufficiently small inductance will generate a voltage that is directly proportional to the time-changing magnetic flux. From the Ampère-Maxwell law, a current flowing down the MELBA-C cathode stalk will generate an azimuthal magnetic field. It therefore follows that a suitable Rogowski coil, which has a sufficiently small inductance and loops oriented to respond to the time-changing azimuthal magnetic field, will produce a voltage directly proportional to the time rate of change of the MelbA-C current. Time integration of the Rogowski coil voltage therefore provides a means of current measurement. In addition to the calibration, Appendix D.4 also documents the design of the ENTC Rogowski coil, which needed replacement during the course of the project.

To determine the MELBA-C current, any DC voltage offset was first removed from the ENTC signal. Due to the presence of substantial noise, the signal was also filtered using a moving-window average. Integration was performed numerically using a cumulative trapezoidal approximation. The integrated signal was then corrected using the calibration described in the appendix.

\subsubsection{Voltage Measurement}

The magnetron diode voltage was monitored using a $\mathrm{CuSO}_{4}$ resistive voltage divider located in the MELBA-C oil tank. Because of the relatively low inductance between the MELBA-C tank and the magnetron, as well as the relatively long MELBA-C voltage risetime, no inductive voltage correction was required. Design specifics for the diagnostic can be found in [102]. Appendix D.3 describes the calibration of the voltage monitor, which is occasionally abbreviated as VOLM. Digital processing involved the removal of any DC voltage offset and the application of a moving-window average to remove the small amount of noise present on the signal. 


\subsubsection{Marx Switch Diagnostic}

MELBA-C switch closure times were monitored using fiber optic lines and Photomultiplier Tubes (PMTs). The switch housing was made of a clear plastic, which enabled light to be easily collected by carefully aligning the fibers. The PMT signals were captured using two Tektronix 2014 scopes using $\mathrm{M} \Omega$ termination. Appendix $\mathrm{C}$ shows many examples of MELBA-C voltage pulses and how they relate to the results from the switch diagnostic.

\subsubsection{Microwave Diagnostics}

Two microwave diagnostics were used to measure the performance of the magnetron. The first measured the frequency spectrum produced by the oscillators, and the second measured the microwave powers. Figure 4.11 shows the different configurations used for the microwave diagnostics.

Two microwave loads were used, which are designated the standard load and the taper load. The standard load, shown in Fig. 4.11b, was used to evaluate magnetron operation using normal, matched microwave loads. The waveguide taper load, shown in Fig. 4.11d, was used to measure the frequencies and powers of the LBO frequency harmonics. The taper configuration was necessary to isolate the individual frequency components to ensure accurate power measurements and to determine which closely-spaced frequencies were dominant or competing. Figure 4.11c shows a block diagram illustrating the isolation of the different frequencies, and Appendix D.2.2 documents the characteristics of the microwave loads.

\subsubsection{Spectral Measurement}

Figure 4.11a shows a block diagram illustrating the general configuration used to determine the frequency spectrum of a microwave signal, which was sampled using a waveguide directional coupler. In the standard load configuration, microwave signals were sampled from the LBO (SBO) using WR-650 (WR-340) waveguide loop directional couplers. Coaxial cables were used to propagate the microwave signals to the screen room, where a series of frequency-insensitive in-line attenuators were used to bring the RF voltage amplitudes down to $<5 \mathrm{~V}$. The signal was split, with one line terminating at a fast Agilent 54855A oscilloscope. The Agilent scope enabled direct measurement of the RF voltage up to $5 \mathrm{GHz}$. By recording the unfiltered RF voltage, all spectral components sampled by the directional coupler could be measured. In the waveguide taper load configuration, the second and fourth LBO frequency harmonics were isolated for measurement using WR-340 and WR-187 waveguides and bandpass filters. 


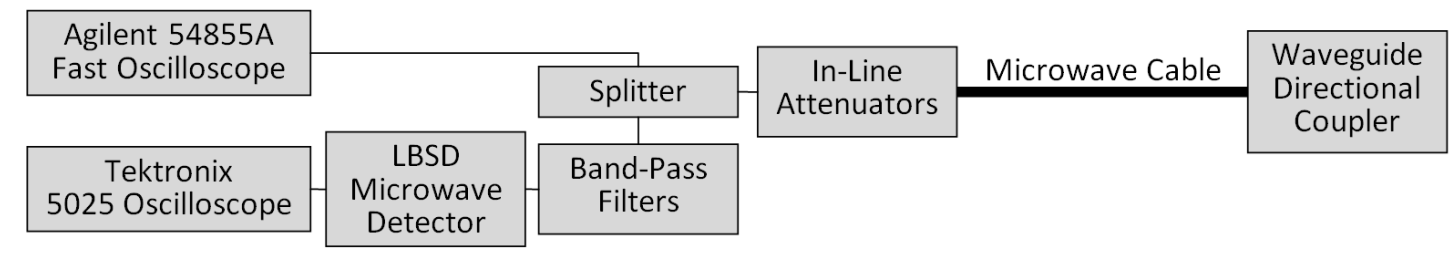

(a) Block diagram illustrating the general configuration used for microwave measurements sampled from a waveguide directional coupler. All oscilloscope connections terminate at $50 \Omega$.

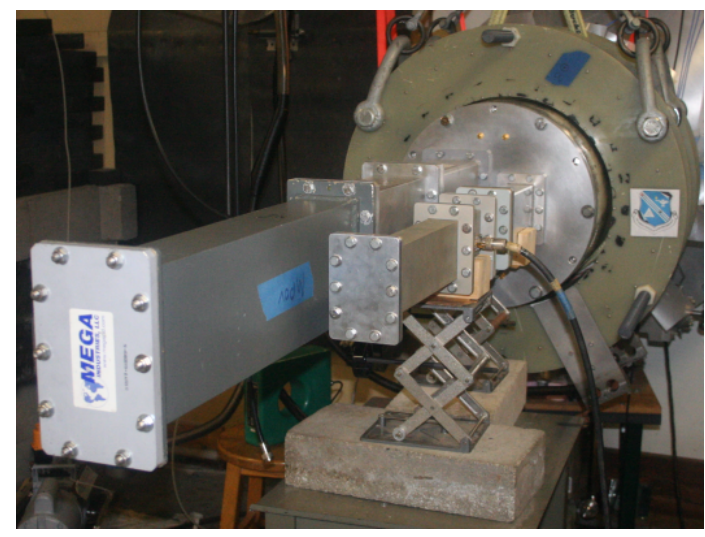

(b) Photograph of the waveguide standard load configuration.

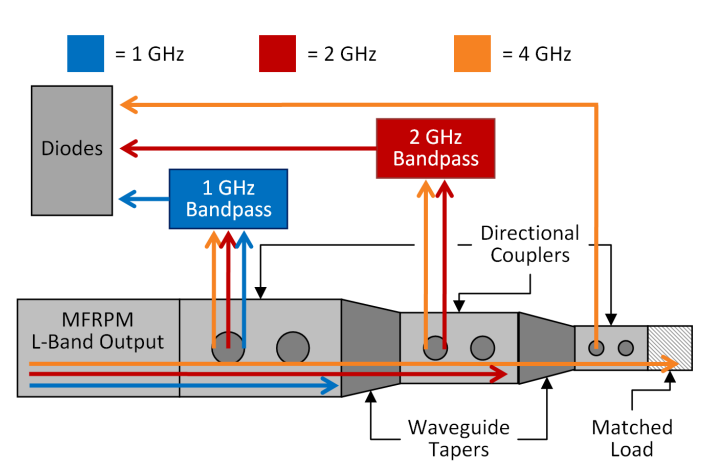

(c) Block diagram illustrating the LBO waveguide taper load configuration used to isolate individual frequency components for power measurements.

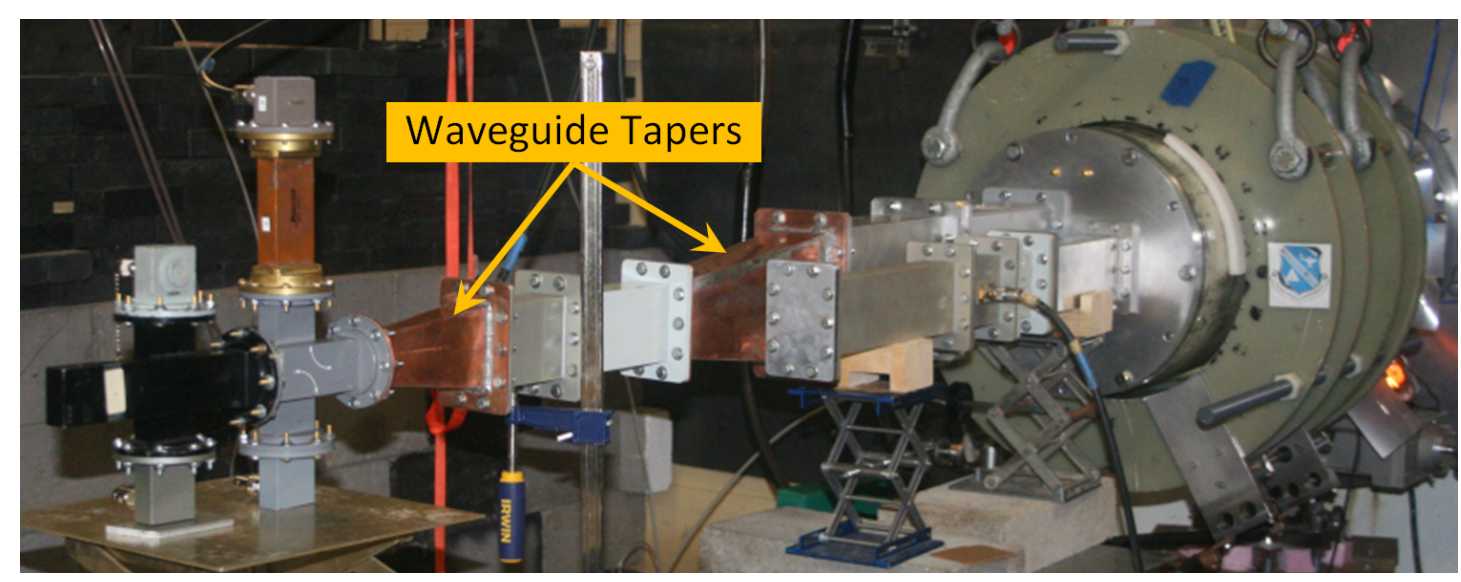

(d) Photograph of the LBO waveguide taper load configuration. The SBO load was not modified.

Figure 4.11: Microwave diagnostic configurations. 
Digital processing involved first removing any DC voltage offset, followed by application of an FFT to obtain the time-integrated Fourier transform. The frequency window was then restricted to a range appropriate for the diagnostic and SWS of interest (e.g., 0.9-1.1 GHz for the LBO, 1.9-2.1 GHz for the SBO, etc.). Within the window, the amplitudes were normalized to the largest peak, slightly smoothed to reduce excessive noise, and passed to a peak-finder function to determine and record the frequencies corresponding to the first- and second-highest peaks. These peaks were treated as the dominant and competing frequencies in the frequency window of interest. This process was applied to all channels sampled in each configuration (LBO WR-650 and SBO WR-340 for the standard load, and LBO WR-650, WR-340, and WR-187, and the SBO WR-340 for the waveguide taper load).

\subsubsection{Power Measurement}

Microwave power measurements were obtained by passing the remaining split signal from Fig. 4.11a through a series of band-pass filters to calibrated Low-Barrier Schottky Diodes (LBSDs), also called microwave detectors or crystal diodes. The calibration procedure for the diodes is described in Appendix D.1. In all cases, the configuration was designed to pass an isolated frequency to each diode. This was necessary to accurately estimate the waveguide microwave power using attenuation measurements obtained for each component (directional coupler, cable, in-line attenuators, filters, and splitter) at a single frequency $(1,2$, or $4 \mathrm{GHz})$. If a broad frequency range was passed to the diodes, the procedure would be unreliable because many of the attenuations (e.g. cables) are frequencydependant, meaning a single representative attenuation number would be inaccurate. Thus, using the spectrum measurements as confirmation, only a narrow range of frequencies were fed to each diode. The frequency range for each channel was confirmed by the calibrations to be sufficiently well-described by a single attenuation number for each component in the diagnostic chain. Calibration details can be found in Appendix D.2.

Digital signal processing of the microwave diode signals involved first removing any DC voltage offset. Due to the noise on the signal, any negative voltages (which would imply negative powers) were replaced with zeros. This replacement was also necessary due to the mathematical form of the calibration function. The diode calibration was then applied to determine the microwave power fed to the diode. Once the diode input power was known, the waveguide power could be estimated by accounting for the attenuation of each component in the configuration chain in Fig. 4.11a. For clarity, these attenuations were determined at the frequency of the input signal. 


\subsection{Pulse-Shortening Imaging Configuration}

Two imaging studies were conducted to observe plasma-related microwave pulse-shortening in each of the isolated oscillator configurations using a uniform magnetic field. The imaging configurations repurposed the MELBA-C vacuum chamber flange from the MFRPM microwave power extraction assembly to enable direct observation of the anode and cathode. For each anode configuration, the extraction antenna, coaxial outer conductor, and waveguide were removed from the microwave power extraction assembly. The MELBA-C vacuum flange was rotated such that the extraction port normally used exclusively by the LBO was aligned with the oscillator being imaged, and the extraction port was sealed using the transparent Lexan LBO microwave window. The window was then covered with wire mesh and metal tape to create an electrical seal that shielded the imaging cameras from any high-power microwaves. Figure 4.12a shows a photograph of the shielded observation port. To correlate the images with the microwave pulse-shortening, a small antenna was placed in the chamber to detect microwave oscillations. Since no microwave load was used, the anode configurations had high $Q$-factors and slightly upshifted frequencies for the different electromagnetic modes.

To assess the safety of using an expensive fast framing camera in front of the anode structures, and to assess the amount of light produced by the plasma, a Canon EOS Digital Rebel Xsi SLR camera was used for initial imaging. The camera settings used an F 5.6 aperture, $2.5 \mathrm{~s}$ exposure triggered $500 \mathrm{~ms}$ before the electromagnet trigger, and an ISO speed that was typically 400 . Figure $4.12 \mathrm{~b}$ shows the SLR camera positioned in front of the imaging port. After determining that the risk to the framing camera was low, and after verifying that shots produced a sufficiently high light yield, the configuration was modified to use the fast framing camera.

Figure 4.12c shows the fast framing camera positioned in front of the observation port. The camera was an Invisible Vision UHSi-12 fast framing camera capable of capturing 12 frames with exposure times as low as $5 \mathrm{~ns}$. Due to the relatively low plasma light yield, exposure times were $25 \mathrm{~ns}$ at minimum. The camera trigger and exposure times were adjusted to ensure the microwave pulse was captured.

Before imaging was performed for either oscillator, the results of the isolated LBO and SBO configurations were processed (as presented in Chapter 5) to identify different magnetic field regions of interest. These regions were then investigated using both the SLR and fast framing camera. The results are presented and discussed in $\S 5.8$ of Chapter 5 . 


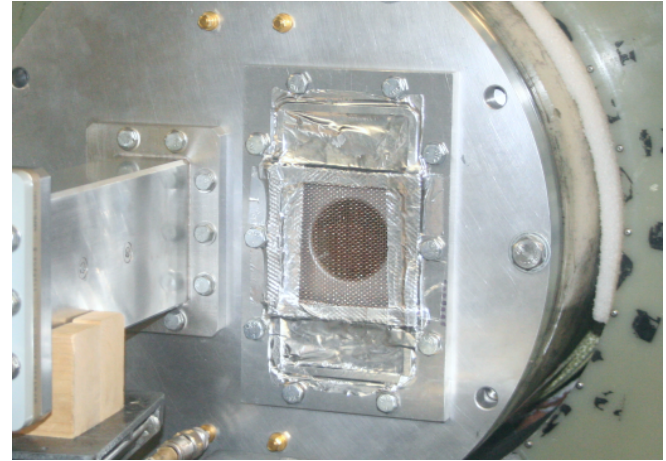

(a) Shielded LBO microwave extraction port used for direct plasma observation.

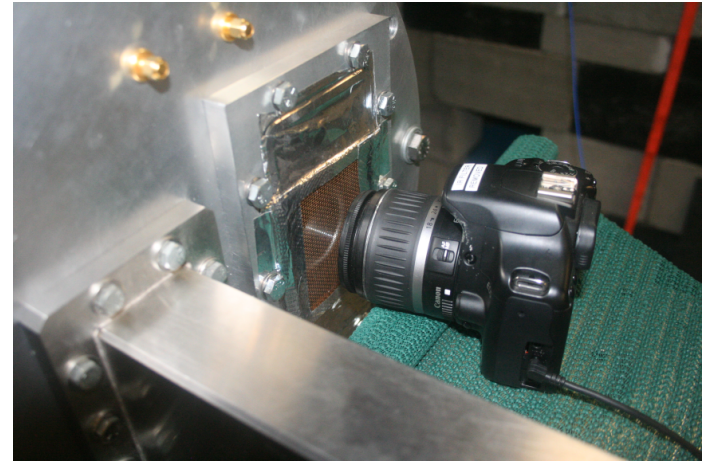

(b) SLR camera positioned in front of observation port.

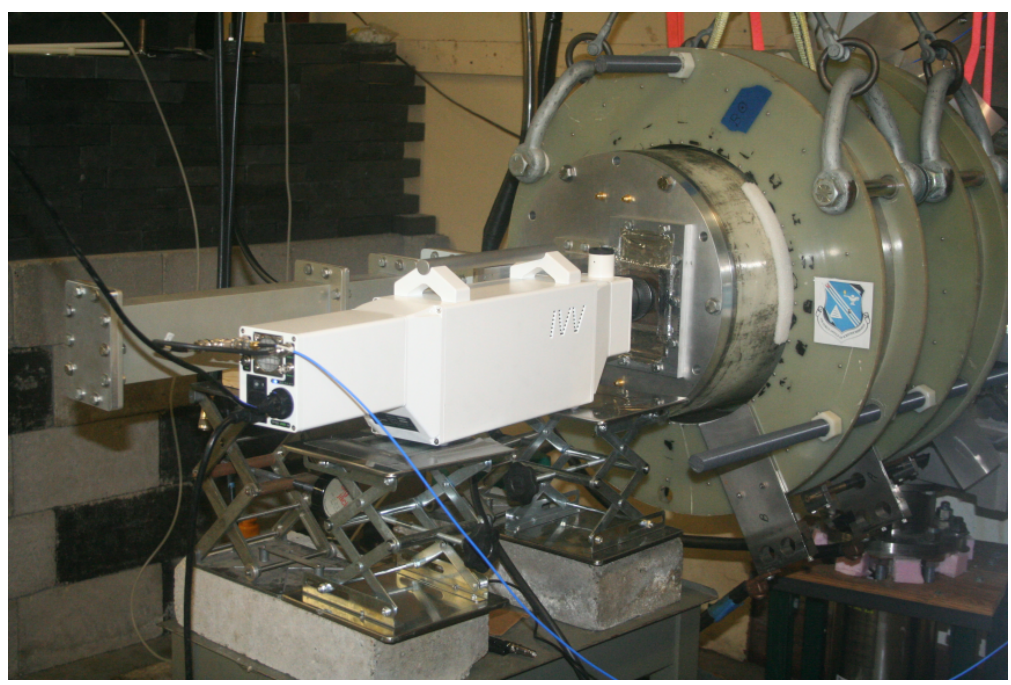

(c) Fast framing camera positioned in front of observation port.

Figure 4.12: Photographs of the pulse-shortening imaging configuration. 


\section{CHAPTER 5}

\section{Experimental Results for the Standard Load}

This chapter details the results and analysis of shots conducted using the standard load described in $\S 4.5 .2$. Four experimental configurations were tested. The first two configurations ran each oscillator in isolation, wherein the omitted oscillator was replaced with the smooth drift region described in $\S 4.1 .1$. The last two configurations tested the MFRPM with two different magnetic field profiles: a nonuniform axial magnetic field, and an axial magnetic field that was optimized to be approximately uniform throughout the Multi-Frequency Recirculating Planar Magnetron (MFRPM). The different magnetic field configurations were described in $\S \S 4.2 .1$ and 4.2.2. Note that the isolated oscillator configurations used the uniform magnetic field.

The chapter begins with a Summary of Findings section, which contains all the result tables that summarize the notable aspects of operation. Brief comments accompany each table to provide context and condense the table results into the most important points. The next section is the Operation Metrics section, which addresses the choice of plotted parameters, method of visualization, and structure of result discussion. The following sections present and discuss the experimental results in detail for the aforementioned configurations. The effects of optimizing the magnetic field for axial uniformity are then summarized and discussed. The chapter concludes with some preliminary results from the pulse-shortening imaging configuration described in $\S 4.6$.

\subsection{Summary of Findings}

In the following tables, the powers, impedances, and efficiencies were determined at the instant of peak total power. For the MFRPM anode, the total power was the summed power from both oscillators. The peak power $|\Delta t|$ was defined as $|\Delta t|=\left|t_{2}-t_{1}\right|$, where $t_{1}$ was the time at which peak power was observed from the $\mathrm{LBO}$, and $t_{2}$ was the time at which peak power was observed from the SBO. 


\subsubsection{Isolated LBO with Standard Load and Uniform Magnetic Field}

Analysis of the isolated LBO configuration produced three tables corresponding to three regions of operation. Table 5.1 (the first region) shows the performance near $0.16 \mathrm{~T}$, which was the intended magnetic field for operation. As expected, the $\pi$-mode was the dominant mode. Shots in this region exhibited low variability in frequency and power. Table 5.2 (the second region) operated in the $5 \pi / 6$-mode, which exhibited lower microwave power than the $\pi$-mode (as expected based on simulations). Table 5.3 (the third region) shows the performance near $0.23 \mathrm{~T}$, also in the $\pi$-mode, where the highest microwave powers (32 MW) were observed. The optimal operating magnetic field for the isolated LBO was therefore $0.23 \mathrm{~T}$. Shots in this region exhibited low variability in frequency and power. The $\pi$-mode operation at this unusually high magnetic field (relative to the Buneman-Hartree threshold of $0.16 \mathrm{~T}$, see Fig. 2.6) may be due to synchronism with a $\pi$-mode space harmonic.

Table 5.1: Isolated LBO: Summarized performance metrics near 0.16 T.

\begin{tabular}{lrlll}
\hline \hline$\pi$-Mode Power: & 25 & \pm & 2 & $\mathrm{MW}$ \\
$\pi$-Mode Frequency: & 0.986 & \pm & 0.003 & $\mathrm{GHz}$ \\
Impedance: & 124 & \pm & 8 & $\Omega$ \\
Efficiency: & 5 & \pm & 1 & $\%$ \\
\hline \hline
\end{tabular}

Table 5.2: Isolated LBO: Summarized performance metrics for the $5 \pi / 6$ mode near $B=$ $0.19 \mathrm{~T}$.

\begin{tabular}{lrlcl}
\hline \hline $5 \pi / 6$-Mode Power: & 17 & \pm & 2 & $\mathrm{MW}$ \\
$5 \pi / 6$-Mode Frequency: & 0.972 & \pm & 0.005 & $\mathrm{GHz}$ \\
Impedance: & 154 & \pm & 21 & $\Omega$ \\
Efficiency: & 4 & \pm & 1 & $\%$ \\
\hline \hline
\end{tabular}

Table 5.3: Isolated LBO: Summarized performance metrics for the region of optimal operation in $B=0.22-0.25 \mathrm{~T}$.

\begin{tabular}{lclcl}
\hline \hline$\pi$-Mode Power: & 32 & \pm & 4 & $\mathrm{MW}$ \\
$\pi$-Mode Frequency: & 0.985 & \pm & 0.003 & $\mathrm{GHz}$ \\
Impedance: & 200 & \pm & 30 & $\Omega$ \\
Efficiency: & 8 & \pm & 2 & $\%$ \\
\hline \hline
\end{tabular}

\subsubsection{Isolated SBO with Standard Load and Uniform Magnetic Field}

Analysis of the isolated SBO configuration produced one table corresponding to the region of optimal operation (Table 5.4). The optimal magnetic field was $0.17 \mathrm{~T}$, which was 
in good agreement with the intended magnetic field for operation $(0.16 \mathrm{~T})$. As expected, the $\pi$-mode was the dominant mode, which produced $18 \mathrm{MW}$. Shots in this region exhibited low variability in frequency and power. The other identified modes from the analysis were the $6 \pi / 8$-mode $(1.94 \mathrm{GHz})$ and the $7 \pi / 8$-mode $(2 \mathrm{GHz})$.

Table 5.4: Isolated SBO: Summarized performance metrics for the region of optimal operation near $B=0.16 \mathrm{~T}$.

\begin{tabular}{lcccl}
\hline \hline$\pi$-Mode Power: & 18 & \pm & 2 & $\mathrm{MW}$ \\
$\pi$-Mode Frequency: & 2.022 & \pm & 0.004 & $\mathrm{GHz}$ \\
Impedance: & 236 & \pm & 21 & $\Omega$ \\
Efficiency: & 7 & \pm & 2 & $\%$ \\
\hline \hline
\end{tabular}

\subsubsection{MFRPM with Standard Load and Nonuniform Magnetic Field}

Analysis of the MFRPM with the standard load and nonuniform magnetic field produced three tables corresponding to two regions of operation and a separate analysis of the parasitic chamber mode. Table 5.5 (the first region) shows the performance in $B=0.21-$ $0.23 \mathrm{~T}$, where the highest microwave powers were observed. Both oscillators operated in the $\pi$-mode. The optimal magnetic field was $B=0.23 \mathrm{~T}$. Shots exhibited high variability in frequency and power. Table 5.6 (the second region) shows the performance in $B=0.14-$ $0.17 \mathrm{~T}$, where the SBO consistently operated in the $7 \pi / 8$-mode. This region characterized the $7 \pi / 8$-mode with high confidence, and confirmed that the $7 \pi / 8$-mode produces lower power than the $\pi$-mode. The parasitic chamber mode (Table 5.7) was observed at all tested magnetic fields, and was evidently capable of producing powers near $15 \mathrm{MW}$, which contradicted the results from simulations. This experimental configuration provided valuable evidence supporting the conclusion that harmonic frequency-locking could occur between the LBO $\pi$-mode and the SBO $6 \pi / 8$-mode, although the locked state never dominated in this configuration. The free-running SBO $6 \pi / 8$-mode frequency was observed at $1.94 \mathrm{GHz}$. 
Table 5.5: Standard Load and Nonuniform Magnetic Field: Summarized performance metrics for the region of optimal operation in $B=0.21-0.23 \mathrm{~T}$.

\begin{tabular}{lclll}
\hline \hline LBO $\pi$-Mode Power: & 21 & \pm & 9 & $\mathrm{MW}$ \\
LBO $\pi$-Mode Frequency: & 0.980 & \pm & 0.021 & $\mathrm{GHz}$ \\
SBO $\pi$-Mode Power: & 12 & \pm & 4 & $\mathrm{MW}$ \\
SBO $\pi$-Mode Frequency: & 2.012 & \pm & 0.013 & $\mathrm{GHz}$ \\
Total Power: & 27 & \pm & 10 & $\mathrm{MW}$ \\
Peak Power $|\Delta t|:$ & 25 & \pm & 19 & $\mathrm{~ns}$ \\
Impedance: & 94 & \pm & 15 & $\Omega$ \\
Efficiency: & 4 & \pm & 1 & $\%$ \\
\hline \hline
\end{tabular}

Table 5.6: Standard Load and Nonuniform Magnetic Field: Summarized performance metrics in $B=0.14-0.17 \mathrm{~T}$, wherein the SBO operated in the $7 \pi / 8$-mode.

\begin{tabular}{lclll}
\hline \hline LBO $\pi$-Mode Power: & 10 & \pm & 3 & $\mathrm{MW}$ \\
LBO $\pi$-Mode Frequency: & 0.988 & \pm & 0.021 & $\mathrm{GHz}$ \\
SBO 7 $\pi$ /8-Mode Power: & 3 & \pm & 1 & $\mathrm{MW}$ \\
SBO 7 $\pi$ /8-Mode Frequency: & 1.999 & \pm & 0.003 & $\mathrm{GHz}$ \\
Total Power: & 12 & \pm & 3 & $\mathrm{MW}$ \\
Peak Power $|\Delta t|:$ & 18 & \pm & 14 & $\mathrm{~ns}$ \\
Impedance: & 50 & \pm & 4 & $\Omega$ \\
Efficiency: & 1 & \pm & 1 & $\%$ \\
\hline \hline
\end{tabular}

Table 5.7: Standard Load and Nonuniform Magnetic Field: Summarized performance metrics for the LBO parasitic chamber mode.

\begin{tabular}{lclll}
\hline \hline LBO Chamber Mode Power: & 15 & \pm & 5 & $\mathrm{MW}$ \\
LBO Chamber Mode Frequency: & 1.040 & \pm & 0.001 & $\mathrm{GHz}$ \\
\hline \hline
\end{tabular}

\subsubsection{MFRPM with Standard Load and Uniform Magnetic Field}

Analysis of the MFRPM with the standard load and uniform magnetic field produced two tables. Table 5.8 shows the performance of the MFRPM in the region of optimal performance in $B=0.16-0.18 \mathrm{~T}$. The optimal magnetic field was $0.17 \mathrm{~T}$. A novel harmonic frequency-locked state was observed wherein the SBO $6 \pi / 8$-mode frequency-locked to the second harmonic frequency of the LBO. The locked state was characterized by remarkably low variability in powers and frequencies. The results of a phase analysis are shown in Table 5.9. The shot-to-shot variability in the phase difference between the LBO and SBO was too great to be considered phase-locking. However, the phase difference did correlate 
with changes in the magnetic field, which suggested the coupling between the LBO and SBO was related to the electron beam kinetics.

Table 5.8: Standard Load and Uniform Magnetic Field: Summarized performance metrics in $B=0.16-0.18 \mathrm{~T}$ wherein consistent frequency-locking occurred.

\begin{tabular}{lclll}
\hline \hline LBO $\pi$-Mode Power: & 32 & \pm & 3 & $\mathrm{MW}$ \\
LBO $\pi$-Mode Frequency: & 0.984 & \pm & 0.001 & $\mathrm{GHz}$ \\
SBO 6 $\pi$ /8-Mode Power: & 13 & \pm & 2 & $\mathrm{MW}$ \\
SBO 6 $\pi$ /8-Mode Frequency: & 1.970 & \pm & 0.002 & $\mathrm{GHz}$ \\
Total Power: & 40 & \pm & 4 & $\mathrm{MW}$ \\
Peak Power $|\Delta t|:$ & 4 & \pm & 3 & $\mathrm{~ns}$ \\
Impedance: & 139 & \pm & 7 & $\Omega$ \\
Efficiency: & 10 & \pm & 1 & $\%$ \\
\hline \hline
\end{tabular}

Table 5.9: Standard Load and Uniform Magnetic Field: Phase metrics in $B=0.16-0.17 \mathrm{~T}$ encompassing peak power production, wherein consistent frequency-locking and preferential selection of a relative phase difference occurred.

\begin{tabular}{lrlrl}
\hline \hline Phase Difference: & 93 & \pm & 17 & $\circ$ \\
Phase Variation: & 8 & \pm & 4 & $\circ$ \\
Duration: & 14 & \pm & 3 & $\mathrm{~ns}$ \\
\hline \hline
\end{tabular}

\subsubsection{Comparison of Metrics}

Two tables compare the operation of the isolated oscillators with the MFRPM and summarize the impact of the magnetic field optimization. All metrics were taken from the regions of optimal operation for each respective configuration.

Table 5.10 compares the performance metrics for the individual oscillators. The LBO operated similarly in both the isolated configuration and in the MFRPM using the uniform magnetic field, whereas the SBO generated $28 \%$ less power (13 MW) in a different mode (the harmonic frequency-locked $1.970 \mathrm{GHz} 6 \pi / 8$-mode) relative to the isolated configuration (18 MW in the $2.022 \mathrm{GHz} \pi$-mode). In the harmonic frequency-locked state, the variability of the powers and frequencies was the lowest of any tested configuration. Across all tested metrics, the individual oscillators performed better using the uniform magnetic field compared to the nonuniform magnetic field. The nonuniform magnetic field yielded lower microwave powers with higher variability, and the shot-to-shot frequency variability was also higher.

Table 5.11 compares the performance metrics for the MFRPM as a whole. Here again, the MFRPM performance was improved by the uniform magnetic field. Notably, in the 
harmonic frequency-locked state, the time difference between peak powers from the two oscillators (Peak Power $|\Delta t|$ ) was very small, which indicates the MFRPM operated as a single unit rather than two independent oscillators.

Table 5.10: Standard Load: Comparison of metrics for individual oscillators.

\begin{tabular}{llcccl}
\hline \hline CONFIGURATION & METRIC & VALUE & & STD. DEV. & UNITS \\
\hline Unif. $B$, Iso. LBO & LBO $\pi$-Mode Power: & 32 & \pm & 4 & MW \\
Nonunif. $B$, MFRPM & LBO $\pi$-Mode Power: & 21 & \pm & 9 & MW \\
Unif. $B$, MFRPM & LBO $\pi$-Mode Power: & 32 & \pm & 3 & $\mathrm{MW}$ \\
Unif. $B$, Iso. LBO & LBO $\pi$-Mode Frequency: & 0.985 & \pm & 0.003 & $\mathrm{GHz}$ \\
Nonunif. $B$, MFRPM & LBO $\pi$-Mode Frequency: & 0.980 & \pm & 0.021 & $\mathrm{GHz}$ \\
Unif. $B$, MFRPM & LBO $\pi$-Mode Frequency: & 0.984 & \pm & 0.001 & $\mathrm{GHz}$ \\
Unif. $B$, Iso. SBO & SBO $\pi$-Mode Power: & 18 & \pm & 2 & $\mathrm{MW}$ \\
Nonunif. $B$, MFRPM & SBO $\pi$-Mode Power: & 12 & \pm & 4 & $\mathrm{MW}$ \\
Unif. $B$, MFRPM & SBO $6 \pi / 8$-Mode Power: & 13 & \pm & 2 & $\mathrm{MW}$ \\
Unif. $B$, Iso. SBO & SBO $\pi$-Mode Frequency: & 2.022 & \pm & 0.004 & $\mathrm{GHz}$ \\
Nonunif. $B$, MFRPM & SBO $\pi$-Mode Frequency: & 2.012 & \pm & 0.013 & $\mathrm{GHz}$ \\
Unif. $B$, MFRPM & SBO $6 \pi / 8$-Mode Frequency: & 1.970 & \pm & 0.002 & $\mathrm{GHz}$ \\
\hline \hline
\end{tabular}

Table 5.11: Standard Load: Comparison of MFRPM metrics.

\begin{tabular}{llrrrl}
\hline \hline ConfigurATION & METRIC & VAluE & & StD. DeV. & UNITS \\
\hline Nonunif. $B$ & Total Power: & 27 & \pm & 10 & MW \\
Unif. $B$ & Total Power: & 40 & \pm & 4 & MW \\
Nonunif. $B$ & Peak Power $|\Delta t|:$ & 25 & \pm & 19 & $\mathrm{~ns}$ \\
Unif. $B$ & Peak Power $|\Delta t|:$ & 4 & \pm & 3 & $\mathrm{~ns}$ \\
Nonunif. $B$ & Impedance: & 94 & \pm & 15 & $\Omega$ \\
Unif. $B$ & Impedance: & 139 & \pm & 7 & $\Omega$ \\
Nonunif. $B$ & Efficiency: & 4 & \pm & 1 & $\%$ \\
Unif. $B$ & Efficiency: & 10 & \pm & 1 & $\%$ \\
\hline \hline
\end{tabular}




\subsection{Operation Metrics}

The results for each experimental configuration are divided into two sections: Standard Metrics and Additional Analysis. The standard metrics were shared diagnostics that were applied to all experimental configurations for later comparison. The Additional Analysis sections include plots and metrics specific to the configuration and discussion of operating behavior that was unique to the shot series.

\subsubsection{Standard Metrics}

The standard metrics were those parameters common to all experiments conducted in this chapter. The selection of plotted quantities and method of visualization used for each plot were chosen to elucidate the physics of operation. A description of each follows, along with examples of interpretation. The standard metrics include the following plots: 1) Power vs. Magnetic Field, 2) Frequency vs. Magnetic Field, 3) Frequency Histograms, 4) Power vs. Frequency, 5) Current vs. Magnetic Field, 6) Impedance vs. Magnetic Field, 7) Start and Peak Power Voltages vs. Magnetic Field, 8) Efficiency vs. Magnetic Field, 9) Energy vs. Magnetic Field, 10) Start and Peak Power Times vs. Magnetic Field, and 11) Peak Power $|\Delta t|$ vs. Magnetic Field.

The first four plots establish the relation between the magnetic field, the different operating electromagnetic modes, and the modes' respective frequencies and powers. The Power vs. Magnetic Field plot is the most important metric because the objective of the project was to develop a dual-frequency, high-power microwave source. From this plot (and, in part, the Power vs. Frequency plot), different regions of operation were identified wherein consistent and distinct operation was observed. These regions of operation were used to obtain the summary of parameters tabulated in the standard metric tables, which were presented in the Summary of Findings in $\S 5.1$. The Frequency vs. Magnetic Field plot shows both the dominant and competing frequencies observed from each oscillator for each shot vs. magnetic field, where the dominant and competing frequencies are defined as the highest and second highest peaks observed in the time-integrated Fourier transform of the RF voltage signal. This illustrates the stability of an electromagnetic mode's frequency, the consistency and degree of mode competition, and regions of transition from one operating mode to another. When compared with the Current vs. Magnetic Field plot, the degree of beam-loading on the frequency can also be determined. Described earlier in $\S 2.1$, frequency-pushing or beam-loading is a phenomenon where the resonant frequency changes with current [6]. The Frequency Histogram essentially collapses the Frequency vs. Magnetic Field plot onto the vertical axis. It provides a visually convenient method of 
determining which frequencies exist and how they distribute, thereby aiding the identification of the different electromagnetic modes. In isolation, the histogram can be misleading if a single electromagnetic mode's frequency changes substantially with magnetic field due to beam-loading or other effects, so comparison with the Frequency vs. Magnetic Field plot is essential. The last plot of this group is the Power vs. Frequency plot, which relates the extracted microwave power to the dominant operating electromagnetic mode. This plot quickly identifies frequencies that do and do not couple effectively to the microwave extractor, which suggests an even- or odd-mode electric field geometry for that frequency. Field geometries were previously discussed in $\S 3.3 .1$. Other factors, such as the electronic efficiency and impedance of the electromagnetic mode, can also affect the power. This plot can also be misleading if a single electromagnetic mode's frequency changes substantially with magnetic field, so comparison with the Frequency vs. Magnetic Field plot is necessary.

The next three plots show the basic electrical characteristics vs. magnetic field: current, impedance, and voltage. Currents and impedances were determined at the time of peak total output power. As previously stated, the Current vs. Magnetic Field plot can be compared with the Frequency vs. Magnetic Field plot to determine the degree of beam-loading. The Impedance vs. Magnetic Field plot diagnoses whether the operating impedance at a given magnetic field was matched to the design impedance of the MELBA-C pulser. As described in $\S 4.3$, the pulsed-power source was designed for 100-150 $\Omega$ [98]. Low impedance can be particularly detrimental to voltage pulse rise-times, as shown by Figs. C.6a vs. C.6b on page 188 in the appendix. Poor impedance matching can lead to poor voltage pulse shapes during the rise-time, which can have a detrimental effect on magnetron start-up, mode stability, and microwave pulselengths [103]. Finally, the plot of Start and Peak Power Voltages vs. Magnetic Field provides information about beam synchronism and offers insight into the operation of the different electromagnetic modes. For instance, linear scaling of voltage at peak power with magnetic field may indicate a favorable beam velocity that is well-synchronized with a given mode. This is due to the electron beam velocity $v \simeq E / B \propto V / B$, so plots of voltage vs. magnetic field having a linear distribution of points suggest a constant beam velocity $v$ was favored for start-up or peak microwave power generation. Low start-up voltages at low magnetic fields might be due to the larger electron hub height. When the magnetic field is low, the hub edge is closer to the anode (see Eq. 2.7 on page 16), which may increase beam coupling to the anode fields, thereby reducing start times. If the magnetron starts at a very different voltage compared to the peak power voltage, it may indicate a region of mode competition where the start-up mode is different from the high-power mode. The start voltage was defined as the voltage at which $10 \%$ of peak power was first observed. Shot series using the MFRPM use the same voltage 
scales for both the LBO and SBO plots for easy comparison.

The next two plots show the energy conversion metrics. The Efficiency vs. Magnetic Field plot illustrates the peak total efficiency, where the total efficiency is derived from the total output power (defined as the summed powers from each output waveguide) and total current entering the magnetron. The total efficiency is defined differently from the electronic efficiency, which is derived using only the current collected by the anode [66]. The differences in current are largely due to endloss, and because the experimental configuration was not conducive to measurement of forward- and backstreaming-endloss currents, only the total efficiency could be calculated. The Energy vs. Magnetic Field plot shows the total microwave energy produced over the duration of a shot. The output energy offers some insight into the electron emission physics, microwave pulse-shortening mechanisms, and the consistency of operation. It has been postulated in the literature that cathode plasma expansion from explosive-emission cathodes leads to a constant-energy limit $[89,104]$, though more compelling models show scaling of pulse energy limits with pulselength $[17,105]$. If the MFRPM results disagreed with these models, it would suggest a different pulse-shortening mechanism was responsible for the observed behavior.

The last standard plots illustrate the temporal metrics. The first of these is the Start and Peak Power Times vs. Magnetic Field plot. Like the start voltage, the start time of an oscillator was defined as the time at which $10 \%$ of peak power was first observed. This provides insight into the oscillators' different degrees of beam coupling. As discussed in $\S 2.1$, the analytic model of the planar cavity arrays shows that the LBO has stronger fringing cavity fields in the AK gap relative to the SBO, which may lead to stronger LBO beam coupling and faster start-up (although the effects of stronger coupling on start-up may be diminished or negated by the stronger LBO coupling to the extractor relative to the SBO). The times at which peak powers were observed factor into the evaluation of operational consistency, and can indirectly provide evidence for the degree of coupling between the oscillators by determining the peak power $|\Delta t|$ described below. As discussed at length in Appendix $\mathrm{C}$, the MELBA-C pulser has significant jitter in its start time (typically $<100 \mathrm{~ns}$ ). To compensate for this fact, all plotted traces were shifted such that a bank voltage $V=100 \mathrm{kV}$ always corresponded to time $t=100 \mathrm{~ns}$. Figure 5.1 illustrates several overlaid shots with the applied time shifts. Shot series using the MFRPM use the same time scales for both the LBO and SBO plots for easy comparison. The second temporal plot shows the Peak Power $|\Delta t|$ vs. Magnetic Field, which measures how well the two oscillators operated as a single unit. The peak power $|\Delta t|$ was defined as $|\Delta t|=\left|t_{2}-t_{1}\right|$, where $t_{1}$ was the time at which peak power was observed from the LBO, and $t_{2}$ was the time at which peak power was observed from the SBO. Pulselengths were not chosen as a metric because the rel- 


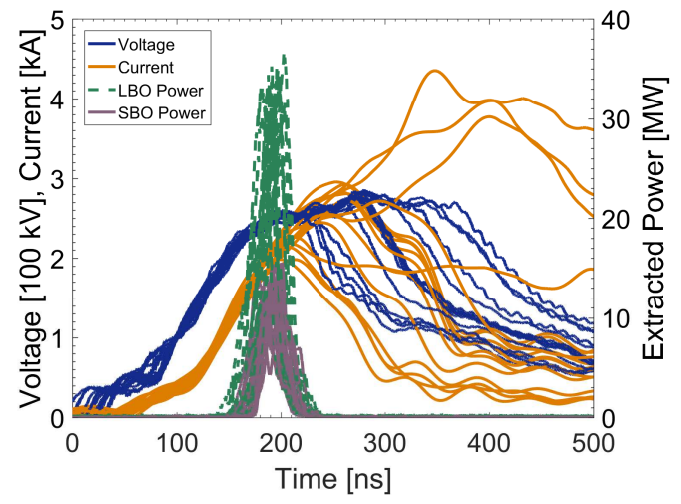

Figure 5.1: Overlay of multiple time-shifted shots compensating for jitter in the MELBA-C start time.

atively high- $Q$ configuration of the experiment means the best shots experience excessive pulse-shortening due to abnormally high electric fields that would be lower in a design with an optimized extractor. These shots therefore exhibit the shortest pulselengths, making the pulselength metric a poor one for this dissertation. Pulse-shortening will be discussed in $\S 5.8$.

For clarity, the inclusion or omission of error bars in plots was based on whether the plot was intended to show a pattern facilitated by averaging, and whether the averaging would conceal a significant and noteworthy spread in the data (e.g. in the case of competing frequencies) or discontinuities (e.g. mode hops in frequency). The points on plots that have error bars were generated using a fixed number of shots per point. The number of shots per point was chosen to produce 10-12 points on the plots, provided that the number of shots per point was at least five. The error bars indicate the standard deviation for that group of shots represented by each point. A range of magnetic fields that was investigated more closely than others will therefore have closer spacing between points with error bars. The limited number of shots that can be practicably obtained using non-rep-rated pulsed power drivers leads to small data sets with poor statistics relative to many other fields of study. Despite this fact, visualizing the results using the aforementioned process conveys the presence or absence of shot-to-shot consistency.

Finally, the most important metrics were summarized in tables for each region of operation, which were presented in the Summary of Findings section at the beginning of the chapter. These tables include the power and frequency for each observed mode for each oscillator, as well as the impedance and efficiency. For shot series using the MFRPM, the total power and peak power $|\Delta t|$ is also tabulated.

The identified regions of operation include regions of optimal operation and regions in 
which unusual behavior was observed. The regions of optimal operation are identified in the Standard Metrics sections based on analysis of the various plots, and the results are compared to determine the operational impact of the different magnetic field configurations in $\S 5.7$. The regions of operation in which unusual behavior was observed, such as operation in a mode other than the $\pi$-mode, are identified in the Additional Analysis sections.

\subsubsection{Additional Analysis}

The Additional Analysis sections present the analysis of performance specific to a shot series and they identify the observed operating modes. A few interesting shots may be discussed using shot plots, Fourier transforms, or Time-Frequency Analysis (TFA) plots (discussed below). Shot plots show the MELBA-C voltage, current, and microwave powers, and the Fourier transform plots show the time-integrated Fast Fourier Transform (FFT) of the RF voltage signal from the oscillators.

\subsubsection{Time-Frequency Analysis}

Shots exhibiting particularly interesting behavior, such as mode competition or generation of high microwave powers at very different cathode voltages, were also examined using a TFA [106]. A TFA is the Fourier transform of the local autocorrelation function with a Reduced Interference Kernel (RIK) applied to the signal, which can be either the original $\mathrm{RF}$ voltage or the analytic representation of the signal [107,108]. A window function is then applied to the autocorrelation function prior to application of the Fourier transform. A detailed explanation of the underlying mathematics and implementation of the TFA can be found in [109]. Unless otherwise specified, all TFA plots in this dissertation were generated using either the Zhao-Atlas-Marks or Born-Jordan distribution as the RIK, and a Hanning window function applied to the analytic representation of the original RF voltage signal.

Examples of different RIKs and resolutions are shown in Fig 5.2. The need for a RIK is apparent from Fig. 5.2a, which shows clear contamination from cross terms leading to artificial frequencies at $1.5,2.5$, and $3 \mathrm{GHz}$. The Wigner distribution has a kernel function equal to one, meaning it functionally has no RIK. Using the same resolution settings, Figs.5.2b-5.2d were generated using other RIKs. Under these conditions, some RIKs are more effective than others at reducing the contamination due to cross terms.

In TFA plots, the ability to resolve the frequencies is closely related to the width of the temporal processing window in the discretized representation of the mathematics. High frequency resolution can only be realized at the expense of temporal resolution, and vice- 


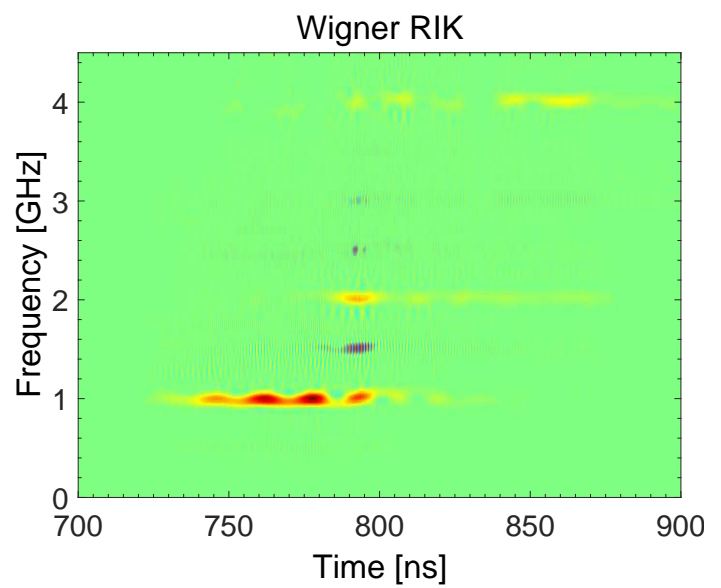

(a) TFA with high time resolution and the Wigner RIK.

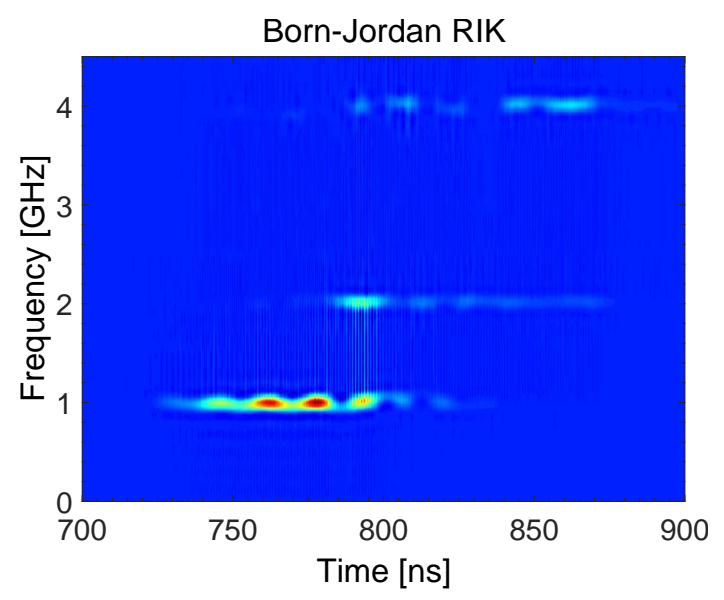

(c) TFA with high time resolution and the BornJordan RIK.

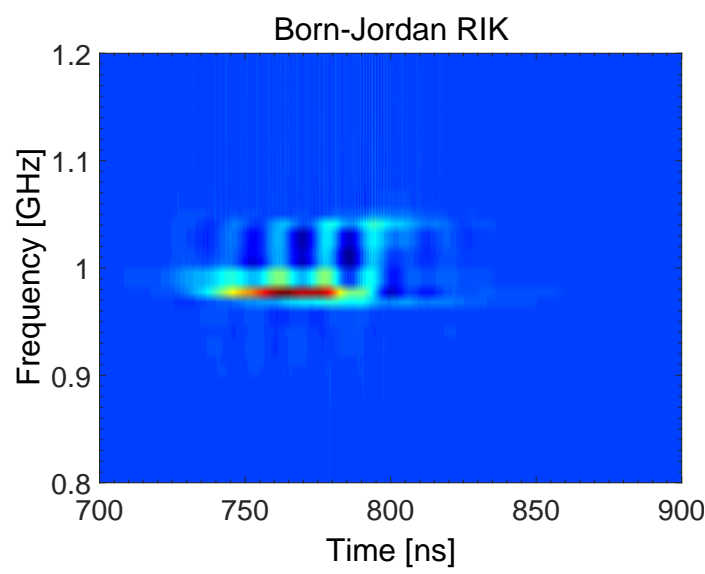

(e) TFA with high frequency resolution and the Born-Jordan RIK.

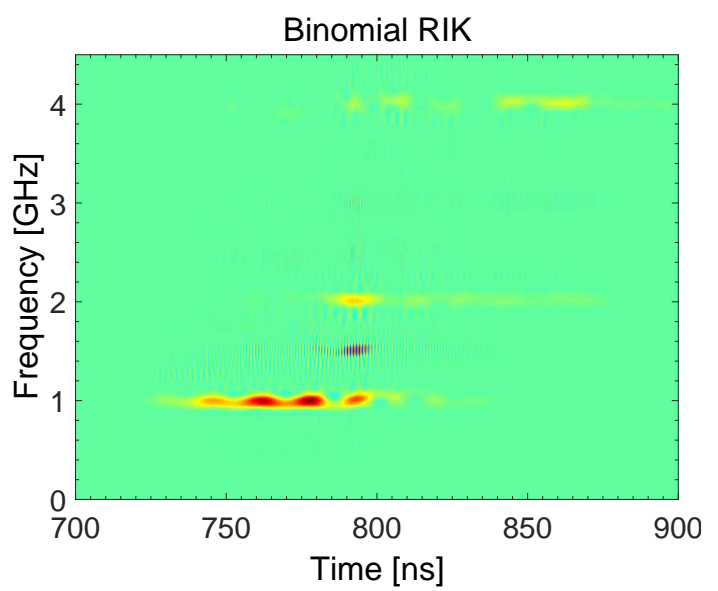

(b) TFA with high time resolution and the binomial RIK.

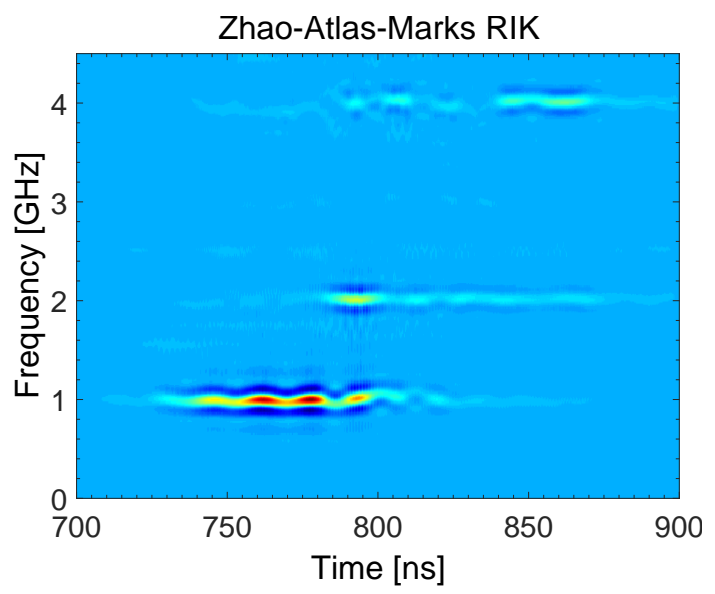

(d) TFA with high time resolution and the ZhaoAtlas-Marks RIK.

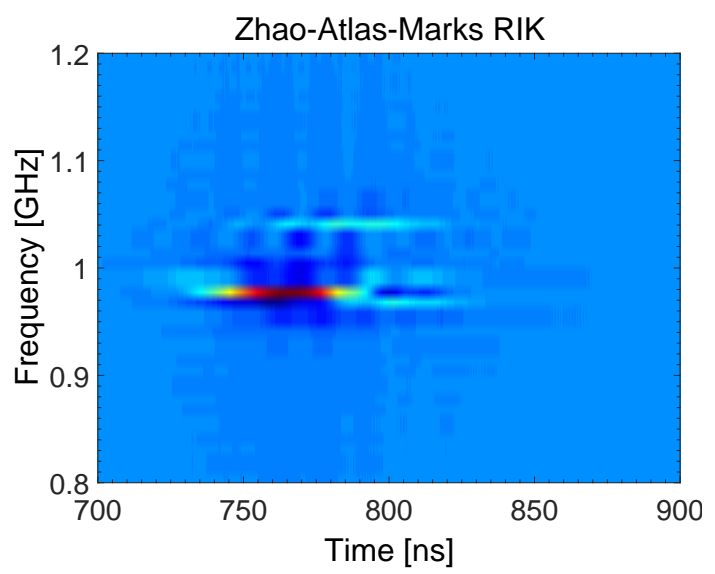

(f) TFA with high frequency resolution and the Zhao-Atlas-Marks RIK.

Figure 5.2: TFA plots of the RF voltage from the LBO on shot 13597 using different resolutions and RIKs. 
versa. In shot 13597 , the LBO initially oscillates in both the $\pi$-mode $(0.98 \mathrm{GHz})$ and the parasitic chamber mode $(1.04 \mathrm{GHz})$. This causes beating in the signal amplitude, which can be seen in Figs. 5.2c and 5.2d. However, this high time resolution results in low frequency resolution, and it is not clear that two frequencies are present. Processing using a wider temporal window results in Figs. 5.2e and 5.2f, which clearly show the two frequencies, but the beating is considerably less obvious (the beating visible in Fig. 5.2e is easily interpreted as a cross term). Generally, for time-resolved plots, the Born-Jordan RIK yielded better results, whereas the Zhao-Atlas-Marks RIK was more effective for frequency-resolved plots, though obtaining the best visualization required generating plots using both RIKs with different temporal resolutions.

\subsubsection{Phase Analysis}

For shots exhibiting harmonic frequency-locked operation between the dominant frequencies produced by the two oscillators, a phase analysis was performed to determine whether the relative phase difference between the oscillators was consistent on a shot-toshot basis. This property is termed phase-locking, which is a more stringent criterion than frequency-locking [13].

Determination of the relative phase difference between the two oscillators required several processing steps. First, the unprocessed RF voltage signals from each oscillator were digitally filtered using a low-pass filter with a threshold frequency slightly higher than the dominant frequency. This was particularly important for the LBO signal, which typically suffered heavy contamination due to overcoupling of frequency harmonics by the WR-650 loop directional coupler. Next, a polynomial spline was fit to further filter the signal and increase the number of samples per unit time to produce more continuous traces [110]. Finally, a 5 ns moving window average was applied. An example is shown by Fig. 5.3, which shows the raw signal and the processed signal. Despite the significant filtering and digital processing, the processed signal remains representative of the original signal. After processing, the signal contains more samples per unit time and has a significant reduction in high-frequency contamination.

The phases of the signals were then extracted from their analytic representation obtained using the Hilbert transform $[107,108]$. The phases were then converted from radians to degrees. However, a subtle point of comparison between the two oscillators lies in determining a reference point of $0^{\circ}$ for one cycle of the LBO and two cycles of the SBO, since the SBO frequency is twice that of the LBO. Ordinarily, to compare the phases of two signals, the modulo operation is applied after subtracting the phases to obtain the phase difference modulo $360^{\circ}$, which produces a set of values between $0-360^{\circ}$. A phase differ- 


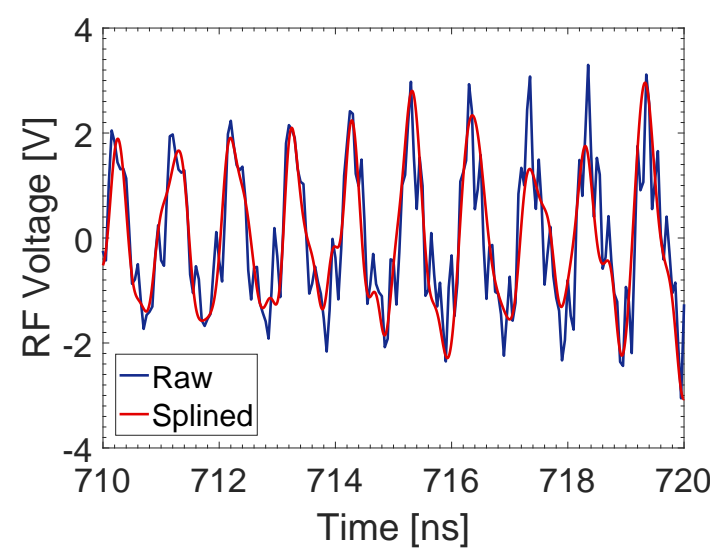

Figure 5.3: Sample of the RF voltage signal from the LBO on shot 14610 before and after splining and filtering.

ence having a slipping value near zero would be problematic because it wraps to a number slightly less than 360 , which produces artificially large phase jumps despite small changes in the actual phase difference, so the final step involves reflecting values about $180^{\circ}$, where any phase difference $\Delta \phi>180$ is mapped to $\Delta \phi \rightarrow 360-\Delta \phi[40]$.

The problem of comparing the phases of two signals having different frequencies becomes apparent when considering that two sets of phases between $0-360^{\circ}$ exist for the SBO per LBO cycle. Simply dividing the phase of the SBO by two prior to performing both subtraction and the modulo operation fails to produce a unique set of phase differences because, intuitively, two identical phase points in the SBO signal per LBO cycle can produce two different answers for the phase difference relative to the LBO. This is incorrect because a $360^{\circ}$ shift of the SBO signal should produce an identical phase difference relative to the LBO. The procedure chosen to uniquely compare the phases was to multiply the LBO phase by two prior to applying the modulo operation, which establishes a unique phase difference between the LBO and SBO per single LBO cycle (or, equivalently, per two SBO cycles). The phase difference was then determined by subtracting the LBO phase from the SBO phase, applying the modulo 360 operation, and wrapping the phase to a set of values between $0-180^{\circ}$.

To determine whether a shot demonstrated harmonic frequency- or phase-locking, the mean and standard deviation of the phase difference were extracted over a specified time window. The time window was determined by choosing the time during which both oscillators were operating at high power. The threshold for "high power" was set at $67 \%$ of the oscillators' respective peak power, and the window was determined by the latest start time and earliest end time of the two oscillators. If no window existed satisfying these criteria, 
the shot was obviously unlocked because the oscillators turned on and off at very different times, and the MFRPM failed to operate as a single unit. Locked cases have small standard deviations in the phase difference, and unlocked cases have large standard deviations in the phase difference. The section where a phase analysis was performed discusses an example in depth to further clarify the phase analysis process.

\subsection{Isolated LBO with Standard Load and Uniform Mag- netic Field}

This shot series tested the LBO in isolation to characterize operation of the planar cavity array without interference from the SBO or a mismatched microwave load. The SBO was replaced with the smooth drift region previously described in $\S 4.1 .1$, and all shots were conducted using the uniform magnetic field discussed in $\S 4.2 .2$. A total of 54 shots were obtained with no MELBA-C self-triggers, magnet triggering failures, or failed crowbars. The range of magnetic fields spanned $0.12-0.27 \mathrm{~T}$, and the base pressure was $0.7 \mu$ torr. While no antenna was connected to the omitted SBO, the copper outer conductor of the microwave extractor coaxial transmission line was left in place, as was the WR-340 output waveguide and absorbing load.

\subsubsection{Standard Metrics}

Figure 5.4 shows the peak microwave output power from the LBO vs. magnetic field. Immediately apparent was the surprising double peak appearing over the range of tested magnetic fields. As expected, the first peak at $24 \mathrm{MW}$ occurred near the Buneman-Hartree

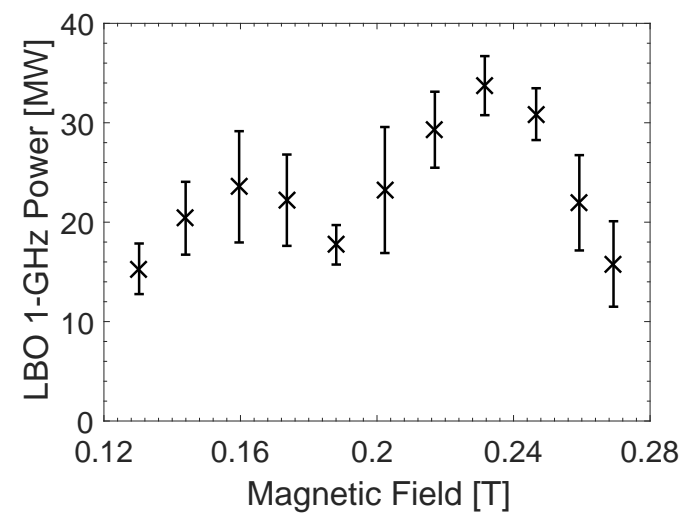

Figure 5.4: Isolated LBO: Peak microwave output powers near $1 \mathrm{GHz}$ vs. magnetic field. 
threshold magnetic field $B_{\mathrm{BH}} \simeq 0.16 \mathrm{~T}$ for $\pi$-mode operation. However, the second peak at $34 \mathrm{MW}$ near $B=0.23 \mathrm{~T}$ was unexpected. One explanation for this behavior is synchronism with the next space harmonic of the $\pi$-mode, also termed the $3 \pi$-mode. However, the expected magnetic field for $3 \pi$-mode operation based on theory would be approximately three times that of the Buneman-Hartree magnetic field $B_{\mathrm{BH}}=0.16 \mathrm{~T}$. While the experimental geometry does deviate from the assumptions made by the theory, the substantial discrepancy between the theory and the experiment at high magnetic fields makes the explanation of synchronism with a higher $\pi$-mode space harmonic unlikely. The precise origin of the second power peak is therefore unknown. Across the range of tested magnetic fields, output powers were notably lower than the power per oscillator in the dual-LBO RPM-12A, which typically produced $50 \mathrm{MW}$ per oscillator [40,41]. The LBO therefore appears to benefit from the dual-structure configuration of the RPM-12A when paired with

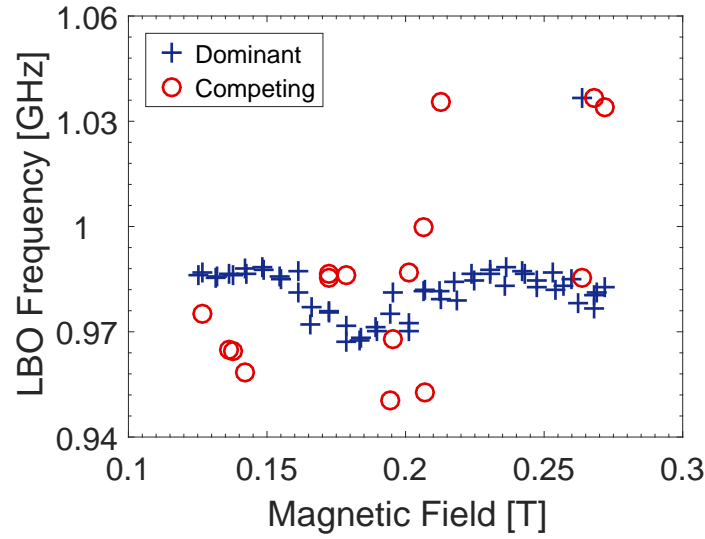

(a) Dominant and competing frequencies vs. magnetic field.

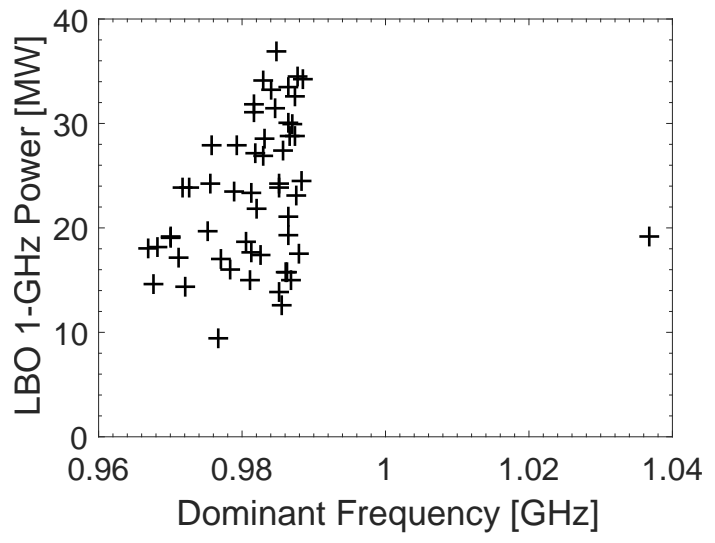

(b) Peak LBO power vs. dominant operating frequency.

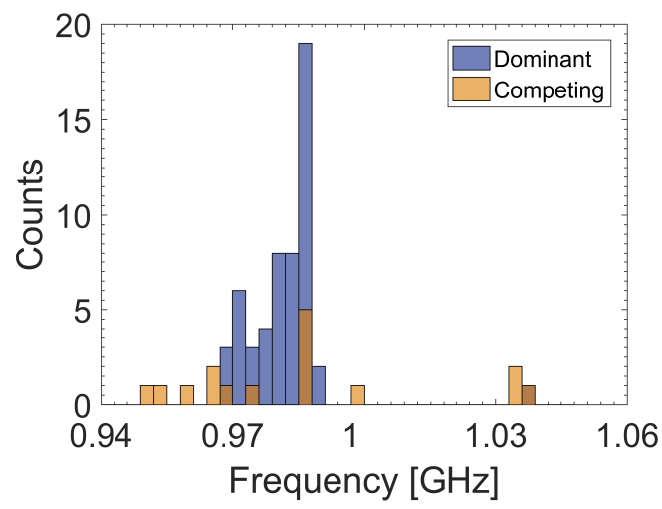

(c) Dominant and competing frequency histogram.

Figure 5.5: Isolated LBO: Frequency metrics. 
the MCC-2 cathode.

Frequency metrics are shown in Fig. 5.5. Another surprising result appears in Fig. 5.5a, where a single, relatively stable operating frequency near $0.985 \mathrm{GHz}$ was observed at magnetic fields other than $B \simeq 0.19 \mathrm{~T}$. Near this magnetic field, the dominant frequency stabilized at a minimum of approximately $0.970 \mathrm{GHz}$. A few shots in the vicinity of $0.19 \mathrm{~T}$ showed competition with the higher frequency (or the higher frequency competing with the lower frequency), but on first inspection, the transition between frequencies appears to be smooth, rather than abrupt as might be expected for discrete electromagnetic modes. The small number of competing frequencies near $1.04 \mathrm{GHz}$ corresponded to the chamber mode.

The expectation based on analytic theory was that two frequencies would be observed corresponding to the $\pi$-mode and $5 \pi / 6$-mode, with the former, higher-frequency mode appearing at higher magnetic fields than the latter, lower-frequency mode. Contrary to this expectation, simulations (the results of which are compared with the experiment in a later section on page 82) qualitatively replicate the experimental behavior, so the results are

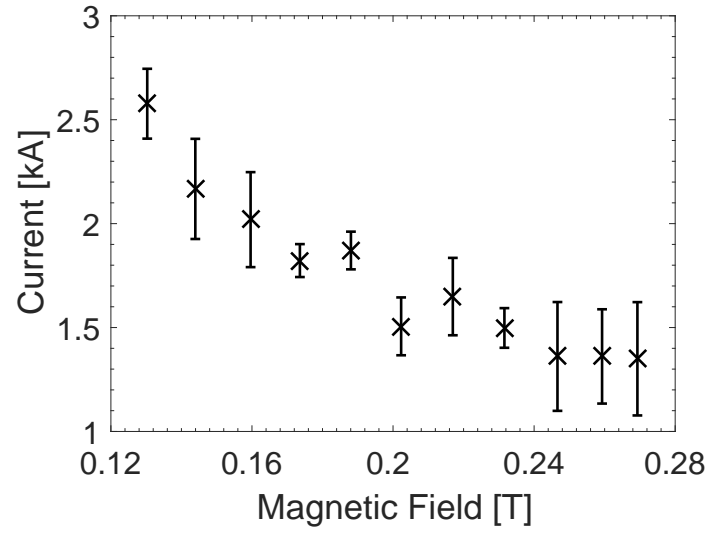

(a) Current at peak power vs. magnetic field.

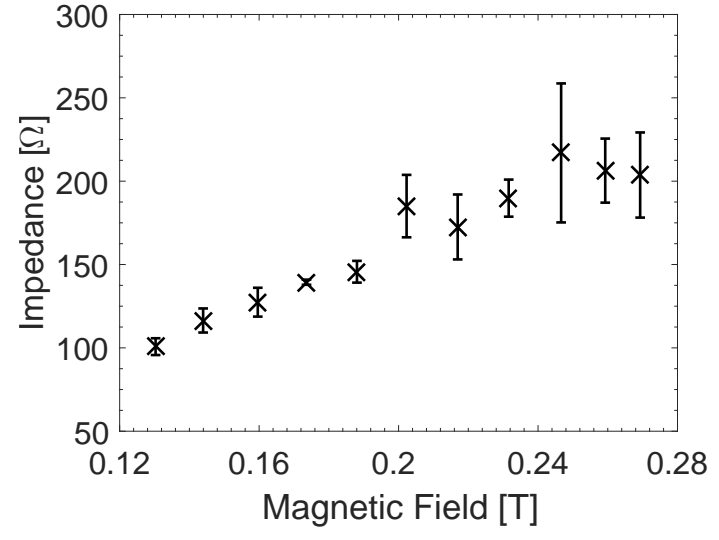

(b) Impedance at peak power vs. magnetic field.

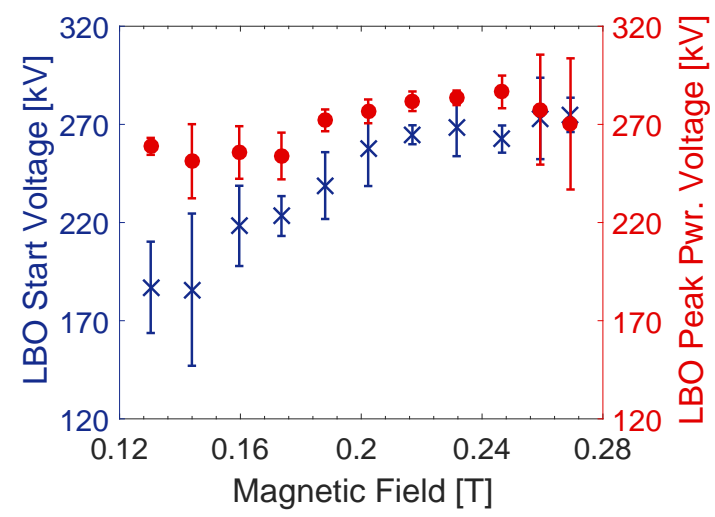

(c) Voltage at time of LBO start and peak power.

Figure 5.6: Isolated LBO: Electrical characteristics vs. magnetic field. 
possibly a consequence of the complicated geometry of the experiment, which was omitted from the analytic treatment to make the problem tractable. Features that were omitted from the analytic model, such as the MCC, and the fact that the $5 \pi / 6$-mode actually manifests as a standing wave with $\pi$-mode-like fields on the ends of the planar cavity array and a null in the center, might explain the deviation from analytic expectations.

The frequency distribution in Fig. 5.5c confirms the existence of two discrete modes with some spread between them. In the figure, the darker, brown shade represents overlap between the dominant and competing frequencies. In other words, that frequency bin had shots that were competing, and shots that were dominant. The bars were overlaid because the use of separate bars at the same frequency led to plots that were visually more challenging to interpret. The higher-frequency mode was $0.985 \mathrm{GHz}$, and the lower-frequency mode was $0.970 \mathrm{GHz}$. Comparison with Fig. 5.5b suggests the $0.985 \mathrm{GHz}$ mode produced, on average, higher powers relative to the $0.970 \mathrm{GHz}$ mode. Based on the good agreement with the simulations, the high- and low-frequency modes appear to be the $\pi$-mode and $5 \pi / 6$-mode, respectively. The region of designed operation was the range of magnetic fields slightly less than $B=B_{\mathrm{BH}}$, where the electron beam velocity was sufficient to synchronize with the $\pi$-mode, stable frequencies of $0.985 \mathrm{GHz}$ were observed, and power was locally maximized. Though not the magnetic field for which the device was designed, the region of optimal performance was near $B=0.23 \mathrm{~T}$.

Electrical characteristics are shown in Fig. 5.6. Comparison of Fig. 5.6a with Fig. 5.5a shows a very minor reduction in the $\pi$-mode frequency as current was increased, but the effect is not pronounced, and the opposite was true at the highest magnetic fields tested. Impedances in Fig. 5.6b confirm that the structure was consistently well-matched to the 100-150 $\Omega$ range for MELBA-C at $B<B_{\mathrm{BH}}$, which corresponds to electron beam ve-

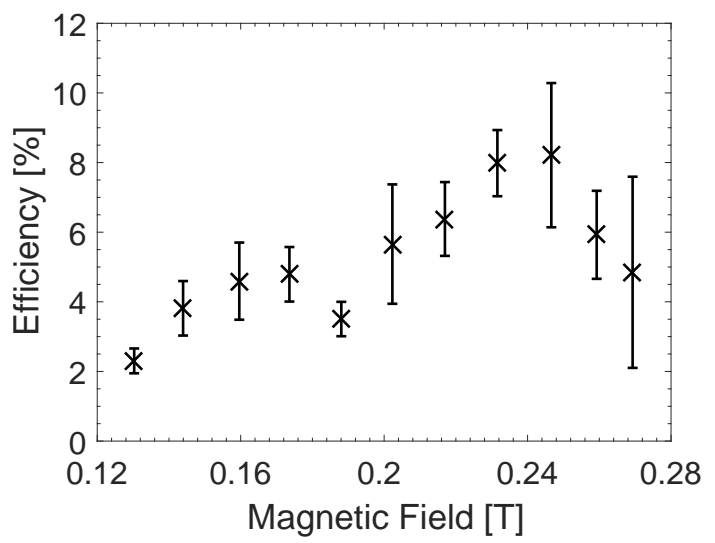

(a) Peak total efficiency vs. magnetic field.

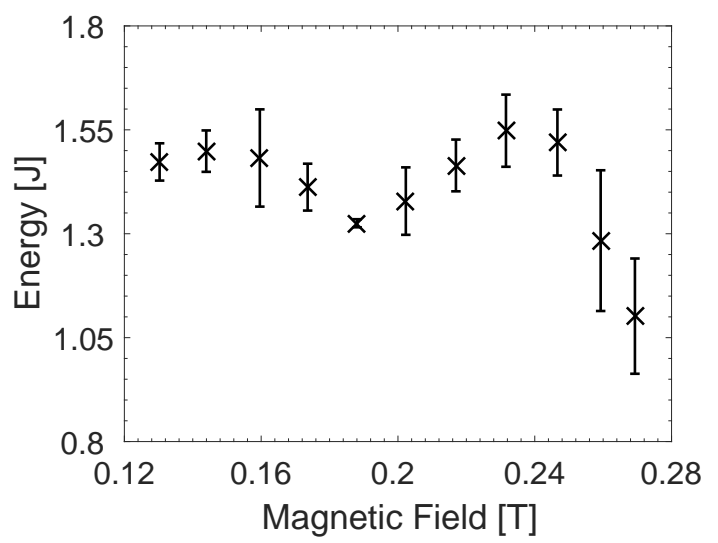

(b) Total pulse energy vs. magnetic field.

Figure 5.7: Isolated LBO: Energy conversion metrics vs. magnetic field. 
locities equal to or greater than that required for $\pi$-mode operation. At $B \gtrsim 0.19 \mathrm{~T}$, higher impedances near $200 \Omega$ with greater variance were observed. Peak power voltages in Fig. 5.6c appear to fall along a reasonably well-defined line for shots that exhibited a dominant frequency of $0.985 \mathrm{GHz}$, suggesting the $\pi$-mode repeatedly operated near the same favorable beam velocity in $B \lesssim 0.22 \mathrm{~T}$. In $B \gtrsim 0.22 \mathrm{~T}$, both the start and peak power voltages are approximately constant, suggesting operation during the flat-top voltage pulse. At low magnetic fields, the difference between the start-up voltage and peak power voltage was greater. This is not surprising considering the larger electron hub, which evidently excited microwave oscillations earlier during the voltage rise. This is confirmed by the time metrics shown by Fig. 5.8, which is discussed shortly.

Energy conversion metrics are shown in Fig. 5.7. As expected, higher peak powers correlate with the higher peak total efficiencies in Fig. 5.7a. However, the results of Fig. 5.7b are somewhat surprising. The dual power peaks of Fig. 5.4, despite having different maxima, appear to produce pulses with comparable total energies in Fig. 5.7b. This appears to be consistent with cathode plasma expansion, as postulated in [104].

Figure 5.8 shows consistent scaling of the onset of microwave oscillations with magnetic field, although there was some variability in the time of peak microwave power. The $\pi$-mode in $B \lesssim 0.17 \mathrm{~T}$ produced peak power earlier than the $\pi$-mode in $B \gtrsim 0.21 \mathrm{~T}$, which is logical given that higher magnetic fields would require higher voltages to reach the same synchronous beam velocity, and therefore delayed times as the voltage rises. The greater variation in the times relative to the voltages is likely due to shot-to-shot variability of the MELBA-C pulse shapes. Tables 5.1 and 5.3, which can be found in the Summary of Findings section on page 62, summarize the standard metrics observed in the two distinct $\pi$-mode regions of operation near the Buneman-Hartree magnetic field and at higher magnetic fields.

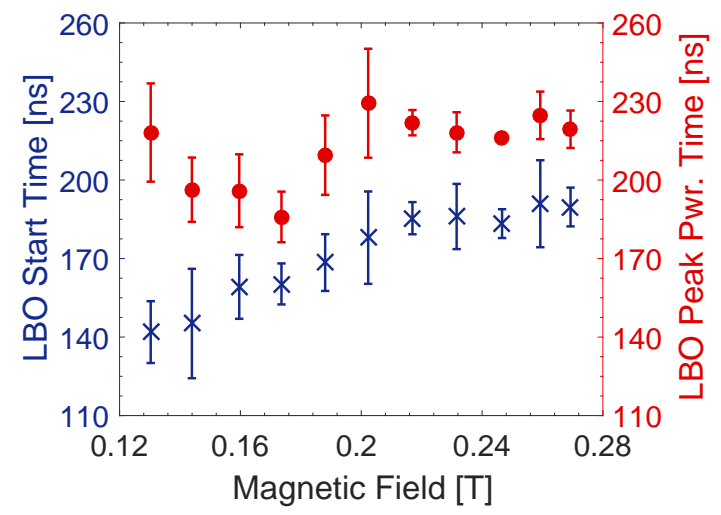

Figure 5.8: Microwave start and peak power times. 


\subsubsection{Additional Analysis}

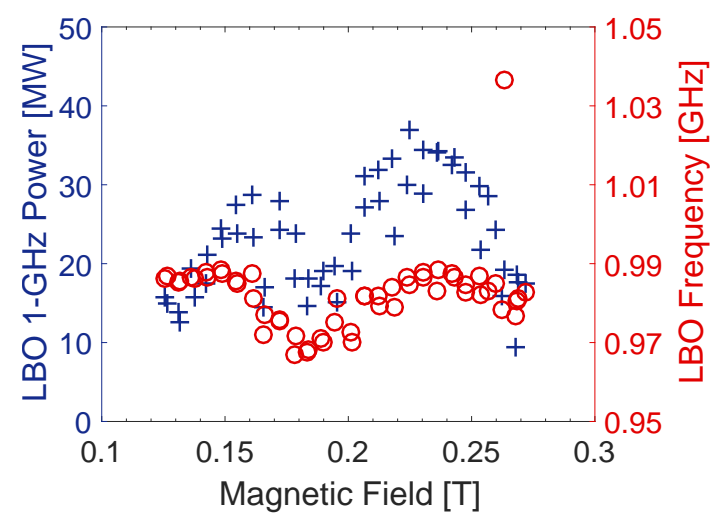

Figure 5.9: Isolated LBO: Power and dominant frequency vs. magnetic field.

The previous section noted the appearance of a narrow range of magnetic fields wherein consistent $5 \pi / 6$-mode oscillations were observed. This region can be illustrated clearly by plotting powers and frequencies together vs. magnetic field, which is shown in Fig. 5.9. Consistent, relatively low powers and frequencies were observed near $B=0.19 \mathrm{~T}$. By selecting shots in the vicinity of this magnetic field, operating parameters of the $5 \pi / 6$ mode could be determined. The results are summarized in Table 5.2 on page 62 .

While the transition between $\pi$-mode and $5 \pi / 6$-mode frequencies appears smooth, the two modes are nonetheless distinct, and the smooth transition may be a misleading byproduct of the time-integrated Fourier transform with overlapping peaks. The distinction between the modes is illustrated by an analysis of a shot in the transition region shown by Fig. 5.10.

Figure 5.10a shows the shot plot for shot 14924. Microwave oscillations begin at low power, then later jump to higher power before microwave pulse-shortening and oscillation cessation. The time-integrated Fourier transform in Fig. 5.10b reveals the presence of two distinct modes. A TFA of the shot is shown by Figs. 5.10c and 5.10d, which suggest the initial, low-power oscillations correspond to the $5 \pi / 6$-mode, and the later, high-power oscillations correspond to the $\pi$-mode. This result is consistent with simulation predictions, where the $5 \pi / 6$-mode coupled poorly to the microwave extractor and therefore produced low power. 


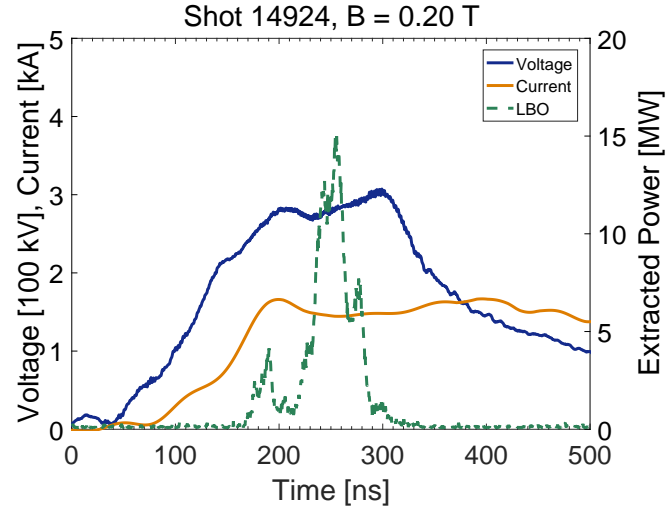

(a) Plot of voltage, current, and RF power for shot 14924.

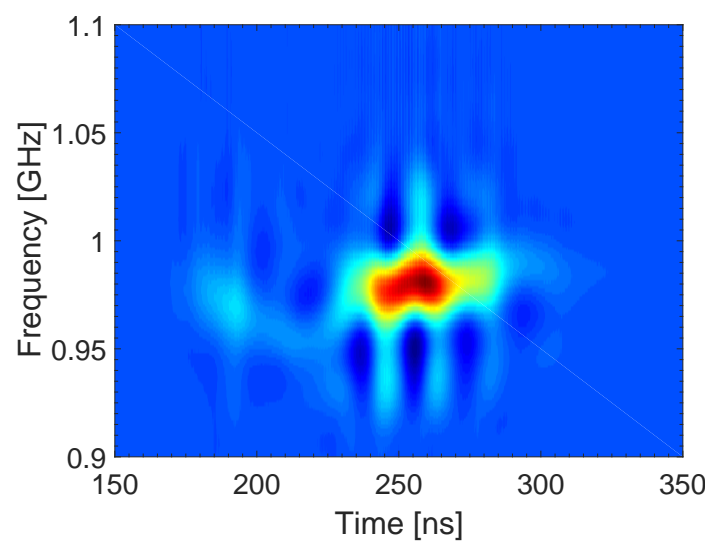

(c) Time-resolved TFA of the LBO RF voltage signal from shot 14924 using the Born-Jordan RIK.

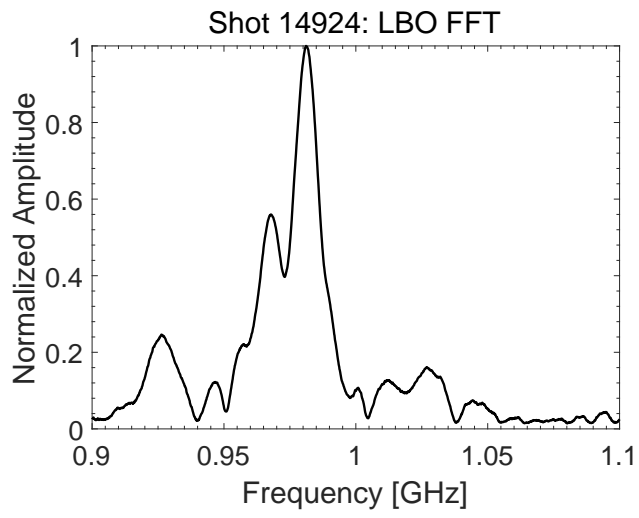

(b) Time-integrated Fourier transform of the LBO RF voltage signal from shot 14924 .

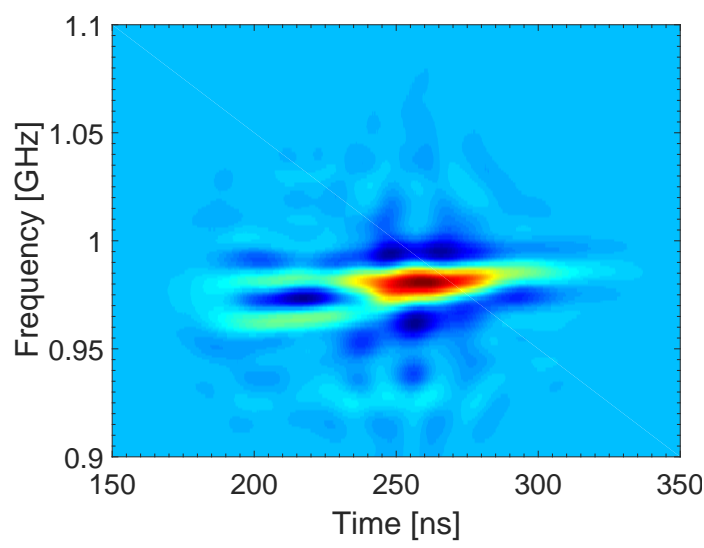

(d) Frequency-resolved TFA of the LBO RF voltage signal from shot 14924 using the Zhao-AtlasMarks RIK.

Figure 5.10: Isolated LBO: Analysis of shot 14924. 


\subsubsection{Comparison with Simulation}

Figure 5.11 compares the results of several metrics from simulation and experiment. As outlined in $\S 3.3 .2$, hot tube simulations of the isolated LBO configuration were conducted using voltage correction and full emission from the cathode. In all plots, the simulated parameters fell on magnetic field values lower than those observed in the experiment, which is very reasonable given the long-accepted explanation of cathode plasma formation, which makes the AK gap appear functionally smaller to the magnetron, thereby requiring higher magnetic fields [17].

Figure 5.11a compares the powers, which exhibit the greatest discrepancy of any metric. The simulations generated very high powers in $B<0.15 \mathrm{~T}$, which was highly unlikely in an experimental setting given the intense electron bombardment of the anode and surrounding structures, likely causing significant liberation of adsorbed surface contaminants and rapid anode plasma formation leading to pulse-shortening [111]. Because these simulations also used voltage correction, the low impedances at low magnetic fields did not affect the simulation voltage, whereas the voltage in the experiment would droop significantly. In addition, the unexpected double-peak in power output vs. magnetic field was not reproduced in simulation. However, simulated powers were reasonable at magnetic fields $B \gtrsim B_{\mathrm{BH}}=0.16 \mathrm{~T}$.

The most striking agreement is shown in Fig. 5.11b, which shows the dominant operating frequency vs. magnetic field. Simulations accurately predicted the qualitative behavior across the range of magnetic fields, including the jump to the $5 \pi / 6$-mode. Quantitatively, agreement was also good, with both the $\pi$-mode and $5 \pi / 6$-mode frequencies differing from experimental observations by approximately $5-10 \mathrm{MHz}$, or less than $2 \%$. Notably, the jump in the simulation results to the $5 \pi / 6$-mode (which had a simulated frequency of approximately $0.955 \mathrm{GHz}$ ) corresponded to a substantial drop in the simulated power, as shown by Fig. 5.11a. From the previous discussion of the field geometry of the different modes

in Chapter 3, this result is consistent with expectations because the $5 \pi / 6$-mode was not expected to couple effectively to the microwave extraction assembly.

With the exception of the efficiency plot in Fig. 5.11e, which was closely related to the powers, the remaining metrics showed good agreement with the experiment. Figures 5.11c and 5.11d show the currents and impedances, respectively. In both cases, when accounting for the previously described shift in magnetic field between simulation and experiment, the scaling agrees remarkably well, both qualitatively and quantitatively. 


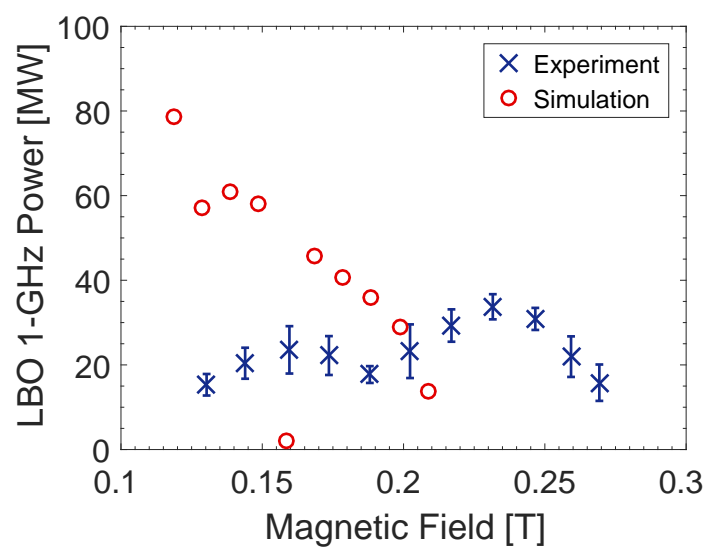

(a) Powers vs. magnetic field.

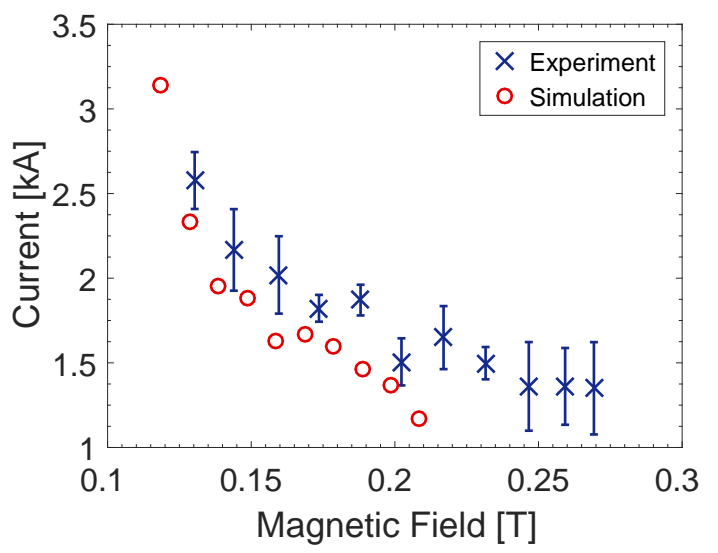

(c) Currents vs. magnetic field.

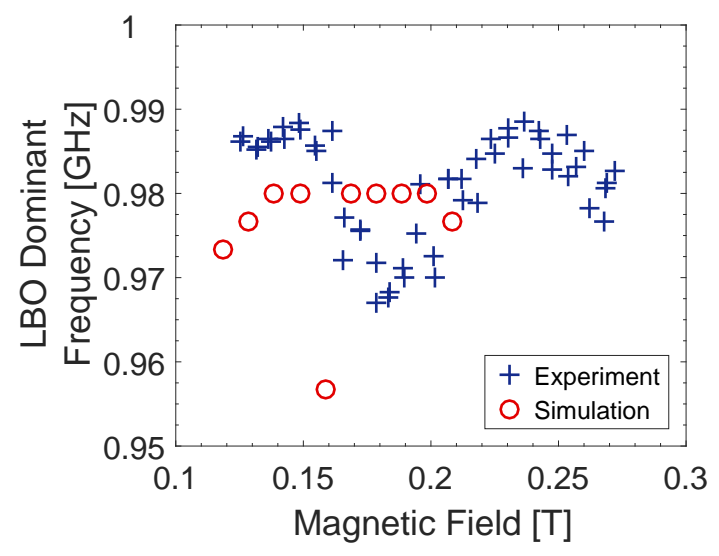

(b) Frequencies vs. magnetic field.

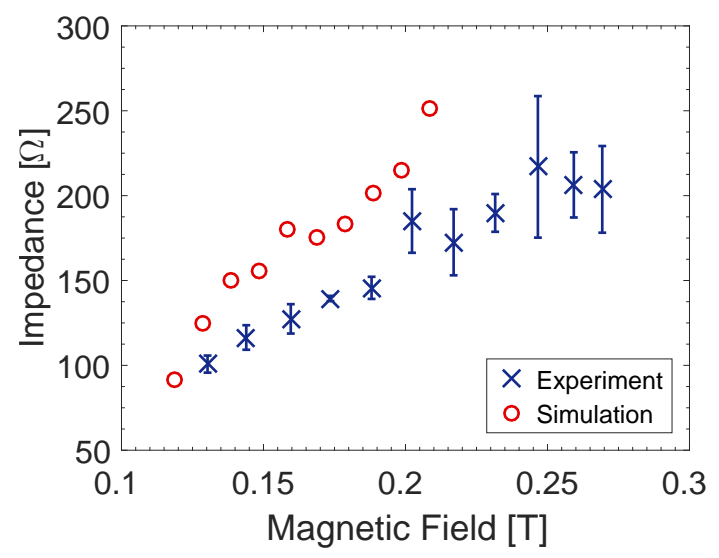

(d) Impedances vs. magnetic field.

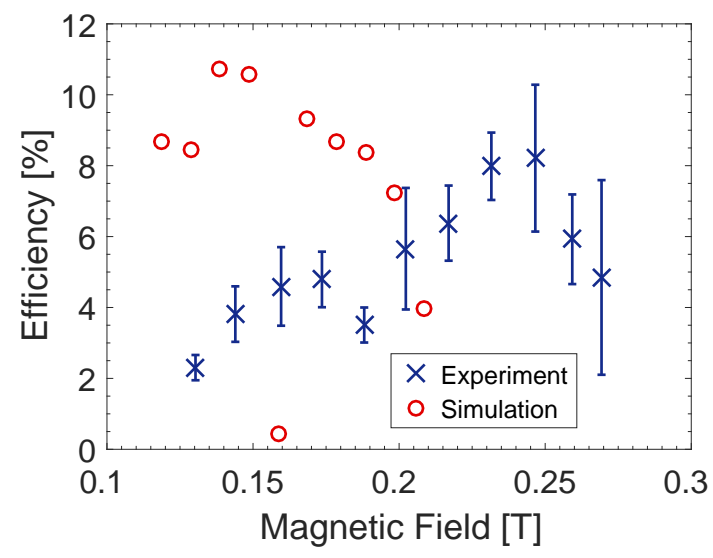

(e) Efficiencies vs. magnetic field.

Figure 5.11: Isolated LBO: Comparison between simulation and experiment. 


\subsection{Isolated SBO with Standard Load and Uniform Mag- netic Field}

This shot series tested the SBO in isolation to characterize operation of the planar cavity array without interference from the LBO. The LBO was replaced with the smooth drift region previously described in $\S 4.1 .1$, and all shots were conducted using the uniform magnetic field discussed in $\S 4.2 .2$. A total of 55 shots were obtained with no MELBA-C self-triggers, magnet triggering failures, or failed crowbars. The range of magnetic fields spanned $0.12-0.27 \mathrm{~T}$, and the base pressure was $1 \mu$ torr. While no antenna was connected to the omitted LBO, the copper outer conductor of the extractor coaxial transmission line was left in place, as was the WR-650 output waveguide and absorbing load.

\subsubsection{Standard Metrics}

Figure 5.12 shows the peak microwave output power from the SBO vs. magnetic field. For this series of shots, the SBO behaved ideally. Power was maximized at $B \simeq 0.17 \mathrm{~T}$, which was slightly greater than the Buneman-Hartree magnetic field $B_{\mathrm{BH}} \simeq 0.16 \mathrm{~T}$, which is in very good agreement with analytic expectations. As shown by the error bars, shot-toshot consistency was very good near $B_{\mathrm{BH}}$ up to approximately $0.2 \mathrm{~T}$, where powers dropped and shot-to-shot variability increased.

Frequency metrics are shown in Fig. 5.13. The SBO exhibited remarkably good frequency stability over a very wide range of magnetic fields in Fig. 5.13a, which was likely due to its high- $Q$ configuration. Inspection of Fig. 5.13c reveals a single dominant mode and what appears to be two competing modes near approximately $1.94 \mathrm{GHz}$ and $2 \mathrm{GHz}$. The frequency distribution of the $1.94 \mathrm{GHz}$ mode was relatively broad, and the number

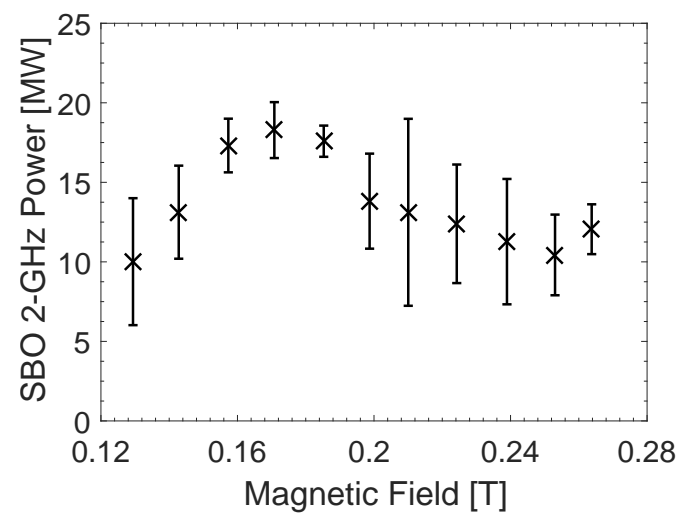

Figure 5.12: Isolated SBO: Peak microwave output powers near $2 \mathrm{GHz}$ vs. magnetic field. 


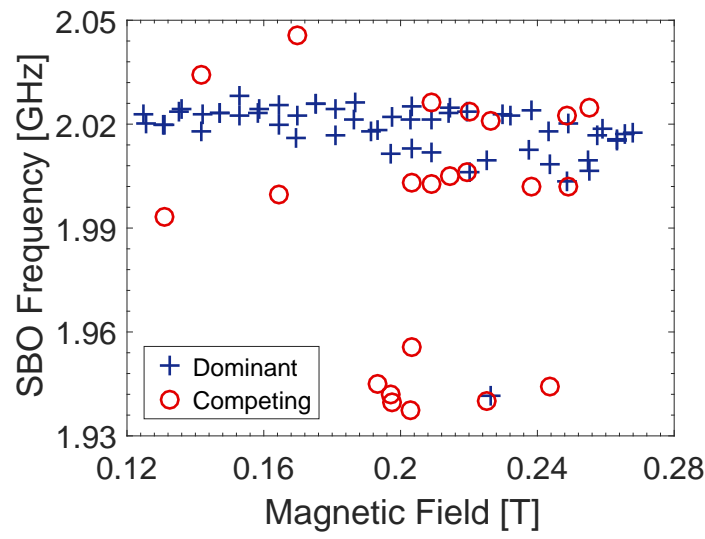

(a) Dominant and competing frequencies vs. magnetic field.

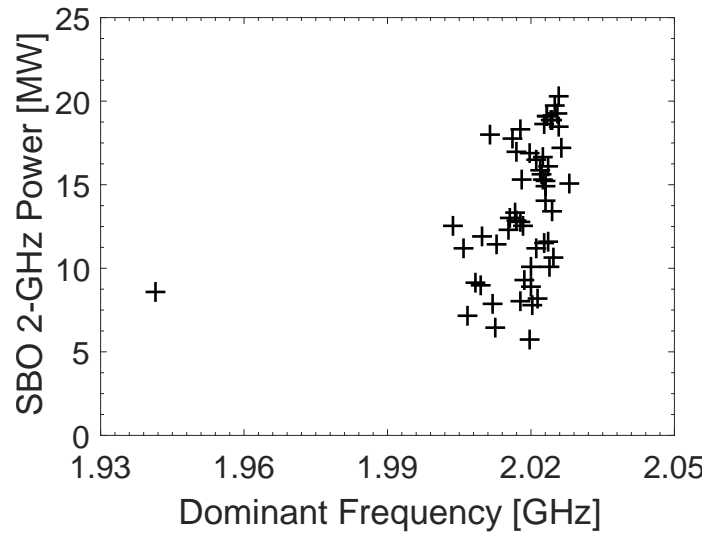

(b) Peak SBO power vs. dominant operating frequency.

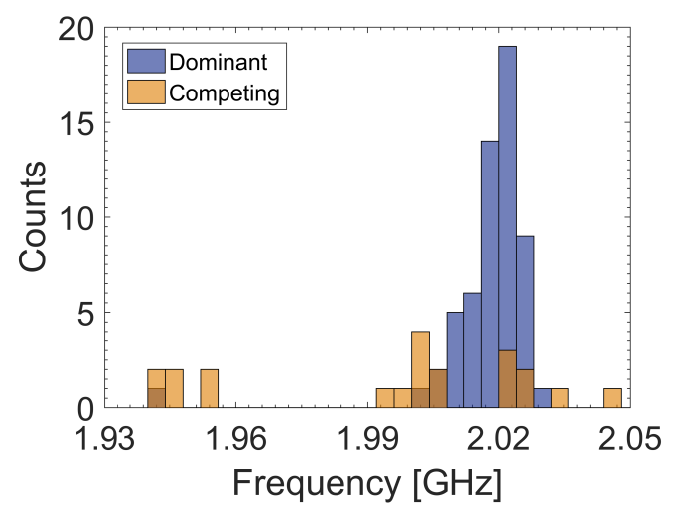

(c) Dominant and competing frequency histogram.

Figure 5.13: Isolated SBO: Frequency metrics.

of instances of both the $1.94 \mathrm{GHz}$ and $2 \mathrm{GHz}$ frequencies was small. However, this interpretation appears reasonable when comparing with Fig. 5.13a. A small number of shots had a competing mode near $1.94 \mathrm{GHz}$, and at magnetic fields $B \gtrsim 0.2 \mathrm{~T}$, two modes at approximately $2 \mathrm{GHz}$ and $2.02 \mathrm{GHz}$ appear to be consistently in competition with each other.

Similar to the discussion in the previous section, the expectation based solely on analytic theory was that two frequencies would appear corresponding to the higher-frequency $\pi$-mode and lower-frequency $7 \pi / 8$-mode, with the former appearing at higher magnetic fields than the latter. Figure 5.13a actually shows the opposite trend, with the higherfrequency mode manifesting at $B \lesssim 0.2 \mathrm{~T}$, and the lower-frequency mode beginning to appear at $B \gtrsim 0.2 \mathrm{~T}$, though a full transition to operation at $2 \mathrm{GHz}$ never occurs. One possible explanation for this discrepancy is that the observed $2 \mathrm{GHz}$ frequency actually 


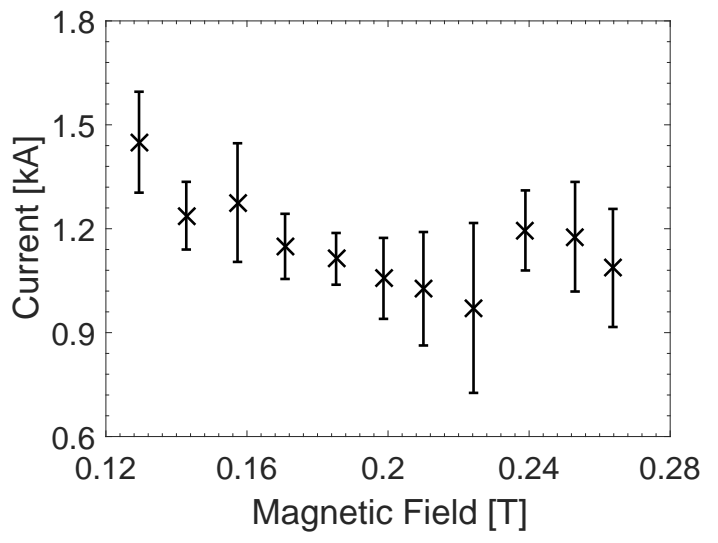

(a) Current at peak power vs. magnetic field.

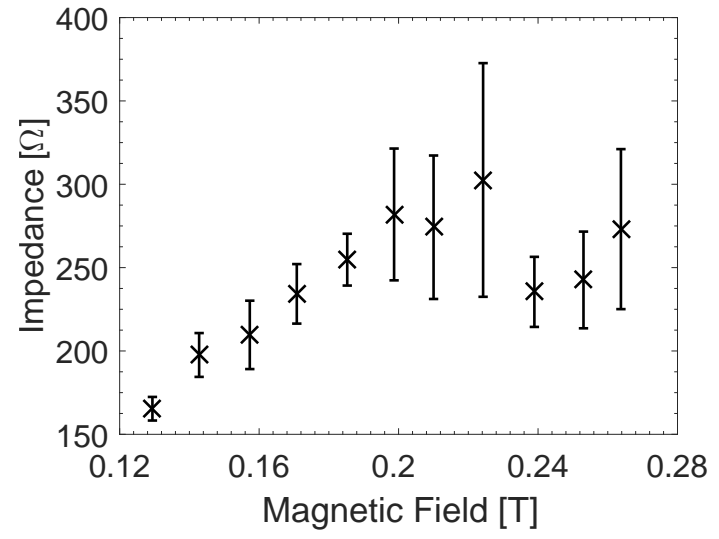

(b) Impedance at peak power vs. magnetic field.

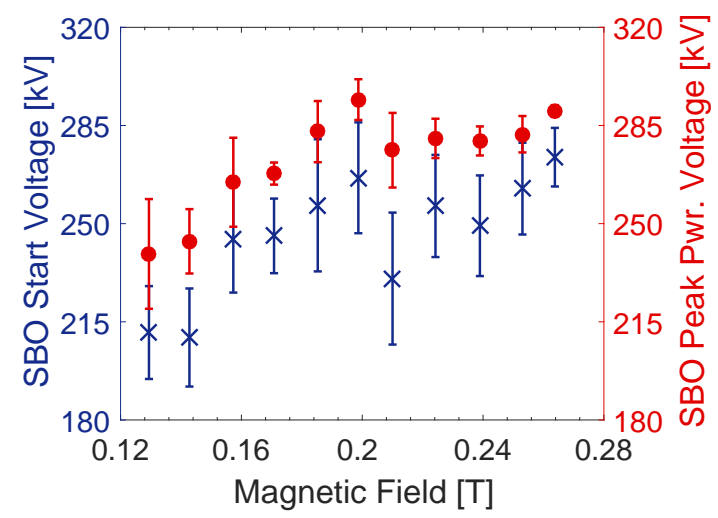

(c) Voltage at time of SBO start and peak power.

Figure 5.14: Isolated SBO: Electrical characteristics vs. magnetic field.

corresponds to the $9 \pi / 8$-mode, which is frequency-degenerate with the $7 \pi / 8$-mode and corresponds to a backward-wave interaction instead of a forward-wave interaction in an infinitely long planar cavity array. An alternative explanation is that the observed $2 \mathrm{GHz}$ mode is interacting at the next space harmonic, although this should occur at much higher magnetic fields.

Figure 5.13b shows the powers vs. dominant frequency. With the exception of the single shot operating near $1.94 \mathrm{GHz}$, there were a sufficient number of shots near $2.02 \mathrm{GHz}$ and $2 \mathrm{GHz}$ to conclude that the former mode is certainly higher-power than the latter. Considering the comparison of these results with simulations (which are discussed later on page 91), these results support the interpretation that the $2 \mathrm{GHz}$ and $2.02 \mathrm{GHz}$ modes are the $7 \pi / 8$-mode and $\pi$-mode, respectively. The region of optimal operation was near $B \simeq 0.17 \mathrm{~T}$, where power was maximized and consistent $\pi$-mode operation was observed.

Electrical characteristics are shown in Fig. 5.14. When comparing Fig. 5.13a to the currents in Fig. 5.14a, the impact of beam-loading appears minimal. In the region of opti- 
mal operation, shot-to-shot variability in the current was relatively low compared to shots conducted at higher and lower magnetic fields. Scaling of the current with magnetic field was consistent with expectations up to approximately $0.23 \mathrm{~T}$, where the current at peak power increases. Inspection of Fig. 5.16 reveals that this is likely due to achieving peak power generation at later times, when diode closure resulted in greater currents relative to those observed during peak power at lower magnetic fields. Impedances in Fig. 5.14b were consistently higher than the optimal 100-150 $\Omega$ for MELBA-C, though this does not appear to have adversely affected operation in the region of optimal operation.

Figure 5.14c shows the voltages at microwave start-up and peak power. As discussed previously, the linear progression of the peak power voltages with magnetic field in $B \lesssim$ $0.2 \mathrm{~T}$ supports the existence of a favorable beam velocity for the $\pi$-mode, which clearly dominated in this magnetic field range according to Fig. 5.13a on page 85. However, once the magnetic field is sufficiently high, the magnetron start time (Fig. 5.16) is late enough to occur during the flat-top portion of the MELBA-C pulse. In those cases, the peak power voltage does not change appreciably (see $B \gtrsim 0.2 \mathrm{~T}$ on Fig. $5.14 \mathrm{c}$ ). For the start voltage, a similar linear progression is observed in $B \lesssim 0.2 \mathrm{~T}$. However, at $B \gtrsim 0.2 \mathrm{~T}$, there is a shift to a different linear progression of the start voltage. This suggests a different mode (having a different synchronous beam velocity) is starting first, which is consistent with the previous analysis suggesting this range of magnetic fields supported oscillation in the $7 \pi / 8$-mode in addition to the $\pi$-mode (see Fig. $5.13 \mathrm{a}$ ). The earlier start of the $7 \pi / 8$-mode relative to the $\pi$-mode is shown explicitly during the analysis of a specific shot shown later.

Energy conversion metrics are shown in Fig. 5.15. As expected, both efficiency and pulse energy shown in Figs. 5.15a and 5.15b are maximized at the point of maximized peak power generation. Pulse energies were remarkably consistent, with less than $10 \%$ variation

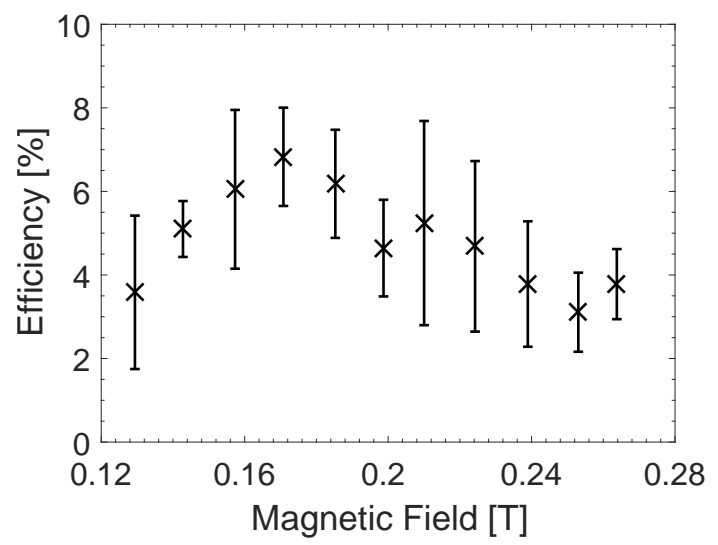

(a) Peak total efficiency vs. magnetic field.

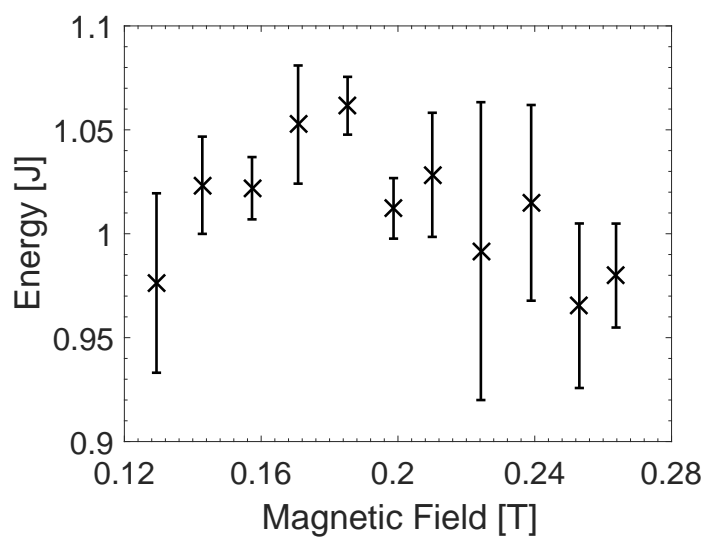

(b) Total pulse energy vs. magnetic field.

Figure 5.15: Isolated SBO: Energy conversion metrics vs. magnetic field. 


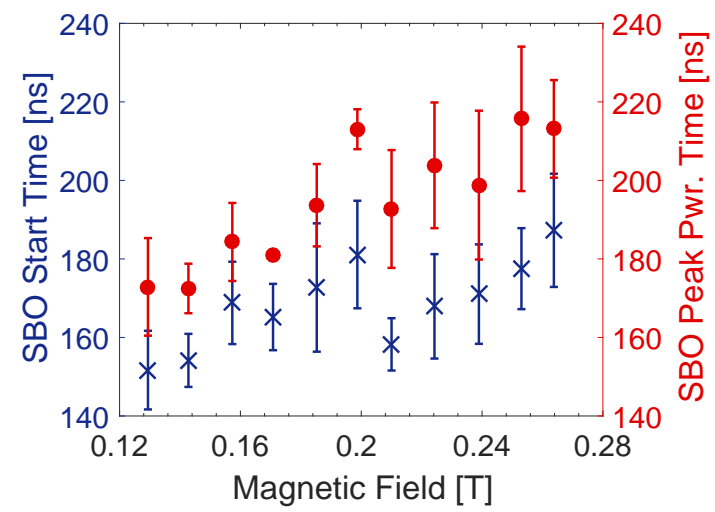

Figure 5.16: Microwave start and peak power times.

across the range of magnetic fields tested. Despite significant variations in peak power, this consistency hints at a consistent source of pulse-shortening in the SBO, possibly cathode plasma expansion, as postulated in [104]. The topic will be discussed in a later section.

Figure 5.16 shows the temporal metrics. While variable, there is a clear trend in the time of peak power production toward increasingly late times. From the previous voltage analysis, in $B \lesssim 0.2 \mathrm{~T}$, the magnetron achieved peak power during the voltage rise, and in $B \gtrsim 0.2 \mathrm{~T}$, the magnetron reached peak power during the flat-top voltage. Here, higher magnetic fields led to longer delays before peak power was observed, but synchronism was still possible despite the fixed voltage at the flat-top of the pulse. This was due to cathode plasma gap closure, which led to an effectively smaller AK gap. The gap closure increased the DC electric field with time. Eventually, synchronism was achieved despite the higher magnetic field [17]. Thus, the peak power times scale linearly with magnetic field. The most notable feature of the plot is the two distinct linear scalings in the oscillation start times. If the same mode was starting in all cases, the progression of the trend at $B \lesssim 0.2 \mathrm{~T}$ might be expected to continue, with microwaves starting at later times corresponding to higher voltages given the relatively slow MELBA-C rise-time or, due to AK gap closure, higher gap electric fields facilitating synchronism at higher magnetic fields. Instead, the different trend (in $B \gtrsim 0.2 \mathrm{~T}$ ) suggests the appearance of a new mode starting, which is consistent with the previous discussion of the $7 \pi / 8$-mode. Since this region supports the $7 \pi / 8$-mode (whereas the previous region did not), it may be more synchronous than the $\pi$-mode, which may explain the earlier start times. Table 5.4 on page 63 summarizes the standard metrics observed in the region of optimal operation. 


\subsubsection{Additional Analysis}

One of the primary objectives of running the SBO over a wide range of magnetic fields was to identify the frequencies of the competing modes supported by the SBO anode. Simulations suggested the SBO might support two competing modes (the $6 \pi / 8$-mode and $7 \pi / 8$ mode) in addition to the intended $\pi$-mode. The models also suggested that shots exhibiting pure operation in the $7 \pi / 8$-mode could be expected to generate considerably lower output powers than the $\pi$-mode due to the differences in interaction efficiency and coupling efficacy to the microwave extractor.

As previously discussed, inspection of Fig. 5.13a on page 85 showed that the SBO operated primarily in the $\pi$-mode, though a small number of shots had some competing mode frequencies. Shots 14970 and 14971 are representative of these shots with competing mode frequencies. Figures 5.17 and 5.18 illustrate their analysis. In both cases, conditions

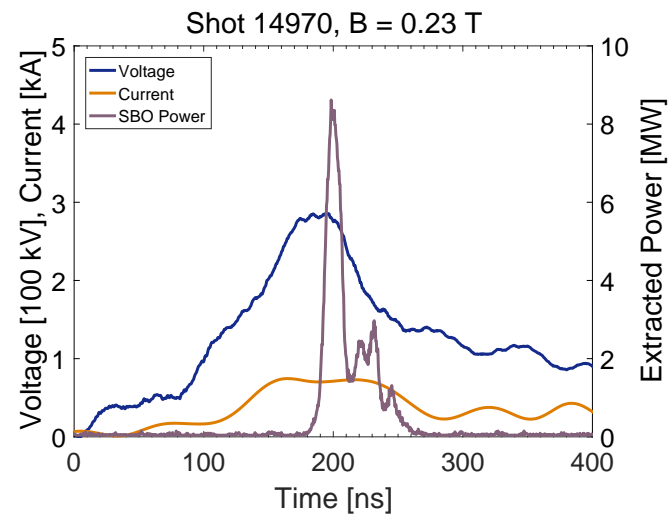

(a) Plot of voltage, current, and RF power for shot 14970.

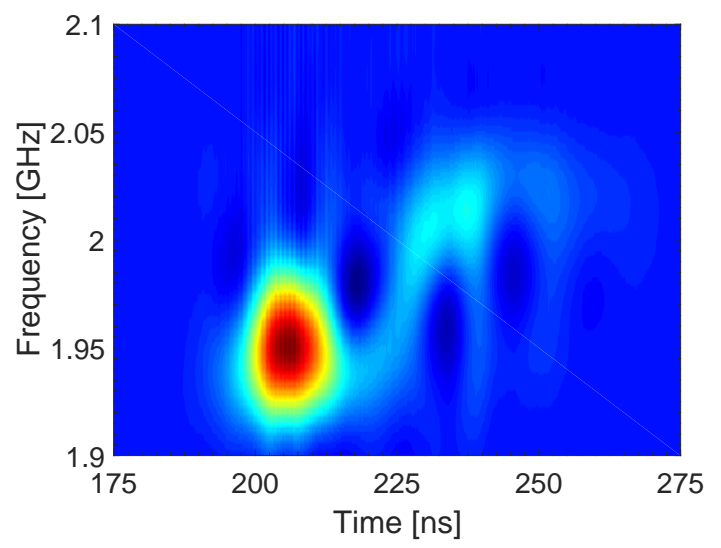

(c) Time-resolved TFA of the SBO RF voltage signal from shot 14970 using the Born-Jordan RIK.

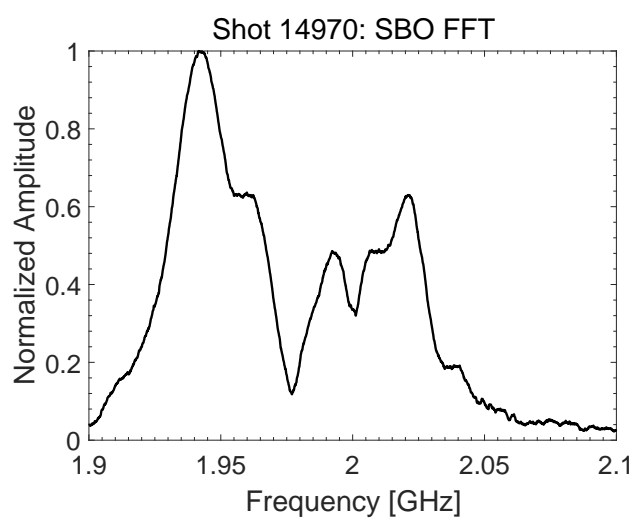

(b) Time-integrated Fourier transform of the SBO RF voltage signal from shot 14970 .

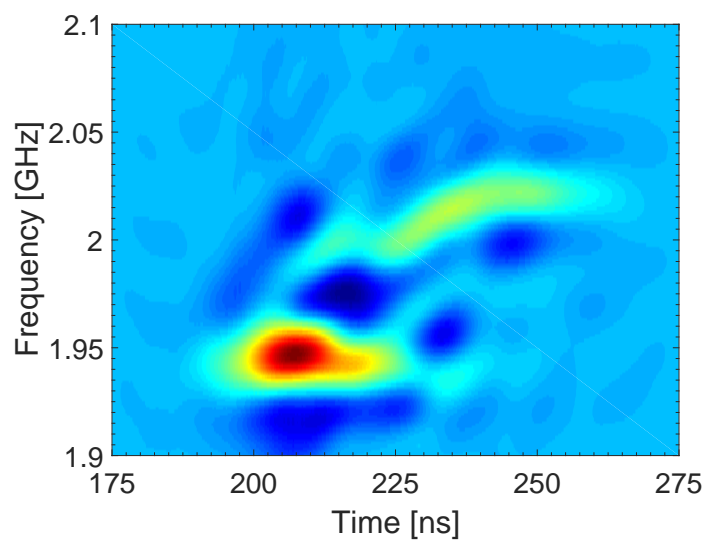

(d) Frequency-resolved TFA of the SBO RF voltage signal from shot 14970 using the Zhao-AtlasMarks RIK.

Figure 5.17: Isolated SBO: Analysis of shot 14970. 
were favorable to supporting multiple modes of operation at some point during the shot, which simplifies the process of positively identifying an operating mode and its respective frequency.

Figure 5.17a shows that the crowbar occurred during microwave generation, which led to a sweep of the voltage from high to low during conditions favorable to microwave generation prior to plasma-related microwave pulse-shortening. Consequently, different modes are excited that ordinarily would not be observed, as shown by the time-integrated FFT in Fig. 5.17b. Since the time-integrated FFT provides no information about when different modes are excited, TFA plots were generated, which are shown in Figs. 5.17c and 5.18c. Shot 14970 clearly started at a low-frequency mode near $1.94 \mathrm{GHz}$ and jumped to a higher frequency mode near $2.02 \mathrm{GHz}$, as shown by the time-resolved TFA plot in Fig. 5.17c. The frequency-resolved TFA plot in Fig. 5.17d reveals that this is true, but that

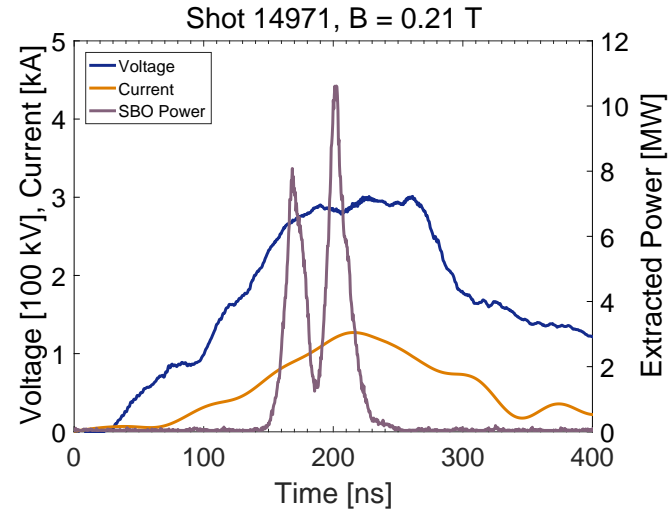

(a) Plot of voltage, current, and RF power for shot 14971.

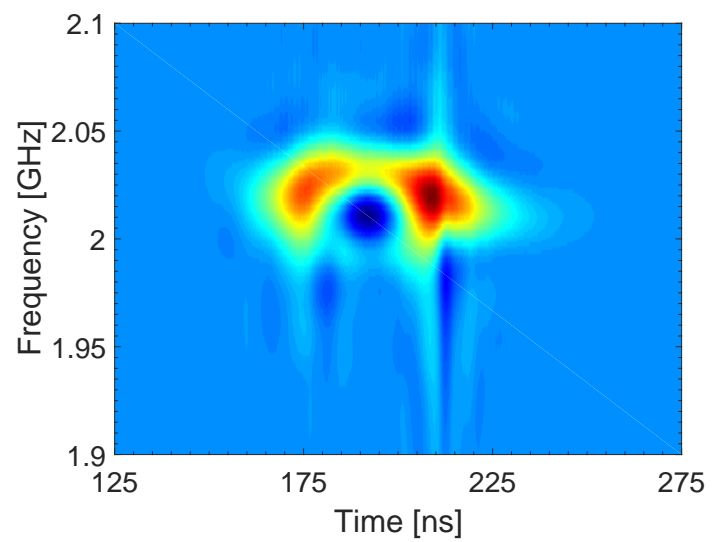

(c) Time-resolved TFA of the SBO RF voltage signal from shot 14971 using the Born-Jordan RIK.

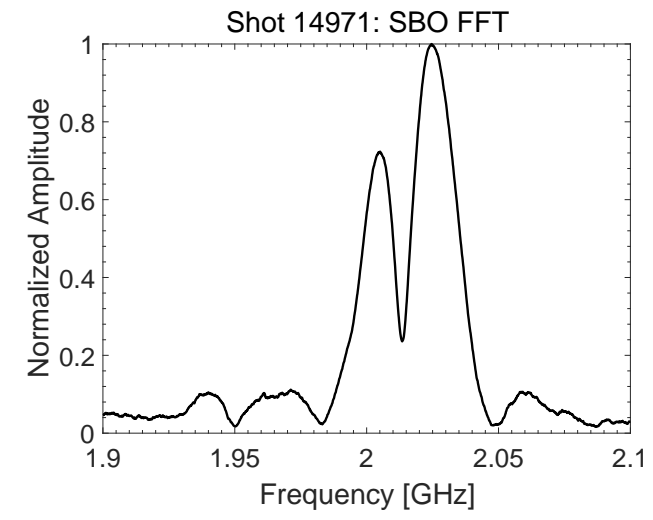

(b) Time-integrated Fourier transform of the SBO RF voltage signal from shot 14971.

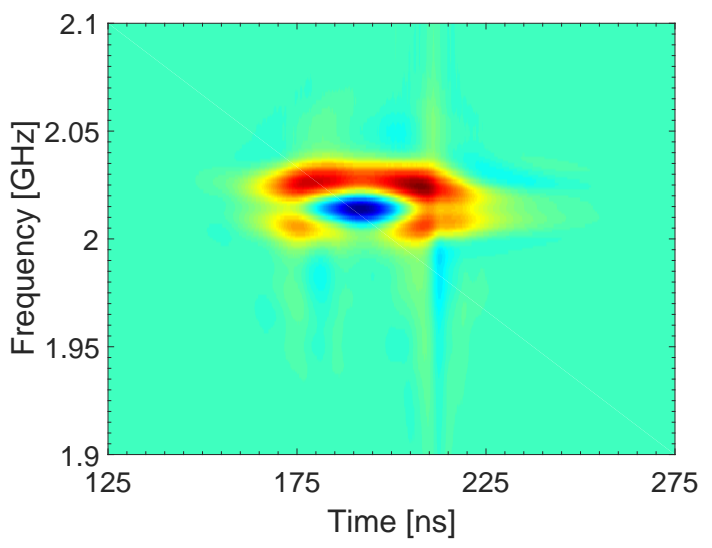

(d) Frequency-resolved TFA of the SBO RF voltage signal from shot 14971 using the Born-Jordan RIK.

Figure 5.18: Isolated SBO: Analysis of shot 14971. 
there are actually two jumps in quick succession from $1.94 \mathrm{GHz}$ to $2 \mathrm{GHz}$, and $2 \mathrm{GHz}$ to $2.02 \mathrm{GHz}$.

For the second shot, Fig. 5.18a shows considerable fluctuations in the output power. This fact, coupled with the time-integrated FFT in Fig. 5.18b, clearly shows evidence of either mode competition, wherein two modes exist simultaneously, or mode hopping, wherein the oscillator jumps from operation in one mode to another. The time-resolved TFA plot in Fig. 5.18c clearly exhibits beating consistent with the power fluctuation in Fig. 5.18a, but it does not appear to be a case where the mode jumps from the lower frequency to the higher frequency. The frequency-resolved TFA plot in Fig. 5.18d, which does retain some temporal resolution, confirms this while resolving the two very closely spaced $2 \mathrm{GHz}$ and $2.02 \mathrm{GHz}$ modes. Furthermore, the $2 \mathrm{GHz}$ mode is considerably weaker than the $2.02 \mathrm{GHz}$ mode, which is consistent with the interpretation that $2 \mathrm{GHz}$ and $2.02 \mathrm{GHz}$ correspond to the $7 \pi / 8$-mode and $\pi$-mode, respectively, due to the differences between the modes in extractor coupling efficacy.

Considering the results of the simulations and shots like 14970 and 14971, the best explanation for these modes is that the $6 \pi / 8$-mode and $7 \pi / 8$-mode approximately correspond to $1.94 \mathrm{GHz}$ and $2 \mathrm{GHz}$, respectively. These findings reasonably explain the frequency distribution observed in Fig. 5.13c and the absence of significant dominant operation at $2 \mathrm{GHz}$, since the $7 \pi / 8$-mode couples very poorly to the microwave extractor and therefore would not manifest as a dominant frequency component of the output signal. Those few shots having dominant operation near $2 \mathrm{GHz}$ do have lower output powers relative to $2.02 \mathrm{GHz}$ shots, as shown by Fig. $5.13 \mathrm{~b}$ on page 85 . Due to the absence of a significant number of shots having dominant operation at either $6 \pi / 8$-mode or $7 \pi / 8$-mode, no associated power, impedance, or efficiency can be determined with confidence for those modes based on this set of data.

\subsubsection{Comparison with Simulation}

Figure 5.19 compares several metrics for simulation and experiment. As outlined in $\S 3.3 .2$, hot tube simulations of the isolated SBO configuration were conducted using voltage correction and full emission from the cathode. Agreement was excellent for the predicted powers shown in Fig. 5.19a. At lower magnetic fields, the SBO operates in the $7 \pi / 8$ mode, which couples poorly to the extractor and leads to low extracted powers. As with the LBO, the experimental results for the SBO appear to be shifted to a higher magnetic field, a result that is consistent with cathode plasma formation, which leads to a functionally smaller AK gap, therefore requiring higher magnetic fields [17]. Due to the relation be- 
tween power and efficiency, it is not surprising to see similar efficiency agreement (within the error bars) between simulation and experiment, as shown by Fig. 5.19e.

A comparison of the operating frequencies is shown in Fig. 5.19b. The simulated $7 \pi / 8$ mode and $\pi$-mode frequencies were accurate to within $20 \mathrm{MHz}$, or about $1 \%$. The lower simulated frequencies are a consequence of the excessively coarse mesh, which leads to overestimation of the effects of beam-loading on the frequencies (see Appendix B). The mode transition point observed in the experiment was not reproduced, viz. that the $7 \pi / 8$ mode was observed at lower magnetic fields in simulation instead of higher magnetic fields in the experiment. This is not altogether surprising because achieving the conditions for the $7 \pi / 8$-mode in the experiment using the uniform magnetic field was nearly impossible because it required excessively low magnetic fields, which resulted in low impedances and very high currents, which led to a reduction in the applied voltage and therefore a failure to achieve the high beam velocity needed to synchronize with the $7 \pi / 8$-mode. This problem was easily overcome in simulations using the applied voltage correction. At $B>$ $0.2 \mathrm{~T}$, simulations failed to start up because most of the current was lost in the form of endloss current, and the tight electron hub failed to excite any mode on the planar cavity arrays. In the experiment, expansion of the cathode plasma eventually led to a functionally smaller AK gap and, consequently, a higher beam velocity capable of exciting microwave oscillations. It should be noted that a competing mode was occasionally observed in the simulations at $1.94 \mathrm{GHz}$, which was the $6 \pi / 8$-mode.

Simulations roughly reproduced the trends in the SBO currents and impedances shown by Figs. 5.19c and 5.19d. In the simulations, the dip in drawn current at low magnetic fields occurred due to the transition between the $7 \pi / 8$-mode and $\pi$-mode. In the transition region, mode competition prevented well-formed oscillations from stabilizing, and the magnetron failed to draw as much current as would be the case in pure-mode operation. Across the range of simulated and experimentally tested magnetic fields, the simulated currents exceeded those of the experiment by approximately $50 \%$ due to the fact that full emission was specified in simulations, whereas only about $10 \%$ of the cathode area was configured to emit in the experiment. This also illustrates the discrepancy between emission area and drawn current in simulations compared to the experiment, a disagreement that is reasonable given the omitted physics in the simulations, such as ion formation, the presence of bipolar flow, and plasma diode closure $[99,112,113]$. Apart from the increased impedance in the mode transition region at low magnetic fields, the scaling of impedances in simulations was in qualitative agreement with the experiment. 


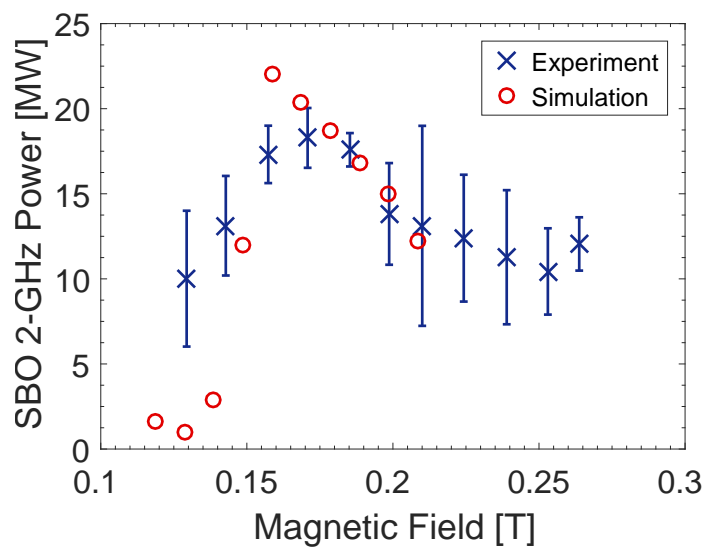

(a) Powers vs. magnetic field.

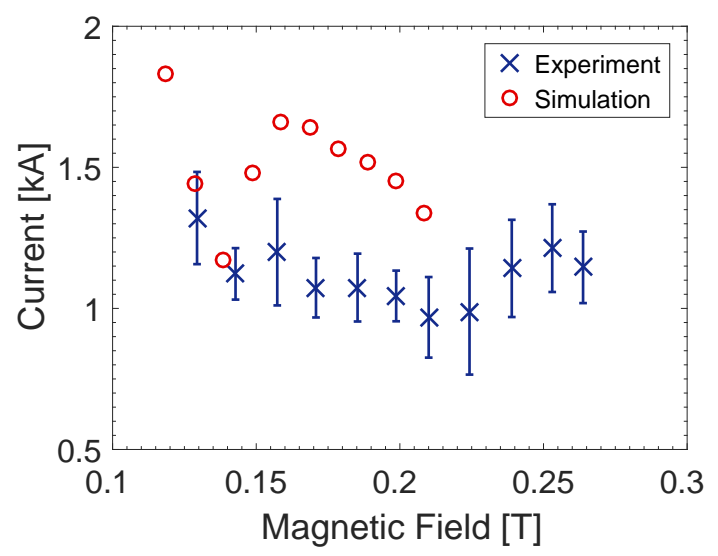

(c) Currents vs. magnetic field.

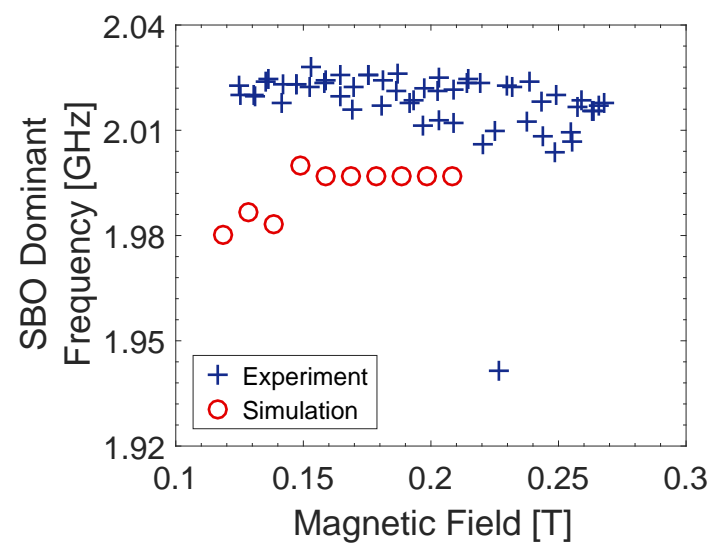

(b) Frequencies vs. magnetic field.

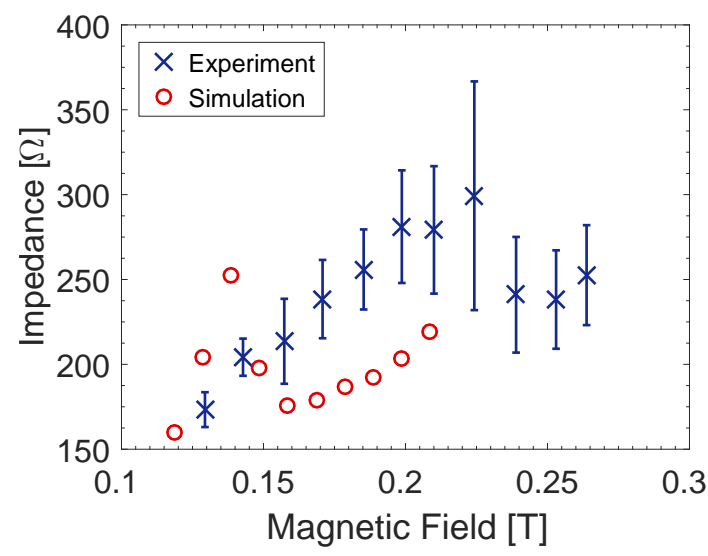

(d) Impedances vs. magnetic field.

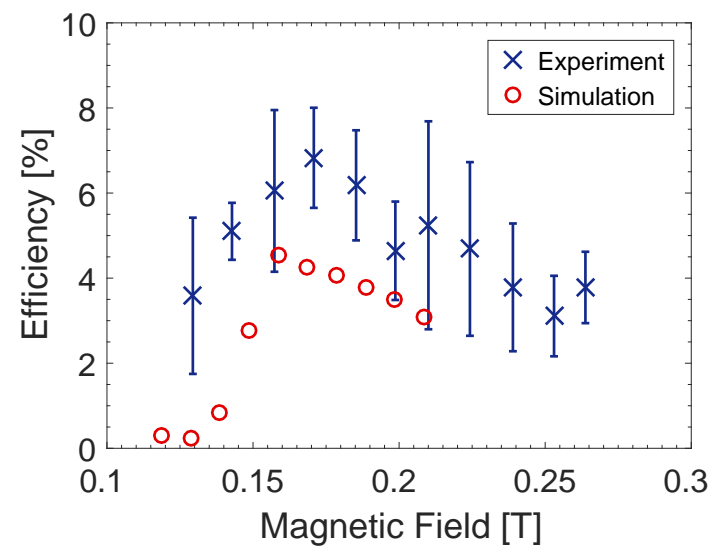

(e) Efficiencies vs. magnetic field.

Figure 5.19: Isolated SBO: Comparison between simulation and experiment. 


\subsection{MFRPM with Standard Load and Nonuniform Mag- netic Field}

This shot series tested the MFRPM using the nonuniform magnetic field described in $\S 4.2 .1$. The purpose was to obtain the first of two data sets necessary to determine how the magnetic field variation along the planar cavity arrays impacted operation of the MFRPM under ideal operating conditions with well-matched microwave loads. A total of 118 shots were obtained at a base pressure of $0.8 \mu$ torr. The range of magnetic fields was $0.14-0.24 \mathrm{~T}$.

These shots were conducted before the crowbar and procedural improvements outlined in Appendix H. Consequently, several shots caused magnetron damage, including two MELBA-C pre-triggers and six crowbar failures. Damage manifests as an accelerated collapse in diode impedance relative to operation before the first damaging shot. Subsequent damage tends to exacerbate the effect. Figure 5.20 shows the current at peak power vs. series shot number for this data set. The set of shots where most of the damage occurred is readily apparent, with the first damage-inducing shot at 37 on the plot. Note that the jump in current was not due to the nonuniform magnetic field in the cylindrical recirculation bends dropping below the Hull cutoff condition for magnetic insulation. The large fluctuation in current with shot number over the entire data set is due to variation of the magnetic field over a wide range during acquisition. Data interpretation for this shot series therefore must be made with the caveat that damage leads to the magnetron drawing more current than might otherwise have been the case.

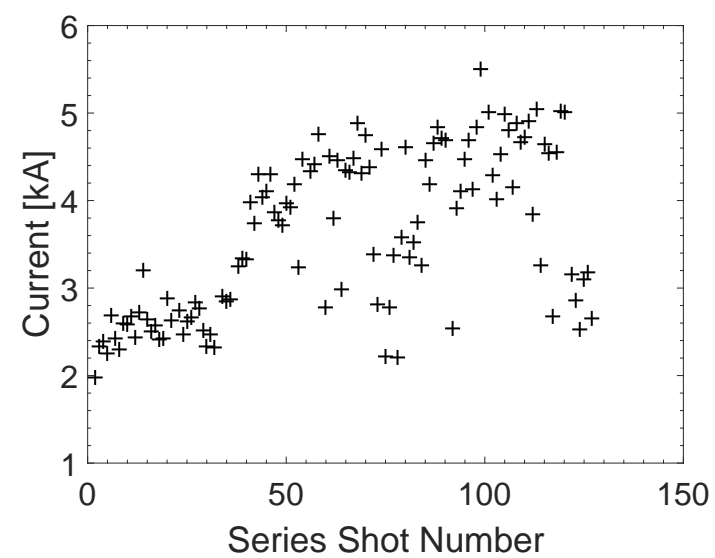

Figure 5.20: Current at peak power vs. series shot number. 


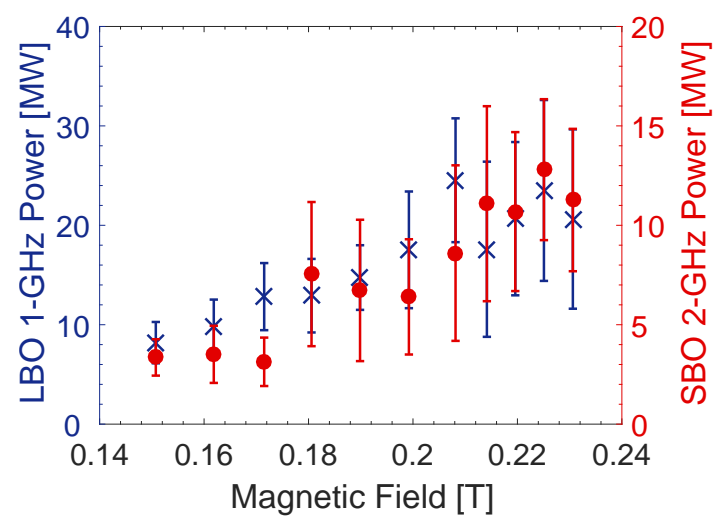

Figure 5.21: Standard Load and Nonuniform Magnetic Field: Peak microwave output powers for both oscillators vs. magnetic field.

\subsubsection{Standard Metrics}

As illustrated in $\S 4.2 .1$, the magnetic field variation along the planar cavity arrays was considerable, with the ratio of the field maximum (located in the center of the planar cavity arrays) to the field minimum (located in the cylindrical recirculation bends) equal to 1.4 . This $40 \%$ difference leads to a large variation in both the electron hub height and $\vec{E} \times \vec{B}$ drift velocity, making prediction of the optimal magnetic field for $\pi$-mode operation difficult. Rather, the spread in velocities can be interpreted as having some population of electrons that are always synchronous with the $\pi$-mode, with the hope that a possibly dominant $\pi$-mode would be preferentially selected regardless of magnetic field due to the velocity spread along the planar cavity arrays.

Figure 5.21 shows the peak microwave output powers from both oscillators vs. magnetic field. Optimal operation was observed at the limits of electromagnet operation, although even at these high magnetic fields, considerable shot-to-shot variation was observed. These data are not consistent with the theoretical predictions because neither consistent nor high power operation was observed near the Buneman-Hartree threshold magnetic field for $\pi$ mode operation at $B_{\mathrm{BH}}=0.16 \mathrm{~T}$. The region of optimal operation lay instead in $B=0.21-$ $0.23 \mathrm{~T}$.

Frequency metrics for both oscillators are shown in Fig. 5.22, with the results for the LBO shown in Fig. 5.22a. The LBO displayed surprising operation because it exhibited a smooth reduction in frequency as the magnetic field was increased (thereby decreasing the current) up to approximately $0.21 \mathrm{~T}$. This behavior contradicts the predictions of $\S 2.1$, which explains the physical basis for the beam-loading effect observed in traditional magnetrons in which an increase in current leads to a reduction in frequency [6]. Comparing 
the plot to Fig. 5.25a on page 99 clearly shows that the opposite trend occurred here. No theoretical basis exists for this behavior, but it is very plausible that the variation in the hub height across the planar cavity array affected the resonant frequency. Unlike the case of a uniform magnetic field, the magnetic field variation along the planar cavity array leads to larger electron hub heights on the ends, and a smaller hub in the center (see Eq. 2.7 on page 16). To the cavities, this functionally looks like a peanut-shaped cathode with a smaller AK gap on the ends relative to the center. While the effect of such a cathode shape on cold-tube frequencies was not investigated, it is conceivable that it would lead to an increase in the resonant frequency, as changes in the geometry of the complicated MFRPM structure frequently produced counter-intuitive simulation results during the design process. This smooth frequency reduction transitions to bifurcation at $B \gtrsim 0.22 \mathrm{~T}$, where two distinct modes appear with different frequencies. This will be discussed in greater detail in the Additional Analysis section.

Given the results of the isolated LBO in $\S 5.3$ and the relatively smooth scaling of appreciable LBO powers with magnetic field in Fig. 5.21, the $\pi$-mode was likely the dominant mode observed in nearly all shots. If the magnetron operated primarily in the $5 \pi / 6$-mode, it is unlikely that the output powers would be higher than those observed with the uniform magnetic field in the isolated configuration. Furthermore, the frequency bifurcation at higher magnetic fields is more consistent with both expectations and observed operation in the isolated LBO configuration (since two modes were observed). It is therefore conceivable that the effect of the previously discussed hub height variation on the resonant frequency of the $\pi$-mode diminishes below a threshold near $B=0.21 \mathrm{~T}$ leading to $\pi$-mode and $5 \pi / 6$-mode frequencies that were similar to those observed in the isolated LBO shots.

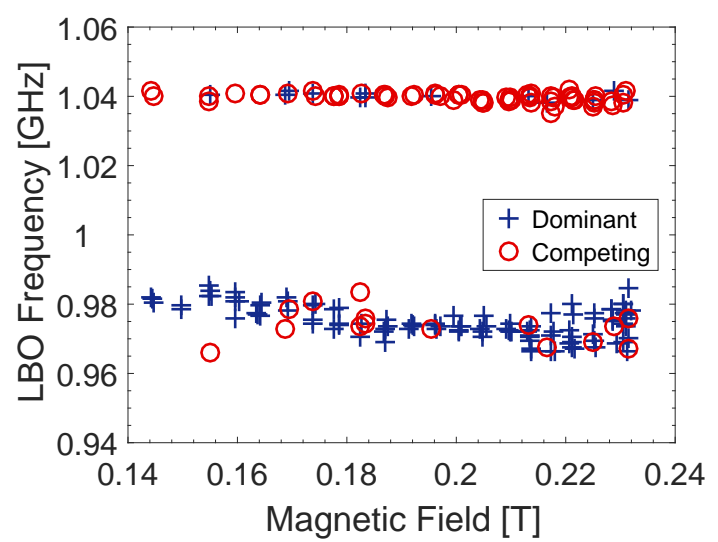

(a) LBO frequencies vs. magnetic field.

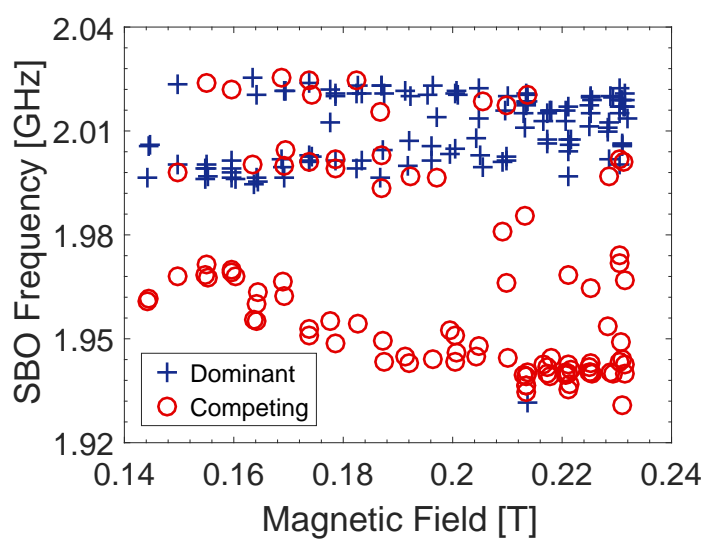

(b) SBO frequencies vs. magnetic field.

Figure 5.22: Standard Load and Nonuniform Magnetic Field: Dominant and competing frequencies of both oscillators vs. magnetic field. 


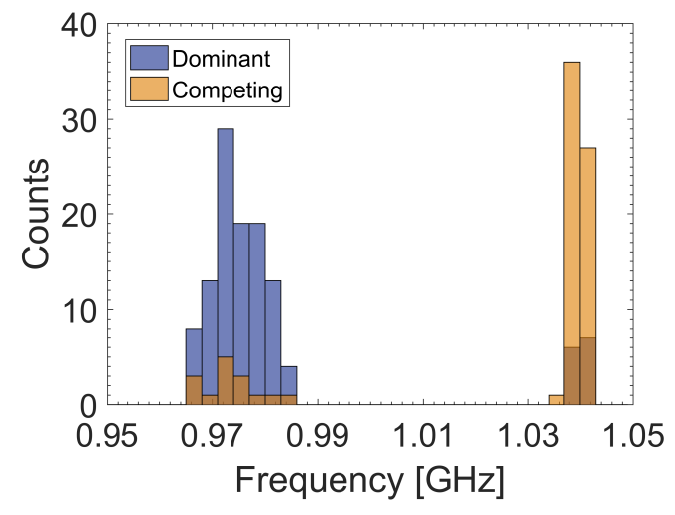

(a) LBO frequency histogram.

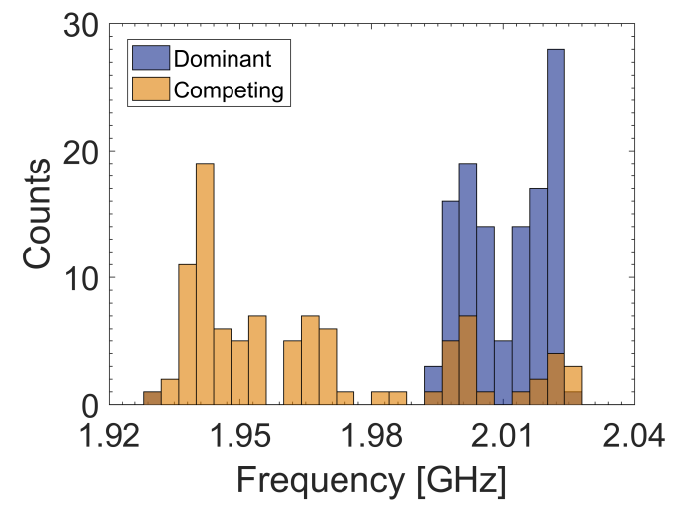

(b) SBO frequency histogram.

Figure 5.23: Standard Load and Nonuniform Magnetic Field: Dominant and competing frequency histograms for both oscillators.

This interpretation is reasonable because the electron hub height at high magnetic fields is smaller and the impact of the variation would be less pronounced. In nearly all shots, the LBO experienced mode competition with the $1.04 \mathrm{GHz}$ parasitic chamber mode described in $\S 3.3 .1$.

Figure 5.22b shows the SBO frequencies vs. magnetic field. SBO operation was more consistent with both theoretical expectations and the experimentally observed operation in the isolated configuration. Operation near $2.02 \mathrm{GHz}$ was observed at higher magnetic fields with a gradual transition to a $2 \mathrm{GHz}$ mode at lower magnetic fields. Most mode competition occurred near $1.94 \mathrm{GHz}$ at magnetic fields above approximately $0.21 \mathrm{~T}$. Below this magnetic field, a smooth frequency increase occurred with decreasing magnetic field, which was similar to the behavior observed in the LBO. The possible relation between the oscillators and origin of this trend is explored in the Additional Analysis section below. From the agreement between the observed frequencies and the results of both simulations and the experimental isolated SBO configuration, it is likely that the $1.94,2.00$, and $2.02 \mathrm{GHz}$ frequencies correspond to the $6 \pi / 8$-mode, $7 \pi / 8$-mode, and $\pi$-mode, respectively.

The corresponding LBO and SBO frequency histograms are shown in Fig. 5.23. The results for the LBO shown in Fig. 5.23a show that any distinct identification of the $5 \pi / 6$ mode and $\pi$-mode based on the frequency distribution is impossible. However, the SBO shows clearly defined, distinct peaks at the expected frequencies for the $6 \pi / 8$-mode, $7 \pi / 8$ mode, and $\pi$-mode, although the previously described smooth frequency scaling of the $6 \pi / 8$-mode with magnetic field led to considerable spread in its frequency distribution.

Figure 5.24 shows the microwave output powers vs. dominant frequency for the oscillators. For the LBO results shown in Fig. 5.24a, the parasitic chamber mode was evidently 


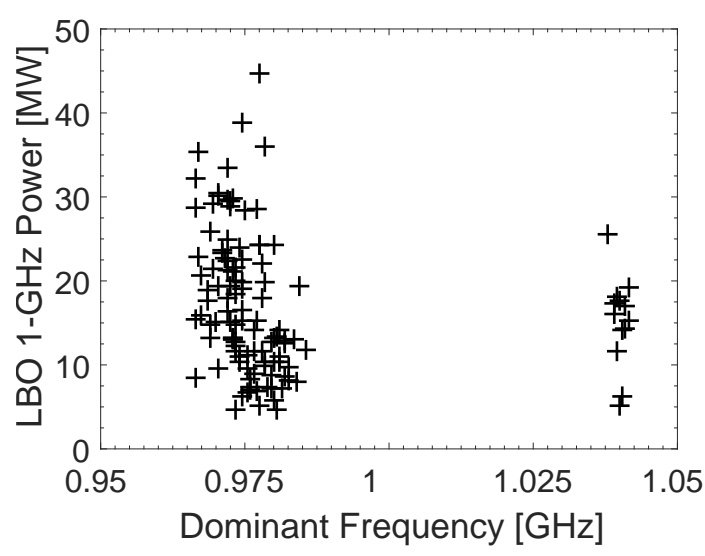

(a) Peak LBO power vs. dominant operating frequency.

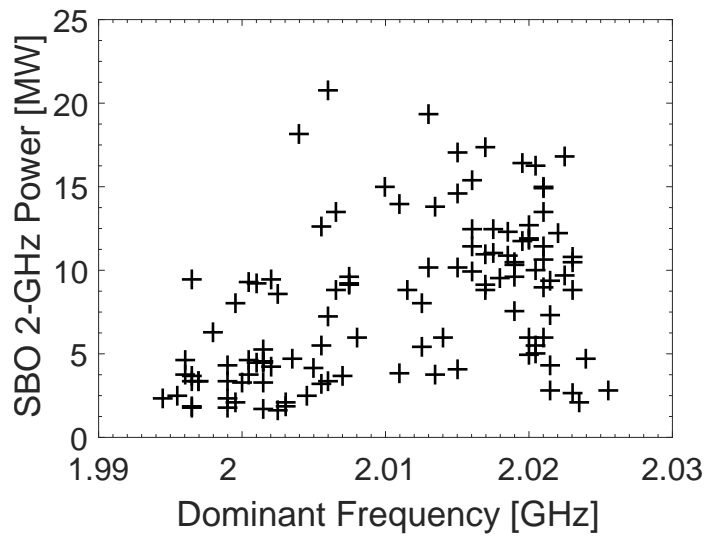

(b) Peak SBO power vs. dominant operating frequency.

Figure 5.24: Standard Load and Nonuniform Magnetic Field: Peak oscillator powers vs. dominant operating frequencies.

capable of producing significant microwave power at $1.04 \mathrm{GHz}$ and coupling to the extractor, which was not consistent with the simulations. Further analysis of the chamber mode performance will be performed in the Additional Analysis section. The previously described smooth scaling of the $\pi$-mode frequency with magnetic field makes interpretation of the plot difficult, particularly because the frequency bifurcation observed at $B \gtrsim 0.21 \mathrm{~T}$ leads to two frequencies falling in the span observed during the smooth scaling observed at lower magnetic fields. Further analysis of the shots at higher magnetic fields is left to the Additional Analysis section.

The SBO power vs. dominant frequency is shown in Fig. 5.24b. These results were perfectly consistent with expectations, with the $2 \mathrm{GHz} 7 \pi / 8$-mode consistently generating significantly less extracted microwave power than the $2.02 \mathrm{GHz} \pi$-mode. This is consistent with the results from simulations and the isolated SBO configuration.

Electrical characteristics for the MFRPM are shown in Fig. 5.25. As discussed previously, the currents (and therefore impedances) in Figs. 5.25a and 5.25b were disrupted relatively early in the series by damaging shots. Shot-to-shot variability was considerable, and while it is difficult to say whether that was due primarily to damage or the nonuniform magnetic field, a limited shot series conducted after component refurbishment produced similar results at lower average currents. It is therefore likely that the variability was due primarily to the nonuniform magnetic field. The higher average currents lead to impedances that were uniformly less than the 100-150 $\Omega$ MELBA-C specification, which negatively impacts voltage pulse rise-times, as described in Appendix C.

The LBO start and peak power voltages vs. magnetic field are shown in Fig. 5.25c. 


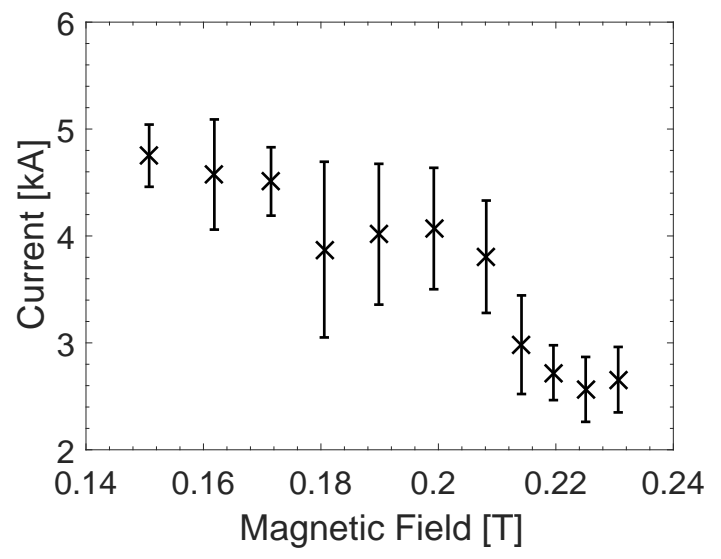

(a) Current at peak power vs. magnetic field.

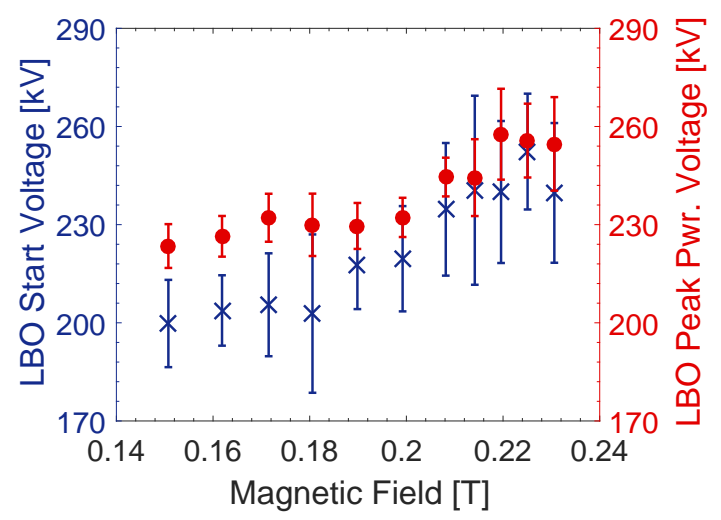

(c) Voltage at time of LBO start and peak power.

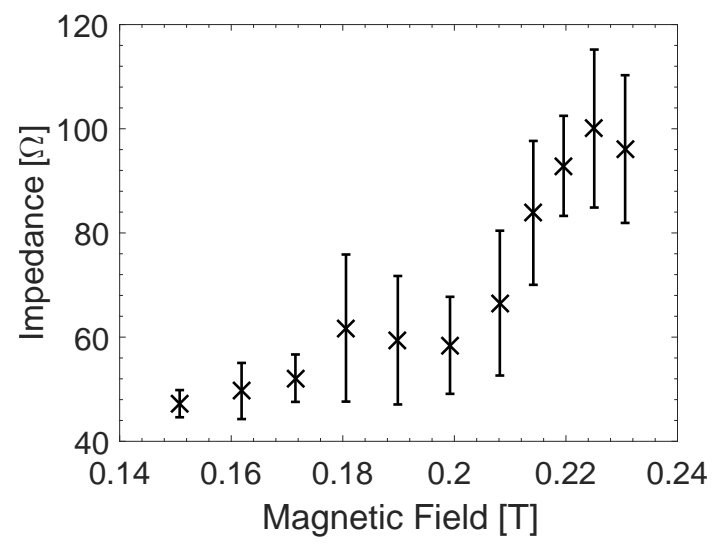

(b) Impedance at peak power vs. magnetic field.

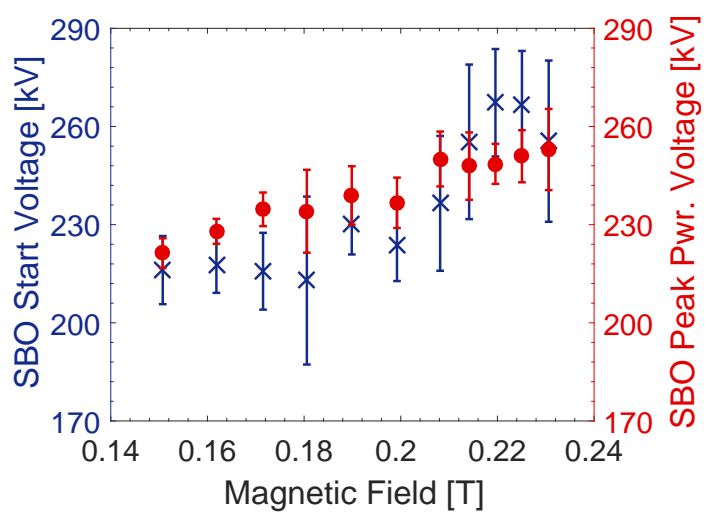

(d) Voltage at time of SBO start and peak power.

Figure 5.25: Standard Load and Nonuniform Magnetic Field: Electrical characteristics vs. magnetic field.

Linear scaling of the peak power voltage changes at $B \simeq 0.21 \mathrm{~T}$, which is the point at which frequency bifurcation begins. This is consistent with a change in operating mode or a region of mode competition, which was precisely what was observed at $B \gtrsim 0.21 \mathrm{~T}$. This was also reflected in the start voltages, although the change in start voltage manifests at a lower magnetic field of approximately $0.19 \mathrm{~T}$, which is not surprising given that a competing mode might start earlier, but not be the dominant mode at a given magnetic field.

Similar behavior was observed for the SBO in Fig. 5.25d. The jump in linear scaling for the peak power voltage occurred at $B \simeq 0.21 \mathrm{~T}$, which is consistent with the results of Fig. 5.22b on page 96, which shows that $B \gtrsim 0.21 \mathrm{~T}$ was the range in which the $\pi$ mode was the consistently dominant mode. However, the start voltages in this magnetic field range were higher than the peak power voltages. As shown by Fig. 5.27b (discussed shortly), this was due to the significantly later times at which peak power was observed. The 


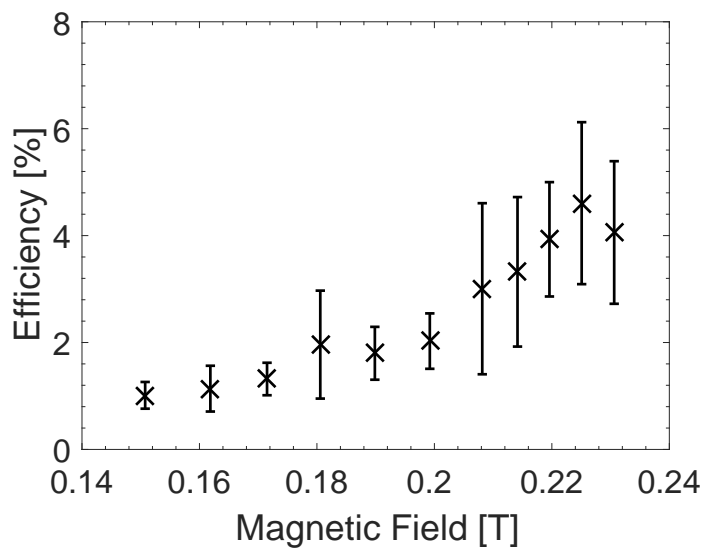

(a) Peak total efficiency vs. magnetic field.

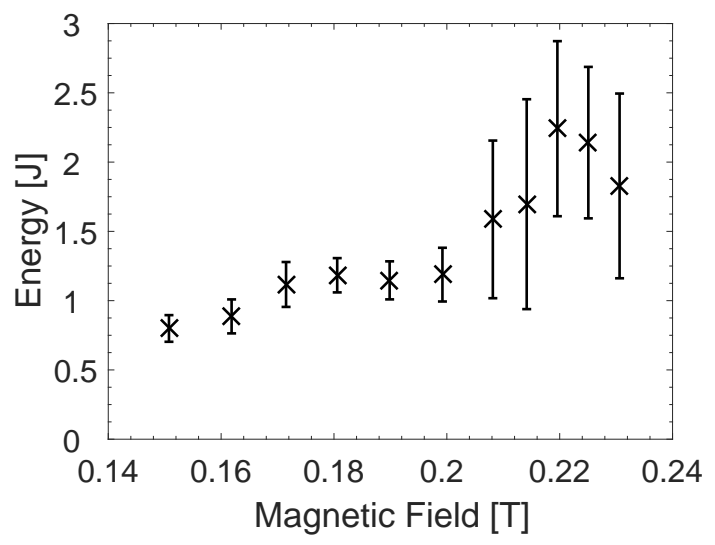

(b) Total pulse energy vs. magnetic field.

Figure 5.26: Standard Load and Nonuniform Magnetic Field: Energy conversion metrics vs. magnetic field.

later times led to greater diode closure and therefore higher current, which is the origin of the lower voltage at later times. An example of a shot exhibiting this behavior is discussed in the Additional Analysis section.

Energy conversion metrics are shown in Fig. 5.26. Efficiencies were generally low at $B \lesssim 0.21 \mathrm{~T}$, although even in the region of optimal operation, efficiencies were less than $5 \%$ and exhibited considerable shot-to-shot variability. This is reflected in the total pulse energies, although it is interesting that there was considerable variability at $B \gtrsim$ $0.21 \mathrm{~T}$, and a high degree of consistency at $B \lesssim 0.21 \mathrm{~T}$. It appears likely that the primary pulse-shortening mechanism changed at $B \simeq 0.21 \mathrm{~T}$. Cathode plasma expansion appears to be the source of pulse-shortening at $B \lesssim 0.21$, which is consistent with constant-energy behavior [104]. At $B \gtrsim 0.21 \mathrm{~T}$, it is likely that the higher magnetic fields help to reduce the closure velocity of the cathode plasma [112], and that the higher observed powers in this range led to RF-induced cavity plasma formation that was observed in the pulse-shortening configurations discussed at the end of this chapter.

Temporal metrics are shown in Fig. 5.27. In most cases, the LBO started before the SBO, which is consistent with expectations based on the discussion of cavity fringe fields in $\S 2.1$. The LBO peak power times in Fig.5.27a scale linearly with magnetic field up to $B \simeq 0.19 \mathrm{~T}$. Later times correspond to greater gap closure during the MELBA-C voltage flat-top. At $B \gtrsim 0.21 \mathrm{~T}$, there was high variation in the peak power time.

The SBO exhibited similar behavior, although it is interesting to note that the peak power times in Fig. 5.27b actually decrease with increasing magnetic field up to $B \simeq 0.21 \mathrm{~T}$. This result is difficult to explain because the region of magnetic fields $B \lesssim 0.21 \mathrm{~T}$ corresponds to considerable mode competition between the $7 \pi / 8$-mode and $\pi$-mode, although 


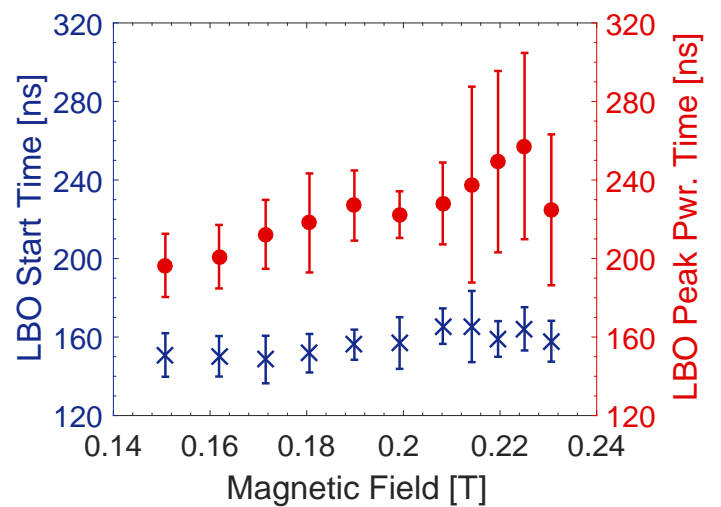

(a) LBO microwave start and peak power times.

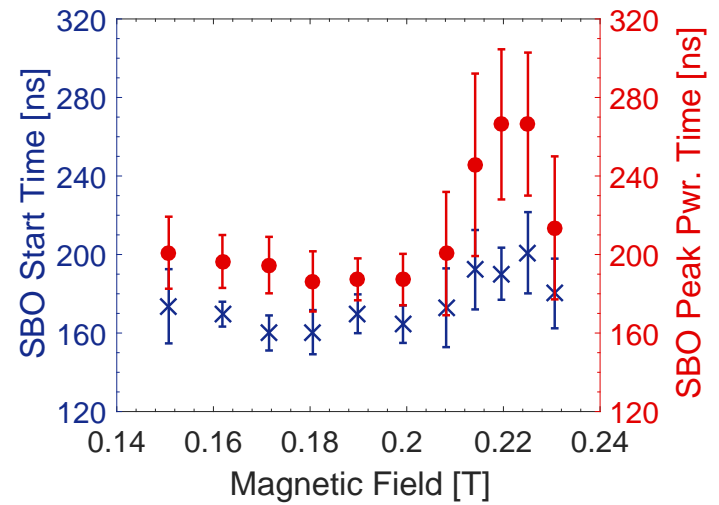

(b) SBO microwave start and peak power times.

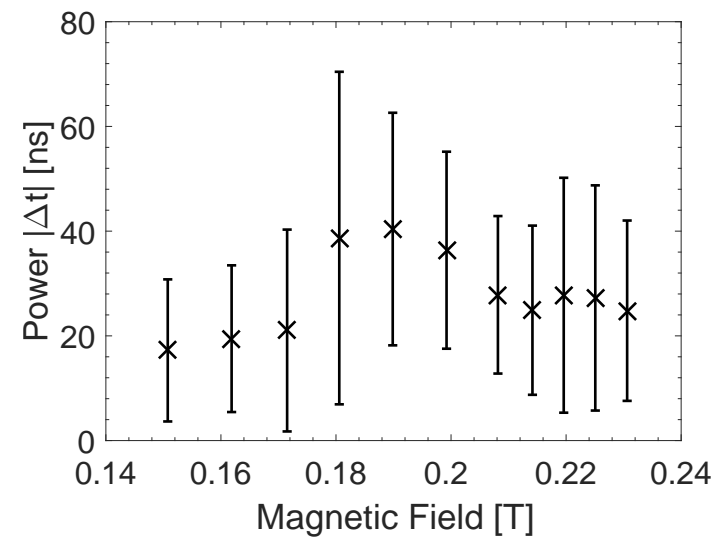

(c) Absolute time difference $|\Delta t|$ between oscillator peak powers vs. magnetic field.

Figure 5.27: Standard Load and Nonuniform Magnetic Field: Temporal metrics vs. magnetic field.

Fig. 5.22b shows that $7 \pi / 8$-mode was generally the more dominant of the two. This fact, coupled with both the magnetic field variation along the planar cavity arrays and the unusual electric field pattern of the $7 \pi / 8$-mode (discussed in greater detail in $\S 3.3 .1$ ), makes an intuitive explanation exceptionally difficult, but it is clear that the optimal beam velocity (determined by the voltage and magnetic field) leading to peak power production at the $7 \pi / 8$-mode that does not scale in a way that is consistent with the theory presented in $\S 2.2$.

Finally, Fig. 5.27c shows the scaling of the peak power $|\Delta t|$ with magnetic field. It is clear that the oscillators did not behave as a coupled unit at any magnetic field magnitude. All shots saw a significant difference between the times at which peak power was observed for the two oscillators, and shot-to-shot variation was very large. Table 5.5 on page 64 summarizes the standard metrics in the region of optimal operation. 


\subsubsection{Additional Analysis}

Several interesting operational attributes were observed in this data set, including: 1) several shots in which the SBO operated in the $7 \pi / 8$-mode, 2) several shots where the LBO operated in the parasitic chamber mode, 3) the frequency bifurcation of the LBO at $B \gtrsim 0.21 \mathrm{~T}, 4)$ the harmonic relation between the dominant LBO and competing SBO modes, and 5) individual shots exhibiting clear mode competition. These points will be addressed in order.

Unlike shots conducted using the isolated SBO configuration, this data set provided an ample number of shots in which the SBO operated in the $7 \pi / 8$-mode, enabling accurate frequency and power determination. The range of magnetic fields best suited for $7 \pi / 8$ mode operation was identified by inspecting Fig. 5.22b on page 96 and locating the region of minimal mode competition with the $\pi$-mode, which was $B \simeq 0.14-0.17$ T. Table 5.6 on page 64 summarizes the performance metrics for the MFRPM in this magnetic field range. The analysis enabled the identification of the SBO $7 \pi / 8$-mode frequency with high confidence, and also proved that the $7 \pi / 8$-mode powers, even during pure operation, were consistently lower than the $\pi$-mode powers.

For the parasitic chamber mode, no single range of magnetic fields produced consistent, dominant operation. Instead, the LBO occasionally exhibited dominant operation in the parasitic chamber mode across the entire range of tested magnetic fields. Since this range covers different regions of operation for the SBO, only the LBO power and frequency are reported in the summary provided by Table 5.7 on page 64 . The appearance of the mode at all magnetic fields with powers as high as $15 \mathrm{MW}$ was not expected based on the simulation

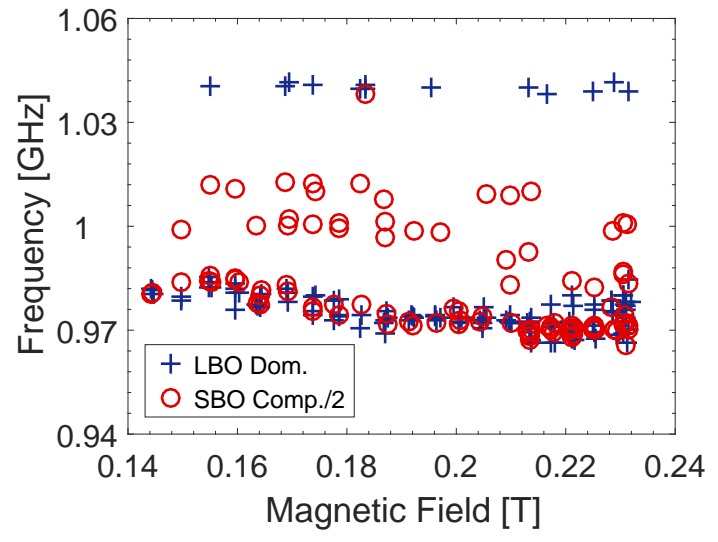

(a) Standard view.

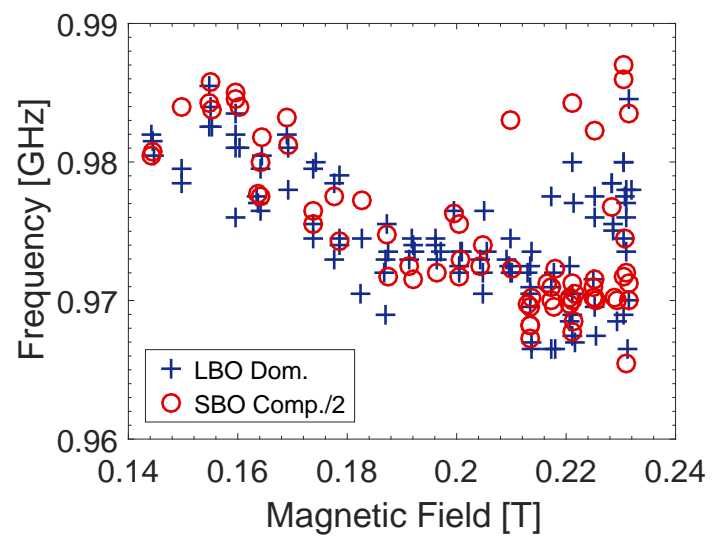

(b) Expanded frequency view.

Figure 5.28: Standard Load and Nonuniform Magnetic Field: LBO dominant frequency and $0.5 \times$ SBO competing frequency vs. magnetic field. 
results.

As previously mentioned, the LBO exhibited frequency bifurcation at $B \gtrsim 0.21 \mathrm{~T}$, as shown in Fig. 5.28b. This phenomenon is likely related to the harmonic relation between the dominant LBO and competing SBO modes. These modes clearly show identical scaling in the same figure. This effect was not observed in simulations, so it may relate to the magnetic field variation along the planar cavity arrays because the magnetic field variation was omitted from simulations. In any case, it is apparent that a mode supported by the SBO was capable of coupling to the LBO and producing harmonic frequency-locked behavior, though a phase analysis cannot be performed because the competing SBO mode was not dominant.

As discussed in the Standard Metrics section, the $6 \pi / 8$-mode of the SBO likely falls near $1.94 \mathrm{GHz}$, but a competing mode consistently near this frequency was only observed in $B \gtrsim 0.21 \mathrm{~T}$. Given the smooth scaling of the frequency with magnetic field at lower magnetic fields, it appears that the SBO $6 \pi / 8$-mode frequency-locked to the second harmonic frequency of the dominant LBO mode only when $B \lesssim 0.21 \mathrm{~T}$. The conditions under which this occurred were probably related to the conditions that led to the smooth frequency scaling of the LBO $\pi$-mode, viz. that the hub height variation effect on the resonant frequency of the LBO $\pi$-mode was sufficiently strong in $B \lesssim 0.21 \mathrm{~T}$. In $B \gtrsim 0.21 \mathrm{~T}$, the effect is weak, leading to distinct $\pi$-mode and $5 \pi / 6$-mode frequencies similar to those observed in the isolated LBO shots. Additionally, in this higher magnetic field range, a decoupling of the SBO $6 \pi / 8$-mode from the second harmonic frequency of the LBO $\pi$-mode appears to occur, leading to the "free-running" SBO $6 \pi / 8$-mode frequency observed in the shots conducted using the isolated SBO configuration. Thus, $B=0.21 \mathrm{~T}$ marks a transition point below which a competing SBO mode (likely the $6 \pi / 8$-mode) frequency-locks to the second harmonic frequency of the dominant LBO mode (likely the $\pi$-mode).

Two shots illustrate the existence of the three distinct modes for the two oscillators, namely the $5 \pi / 6$-mode, $\pi$-mode, and chamber mode for the LBO, and the $6 \pi / 8$-mode, $7 \pi / 8$-mode, and $\pi$-mode for the SBO. The first shot, shown in Fig. 5.29, focuses on the LBO. Figure 5.29a shows the shot plot, where the LBO initially shows considerable swings in output power, followed by a relatively constant power output at a much lower magnitude prior to oscillation cessation. The time-integrated Fourier transform in Fig. 5.29b reveals the presence of all three modes in this single shot. The time-resolved TFA in Fig. 5.29c supports the interpretation of the power trace, where the $\pi$-mode and chamber mode appear to be in competition during the first part of the pulse, followed by stabilization at a lower frequency corresponding to the low-power $5 \pi / 6$-mode later in the pulse. Furthermore, the frequency-resolved TFA in Fig. 5.29d suggests the beginning of the pulse actually saw 


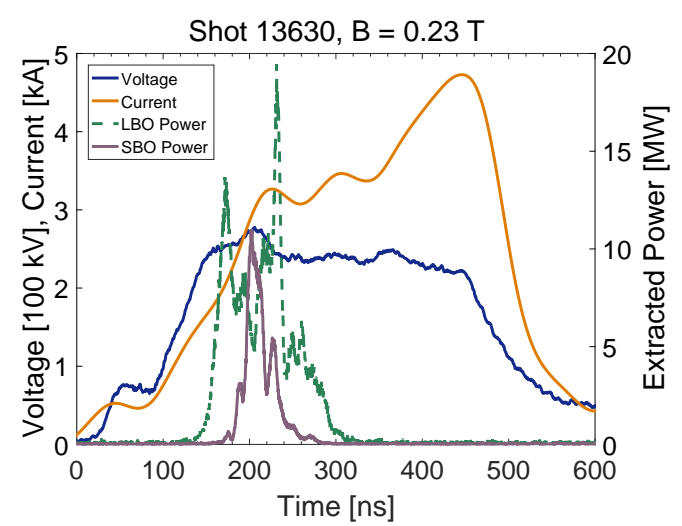

(a) Plot of voltage, current, and RF power for shot 13630.

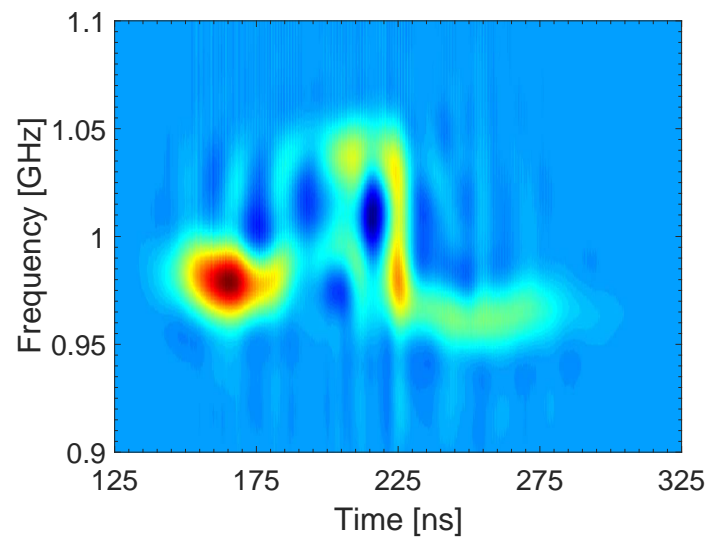

(c) Time-resolved TFA of the LBO RF voltage signal from shot 13630 using the Born-Jordan RIK.

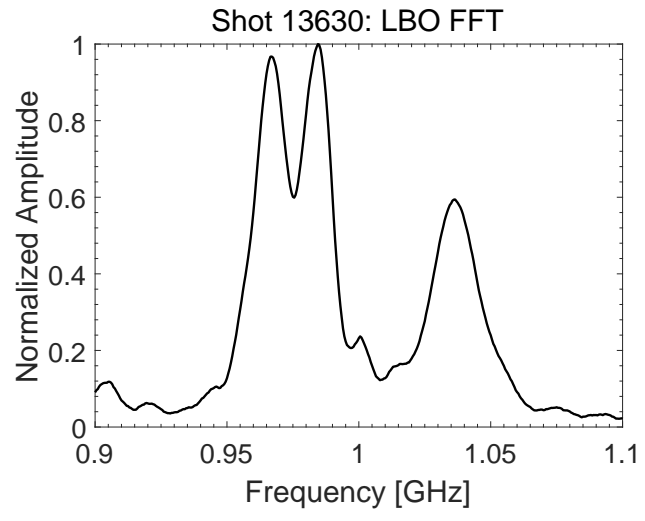

(b) Time-integrated Fourier transform of the LBO RF voltage signal from shot 16320.

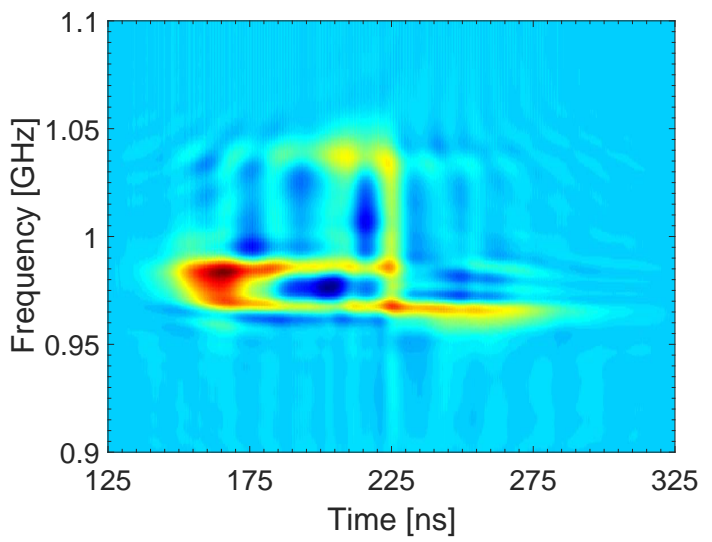

(d) Frequency-resolved TFA of the LBO RF voltage signal from shot 13630 using the Born-Jordan RIK.

Figure 5.29: Standard Load and Nonuniform Magnetic Field: Analysis of the LBO on shot 13630.

competition between all three modes, not just the $\pi$-mode and parasitic chamber mode.

Figure 5.30 shows the analysis of a shot focusing on the SBO. Again, within the single shot, all three SBO-supported modes were observed. Figure 5.30a shows the shot plot, which reveals a smooth, beating increase in the SBO power output, followed by a significant drop in output power before a second burst followed by oscillation cessation. The time-integrated Fourier transform in Fig. 5.30b confirms the presence of the three modes. A time-resolved TFA shows that the initial beating in the microwave pulse was consistent with simultaneous operation in different modes, although it does not have enough frequency resolution to say whether the mode competing with the $6 \pi / 8$-mode was the $7 \pi / 8$-mode or $\pi$-mode. Figure 5.30d suggests the competing mode was the $\pi$-mode, with the $7 \pi / 8$ mode starting once the $6 \pi / 8$-mode ceases. Subsequent operation was bimodal, and the two 


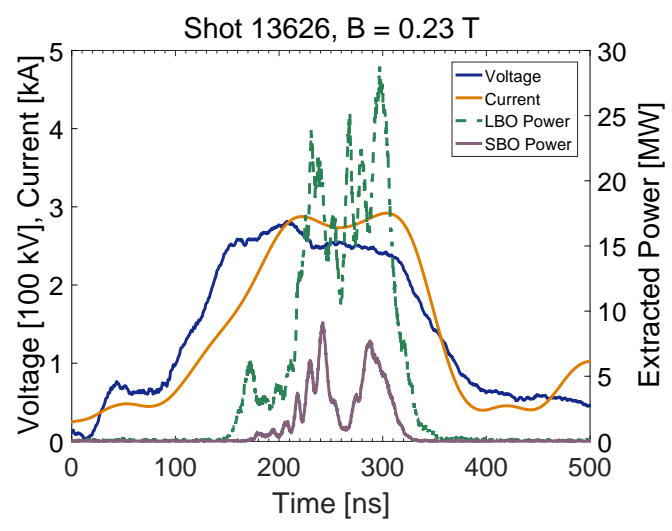

(a) Plot of voltage, current, and RF power for shot 13626.

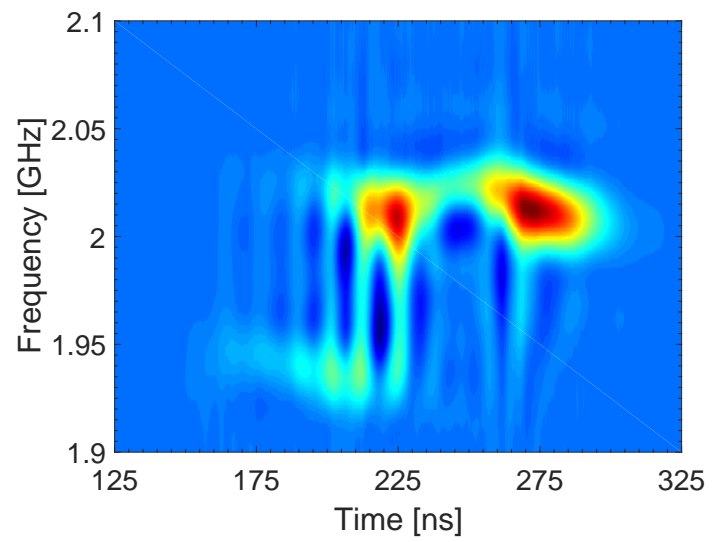

(c) Time-resolved TFA of the SBO RF voltage signal from shot 13626 using the Born-Jordan RIK.

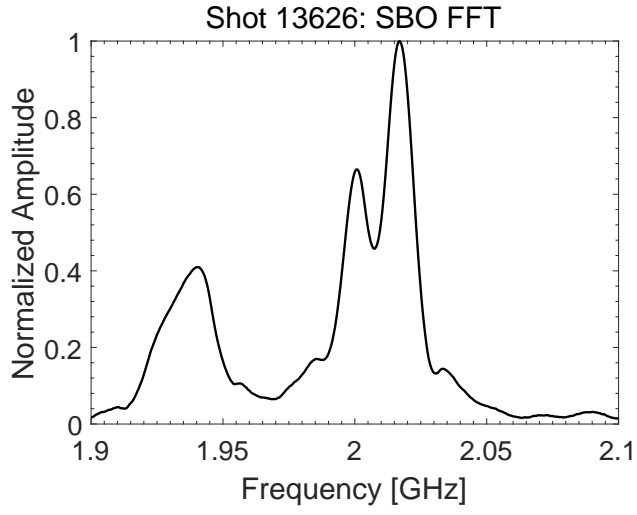

(b) Time-integrated Fourier transform of the SBO RF voltage signal from shot 13626.

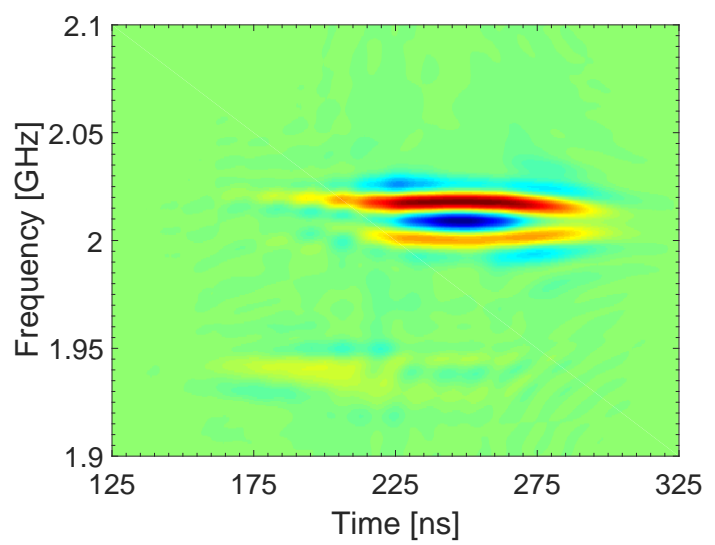

(d) Frequency-resolved TFA of the SBO RF voltage signal from shot 13626 using the Zhao-AtlasMarks RIK.

Figure 5.30: Standard Load and Nonuniform Magnetic Field: Analysis of the SBO on shot 13626.

modes, which have very closely-spaced frequencies, explain the low beat frequency. 


\subsection{MFRPM with Standard Load and Uniform Magnetic Field}

This shot series tested the MFRPM using the uniform magnetic field described in $\S 4.2 .2$. The purpose was to obtain the second of two data sets necessary to determine how the magnetic field variation along the planar cavity arrays impacted operation of the MFRPM under ideal operating conditions with well-matched microwave loads. A total of 95 shots were obtained with no MELBA-C self-triggers, magnet triggering failures, or failed crowbars. The base pressure was $1.1 \mu$ torr, and a residual gas analyzer indicated the background gas composition was $60 \% \mathrm{H}_{2}, 15 \% \mathrm{He}, 12 \%$ air, $7 \% \mathrm{H}_{2} \mathrm{O}$, and $6 \%$ other trace gasses. The range of tested magnetic fields was $0.12-0.26 \mathrm{~T}$.

\subsubsection{Standard Metrics}

Figure 5.31 shows the peak output powers from both oscillators vs. magnetic field. Operation in the range of magnetic fields $B \lesssim 0.17 \mathrm{~T}$ was excellent from the standpoint of consistency, with the region of optimal operation falling in the range of $B=0.16-0.18 \mathrm{~T}$. The Buneman-Hartree magnetic field $B_{\mathrm{BH}}$ for synchronism with the $\pi$-mode is approximately $0.16 \mathrm{~T}$, so these data were also in good agreement with theoretical predictions. Operation at higher magnetic fields displayed greater variability, and power production from the SBO was considerably lower, both of which were expected due to the insufficient velocity of the electron beam to synchronize with the $5 \pi / 6$-mode or $\pi$-mode. Interestingly, at the limits of electromagnet operation, the SBO returned to high power operation, albeit with greater variability. One possibility for this behavior is synchronism with the next

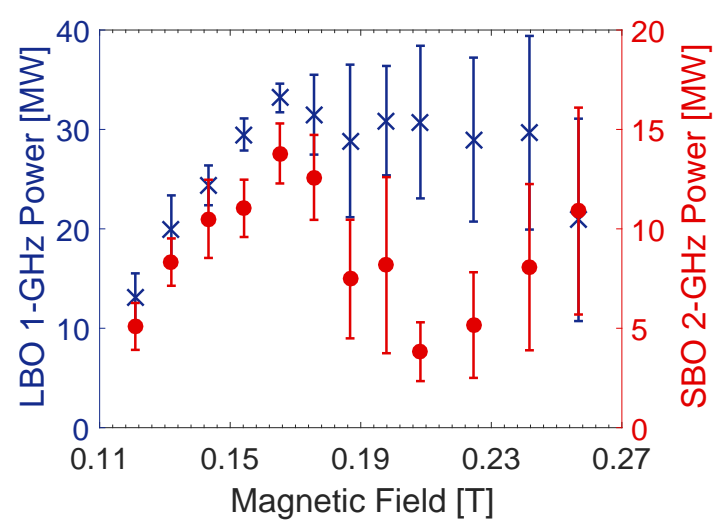

Figure 5.31: Standard Load and Uniform Magnetic Field: Peak output powers for both oscillators vs. magnetic field. 


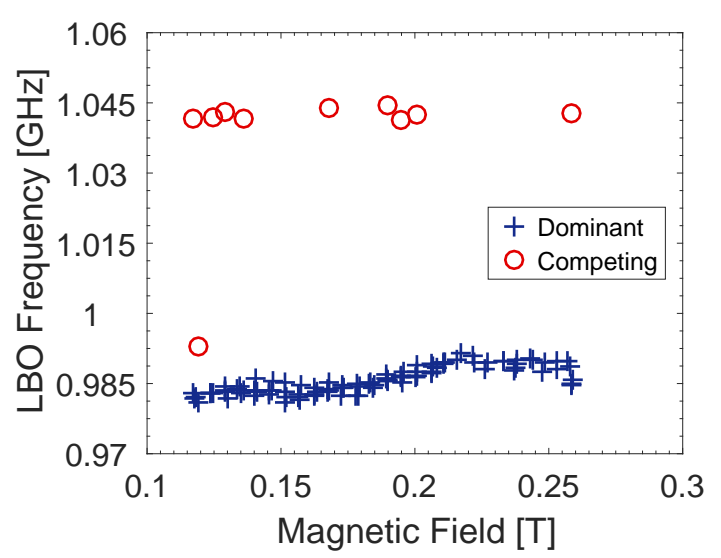

(a) LBO frequencies vs. magnetic field.

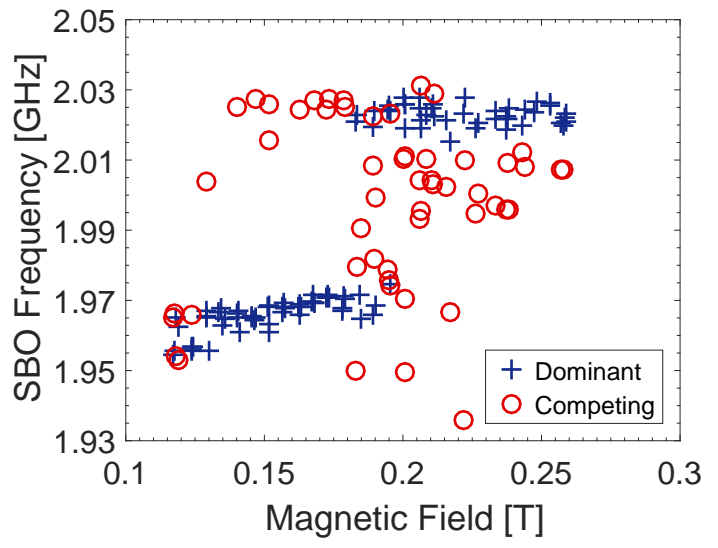

(b) SBO frequencies vs. magnetic field.

Figure 5.32: Standard Load and Uniform Magnetic Field: Dominant and competing frequencies of both oscillators vs. magnetic field.

space harmonic of the $\pi$-mode, also termed the $3 \pi$-mode. However, the expected magnetic field for $3 \pi$-mode operation based on theory would be approximately three times that of the Buneman-Hartree magnetic field $B_{\mathrm{BH}}=0.16 \mathrm{~T}$, so this explanation is not compelling.

The frequency metrics are shown in Fig. 5.32. The range of LBO frequencies shown by Fig. 5.32a was remarkably consistent at all tested magnetic fields. There did not appear to be any transitions to other operating modes, though a few shots occasionally had mode competition with the parasitic chamber mode near $1.04 \mathrm{GHz}$. Since the dominant operating frequency was consistently near $0.985 \mathrm{GHz}$, the LBO likely operated in the $\pi$-mode on every shot. However, the $\pi$-mode frequency was susceptible to variation, with one consistent frequency in the range of $B \lesssim 0.18 \mathrm{~T}$, and another consistent frequency in the range of $B \gtrsim 0.22 \mathrm{~T}$, with a gradual transition region between the two. This property appears to be closely related to the operation of the SBO.

The range of SBO frequencies is shown in Fig. 5.32b. The results illustrate a clear transition point between two different operating frequencies at $B \simeq 0.18 \mathrm{~T}$. There were three distinct regions of mode competition. The first appears at the lowest tested magnetic fields, where a mode near $1.95 \mathrm{GHz}$ was in competition with a mode near $1.97 \mathrm{GHz}$. In this range, some shots exhibited the $1.97 \mathrm{GHz}$ frequency as the dominant frequency and the $1.95 \mathrm{GHz}$ frequency as the competing frequency, and some shots saw the $1.95 \mathrm{GHz}$ frequency as the dominant frequency and the $1.97 \mathrm{GHz}$ frequency as the competing frequency. The second region was at $B \lesssim 0.18 \mathrm{~T}$, where the $1.97 \mathrm{GHz}$ mode was in competition with a $2.02 \mathrm{GHz}$ mode, though the competition was not extreme and the number of instances was relatively small. The third region was at $B \gtrsim 0.18 \mathrm{~T}$, where the $2.02 \mathrm{GHz}$ mode is in consistent competition with a mode having a spread of frequencies near $2 \mathrm{GHz}$. Representative fre- 


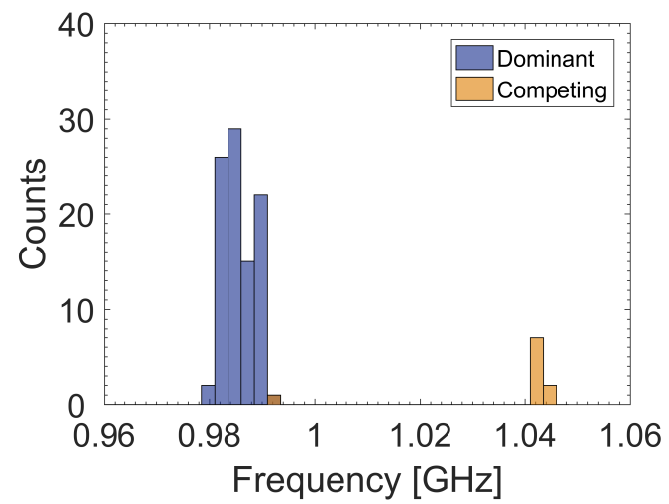

(a) LBO frequency histogram.

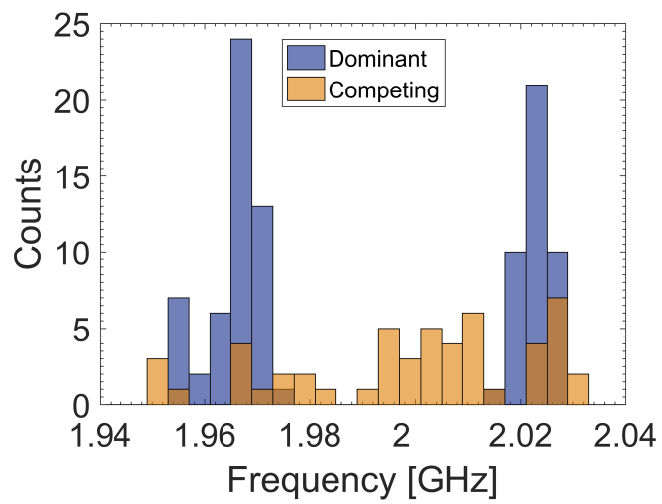

(b) SBO frequency histogram.

Figure 5.33: Standard Load and Uniform Magnetic Field: Dominant and competing frequency histograms for both oscillators.

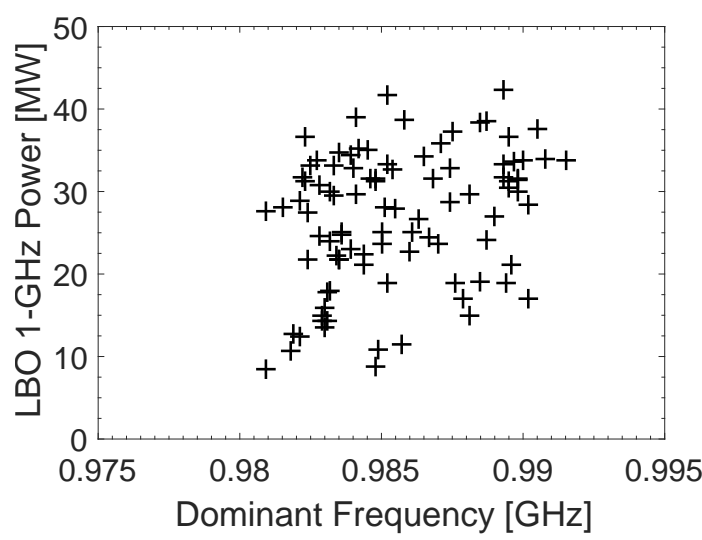

(a) Peak LBO power vs. dominant operating frequency.

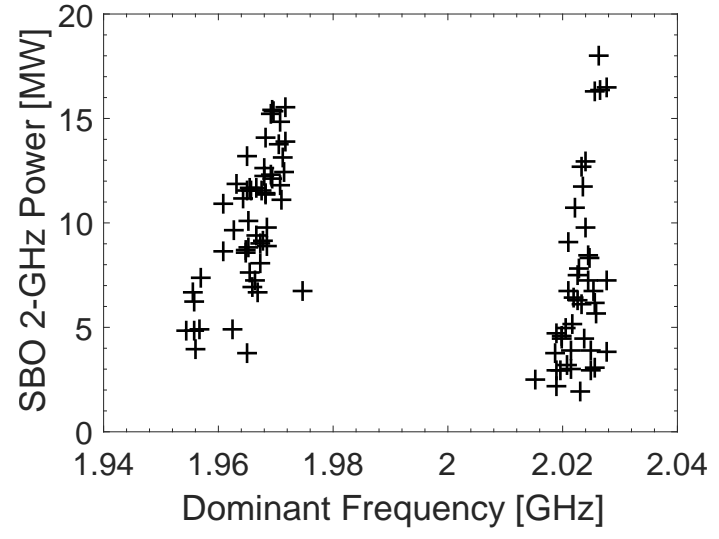

(b) Peak SBO power vs. dominant operating frequency.

Figure 5.34: Standard Load and Uniform Magnetic Field: Peak oscillator powers vs. dominant operating frequencies.

quencies for these four modes can be determined using the frequency histograms discussed next.

The two dominant peaks in the LBO frequency histogram (Fig. 5.33a) are simply due to the gradual transition in the $\pi$-mode operating frequency with changes in the magnetic field (Fig. 5.32a), which was likely related to the SBO transition from $1.970 \mathrm{GHz}$ to $2.02 \mathrm{GHz}$. The competing frequencies near $1.04 \mathrm{GHz}$ are consistent with the parasitic chamber mode identified in $\S 3.3 .1$. The SBO frequency histogram in Fig. 5.33b confirms the presence of four frequencies. Three manifest as dominant peaks (1.95 GHz, $1.97 \mathrm{GHz}$, and $2.02 \mathrm{GHz}$ ), and the fourth has a spread of frequencies between $2 \mathrm{GHz}$ and $2.01 \mathrm{GHz}$.

The relation between operating power and dominant frequency is shown in Fig. 5.34. 


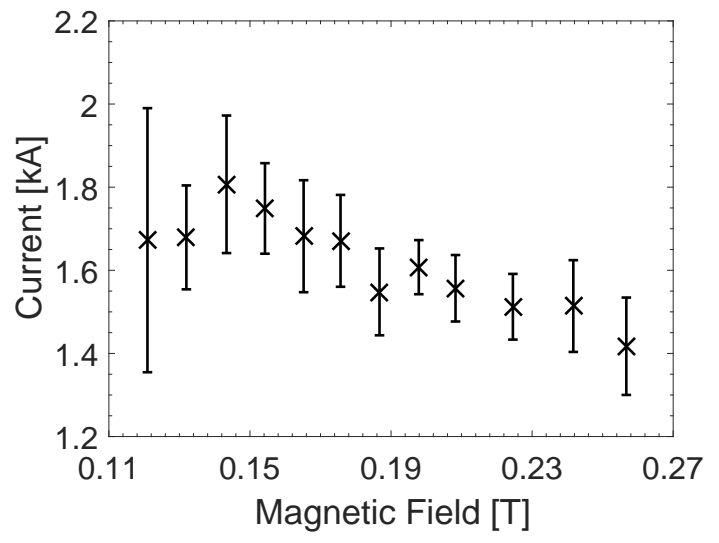

(a) Current at peak power vs. magnetic field.

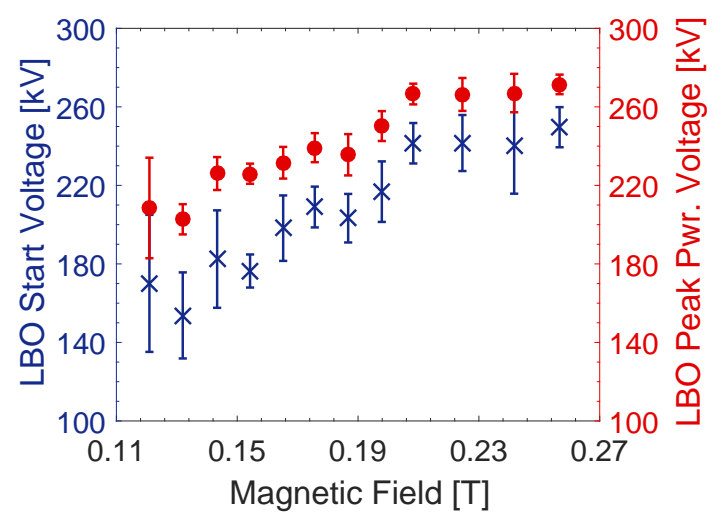

(c) Voltage at time of LBO start and peak power.

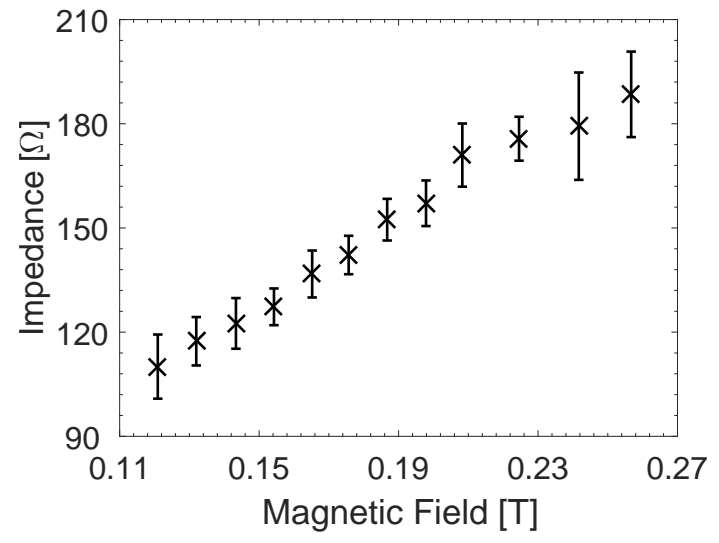

(b) Impedance at peak power vs. magnetic field.

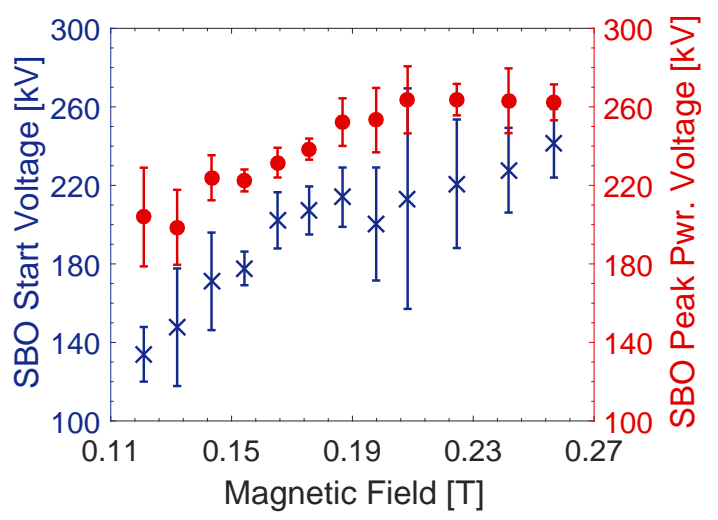

(d) Voltage at time of SBO start and peak power.

Figure 5.35: Standard Load and Uniform Magnetic Field: Electrical characteristics vs. magnetic field.

For the LBO, Fig. 5.34a shows no significant difference between the powers produced by the different $\pi$-mode frequencies despite evidence of SBO influence on LBO operation (where the LBO $\pi$-mode frequency changed slighly when the SBO operating mode shifted). The same cannot be said of the SBO in Fig. 5.34b, which clearly shows the $1.97 \mathrm{GHz}$ mode producing the highest power (on average), followed by the $2.02 \mathrm{GHz}$ mode (albeit with significant variation in power), followed by the few shots exhibiting dominant operation close to $1.95 \mathrm{GHz}$. Given the similarities in SBO operation between this configuration and the results from both simulations and experiment for the isolated SBO configuration, it appears that the $1.955 \mathrm{GHz}, 2.00 \mathrm{GHz}$, and $2.02 \mathrm{GHz}$ modes correspond to the $6 \pi / 8$ mode, $7 \pi / 8$-mode, and $\pi$-mode, respectively. Discussion of the origin of the $1.97 \mathrm{GHz}$ mode, which was observed as neither a dominant nor a competing mode in the isolated SBO configuration, is left to the Additional Analysis section.

Electrical characteristics are shown in Fig. 5.35. With the exception of the lowest and 
highest magnetic fields tested, the currents shown in Fig. 5.35a exhibit excellent shot-toshot consistency. The gradual reduction in current vs. magnetic field was consistent with expectations. This outcome was also reflected in the impedances shown in Fig. 5.35b. The results show that the MFRPM was nearly perfectly matched to the 100-150 $\Omega$ specification for MELBA-C in $B \lesssim 0.18 \mathrm{~T}$, which includes the region of optimal operation. Shot-toshot impedance variability was exceptionally low, and the scaling with magnetic field was smooth and consistent with expectations.

The start and peak power voltages for the LBO are shown in Fig. 5.35c. Both quantities exhibit two clearly defined linear regions which, as discussed previously, suggest two different modes of operation or a transition from operation during the voltage rise to operation during the flat-top portion of the voltage pulse. Given the appreciable powers observed from the LBO over the range of magnetic fields tested, and given the proximity of the range of observed dominant frequencies to the $\pi$-mode frequencies observed in the experiment and in the isolated LBO experimental configuration, it is unlikely that a transition to the $5 \pi / 6$-mode occurred in this data set. Low power production by the LBO operating in the $5 \pi / 6$-mode was demonstrated in both simulation and in the isolated LBO experimental configuration. Instead, it appears that the SBO transition from the $1.97 \mathrm{GHz}$ mode to the $2.02 \mathrm{GHz}$ mode affected the synchronous velocity of the LBO $\pi$-mode, which is reasonable given the change in the dominant LBO frequency from approximately $0.984 \mathrm{GHz}$ to $0.989 \mathrm{GHz}$. From the discussion in $\S 2.2$, a change in frequency necessitates a change in synchronous velocity for dispersive structures, which includes planar cavity arrays, so this result supports the interpretation that the LBO $\pi$-mode underwent a change in coupling to the SBO, which slightly modified the LBO $\pi$-mode frequency. While this is a possible explanation for the shift in the LBO $\pi$-mode frequency, it should be noted that the SBO frequency transition was sharper than the LBO frequency transition, which suggests that other factors were also involved.

For the SBO, the clear transition in operating frequency illustrated in Fig. 5.32b on page 107 is also reflected in Fig. 5.35d. The trends are similar to those observed for the LBO, as shown by Fig. 5.35c. A region of transition between the linear scaling for both the start and peak power voltages in $B \simeq 0.19-0.21 \mathrm{~T}$ coincides with the region of transition in Fig. 5.32b. The peak powers in $B \gtrsim 0.21 \mathrm{~T}$ are constant, suggesting operation during the flat-top portion of the voltage pulse. However, the start voltages (which are lower than the peak power voltages) continue a linear increase with a different slope than was observed in $B \lesssim 0.21 \mathrm{~T}$, which is evidence of transition to a different mode having a different synchronous velocity.

The energy conversion metrics are shown in Fig. 5.36. Efficiency peaked near $10 \%$ in 


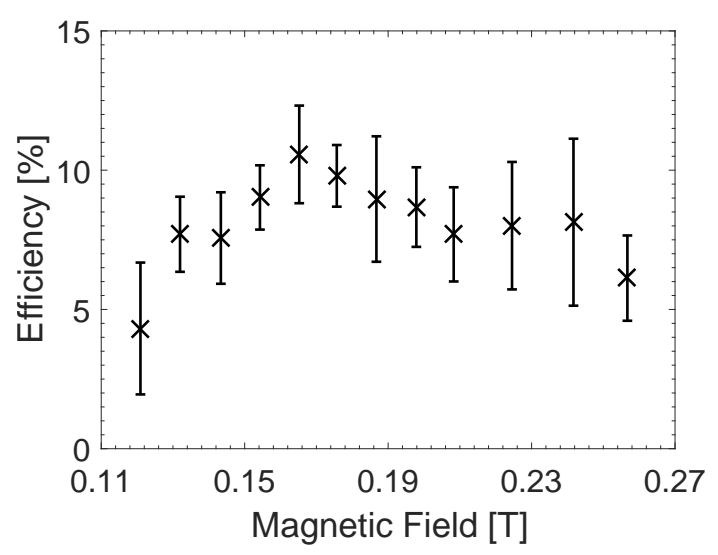

(a) Peak total efficiency vs. magnetic field.

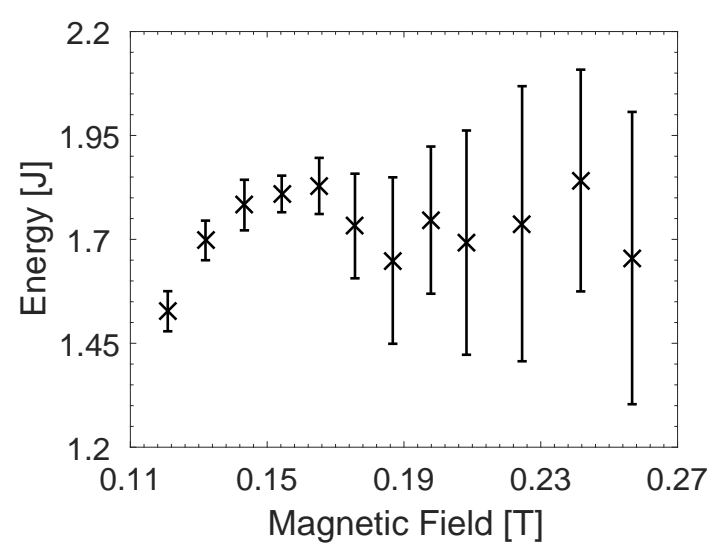

(b) Total pulse energy vs. magnetic field.

Figure 5.36: Standard Load and Uniform Magnetic Field: Energy conversion metrics vs. magnetic field.

the region of optimal operation, and shot-to-shot consistency was acceptable. As with the powers in Fig. 5.31 on page 106, efficiencies at higher magnetic fields were less consistent.

Figure 5.36b shows total pulse energies vs. magnetic field. It is interesting to note that the region of $B \simeq 0.14-0.17$, which includes most of the region of optimal operation, exhibited nearly constant pulse energies, with an increase over the magnetic field range of only about $3 \%$. This conflicts with the increase in powers over the same range, where both oscillators produced 31-35\% greater power at the upper end of the magnetic field range relative to the lower end of the magnetic field range. As with the previous experimental configurations, the near constant-energy phenomenon is consistent with cathode-plasma expansion leading to pulse-shortening, as postulated in the literature [104]. However, the smooth increase in pulse energies at lower magnetic fields up to the peak near $B \simeq 0.17 \mathrm{~T}$ suggests the cause of pulse-shortening may be different in the MFRPM. One possible explanation is oscillation disruption at high powers due to RF-induced cavity plasma formation, which is discussed in the pulse-shortening imaging section at the end of this chapter. These data do not conclusively support one pulse-shortening mechanism over the other.

Temporal metrics are illustrated in Fig. 5.37. The approximately constant scaling of the start and peak power times of the LBO in Fig. 5.37a may appear to be at odds with the previous discussion of the start and peak power voltages (which did not have a constant scaling across the entire range of magnetic fields), but the discrepancy is easily explained by recognizing that the MFRPM generally starts before the MELBA-C voltage pulse reaches flat-top at lower magnetic fields, and starts during the flat-top at higher magnetic fields. Thus, the voltage scaling changed with magnetic field, but the scaling of the times with magnetic field did not. 


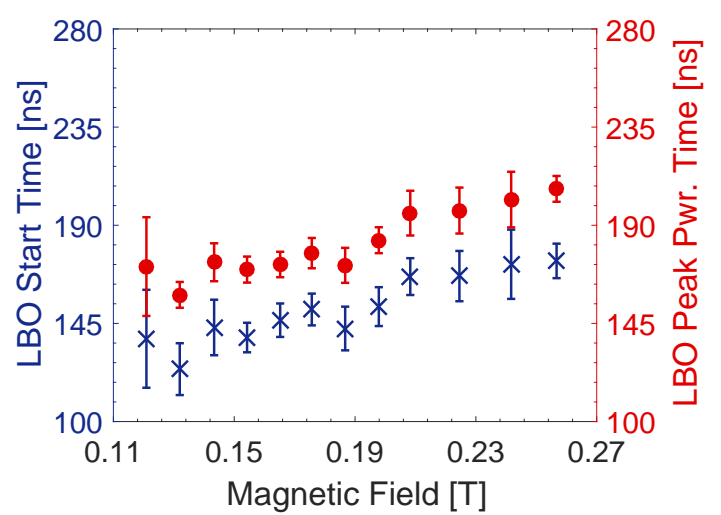

(a) LBO microwave start and peak power times.

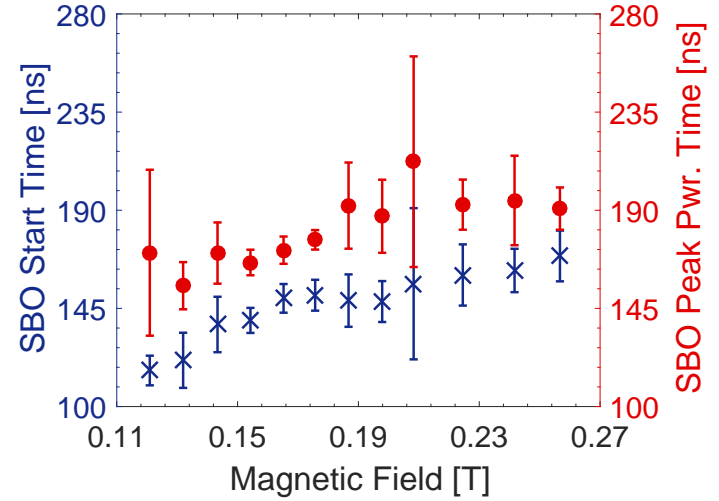

(b) SBO microwave start and peak power times.

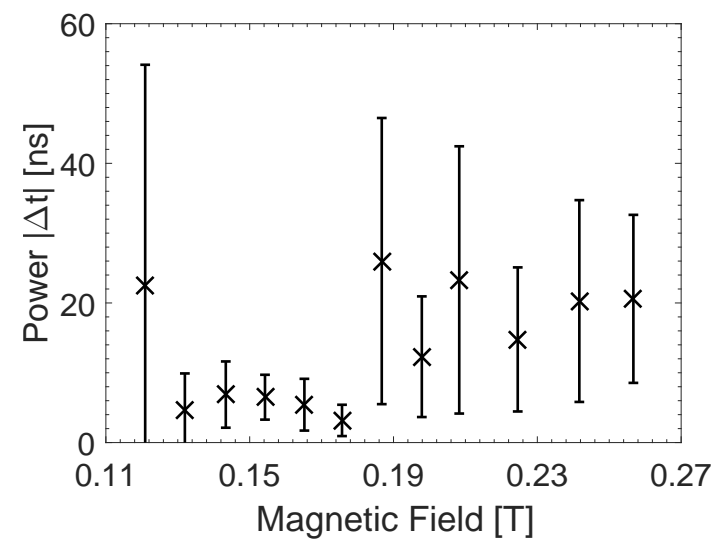

(c) Absolute time difference $|\Delta t|$ between oscillator peak powers vs. magnetic field.

Figure 5.37: Standard Load and Uniform Magnetic Field: Temporal metrics vs. magnetic field.

The performance of the SBO was slightly different because some changes occurred in the scaling of the start and peak power times with magnetic field. This change in behavior at $B \simeq 0.18 \mathrm{~T}$ is explained by the fact that the times of SBO operation no longer coincided with the LBO, which is clearly evident in Fig. 5.37c. Also shown by Fig. 5.37c is the remarkably small peak power $|\Delta t|$ in the region of optimal operation, which suggests the MFRPM operated not as two independent oscillators, but as a coupled system. This observation reinforces the discussion of the harmonic frequency-locked state in the Additional Analysis section below. Standard performance metrics for the region of optimal operation are summarized in Table 5.8 on page 65. 


\subsubsection{Additional Analysis}

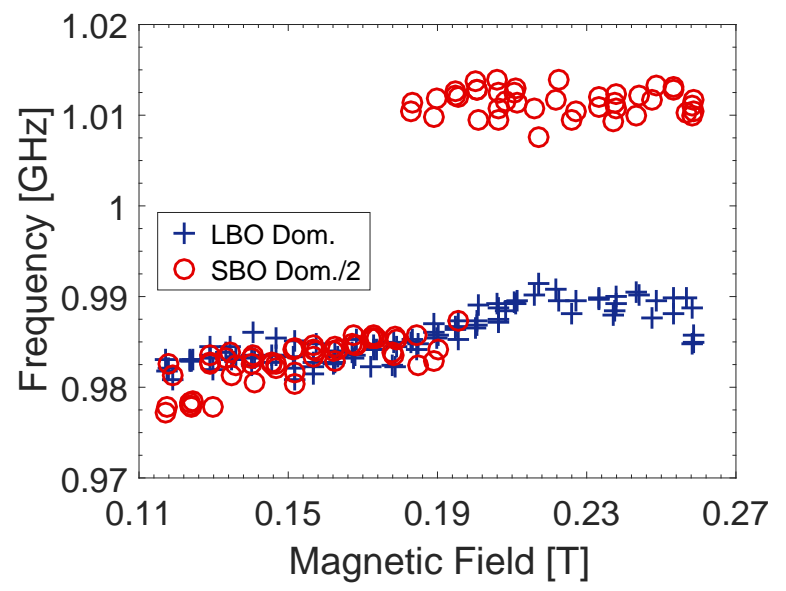

Figure 5.38: Standard Load and Uniform Magnetic Field: LBO dominant frequency and $0.5 \times \mathrm{SBO}$ dominant frequency vs. magnetic field.

Figure 5.38 shows the relation between the LBO and SBO frequencies, with the SBO frequencies divided by two in the plot for easy comparison. Immediately apparent is the integer-multiple relation between the dominant LBO frequency and dominant SBO frequency in $B \simeq 0.14-0.18 \mathrm{~T}$, which shows locking between the $\mathrm{LBO}$ second harmonic frequency and the SBO fundamental frequency. In a transition region $B \simeq 0.18-0.22 \mathrm{~T}$, the SBO switches to operation at $2.02 \mathrm{GHz}$. In the same transition region, the LBO also undergoes a change in operating frequency from approximately $0.984 \mathrm{GHz}$ to a new consistent, higher frequency near $0.990 \mathrm{GHz}$, but far less abrupt. Considering the exceptionally low peak power $|\Delta t|$ shown in Fig. 5.37c, the MFRPM evidently operated as a single device with both oscillators in a state of harmonic frequency-locked operation in $B \simeq 0.14-0.18 \mathrm{~T}$. This condition was dominant throughout the region of optimal operation.

The complete absence of the locked $1.970 \mathrm{GHz}$ SBO frequency in both simulations and shots obtained using the isolated SBO configuration makes identification of the $1.970 \mathrm{GHz}$ mode particularly difficult. If there existed a resonant mode at $1.970 \mathrm{GHz}$ capable of producing the high powers observed in this data set, it should have been observed in a shot such as 14970, which was shown in Fig. 5.17 on page 89 and previously discussed in $\S 5.4$. Despite the uncertainty in the identity of the $1.970 \mathrm{GHz}$ SBO mode, it must have $\pi$-modelike fields at the microwave extractor due to the high powers generated by the oscillator. The odd modes, such as the $5 \pi / 6$-mode for the LBO and the $7 \pi / 8$-mode for the SBO, have zero phase advance per vane at the extractor, and therefore couple very poorly to the output. This assertion is supported by both simulations and the previously discussed experimental 
configurations. High SBO powers therefore constitute strong evidence that the $1.970 \mathrm{GHz}$ mode must be either the $6 \pi / 8$-mode or $\pi$-mode pulled to the second frequency harmonic of the LBO $\pi$-mode in the locked state. Notably, the LBO $\pi$-mode frequency in the locked state was approximately $0.984 \mathrm{GHz}$, which is very close to the free-running $0.986 \mathrm{GHz}$ $\pi$-mode frequency, meaning it had a far greater effect on the operation of the SBO than the SBO had on the LBO. An alternate possibility is that a new mode supported by the SBO was introduced by replacing the smooth drift section with the LBO in the MFRPM configuration, but no evidence for such a mode was found in either cold- or hot-tube simulations. Instead, these data support the existence of the same modes observed in the isolated configurations with minor changes in frequency.

Harmonic frequency-locking between the SBO $6 \pi / 8$-mode and LBO $\pi$-mode second frequency harmonic was demonstrated in $\S 5.5$ using the nonuniform magnetic field. However, in that configuration, the $6 \pi / 8$-mode manifested exclusively as a competing mode to the more dominant $7 \pi / 8$-mode and $\pi$-mode, and a cursory comparison of the standard metric plots clearly shows that the nonuniform magnetic field drastically affected operation of the MFRPM. Any direct comparison between $\S 5.5$ and the present configuration would therefore be premature, but those results proved that the SBO was capable of supporting oscillation at the second frequency harmonic of the dominant LBO frequency, and that the SBO mode in question was likely the $6 \pi / 8$-mode.

Further evidence supporting the SBO $6 \pi / 8$-mode as the locked mode can be found in the quality factors from the cold test discussed in $\S 4.1 .3$. While the hot $Q$-factors are not exactly known, it is very likely that the loaded $Q$ of the $\mathrm{SBO} \pi$-mode was too high, and the frequency difference between the SBO $\pi$-mode and the LBO second frequency harmonic too great, to support locking of the SBO $\pi$-mode off-resonance at $1.97 \mathrm{GHz}$. By constrast, the $Q$ of the $6 \pi / 8$-mode appeared to be lower in the cold test, and its freerunning frequency was much closer to the second LBO frequency harmonic. Considering the analogous behavior to the previously discussed results from the nonuniform magnetic field configuration and the likely suitable locking conditions of the $6 \pi / 8$-mode free-running frequency and $Q$-factor, the $1.97 \mathrm{GHz}$ locked mode was most likely the $6 \pi / 8$-mode.

Evidently, the MFRPM demonstrated consistent, harmonic frequency-locked operation as a single device, with both oscillators producing peak output power nearly simultaneously within experimental uncertainty. The remaining question of relative phase needed to be answered to fully characterize the locked state as one of frequency-locked operation, wherein the relative phase between the oscillators was randomly selected on a shot-to-shot basis, or whether phase-locking occurred, wherein a consistent, repeatable phase difference between the oscillators was observed for each shot. 
The procedure used to conduct the phase analysis was described earlier in $\S 5.2$. To illustrate and validate the approach, several plots were generated. Figure 5.39 illustrates the results for an example of a locked shot, with Fig. 5.39a showing the standard shot plot. The power traces suggest a high degree of mode purity due to the absence of a beat frequency, and the power for each oscillator peaked nearly simultaneously. The timeintegrated Fourier transforms for the LBO and SBO, shown in Figs. 5.39b and 5.39c, confirm the harmonic relation between the frequencies and the absence of competing modes. To analyze the relative phase difference between the oscillators, a processing time window was determined during which both oscillators were operating at powers $\geq 67 \%$ of their respective peak powers. This temporal window forms the horizontal axis limits of Figs. 5.39d and $5.39 \mathrm{e}$, with the former showing the splined and filtered RF voltage traces for the oscillators, and the latter showing the relative phase difference after extracting, processing, and filtering the phases from the Hilbert transform.

To aggregate the results from the individual shots, a metric was needed to determine a representative number for the phase difference over the course of the processing windows, and to quantify the degree of "lockedness," since not all shots with nonzero temporal processing windows demonstrated locked operation. The representative phase difference $\overline{\Delta \phi}$ of a shot was defined as the mean of the values of the phase difference $\Delta \phi$ within the processing window. A measure of "lockedness" for each shot was defined as the standard deviation $\sigma_{\Delta \phi}$ of the phase difference $\Delta \phi$ in the respective processing windows of the shots. To avoid confusion between this quantity and standard deviations obtained for other plotted quantities, the standard deviation of the relative phase difference in the temporal processing window, $\sigma_{\Delta \phi}$, is termed the phase variation.

If a shot had a temporal processing window width less than $5 \mathrm{~ns}$, it was not considered in the phase analysis because very small windows almost always appear locked. The "locked" description is not meaningful over these small timescales, and in most of these instances, the MFRPM failed to operate as a single device because the oscillators behaved independently, with peak power production at very different times (hence the small temporal processing window).

Figure 5.40 shows several plots of the representative phase differences $\overline{\Delta \phi}$ vs. magnetic field. To ensure the digital filtering and processing of the phase was performing as intended, artificial phase shifts were introduced to the LBO signal. These shifts were produced by shifting the signal by a specific time relative to the SBO signal. Shifts of approximately $45^{\circ}, 90^{\circ}$, and $180^{\circ}$ were used to produce the plots shown in Figs. 5.40b-5.40d. The shifts were approximate because they were determined from fractions of a period of a $1 \mathrm{GHz}$ signal, which does not exactly correspond to the LBO frequency. Nonetheless, the plots 


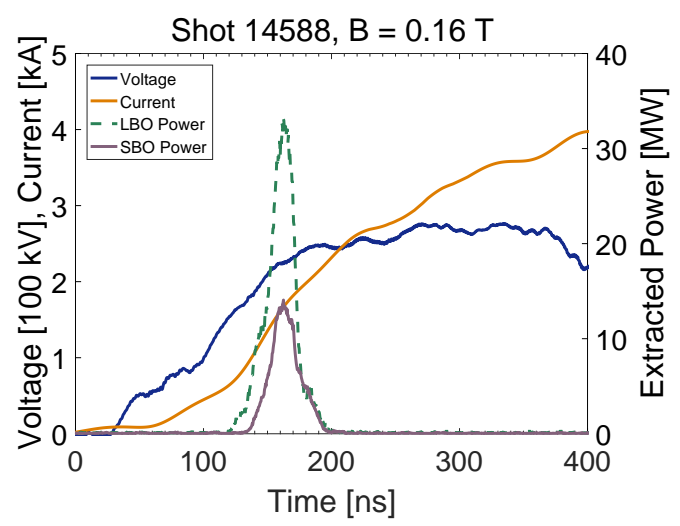

(a) Plot of voltage, current, and RF power.

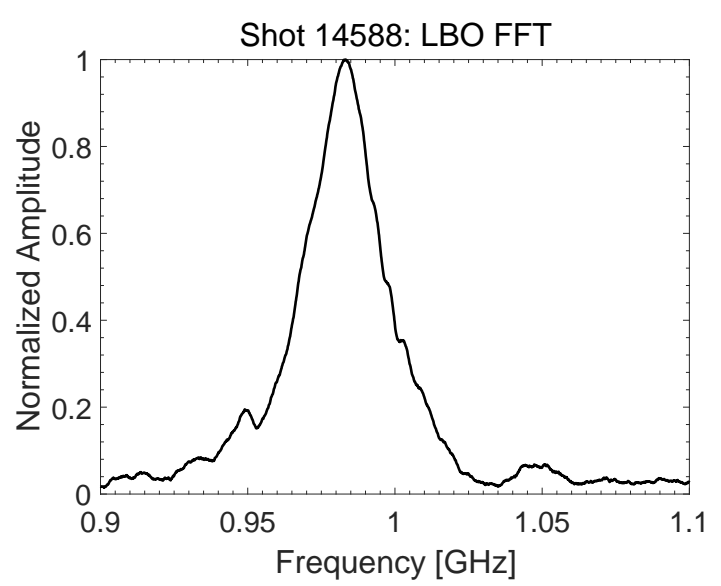

(b) Time-integrated Fourier transform of the LBO RF voltage signal.

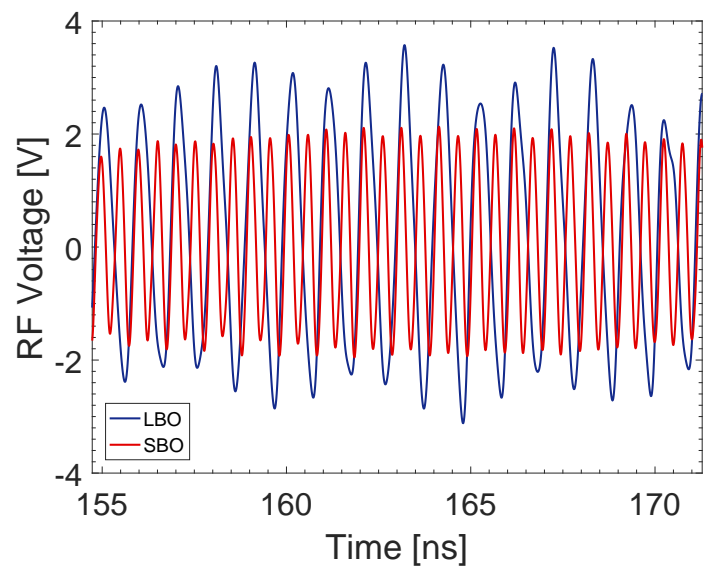

(d) Splined and filtered RF voltage traces from both oscillators in the high-power time window.

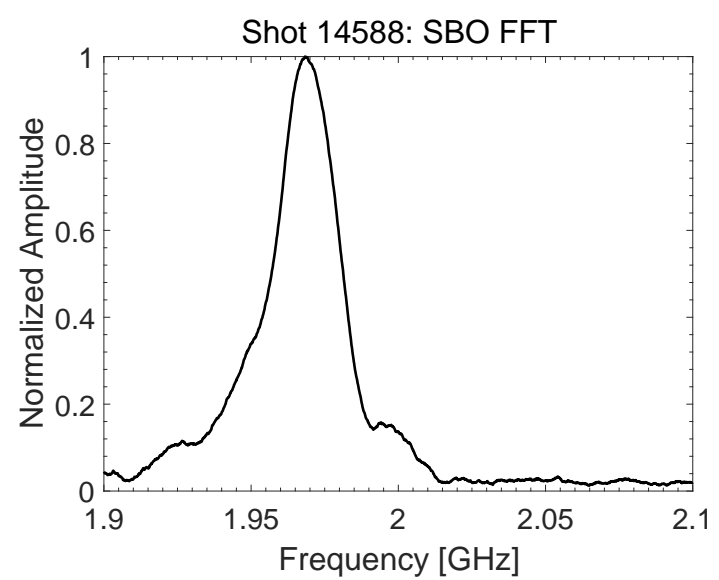

(c) Time-integrated Fourier transform of the SBO RF voltage signal.

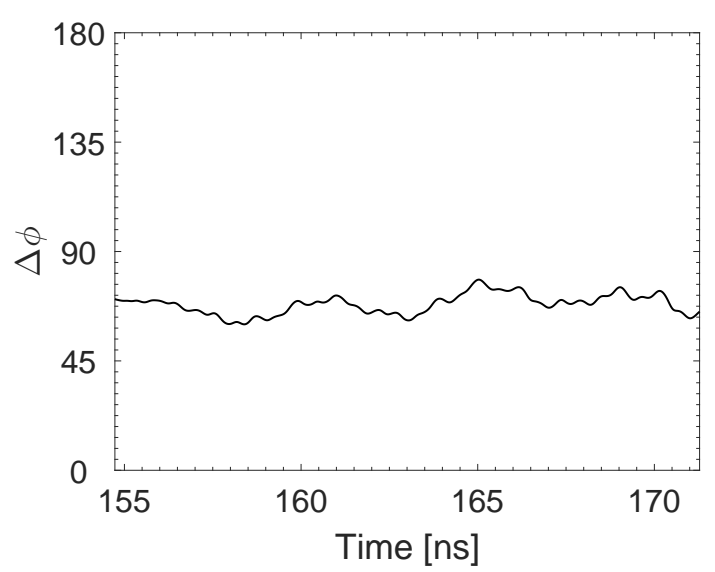

(e) Phase difference $\Delta \phi$ between the oscillators in the high-power time window.

Figure 5.39: Standard Load and Uniform Magnetic Field: Analysis of shot 14588. 
show the processor was operating as intended. From the discussion in $\S 5.2$, the phase difference $\Delta \phi$ was obtained by subtracting the LBO phase from the SBO phase. Note that, prior to the subtraction, the LBO phase was multiplied by two prior to the modulo 360 operation in order to obtain unique phase differences per LBO cycle. The expectation based on this processing method was that a $45^{\circ}$ shift in the LBO phase should produce a $90^{\circ}$ phase difference. Figure 5.40b confirms this expectation. In the plot, values that dropped below $0^{\circ}$ due to the phase shift are wrapped to the negative value plus 360 , then reflected about 180 , as expected. Similarly, a $90^{\circ}$ shift should appear as a $180^{\circ}$ change in the plot, or a mirroring of the values about $180^{\circ}$. This is clearly the case in Fig. 5.40c. Finally, a $180^{\circ}$ shift in the raw LBO signal should appear as a $360^{\circ}$ shift in $\overline{\Delta \phi}$, which should produce an identical result to the unshifted values. Figure 5.40d confirms that the original plot is nearly perfectly recovered. Minor differences are due to the aforementioned imperfection in the assumption of a $1 \mathrm{GHz}$ signal to produce the desired shifts.

Phase analysis results for the MFRPM are shown in Fig. 5.41. To better understand the trends and stability of the locked states in Fig. 5.40a, Figs. 5.41a and 5.41b were produced. Each point and error bar was computed using the method outlined in $\S 5.2$. For Fig. 5.41a, a single point represents a collection of eight shots, with the plotted value corresponding to the mean value of $\overline{\Delta \phi}$ for those shots eight, and the error bars representing the standard deviation of $\overline{\Delta \phi}$ for those eight shots. Similarly, for Fig. 5.41b, each point again represents a collection of eight shots, but the plotted value corresponds to the mean of the phase variation $\sigma_{\Delta \phi}$ for those eight shots, and the error bars represent the standard deviation of $\sigma_{\Delta \phi}$ for those eight shots.

Figure 5.41a reveals a clear trend in the phase difference between the oscillators, and it is evident that relative phase selected by the MFRPM was not random. Instead, the preferentially selected phase difference of the locked state was a function of the magnetic field, provided that a shot occurred in the range of $B \simeq 0.12-0.19 \mathrm{~T}$ where harmonic frequencylocking occurred. The magnetic field exhibiting the greatest shot-to-shot consistency in the representative phase difference $\overline{\Delta \phi}$ corresponded exactly to the magnetic field exhibiting the highest peak powers observed in Fig. 5.31 on page 106, indicating superior consistency at the point of optimal operation.

Since the error bars of Fig. 5.41a only communicate the shot-to-shot consistency of the representative phase difference $\overline{\Delta \phi}$, the second plot (Fig. 5.41b) was needed to understand how much the phase difference varied during the temporal processing windows. The results corroborate the observation of consistent operation at the point of optimal operation, with the average variation in the phase not exceeding approximately $10^{\circ}$. This condition meets the standard for strict frequency-locking utilized in similar studies appearing in the litera- 


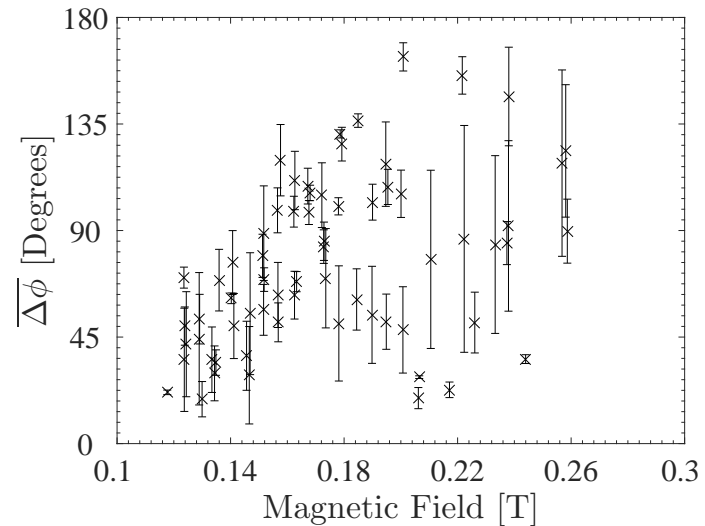

(a) Mean phase differences $\overline{\Delta \phi}$ vs. magnetic field with zero phase shift.

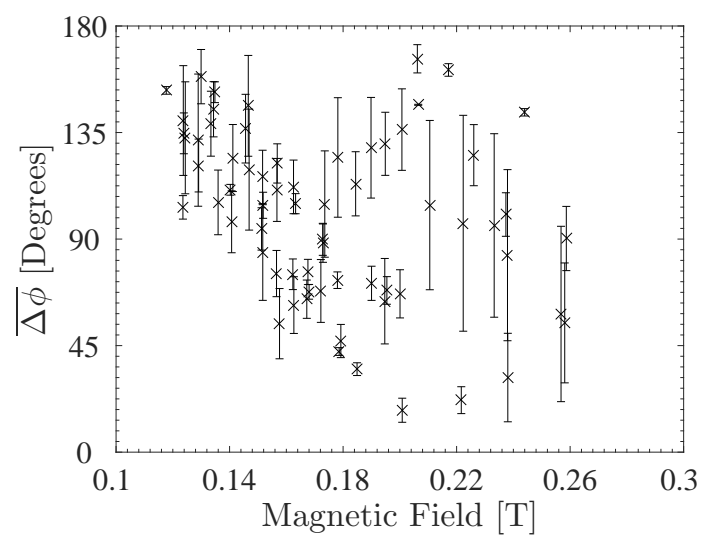

(c) Mean phase differences $\overline{\Delta \phi}$ vs. magnetic field with $90^{\circ}$ phase shift.

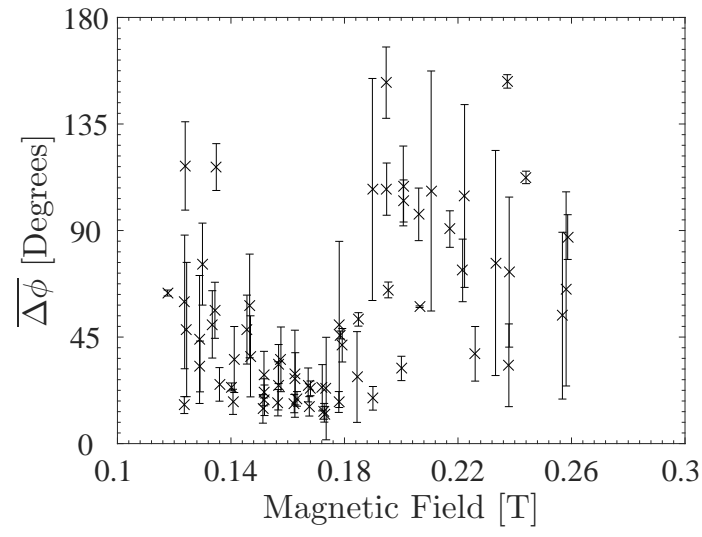

(b) Mean phase differences $\overline{\Delta \phi}$ vs. magnetic field with $45^{\circ}$ phase shift.

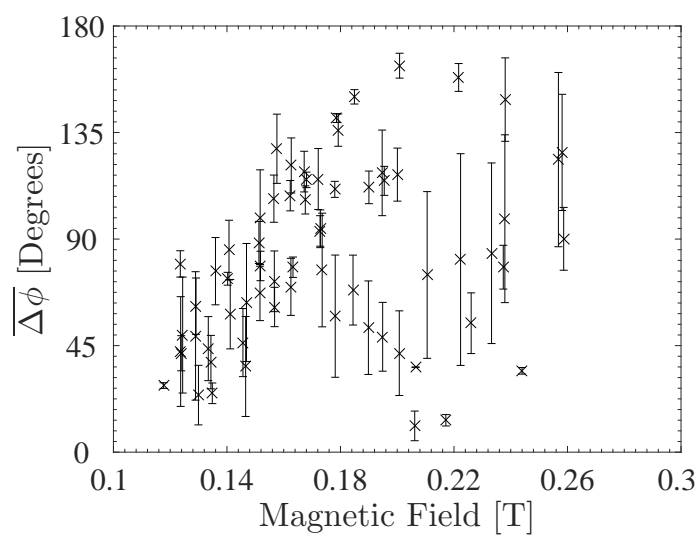

(d) Mean phase differences $\overline{\Delta \phi}$ vs. magnetic field with $180^{\circ}$ phase shift.

Figure 5.40: Standard Load and Uniform Magnetic Field: Mean phase differences $\overline{\Delta \phi}$ vs. magnetic field for a variety of phase shifts applied to the LBO RF voltage signal.

ture $[13,40]$. Thus, the MFRPM conclusively demonstrated repeatable frequency-locked operation and preferential phase selection as a function of magnetic field. Because there was an approximately $17^{\circ}$ shot-to-shot variation in the selected phase difference at constant magnetic field, this does not meet the strict accepted phase-locking criterion of variation $\leq 10^{\circ}$.

Figure 5.41c shows the phase variation vs. frequency ratio. On initial inspection, it appears as though several unlocked shots (near $f_{2} / f_{1}=2.04$ ) underwent locked operation (a phase variation less than 10 degrees), which should be impossible. However, the representative frequencies $f_{1}$ and $f_{2}$ for the LBO and SBO used in the plot were determined from the dominant peak in the time-integrated Fourier transform. A detailed investigation of those shots revealed that segments did indeed lock, but earlier or later operation occurred outside the locking window at a different frequency, usually at a lower power, for long du- 


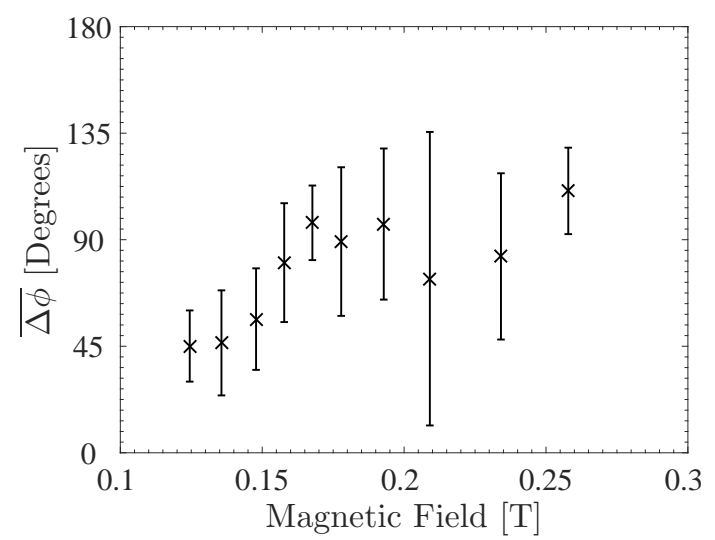

(a) Mean phase differences $\overline{\Delta \phi}$ and associated standard deviations of the means vs. magnetic field.

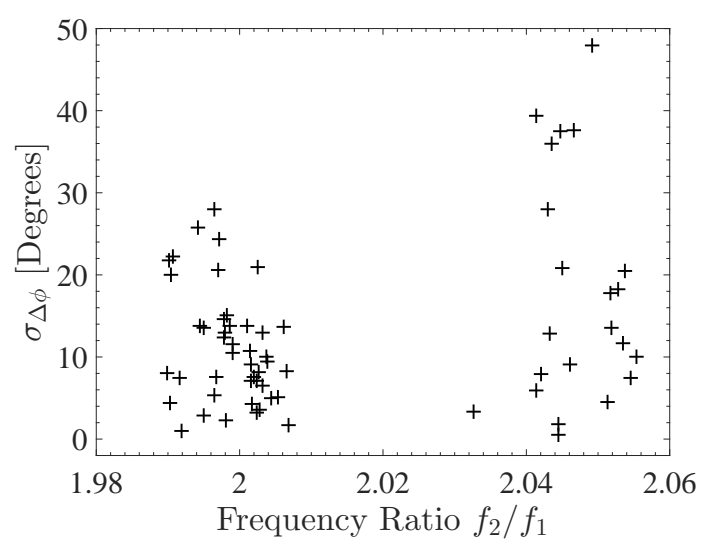

(c) Phase variation $\sigma_{\Delta \phi}$ vs. frequency ratio $f_{2} / f_{1}$.

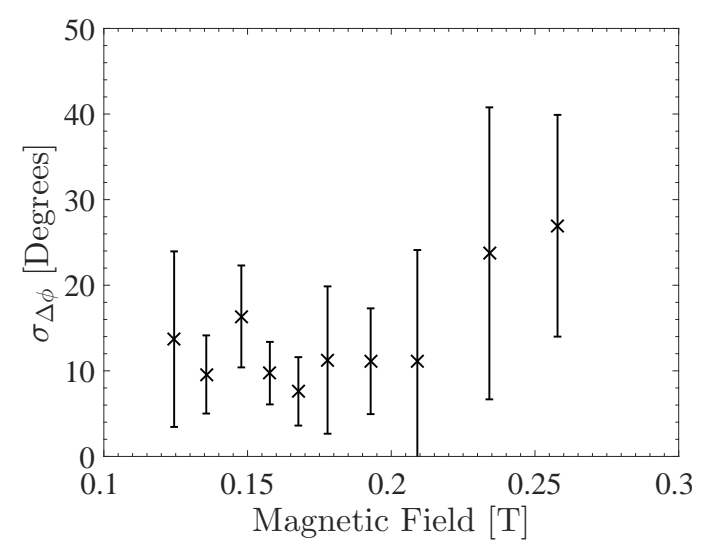

(b) Means and associated standard deviations of the phase variation $\sigma_{\Delta \phi}$ vs. magnetic field.

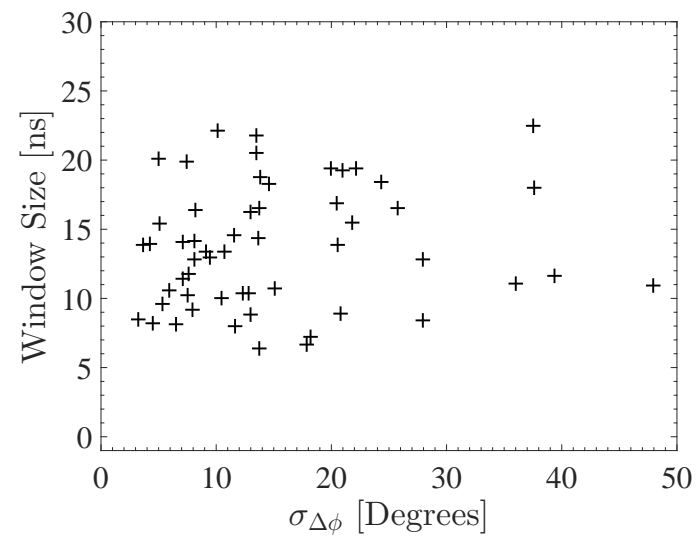

(d) Temporal window sizes vs. phase variation $\sigma_{\Delta \phi}$.

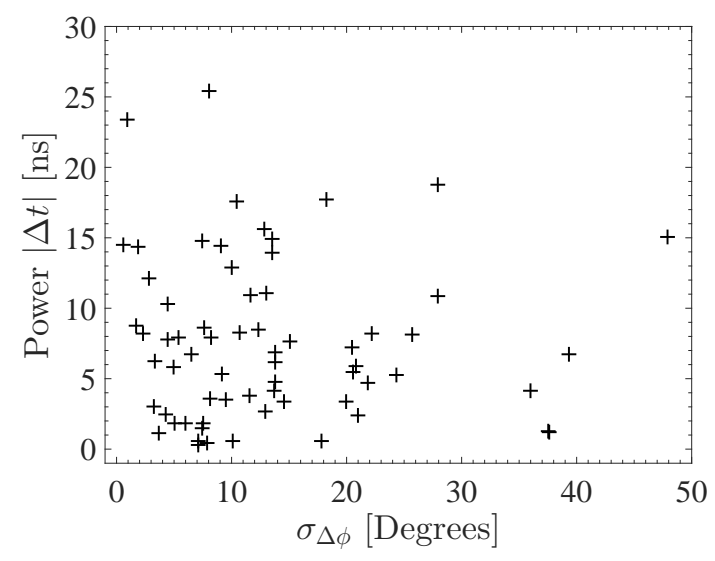

(e) Peak power $|\Delta t|$ vs. phase variation $\sigma_{\Delta \phi}$.

Figure 5.41: Standard Load and Uniform Magnetic Field: Phase analysis of shots having temporal windows $\geq 5 \mathrm{~ns}$ during which each oscillator produced at least $67 \%$ of their respective peak powers. 
rations leading to larger peaks in the time-integrated Fourier transform. As expected, the majority of shots with low phase variation occurred at $f_{2} / f_{1}=2$. Again, minor variation in this ratio near 2 was due to the temporal integration of the Fourier transform.

Further inspection of Fig. 5.41b reveals that locked states were relatively common in the range of magnetic fields $B \simeq 0.12-0.19$ T, provided that the definition of "locked" is modified to include variations up to $20^{\circ}$. However, as discussed previously, the strength of the locked state depends on the duration, with longer locking durations being more desirable than durations of only a few ns. To determine if locked states occurred over meaningful durations, a plot of the size of the processing windows vs. phase variation was generated, which is shown in Fig. 5.41d. In almost all cases, locked states having a phase variation $\lesssim 15$ degrees persisted for durations of 10-20 ns. Typical pulses had a power fullwidth at half-maximum equal to approximately $20 \mathrm{~ns}$, which suggests these data constitute a significant average locking duration relative to an average microwave pulselength.

The relation between the peak power $|\Delta t|$ and phase variation $\sigma_{\Delta \phi}$ is shown in Fig. 5.41e. Most of the points fall in the bottom-left of the plot, which validates the conclusions from Fig. $5.37 \mathrm{c}$ on page 112 . Locked states with small phase variation correlate with minimal temporal separation in peak power production from the two oscillators.

Approximately 10 shots were conducted in the vicinity of the magnetic field range yielding peak power production and optimal frequency-locked behavior. The stringent criterion for phase-locking necessitated the definition of a narrower magnetic field range than the previously defined region of optimal operation in the Standard Metrics section. The results of the analysis within this range were shown in Table 5.9 on page 65 . Results are tabulated for the phase difference, phase variation, and processing window duration as a proxy for the locked duration. To summarize the interpretation of the phase metrics: 1) The phase difference and associated uncertainty reflects the consistency with which the same phase difference was observed between the oscillators on a shot to shot basis; 2) The phase variation and associated uncertainty conveys the stability of the locked states, viz. the variation in the phase difference during the shots' respective temporal processing windows and the standard deviation of that variation; and 3) The mean and associated standard deviation of the temporal processing window size serves as a proxy for the locking duration. The table results show that even the best operation did not meet the criterion for phase-locking, but the evidence for preferential selection of a relative phase is compelling.

The change in the phase difference between the oscillators with changes in the magnetic field suggests the locking mechanism occured through the electron kinetics. If the second frequency harmonic of the LBO-modulated electron beam provided the coupling between the LBO and SBO, then changes in the beam velocity should lead to changes in the phase 
difference between the LBO and SBO. The electron beam velocity (and therefore the transit time of the beam from the LBO, around the cylindrical bend, to the SBO) depends on the magnetic field because $v_{d} \propto E / B \propto V / B$. Dependence on the magnetic field was precisely what was observed in the optimal locking region $B \simeq 0.13-0.17 \mathrm{~T}$ in Fig. 5.41a.

To summarize the frequency-locking effect, the evidence suggests that excitation of the $6 \pi / 8$-mode occurred at a frequency off-resonance. The excitation was possibly driven by the harmonic content of the LBO-modulated electron beam, and the relative phase between the oscillators was controlled by modifying the transit time of the beam from the LBO to the SBO using the magnetic field. The comparison between the analytic locking condition and the experimental results is the subject of the next section.

\subsubsection{Comparison with Theory}

The harmonic frequency-locked operation can be compared with the theory presented in $\S 2.3$. From that discussion, the analytic model predicted that locking should occur when the left-hand side of Eq. 2.23 on page 25, denoted by $\kappa$ (termed the locking parameter), is less than some constant $C$. Furthermore, the free-running power and frequency of both the LBO and SBO need to be known because the theory evaluates whether the coupled oscillators, which independently would run at those powers and frequencies, would be capable of locking.

The power fed from one oscillator to the other is not precisely known because, unlike previous magnetron locking experiments, the locking in the MFRPM occurs internally, not through the extractor. The extracted powers must therefore be used as a proxy for the power fed from one oscillator to the other. Unfortunately, the free-running power of the SBO $6 \pi / 8$-mode is also unknown known because, in the isolated configuration, only a single shot exhibited dominant operation in the $6 \pi / 8$-mode. A single shot is not sufficient to characterize the power, and it occurred at a magnetic field outside the range where locking was observed in the present configuration. For these reasons, a strictly valid comparison with the experimental theory was not possible with this experimental configuration. However, an attempted comparison was nonetheless made using the free-running LBO and SBO frequencies from their respective isolated configurations and the locked powers in the present configuration. Note that the free-running SBO $6 \pi / 8$-mode frequency is known because several shots in the isolated configuration exhibited some mode competition with that mode.

Using the frequencies from the free-running configurations and the powers from the present configuration, a comparison with the theory still required a set of shots with the 
SBO operating in the same mode, where some shots were locked, and some were not. The reason for this requirement is that the theory was derived under the assumption of single-mode operation, so evaluation of the analytic locking condition using a comparison between experimental results for locked and unlocked states cannot be performed using a set of shots operating in different modes. If there was a threshold constant $C$ for which $\kappa<C$ corresponded to locked operation, and for which $\kappa>C$ corresponded to unlocked operation, then determination of the threshold requires shots in the same mode, some of which were locked, and some of which were unlocked.

From the discussion of the lowest magnetic fields in Fig. 5.38 on page 113, there appeared to be a set of shots from the present configuration where the SBO operated in the $6 \pi / 8$-mode and exhibited both locked and unlocked operation. These shots were therefore appropriate for comparison with the analytic locking criterion. From $\S 2.3$, the locking parameter $\kappa=\left|\omega_{2}-2 \omega_{1}\right| /\left(P_{1} / \sqrt{P_{2}}\right)$. For the comparison, $\omega_{1}$ and $\omega_{2}$ correspond to the respective free-running LBO $\pi$-mode and SBO $6 \pi / 8$-mode frequencies, whereas the powers $P_{1}$ and $P_{2}$ correspond to the extracted microwave powers from the LBO and SBO in the present configuration.

Figure 5.42 illustrates the comparison between the theory and the experiment. The first plot in Fig. 5.42a shows the phase variation vs. the locking parameter $\kappa$. The phase variation, which was defined in the previous section, is a measure of locking quality. Those shots having $\sigma_{\Delta \phi} \lesssim 10$ exhibit strong locking. As described previously, the quantity $\sigma_{\Delta \phi}$ is defined only for shots having a nonzero temporal processing window, which was used for the phase analysis. Unlocked shots that were excluded from the phase analysis were therefore included at the top of Figs. 5.42a and 5.42b, a region indicative of an unlocked state (corresponding to large $\sigma_{\Delta \phi}$ ).

Good agreement with theory would manifest as a cluster of points in the lower-left and upper-right of Fig. 5.42a. The lower-left region should correspond to locked shots having a small phase variation $\sigma_{\Delta \phi}$ and a locking parameter $\kappa$ that is smaller than some constant. The upper-right region should correspond to unlocked shots having a large phase variation $\sigma_{\Delta \phi}$ and a locking parameter $\kappa$ that is larger than the same constant. While the results do suggest a weak trend in which larger phase uncertainties correlate with larger $\kappa$, there are several shots that deviate from the trend. Specifically, some unlocked shots should be locked due to their small $\kappa$, and some locked shots should be unlocked due to their large $\kappa$. Furthermore, there does not appear to be a clear transition point $\kappa=C$ below which locking occurs, and above which locking does not occur. However, despite the deviations from expectations, these results do not appear to conclusively invalidate the analytic model.

Figure $5.42 \mathrm{~b}$ shows the phase variation vs. the dominant SBO frequency. Most shots 


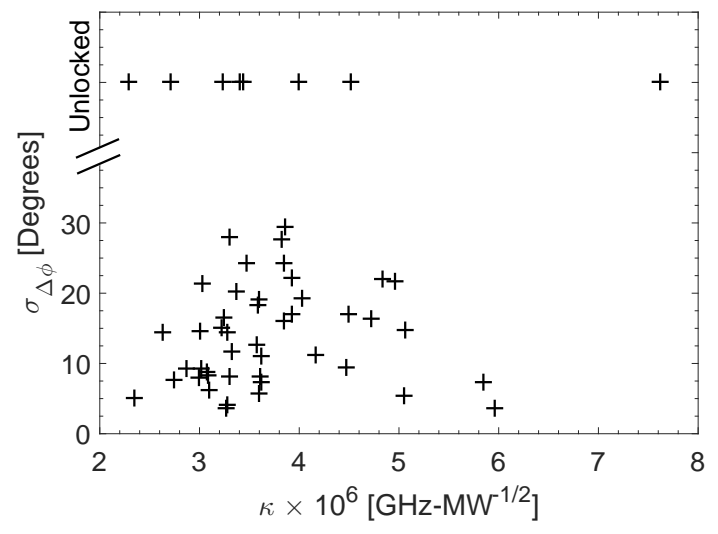

(a) Phase variation $\sigma_{\Delta \phi}$ vs. locking parameter $\kappa$.

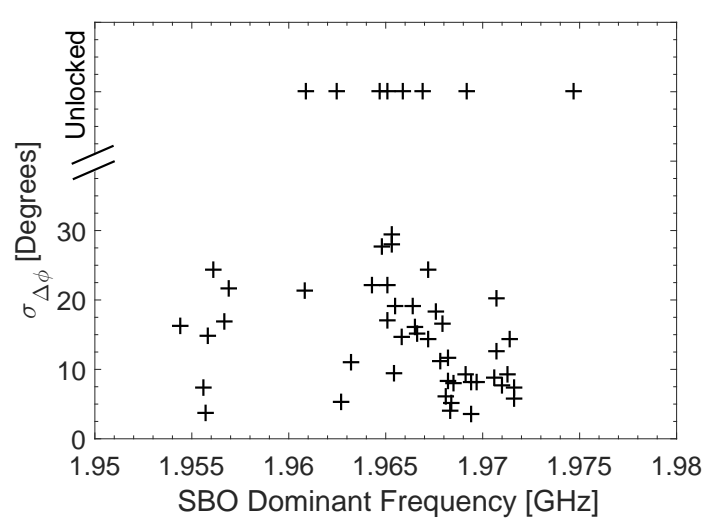

(b) Phase variation $\sigma_{\Delta \phi}$ vs. SBO dominant frequency.

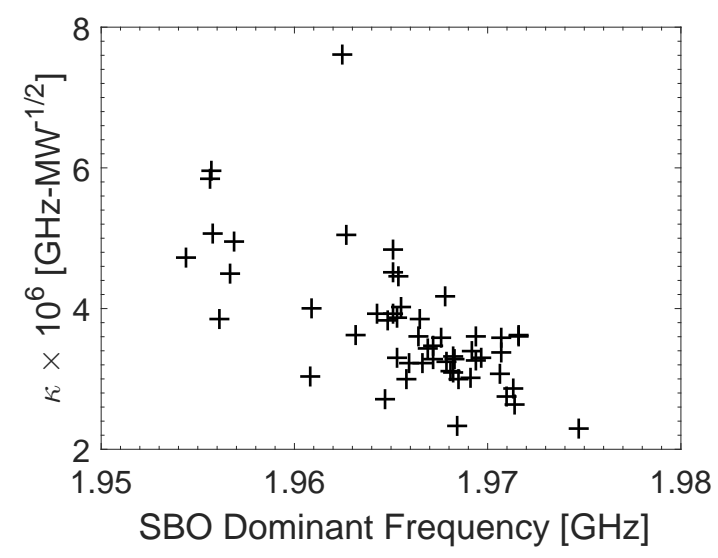

(c) Locking parameter $\kappa$ vs. SBO dominant frequency.

Figure 5.42: Comparison between operation metrics and the analytic locking condition.

fell between 1.965-1.975 GHz. However, a few shots fell near $1.955 \mathrm{GHz}$, which appeared to be close to the free-running SBO $6 \pi / 8$-mode. The expectation was that these shots would be clearly unlocked. Of the seven shots near the free-running frequency, two fell under the approximate threshold for strong locking, where $\sigma_{\Delta \phi} \lesssim 10$. This outcome was particularly surprising given the results of Fig. 5.42c, which shows that these near-freerunning frequencies correspond to larger values for $\kappa$ relative to the average $\kappa$ associated with locked shots. These ususual shots corresponded to instances where the SBO exhibited locked operation during part of the pulse, and unlocked operation during a different part of the pulse. The time-integrated FFT was not restricted to the locked portion only, which is why these frequency metrics appeared to show locking for two cases where the frequencies did not appear to exhibit a harmonic relation.

As previously stated, a strictly valid comparison with the analytic theory should use the power fed between the oscillators. However, determination of those powers is functionally 
impossible and further confounded by the beam kinetics, which likely modify the degree of coupling between the structures as the magnetic field is changed. The reasoning for this assertion is that higher magnetic fields, which correspond to a slower electron drift velocity, allow greater time for electrostatic forces to demodulate the electron beam as it circulates from the LBO to the SBO. Yet another confounding factor is the dependence of the beam interaction efficiency on magnetic field. Finally, the $Q$-factors could be different for the isolated LBO and SBO configurations relative to the MFRPM. These results and the aforementioned considerations mean a compelling statement cannot be made regarding the agreement or disagreement between these data and the analytic theory. Adequate validation or invalidation would require a controlled experiment specifically designed for such testing.

\subsubsection{Comparison with Simulation}

As described in $\S 3.3 .2$, three simulation configurations were run over a range of magnetic fields to model the MFRPM. These sets varied the applied DC voltage and the electron emission area. Specifically, two DC voltage excitation models were used: Dynamic Voltage (DV) and Constant Voltage (CV). The DV model allows the voltage to vary with changes in magnetron impedance, which depends on the operating mode and magnetic field, among other factors. The CV model eliminates the applied voltage as a variable by using a feedback function to apply a constant voltage regardless of changes in magnetron impedance. The different emission areas included a Limited Emission (LE) model, which duplicated the experimental configuration of electron emission from $10 \%$ of the cathode area, and a Full Emission (FE) model, which emits from the full cathode area.

Figure 5.43 compares the individual oscillator performance metrics from simulations and the experiment. Figures 5.43a and 5.43b show the powers for the LBO and SBO, respectively. No single set of simulations accurately duplicated the experimentally observed powers over the tested range of magnetic fields. For the LBO, both DV models qualitatively reproduced the experimentally observed reduction in microwave power at low magnetic fields relative to the peak output powers, which should be the case as the magnetron impedance is reduced due to the reduction in applied magnetic field. The CV model does not reproduce this behavior because the DC excitation magnitude was increased at low magnetic fields to maintain the intended voltage despite the high currents pulled by the magnetron, hence the unrealistically high output powers. Experimentally observed powers lay between the simulation results for the LE and FE configurations using the DV excitation, suggesting the existence of an "experimentally equivalent" emission area that would bring simulated powers into closer agreement with the experiment. However, because the 


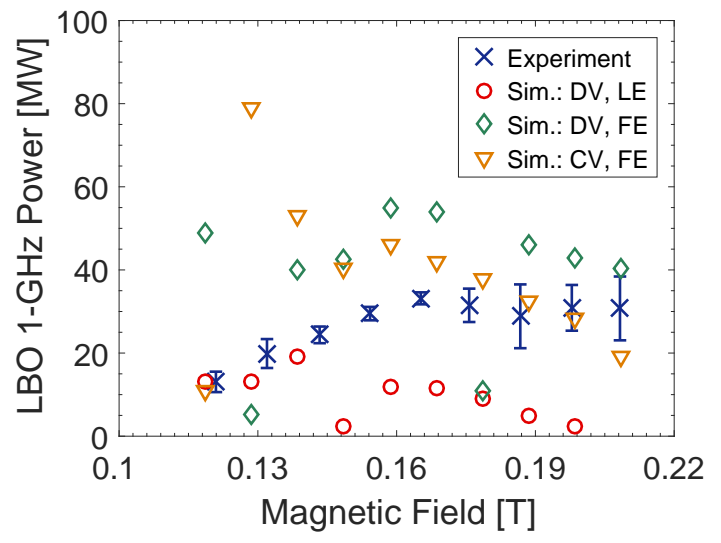

(a) LBO powers vs. magnetic field.

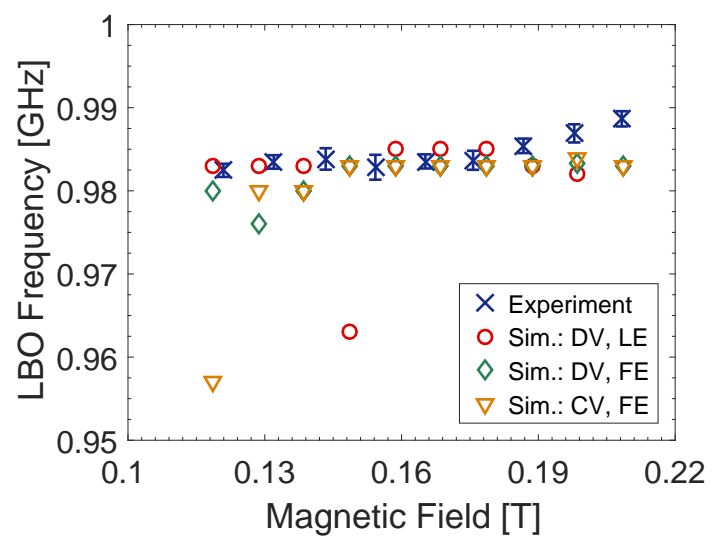

(c) LBO frequencies vs. magnetic field.

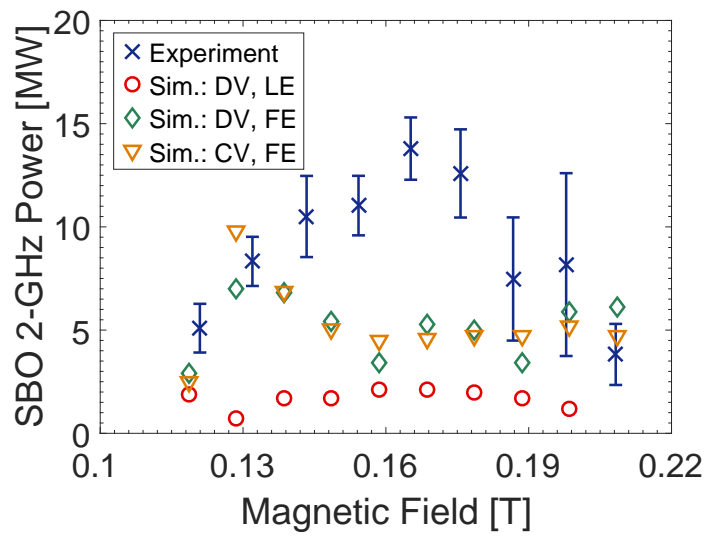

(b) SBO powers vs. magnetic field.

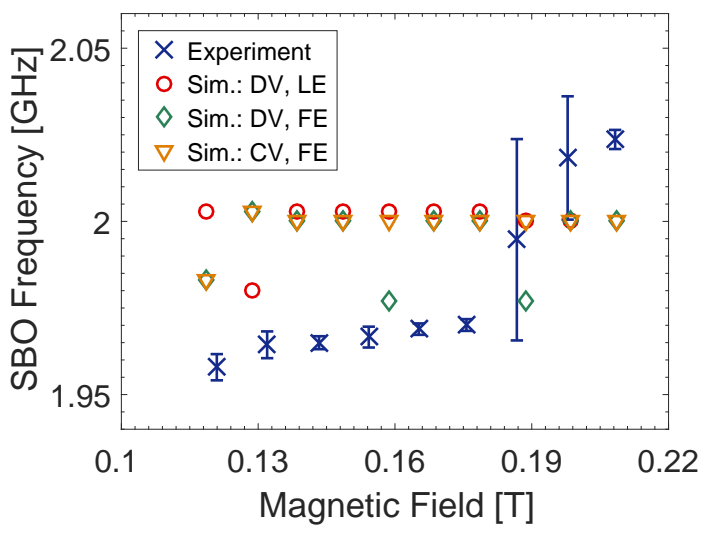

(d) SBO frequencies vs. magnetic field.

Figure 5.43: Standard Load and Uniform Magnetic Field: Individual oscillator performance comparison between simulation and experiment.

simulated currents did not increase linearly with the increase in emission area, it is difficult to predict what simulated emission area would yield the best agreement with the experiment. In all simulation sets, extreme dips in the LBO powers at some magnetic fields were due to cases exhibiting extreme mode competition or operation in the $5 \pi / 6$-mode.

As with the LBO, the SBO power comparison plot shown in Fig. 5.43b reveals generally poor agreement. Qualitatively, the best simulation set used the DV excitation and LE for the cathode area. With the exception of the lowest simulated magnetic field, the trend in microwave powers for the DV+LE model over the range of magnetic fields was reasonably close to the experimentally observed trend. However, unlike the LBO, simply increasing the emission area did not bring the results into closer agreement with experiment. Both FE sets did yield higher output powers, but in some cases, these were less than one-third of the powers observed in the experiment. As with the LBO, the dips in simulated SBO powers were a result of mode competition or operation in the $7 \pi / 8$-mode. 


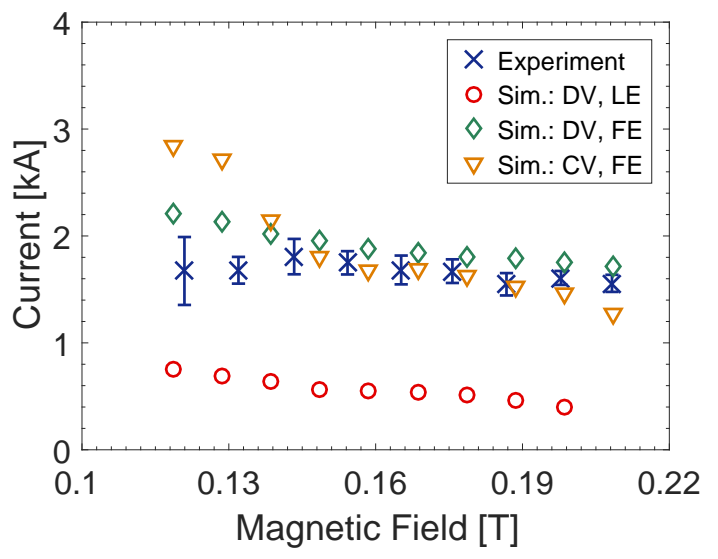

(a) Currents vs. magnetic field.

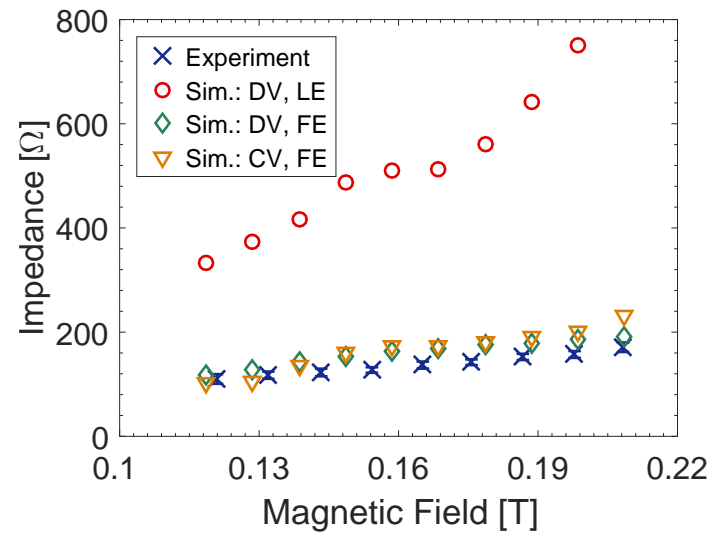

(b) Impedances vs. magnetic field.

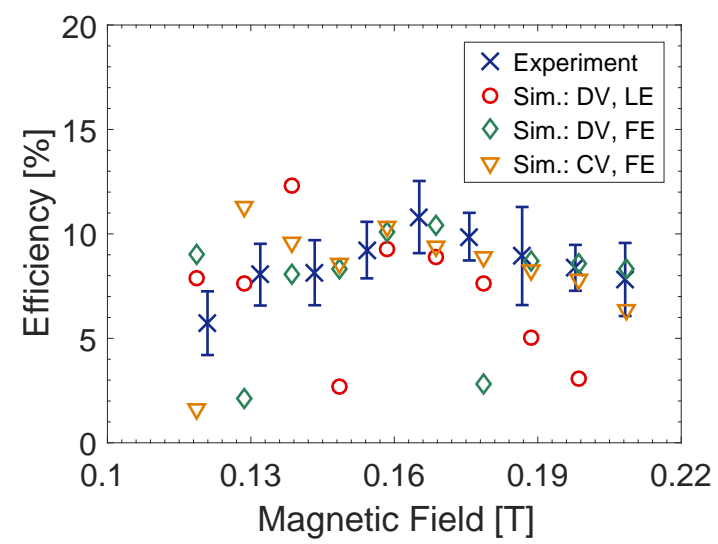

(c) Efficiencies vs. magnetic field.

Figure 5.44: Standard Load and Uniform Magnetic Field: Overall MFRPM performance comparison between simulation and experiment.

Figures 5.43c and 5.43d compare the dominant frequencies observed in simulation and experiment for the LBO and SBO, respectively. Predicted frequencies for the LBO exhibited good agreement, though at low magnetic fields, the FE sets suffered mode competition or slight $\pi$-mode frequency variation that was not observed in the experiment. Generally, with the exception of one simulation that operated in the $5 \pi / 6$-mode, the LE set yielded a consistent and stable frequency that changed little with magnetic field, a result that is supported by the experiment.

Unlike the LBO, the results for the SBO shown in Fig. 5.43d did not exhibit good agreement. In nearly all simulations, the $\pi$-mode was the preferred operating state. Harmonic frequency-locking was never achieved in any simulation. A few simulations exhibited operation at a non- $\pi$-mode frequency, but all cases were instances of mode competition with, or operation in, the $7 \pi / 8$-mode. As has been discussed at length, the $7 \pi / 8$-mode couples poorly to the microwave extractor, which leads to very low output power. It is therefore 
very unlikely that the experimentally observed harmonic frequency-locked SBO mode was the $7 \pi / 8$-mode despite the closer agreement between the simulated $7 \pi / 8$-mode frequency and experiment vs. the simulated $\pi$-mode frequency and experiment. From the discussion in Appendix B, fully converged simulations were infeasible, so all simulated SBO frequencies were lower by approximately $20 \mathrm{MHz}$ than would be the case if full convergence was achieved. Therefore, the apparently close agreement between the simulated $7 \pi / 8$-mode frequency and the experimentally observed locked SBO frequency is misleading. While the experimentally observed frequency-locked mode was likely the $6 \pi / 8$-mode, the absence of any frequency-locked behavior eliminates simulation results from assisting in the identification of the locked state (apart from ruling out the $7 \pi / 8$-mode).

Figure 5.44 compares simulation results to the experiment for metrics describing the MFRPM as a whole. Currents and impedances are compared in Figs. 5.44a and 5.44b, respectively. The best agreement was obtained using the DV excitation model and the FE cathode area. Figure 5.44c compares simulated and experimental efficiencies. Again, the simulation set using the DV excitation model and the LE cathode area yielded the best agreement, with the exception of two simulations that experienced significant mode competition or operation in one of the odd modes ( $\mathrm{LBO} 5 \pi / 6$-mode or SBO $7 \pi / 8$-mode).

\subsection{Impact of Magnetic Field Optimization}

The impact of the magnetic field optimization for uniformity was significant for all investigated operating metrics. Optimization of the field yielded higher powers with greater consistency, a reduction in shot-to-shot variation in frequencies, and higher efficiencies. Additionally, the harmonic frequency-locking effect between the competing mode on the SBO and the dominant LBO mode in the nonuniform magnetic field configuration was stabilized and became the dominant operating state in the uniform magnetic field configuration.

Table 5.10 on page 66 compares metrics for individual oscillators, and includes the results from the isolated oscillator configurations. All comparisons utilize results from the regions of optimal operation for each configuration. Relative to the isolated oscillator configuration, the LBO appeared to be largely unaffected by the presence of the SBO in the MFRPM configuration with the uniform magnetic field. Outside of harmonic frequencylocked operation, the LBO $\pi$-mode frequency shifted only slightly, and relative to the isolated LBO configuration (in the same range of magnetic fields that produced locked operation in the MFRPM), the LBO frequency was nearly identical. The same cannot be said of the SBO, which suffered a $28 \%$ power reduction from the isolated configuration. It is 
possible that this is due to the different operating mode because the $6 \pi / 8$-mode should have a lower electronic efficiency relative to the $\pi$-mode. Shot-to-shot variation in the SBO operating frequency was reduced by a factor of 2 . The LBO saw a similar reduction in shot-to-shot frequency variation by a factor of 3 .

Table 5.11 on page 66 compares metrics for the MFRPM operating as a whole. The magnetic field optimization produced a $48 \%$ improvement of the total power, a $60 \%$ reduction in shot-to-shot variation, and a $250 \%$ improvement in total efficiency. The optimization also brought the MFRPM into the specified impedance range for MELBA-C. Finally, the improved magnetic field led to the MFRPM operating as a single unit during locked operation, bringing the peak power $|\Delta t|$ to less than $5 \mathrm{~ns}$.

\subsection{Pulse-Shortening Analysis}

This section discusses a series of shots conducted to image plasma-related microwave pulse-shortening in the MFRPM anodes. While these results are preliminary and tangentially related to this dissertation, the findings may explain some of the observations both this and the next chapter. Note that, as outlined in $\S 4.6$ of Chapter 4 , the imaging configuration differed from all the other configurations used for this dissertation in that it lacked power extraction. This was necessary to enable axial imaging of the anodes.

Two data sets were acquired using the isolated LBO and SBO configurations. Each set first imaged the anodes using an SLR camera to obtain time-integrated photographs at different magnetic fields of interest, which were determined from the analysis earlier in this chapter (e.g., a field that produced optimal operation for a given anode, or a field that produced lower power due to operation in a different mode). After the SLR images were acquired, the process was repeated using the fast framing camera with exposures ranging from 25 ns to $250 \mathrm{~ns}$.

Figure 5.45 shows some SLR photographs obtained using the isolated SBO configuration. The baseline image (Fig. 5.45a) shows the location of the SBO for context relative to the shot image (Fig. 5.45b). The image shows clear evidence of plasma on the surface of the MCC-2 cathode, as well as plasma in the cavities of the SWS. The depth of the cavity plasma appeared to correlate with the simulated relative amplitudes of the cavity electric fields for the SBO $\pi$-mode, which are shown in Figs. 5.45c and 5.45d. Note the profile across the structure, where the highest fields reside in the center cavities, and the lowest fields reside in the end cavities. The plasma in the SLR images and the observed microwave frequencies depended on the magnetic field, which was expected as the operating mode changed. The $\pi$-mode, which had an unloaded frequency of approximately $2.03 \mathrm{GHz}$, was 


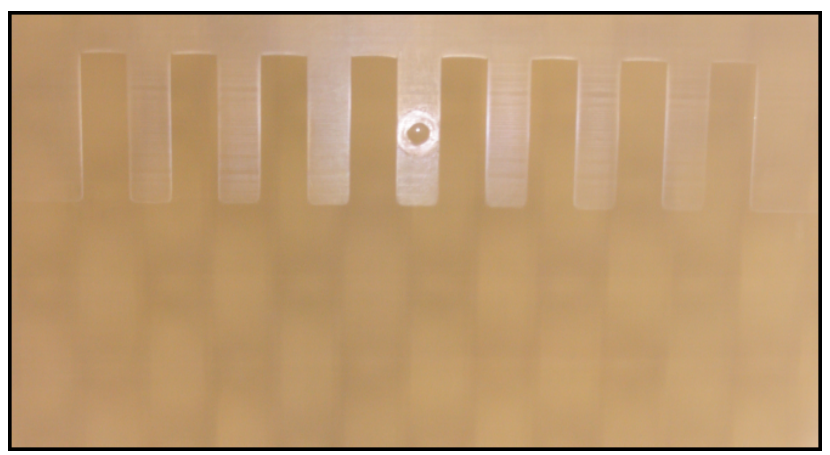

(a) SLR baseline photograph.

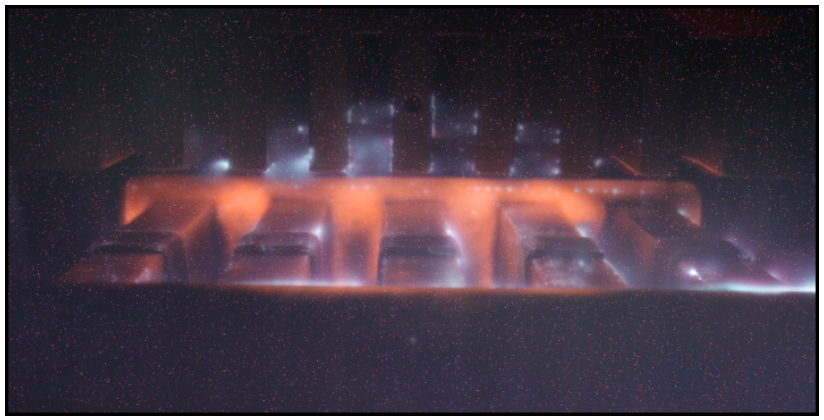

(b) SLR photograph of shot 15023 .

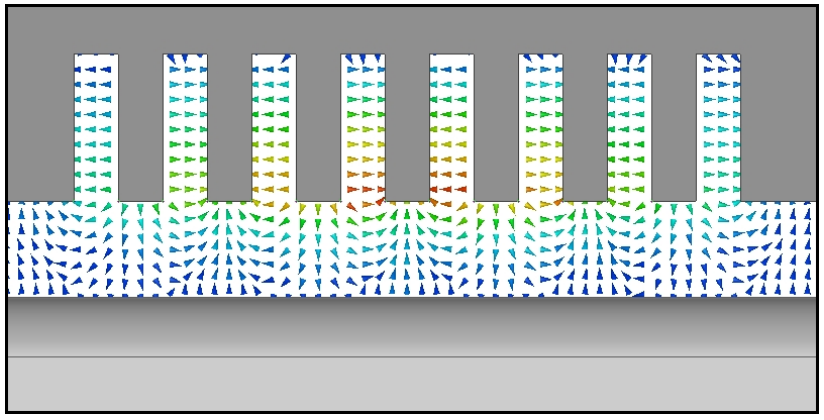

(c) HFSS simulated SBO $\pi$-mode RF electric field.

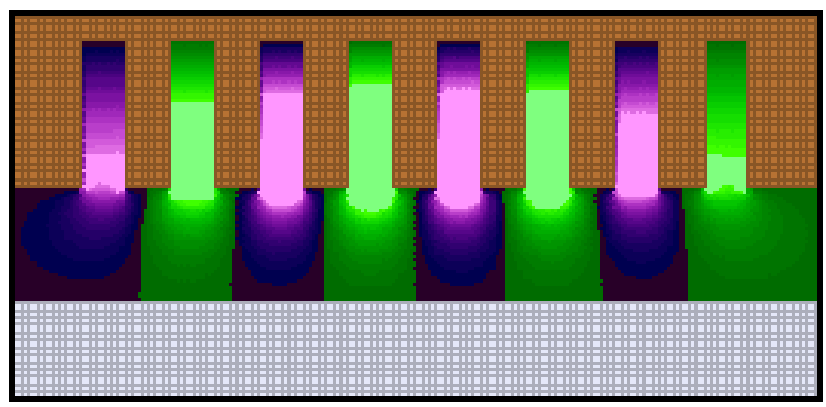

(d) MAGIC simulated SBO $\pi$-mode RF electric field.

Figure 5.45: Comparison between experimental pulse-shortening images and simulated RF electric fields for the SBO $\pi$-mode. 
consistently observed in $B>0.22 \mathrm{~T}$, and had a plasma profile like that of Fig. 5.45b. The $7 \pi / 8$-mode, which had an unloaded frequency of approximately $2 \mathrm{GHz}$, was observed in $B<0.22 \mathrm{~T}$, and had a different plasma profile. The $7 \pi / 8$-mode plasma profile did not match as well with cold-tube simulations compared to the $\pi$-mode, although some hot-tube simulations exhibited similar electric field profiles to the experimental $7 \pi / 8$-mode plasma observations.

Figure 5.46 shows an example of the images obtained using the isolated LBO configuration and the fast framing camera. For this shot, the exposures were 50 ns. Early in the shot, plasma formed on the surface of the cathode, which persisted through the remainder of the pulse. The plasma can be seen on the left side of Fig. 5.46b, where the bright red outlines the surface of the cathode bars. This frame did not indicate the presence of plasma on the anode, which is outlined in yellow, despite being in the middle of the microwave pulse shown by Fig. 5.46a. The next frame occurs during the onset of microwave pulseshortening, which is clearly shown in Fig. 5.46c. The camera image (Fig. 5.46d) indicates that plasma had begun to form in the cavities (shown by the red circle), which suggests a correlation between microwave pulse-shortening and cavity plasma formation. The cavity plasma persists (Fig. 5.46f) after the microwave pulse extinguished (Fig. 5.46e). Due to the angle of the images, it is difficult to make any definitive statements about the expansion of cathode plasma into the AK gap.

While these results are still the subject of ongoing analysis, the presence of cavity plasma was a surprising result that may explain microwave pulse-shortening under some circumstances in the MFRPM. Despite these experiments being conducted using an unloaded configuration, it is conceivable that cavity plasma forms in the MFRPM when high microwave powers are observed because simulations suggested the cavity fields remain high due to the relatively low power extracted from the anode structures by the axial extractors, as indicated by their relatively high $Q$-factors. Cavity breakdown is known to occur in magnetrons extracting powers $>2 \mathrm{GW}$ [105]. However, at lower powers (such as those observed using a de-tuned electron beam velocity for a given mode), the widely accepted explanation of cathode plasma expansion from the velvet emitters remains the best explanation $[17,104,105,112]$. 


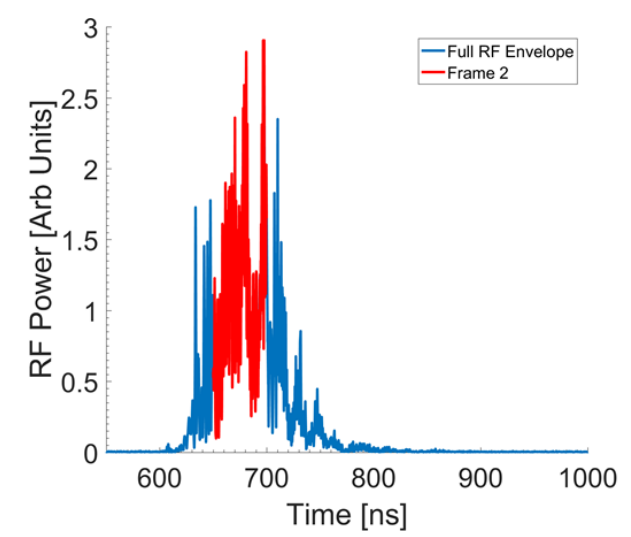

(a) Frame 2: Mid-pulse microwave amplitudes.

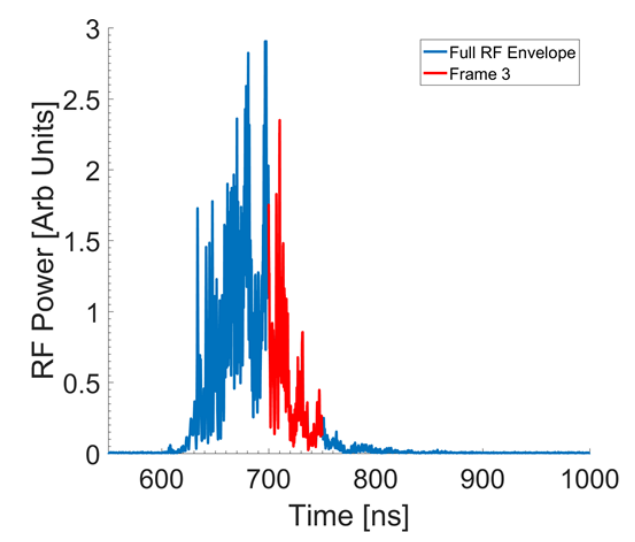

(c) Frame 3: Microwave amplitudes at onset of pulse-shortening.

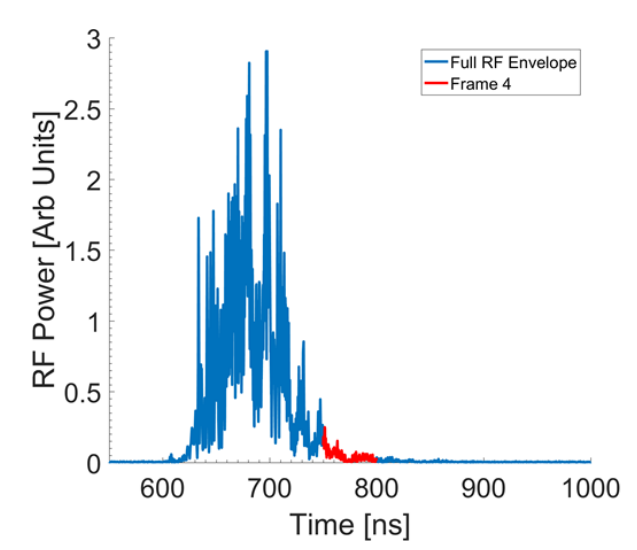

(e) Frame 4: Extinguished microwave pulse.

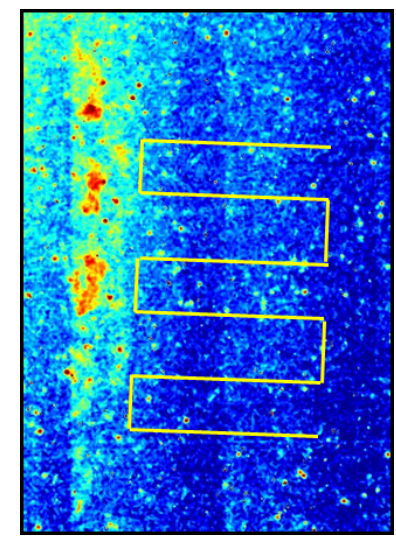

(b) Frame 2: Camera image showing formation of cathode plasma.

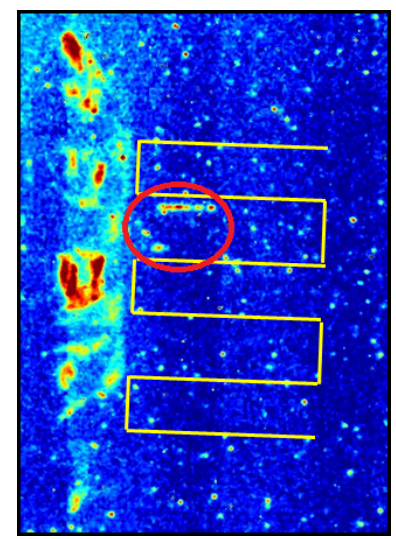

(d) Frame 3: Camera image showing first cavity plasma formation in red circle during onset of pulse-shortening.

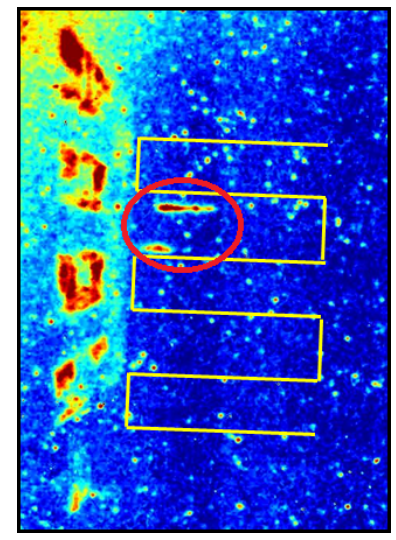

(f) Frame 4: Camera image showing persistent cavity plasma in red circle.

Figure 5.46: Microwave amplitudes and corresponding colorized fast framing camera images during shot 15104. Images were provided by Dr. Nick Jordan. 


\section{CHAPTER 6}

\section{Experimental Results for the Taper Load}

This chapter reports the results and analysis of shots conducted using the waveguide taper load described in $\S$ 4.5.2. Two MFRPM configurations were tested. The first used a nonuniform axial magnetic field, and the second used a magnetic field that was optimized for uniformity throughout the MFRPM. The different magnetic field configurations were described in $\S \S 4.2 .1$ and 4.2.2.

The chapter begins with a Summary of Findings section, which contains all the results tables that summarize the notable aspects of operation. Brief comments accompany each table to provide context and condense the table results into the most important points. The choice of plotted parameters, method of visualization, and structure of result discussion were addressed in $\S 5.2$ in the preceding chapter. However, configuration differences and emphasis on the analysis of frequency harmonics necessarily modify how the metrics are interpreted, and the following Operation Metrics section of this chapter addresses these differences. That section is followed by the experimental results for the aforementioned configurations, and a discussion of the effects of the magnetic field optimization on operation concludes the chapter.

\subsection{Summary of Findings}

\subsubsection{MFRPM with Taper Load and Nonuniform Magnetic Field}

Analysis of the MFRPM with the taper load and nonuniform magnetic field produced four tables for two regions of operation and a separate analysis of the SBO $6 \pi / 8$-mode. Table 6.1 (the first region) shows the performance of the fundamental modes near $B=0.17 \mathrm{~T}$, as well as the MFRPM as a whole. The LBO operated in the $\pi$-mode, and SBO was a mix between the $\pi$-mode and the $6 \pi / 8$-mode. The $6 \pi / 8$-mode frequency was a harmonic of the dominant LBO fundamental mode, suggesting some operation in a harmonic frequencylocked state. The SBO $6 \pi / 8$-mode power was $28 \%$ lower than the SBO $\pi$-mode power, 
which was the same reduction seen in Chapter 5. Shots exhibited high variability in power and moderate variability in frequency. The LBO frequency was strongly affected (specifically, reduced from the typical $0.980-0.985 \mathrm{GHz}$ to $0.969 \mathrm{GHz}$ ) by the presence of the waveguide taper load. Table 6.2 shows the performance of the LBO harmonics in this region. The second harmonic powers were $\simeq 200 \mathrm{~kW}$, and the fourth harmonic powers were $\simeq 10 \mathrm{~kW}$.

Table 6.3 (the second region) shows the performance of the fundamental modes in the region of optimal operation in $B=0.22-0.23 \mathrm{~T}$. The optimal magnetic field was $0.23 \mathrm{~T}$. Both oscillators operated in the $\pi$-mode. There was moderate variability in the powers and frequencies. There was some harmonic correlation between the competing frequencies on the oscillators, but no dominant, harmonic frequency-locked operation was observed. Table 6.4 shows the performance of the LBO harmonics in this region. The second harmonic powers were $\simeq 300 \mathrm{~kW}$, and the fourth harmonic powers were $\simeq 4 \mathrm{~kW}$.

Consistent, dominant SBO $6 \pi / 8$-mode operation was observed in $B=0.13-0.17 \mathrm{~T}$, which enabled accurate determination of the frequency and power. Table 6.5 shows the results. The average frequency for the SBO $6 \pi / 8$-mode $(1.945 \mathrm{GHz})$ was near the freerunning 6 $6 \pi / 8$-mode frequency from Chapter $5(1.94 \mathrm{GHz})$.

In general, analysis of this configuration provided further evidence of coupling between the LBO $\pi$-mode (and possibly the $5 \pi / 6$-mode) and the SBO $6 \pi / 8$-mode. In addition, high-power operation of the LBO at its fundamental frequencies correlated with lower power generated at its harmonic frequencies.

Table 6.1: Taper Load and Nonuniform Magnetic Field: Summarized performance metrics near $0.17 \mathrm{~T}$.

\begin{tabular}{lclll}
\hline \hline LBO $\pi$-Mode Power: & 24 & \pm & 6 & $\mathrm{MW}$ \\
LBO $\pi$-Mode Frequency: & 0.969 & \pm & 0.003 & $\mathrm{GHz}$ \\
SBO $\pi$-Mode Power: & 7 & \pm & 2 & $\mathrm{MW}$ \\
SBO $\pi$-Mode Frequency: & 2.019 & \pm & 0.004 & $\mathrm{GHz}$ \\
SBO 6 $\pi$ /8-Mode Power: & 5 & \pm & 1 & $\mathrm{MW}$ \\
SBO 6 $\pi$ /8-Mode Frequency: & 1.937 & \pm & 0.007 & $\mathrm{GHz}$ \\
Total Power: & 26 & \pm & 7 & $\mathrm{MW}$ \\
Peak Power $|\Delta t|:$ & 52 & \pm & 25 & $\mathrm{~ns}$ \\
Impedance: & 114 & \pm & 15 & $\Omega$ \\
Efficiency: & 5 & \pm & 2 & $\%$ \\
\hline \hline
\end{tabular}


Table 6.2: Taper Load and Nonuniform Magnetic Field: Summarized performance metrics near $0.17 \mathrm{~T}$ for the LBO harmonics.

\begin{tabular}{lcccl}
\hline \hline LBO Second Harmonic Power: & 218 & \pm & 65 & $\mathrm{~kW}$ \\
LBO Second Harmonic Frequency: & 1.986 & \pm & 0.038 & $\mathrm{GHz}$ \\
LBO Fourth Harmonic Power: & 13 & \pm & 5 & $\mathrm{~kW}$ \\
LBO Fourth Harmonic Frequency: & 3.913 & \pm & 0.052 & $\mathrm{GHz}$ \\
\hline \hline
\end{tabular}

Table 6.3: Taper Load and Nonuniform Magnetic Field: Summarized performance metrics in the region of optimal operation in $B=0.22-0.23 \mathrm{~T}$.

\begin{tabular}{lclll}
\hline \hline LBO $\pi$-Mode Power: & 28 & \pm & 5 & $\mathrm{MW}$ \\
LBO $\pi$-Mode Frequency: & 0.972 & \pm & 0.002 & $\mathrm{GHz}$ \\
SBO $\pi$-Mode Power: & 11 & \pm & 3 & $\mathrm{MW}$ \\
SBO $\pi$-Mode Frequency: & 2.021 & \pm & 0.005 & $\mathrm{GHz}$ \\
Total Power: & 32 & \pm & 7 & $\mathrm{MW}$ \\
Peak Power $|\Delta t|:$ & 27 & \pm & 12 & $\mathrm{~ns}$ \\
Impedance: & 206 & \pm & 15 & $\Omega$ \\
Efficiency: & 9 & \pm & 2 & $\%$ \\
\hline \hline
\end{tabular}

Table 6.4: Taper Load and Nonuniform Magnetic Field: Summarized performance metrics for the LBO harmonics in the region of optimal operation in $B=0.22-0.23 \mathrm{~T}$.

\begin{tabular}{lcccl}
\hline \hline LBO Second Harmonic Power: & 291 & \pm & 143 & $\mathrm{~kW}$ \\
LBO Second Harmonic Frequency: & 1.986 & \pm & 0.041 & $\mathrm{GHz}$ \\
LBO Fourth Harmonic Power: & 4 & \pm & 2 & $\mathrm{~kW}$ \\
LBO Fourth Harmonic Frequency: & 4.009 & \pm & 0.022 & $\mathrm{GHz}$ \\
\hline \hline
\end{tabular}

Table 6.5: Taper Load and Nonuniform Magnetic Field: Summarized performance metrics for the SBO $6 \pi / 8$-mode in $B=0.13-0.17 \mathrm{~T}$.

\begin{tabular}{lllll}
\hline \hline $6 \pi / 8$-Mode Power: & 5 & \pm & 1 & $\mathrm{MW}$ \\
$6 \pi / 8$-Mode Frequency: & 1.945 & \pm & 0.009 & $\mathrm{GHz}$ \\
\hline \hline
\end{tabular}




\subsubsection{MFRPM with Taper Load and Uniform Magnetic Field}

Analysis of the MFRPM with the taper load and uniform magnetic field produced two tables for the region of optimal operation, which occurred near 0.17 T. Table 6.6 summarizes the results for the fundamental modes of the individual oscillators, as well as the MFRPM as a whole. Harmonic frequency-locked operation was observed between the LBO first, second, and fourth harmonics frequencies, and the SBO fundamental frequency. The LBO operated in the $\pi$-mode, and the SBO operated in the $6 \pi / 8$-mode. The harmonic frequency-locked state was associated with reductions in the variability of both power and frequency, and the MFRPM operated more like a single device due to the low time delay between peak powers from each oscillator. Microwave pulse-shortening in the LBO reduced some of the effect of the waveguide taper load on the operating frequency. The analysis also suggested the SBO affects the LBO second frequency harmonic.

Table 6.7 shows the performance of the LBO harmonics. The second harmonic powers were $\simeq 180 \mathrm{~kW}$, and the fourth harmonic powers were $\simeq 5 \mathrm{~kW}$. The highest LBO second harmonic powers were produced at the same frequency as the free-running SBO $6 \pi / 8$ mode, and occurred when the SBO competing mode corresponded to the same.

Table 6.6: Taper Load and Uniform Magnetic Field: Summarized performance metrics near $0.17 \mathrm{~T}$.

\begin{tabular}{lclll}
\hline \hline LBO $\pi$-Mode Power: & 39 & \pm & 4 & $\mathrm{MW}$ \\
LBO $\pi$-Mode Frequency: & 0.980 & \pm & 0.003 & $\mathrm{GHz}$ \\
SBO 6 $\pi$ /8-Mode Power: & 10 & \pm & 2 & $\mathrm{MW}$ \\
SBO 6 $\pi$ /8-Mode Frequency: & 1.969 & \pm & 0.003 & $\mathrm{GHz}$ \\
Total Power: & 47 & \pm & 4 & $\mathrm{MW}$ \\
Peak Power $|\Delta t|:$ & 10 & \pm & 8 & $\mathrm{~ns}$ \\
Impedance: & 132 & \pm & 15 & $\Omega$ \\
Efficiency: & 12 & \pm & 1 & $\%$ \\
\hline \hline
\end{tabular}

Table 6.7: Taper Load and Uniform Magnetic Field: Summarized performance metrics near $0.17 \mathrm{~T}$ for the LBO harmonics.

\begin{tabular}{lcccl}
\hline \hline LBO Second Harmonic Power: & 178 & \pm & 60 & $\mathrm{~kW}$ \\
LBO Second Harmonic Frequency: & 1.962 & \pm & 0.013 & $\mathrm{GHz}$ \\
LBO Fourth Harmonic Power: & 5 & \pm & 1 & $\mathrm{~kW}$ \\
LBO Fourth Harmonic Frequency: & 3.916 & \pm & 0.018 & $\mathrm{GHz}$ \\
\hline \hline
\end{tabular}




\subsubsection{Comparison of Metrics}

Three tables compare the operation of the MFRPM and the LBO frequency harmonics using the uniform and nonuniform magnetic fields. All metrics were taken from the regions of optimal operation for the respective configurations.

Table 6.8 compares the performance metrics for the individual oscillators. The LBO saw a 39\% improvement in microwave output power using the uniform magnetic field, whereas the SBO saw a $9 \%$ reduction in microwave output power. This was likely due to the shift in the SBO operating mode from the $\pi$-mode to the harmonic frequency-locked $6 \pi / 8$-mode. The choice of magnetic field configuration did not substantially change the variability of the powers and frequencies.

Table 6.9 compares the performance metrics for the MFRPM as a whole. Performance of the MFRPM was improved by the use of the uniform magnetic field. The total power increased by $47 \%$, and the time difference between peak powers from the two oscillators (Peak Power $|\Delta t|$ ) was reduced from $27 \mathrm{~ns}$ to $10 \mathrm{~ns}$.

Table 6.10 compares the performance of the LBO frequency harmonics. The second LBO harmonic power was reduced by $39 \%$, and the fourth harmonic power increased by $25 \%$. However, for both magnetic field configurations, the variability was high.

Table 6.8: Taper Load: Comparison of metrics for individual oscillators.

\begin{tabular}{llcccl}
\hline \hline Configuration & METRIC & VAlue & & StD. DeV. & Units \\
\hline Nonunif. $B$ & LBO $\pi$-Mode Power: & 28 & \pm & 5 & $\mathrm{MW}$ \\
Unif. $B$ & LBO $\pi$-Mode Power: & 39 & \pm & 4 & $\mathrm{MW}$ \\
Nonunif. $B$ & LBO $\pi$-Mode Frequency: & 0.972 & \pm & 0.002 & $\mathrm{GHz}$ \\
Unif. $B$ & LBO $\pi$-Mode Frequency: & 0.980 & \pm & 0.003 & $\mathrm{GHz}$ \\
Nonunif. $B$ & SBO $\pi$-Mode Power: & 11 & \pm & 3 & $\mathrm{MW}$ \\
Unif. $B$ & SBO $6 \pi / 8$-Mode Power: & 10 & \pm & 2 & $\mathrm{MW}$ \\
Nonunif. $B$ & SBO $\pi$-Mode Frequency: & 2.021 & \pm & 0.005 & $\mathrm{GHz}$ \\
Unif. $B$ & SBO $6 \pi / 8$-Mode Frequency: & 1.969 & \pm & 0.003 & $\mathrm{GHz}$ \\
\hline \hline
\end{tabular}


Table 6.9: Taper Load: Comparison of MFRPM metrics.

\begin{tabular}{llrrrl}
\hline \hline CONFIGURATION & METRIC & VALUE & & STD. DEV. & UNITS \\
\hline Nonunif. $B$ & Total Power: & 32 & \pm & 7 & MW \\
Unif. $B$ & Total Power: & 47 & \pm & 4 & MW \\
Nonunif. $B$ & Peak Power $|\Delta t|:$ & 27 & \pm & 12 & ns \\
Unif. $B$ & Peak Power $|\Delta t|:$ & 10 & \pm & 8 & ns \\
Nonunif. $B$ & Impedance: & 206 & \pm & 15 & $\Omega$ \\
Unif. $B$ & Impedance: & 132 & \pm & 15 & $\Omega$ \\
Nonunif. $B$ & Efficiency: & 9 & \pm & 2 & $\%$ \\
Unif. $B$ & Efficiency: & 12 & \pm & 1 & $\%$ \\
\hline \hline
\end{tabular}

Table 6.10: Taper Load: Comparison of metrics for the LBO frequency harmonics.

\begin{tabular}{llcccl}
\hline \hline CONFIGURATION & METRIC & VAlUE & & STD. DEV. & UNITS \\
\hline Nonunif. $B$ & LBO Second Harmonic Power: & 291 & \pm & 143 & $\mathrm{~kW}$ \\
Unif. $B$ & LBO Second Harmonic Power: & 178 & \pm & 60 & $\mathrm{~kW}$ \\
Nonunif. $B$ & LBO Second Harmonic Frequency: & 1.986 & \pm & 0.041 & $\mathrm{GHz}$ \\
Unif. $B$ & LBO Second Harmonic Frequency: & 1.962 & \pm & 0.013 & $\mathrm{GHz}$ \\
Nonunif. $B$ & LBO Fourth Harmonic Power: & 4 & \pm & 2 & $\mathrm{~kW}$ \\
Unif. $B$ & LBO Fourth Harmonic Power: & 5 & \pm & 1 & $\mathrm{~kW}$ \\
Nonunif. $B$ & LBO Fourth Harmonic Frequency: & 4.009 & \pm & 0.022 & $\mathrm{GHz}$ \\
Unif. $B$ & LBO Fourth Harmonic Frequency: & 3.916 & \pm & 0.018 & $\mathrm{GHz}$ \\
\hline \hline
\end{tabular}

\subsection{Operation Metrics}

Like the previous chapter, the presentation and discussion of the results is divided into two sections: Standard Metrics and Additional Analysis. The standard metrics were shared diagnostics that were applied to both experimental configurations for later comparison. However, due to the differences between the present configuration and that of the previous chapter, the interpretation and meaning of some of the metrics change. Also like the previous chapter, the Additional Analysis sections include plots and metrics that were specific to the individual configurations, or discuss operating behavior that was unique to the shot series. The Additional Analysis sections also discuss the analysis of the frequency harmonics.

For brevity, much of the analysis from Chapter 5 is either referenced or the conclusions and interpretations are restated, rather than explained for a second time. An example would be the analysis and reasoning that supports the conclusion that the $1.04 \mathrm{GHz}$ mode corresponds to the parasitic chamber mode. Whereas the previous chapter described the analysis of the mode, this chapter includes a reference to the previous analysis and treats the identity of the mode as an accepted conclusion. 


\subsubsection{Standard Metrics}

The same plotted quantities were analyzed as those in the previous chapter. These were common to both experiments conducted in this chapter. The standard metrics include the following plots: 1) Power vs. Magnetic Field, 2) Frequency vs. Magnetic Field, 3) Frequency Histograms, 4) Power vs. Frequency, 5) Current vs. Magnetic Field, 6) Impedance vs. Magnetic Field, 7) Start and Peak Power Voltages vs. Magnetic Field, 8) Efficiency vs. Magnetic Field, 9) Energy vs. Magnetic Field, 10) Start and Peak Power Times vs. Magnetic Field, and 11) Peak Power $|\Delta t|$ vs. Magnetic Field.

Although these metrics are the same as those found in the previous chapter, their interpretation changes because of the differences between the configurations. This is because the LBO waveguide taper load used in the configurations investigated in this chapter reflected $>99.9 \%$ of the power back to the magnetron, which limited the mechanisms for power dissipation prior to pulse-shortening to ohmic resistance in the circuit and kinetic losses through acceleration of electrons into the anode. The LBO waveguide taper modification therefore constituted an extremely high- $Q$ configuration relative to the standard load despite good coupling to the microwave extraction assembly. Modification of the oscillator loaded quality factor $Q_{L}$ and load characteristics has a significant impact on the operation of magnetrons $[6,7,49,51,66,114,115]$. The consequences of this fact for LBO operation include artificially higher powers, less informative pulse energies and efficiencies, longer pulselengths, differences in operating frequencies, and possible changes in current due to the modified RF voltage amplitudes across the cavities [116]. Because of these differences, direct comparison with the results of the previous chapter would be unsound.

Despite these caveats, the affected metrics still convey useful information. Powers, energies, and efficiencies remain useful for comparing the effects of the magnetic field optimization and understanding the results of the previous pulse-shortening study, and longer pulselengths can be beneficial for precise frequency identification, especially when attempting to establish the relation between the frequencies of the fundamental modes and the harmonics.

\subsubsection{Additional Analysis}

In addition to any behavior unique to a shot series, the Additional Analysis sections also discuss the results for the MFRPM harmonics, including the powers, frequencies, and the relation between the different oscillators. Due to the complexity introduced by having both dominant and competing frequencies at multiple harmonics for two oscillators, the figures that present different frequencies include explicit labeling of the oscillator, the approximate 
frequency $(1,2$, or $4 \mathrm{GHz})$, and whether the quantity was competing or dominant. Dominant and competing frequencies are defined as the first- and second-highest peaks near the frequency of interest $(1,2$, or $4 \mathrm{GHz})$ in the time-integrated Fourier transform of an RF voltage signal. For clarity, modes near $1 \mathrm{GHz}$ for the LBO are termed the fundamental modes, which are also called the first harmonics, and those near $2 \mathrm{GHz}$ or $4 \mathrm{GHz}$ are the second and fourth harmonics, respectively. Similarly, for the SBO, modes having frequencies near $2 \mathrm{GHz}$ are the fundamental modes of the oscillator. Higher frequencies were not observed for the SBO, so this dissertation contains no discussion of SBO harmonics.

\subsection{MFRPM with Taper Load and Nonuniform Magnetic Field}

This shot series tested the MFRPM using the nonuniform magnetic field described in $\S$ 4.2.1. The purpose was to obtain the first of two data sets necessary to determine how the magnetic field variation along the planar cavity arrays impacted operation of the LBO frequency harmonics. A total of 81 shots were obtained with no MELBA-C self-triggers, magnet triggering failures, or failed crowbars. The base pressure was $3 \mu$ torr, and the range of tested magnetic fields was $0.13-0.23 \mathrm{~T}$.

\subsubsection{Standard Metrics}

As discussed in both the previous chapter and in $\S 4.2 .1$, the variation in the magnitude of the nonuniform magnetic field along the planar cavity arrays was $40 \%$, with a field

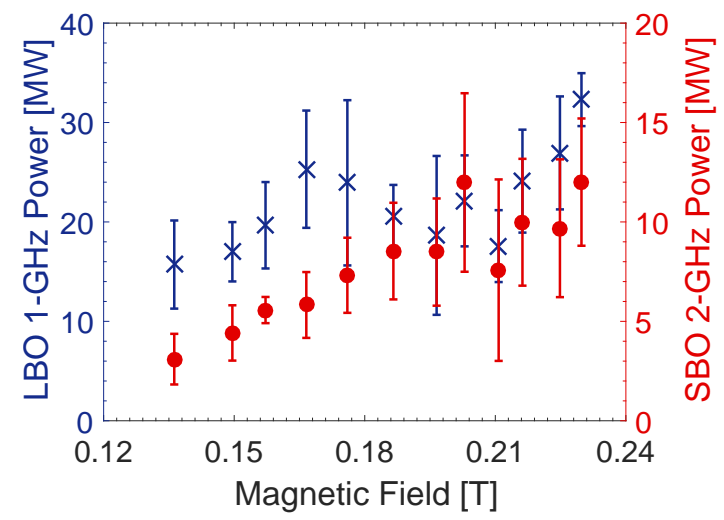

Figure 6.1: Taper Load and Nonuniform Magnetic Field: Peak microwave output powers for both oscillators vs. magnetic field. 
maximum in the center of the planar cavity arrays, and a field minimum in the cylindrical recirculation bends. Differences in the magnitude of the magnetic field led to a large variation in both the electron hub height and $\vec{E} \times \vec{B}$ drift velocity. Thus, the Buneman-Hartree prediction of the threshold magnetic field for operation was not expected to be supported by the experiment because the analytic derivation assumed that the magnetic field was uniform, which was not the case for the nonuniform experimental magnetic field. In addition, as previously discussed, changes to the LBO load were also expected to have a considerable impact on LBO operation. Considering these different aspects of the configuration, relative to the results from Chapter 5 for the nonuniform magnetic field, the LBO was expected to operate differently. By comparison, the SBO was not expected to run differently compared to the standard load and uniform magnetic field configuration because the load and magnetic field were not changed.

Figure 6.1 shows the peak microwave output powers from both oscillators vs. magnetic field. The powers from the SBO were not appreciably different from the results shown by Fig. 5.21 on page 95 in Chapter 5, as expected. However, the LBO results were indeed different. Here, the LBO exhibited two magnetic fields at which power was maximized. The first was at $B \simeq 0.17 \mathrm{~T}$, which was relatively close to the Buneman-Hartree magnetic field $B_{\mathrm{BH}}=0.16 \mathrm{~T}$. This result was surprising for two reasons. First, the experimental agreement with the Buneman-Hartree condition was good despite experimental violation of the derivation's assumption of a uniform magnetic field. Second, a similar peak was not observed in the analogous configuration found in Chapter 5. This fact implies that the dualpeak power effect is probably due to the addition of the waveguide taper load. It is unlikely that the variation in the magnetic field precisely negates the effects from the waveguide taper load such that the analytic predictions remained accurate.

The second LBO power peak was at $B=0.23 \mathrm{~T}$, which was the experimental magnetic field limit. In Chapter 5, the LBO also exhibited dual-peak powers in the isolated configuration. However, that configuration used the uniform magnetic field and standard load, so the analogous behavior was likely due to a different effect. In the case of the isolated LBO configuration, the reduction in output power was consistent with operation in the $5 \pi / 6$ mode. Similar behavior was not observed in the present configuration, as addressed in the discussion of the frequencies below. It remains possible that the high power at magnetic fields $B>0.22 \mathrm{~T}$ was due to synchronism with the next space harmonic of the $\pi$-mode, also termed the $3 \pi$-mode. Periodic peaks in output power with increasing magnetic field are consistent with synchronism with $\pi$-mode space harmonics. However, as was the case at $B=0.17 \mathrm{~T}$, direct comparison with the theoretically predicted values of the magnetic fields for these output power peaks would be unsound because the theoretical assumption 


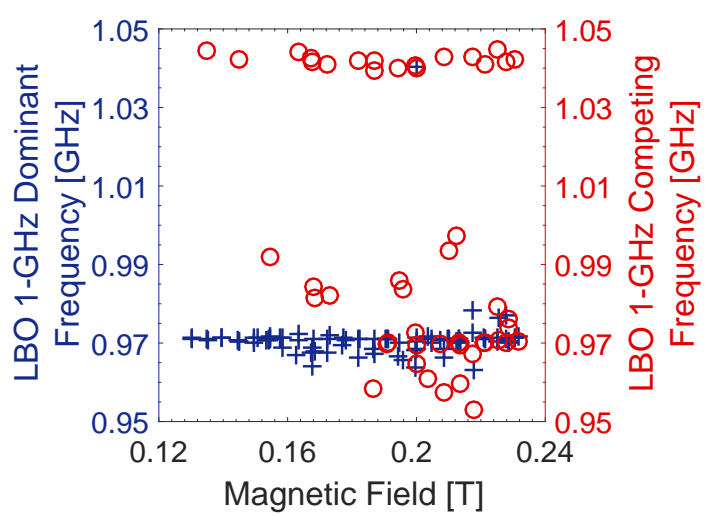

(a) LBO frequencies near $1 \mathrm{GHz}$ vs. magnetic field.

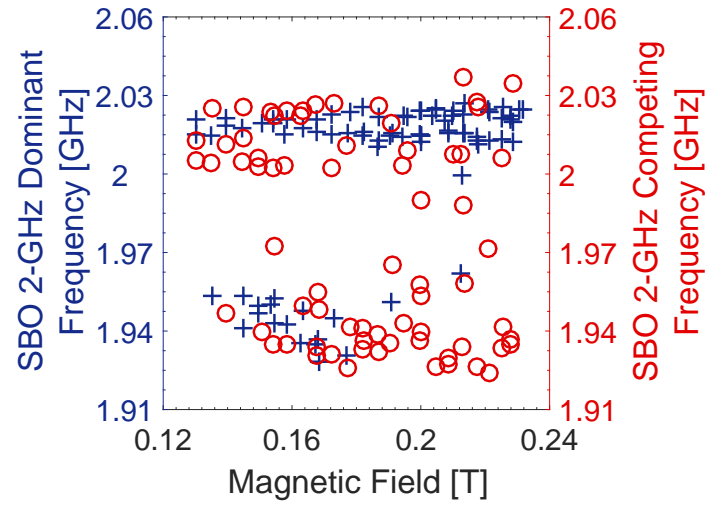

(b) SBO frequencies vs. magnetic field.

Figure 6.2: Taper Load and Nonuniform Magnetic Field: Dominant and competing frequencies of both oscillators vs. magnetic field.

of a uniform magnetic field is significantly violated by the experimental configuration. For both oscillators, the region of optimal operation lay at the highest magnetic fields tested, a narrow range between $B=0.22-0.23 \mathrm{~T}$.

Figure 6.2 shows the dominant and competing frequencies of the fundamental modes of both oscillators vs. magnetic field. The LBO results in Fig. 6.2a were significantly different compared to the results of Chapter 5. The inclusion of the waveguide tapers, which resulted in a high- $Q$ configuration, had a stabilizing effect on the frequency. The sensitivity of a magnetron's operating frequency to changes in the electrical characteristics of the load is a well-known phenomenon, and is termed frequency-pulling [6]. Note that frequency-pulling is not the same as frequency-pushing, also known as beam-loading, which is a different effect. Nearly all shots oscillated near $0.970 \mathrm{GHz}$, although at $B \gtrsim 0.17 \mathrm{~T}$, some shots occurred at slightly lower frequencies. With one exception, the parasitic chamber mode was observed only as a competing mode near $1.04 \mathrm{GHz}$. The frequency of the parasitic chamber mode was consistent with the results of the previous chapter, which suggests that it was not affected by the modifications to the LBO load.

SBO frequencies vs. magnetic field are shown by Fig. 6.2b. These results were qualitatively similar to the observations from the isolated SBO configuration with the uniform magnetic field, where both the $7 \pi / 8$-mode and $\pi$-mode were seen at relatively high magnetic fields $B>0.2 \mathrm{~T}$. For the present configuration, in the lower range of magnetic fields $B \lesssim 0.17 \mathrm{~T}$, the distinction between the $7 \pi / 8$-mode and the $\pi$-mode was less pronounced. On average, the $2.02 \mathrm{GHz} \pi$-mode was the dominant mode, and the $2 \mathrm{GHz} 7 \pi / 8$-mode was the competing mode. The SBO also demonstrated several instances of both dominant and competing operation in the $6 \pi / 8$-mode near $1.94 \mathrm{GHz}$. In most cases, dominant $6 \pi / 8$ - 


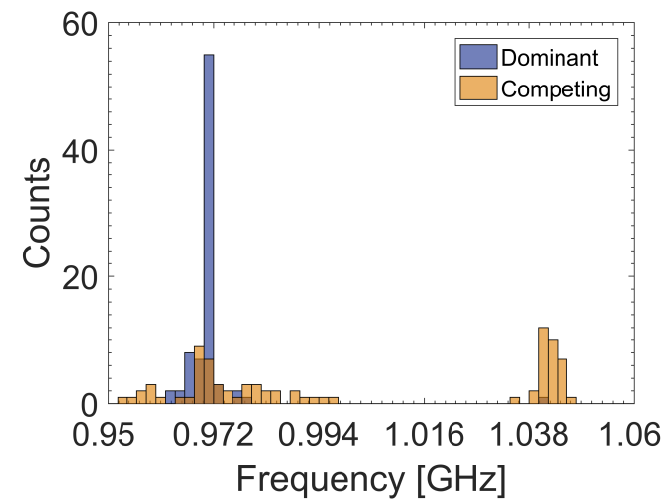

(a) LBO frequency histogram near $1 \mathrm{GHz}$.

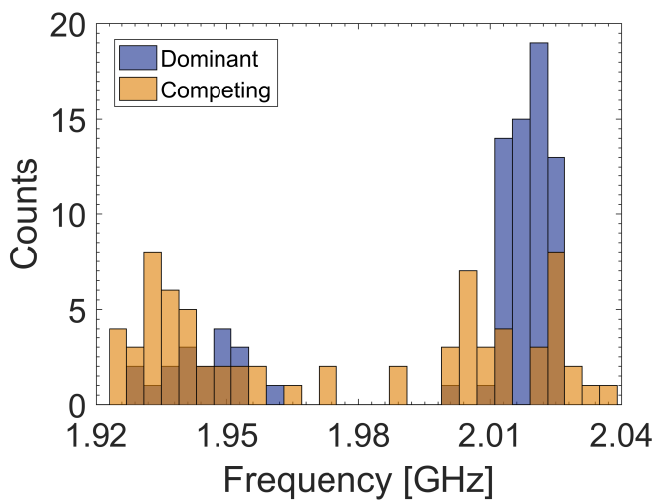

(b) SBO frequency histogram.

Figure 6.3: Taper Load and Nonuniform Magnetic Field: Dominant and competing frequency histograms for both oscillators.

mode operation occurred at $B \lesssim 0.17 \mathrm{~T}$, and competing $6 \pi / 8$-mode operation occurred at $B \gtrsim 0.17 \mathrm{~T}$.

Frequency distributions are shown by Fig. 6.3. In the figure, the darker, brown shade represents overlap between the dominant and competing frequencies. In other words, that frequency bin had shots that were competing, and shots that were dominant. The bars were overlaid because the use of separate bars at the same frequency led to plots that were visually more challenging to interpret. In both cases, the distributions confirm the impressions given by Fig. 6.2. The LBO results in Fig. 6.3a show the very narrow dominant $\pi$-mode frequency range and competition from the $1.04 \mathrm{GHz}$ parasitic chamber mode. Notably, there are a few instances of dominant LBO shots occurring at slightly lower frequencies relative to the large peak. These few instances may correspond to the $5 \pi / 6$-mode, but the lack of a discrete peak precludes any conclusive statements.

The SBO results in Fig. 6.3b show that, despite the reduced clarity between the $\pi$-mode and $7 \pi / 8$-mode due to slight variations in their respective frequencies with magnetic field, a noticeable peak for the $7 \pi / 8$-mode exists at $2 \mathrm{GHz}$ that is distinct from the $2.02 \mathrm{GHz} \pi$ mode peak. Relative to both the $7 \pi / 8$-mode and the $\pi$-mode, the $6 \pi / 8$-mode had a broad distribution that was comparable to the results for the nonuniform magnetic field discussed in the previous chapter. However, closer inspection of the distribution for the $6 \pi / 8$-mode suggests it actually consists of two overlapping distributions: one for shots where the $6 \pi / 8$ mode was the dominant mode, and one for shots where the $6 \pi / 8$-mode was the competing mode. Further analysis of this observation occurs in the following Additional Analysis section.

Figure 6.4 illustrates the relation between the oscillators' output powers and their re- 


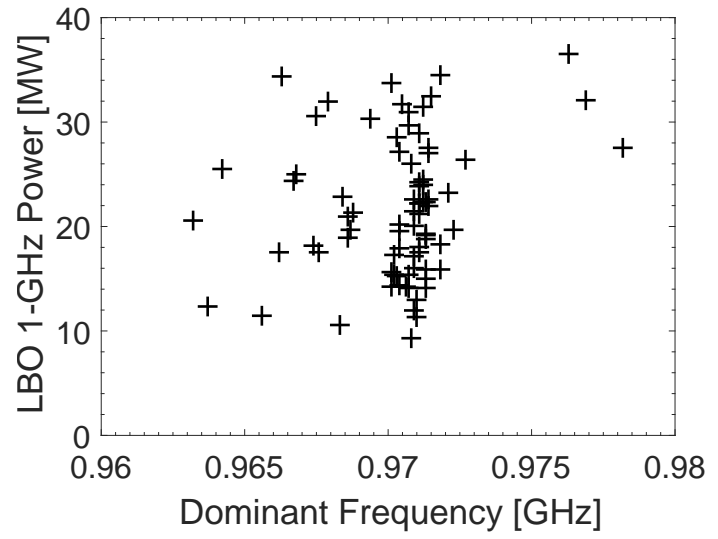

(a) Peak LBO power vs. dominant operating frequency.

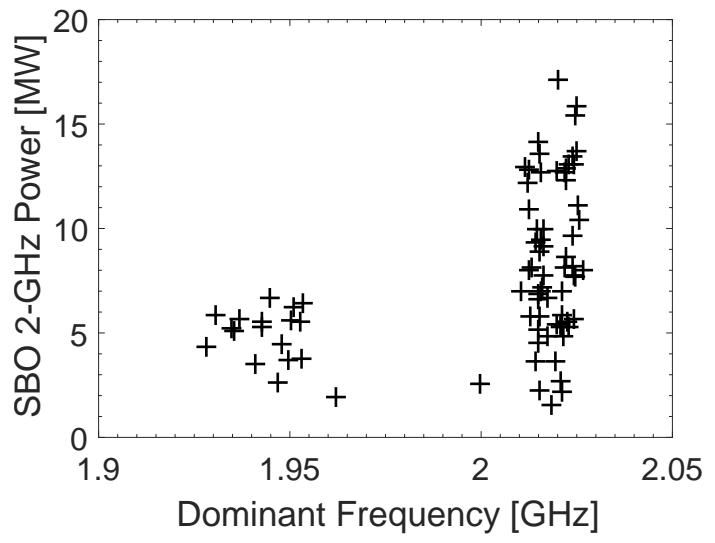

(b) Peak SBO power vs. dominant operating frequency.

Figure 6.4: Taper Load and Nonuniform Magnetic Field: Peak oscillator powers vs. dominant operating frequencies.

spective dominant frequencies. As expected from the previous discussion, the results for the LBO in Fig. 6.4a reveal that most shots occurred near $0.97 \mathrm{GHz}$ and that there was a significant range of powers produced at that single frequency due to the variation in output power with magnetic field. Figure $6.4 \mathrm{~b}$ shows the results for the SBO. Due to the frequency variation of both the $7 \pi / 8$-mode and $\pi$-mode with magnetic field, it is difficult to make any definitive statements about the powers of the two modes. However, the histogram results showed that the $7 \pi / 8$-mode existed primarily as a competing mode, which explains the absence of $7 \pi / 8$-mode frequencies in Fig. 6.4b, which shows only the dominant frequencies. This result is consistent with the poor coupling between the $7 \pi / 8$-mode and the microwave extractor. This configuration also demonstrated a collection of shots operating primarily in the $6 \pi / 8$-mode, all of which exhibited lower powers than the $\pi$-mode. Further analysis of $6 \pi / 8$-mode operation can be found in the Additional Analysis section.

Electrical characteristics for the MFRPM are shown by Fig. 6.5. $B \simeq 0.17 \mathrm{~T}$ represented a point of operational transition, which agrees with the results for the power of the LBO and frequencies of the SBO. The currents at peak power, which are illustrated in Fig. 6.5a, had approximately consistent means with high variability in $B \lesssim 0.17 \mathrm{~T}$, whereas $B \gtrsim 0.17 \mathrm{~T}$ corresponded to a gradual, smooth reduction in drawn current and a significant reduction in shot-to-shot variability. The results for the impedances in Fig. 6.5b were analogous. At $B \lesssim 0.17 \mathrm{~T}$, the impedance at peak power of the MFRPM was, on average, well-matched to MELBA-C specifications. While this magnetic field range does include the first power peak for the LBO, the MFRPM did not appear to benefit materially from the good match to the pulsed power source. 


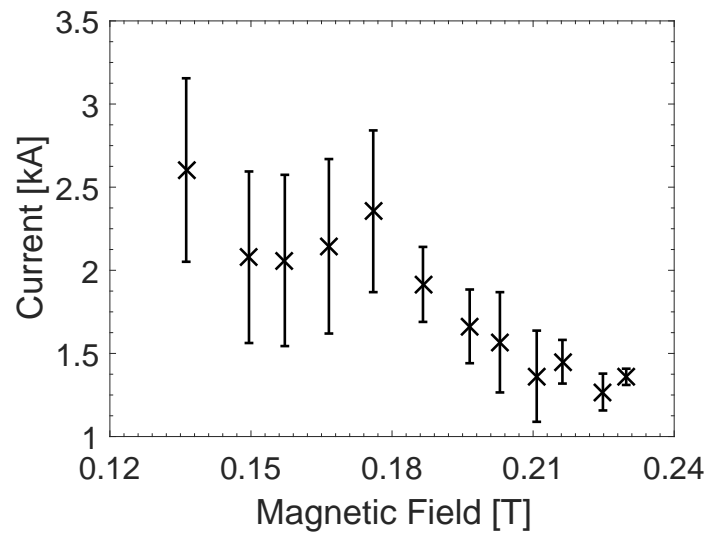

(a) Current at peak power vs. magnetic field.

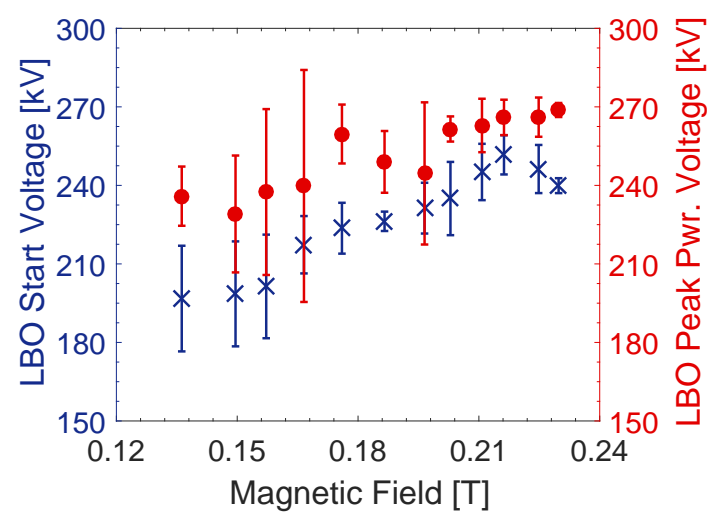

(c) Voltage at time of LBO start and peak power.

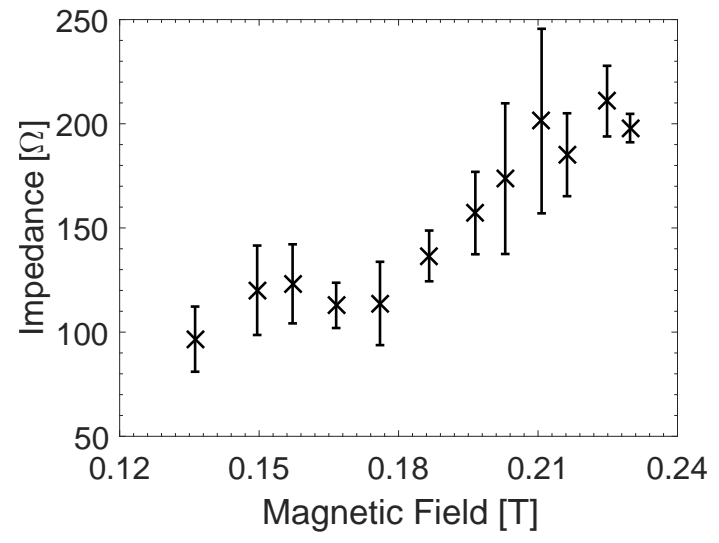

(b) Impedance at peak power vs. magnetic field.

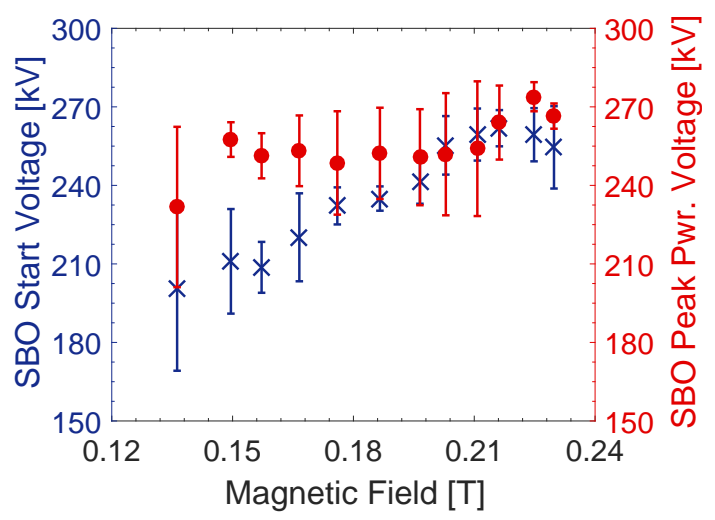

(d) Voltage at time of SBO start and peak power.

Figure 6.5: Taper Load and Nonuniform Magnetic Field: Electrical characteristics vs. magnetic field.

Start and peak power voltages for the LBO are shown in Fig. 6.5c. The start voltage increase was approximately linear with increasing magnetic field, which was the expected result considering the LBO consistently operated near the same frequency, and therefore likely operated in the same mode on the majority of shots. While a similar linear scaling was observed for the LBO peak power voltage, there was considerably greater shot-to-shot variation, particularly in the range of $B \lesssim 0.17 \mathrm{~T}$. The lower consistency of the peak power voltages relative to the start voltages is reasonable when the waveguide taper load is viewed as a disruptive element. Initially, the influence of the reflected power is low, meaning the LBO operates somewhat normally during start-up. At peak power production, the reflected power is nearly equal to the produced power, which explains the variable operation. A temporal delay is expected before the reflected power disrupts operation because the output waveguide, acting like a high- $Q$ cavity, takes time to fill with electromagnetic energy. The fill time is proportional to $Q$ [3], so the high- $Q$ configuration implies a relatively long fill 


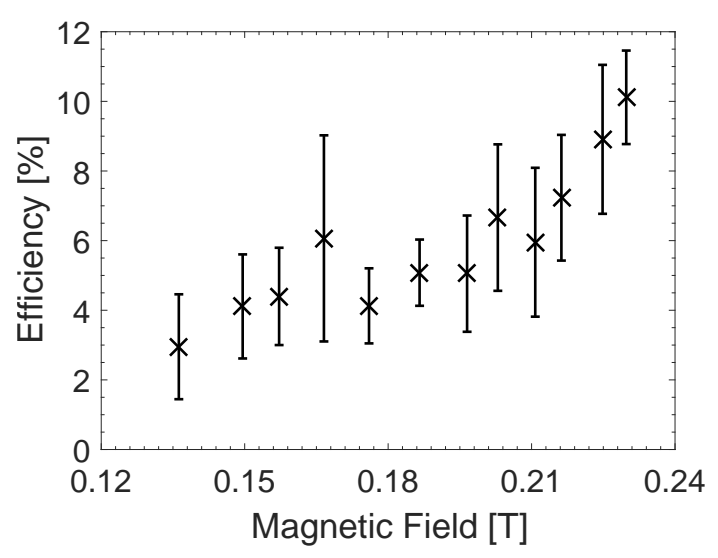

(a) Peak total efficiency vs. magnetic field.

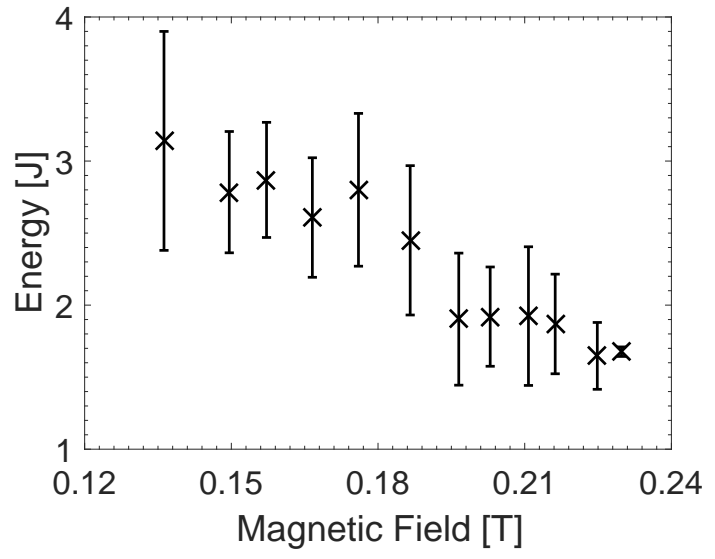

(b) Total pulse energy vs. magnetic field.

Figure 6.6: Taper Load and Nonuniform Magnetic Field: Energy conversion metrics vs. magnetic field.

time. Further evidence of this effect can be found in Fig. 6.8 on page 148, which will be discussed later in the Additional Analysis section.

The voltages for the SBO are shown in Fig. 6.5d. Start voltages were approximately linear, although the region of $B \lesssim 0.17 \mathrm{~T}$ corresponded to higher shot-to-shot variation. This is not surprising considering the conclusions from Fig. 6.2b on page 141, which revealed this magnetic field range to be susceptible to mode competition between the $6 \pi / 8$-mode, $7 \pi / 8$-mode, and $\pi$-mode, with both $6 \pi / 8$-mode and $\pi$-mode frequencies occurring as the dominant frequencies in roughly equal proportion. Interestingly, the mean peak power voltage was approximately constant for the SBO. Near the upper limit of tested magnetic fields, several shots saw the SBO produce peak power at a lower voltage than the start voltage. This was due to relatively late peak power production by the SBO. At these late times, greater diode closure led to higher currents and subsequently lower voltages.

Energy conversion metrics are shown by Fig. 6.6. The efficiencies depicted in Fig. 6.6a suggest that, under the best circumstances, the MFRPM was as efficient as the best results observed in Chapter 5. However, this was largely due to the LBO, which effectively had no microwave load. Similar caveats apply for the pulse energy results in Fig. 6.6b. The scaling of the efficiency with magnetic field was consistent with expectations based on the results from the plots of the electrical characteristics in Fig. 6.5 and the microwave powers in Fig. 6.1 on page 139. The range of magnetic fields $B \lesssim 0.19 \mathrm{~T}$ corresponded to pulse energies that were approximately $50 \%$ greater than at $B \gtrsim 0.19 \mathrm{~T}$. These results can be interpreted using the results from the pulse-shortening sections in Chapter 5 . In those cases, high cavity RF fields resulted in plasma formation in the microwave cavities. Here, it appears that the relatively low peak powers in $B \lesssim 0.19 \mathrm{~T}$ likely failed to generate 


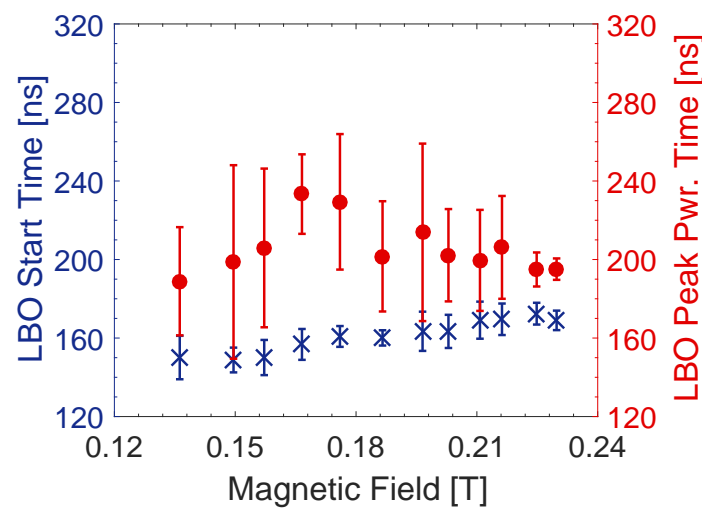

(a) LBO microwave start and peak power times.

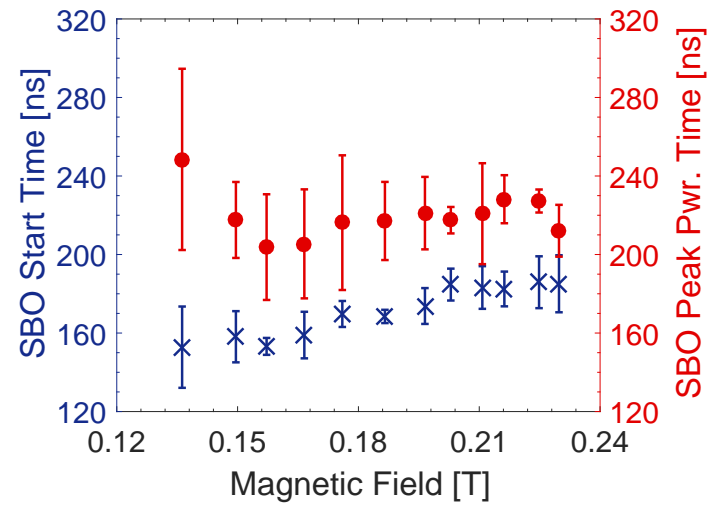

(b) SBO microwave start and peak power times.

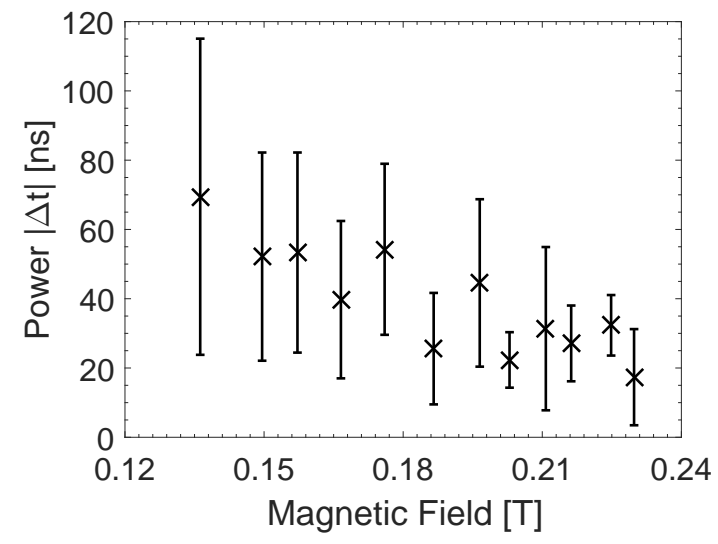

(c) Absolute time difference $|\Delta t|$ between oscillator peak powers vs. magnetic field.

Figure 6.7: Taper Load and Nonuniform Magnetic Field: Temporal metrics vs. magnetic field.

cavity plasma, which acts as a microwave absorber (or a short, depending on the plasma density). In the absence of cavity plasma, normal pulse-shortening due to cathode plasma expansion led to the cessation of constant power production, but oscillations continued to be detected for a long duration due to the high- $Q$ configuration and the absence of a microwave load. An example of these long-duration oscillations with decaying amplitude is shown in the Additional Analysis section. At $B \gtrsim 0.19 \mathrm{~T}$, it is plausible that the cavity plasma rapidly disrupted oscillations and absorbed the microwave energy, which resulted in lower-energy microwave pulses detected by the extraction assembly. This also explains why, in the extreme case at the highest magnetic fields, some shots exhibited LBO operation near $0.980 \mathrm{GHz}$, which is more consistent with normal LBO $\pi$-mode operation based on the results of Chapter 5. A more thorough explanation occurs in the Additional Analysis section, and further clarity was provided by the uniform magnetic field configuration in $\S 6.4$ of this chapter. 
Temporal metrics are shown in Fig. 6.7. The LBO results in Fig. 6.7a were consistent with the previous discussion of the voltages. The start times were consistent due to the delayed disruption by the reflected power, whereas the combination of the nonuniform magnetic field and large reflected power led to inconsistent peak power times. Figure 6.7b shows that the results for the SBO were more consistent, as expected given that the load was not modified. However, like the results for the SBO in Chapter 5, the nonuniform magnetic field led to relatively high shot-to-shot variability. The peak power $|\Delta t|$ for the oscillators is shown in Fig. 6.7c. No range of magnetic fields saw ideal MFRPM operation as a single device, where the peak powers from both oscillators were nearly simultaneous. The time difference between peak powers was routinely $>20 \mathrm{~ns}$, and across the range of tested magnetic fields, shot-to-shot consistency of $|\Delta t|$ was low. The region of optimal operation at the highest magnetic fields corresponded to a lower average $|\Delta t|$, but the results were still variable.

The standard metrics were summarized for two regions of operation. The first was the region near the Buneman-Hartree magnetic field (approximately $0.16 \mathrm{~T}$ ), where the first power peak for the LBO was observed at $0.17 \mathrm{~T}$. That region saw mixed operation by the SBO, with some shots in the $6 \pi / 8$-mode and some shots in the $\pi$-mode. Results for the two modes were analyzed separately. The outcome of the analysis can be found in Table 6.1 on page 133. The second region of operation was the region of optimal operation in $B=0.22-$ $0.23 \mathrm{~T}$, where maximum powers were observed from both oscillators. These results were listed in Table 6.3 on page 134.

\subsubsection{Additional Analysis}

Several observations in the previous section were referenced for additional discussion, including the dual-peak distribution of $6 \pi / 8$-mode frequencies, analysis of $6 \pi / 8$-mode powers, and the high- $Q$ configuration of the taper load leading to long-duration oscillations. This section also analyses the LBO harmonics. The high- $Q$ configuration will be discussed first because it reveals an important effect that must be considered during the analysis of the other observations.

As discussed previously, the waveguide taper load led to reflection of $>99.9 \%$ of the extracted microwave power from the LBO, which produced a high- $Q$ configuration where the extraction waveguide functioned like a high- $Q$ cavity. Figure 6.8 shows an example of the consequences of this configuration. Figure 6.8a was generated using a Hilbert transform of the RF voltage to extract the amplitude envelope, which was then normalized to the maximum and slightly smoothed. As shown by previous shot plots, typical microwave pulse 


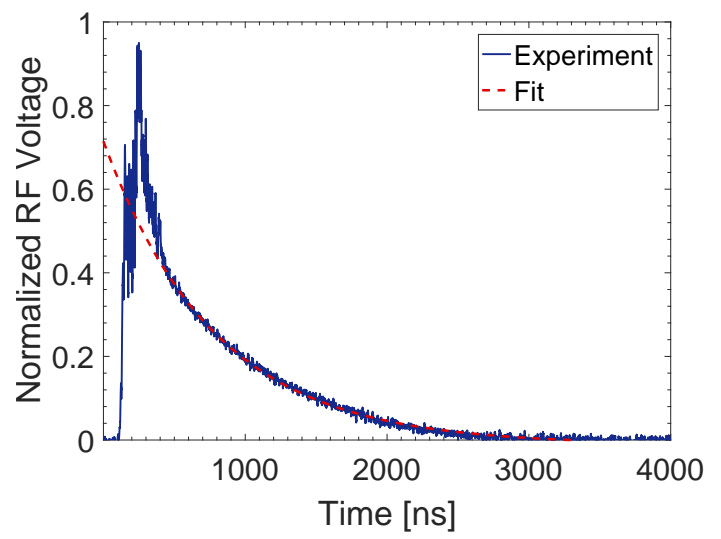

(a) Normalized RF voltage amplitude vs. time for shot 14733 and associated fit to determine the quality factor.

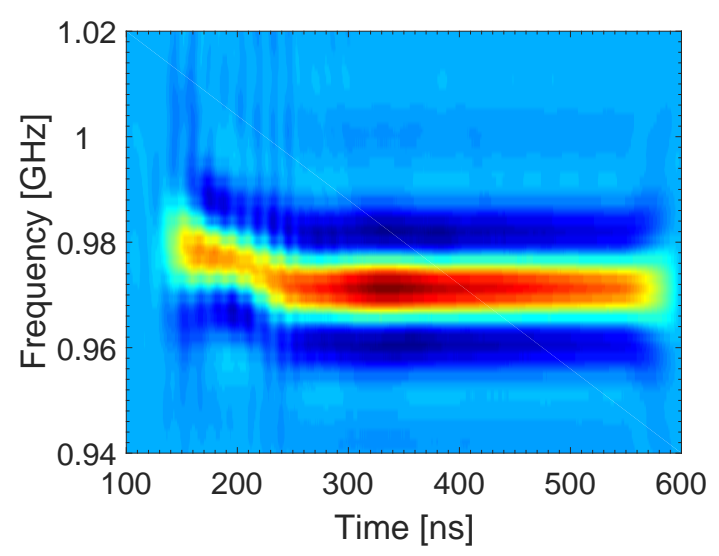

(b) TFA of the LBO RF voltage signal from shot 14733 using the Zhao-Atlas-Marks RIK.

Figure 6.8: Taper Load and Nonuniform Magnetic Field: Effects of the waveguide taper load on LBO operation.

durations were approximately $40 \mathrm{~ns}$. Here, oscillations continue for an extremely long duration, with detectable RF voltage out to nearly $3 \mu \mathrm{s}$. This result clearly illustrates the exponential decay of the oscillation amplitude due to ohmic loss. Assuming $V=V_{0} e^{-\alpha t}$, where $\alpha=2 \pi f_{0} / 2 Q$ and $f_{0}=0.971 \mathrm{GHz}$ from the time-integrated Fourier transform, the $Q$-factor was determined to be 2396 with a fit quality $R^{2}=0.996$. The data used for the fit began at approximately $430 \mathrm{~ns}$. Since most magnetrons use $Q$-factors around 200 or less, this conclusively proves that the waveguide taper configuration is indeed characterized by an extremely high $Q$.

Figure $6.8 \mathrm{~b}$ shows a TFA for the shot. Here, it is clear that the actual $\pi$-mode frequency during microwave production is closer to the $0.980 \mathrm{GHz}$ frequency observed in previous data sets, and that the long-duration oscillations occur at the lower frequency near $0.97 \mathrm{GHz}$. In this shot, crowbar occurred at approximately $350 \mathrm{~ns}$. Note that the TFA appears to indicate that oscillations terminate at $600 \mathrm{~ns}$. This is an artifact of the time window used to generate the plot, which was chosen to reduce TFA computation time; oscillations actually continue much longer. Since the time-integrated FFT was used in the post-processor code written for this dissertation to determine the dominant and competing operating frequencies, the long-duration oscillation frequency appeared as the dominant frequency, so $L B O \pi$-mode shots routinely appeared to have slightly lower frequencies than in reality during the interaction between the electron beam and the $L B O$. This nuanced point must be kept in mind when interpreting the LBO frequencies from the waveguide taper configuration. 


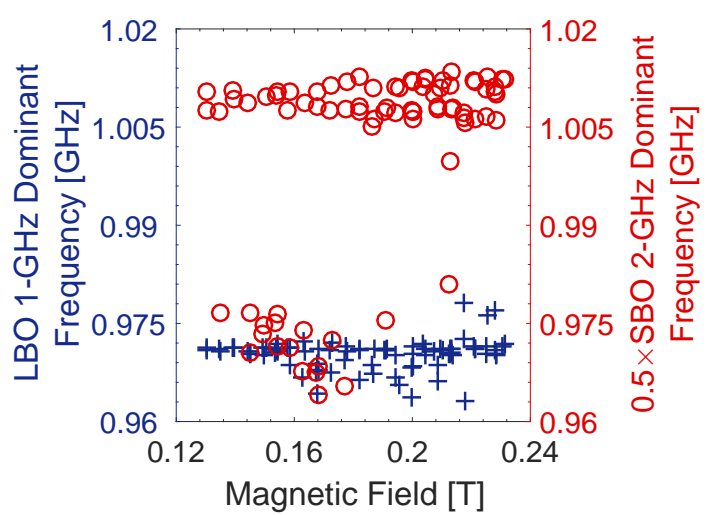

(a) LBO dominant $1 \mathrm{GHz}$ and SBO dominant $0.5 \times 2 \mathrm{GHz}$ frequencies vs. magnetic field.

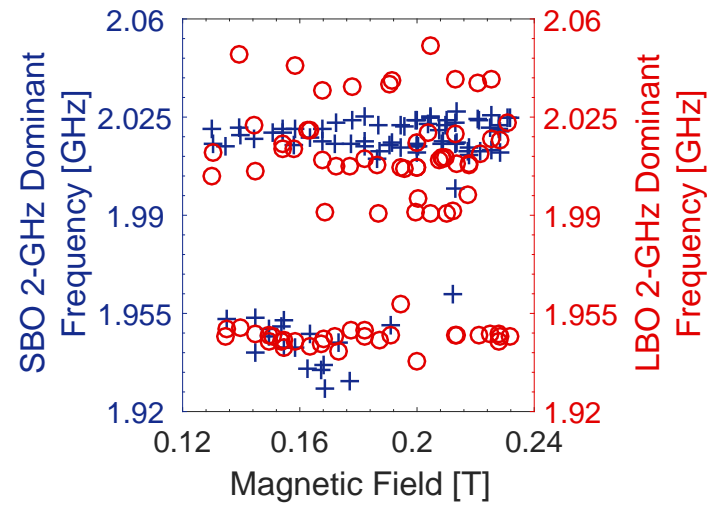

(b) SBO dominant $2 \mathrm{GHz}$ and LBO dominant $2 \mathrm{GHz}$ frequencies vs. magnetic field.

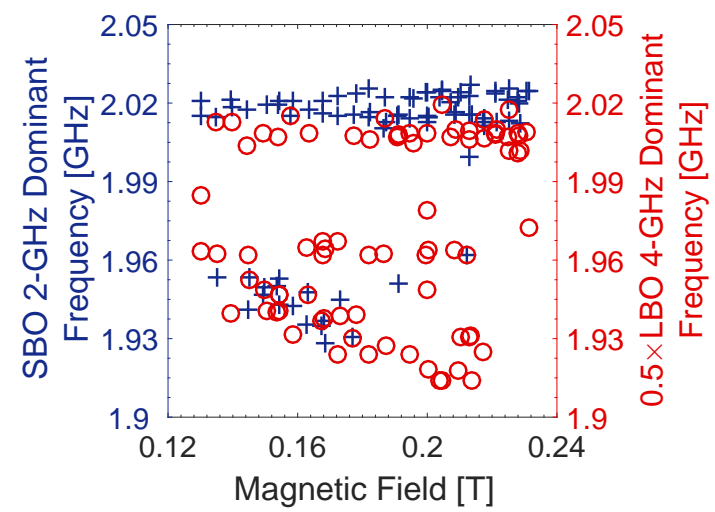

(c) SBO dominant $2 \mathrm{GHz}$ and LBO dominant

$0.5 \times 4 \mathrm{GHz}$ frequencies vs. magnetic field.

Figure 6.9: Taper Load and Nonuniform Magnetic Field: First comparison set of dominant and competing frequencies vs. magnetic field.

Inspection of Fig. $6.3 \mathrm{~b}$ on page 142 revealed that there appeared to be two overlapping frequency distributions for the $6 \pi / 8$-mode, with the higher-frequency distribution near $1.945 \mathrm{GHz}$ appearing to correspond to dominant $6 \pi / 8$-mode operation, and the lowerfrequency distribution near $1.935 \mathrm{GHz}$ appearing to correspond to competing $6 \pi / 8$-mode operation. Determining the relation between dominant and competing fundamental and harmonic frequencies is difficult, but several relations appear to exist for the two different SBO $6 \pi / 8$-mode frequency distributions. As with the fundamental modes, relations between frequencies can be determined using plots of frequency vs. magnetic field, and with frequency histograms that collapse the aforementioned plots onto the vertical axis to determine the frequency distributions. Plots of frequency vs. magnetic field will be discussed first.

Dominant SBO $6 \pi / 8$-mode operation occurred primarily at $B \lesssim 0.17$ T. Figure $6.9 \mathrm{a}$ suggests that this magnetic field range demonstrated dominant $6 \pi / 8$-mode SBO operation 


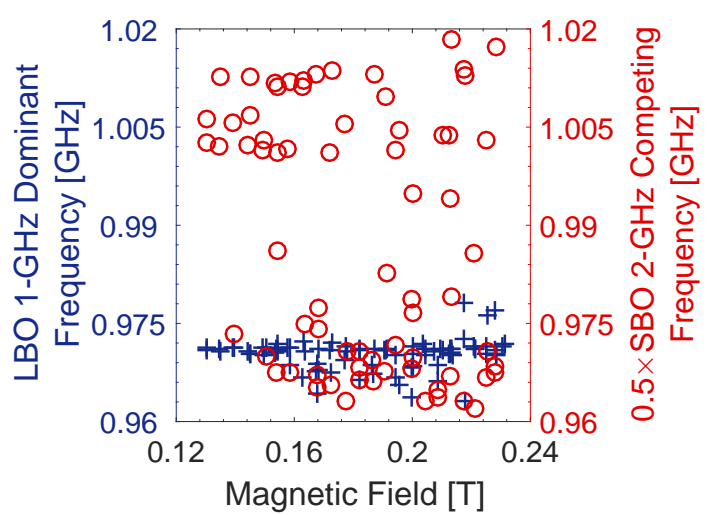

(a) LBO dominant $1 \mathrm{GHz}$ and SBO competing $0.5 \times 2 \mathrm{GHz}$ frequencies vs. magnetic field.

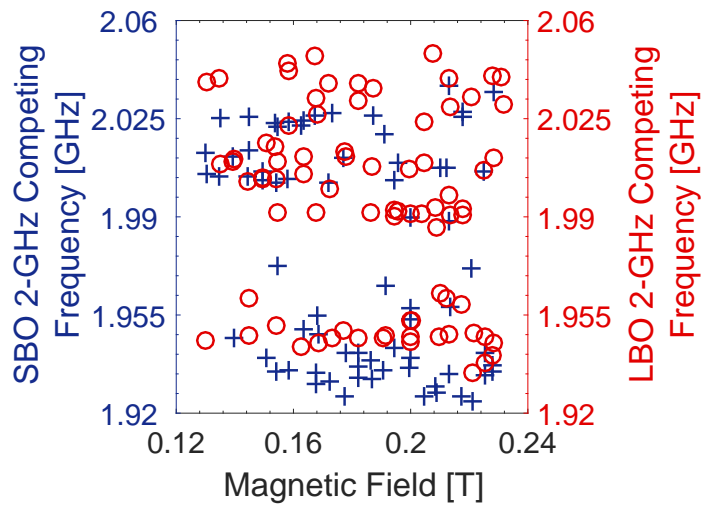

(c) SBO competing $2 \mathrm{GHz}$ and $\mathrm{LBO}$ competing $2 \mathrm{GHz}$ frequencies vs. magnetic field.

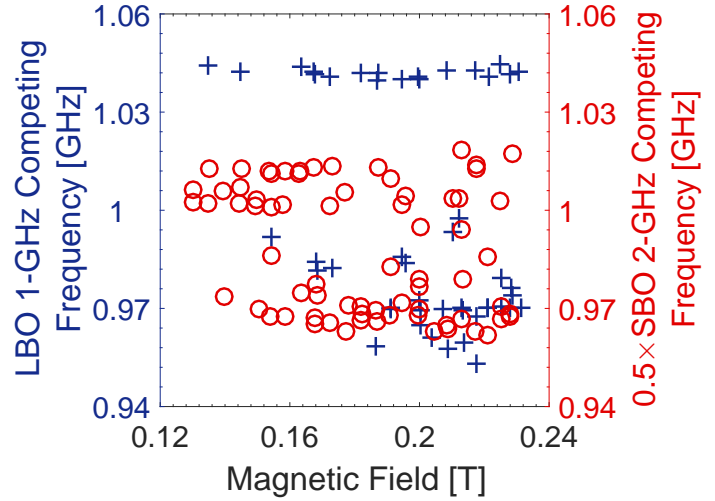

(b) LBO competing $1 \mathrm{GHz}$ and SBO competing $0.5 \times 2 \mathrm{GHz}$ frequencies vs. magnetic field.

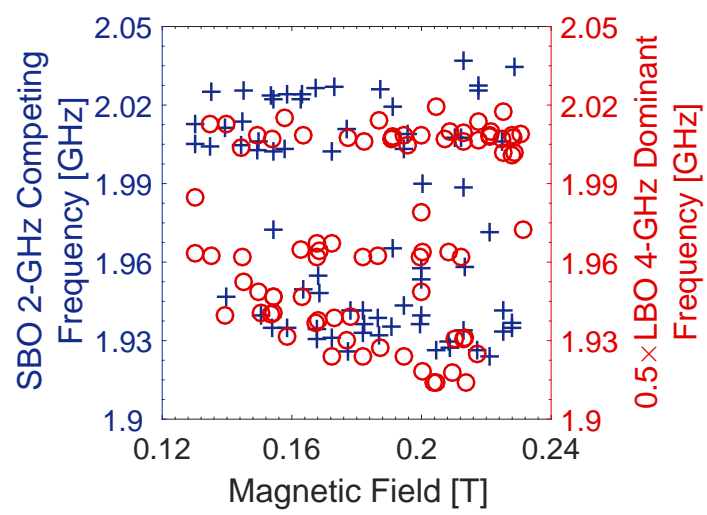

(d) SBO competing $2 \mathrm{GHz}$ and LBO dominant $0.5 \times 4 \mathrm{GHz}$ frequencies vs. magnetic field.

Figure 6.10: Taper Load and Nonuniform Magnetic Field: Second comparison set of dominant and competing frequencies vs. magnetic field.

at a fundamental frequency that was approximately a harmonic of the dominant LBO fundamental frequency. Some correlation was also observed in Figs. 6.9b and 6.9c, which show approximate harmonic relations between the SBO dominant $6 \pi / 8$-mode frequency and both the dominant LBO second and fourth harmonic frequencies, respectively.

At $B \gtrsim 0.17 \mathrm{~T}$, the SBO $6 \pi / 8$-mode manifested as a competing mode because the dominant modes shifted to the $7 \pi / 8$-mode and $\pi$-mode. In this magnetic field range, there also appeared to be several correlating metrics, although these were less compelling than those for the dominant $6 \pi / 8$-mode operation. Figure 6.10 a shows that the harmonic relation between the $6 \pi / 8$-mode and the dominant LBO fundamental frequency continued, though now with the SBO competing $6 \pi / 8$-mode frequency. The average frequency of the correlation in $B \gtrsim 0.17 \mathrm{~T}$ was near $0.966 \mathrm{GHz}$, which was lower than the $0.975 \mathrm{GHz}$ (from $1.95 \mathrm{GHz} / 2$ ) average SBO $6 \pi / 8$-mode correlation frequency in $B \lesssim 0.17 \mathrm{~T}$. As previously discussed, the lower $0.966 \mathrm{GHz}$ LBO frequency may be the $5 \pi / 6$-mode, whereas the 
higher LBO frequency was very likely the $\pi$-mode. Some correlation in $B \gtrsim 0.17 \mathrm{~T}$ was also observed between the competing $6 \pi / 8$-mode and the fundamental LBO competing frequency, as shown by Fig. 6.10b. Correlation with the competing LBO second harmonic in Fig. 6.10c and with the dominant LBO fourth harmonic in Fig. 6.10d was considerably less pronounced. In general, these correlations did not appear to be as strong as those observed for $B \lesssim 0.17 \mathrm{~T}$.

These results can also be interpreted using frequency histograms. From the previous section, Fig. $6.3 \mathrm{~b}$ on page 142 showed that the dominant and competing SBO $6 \pi / 8$-mode frequencies were approximately $1.945 \mathrm{GHz}$ and $1.935 \mathrm{GHz}$, respectively. The fundamental frequency histogram for the LBO, shown by Fig. 6.3a, suggests that the dominant SBO $6 \pi / 8$-mode frequency was, on average, a near harmonic of the most frequently observed fundamental LBO frequency, which was likely the LBO $\pi$-mode. The competing SBO $6 \pi / 8$-mode frequency also appeared to be a harmonic of a fundamental LBO frequency, but at a frequency slightly below the peak in Fig. 6.3a. In previously observed configurations discussed in Chapter 5, those frequencies were usually determined to correspond to the LBO $5 \pi / 6$-mode.

Thus, to summarize, the best explanation for the dual-peak distribution of SBO $6 \pi / 8$ mode frequencies is as follows: 1) The dominant $1.945 \mathrm{GHz}$ mode was observed in a magnetic field range that was sychronous with the $6 \pi / 8$-mode, and arose primarily during LBO $\pi$-mode operation, and 2) the competing $1.935 \mathrm{GHz}$ mode was observed in a magnetic field range that was not synchronous with the $6 \pi / 8$-mode, and arose primarily during LBO $5 \pi / 6$-mode operation. Based on the results of Chapter 5 , it is plausible that the mechanism for the coupling between the LBO modes and the SBO $6 \pi / 8$-mode was the LBO-modulated electron beam, and that it continued to affect the SBO $6 \pi / 8$-mode even when the $6 \pi / 8$ mode was not synchronous with the electron beam.

Figure 6.12 on page 153 (shown later during the harmonic analysis) shows the frequency histograms for the LBO second and fourth harmonics. Comparison with Fig. 6.3b on page 142 suggests the dominant SBO $6 \pi / 8$-mode was equal to the most frequently observed frequency for the LBO second harmonic, whereas the competing SBO $6 \pi / 8$-mode frequency does not appear to correspond to any LBO second harmonic histogram peaks. This reinforces the observation of a lack of compelling correlation between the competing SBO $6 \pi / 8$-mode and the quantities in Figs. 6.10c and 6.10d. Finally, both dominant and competing SBO $6 \pi / 8$-mode frequencies have corresponding points on the LBO fourth harmonic histogram in Fig. 6.12b, though distinct distributions with corresponding peaks were not observed due to the relatively large spread of frequencies for the LBO fourth harmonic.

These data suggest some harmonic frequency-locking was evident between the LBO $\pi$ - 
mode and SBO $6 \pi / 8$-mode, and that the locking was also observed through measurement of the LBO harmonic frequencies. While precise identification of the LBO $5 \pi / 6$-mode frequency was infeasible, it seems likely that some LBO $5 \pi / 6$-mode competition occurred at $B \gtrsim 0.17 \mathrm{~T}$. Both the LBO $5 \pi / 6$-mode and $\pi$-mode appear to have excited the SBO $6 \pi / 8$-mode at a frequency off its natural resonance, and frequency-locking occasionally occurred as a result. The strongest interaction between the LBO and SBO occurred at $B \lesssim$ $0.17 \mathrm{~T}$, where nearly pure LBO $\pi$-mode was observed alongside instances of dominant SBO $6 \pi / 8$-mode operation. Thus, the overlapping frequency distributions of the SBO $6 \pi / 8$ mode appear to be a consequence of SBO excitation at a frequency off of the natural SBO $6 \pi / 8$-mode resonance by two different LBO modes. As stated earlier, these observations appear to be consistent with the interpretations and conclusions of Chapter 5 . At $B \lesssim$ $0.17 \mathrm{~T}$, the dominant SBO $6 \pi / 8$-mode shots were analyzed to determine a representative power and frequency. The results were shown in Table 6.5 on page 134 .

The final portion of this additional analysis section analyzes the LBO frequency harmonics. Figure 6.11 shows the extracted microwave powers for the second and fourth harmonics vs. magnetic field. Unlike plots for the fundamental frequencies, error bars have been omitted to better illustrate the distinct jumps between regions of operation and spread of results. Comparison with Fig. 6.1 on page 139 reveals several observations corresponding to distinct regions of operation. First, the region for $B \gtrsim 0.17 \mathrm{~T}$ was characterized by consistent LBO $\pi$-mode operation with a local power maximum at $B \simeq 0.17 \mathrm{~T}$, and the SBO was characterized by roughly equal numbers of shots exhibiting dominant operation in the $6 \pi / 8$-mode and $\pi$-mode, with some $7 \pi / 8$-mode competition. In this region, LBO second harmonic powers were consistent and relatively low, typically $<400 \mathrm{~kW}$. The LBO fourth harmonic was comparatively less consistent, exhibiting a large relative spread of powers that were approximately $5 \mathrm{~kW}$ on average.

The next region, $0.17 \lesssim B \lesssim 0.19 \mathrm{~T}$, appeared to be one of transition. Here, the LBO second harmonic began to exhibit powers approximately four times greater than in $B \lesssim 0.17 \mathrm{~T}$, and the LBO fourth harmonic exhibited peak powers as high as $18 \mathrm{~kW}$. This region was associated with the first observations of lower LBO fundamental frequencies, which were possibly due to the $5 \pi / 6$-mode, and the transition by the SBO from $6 \pi / 8$-mode and $\pi$-mode operation to competition between the $7 \pi / 8$-mode and $\pi$-mode.

In $0.19 \lesssim B \lesssim 0.22 \mathrm{~T}$, maximum LBO second harmonic power was observed near 1.3 MW, though there was considerable spread in the extracted powers. This region was also associated with a local minimum in LBO power at the fundamental frequency, as shown by Fig. 6.1 on page 139. LBO fourth harmonic powers consistently decreased as $B$ was increased. 


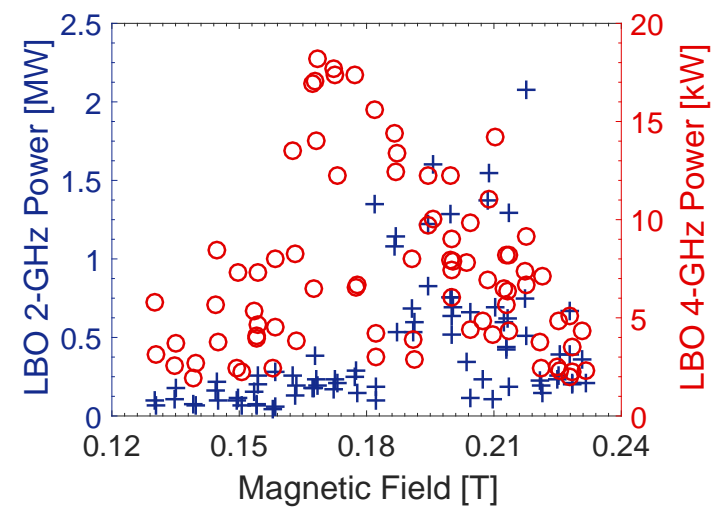

Figure 6.11: Taper Load and Nonuniform Magnetic Field: Peak LBO output powers at harmonic frequencies vs. magnetic field.

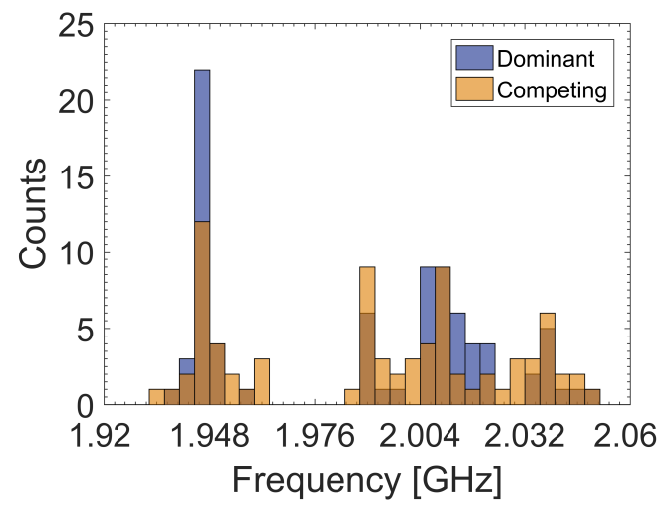

(a) LBO frequency histogram near $2 \mathrm{GHz}$.

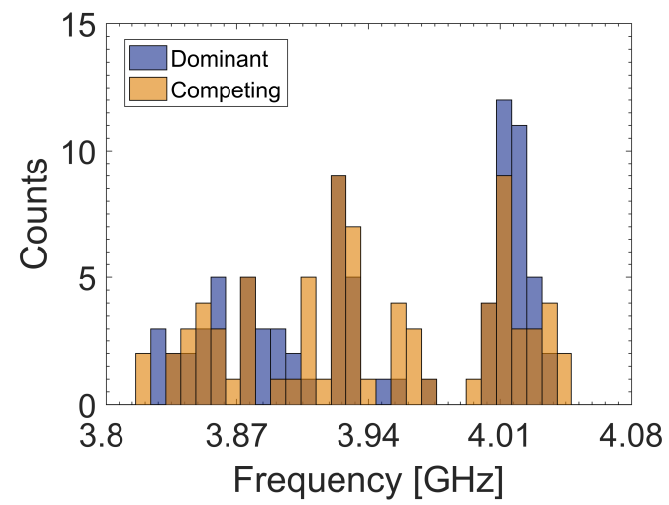

(b) LBO frequency histogram near $4 \mathrm{GHz}$.

Figure 6.12: Taper Load and Nonuniform Magnetic Field: Dominant and competing LBO harmonic frequency histograms.

The final region corresponded to $B \gtrsim 0.22 \mathrm{~T}$, which was the highest tested range. Here, powers at the fundamental frequencies from both oscillators were maximized, and powers for both LBO harmonics were minimized. High-power operation by the LBO appeared to correlate with relatively low harmonic power generation, whereas regions of transition were associated with higher harmonic powers.

Figure 6.12 shows the frequency histograms for the LBO harmonics. The LBO second harmonic frequencies will be discussed first. The largest LBO second harmonic frequency peak at $1.946 \mathrm{GHz}$ in Fig. 6.12a correlates best as a near-harmonic of the $0.972 \mathrm{GHz} \mathrm{LBO}$ fundamental frequency. Another near-harmonic correlation exists in Fig. 6.12b for the fourth harmonic at $3.892 \mathrm{GHz}$, although the distribution of frequencies was broader and the number of counts relatively few. As previously discussed, this $1.946 \mathrm{GHz}$ second harmonic 


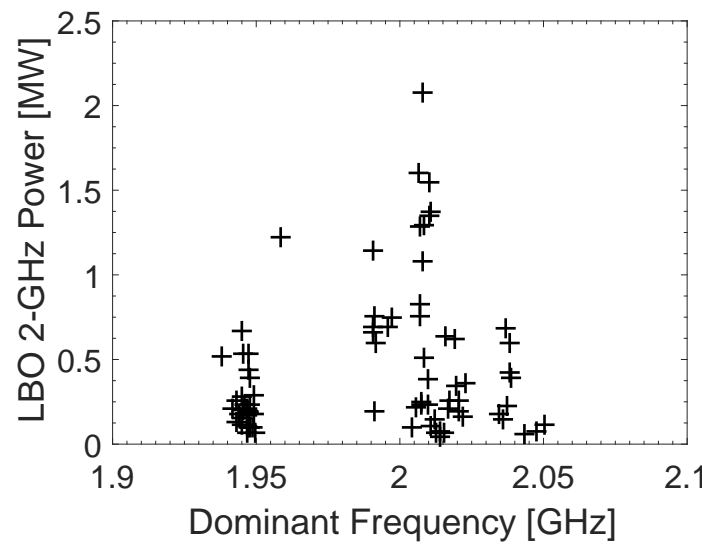

(a) LBO $2 \mathrm{GHz}$ harmonic power vs. dominant operating frequency.

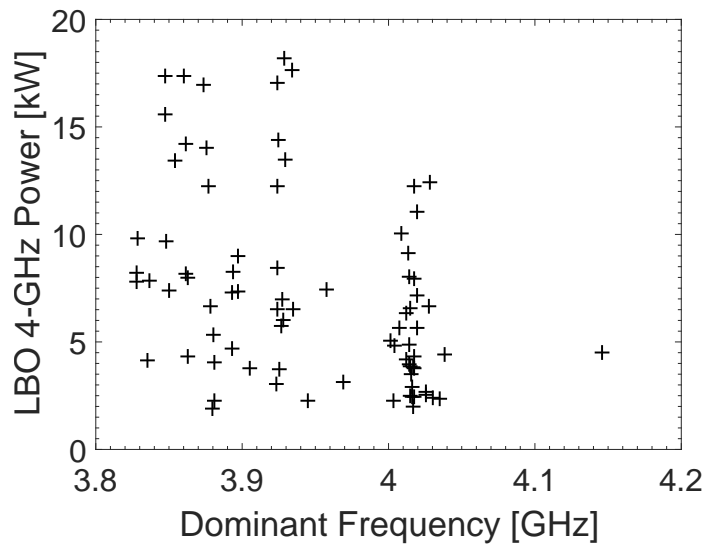

(b) $\mathrm{LBO} 4 \mathrm{GHz}$ harmonic power vs. dominant operating frequency.

Figure 6.13: Taper Load and Nonuniform Magnetic Field: Peak LBO harmonic powers vs. dominant operating frequencies.

frequency was also nearly identical to the SBO $6 \pi / 8$-mode when the $6 \pi / 8$-mode was the dominant operating SBO mode.

The other notable distribution of LBO second harmonic frequencies occurred near $2.008 \mathrm{GHz}$. This distribution appears to be half the $4.016 \mathrm{GHz}$ frequency of the peak LBO fourth harmonic frequency distribution in Fig. 6.12b. The distribution also appears to correlate with instances of dominant SBO $\pi$-mode operation in Fig. $6.3 \mathrm{~b}$ on page 142. A corresponding peak was not observed in the distribution of fundamental LBO frequencies in Fig. 6.3a, which suggests both the $2.008 \mathrm{GHz}$ and $4.016 \mathrm{GHz}$ peaks in the LBO second and fourth harmonic histograms were the result of excitation by the $S B O \pi$-mode. This reinforces the previous discussion of Figs. $6.9 \mathrm{~b}$ and $6.9 \mathrm{c}$ on page 149. Analogous to the results discussed at length in Chapter 5, it is possible that oscillations on the LBO at the fundamental and second harmonic frequencies of the $S B O$ were driven by the SBO-modulated beam exiting the SBO and entering the LBO.

The LBO fourth harmonic peak near $3.924 \mathrm{GHz}$ does not appear to correlate with any other modes, and therefore is likely a competing mode supported by the structure. Cold simulations conducted using HFSS suggested many such modes exist, as do the cold tests discussed in $\S$ 4.1.3. Finally, a distribution of LBO fourth harmonic frequencies near $3.852 \mathrm{GHz}$ approximately correlates with frequencies for the $5 \pi / 6$-mode, although no corresponding peak exists for the second harmonic.

Figure 6.13 shows power vs. dominant frequency for the LBO harmonics. The powers for the second harmonic, shown by Fig. 6.13a, conclusively illustrate that the second harmonic of the LBO $\pi$-mode frequency was not associated with high harmonic power 
generation. Instead, the powers generated at $2.008 \mathrm{GHz}$ were observed to be the highest. As previously discussed, this frequency appears to be strongly correlated with SBO operation, though it cannot be stated conclusively whether this was due to the $7 \pi / 8$-mode or the $\pi$-mode because of the minimal frequency separation between the modes.

Figure $6.13 \mathrm{~b}$ shows the fourth harmonic powers for the LBO. Maximum power appeared to be generated by modes near $3.860 \mathrm{GHz}$ and $3.924 \mathrm{GHz}$. The former frequency is most nearly associated with the $5 \pi / 6$-mode, and the latter with the competing mode that relates with neither the LBO fundamental frequency, nor the second harmonic frequency, nor a harmonic of the SBO.

Figures F.1, F.2, and F.3 in the appendix show all the plot sets comparing dominant and competing fundamental and harmonic frequencies for both oscillators. They were included for completeness, and the aforementioned conclusions were drawn from a combination of these figures and the previously discussed histograms and other harmonic metrics. Most plots were used to confirm the absence of any meaningful integer-multiple correlation between different frequencies. Note that the plots omit SBO harmonics because they did not appear to have been generated at detectable power levels. Also omitted were plots including the competing LBO fourth harmonic because, from the histogram in Fig. 6.12b, the competing frequencies were generally noisy and spread over a broad frequency range, whereas the dominant frequencies were more meaningful. Generally, the trends and patterns exhibited in Figs. F.1, F.2, and F.3 were noisier and less compelling than the results of Chapter 5.

To remain consistent with the analysis of the Standard Metrics section, characterization of the LBO harmonics was performed for the previously identified regions of operation described in that section. The results are shown in Tables 6.2 and 6.4 on page 134 .

To summarize the behavior analyzed by this section, 1) the LBO $\pi$-mode (and possibly also the $5 \pi / 6$-mode) interacted with the SBO $6 \pi / 8$-mode, 2) the high- $Q$ LBO load configuration and the time-integrated FFT used in the post-processor led to misleadingly low LBO frequencies, 3) high-power operation of the LBO appeared to correlate with lower LBO harmonic powers, and 4) during high power SBO $\pi$-mode operation, the LBO second and fourth harmonic appear to be affected by the SBO.

\subsection{MFRPM with Taper Load and Uniform Magnetic Field}

This shot series tested the MFRPM using the uniform magnetic field described in $\S 4.2 .2$. The purpose was to obtain the second of two data sets necessary to determine how the magnetic field variation along the planar cavity arrays impacted operation of the LBO 
harmonics. A total of 47 shots were obtained with one crowbar failure on the sixth shot. The base pressure was $5 \mu$ torr, and the range of tested magnetic fields was $0.14-0.26 \mathrm{~T}$.

\subsubsection{Standard Metrics}

Figure 6.14 shows the peak output powers from both oscillators vs. magnetic field. At $B \lesssim 0.17 \mathrm{~T}$, the LBO results were comparable to those in Fig. 5.31 on page 106 in Chapter 5 for the standard load and uniform magnetic field, although the LBO powers were higher in the present data set due to the high- $Q$ waveguide taper configuration. Relative to the LBO microwave powers for the nonuniform magnetic field and waveguide taper load in Fig. 6.1 on page 139 in the previous section, the LBO powers were uniformly higher across all tested magnetic fields (with the exception of the highest tested magnetic field in the nonuniform field configuration). Performance of the SBO was also comparable, which was expected because neither the magnetic field nor the microwave extractor were modified in this configuration relative to the analogous configuration in Chapter 5. For both oscillators, the power smoothly increased from the lowest magnetic field tested near $0.14 \mathrm{~T}$ to a magnetic field near $0.17 \mathrm{~T}$, which was slightly greater than the Hartree magnetic field $B_{\mathrm{BH}}=0.16 \mathrm{~T}$. Near $B \simeq 0.17 \mathrm{~T}$, power from both oscillators was maximized, which is in good agreement with the analytic model from $\S 2.2$. The region of optimal operation therefore lay at $0.17 \mathrm{~T}$. In $B \gtrsim 0.18 \mathrm{~T}$, neither oscillator exhibited much shot-to-shot consistency in extracted microwave powers, although the LBO did appear to settle near a steady average power near $37 \mathrm{MW}$. The field $B \simeq 0.18 \mathrm{~T}$ therefore represented a point of transition from a region of consistent, predictable operation, to a region of reduced microwave powers and greater operational variability.

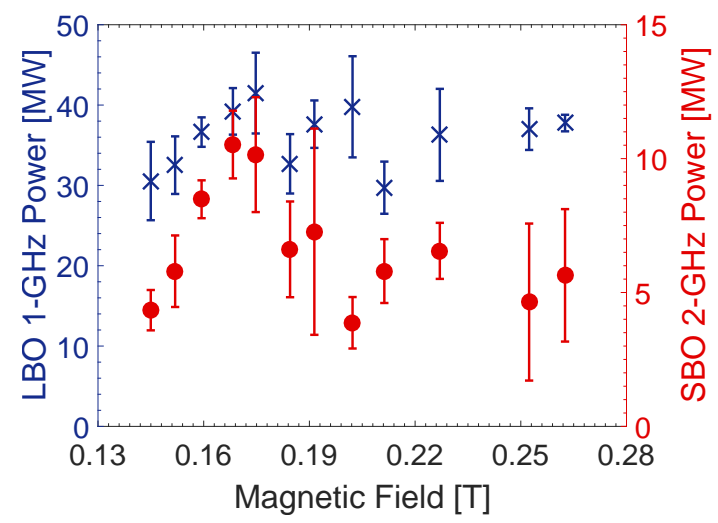

Figure 6.14: Taper Load and Uniform Magnetic Field: Peak output powers for both oscillators vs. magnetic field. 


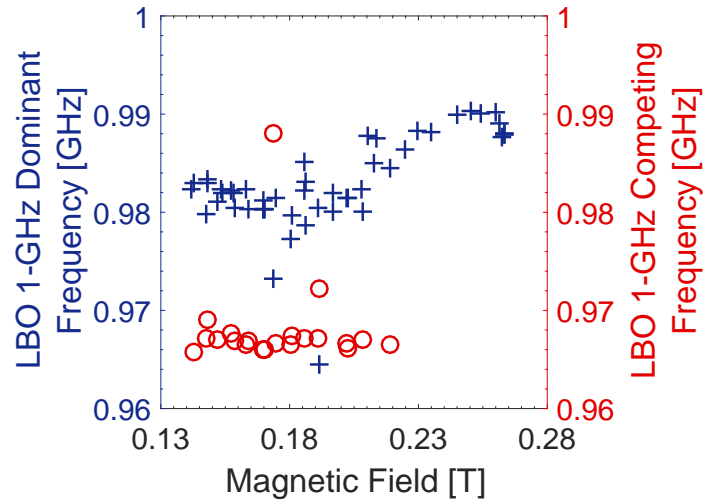

(a) LBO frequencies near $1 \mathrm{GHz}$ vs. magnetic field.

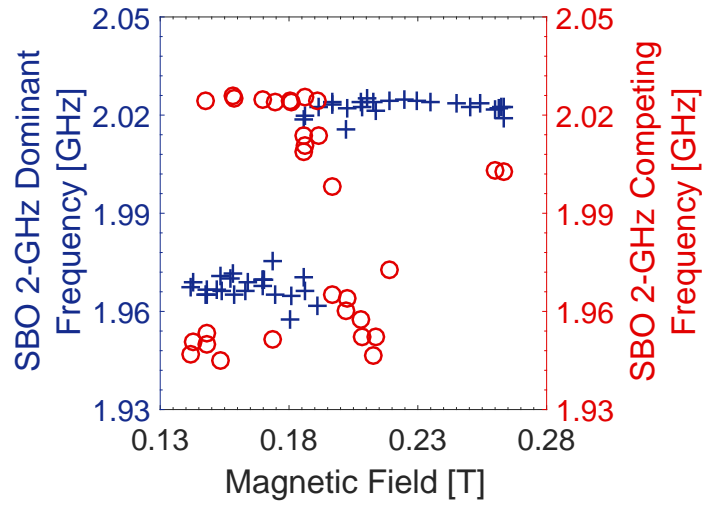

(b) SBO frequencies vs. magnetic field.

Figure 6.15: Taper Load and Uniform Magnetic Field: Dominant and competing frequencies of both oscillators vs. magnetic field.

Figure 6.15 illustrates the frequencies vs. magnetic field for the oscillators. The LBO results in Fig. 6.15a show relatively consistent operation in $B \lesssim 0.20 \mathrm{~T}$. In this region, dominant operation was consistently near $0.98 \mathrm{GHz}$, with a minor decrease as the magnetic field was increased. The competing frequency was consistently near $0.967 \mathrm{GHz}$. Given the previous experimental discussion of Chapter 5 and the simulation results discussed in Chapter 5, the $0.98 \mathrm{GHz}$ and $0.967 \mathrm{GHz}$ modes were likely the $\pi$-mode and $5 \pi / 6$-mode, respectively. At $B \gtrsim 0.2 \mathrm{~T}$, the $\pi$-mode frequency increased to a maximum near $0.99 \mathrm{GHz}$. This may be explained by beam-loading because the trend approximately follows the currents shown by Fig. 6.18a. From the discussion in $\S 2.1$, a decrease in current (which occurs as the magnetic field is increased) should result in an increase in the operating frequency [6].

The results for the SBO are shown in Fig. 6.15b. The plot reinforces the observations from Fig. 6.14 in that $B \simeq 0.18 \mathrm{~T}$ represented a point of operational transition. In $B \lesssim 0.18 \mathrm{~T}$, the dominant operating frequency was consistently near $1.97 \mathrm{GHz}$. Some competition was observed near $1.95 \mathrm{GHz}$ at the lowest magnetic fields, and near $2.02 \mathrm{GHz}$ closer to $B \simeq 0.18 \mathrm{~T}$. In $B \gtrsim 0.18 \mathrm{~T}$, the dominant operating frequency was consistently near $2.02 \mathrm{GHz}$. From the discussion of Chapter 5, the best explanation is that the 1.95 GHz, $1.97 \mathrm{GHz}$, and $2.02 \mathrm{GHz}$ modes correspond to the free-running 6 6 /8-mode, harmonic frequency-locked $6 \pi / 8$-mode, and $\pi$-mode, respectively. Here, free-running is used to distinguish the operating frequency of the unlocked mode from the harmonic frequencylocked mode, wherein the operating frequency of the SBO was a harmonic of the operating LBO frequency. The observation of harmonic frequency-locked operation between the SBO and LBO will be discussed in greater detail in the Additional Analysis section. Given 
the results from Chapter 5, the observation of harmonic frequency-locked operation was not surprising because the magnetic field and microwave load were not changed for the SBO, and the addition of the waveguide taper load to the LBO appeared to only increase the detected RF power with relatively little impact on the operating frequency.

It is interesting to note that the waveguide taper load had a substantial impact on the LBO frequency in the previous section for the nonuniform magnetic field, which was not the case for the uniform magnetic field. One possible explanation is the formation of cavity plasma at high microwave powers. A comparison with the results of the previous section using the nonuniform magnetic field yields two observations. The first is that, as previously mentioned, the performance of the LBO using the uniform magnetic field was, with the exception of the highest magnetic fields, consistently higher-power than the performance of the LBO using the nonuniform magnetic field. The second observation is that, as discussed in the previous section, the distinct regions of pulse energies in Fig. 6.6b on page 145 likely corresponded to two different mechanisms that were responsible for the microwave pulse-shortening, depending on the magnetic field. Those results suggested cavity plasma formation occurred at the highest powers observed in that configuration. Since the powers in the present configuration are as high or higher than those that possibly produced cavity plasma in the previous configuration, it is plausible that cavity plasma formed consistently in the present configuration as well.

The presence of cavity plasma may explain why the present configuration did not exhibit the same frequency-stabilizing effect that was observed in the previous data set obtained using the nonuniform magnetic field. In that configuration, the powers at lower magnetic fields were hypothesized to be too low to form cavity plasma, which would allow RF to continue propagating between the slow-wave structure and the WR-650 to WR-340 waveguide taper because the only mechanism to dissipate power would be ohmic losses, even if cathode plasma expansion disrupted interaction between the electron beam and the fields on the slow-wave structure. This would lead to the effect shown by Fig. 6.8 on page 148 , where the LBO frequency was close to the normal $0.980 \mathrm{GHz}$ frequency during the interaction between the SWS and the electron beam, and close to the reduced $0.970 \mathrm{GHz}$ frequency during the long-duration decay of oscillations. However, when cavity plasma is present, the plasma may act as a microwave absorber. Some or all of the power reflected back to the slow-wave structure could be absorbed. If this was the case, any shift in frequency due to the waveguide taper load functioning as a high- $Q$ cavity might be negated by the rapid power absorption due to cavity plasma. This is precisely the effect observed in the TFA shown by Fig. $6.8 \mathrm{~b}$ on page 148. Thus, cavity plasma formation, which occurs at high microwave powers, may be the origin of the discrepancy between the observed 


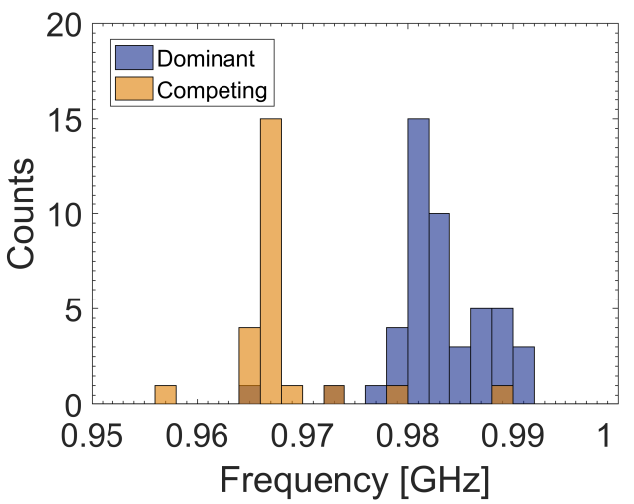

(a) LBO frequency histogram near $1 \mathrm{GHz}$.

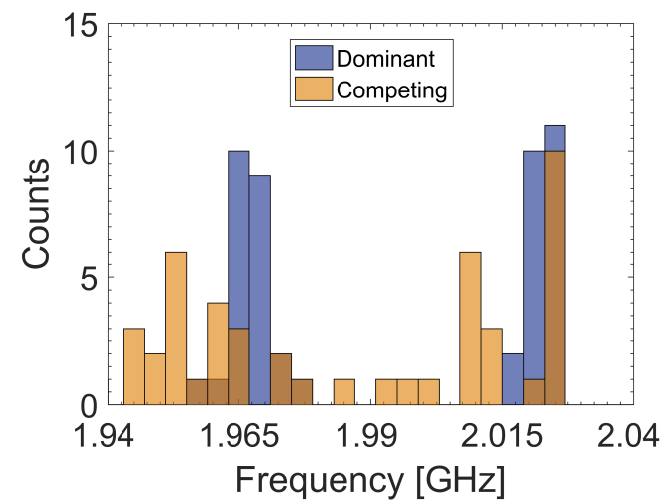

(b) SBO frequency histogram.

Figure 6.16: Taper Load and Uniform Magnetic Field: Dominant and competing frequency histograms for both oscillators.

LBO frequencies in the data sets for the uniform and nonuniform magnetic fields. Plasma formation due to air breakdown in the waveguide at the taper may also be responsible for this behavior, although no diagnostics were present to confirm or eliminate this possibility. Since the formation of cavity plasma was confirmed to occur based on the pulse-shortening imaging analysis in $\S 5.8$, it remains the best explanation.

Histograms for the fundamental frequencies of both oscillators are shown in Fig. 6.16. The LBO results in Fig. 6.16a confirm the impressions from Fig. 6.15a, with distinct peaks occurring at $5 \pi / 6$-mode and $\pi$-mode frequencies, with the former and latter manifesting as competing and dominant modes, respectively. The apparent dual-peak distribution for the $\pi$-mode at approximately $0.980 \mathrm{GHz}$ and $0.988 \mathrm{GHz}$ was due to the gradual increase in the $\pi$-mode frequency as the magnetic field was increased. Figure 6.15a showed that this frequency increase stabilized at high magnetic fields, likely because the drawn current in Fig. 6.18a also stabilized. This resulted in several shots oscillating consistently at the higher $0.988 \mathrm{GHz}$ frequency, thereby producing the second, smaller $\pi$-mode frequency peak in the LBO frequency histogram.

Figure $6.16 \mathrm{~b}$ shows the frequency histogram for the SBO. The histogram visualization reveals the presence of competing frequencies near $2 \mathrm{GHz}$, which was not immediately apparent from Fig. 6.15b. Based on the results and discussion in Chapter 5, this was likely the $7 \pi / 8$-mode. The dominant peaks were near $2.02 \mathrm{GHz}$ (the $\pi$-mode) and $1.97 \mathrm{GHz}$ (the harmonic frequency-locked $6 \pi / 8$-mode), as expected from Fig. 6.15b. An additional set of competing frequencies appeared to be distributed near $1.95 \mathrm{GHz}$, likely corresponding to the free-running $6 \pi / 8$-mode. This conclusion is based on the relatively good agreement between the experimental results and simulations discussed in detail in $\S 5.4 .3$ of Chapter 5 . 


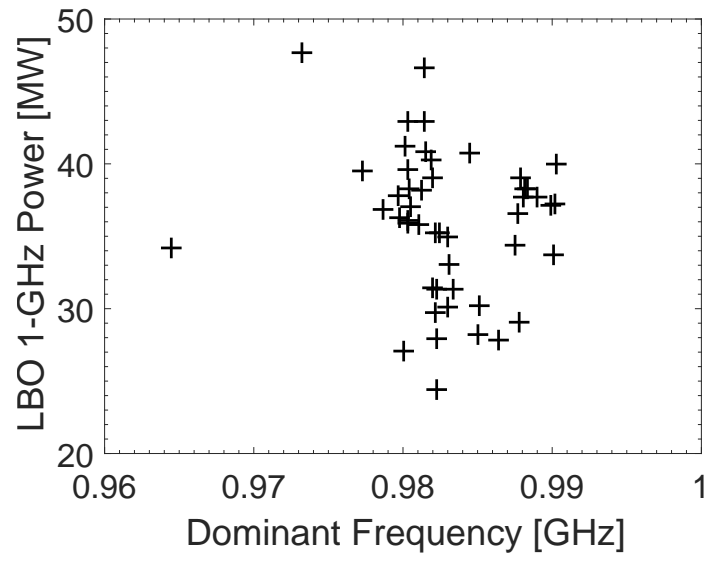

(a) Peak LBO power vs. dominant operating frequency.

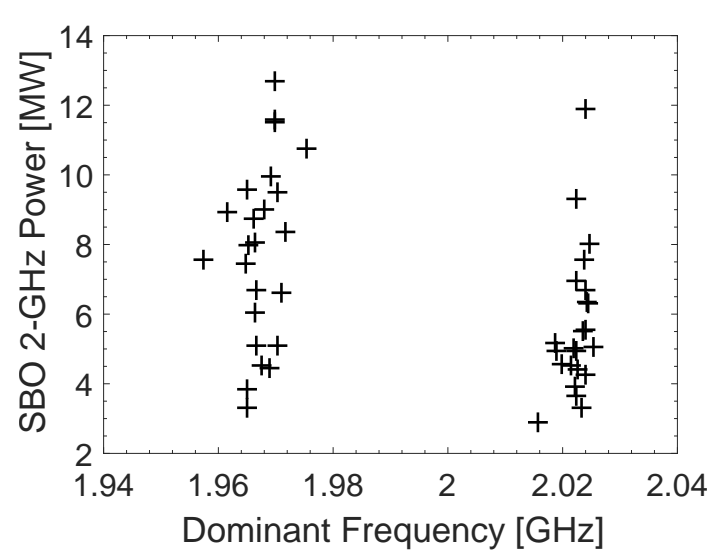

(b) Peak SBO power vs. dominant operating frequency.

Figure 6.17: Taper Load and Uniform Magnetic Field: Peak oscillator powers vs. dominant operating frequencies.

The relation between the microwave powers and the dominant operating frequencies is shown in Fig. 6.17. The LBO results in Fig. 6.17a reveal that the highest powers were associated with the $0.98 \mathrm{GHz}$ operating frequency observed in the harmonic frequency-locked region, and that the second highest powers were observed near $0.988 \mathrm{GHz}$ at the higher range of tested magnetic fields. The set of shots that transitioned from the lower $0.98 \mathrm{GHz}$ to the higher $0.988 \mathrm{GHz}$ were associated with the lowest observed powers, which suggests these two frequencies corresponded to distinct operating states from the perspective of the LBO, with one occurring when harmonic frequency-locked operation with the SBO was observed, and the other occurring when no harmonic frequency-locking was observed.

The SBO results in Fig. 6.17b confirm that the highest SBO powers were observed near $1.97 \mathrm{GHz}$, which corresponds to the harmonic frequency-locked $6 \pi / 8$-mode. The remaining shots occurred near $2.02 \mathrm{GHz}$, which corresponds to the $\pi$-mode. These were associated with lower microwave powers.

Electrical characteristics are shown in Fig. 6.18. As previously referenced, the currents in Fig. 6.18a were crudely constant in the range of $B \lesssim 0.20 \mathrm{~T}$, with an average near $1.8 \mathrm{kA}$. The current then decreased steadily with increasing magnetic field before stabilizing at a lower value of $1.5 \mathrm{kA}$. This behavior mimics the trends observed in the LBO $\pi$-mode frequency observed in Fig. 6.15a, which suggests part of the shift in the LBO frequency may be due to beam-loading, which is also termed frequency-pushing [6].

Impedance vs. magnetic field is shown by Fig. 6.18b. Unlike the currents in Fig. 6.18a, the increase in impedance with magnetic field was fairly linear. This necessarily implies that the voltage at peak power, which will be discussed shortly, must scale differently to 


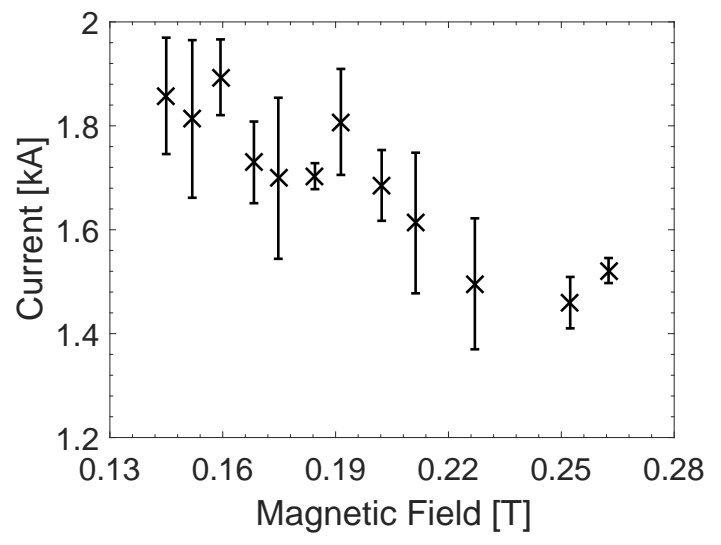

(a) Current at peak power vs. magnetic field.

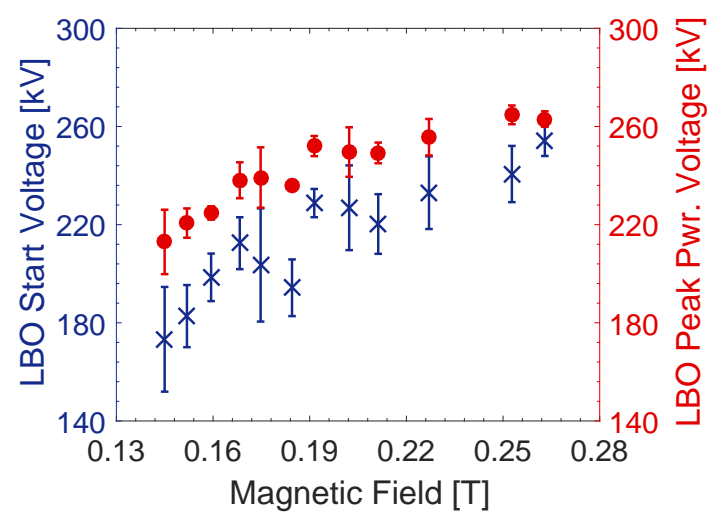

(c) Voltage at time of LBO start and peak power.

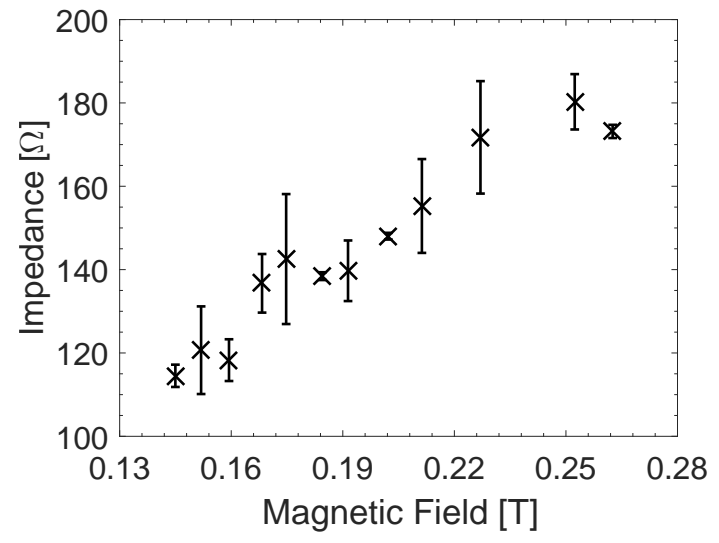

(b) Impedance at peak power vs. magnetic field.

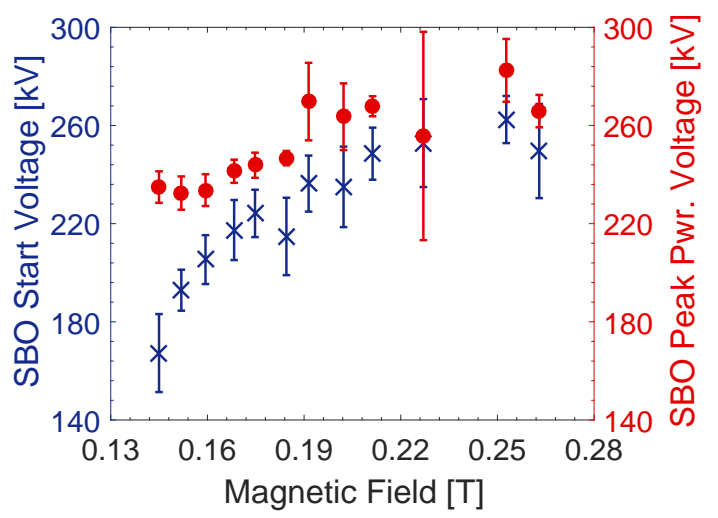

(d) Voltage at time of SBO start and peak power.

Figure 6.18: Taper Load and Uniform Magnetic Field: Electrical characteristics vs. magnetic field.

reconcile the differences between the plots of impedances and currents. The impedances in $B \lesssim 0.18 \mathrm{~T}$, wherein harmonic frequency-locked operation was observed, were wellmatched to the 100-150 $\Omega$ MELBA-C specifications [98].

Start and peak power voltages for the LBO are shown by Fig. 6.18c. The peak power voltages clearly show a distinct change in linear scaling near $B \simeq 0.18 \mathrm{~T}$, which was previously identified to be a point of transition. A similar conclusion results from inspection of the start voltages. As discussed in Chapter 5, a linear scaling in operating voltage with magnetic field implies a constant, synchronous beam velocity favored by the operating electromagnetic mode. Changes in this scaling suggest a change in operation or delayed operation on the flat-top portion of the voltage pulse. However, the peak power voltages were not high enough to occur during the voltage flat-top. This fact, plus the aforementioned evidence from the other metrics, suggests that the operating characteristics of the harmonic frequency-locked LBO $\pi$-mode were distinct from the free-running $\pi$-mode characteristics 


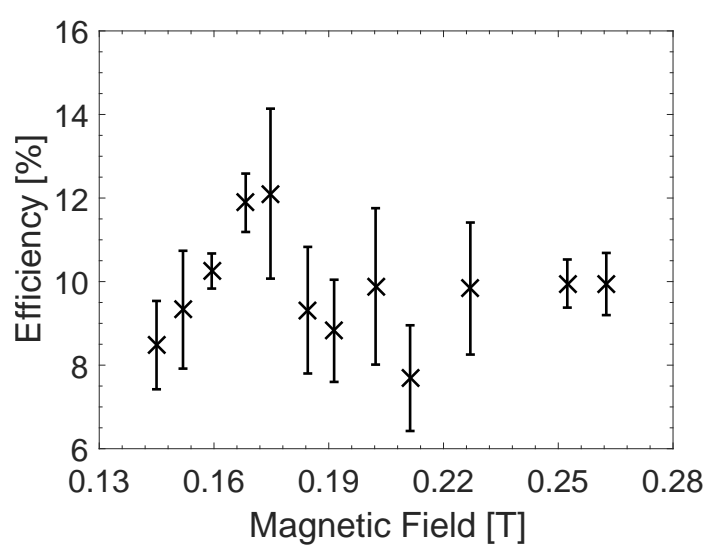

(a) Peak total efficiency vs. magnetic field.

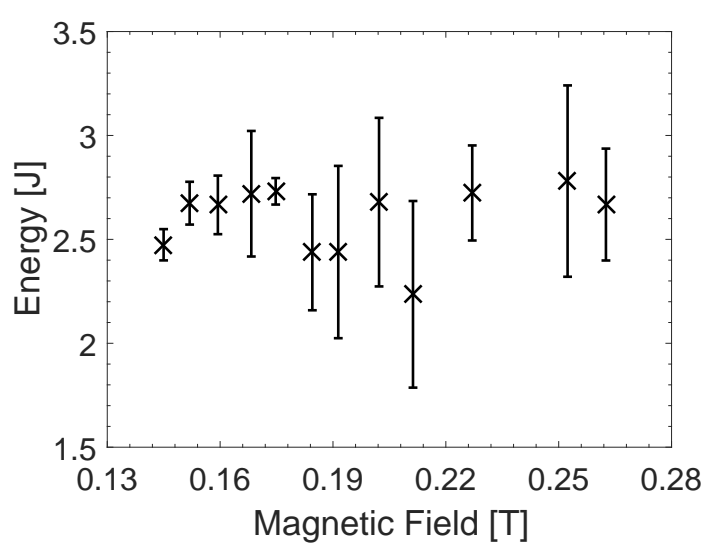

(b) Total pulse energy vs. magnetic field.

Figure 6.19: Taper Load and Uniform Magnetic Field: Energy conversion metrics vs. magnetic field.

despite being the same electromagnetic mode.

Start and peak power voltages for the SBO are shown by Fig. 6.18d. The distinct operating regions separated by $B \simeq 0.18 \mathrm{~T}$ are apparent, but unlike the LBO results, they are not distinguished by changes in linear scaling of the voltage with magnetic field. Instead, the peak power voltages transition from a constant, linear scaling in $B \lesssim 0.18 \mathrm{~T}$, to a region of highly variable peak power voltages that cannot conclusively be described as linear. This result is not unexpected given the inconsistent SBO powers and variable operation observed in that magnetic field region in Fig. 6.14. A similar conclusion can be drawn from the start voltages, which also exhibit relatively constant, consistent scaling in $B \lesssim 0.18 \mathrm{~T}$, and transition to a region of greater variability in $B \gtrsim 0.18 \mathrm{~T}$.

Energy conversion metrics are shown in Fig. 6.19. The peak total efficiency vs. magnetic field shown by Fig. 6.19a reveals a result that is consistent with expectations based on the powers observed in Fig. 6.14. Peak efficiency near 12\% occurred at the same magnetic field as the peak output power, though as previously discussed, this result cannot be compared directly to the performance of the MFRPM configuration using the standard load.

Total microwave pulse energies vs. magnetic field are shown by Fig. 6.19b. The results are surprising because, unlike the plots of powers and efficiencies, the pulse energies remain largely constant across the range of magnetic fields tested. From prior discussions in Chapter 5, constant pulse energy despite changing peak powers suggests cathode-plasma expansion was responsible for the pulse-shortening. However, cavity plasma was also identified as a pulse-shortening explanation when operating at high power, and seems especially likely given the prior analysis of the LBO frequency shifts compared to the nonuniform magnetic field results. It appears that cavity plasma may also lead to constant-energy 


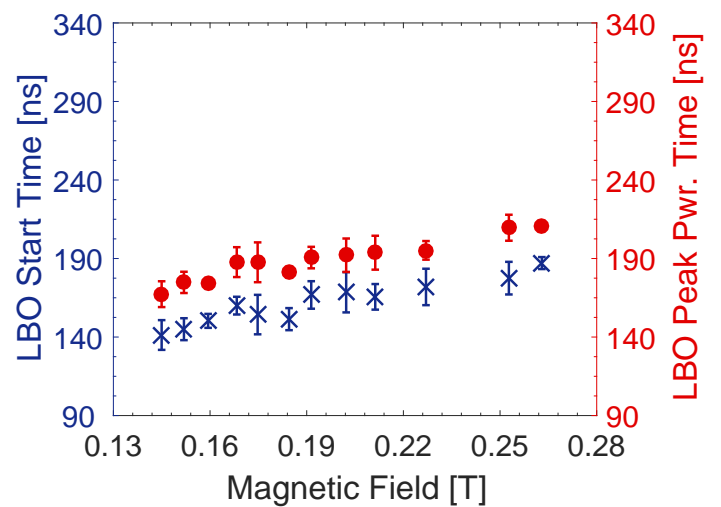

(a) LBO microwave start and peak power times.

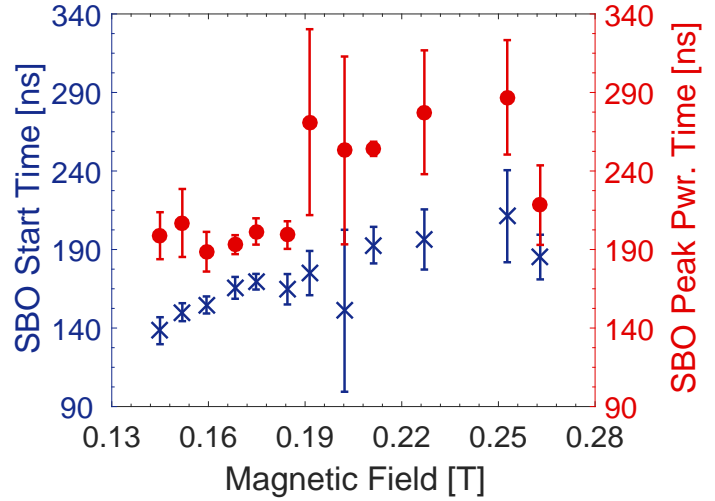

(b) SBO microwave start and peak power times.

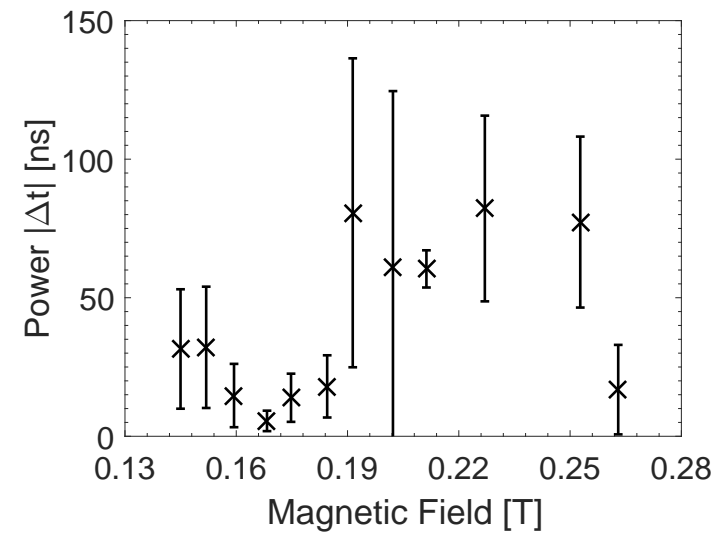

(c) Absolute time difference $|\Delta t|$ between oscillator peak powers vs. magnetic field.

Figure 6.20: Taper Load and Uniform Magnetic Field: Temporal metrics vs. magnetic field.

pulses.

Temporal metrics are shown in Fig. 6.20. While the scale in Fig. 6.20a makes it difficult to discern because the axes limits were set to be the same between the LBO and SBO plots for comparison, there is a slightly different linear progression in the start and peak power times for the LBO in $B \lesssim 0.18 \mathrm{~T}$ compared to $B \gtrsim 0.18 \mathrm{~T}$, which agrees with the prior voltage analysis. By comparison, the SBO results in Fig. 6.20b show clear evidence of the transition from the frequency-locked $6 \pi / 8$-mode to the $\pi$-mode, with the latter achieving peak power at much later times compared to the LBO or SBO frequency-locked $6 \pi / 8$-mode. The SBO $\pi$-mode also experienced considerably more shot-to-shot variability. Interestingly, the SBO start times did not undergo the same jump to later times in the region of $\pi$-mode operation, possibly because early SBO oscillations are induced by the harmonic content of the LBO-modulated beam before full synchronism was achieved via gap closure.

Figure 6.20c shows the absolute time difference $|\Delta t|$ between the peak powers from 
the oscillators vs. magnetic field. Relative to the results for the nonuniform magnetic field in Fig. 6.7c, the results of the present configuration represent a substantial improvement through the reduction of $|\Delta t|$. During optimal harmonic frequency-locked operation at $B \simeq$ $0.17 \mathrm{~T}$, the oscillators reached peak power nearly simultaneously, which is analogous to the results of Chapter 5. Also like the Chapter 5 results, the differences between harmonic frequency-locked operation and unlocked operation are substantial according to this metric.

As mentioned earlier, the region of optimal operation was near the $0.17 \mathrm{~T}$. The standard metrics for this region are summarized in Table 6.6 on page 135.

\subsubsection{Additional Analysis}

Figure 6.21 shows the second and fourth LBO harmonic powers vs. magnetic field. The results can be divided into three operating regions. The first corresponds to $B \lesssim 0.18 \mathrm{~T}$, wherein frequency-locked operation was observed. Here, the mean second harmonic power was $198 \mathrm{~kW}$, and the mean fourth harmonic power was $5.0 \mathrm{~kW}$. The second region lay in $0.18 \lesssim B \lesssim 0.22 \mathrm{~T}$, which corresponded to the same region where the SBO transitioned from the frequency-locked $6 \pi / 8$-mode to the $\pi$-mode, as shown by Fig. $6.15 \mathrm{~b}$ on page 157 . In this region, the mean LBO second harmonic power was $292 \mathrm{~kW}$, which was the highest of the three regions, and the mean fourth harmonic power was $4.9 \mathrm{~kW}$. The last region lay in $B \gtrsim 0.22 \mathrm{~T}$, where the LBO $\pi$-mode frequency steadily increased before stabilizing at the higher $0.99 \mathrm{GHz}$ frequency in Fig. 6.15a. In this region, the mean LBO second harmonic power was $226 \mathrm{~kW}$, and the mean fourth harmonic power was $2.8 \mathrm{~kW}$. In all regions, there was considerable spread in the power of both the second and fourth LBO harmonics.

Figure 6.22 shows the frequency histograms for the LBO harmonics. The histogram

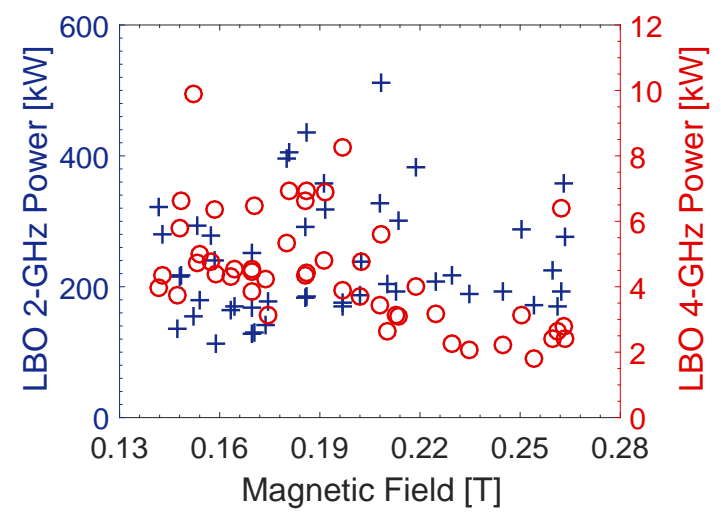

Figure 6.21: Taper Load and Uniform Magnetic Field: Peak LBO output powers at harmonic frequencies vs. magnetic field. 


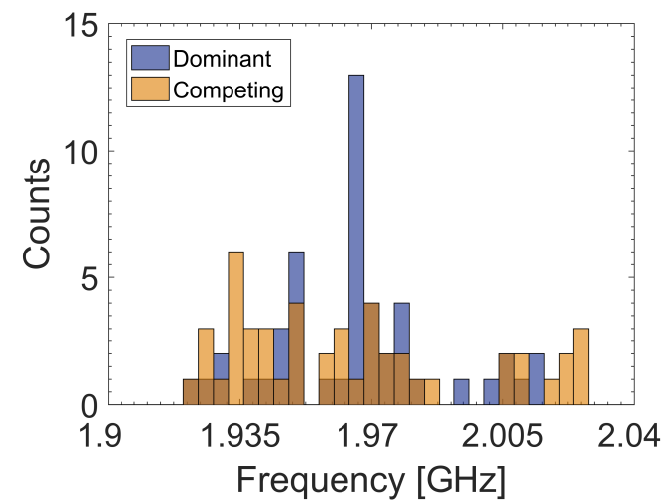

(a) LBO frequency histogram near $2 \mathrm{GHz}$.

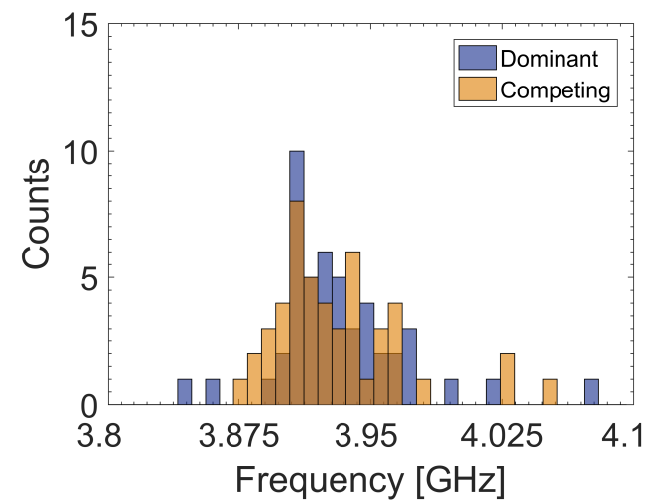

(b) LBO frequency histogram near $4 \mathrm{GHz}$.

Figure 6.22: Taper Load and Uniform Magnetic Field: Dominant and competing LBO harmonic frequency histograms.

for the second harmonic, shown by Fig. 6.22a, shows three significant peaks of interest at $1.934 \mathrm{GHz}, 1.950 \mathrm{GHz}$, and $1.966 \mathrm{GHz}$, with the first corresponding to a competing mode, and the others corresponding to primarily dominant modes. The histogram for the fourth harmonic, shown by Fig. $6.22 \mathrm{~b}$, shows a single peak at $3.908 \mathrm{GHz}$ with a significant spread in the distribution skewing toward higher frequencies.

Comparison of these peaks with the histograms for the fundamental frequencies in Fig. 6.16 on page 159 suggests several relations, although the situations in which they arise are complicated because they depend on the applied magnetic field. Since the histograms are a simplification of this fact, a complete understanding of the relations between these frequencies can only be achieved by analyzing how the different frequencies change with the magnetic field. This analysis will be discussed shortly.

Figure 6.23 shows the relation between the LBO harmonic powers and the dominant harmonic frequencies. The only noteworthy feature of either plot is the fact that the highest LBO second harmonic powers were consistently produced near $1.95 \mathrm{GHz}$, which is the same frequency as the SBO free-running $6 \pi / 8$-mode.

Figures G.1, G.2, and G.3 in Appendix G on page 212 show the dominant and competing fundamental and harmonic frequencies for both oscillators. As with the previous configuration using the nonuniform magnetic field, the plots omit SBO harmonics because they did not appear to have been generated at detectable power levels. Also omitted were plots including the competing LBO fourth harmonic. These plots show the complete, complicated set of relations that exist between these different frequencies, as well as their dependence on the magnetic field. The most relevant plots are duplicated here, and the notable conclusions follow. For convenience, the previously described magnetic field regions will 


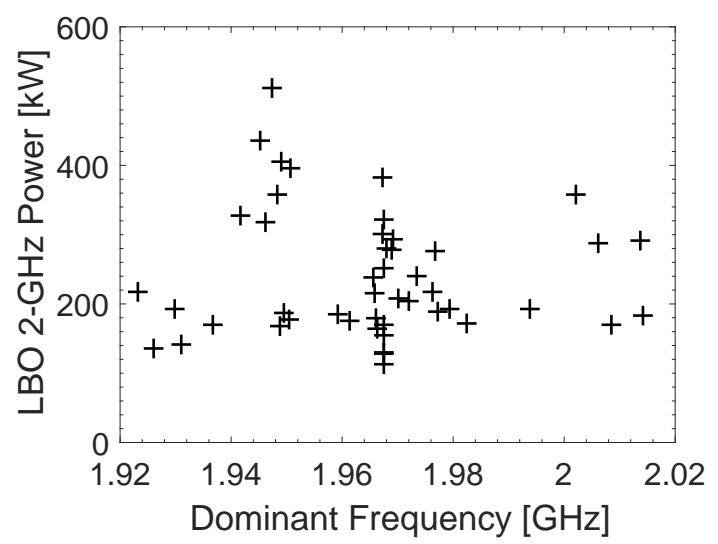

(a) LBO $2 \mathrm{GHz}$ harmonic power vs. dominant operating frequency.

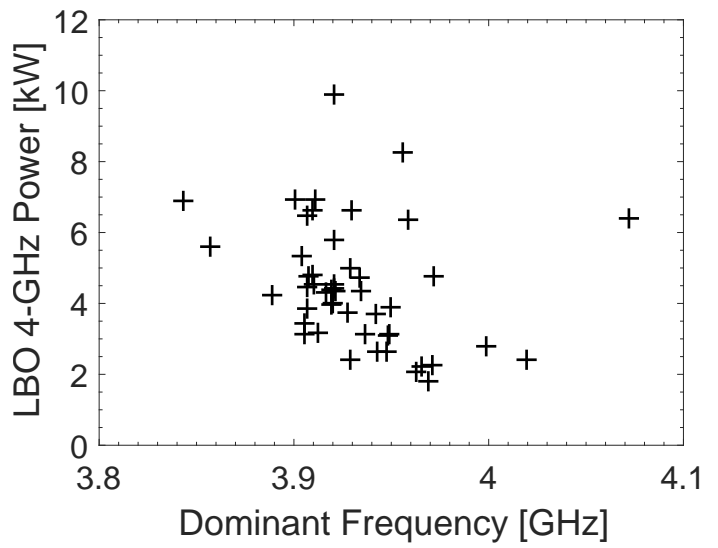

(b) $\mathrm{LBO} 4 \mathrm{GHz}$ harmonic power vs. dominant operating frequency.

Figure 6.23: Taper Load and Uniform Magnetic Field: Peak LBO harmonic powers vs. dominant operating frequencies.

be termed Region 1, 2, and 3, which correspond to $B \lesssim 0.18 \mathrm{~T}, 0.18 \lesssim B \lesssim 0.22 \mathrm{~T}$, and $B \gtrsim 0.22 \mathrm{~T}$, respectively.

A brief note on terminology is required. In the strictest sense, a harmonic is an exact integer multiple of some fundamental frequency [47,117]. Throughout this chapter, the term harmonic has been used as a convenient means of referring to the set of frequencies that is approximately twice that of the fundamental oscillator frequencies. As will be discussed momentarily, there are many integer-multiple relations between the frequencies that are approximate, and many that are exact. Thus, it should be understood that only exact integer-multiple relations prove that a previously termed second- or fourth-harmonic frequency actually correspond to a true harmonic.

Region 1 was of greatest interest, and will be addressed first. Inspection of Fig. 6.24a suggests a near, but not exact, harmonic relation between the fundamental frequencies of the LBO and SBO. From the discussion in the previous section, the waveguide taper load pulls the LBO frequency down from the typical $0.985-0.980 \mathrm{GHz} \pi$-mode frequency to approximately $0.970 \mathrm{GHz}$ during a shot when cavity plasma is absent and long-duration oscillations can occur. However, a careful inspection of the TFA shown in Fig. 6.8b in the previous section on page 148 reveals that, even during the lower power part of the pulse immediately following the HPM burst (prior to the long-duration lower-frequency oscillations), there is some reduction in the frequency. Even with the cavity plasma causing rapid microwave pulse-shortening in the present configuration, which cuts the signals short before the full frequency reduction to $0.970 \mathrm{GHz}$, some frequency reduction still occurs during the low-power part of the pulse prior to full oscillation cessation. Since the results 


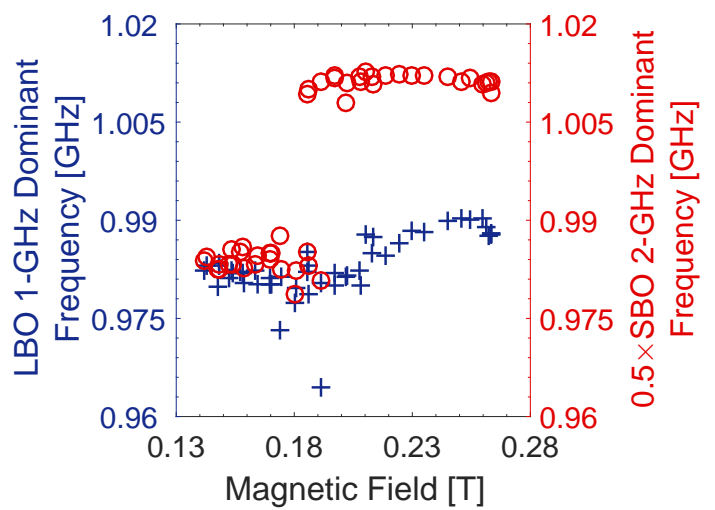

(a) LBO dominant $1 \mathrm{GHz}$ and SBO dominant $0.5 \times 2 \mathrm{GHz}$ frequencies vs. magnetic field.

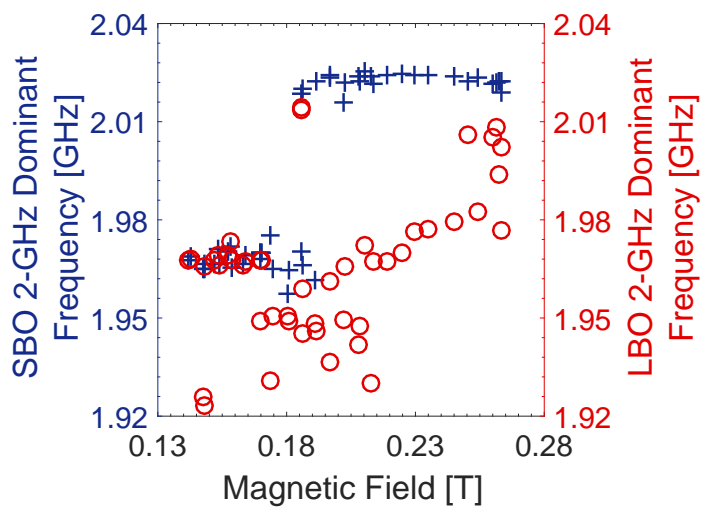

(c) SBO dominant $2 \mathrm{GHz}$ and LBO dominant $2 \mathrm{GHz}$ frequencies vs. magnetic field.

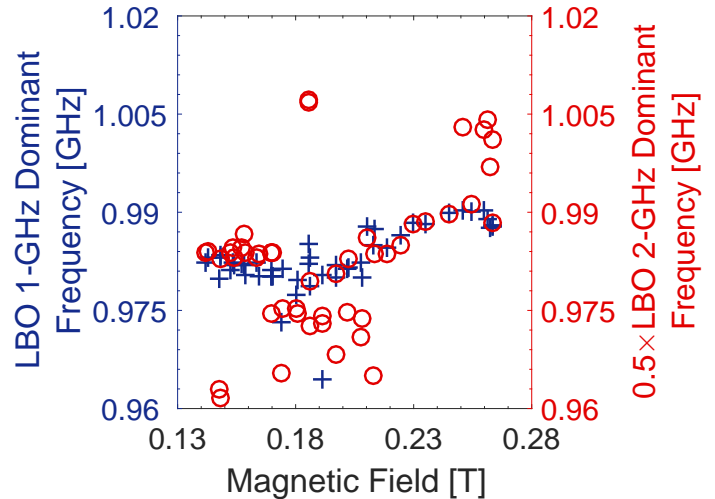

(b) LBO dominant $1 \mathrm{GHz}$ and LBO dominant $0.5 \times 2 \mathrm{GHz}$ frequencies vs. magnetic field.

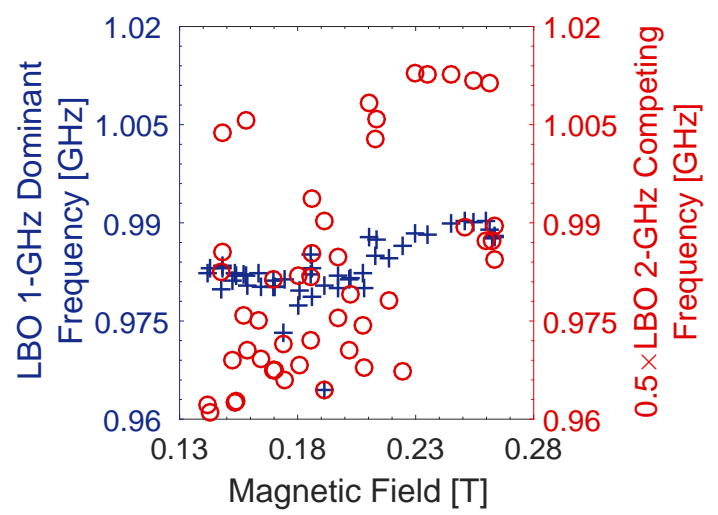

(d) LBO dominant $1 \mathrm{GHz}$ and LBO competing $0.5 \times 2 \mathrm{GHz}$ frequencies vs. magnetic field.

Figure 6.24: Taper Load and Uniform Magnetic Field: First comparison set of dominant and competing frequencies vs. magnetic field.

were derived from the time-integrated FFT, this reduction in the LBO frequencies during the low-power portion late in a pulse was reflected on the plots of frequency vs. magnetic field in this section as a minor frequency reduction. The effect is therefore similar to the observation of the previous section, but nowhere near as severe. Returning to Region 1 in Fig. 6.24a, the appearance of a near-harmonic relation is actually a case of confirmed harmonic frequency-locking during the high-power portion of the pulses based on a closer inspection of the results that accounted for the late-time impact of the waveguide taper load, although the results were not as clean as those of Chapter 5 due to the presence of the taper load.

Figure $6.24 \mathrm{~b}$ reveals a similar near-harmonic relation between the dominant LBO fundamental frequency and dominant LBO second harmonic frequency in Region 1. Since this is a relation between two LBO quantities, the taper load cannot explain the lack of an exact harmonic relation because, if the load were responsible for a reduction in the LBO 
frequency, it should affect both the first and second harmonics. Inspection of Fig. 6.24c confirms that the dominant LBO second harmonic frequency was locked to the dominant SBO fundamental frequency instead of the LBO fundamental frequency. It is likely that the SBO-modulated electron beam, upon entering the LBO, induces oscillations at the SBO modulation frequency that are stronger than oscillations at the true LBO harmonic frequency, and due to the proximity of these two frequencies, a distinction between the two could not be resolved by the time-integrated FFT. This explains why the relation between the fundamental frequencies of the LBO and SBO, as well as the relation between the LBO fundamental and second harmonic frequencies, appear as near-harmonics, whereas the LBO second harmonic and SBO fundamental frequency appeared to be equal. If the LBO true second harmonic were resolvable alongside the dominant SBO frequency, Fig. 6.24d would show an exact harmonic relation between the LBO fundamental and competing LBO second harmonic frequencies in Region 1. The competing quantity should show the relation because the LBO second harmonic should be lower power than the SBO fundamental. A few instances of this are apparent in $0.18 \lesssim B \lesssim 0.19 \mathrm{~T}$ (which lies in Region 2) in Fig. 6.24d because that magnetic field range saw a significant reduction in the SBO power.

Region 1 also exhibited a harmonic relation between the dominant LBO fundamental frequency and the fourth harmonic, as shown by Fig. 6.25a. This further supports the supposition of the previous paragraph, where the true LBO second harmonic was not resolvable due to the strong frequency content at the SBO fundamental frequency induced on the LBO by the SBO-modulated electron beam. The discrepancy between the dominant second and fourth LBO harmonics is shown by Fig. 6.25b, which directly shows the near, but not exact, harmonic relation in Region 1. Also supporting this discussion is the near, but not exact, harmonic relation between the dominant SBO fundamental frequency and the dominant LBO fourth harmonic frequency, which is shown by Fig. 6.25c.

To summarize the interpretation of the results for Region 1, when accounting for the misleading effect of the taper load on the time-integrated FFTs, it appears that harmonic frequency-locking between the LBO fundamental, second, and fourth harmonics, as well as the SBO fundamental frequency, occurred during the high-power portion of the microwave pulses. The analysis also showed that the SBO affected the second harmonic of the LBO, possibly through the SBO-modulated electron beam if the mechanism is similar to the findings from Chapter 5.

Region 2 represented a region of transition between a frequency-locked state and a state where the oscillators operate independently. Since operation was mixed between multiple modes, no concrete conclusions can be drawn because operation was highly variable. 


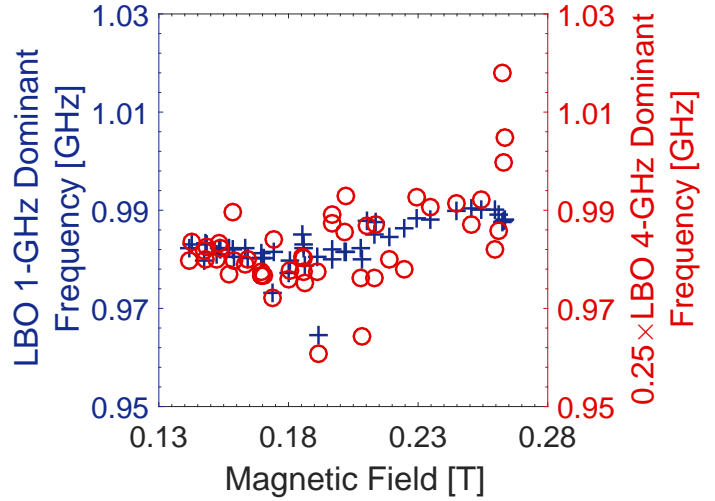

(a) LBO dominant $1 \mathrm{GHz}$ and LBO dominant $0.25 \times 4 \mathrm{GHz}$ frequencies vs. magnetic field.

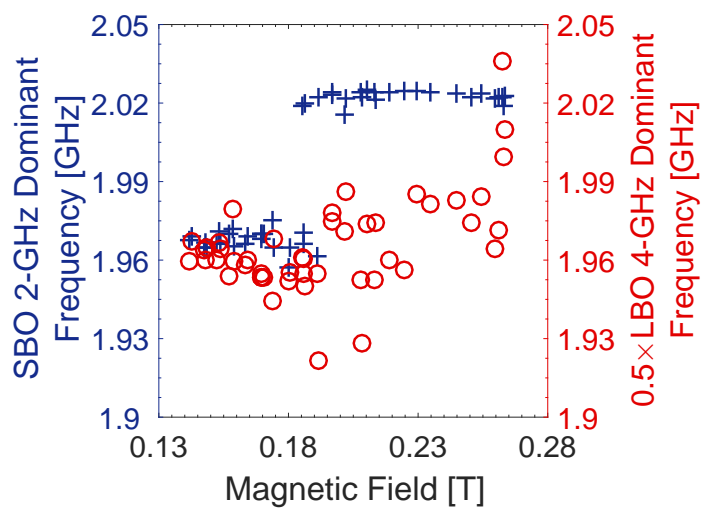

(c) SBO dominant $2 \mathrm{GHz}$ and LBO dominant $0.5 \times 4 \mathrm{GHz}$ frequencies vs. magnetic field.

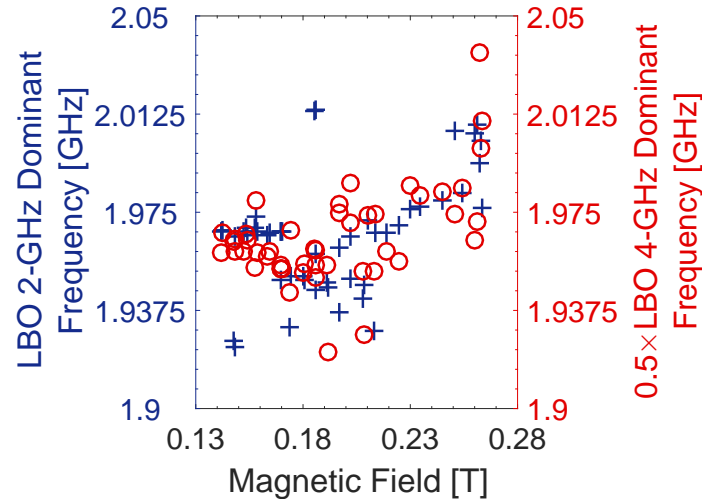

(b) LBO dominant $2 \mathrm{GHz}$ and LBO dominant $0.5 \times 4 \mathrm{GHz}$ frequencies vs. magnetic field.

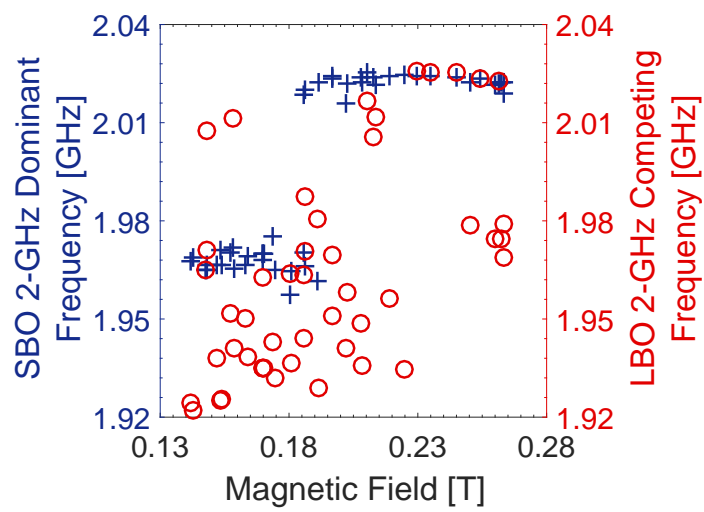

(d) SBO dominant $2 \mathrm{GHz}$ and LBO competing $2 \mathrm{GHz}$ frequencies vs. magnetic field.

Figure 6.25: Taper Load and Uniform Magnetic Field: Second comparison set of dominant and competing frequencies vs. magnetic field.

Region 3 represented a region where the both the LBO and SBO operated in the $\pi$ mode, so no frequency-locking occurred. In Region 3, the SBO powers were considerably lower relative to Region 1, whereas the LBO powers were comparable between the two regions. Figure $6.24 \mathrm{~b}$ clearly shows the harmonic relation between the dominant LBO fundamental frequency and the dominant LBO second harmonic. Some correlation also existed between the dominant LBO fundamental frequency and the dominant LBO fourth harmonic, as shown by Fig. 6.25a, although there is considerable spread in the data. A similar result therefore appeared in Fig. 6.25b, which shows the dominant LBO second and fourth harmonics.

In Region 3, the lower SBO power seemed to have a reduced effect on the second LBO harmonic. From Fig. 6.24c, the dominant LBO second harmonic frequency was never observed to be equal to the dominant SBO fundamental frequency. However, several shots revealed equality between the dominant SBO fundamental frequency and the competing 
LBO second harmonic frequency, as shown by Fig. 6.25d. The SBO therefore still affected the LBO.

To summarize the interpretation of the results for Region 3, it appears that the LBO and its frequency harmonics operated independently of the SBO. The only relation between the two oscillators appeared to be some induction of competing second harmonic oscillations on the LBO by the SBO. In addition to the distinctly different frequencies of the $\pi$-mode for each oscillator, two other factors may be responsible for the absence of any substantial effect by one oscillator on the operation of the other. The first is that the SBO power levels were considerably lower than in Region 1, which explains why it had a reduced impact on the detected LBO second harmonic. The second is that Region 3 was a region of high magnetic fields, which corresponds to a slow electron beam drift velocity. A consequence of this lower velocity is that the electron beam spends more time in transit around the cylindrical recirculation bend from one oscillator to the other. This additional time permits greater demodulation of the electron beam due to repulsive electrostatic forces, so the reduced beam modulation would reasonably be expected to reduce the influence of the oscillators on each other.

To remain consistent with the analysis of the Standard Metrics section, characterization of the LBO harmonics was performed for the previously identified region of optimal operation near $0.17 \mathrm{~T}$. The results were shown in Table 6.7 on page 135.

\subsection{Impact of Magnetic Field Optimization}

Comparisons discussed in this section used results from the regions of optimal operation from each configuration. The impact of the magnetic field optimization for uniformity was significant for some investigated metrics. Table 6.8 on page 136 compared the results of metrics used to evaluate the operation of the individual oscillators. The LBO microwave power was observed to benefit the most from the uniform magnetic field, with an increase of 39\% relative to the nonuniform magnetic field. Surprisingly, the SBO microwave power was not observed to change much, with a comparatively minor decrease of $9 \%$. This was likely due to the higher electronic efficiency of the $\pi$-mode experiencing some performance reduction due to the nonuniform magnetic field, with the result being a minor difference in power when compared to the frequency-locked $6 \pi / 8$-mode in the uniform magnetic field configuration.

As was discussed at length earlier in the chapter, the LBO $\pi$-mode frequency was reduced to $0.972 \mathrm{GHz}$ due to the waveguide taper load in the nonuniform magnetic field configuration, whereas the uniform magnetic field configuration resulted in frequencies that 
were more consistent with the frequencies near $0.980 \mathrm{GHz}$ that were observed throughout Chapter 5. Apart from the different degrees of impact from the waveguide taper load, the LBO operated in the same mode, so the differences in frequency and associated uncertainty were minor. By comparison, the SBO frequency changed substantially due to a change in operating mode from the $\pi$-mode in the nonuniform magnetic field configuration to the $6 \pi / 8$-mode in the uniform magnetic field configuration. The associated standard deviation also saw a minor improvement, which was indicative of reduced shot-to-shot variability.

Table 6.9 on page 137 compared the metrics for the MFRPM as a whole. The magnetic field optimization for axial uniformity improved the total power by $47 \%$ and resulted in a $33 \%$ improvement in efficiency. The change in peak power $|\Delta t|$ was also significant, with $17 \mathrm{~ns}$ reduction observed due to the uniform magnetic field, as well as a reduction in the standard deviation. The impedance also benefited from the uniform magnetic field, which brought the MFRPM closer to MELBA-C specifications.

Table 6.10 on page 137 compared the LBO harmonic frequency metrics for the two magnetic field configurations. Second harmonic powers were reduced by $39 \%$, and shotto-shot variability was reduced by $58 \%$. The fourth harmonic powers were approximately the same given the large associated standard deviation of the two measurements. The second harmonic frequency was reduced from $1.986 \mathrm{GHz}$ to $1.962 \mathrm{GHz}$, with a significant reduction in shot-to-shot variability from $0.041 \mathrm{GHz}$ to $0.013 \mathrm{GHz}$. These changes were likely due to the stabilizing effect of harmonic frequency-locked operation, and the fact that the SBO was operating in the lower-frequency $6 \pi / 8$-mode in the uniform magnetic field configuration. A similar effect was observed for the fourth harmonic, although shot-to-shot variability remained largely unaffected. 


\section{CHAPTER 7}

\section{Summary and Conclusions}

This chapter provides a summary of the most significant findings and the conclusions drawn from the MFRPM experiment. The chapter concludes with suggestions for future work.

\subsection{Summary}

Through a combination of analytic theory, computational modeling, and laboratory experiments, a prototype MFRPM was designed, fabricated, and characterized to determine: 1) the identity of the observed fundamental operating electromagnetic modes, 2) the frequencies, powers, and other electrical characteristics associated with those modes and the LBO frequency harmonics, 3) the magnetic fields corresponding to optimal operation, 4) the operational impact of a nonuniform axial magnetic field, and 5) the origin and performance characteristics of the harmonic frequency-locked operating state. Six experimental configurations were tested using three anodes (the isolated LBO, the isolated SBO, and the MFRPM), two microwave loads (the standard load and the waveguide taper load), and two axial magnetic fields (uniform and nonuniform). The configurations were: 1) the isolated LBO with the uniform magnetic field and the standard load, 2) the isolated SBO with the uniform magnetic field and the standard load, 3) the MFRPM with the nonuniform magnetic field and the standard load, 4) the MFRPM with the uniform magnetic field and the standard load, 5) the MFRPM with the nonuniform magnetic field and the waveguide taper load, and 6) the MFRPM with the uniform magnetic field and the waveguide taper load.

The standard load was used to characterize the fundamental modes of the magnetron. Baseline operation of the individual oscillators was determined using the isolated LBO and SBO configurations. For the LBO, the $5 \pi / 6$-mode and $\pi$-mode frequencies were $0.972 \mathrm{GHz}$ and $0.985 \mathrm{GHz}$, respectively. Optimal LBO operation produced $32 \mathrm{MW}$ in the $\pi$-mode at approximately $0.23 \mathrm{~T}$. For the SBO, the $6 \pi / 8$-mode, $7 \pi / 8$-mode, and $\pi$ - 
mode frequencies were $1.94 \mathrm{GHz}, 2.00 \mathrm{GHz}$, and $2.022 \mathrm{GHz}$, respectively. Optimal SBO operation produced $18 \mathrm{MW}$ in the $\pi$-mode at $0.17 \mathrm{~T}$.

For the standard load and the MFRPM, the uniform magnetic field exhibited superior performance by all metrics compared to the nonuniform magnetic field, although the nonuniform field results provided crucial evidence that the harmonic frequency-locked SBO mode was the $6 \pi / 8$-mode. For the MFRPM and the uniform magnetic field, optimal operation was observed at $0.17 \mathrm{~T}$, where LBO produced $32 \mathrm{MW}$ in the $\pi$-mode at $0.984 \mathrm{GHz}$ and the SBO produced $13 \mathrm{MW}$ in the $6 \pi / 8$-mode at $1.970 \mathrm{GHz}$. Within experimental uncertainty, this novel state was a form of harmonic frequency-locked operation in which the SBO locked to the second frequency harmonic of the LBO. In $B=0.16-$ $0.17 \mathrm{~T}$, the phase drift during a typical locked shot was $8 \pm 4^{\circ}$, and the lock duration was $14 \pm 3 \mathrm{~ns}$. The average phase difference between the oscillators was $93 \pm 17^{\circ}$. The minimal change in the LBO microwave power and frequency relative to its isolated configuration, as well as the shift of the SBO operating mode from the $\pi$-mode in the isolated configuration to the $6 \pi / 8$-mode in the locked state, suggested the locked state was an instance of Adler-like master-slave locking, where the LBO was the driving oscillator and the SBO was the driven oscillator. In the locked state, changes in the relative phase difference between the oscillators correlated with changes in the magnetic field, which suggested the locking mechanism occurred through the second harmonic content of the LBO-modulated electron beam recirculating from the LBO to the SBO. The investigated metrics suggested that the locked state was remarkably stable given the unusually small variation in powers, frequencies, impedance, and timing.

A new theory for harmonic frequency-locking was developed by Y. Y. Lau [43], although a comparison to the experimental results proved to be inconclusive. The reason is that the experiment was not suitable for comparison to the theory because the theory required knowledge of the both the LBO $\pi$-mode power injected into the SBO, as well as the SBO $6 \pi / 8$-mode power. Furthermore, a set of results was required that exhibited both locked and unlocked SBO $6 \pi / 8$-mode shots. In this dissertation, the power delivered to the LBO from the SBO was unknown, and an insufficient number of unlocked SBO $6 \pi / 8$ mode shots were obtained. For these reasons, validation of the new theory would require a dedicated experimental configuration designed to isolate and control these variables.

The waveguide taper load was used to characterize the LBO harmonic frequencies and powers. As with the results for the standard load, the standard metrics indicated that the uniform magnetic field exhibited superior performance of the fundamental modes relative to the nonuniform magnetic field. The nonuniform field results provided additional evidence that the LBO harmonic frequency affected operation of the SBO $6 \pi / 8$-mode frequency. 
Together, both magnetic field data sets supported the speculation that cavity plasma may be the dominant pulse-shortening mechanism at high microwave powers in the MFRPM, which was observed in the microwave pulse-shortening imaging study.

Optimal operation of the fundamental modes using the waveguide taper load and uniform magnetic field occurred at $0.17 \mathrm{~T}$. Within experimental uncertainty, harmonic frequencylocking was again observed between the LBO $\pi$-mode, its frequency harmonics, and the SBO $6 \pi / 8$-mode. The LBO second harmonic power and frequency was $178 \mathrm{~kW}$ and $1.962 \mathrm{GHz}$, and the LBO fourth harmonic power and frequency was $5 \mathrm{~kW}$ and $3.916 \mathrm{GHz}$. In general, LBO harmonic powers increased when the fundamental circuit modes were operating at reduced power with considerable mode competition. Harmonic powers were also as much as $150 \%$ higher using the nonuniform magnetic field relative to the uniform magnetic field.

\subsection{Conclusions}

The MFRPM demonstrated the feasibility of generating simultaneous HPM pulses at more than one frequency using a crossed-field device. Furthermore, through the harmonic frequency-locking effect, the MFRPM operated as a stable harmonic frequency generator with some degree of phase control. The analysis of the LBO frequency harmonics revealed that the SBO did not substantially prime harmonic frequency generation by the LBO. Thus, appreciable powers at harmonic frequencies can be generated using a single device composed of coupled structures designed to oscillate in their fundamental circuit modes at frequencies related by an integer multiple.

While the possibility of some performance benefits from minor magnetic field variations cannot be ruled out, the substantial $40 \%$ variation in the nonuniform magnetic field configuration consistently produced poor magnetron operation. Thus, for structures analogous to the RPM and MFRPM, a uniform magnetic field is an advisable baseline configuration.

\subsection{Suggested Future Work}

The MFRPM concept stands to benefit from several lessons learned as a result of this dissertation. Foremost is the need for new designs to have a properly designed microwave power extraction system with control over the loaded quality factor $Q_{L}$. By exerting control over $Q_{L}$, the harmonic frequency-locking mechanism can be studied more rigorously. In addition, the locking mechanism is potentially exploitable. It is well known that very 
powerful, efficient magnetrons having a low $Q_{L}$ need RF priming to start [76]. The beam modulation method could be used as a form of internal priming, and the experiment suggested the effects of priming may be considerable. Furthermore, the beam could be modulated using a small number of cavities designed solely for that purpose. By designing the few beam-modulation cavities to be frequency-tunable and the driven cavities to have a sufficiently low $Q_{L}$, it may be possible to create a high power, high efficiency, frequencyagile microwave source without the considerable complexity introduced by an RF priming system.

Other topics of future study include the consideration of MCC designs as resonant structures, further investigation of the LBO frequency harmonics, and a detailed study of magnetic field variations. During the course of this dissertation, the cathode was discovered to oscillate strongly in the $600-700 \mathrm{MHz}$ frequency range, which possibly impacted operation. Treatment of the cathode as an additional resonant structure is a topic offering potential benefits for RPM operation. Due to time limitations, the isolated LBO was never tested using the waveguide taper loads. The baseline LBO harmonic powers therefore remain an open question. Finally, as previously mentioned, less extreme variations in the magnetic field may be worth systematic exploration in the MFRPM. 


\section{APPENDIX A \\ HFSS Simulation Implementation}

The finite-element, frequency-domain code HFSS [44] was used to simulate the coldtube characteristics of the MFRPM. This appendix section documents some of the options and methods used to obtain the results for this dissertation.

Broadly, HFSS supports a driven solver and an eigenmode solver. The former is useful for performing frequency sweeps, and in the context of the MFRPM, driven modal solutions are the simulation equivalent of $S_{11}$ cold tests using a network analyzer. In general, the driven solver was unable to reproduce most of the features of the experimental cold tests presented in $\S 4.1 .3$ in Chapter 4, especially in the case of the SBO. The main reason was the very weak coupling between the extractor and the structure. Since the main objective of the cold-tube simulations was to identify the resonant frequencies of the different anode electromagnetic modes, the eigenmode solver was used for all HFSS simulations in this dissertation.

For the 2D simulations of the unit cell of a planar cavity array discussed in Chapter 2, a $0.5 \mathrm{~cm}$ thick slice of the cavity was used with perfect $\mathrm{H}$ boundary conditions, which are equivalent to a boundary through which no current can pass. This forces the eigenmode solver to converge on TE solutions and makes the cell look infinitely long from an axial perspective. Using the notation from the chapter, the phase advance per vane $\beta L$ was specified using master-slave boundaries at the AK gap to obtain the solution frequencies $\omega$ corresponding to the fundamental and higher-order circuit modes. For these simulations, HFSS was directed to converge on real frequencies only because the quality factor was not of interest, and mixed-order basis functions were used to better handle sharp corners and field enhancement points [118]. Convergence was defined as a $<0.1 \%$ change in the real frequency when the mesh was refined, and two consecutive, converged mesh refinements were accepted as a completed simulation. The 3D simulations used the same convergence criteria as the 2D simulations. In most cases, convergence was achieved using 300,000400,000 tetrahedra. 


\section{APPENDIX B \\ MAGIC Simulation Implementation}

The 3D, finite-difference time-domain, Particle-In-Cell (PIC) code MAGIC [80] was used to validate some HFSS cold test results and and to estimate hot tube operation. PIC simulations have considerable flexibility in their configuration, so this appendix section notes some of the important considerations and methods used to model the MFRPM.

The notable considerations for simulations included: 1) boundary conditions for the simulation, 2) how the DC voltage excitation was applied to the simulation, and the choice of rise-time, 3) the macroparticle emission model, including creation rate and macroparticle weighting, 4) cathode emission area, 5) duration of the simulation, 6) spatial mesh resolution, 7) validation checks of the physics, and 8) optimization of the configuration used for parallel execution.

Boundary conditions were specified using the standard MAGIC PORT command, where the coaxial power extraction ports were configured as ideal absorbers using a secondorder centered advection equation, although this approach adds constraints to the spatial mesh [119]. The input port, which represents the coaxial transmission line from MELBA-C, is also an ideal absorber of any incident RF, and therefore acts like an infinitely long coaxial transmission line. Both the output and input ports are significant approximations. In reality, the RF loads for the magnetron are imperfect, as shown by Fig. D.5 in Appendix D.2.2, and for shots using the waveguide taper configuration, the LBO actually sees $99 \%$ of output power reflected back from the extractor. Similarly, the input coax line terminates at MELBA-C, so the method used to launch the DC voltage excitation must be implemented carefully to accurately model the voltage applied to the magnetron in the experiment.

Since the input port appears to be infinitely long to the simulation, loads that are highimpedance (relative to the characteristic impedance of the geometry of the input port) exhibit behavior that deviates considerably from reality. For instance, if a model is config-

ured not to emit electrons, the forward-propagating excitation from the input port reflects off the magnetron with identical magnitude and polarity, causing the effective voltage to 
be twice that which was specified. In reality, if MELBA-C were fired into an open circuit, the LTspice model (described in Appendix C) suggests the observed voltage would be $370 \mathrm{kV}$, not the $600 \mathrm{kV}$ that would be observed in MAGIC for a $300 \mathrm{kV}$ excitation. Therefore, two different approaches could be employed to model the applied voltage: 1) a feedback approach wherein the magnitude of the port excitation was adjusted for changes in magnetron impedance to maintain a constant voltage applied to the magnetron, or 2) a carefully chosen constant excitation magnitude such that, for a set of simulations (usually a sweep of magnetic field values), the observed voltage would change in a manner consistent with MELBA-C operation for different magnetron impedances. The former approach is an idealized model that eliminates the applied voltage as a variable, whereas the latter attempts to model the actual physics leading to voltage changes due to the dynamic magnetron impedance, which can be affected by the mode of oscillation, magnetic field magnitude, emission mechanism, and emitting area, among other factors.

Maintaining a constant voltage using a feedback mechanism to compensate for impedance changes was particularly challenging because the feedback can destabilize the simulation or artificially reinforce oscillations at a given frequency. By using a low-pass filter, an excitation model was developed to maintain a nearly invariant voltage across the magnetron. Simulations attempting to realistically account for the dynamic impedance had the input port excitation magnitude adjusted such that the highest and lowest impedances (usually dictated by the highest and lowest magnetic field values in a parameter sweep) would yield voltages consistent with those observed in MELBA-C experiments, even if the simulated current drawn by the magnetron deviated considerably from experiment. Once an excitation magnitude was found that produced realistic voltages at the extremes of tube impedance for a parameter sweep, the same magnitude was used for the remaining simulations in the sweep.

The rise-time of the DC voltage excitation can also lead to unintended consequences. A very fast $(<5 \mathrm{~ns})$ voltage rise-time is occasionally used to shorten the computation time of simulations. Such an excitation is shock-like, which can unintentionally prime the excitation of some modes of oscillation due to the rich frequency content of the shock. This can lead to artificially rapid magnetron start times and misleading mode stability. While some pulsed-power sources do have extremely fast rise-times ( $\simeq 20 \mathrm{~ns}$ ), MELBA-C does not, and simulations must account for the longer $150 \mathrm{~ns}$ rise-time. The shape of the excitation can also unintentionally prime modes if abrupt ramp functions are used, and in extreme instances, numerical destabilization can result. All simulations used the SMOOTH_RAMP function available in MAGIC, which is sufficiently numerically smooth to avoid instability and unintentional shock. 


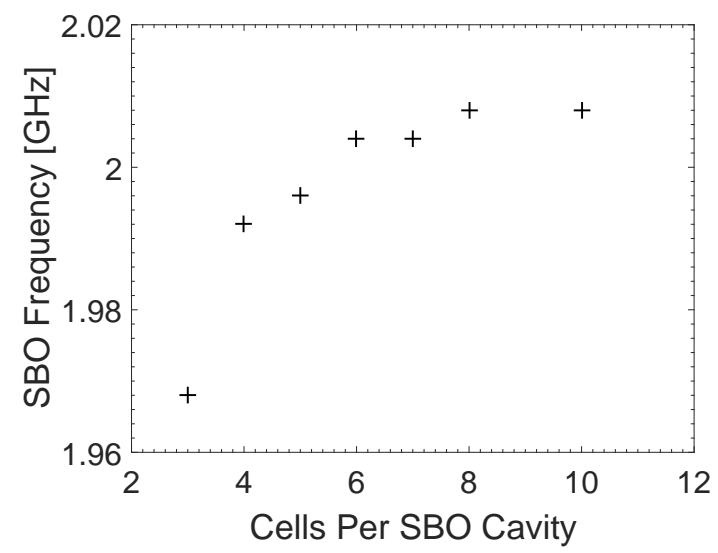

Figure B.1: SBO frequency vs. number of cells per SBO cavity.

The MAGIC emission model used for all simulations was the explosive emission model, which obeys space-charge-limited emission physics [119]. The macroparticle weights in each simulation set were chosen such that the total particle population at steady-state operation was at least 200,000 and never more than 1,000,000. Two cathode emission areas were used. The first emitted from $10 \%$ of the available surface between the endcaps, which was consistent with the experimental configuration, and the second emitted from $100 \%$ of the available surface area. These two extremes were chosen to 1) determine the amount of beam-loading on the oscillator frequencies, 2) to determine how the emitted current might scale with emission area, and 3) to evaluate the agreement between the MAGIC emission model and the experimental results. The results of Chapter 5 showed that the $10 \%$ emission area resulted in simulations that drew far less current than was observed experimentally. Simulated currents were typically between 0.1 and 0.5 times the experimental current. If the full emission area was used, currents were nearly identical to the experiment. Ion formation and bipolar flow can increase emission up to 50\% beyond the Child-Langmuir limit $[113,120]$, which may explain the higher currents observed from the relatively small experimental emission area.

The duration of simulations was chosen to be $250 \mathrm{~ns}$ at minimum. This was long enough to resolve frequencies within $4 \mathrm{MHz}$, since the frequency bin width of the FFT algorithm is given by $d f=1 / T$, where $T$ is the simulation duration. In some instances, simulations did not reach steady state by the end. These simulations were run for longer durations until steady-state operation was observed.

The chosen spatial mesh resolution was a compromise between full simulation convergence and computational feasibility. Due to the small feature sizes relative to the rest of the experiment geometry, the component with the most stringent meshing requirement 
was the SBO. Figure B.1 shows that full convergence was only achieved when eight cells were used per SBO cavity. Simulation CPU times were approximately one week if the full experimental geometry was modeled, which was unacceptably long to perform simulations using different magnetic fields, emission areas, and DC voltage excitation models. Ultimately, all simulations were conducted using only six cells per SBO cavity, which corresponds to a cell size of $1.6 \mathrm{~mm}^{3}$. The most sensitive diagnostic to changes in cell size was the frequency of the SBO, which experienced far greater reductions in frequency due to beam-loading when the mesh was coarse. Thus, while the simulations using $1.6 \mathrm{~mm}^{3}$ cells were not fully converged, the effects were understood and any interpretation of the results was made with those caveats in mind.

$$
\mu=\frac{u_{x} \Delta t}{\Delta x}+\frac{u_{y} \Delta t}{\Delta y}+\frac{u_{z} \Delta t}{\Delta z} \leq 1
$$

The reason for the close relation between simulation CPU time and mesh cell size is the Courant-Friedrichs-Lewy condition shown by Eq. B.1, which requires that the numerical domain of dependence include the mathematical domain of dependence [121]. Here, $\Delta t$ is the time step, $\Delta x$ is the size of a mesh cell along one dimension (and similarly so for the other two dimensions), and $u_{x}$ is the maximum propagation velocity of information dictated by the physics along one dimension (and similarly so for the other two dimensions), which would be the speed of light in vacuum in this case. The Courant number $\mu$ determines the size of the maximum time step that remains stable. As seen by Eq. B.1, the time step $\Delta t$ must decrease if the dimensions of a cell also decrease. Notably, the time step is limited primarily by the smallest dimension of any cell in the simulation. Simply coarsening the mesh elsewhere in the simulation therefore does relatively little to improve simulation $\mathrm{CPU}$ times. Any change in mesh size in MAGIC must be done gradually because resolution errors may result if the cell-to-cell variation exceeds $25 \%$ or if the cell aspect ratio exceeds 5:1 [119]. To avoid this possibility, a uniform mesh was used for all simulations.

Some physics validation checks were included in the simulations because not all commercial PIC codes have self-consistent physics, depending on model approximations and optimizations. The first check confirmed that the net emitted electron current was equal to the input current at the input coaxial transmission line, which was measured using line integration of the magnetic field around the cathode stalk. The integration path needed to be very close to the cathode stalk to avoid enclosing any backstreaming endloss current, which would reduce the apparent input current. A minor discrepancy in the input current at the start of the simulation was due to the magnetron charging like a capacitor during the voltage rise before electron emission began. The second physics check confirmed that 
the input power, calculated by an integration of the Poynting flux over the input port area, agreed with the input power calculated by multiplying the magnetron voltage by the net emitted current.

Since many simulations were required to estimate and understand the operation of the different MFRPM configurations, an optimization study was performed to determine what parallel computing configuration resulted in the shortest CPU times. The CPU used for these simulations had six cores with hyperthreading, and therefore 12 logical CPUs were available for use. Identical simulations were resumed from a saved state for a preset simulation duration to determine how much CPU time was required for completion using different parallel computing configurations. Table B.1 summarizes the results. A shared configuration for one simulation used a single CPU core that was shared by two logical threads of execution, whereas a dedicated configuration only assigned one thread of execution per CPU core. For two or more simultaneous simulations, several configurations were tested. When the total number of threads was less than the number of logical CPUs (12), a shared configuration assigned two threads of execution per CPU core, but each thread corresponded to a different simulation running in parallel, whereas the dedicated configuration assigned only one thread per core. When the total number of threads was equal to the number of logical CPUs (12), a shared configuration assigned two threads per core, where each thread belonged to a different simulation, and a dedicated configuration assigned two threads per core, where each thread belonged to the same simulation.

Two conclusions resulted from the study. The first found that, while MAGIC did support multi-threaded execution, the benefits were not significant compared to simply running multiple simulations simultaneously using a single thread for each. All simulations were therefore configured for single-threaded execution. The second conclusion was that hyperthreading was rarely beneficial, and typically resulted in performance degradation. All simulation configurations therefore assigned only one thread of execution per CPU core. 


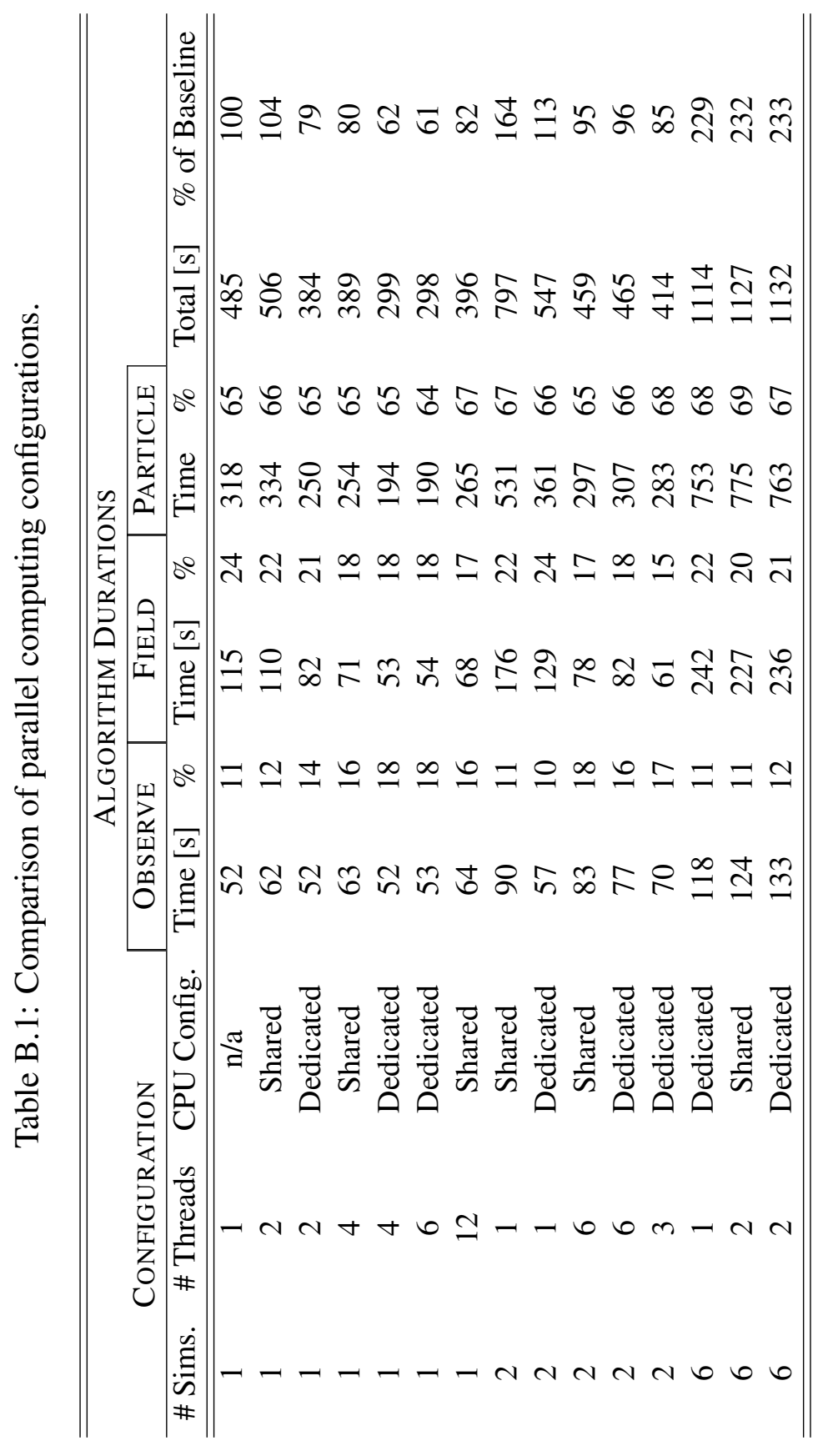




\section{APPENDIX C \\ Simulation of the MELBA-C Marx Generator}

\section{C.1 Overview}

The Michigan Electron Long Beam Accelerator with Ceramic Insulator (MELBA-C) Marx generator involves the coordinated operation of several components. The Marx erection process, which involves breaking down the switches to achieve high-voltage output, can lead to floating potentials and voltage swings on different components due to parasitic capacitances. As component properties change over time (e.g. a resistor absorbing oil leading to infinite resistance), the highly coupled nature of the design can lead to complex behavior that is misleading and difficult to diagnose during the course of data collection, particularly when the operating characteristics of the load, such as the MultiFrequency Recirculating Planar Magnetron (MFRPM), are not fully understood. In an effort to better understand whether the features of MELBA-C voltage traces were the result of proper operation or failed components, several simulations were conducted using the Simulation Program with Integrated Circuit Emphasis developed by Linear Technology Corporation (LTspice) [122] to gain insight into the features of MELBA-C voltage traces.

Prior analysis of simplified MELBA-C circuits has been conducted using various software, including LTspice and its predecessors, by both the original architects (Pulse Sciences, Inc.) and by previous students [98, 123]. While these simplified models could duplicate the overall qualitative behavior, they generally did not produce some of the interesting features observed in nearly every MELBA-C pulse. Examples include the low-voltage "knee" early in the erection of the Marx circuit. Those early models also did not explain what failures might cause egregiously poor pulses, such as very long "knees," ringing superposed on the pulse, or substantial inflection points during the voltage rise, all of which impact the temporal characteristics of the voltage pulse (and therefore affect magnetron operation). 


\section{C.2 Implementation}

The simulation approach was to model all components present in the circuit diagram for the MELBA-C design proposal [98], including changes made between the proposed design and the final product. The model also incorporated features that were not found in any previous model. These features include: 1) the capacitance present between the cases of the capacitors, 2) the transmission line characteristics between the magnetron and the Marx generator output, 3) a switching model that could duplicate the finite breakdown voltage threshold for the switches, and 4) the pick-off trigger network comprising the trigger resistors, switch trigger planes, and the capacitor cases.

Several simplifications were included in the model. One example was the circuit model used to include capacitances between resistor cases. The larger horizontal separation and smaller area between adjacent capacitors led to the omission of their case-to-case capacitance, while the capacitance between the capacitors stacked vertically was included due to their close spacing and large area. The most significant simplification was the assumption of time-invariant load parameters (inductance, capacitance, and resistance). The load characteristics are emphatically not time-invariant; all loads eventually see impedance collapse. However, because only the Marx erection and early voltage pulse stabilization were of interest in this study, the assumption of a time-invariant load was surprisingly consistent with experimental observations. This is due to the fact that it can take several hundred nanoseconds before plasma expansion in the load, whether from the cathode, anode, or both, begin to reduce the effective Anode-Cathode Gap (AK gap) and change the load characteristics. Closure of the AK gap leads to the subsequent, progressive reduction in load impedance on longer timescales. Many studies in the literature confirm this through the study of plasma closure velocities $[17,88,91,99,105,112,113,124,125]$. Thus, on timescales $>300 \mathrm{~ns}$, the model becomes progressively less reliable.

The simulation circuit diagram is shown in Figs. C.1-C.3. Parameters were adjusted to obtain good agreement with experimental observations. While this approach does not imply the model is necessarily correct given the large number of parameters that can be used to fit any given shot, it does provide insight into the effect of a given parameter on the voltage trace once a reasonable range for each parameter is known. Furthermore, the simulation was successfully used to reproduce several experimental voltage traces with different loads, which provides some confidence in its predictive ability. 


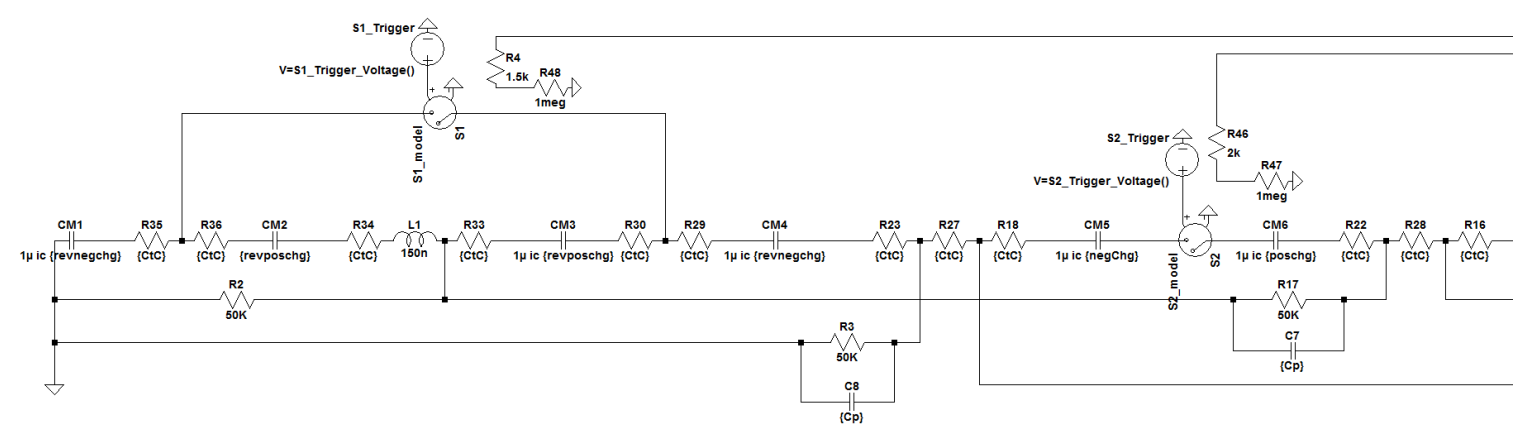

Figure C.1: Left portion of the LTspice MeLBA-C circuit model.

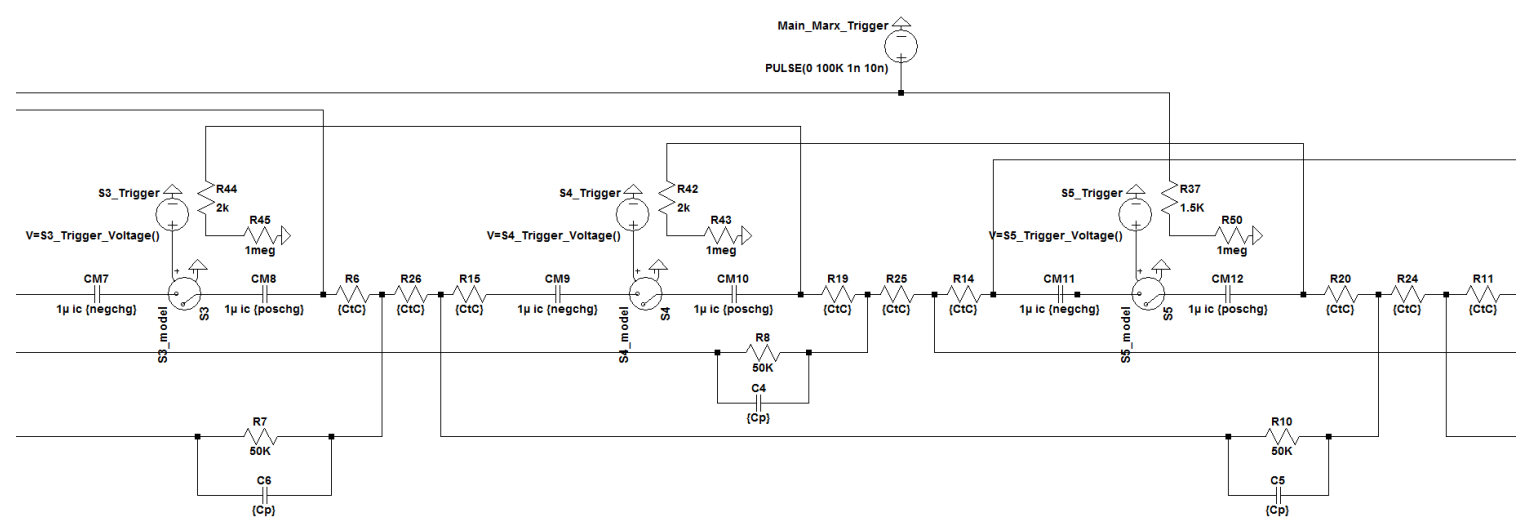

Figure C.2: Middle portion of the LTspice MELBA-C circuit model.

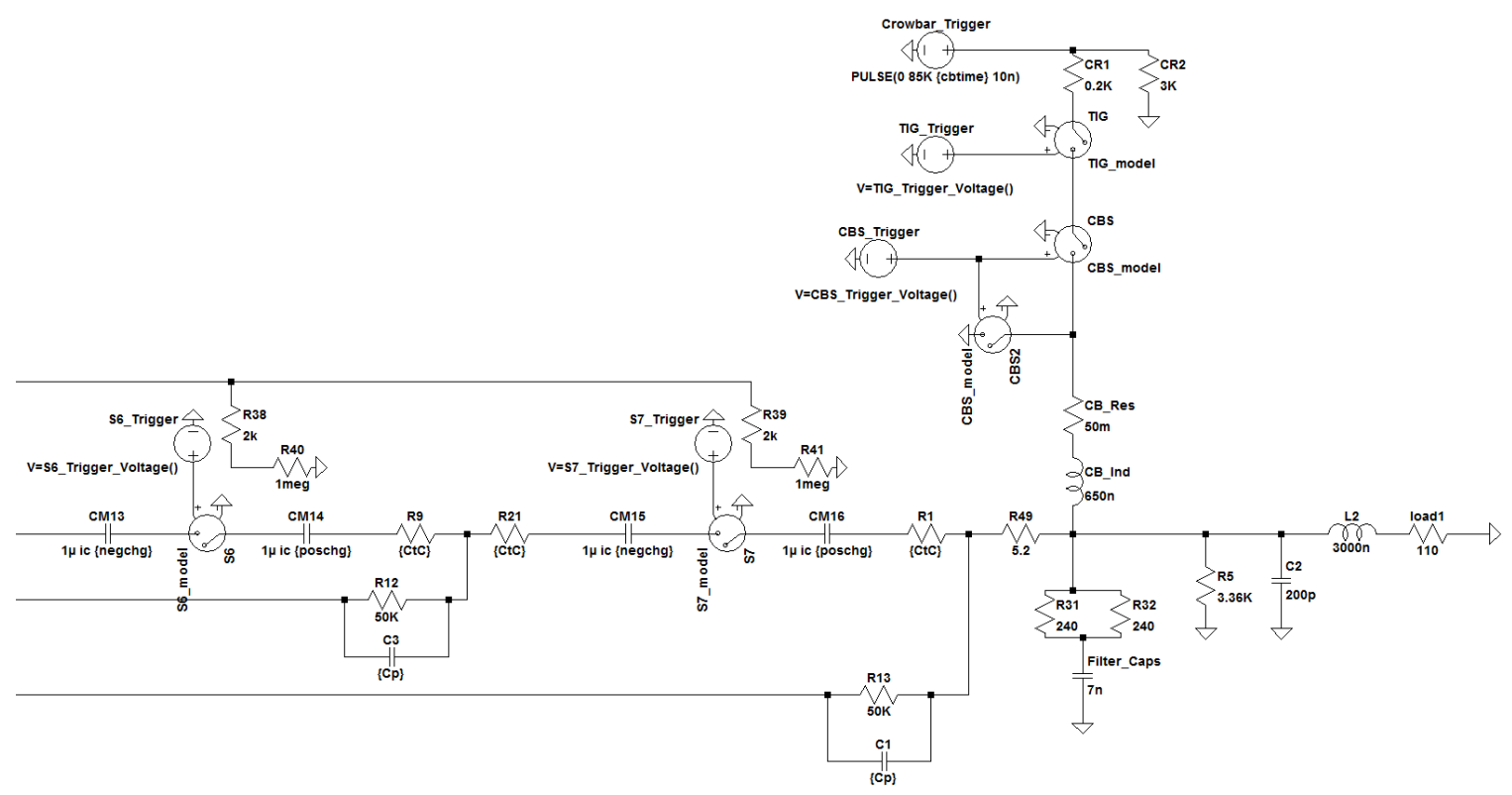

Figure C.3: Right portion of the LTspice MELBA-C circuit model. 


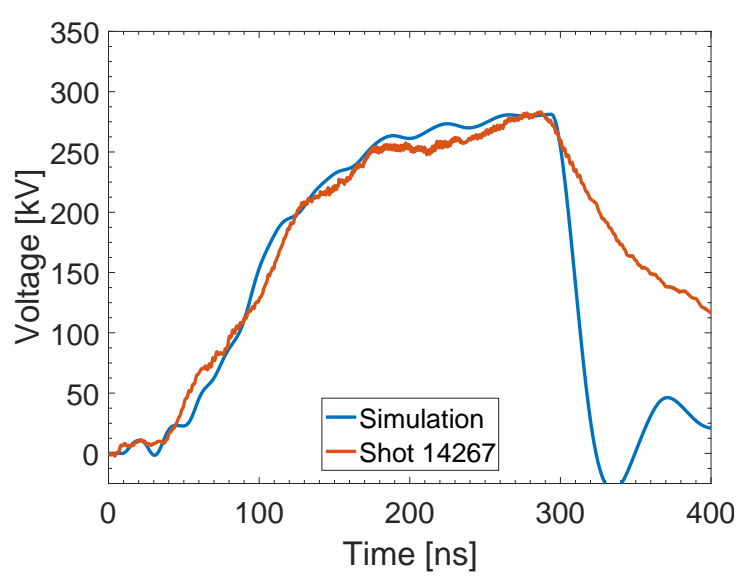

(a) RPM-12A with MCC cathode.

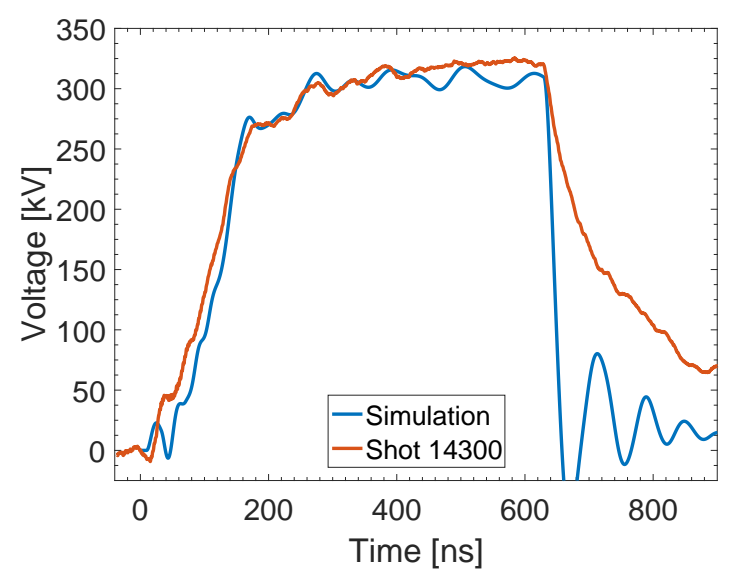

(b) Rod cathode with stainless steel current collector.

Figure C.4: Comparison of LTspice voltage traces with experimental MELBA-C shots.

\section{C.3 Comparison to Experiment}

Figure C.4 shows two comparisons between a simulated voltage trace and an experimental voltage trace using two different loads. In Fig. C.4a, the experimental voltage trace was obtained using the Recirculating Planar Magnetron with Aluminum Anode (RPM-12A) as the load. In Fig. C.4b, the load was a rod cathode emitting an electron beam that was collected directly using a stainless steel plate, which was a configuration used for testing MELBA-C operation, and for calibration of the current diagnostics. After adjusting the characteristics of the different loads in the model, the simulations exhibited good agreement with experiment.

Development of the LTspice model was also motivated by the installation of a new fiber optic switch diagnostic on MELBA-C. By comparing switch closure times in simulation and experiment, a clear trend was observed. Figure C.5 shows a comparison between two simulations. The first trace used a switch model having a high voltage threshold for breakdown and subsequent closure. This was the configuration that was generally consistent with experimental observations. In the model, the voltage on the trigger plane in any switch $\left(\mathrm{V}_{\mathrm{tp}}\right)$ would only initiate breakdown if $\left|\mathrm{V}_{\mathrm{tp}}\right|>85 \mathrm{kV}$. The second trace shows rapid switching with a much lower threshold for switch breakdown, with a breakdown criterion of $\left|\mathrm{V}_{\mathrm{tp}}\right|>10 \mathrm{kV}$. The total time for all switches to close was $90 \mathrm{~ns}$ and $10 \mathrm{~ns}$ for the delayed and rapid models, respectively. The significant observation from this analysis was the elimination of the ringing superposed on the voltage trace. The ringing was due to the transient voltage swings present on the cases of the capacitors due to the case-to-case capacitances. If the switches fail to close with near simultaneity, large oscillations are excited, whereas 


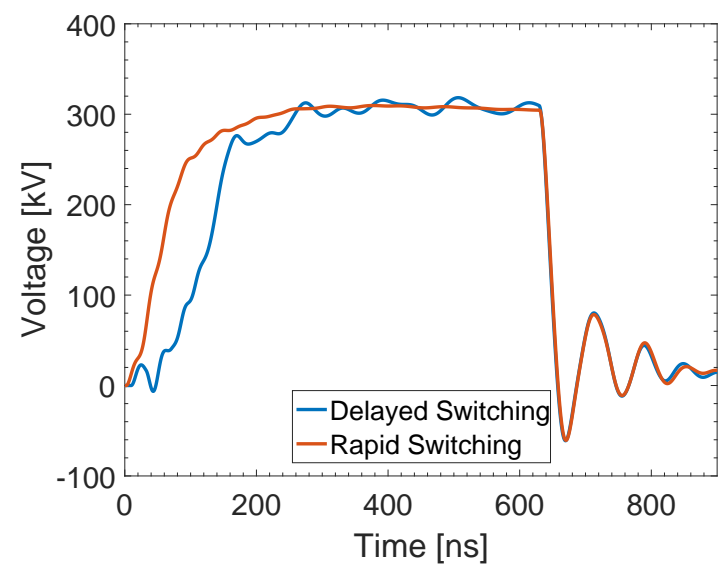

Figure C.5: Comparison of delayed (90 ns) and rapid (10 ns) time to closure of all switches. The delayed simulation is identical to that shown in Fig. C.4b.

rapid closure of all switches produces ideal Marx erection and smooth voltage traces. This model might therefore be useful for exploring alternative trigger resistor placements that maximize the voltage delivered to all switch trigger planes and minimize the time it takes to occur.

\section{C.4 Effect of Switch Timing on Marx Erection}

The effects of the switch closure order and rapidity on the erection of the MELBA-C Marx generator are illustrated by the switch diagnostic comprising fiber optic lines and Photomultiplier Tubes (PMTs) described in $\S$ 4.5.1.3. By comparing the switch closure times (indicated by sharp spikes in the PMT output voltages) with the MELBA-C voltage trace, patterns emerge indicating proper operation and modes of failure.

Four shots were chosen to illustrate the basic types of operation: 1) excellent Marx erection with a low-impedance load, 2) excellent Marx erection with a high-impedance load, 3) normal Marx erection indicative of typical behavior, and 4) poor Marx erection leading to a failed shot. From Figs. C.1-C.3, the proper triggering order of the switches should be $1 \& 5$, followed by $4 \& 6 \& 7$, followed by 3 , followed by 2 .

Figures C.6a and C.6b show the voltage and current traces from ideal shots using a low- and high-impedance load, respectively. The associated switch PMT traces are shown in Figs. C.6c and C.6d. Note that the failure of the voltage to rapidly reach a flat top in Fig. C.6a is due only to the lower impedance of the load and not a switching failure, which illustrates the importance of designing loads matching the 100-150 $\Omega$ specification for MELBA-C. Shot 14772 has a switch closure order of $1 \& 5$, followed by 4, 6, and 7 in 


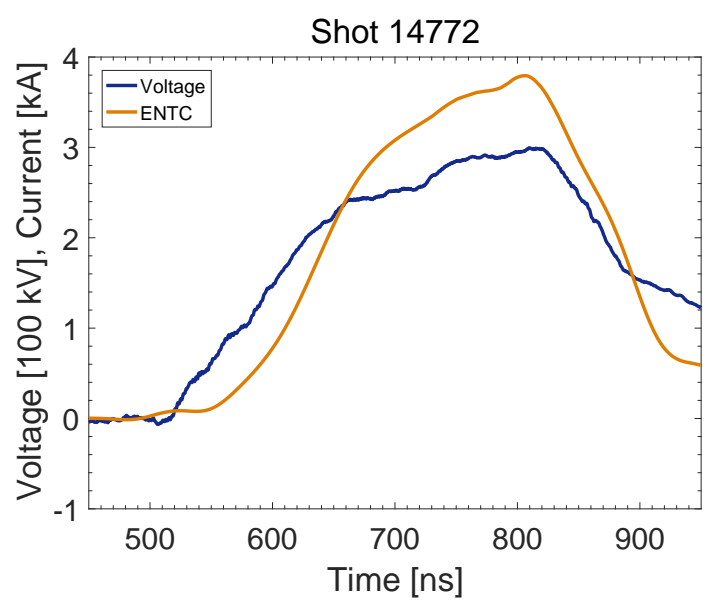

(a) Shot 14772 with a low-impedance load.

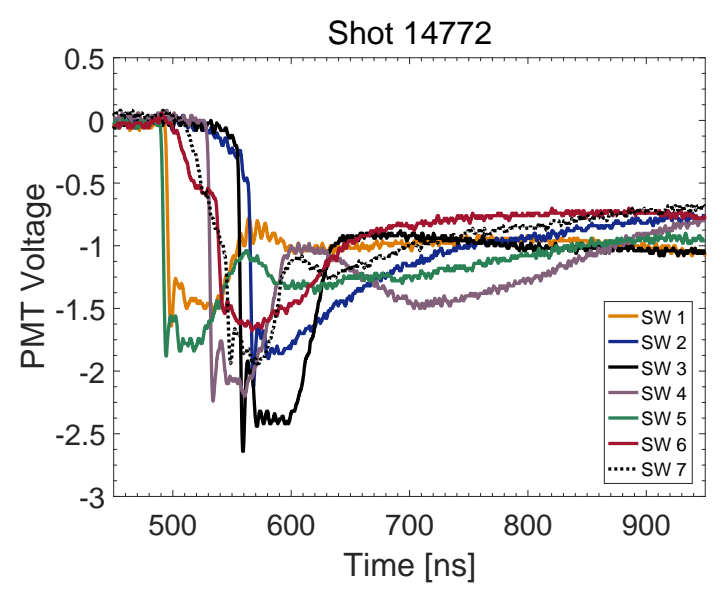

(c) Shot 14772 switch response.

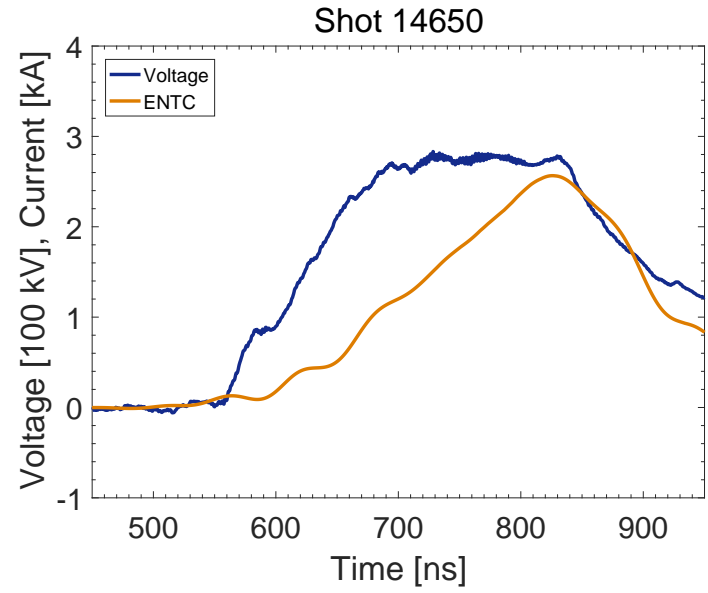

(b) Shot 14650 with a high-impedance load.

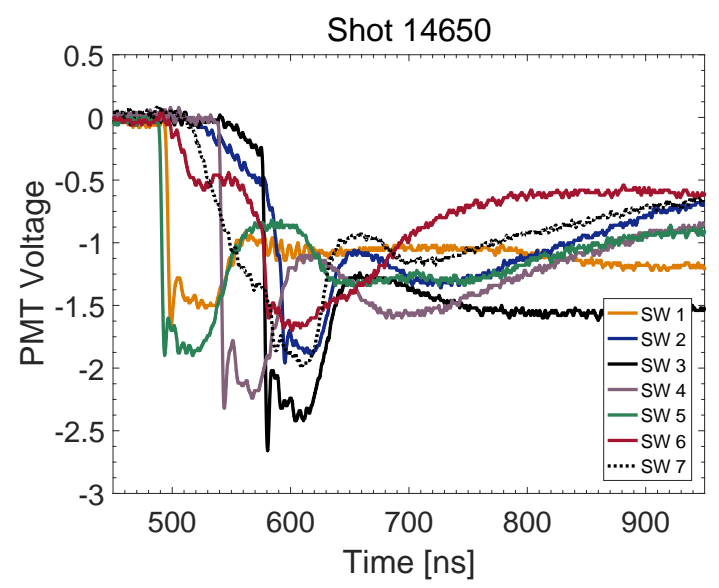

(d) Shot 14650 switch response.

Figure C.6: Sample shots illustrating ideal MELBA-C Marx erection.

rapid succession, followed by 3 and 2 in rapid succession. This precisely matches the ideal switch order, and explains the flawless voltage rise. Shot 14650 has a switch closure order of $1 \& 5$, followed by 4 , followed by a slight delay to $6 \& 3 \& 7$, followed by 2 . The delay is responsible for the small "knee," which is a typical characteristic of MELBA-C voltage traces. Otherwise, the switch order is nearly ideal, leading to very good voltage rise.

Figures C.6a and C.6b show voltage and current traces for standard shots illustrating typical and poor behavior, respectively, with the switch PMT traces in Figs. C.6c and C.6d. The normal characteristics of the "knee" are due to early closure of switches 4, 6, or 7 shortly after switches $1 \& 5$, followed by a delay to closure of the remaining switches. A "knee" can also be produced by switches closing in an incorrect order, although this typically results in a bad voltage pulse rather than the harmless "knee." Shot 14650 is a dramatic illustration of this effect, with a closure order of $1 \& 5$, followed by $4 \& 7$, fol- 


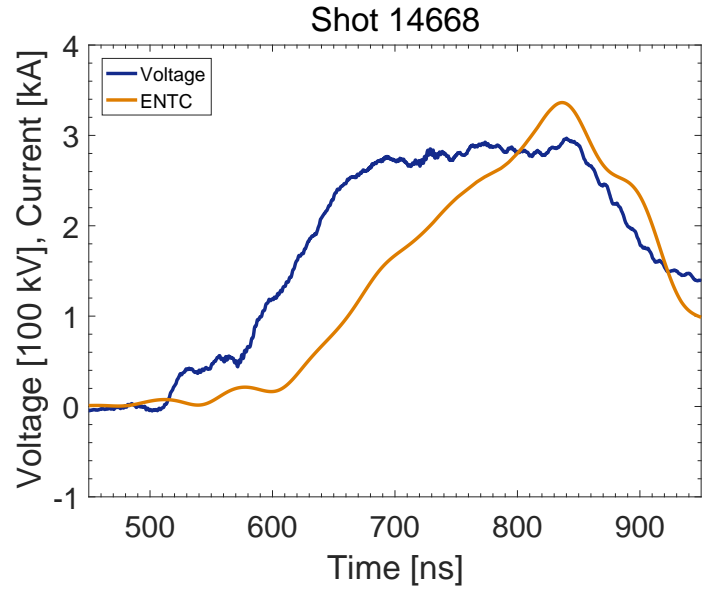

(a) Shot 14668 illustrating typical behavior.

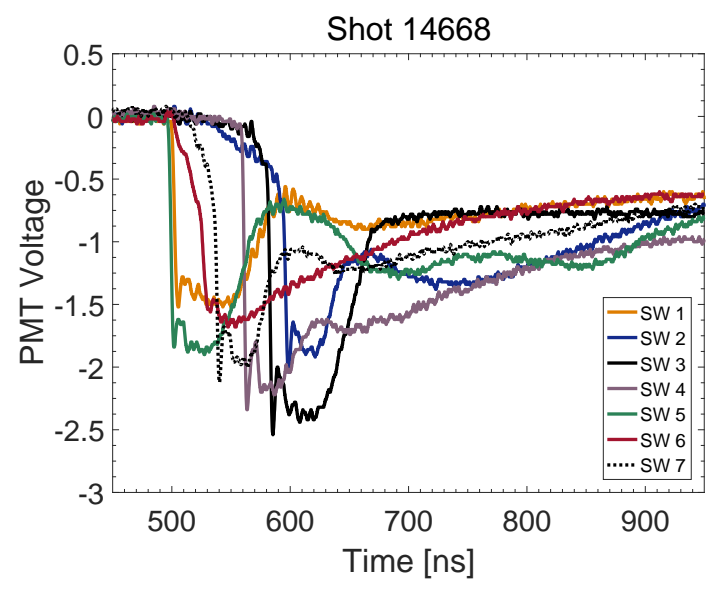

(c) Shot 14668 switch response.

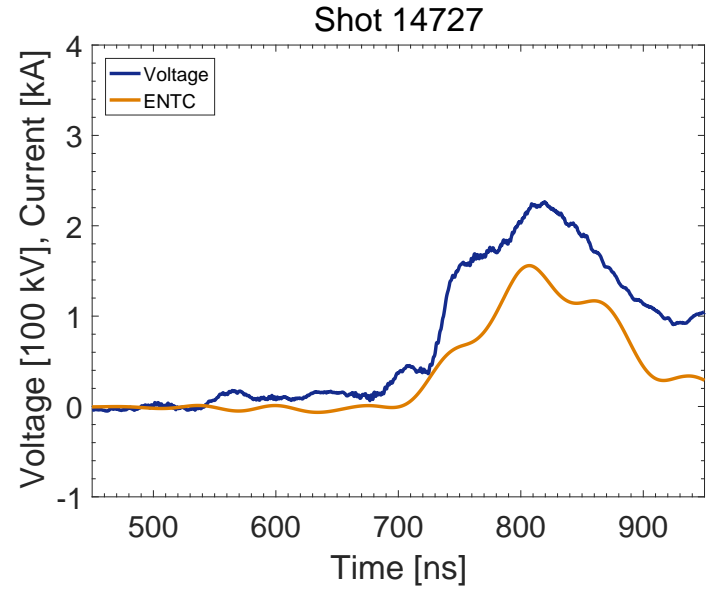

(b) Shot 14727 illustrating poor behavior.

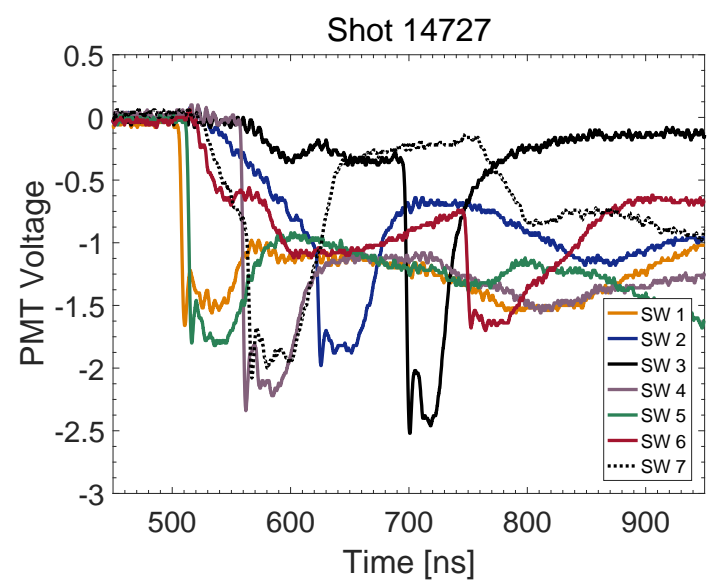

(d) Shot 14727 switch response.

Figure C.7: Sample shots illustrating typical and poor MELBA-C Marx erection.

lowed by 2, 3, 6, and 7 again, with significant time delays between each closure. Switch 7 appears to close twice, with an arc extinguishing due to the very long delay for all switches to close. The poor performance of shot 14727 was likely due to switch 6 failing to close at the correct time. 


\section{APPENDIX D}

\section{Calibrations}

\section{D.1 LBSD Microwave Detectors}

The LBSD microwave detectors were calibrated using the configuration illustrated in Fig. D.1. A digital oscillator was configured to output a $1 \mathrm{GHz}$ signal at low power to a Traveling Wave Tube Amplifier (TWTA), which was isolated from reflections using a three-port circulator. After amplification, the signal was fed to a directional coupler to sample the power using a bolometer. The signal was then fed to the microwave detector, and the detector output was recorded using an oscilloscope terminated at $50 \Omega$. Despite being calibrated under functionally Continuous Wave $(\mathrm{CW})$ conditions, the $50 \Omega$ termination was necessary because it was used during MELBA-C experiments to prevent cable reflections, so the termination must be factored into the calibration. In order to precisely map the power fed to the microwave detector at port 2 using the power measured by the bolometer at port 3, a second bolometer was connected to port 2 to determine the exact

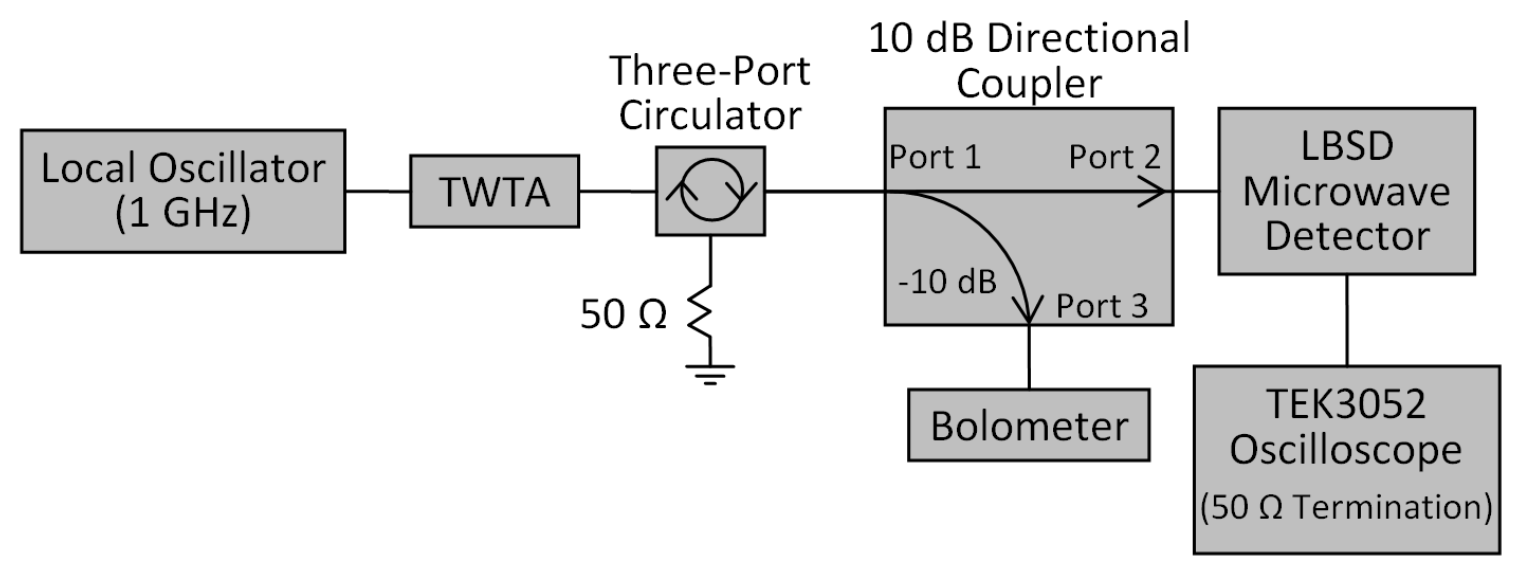

Figure D.1: Block diagram illustrating the LBSD microwave detector calibration configuration. 


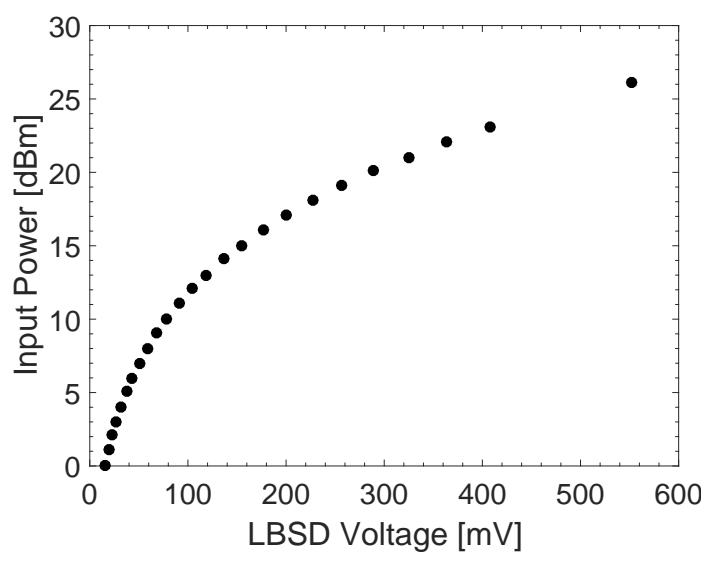

Figure D.2: Sample plot illustrating the calibration points obtained for LBSD HM01 used for MELBA-C shots. The diode output was terminated at $50 \Omega$.

relation between the power measured at port 2 and port 3 . The range of powers fed to the microwave diode (approximately $0-26 \mathrm{dBm}$ ) were chosen so that the diode output voltages recorded by the oscilloscope ranged from the noise floor (approximately $10 \mathrm{mV}$ ) to the voltage output by the diode when fed the maximum short-time safe input power indicated by the diode specifications.

The equipment used was: Agilent E4400B Signal Generator, HP 489A TWTA, JQL JCC0962T1213 N15 Three-Port Ciculators, Narda 3042B-10 Coaxial Directional Coupler, Agilent 8481A Power Sensor, HP 8481A Power Sensor, Agilent E4418B EPM Series Power Meter, HP E4418B EPM Series Power Meter, Tektronix 3052 Oscilloscope, and all diodes were HP 8472B Crystal Diodes. The directional coupler and circulator were confirmed to be functional at the calibration frequency $(1 \mathrm{GHz})$ using the HP 8722D Network Analyzer.

Figure D.2 shows a sample plot illustrating the calibration points for a diode used for MELBA-C shots. A calibration curve was fit to these data using an equation of the form $f(x)=a \ln (x+b)+c x^{d}+e$, where $f(x)$ is the power fed to the diode in $\mathrm{dBm}, x$ is the output voltage from the diode when terminated at $50 \Omega$, and $a, b, c, d$, and $e$ are fit parameters. Fits for all diodes were excellent, with $\mathrm{R}^{2}$ values of 1.000 . Per specifications for the diodes, these fits should be reliable at a broad range of frequencies, with a response $\pm 0.3 \mathrm{~dB}$ from $10 \mathrm{MHz}$ to $12.4 \mathrm{GHz}$. 
Table D.1: Calibration parameters for all diodes.

\begin{tabular}{ccccccc}
\hline & & \multicolumn{5}{c}{ FIT PARAMETERS } \\
\cline { 3 - 7 } DIODE & SERIAL No. & $a$ & $b$ & $c$ & $d$ & $e$ \\
\hline HM01 & 1822A 13237 & 7.252 & 4.703 & $3.540 \mathrm{e}-4$ & 1.441 & -21.39 \\
HM02 & MY4224 2102 & 7.480 & 5.740 & $7.089 \mathrm{e}-5$ & 1.681 & -22.39 \\
ML01 & 1822A 13230 & 7.704 & 6.765 & $1.111 \mathrm{e}-5$ & 1.963 & -23.22 \\
ML03 & MY4224 2127 & 7.902 & 7.732 & $9.044 \mathrm{e}-7$ & 2.304 & -25.03 \\
\hline \hline
\end{tabular}

\section{D.2 Network Analyzer Calibrations}

Several RF components required calibration to determine their frequency-dependent responses. These calibrations were performed using an HP 8722D Network Analyzer.

\section{D.2.1 Directional Couplers}

Four directional couplers were used for this dissertation. In the standard load configuration, WR-650 and WR-340 directional couplers were used to sample the LBO and SBO output signals, respectively. In the configuration used to measure the harmonic power produced by the LBO, additional WR-340 and WR-187 were also used. Figure D.3 shows photographs illustrating the calibration configurations used to obtain the frequency-dependent calibration curves depicted in Fig D.4.

It is important to note that all coupler ports not in use must be terminated using broadband $50 \Omega$ loads, which was especially necessary for the neighboring port on the WR-650 directional coupler used for signal sampling. Failure to do so can affect the measurement by as much as $3 \mathrm{~dB}$. Additionally, the quality of the measurement was impacted by the quality of the waveguide load. In each case, the best load was used. For the WR-650 calibration, this was a coax-to-waveguide antenna terminated using a broadband $50 \Omega$ load. For the WR-340 and WR-187 calibrations, tapered RF-absorbing wedges were used in the waveguide. Load characteristics can be found in Appendix D.2.2. In each case, two calibration curves were obtained using two network analyzer calibration techniques. The first uses an Agilent SMA calibration kit to set the reference planes at the end of the analyzer SMA cables. The second uses a Thru-Reflect-Line (TRL) calibration, which sets the reference planes within the waveguide [126]. Due to the bandwidth limitations of the TRL technique, the curves shown in Fig. D.4 show the results obtained using the SMA calibration. However, agreement was good in all cases between the SMA and TRL calibration methods. 


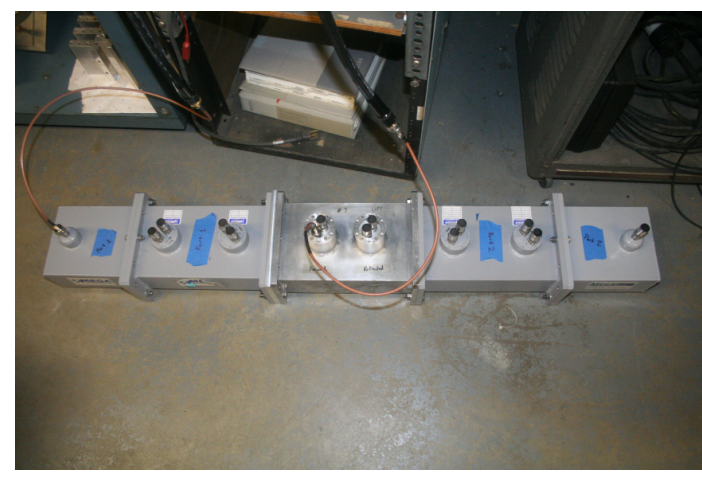

(a) Calibration of the WR-650 directional coupler.

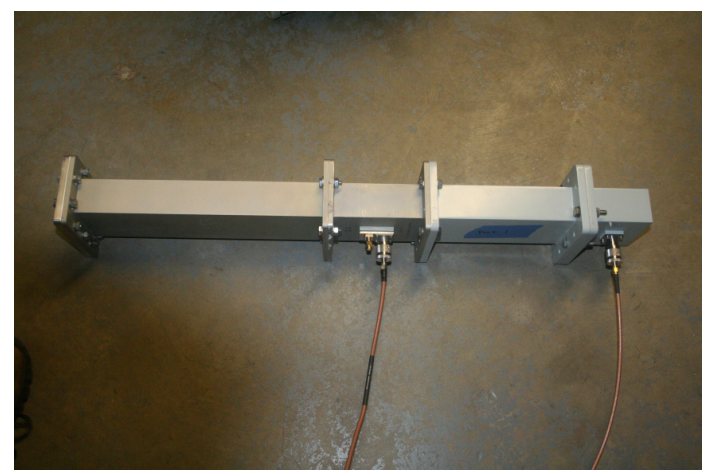

(b) Calibration of a WR-340 directional coupler.

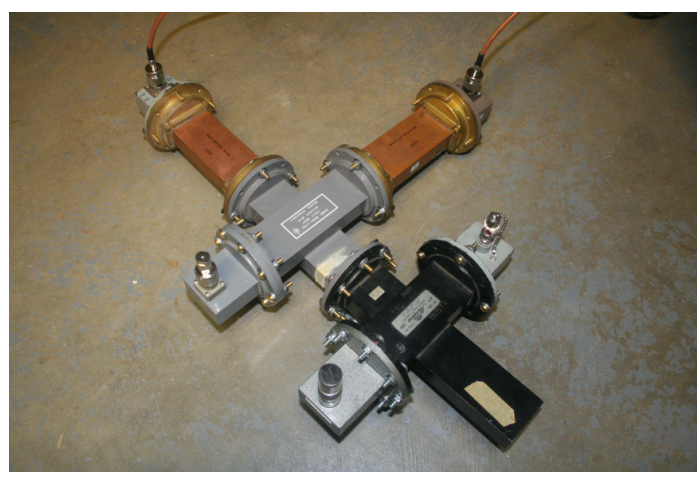

(c) Calibration of the WR-187 directional coupler.

Figure D.3: Photographs illustrating the configurations used to calibrate the directional couplers. 


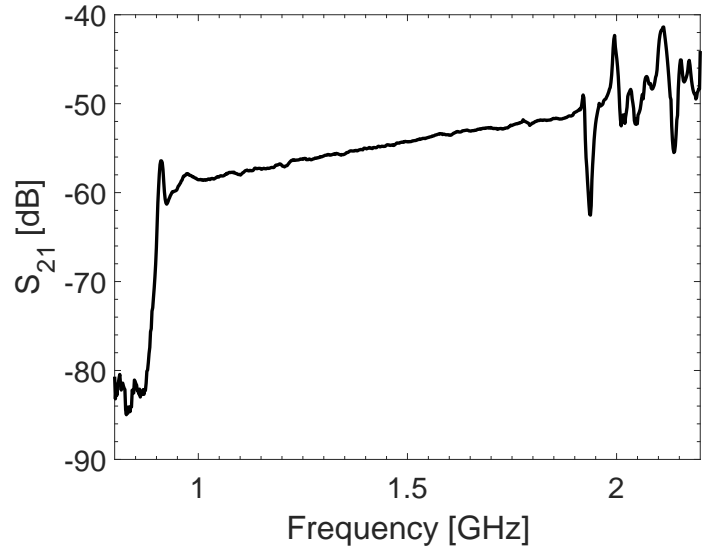

(a) Plot of $\mathrm{S}_{21}$ for the WR-650 directional coupler.

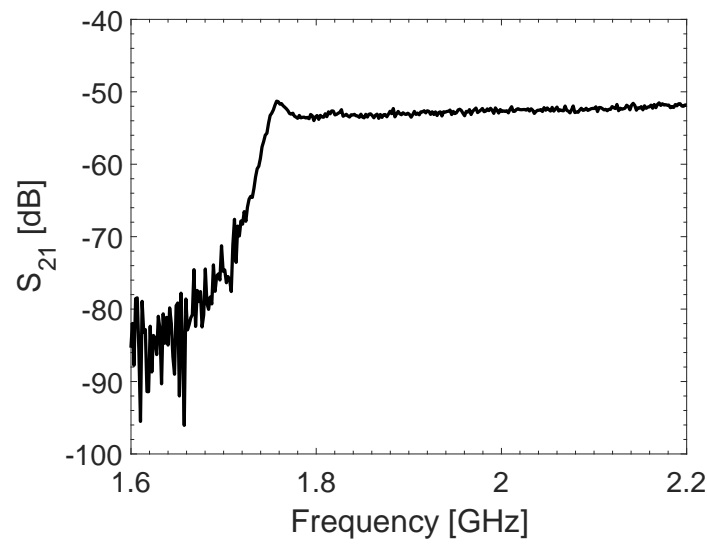

(c) Plot of $\mathrm{S}_{21}$ for the second WR-340 directional coupler.

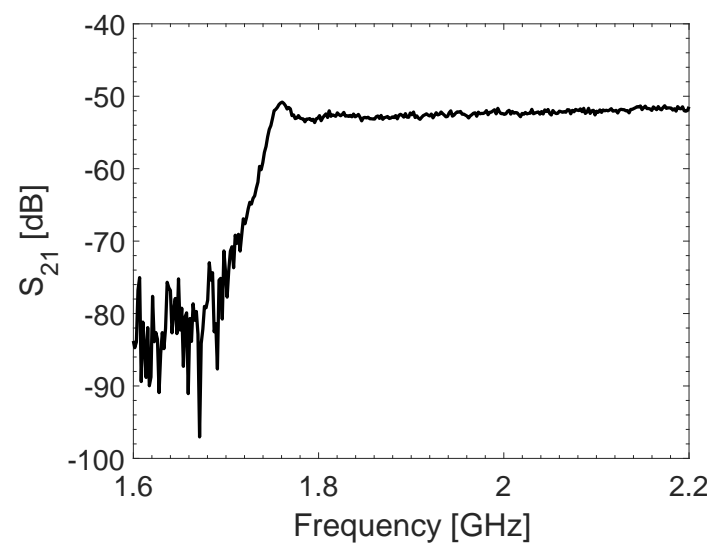

(b) Plot of $\mathrm{S}_{21}$ for the first WR-340 directional coupler.

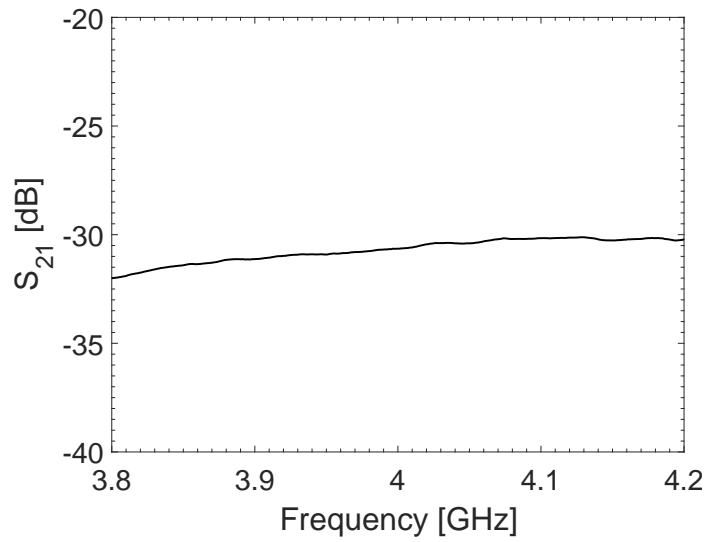

(d) Plot of $\mathrm{S}_{21}$ for the WR-187 directional coupler.

Figure D.4: Frequency-dependent attenuation of waveguide directional couplers. 


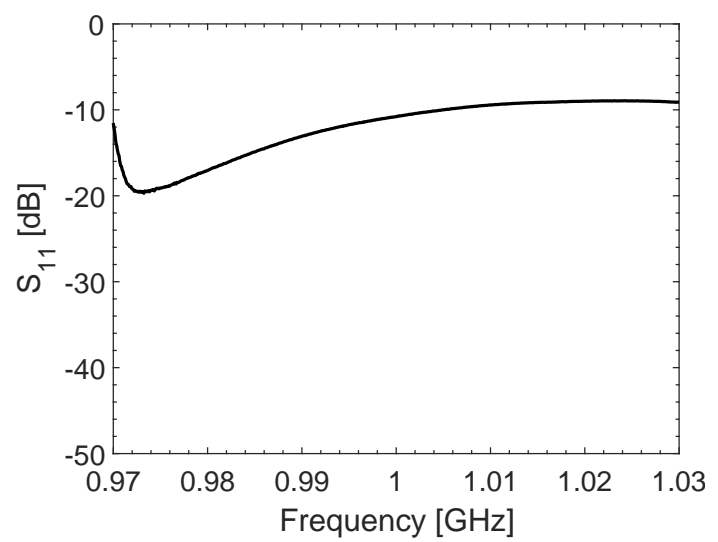

(a) Dark gray WR-650 waveguide load.

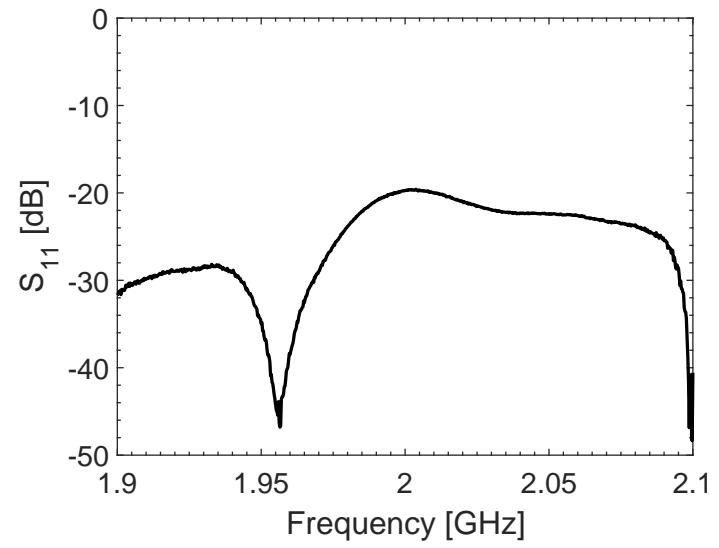

(b) WR-340 waveguide load.

Figure D.5: Examples of waveguide load matches.

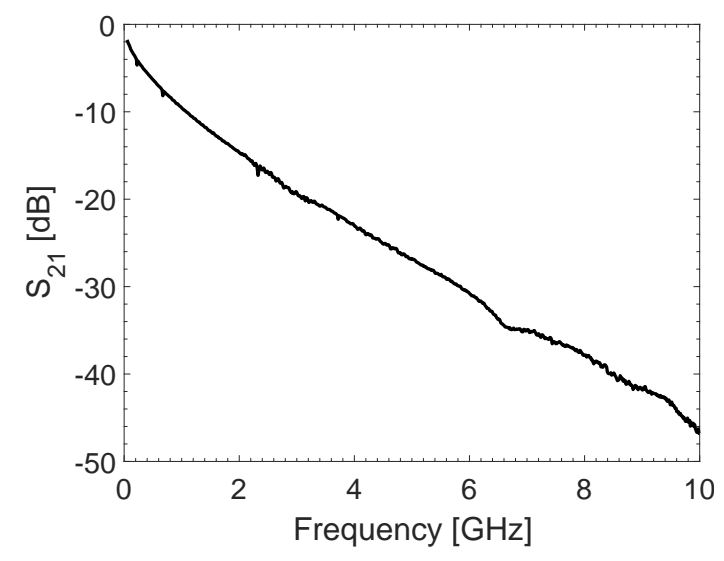

Figure D.6: Sample plot illustrating the attenuation of cables A+B.

\section{D.2.2 RF Load Characteristics}

While not strictly a calibration, it was important to understand the quality of the match of the RF loads over a range of frequencies. The WR-650 waveguide load was filled with fibrous Eccosorb RF absorber. Figures D.5a shows the quality of the match using a measurement of $S_{11}$. The WR-340 load consisted of a tapered wedge of solid Eccosorb RF absorber, which produced a significantly better match relative to the fibrous Eccosorb. Figure D.5b shows the measurement of $S_{11}$.

\section{D.2.3 Cables}

The frequency-dependent attenuation of all cables used to sample the microwave signals was determined by performing four measurements of the attenuation of two cables in series, 
Table D.2: Calibration parameters for all cables.

\begin{tabular}{cccc}
\hline \hline & \multicolumn{3}{c}{ Attenuation [dB] } \\
\cline { 2 - 4 } CABle & $1 \mathrm{GHz}$ & $2 \mathrm{GHz}$ & $4 \mathrm{GHz}$ \\
\hline $\mathrm{A}$ & 4.9 & 7.4 & 11.4 \\
$\mathrm{~B}$ & 4.8 & 7.4 & 11.6 \\
$\mathrm{C}$ & 4.8 & 7.4 & 11.7 \\
$\mathrm{~F}$ & 5.7 & 9.0 & 16.6 \\
\hline \hline
\end{tabular}

Table D.3: Calibrated attenuation for other RF components at frequencies of interest.

\begin{tabular}{cccc}
\hline \hline & \multicolumn{3}{c}{ ATTENUATION [dB] } \\
\cline { 2 - 4 } COMPONENT & $1 \mathrm{GHz}$ & $2 \mathrm{GHz}$ & $4 \mathrm{GHz}$ \\
\hline Splitter 1 & 3.2 & 3.3 & 3.6 \\
Splitter 2 & 3.2 & 3.4 & 3.5 \\
Splitter 3 & 3.2 & 3.3 & 3.6 \\
Splitter 4 & 3.2 & 3.3 & 3.6 \\
LBO Filters & 1.3 & - & - \\
SBO Filters & - & 0.7 & - \\
$90^{\circ}$ Bend & - & - & 0.86 \\
\hline \hline
\end{tabular}

where one cable was connected to another in the screen room, and each cable end was connected to one port on the network analyzer. Figure D.6 shows an example of a result for cables $\mathrm{A}+\mathrm{B}$. The total attenuation of each set was determined at 1,2, and $4 \mathrm{GHz}$. The outcome of this approach is a set of four linear equations in four unknowns, the solution of which provides the attenuation of each cable near 1, 2, and $4 \mathrm{GHz}$. Table D.2 summarizes the results.

\section{D.2.4 Other RF Components}

The attenuation of several other RF components was measured and verified to be relatively frequency-insensitive. Table D.3 shows the results.

\section{D.3 Voltage Monitor}

The MELBA-C voltage divider was calibrated using a pair of Febetron modules to apply negative-polarity high-voltage pulses from approximately $30-80 \mathrm{kV}$ to the cathode stalk with the MELBA-C shorting arm in a retracted position. The calibration standard was a North Star high-voltage probe (PVM-5, 1000:1, Serial No. 1724804, using the paired PVM-5 cable). The probe sampled the cathode stalk voltage from the Marx side of the ceramic insulator, which is the same location from which the voltage monitor samples the 


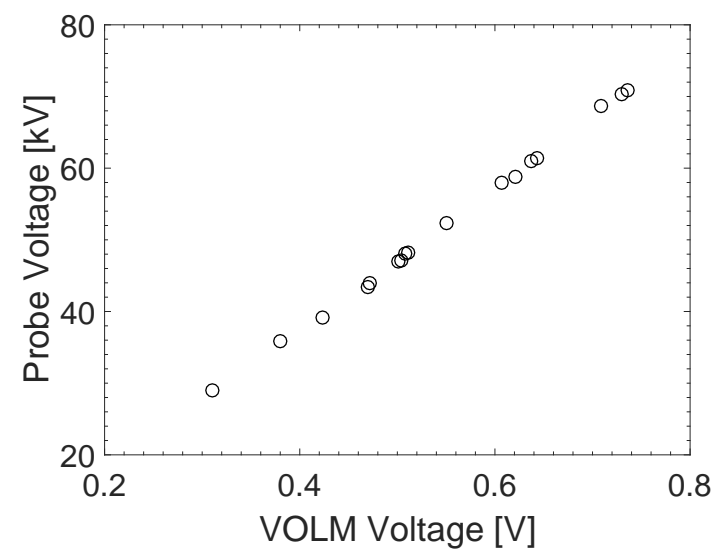

Figure D.7: Plot illustrating the calibration points obtained for the MELBA-C voltage monitor.

MELBA-C pulse. Per specifications, the PVM-5 probe was $\mathrm{M} \Omega$-terminated with no additional attenuation at a Tektronix 3052 oscilloscope, which was used to record the traces. The Voltage Monitor (VOLM) signal was recorded using the same configuration used for MELBA-C shots to incorporate the effects of all components into the calibration. The VOLM cable was connected to a Tektronix 3054 oscilloscope with a 10x attenuator (identification \#6) and terminated at $50 \Omega$ to prevent cable reflections.

Figure D.7 shows the data obtained for the VOLM calibration. These data were fit using a nonzero-offset function. With an $\mathrm{R}^{2}$ value of 0.9990 , the function used for all shots in this dissertation was $f(x)=1.004 \mathrm{E} 5 \times x-3010$, where $f(x)$ is the MELBA-C output voltage in volts, and $x$ is the VOLM voltage in volts. It is apparent that the calibration becomes unreliable at very low voltages due to the non-zero offset of approximately $3 \mathrm{kV}$. Fits with a forced zero offset were relatively poor, so the VOLM response is conclusively linear at high voltages, but some nonlinearity exists at lower voltages outside the range of interest for this dissertation.

\section{D.4 Rogowski Coils}

As discussed in $§ 4.5 .1 .1$, the primary MELBA-C Marx generator diagnostic for measuring the current delivered to a load, called the Entrance Current (ENTC), was a Rogowski coil located in a recessed groove in the main MELBA-C flange. A failure of the coil SMA connector at the beginning of MFRPM experiments led to the fabrication and calibration of a new coil.

The replacement Rogowski coil was 102 inches long, with 1 turn per inch around a 


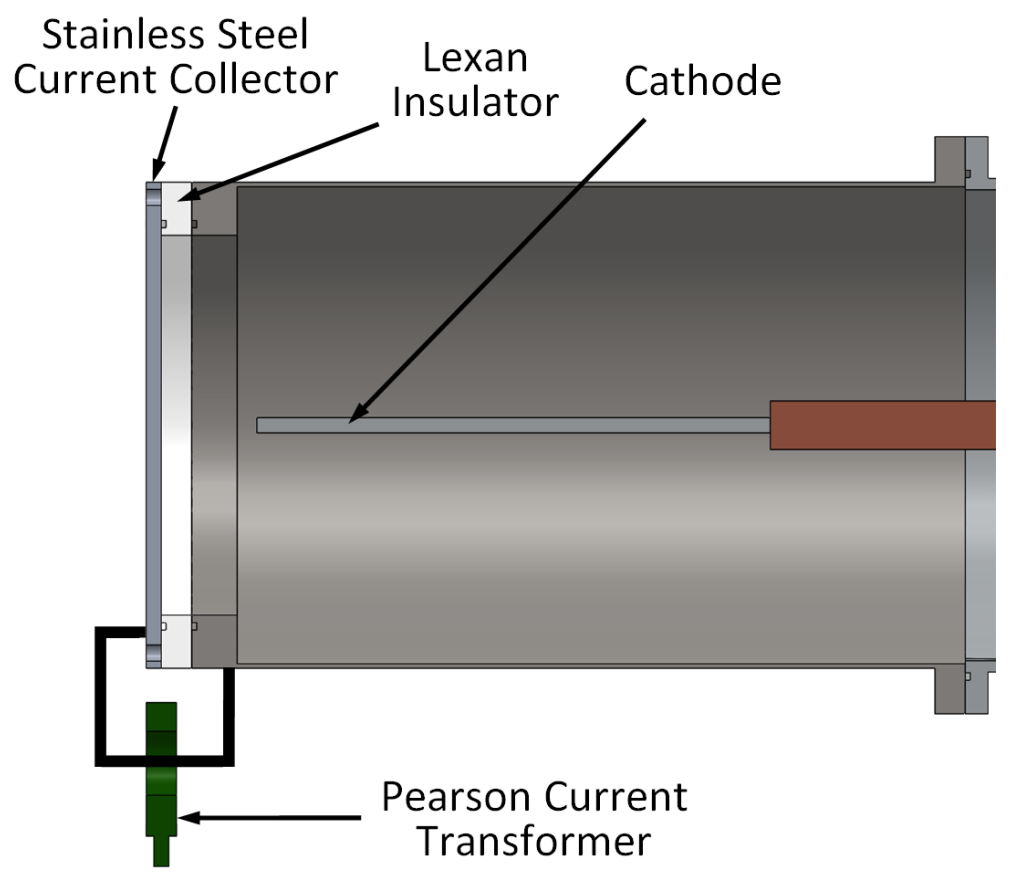

Figure D.8: Calibration configuration for the Rogowski coil current diagnostic.

center conductor composed of RG-58 with the braid and outer jacket removed, leaving only the conductor and dielectric. The coils were formed using 20 AWG stranded wire, which were soldered to the center conductor and insulated using shrink wrap at the far end opposite the connector. At the end with the SMA connector, the coil was soldered to the inner conductor of the SMA cable. The outer SMA braid was soldered to the coil RG-58 center conductor, and all connections were then insulated with shrink wrap.

The calibration standard was a Pearson current transformer (model 110A, label \#4, 0.1 V/A, UM No. 209966), which is a commercial wideband current monitor generally accepted as a reliable diagnostic in the pulsed power and microwave component community [100]. Figure D.8 shows the calibration configuration. A stainless steel current collector was used as the target anode for an electron beam emitted from a velvet surface on the end of an aluminum rod coated in Glyptal insulating enamel. A photograph of the cathode is shown in Fig. D.9. The current was passed through the Pearson current transformer and grounded on the vacuum chamber using the shortest path possible to minimize the inductance. Two additional Rogowski coils, called the Magnetron Entrance Current (MEC) and Endloss Current (ENDL) coils, were placed in the vacuum chamber to confirm the reliability of the ENTC coil, though those coils did not provide reliable measurements in the presence of HPM generated by the magnetron.

All Rogowski signals were captured using a Tektronix 3054 oscilloscope using $50 \Omega$ 


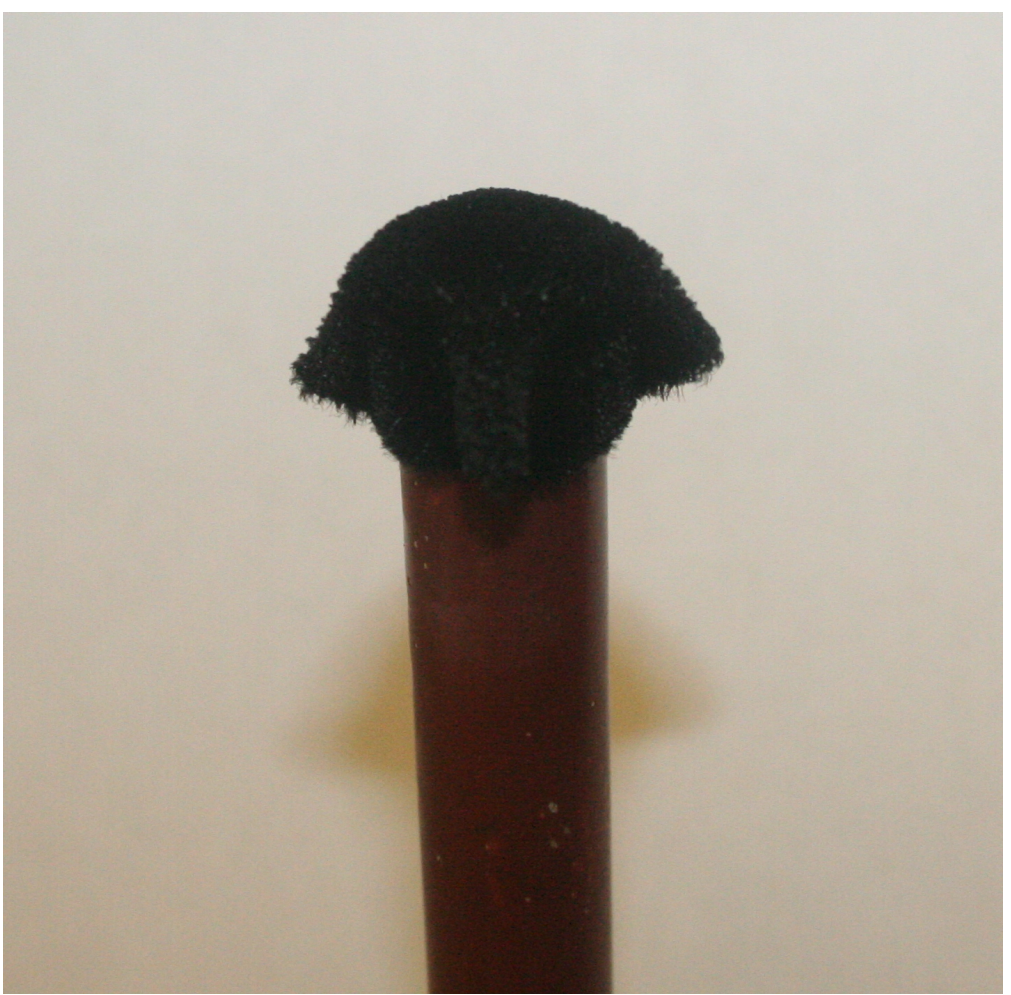

Figure D.9: Photograph of the rod cathode with a velvet electron emitter used for calibrating the MELBA-C Rogowski coils. 


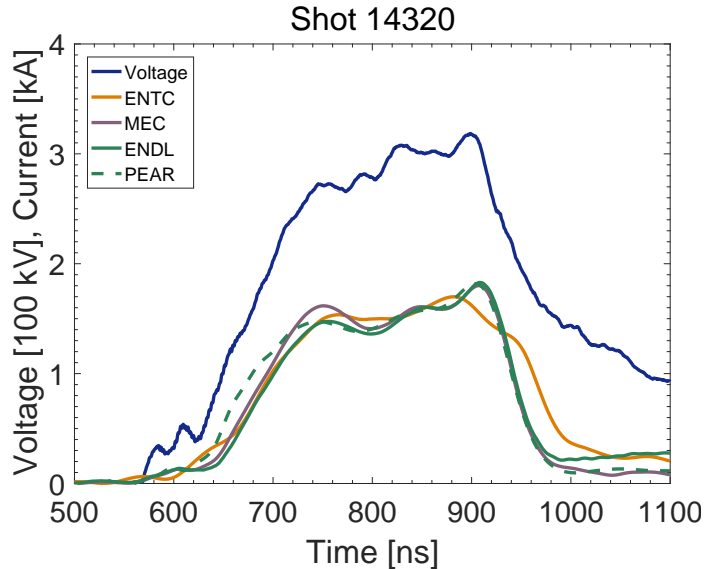

(a) Calibrated currents for shot 14320 (short pulselength).

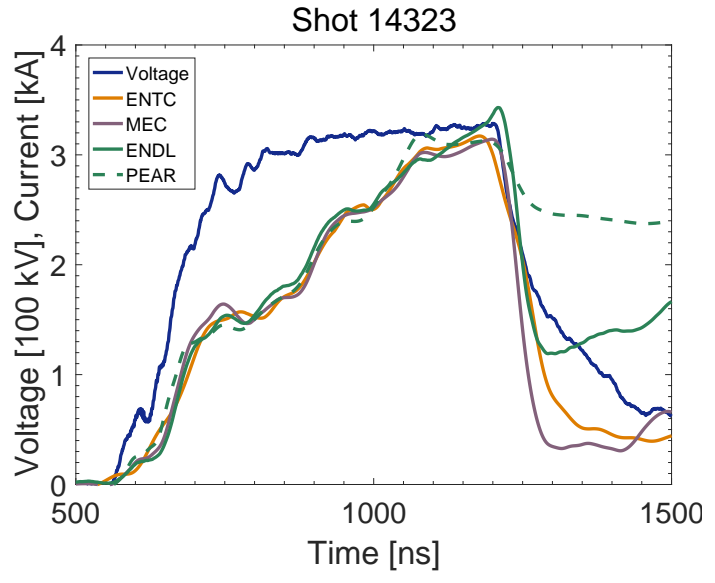

(b) Calibrated currents for shot 14323 (long pulselength).

Figure D.10: Calibrated currents for two MELBA-C shots using a rod cathode and stainless steel current collector.

termination. The oscilloscopes had a sufficiently high sampling rate to resolve frequencies up to $500 \mathrm{MHz}$. To avoid possible frequency artifacts due to higher frequencies, all Rogowski signals were first passed through $500 \mathrm{MHz}$ low-pass filters.

Figure D.10 shows two sample shots used to calibrate the ENTC Rogowski coil (replotted using the new calibration). The calibration was produced by generating a multiplicative factor to adjust the ENTC response to equal the Pearson coil current during the voltage pulse time of interest, which was a temporal processing window defined as the middlemost $75 \%$ set of data points in the range of time between the start and end of the voltage pulse (defined as $70 \%$ of the peak voltage). This temporal range avoids the extremes of voltage rise and fall, as well as unreliable measurements obtained post-crowbar (during which considerable swings in ground potential are observed all over MELBA-C). During the temporal processing window, stable operation of the MELBA-C pulser and consistent current (or rate of current increase) was drawn by the load. Times falling outside of the aforementioned processing window used a multiplicative factor equal to the mean of all multiplicative factors in the processing window. The overall vector of multiplicative factors was then smoothed using a 50-point window width. Correction vectors from multiple shots with a variety of pulselengths were then averaged to obtain a single representative vector of correction factors for each digitized time step.

It is important to note that the validity of the ENTC Rogowski coil calibration is predicated on the MELBA-C stack resistors having a large resistance relative to the magnetron. The reason is that the ENTC coil measures both the current emitted from the cathode and the current through the stack resistors because both current paths lie inside of the coil. As 


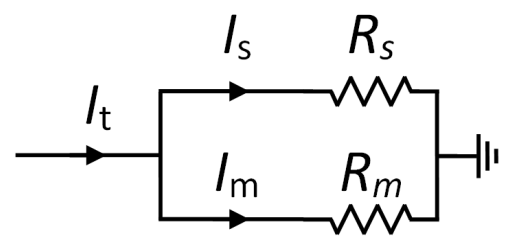

Figure D.11: Circuit diagram showing the paths of the current detected by the ENTC Rogowski coil.

stated, the calibration used a precise measurement of only the cathode current and showed very clear evidence in Fig. D.10 that the final calibration reliably recovered the desired cathode current, but a simple mathematical argument also reinforces the reliability of this calibration despite the stack resistor current.

Figure D.11 shows a simple circuit diagram of the paths of the current detected by the ENTC Rogowski coil. In the diagram, $I_{t}$ is the total current, $I_{s}$ is the current through the stack resistors, $I_{m}$ is the magnetron current, $R_{s}$ is the stack resistance, and $R_{m}$ is the magnetron impedance. During a shot, only the quantity $R_{s}$ is static because the magnetron impedance and MELBA-C voltage change over time. Current conservation implies $I_{t}=$ $I_{s}+I_{m}$, and for a MELBA-C voltage $V$, it follows that

$$
V=I_{s} R_{s}=I_{m} R_{m},
$$

so the total current $I_{t}$ can be rewritten as

$$
I_{t}=V\left(\frac{1}{R_{s}}+\frac{1}{R_{m}}\right) .
$$

Let $V_{c}$ be the Rogowski coil voltage. Then

$$
\begin{aligned}
V_{c} \propto \frac{d I_{t}}{d t} & =\frac{d}{d t}\left[V\left(\frac{1}{R_{s}}+\frac{1}{R_{m}}\right)\right] \\
& =\frac{d V}{d t}\left(\frac{1}{R_{s}}+\frac{1}{R_{m}}\right)+V \frac{d}{d t}\left(\frac{1}{R_{m}}\right) \\
& =\frac{d V}{d t}\left(\frac{R_{m}+R_{s}}{R_{m} R_{s}}\right)+V \frac{d}{d t}\left(\frac{1}{R_{m}}\right) \\
& =\frac{d V}{d t}\left(\frac{R_{s}}{R_{m} R_{s}}\right)+V \frac{d}{d t}\left(\frac{1}{R_{m}}\right) \\
& =\frac{d V}{d t}\left(\frac{1}{R_{m}}\right)+V \frac{d}{d t}\left(\frac{1}{R_{m}}\right)
\end{aligned}
$$




$$
\begin{aligned}
& =\frac{d}{d t}\left(\frac{V}{R_{m}}\right) \\
& =\frac{d I_{m}}{d t} \\
\Rightarrow V_{c} & \propto \frac{d I_{m}}{d t}
\end{aligned}
$$

which is the desired relation for the current diagnostic. In the process of obtaining Eq. D.3, $R_{s}$ was assumed to be a constant, and it was further assumed that $R_{m} \ll R_{s}$. The latter assumption is valid because $R_{m}$ was typically $<200 \Omega$ and $R_{s} \simeq 8 \mathrm{k} \Omega$.

The error can be estimated by finding $d I_{t} / d t-d I_{m} / d t$, since $d I_{t} / d t$ is what the coil measures and $d I_{m} / d t$ is the desired quantity to measure. The error is then

$$
\begin{aligned}
\text { Error } & =\frac{d I_{t}}{d t}-\frac{d I_{m}}{d t} \\
& =\left[\frac{d V}{d t}\left(\frac{1}{R_{s}}+\frac{1}{R_{m}}\right)+V \frac{d}{d t}\left(\frac{1}{R_{m}}\right)\right]-\left[\frac{d V}{d t}\left(\frac{1}{R_{m}}\right)+V \frac{d}{d t}\left(\frac{1}{R_{m}}\right)\right] \\
& =\frac{d V}{d t}\left(\frac{1}{R_{s}}\right)
\end{aligned}
$$

which shows that the error depends on $d V / d t$ and the stack resistance. However, to obtain the current, the Rogowski coil response is integrated. Using $V=300 \mathrm{kV}$, a rise-time of $150 \mathrm{~ns}$, and the stack resistance $R_{s} \simeq 8 \mathrm{k} \Omega$, the integrated error after the voltage plateaus is $300 \mathrm{kV} / 8 \mathrm{k} \Omega$, or $38 \mathrm{~A}$. For a magnetron current of $3 \mathrm{kV}$, this error is $1.3 \%$.

\section{D.5 Magnetic Fields}

Direct magnetic field measurement during magnetron shots was infeasible because the interaction space in the magnetron AK gap was filled with an intense electron beam and subjected to electric fields $>100 \mathrm{kV} / \mathrm{cm}$. By necessity, determination of the magnetic field involved a process that related the electromagnet current to magnetic field magnitudes sampled using a Hall probe during an earlier calibration procedure. This section describes that procedure and reports the calibration functions used for this dissertation.

Due to the absence of cylindrical symmetry in the MFRPM and its configuration variations, the magnetic field was sampled in a variety of locations to determine how the asymmetry affected the magnetic field in the AK gap. The locations were described earlier in Chapter 4 and shown by Fig. 4.8a. At each location, a Hall probe was used to sample the magnetic field vs. time for a variety of magnet capacitor bank charges. Figure D.12a shows 
a photograph of the Hall probe positioned in the cylindrical bend, and Fig. D.12b shows an example illustrating the traces from the Hall probe and electromagnet Pearson coil during an electromagnet pulse. Figures $4.8 \mathrm{~b}$ and $4.8 \mathrm{c}$ in Chapter 4 indicate that the magnetic field profile was highly dependant on the Marx trigger delay separating the electromagnet trigger time from the Marx trigger time. The reason for this dependence stems from the magnetic field diffusion time through metals. Because the amount of metal through which the field needed to diffuse was different depending on the location within the magnetron, different Marx trigger delays produced substantially different magnetic field profiles along the planar cavity arrays. Appendix E describes the process used to determine an optimal Marx trigger delay time that produced a nearly uniform magnetic field.

The primary magnet diagnostic during magnetron shots was the electromagnet Pearson coil, so a calibration curve was derived to relate data from the Pearson coil to the Hall probe results. For each sampled location and electromagnet capacitor bank charge, the magnetic field detected by the Hall probe at the time corresponding to the Marx trigger delay was plotted against the peak electromagnet current. Curve fits were then applied to the data to determine the calibration functions. Figures D.12c-D.12e show some of the results. Therefore, for a given peak electromagnet current, the calibration curves provide a representative magnetic field that is the average of the fields sampled at each location within the magnetron. Note that the presence or absence of the large aluminum vacuum flange on the front of the chamber was not found to affect the magnetic field in the magnetron.

Only the nonuniform magnetic field exhibited significant nonlinearity in the relation between the magnetic field and the electromagnet current. From Figs. D.12c-D.12e, the magnetic fields at the optimized trigger delay times exhibited strong linearity with electromagnet current. Therefore, significantly fewer capacitor bank charges were explored during the magnetic field calibrations for the isolated LBO and SBO configurations. Table D.4 summarizes the calibration functions used for each experimental configuration.

In all configurations, the cathode was the MCC-2. The electromagnet Pearson coil was a Model 301X, $0.01 \mathrm{~V} / \mathrm{A}$, and was terminated at the oscilloscope at $50 \Omega$. The Hall probe was a LakeShore HMNA-1908-VR controlled using a LakeShore 475 Gaussmeter controller. Pin 2 was used to monitor the probe response, and the Gaussmeter was operated in "Peak" mode. The reliability of the Hall probe was confirmed using independent measurements obtained using a $\dot{B}$ loop. 


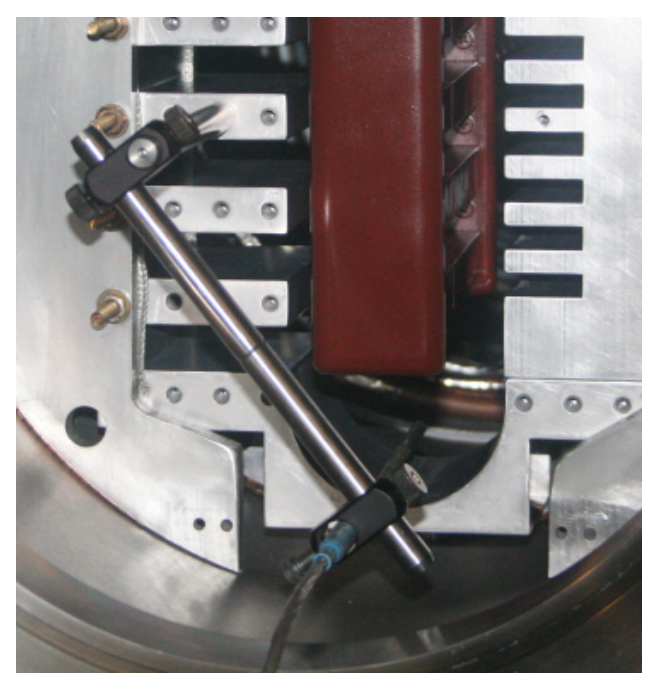

(a) Photograph of the Hall probe positioned in a cylindrical bend during magnetic field measurements.

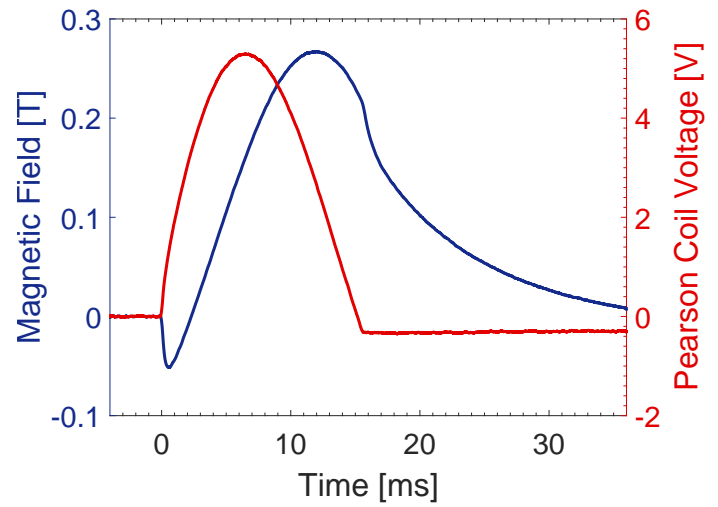

(b) Example Hall probe and electromagnet Pearson coil traces vs. time.

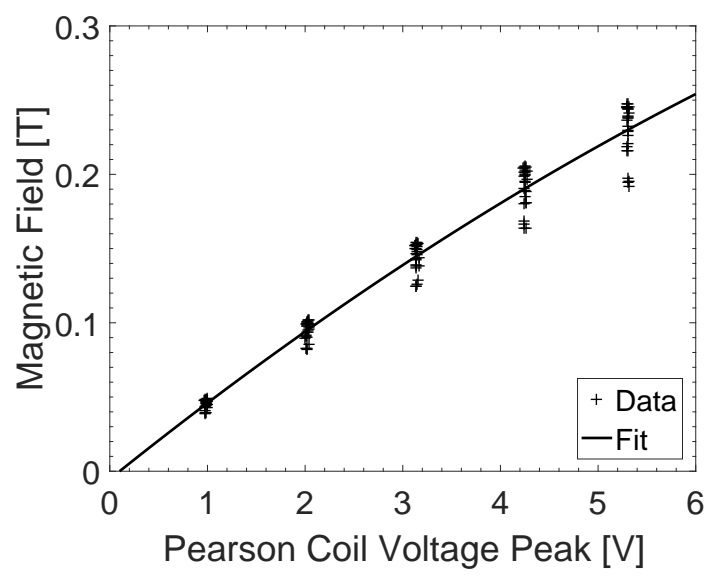

(d) Calibration curve for the nonuniform magnetic field and MFRPM configuration.

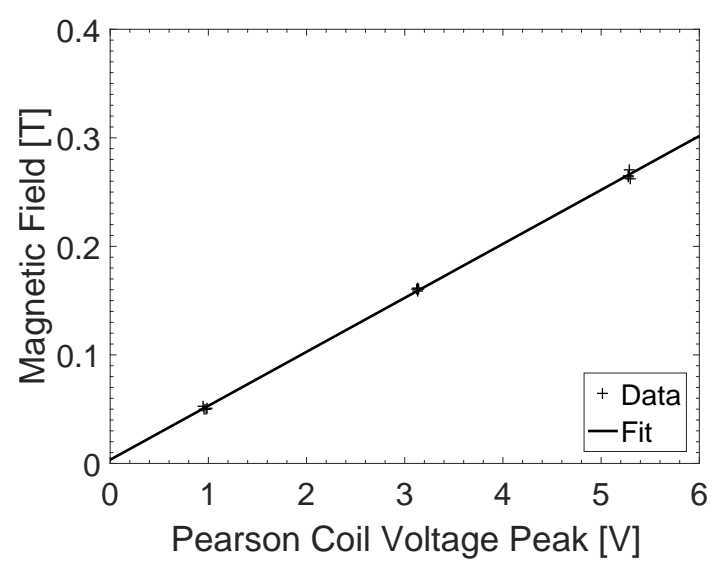

(c) Calibration curve for the uniform magnetic field and isolated SBO configuration.

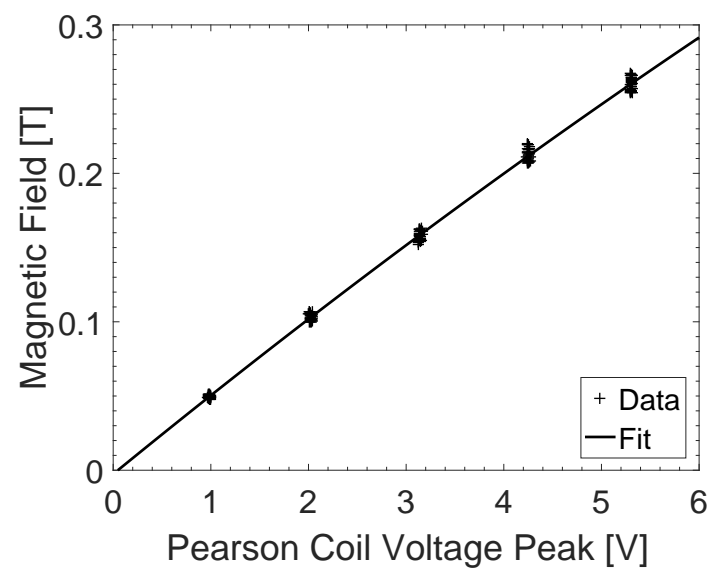

(e) Calibration curve for the uniform magnetic field and MFRPM configuration.

Figure D.12: Calibration of the magnetic field. 
Table D.4: Magnetic field calibration functions $f(x)$ for the different experimental configurations, where $f(x)$ is the magnetic field in Tesla, and $x$ is the peak voltage from the electromagnet Pearson coil.

\begin{tabular}{clcl}
\hline \hline ANODE & FIELD DESC. & TRIGGER DELAY [ms] & $f(x)$ \\
\hline Iso. LBO & Uniform & 12.7 & $0.515 x$ \\
Iso. SBO & Uniform & 11.3 & $0.04972 x+0.003376$ \\
MFRPM & Nonuniform & 9.7 & $-0.001606 x^{2}+0.05287 x-0.005302$ \\
MFRPM & Uniform & 13.7 & $-0.0007918 x^{2}+0.05377 x-0.002589$ \\
\hline \hline
\end{tabular}




\section{APPENDIX E}

\section{Magnetic Field Optimization}

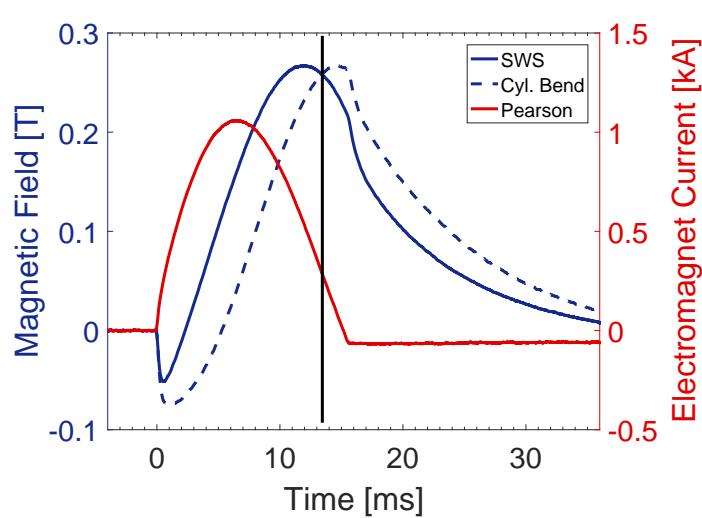

(a) Magnetic fields in two MFRPM locations and electromagnet current vs. time. Current was measured using a Pearson coil.

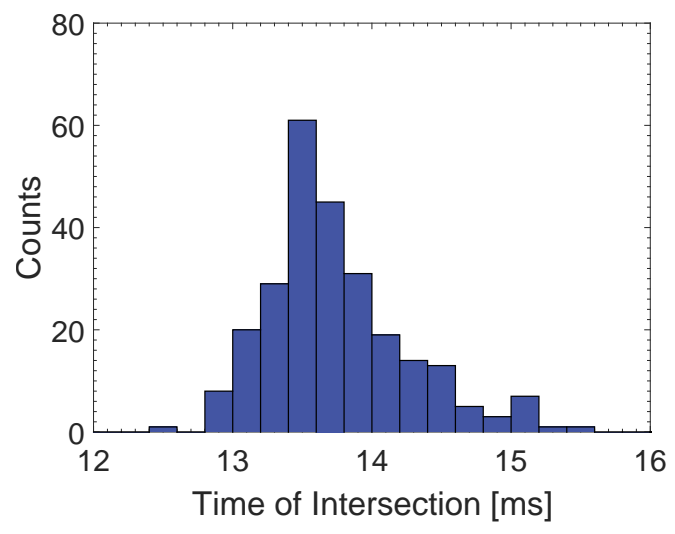

(b) Distribution of times of identical magnetic fields for different MFRPM locations relative to the cylindrical bends.

Figure E.1: Identification of the optimal Marx trigger delay time.

This appendix section describes the process by which a delay time was chosen between the electromagnet trigger and the MELBA-C Marx generator trigger to produce a nearly uniform axial magnetic field in the MFRPM. This process builds on the discussion of the electromagnet in $\S 4.2$ and uses the results from the calibration procedure outlined in Appendix D.5.

Figure E.1a shows the magnetic fields in two different MFRPM locations vs. time. Inspection of the figure reveals the existence of a time at which the magnetic field was the same in both locations, which is shown by the vertical black line. By using the cylindrical bends as the reference locations, a distribution of times was generated by identifying the times at which the magnetic fields were identical in both a cylindrical bend and one of the probed locations discussed previously and shown in Fig. 4.8a. An example distribution for the MFRPM is shown in Fig. E.1b. The mean of the distribution was used as the 
optimal delay time between the electromagnet trigger and the MELBA-C Marx generator trigger, less the delay time for the erection of the Marx generator. Since the timescales of MELBA-C pulses were typically $<300 \mathrm{~ns}$, the magnetic field was functionally static and nearly uniform from the perspective of the magnetron. Any changes to the magnetron configuration, such as the replacement of an oscillator by a smooth drift region for the isolated configurations, required the optimization process to be repeated to determine the optimal delay time. The optimal times were tabulated in the Magnet Calibration section in Table D.4. 


\section{APPENDIX F \\ MFRPM with Taper Load and Unoptimized Magnetic Field: Plots of Frequencies vs. Magnetic Field}

This appendix section contains all the plots comparing the fundamental and harmonic frequencies of the LBO and SBO vs. magnetic field. They are included for completeness. Most plots were used to confirm the absence of any meaningful harmonic correlation between different frequencies. Note that the plots omit SBO harmonics because they did not appear to have been generated at detectable power levels. 


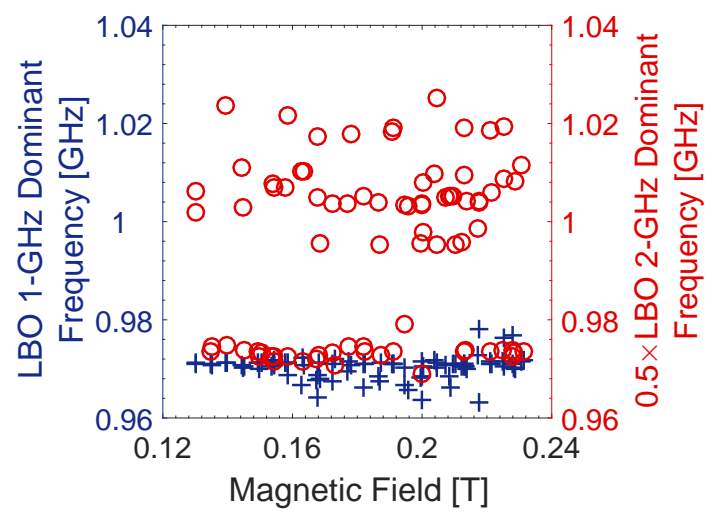

(a) LBO dominant $1 \mathrm{GHz}$ and LBO dominant $0.5 \times 2 \mathrm{GHz}$ frequencies vs. magnetic field.

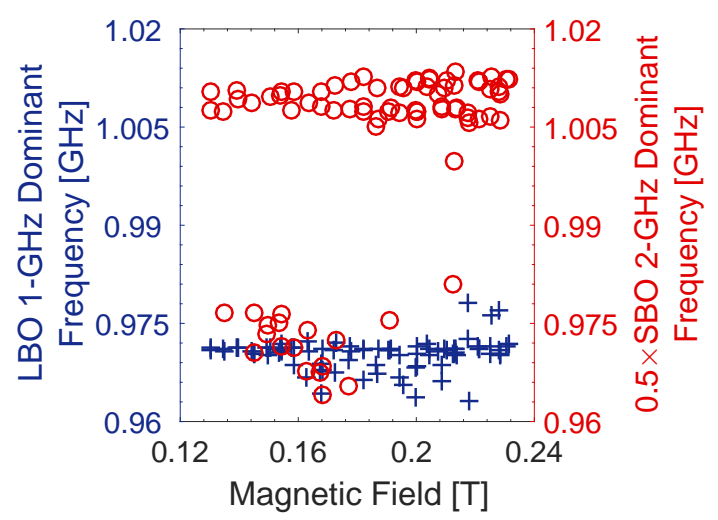

(c) LBO dominant $1 \mathrm{GHz}$ and $\mathrm{SBO}$ dominant $0.5 \times 2 \mathrm{GHz}$ frequencies vs. magnetic field.

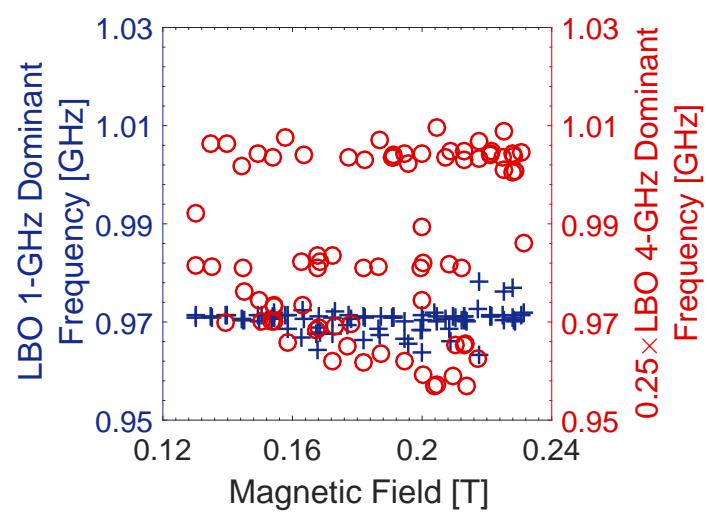

(e) LBO dominant $1 \mathrm{GHz}$ and LBO dominant $0.25 \times 4 \mathrm{GHz}$ frequencies vs. magnetic field.

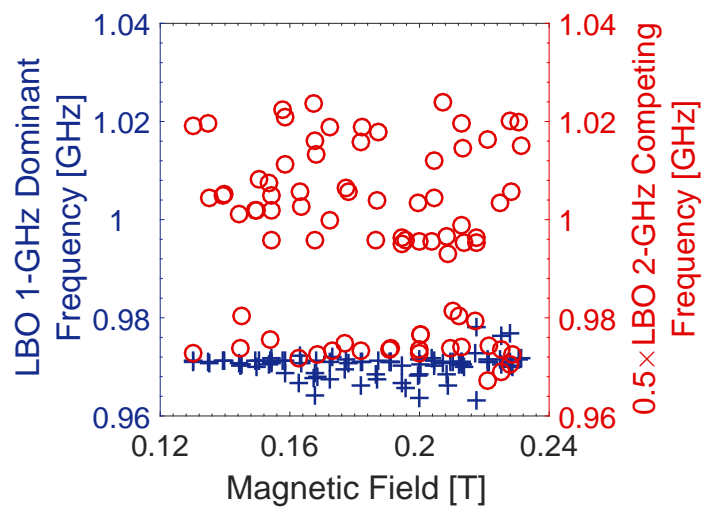

(b) LBO dominant $1 \mathrm{GHz}$ and LBO competing $0.5 \times 2 \mathrm{GHz}$ frequencies vs. magnetic field.

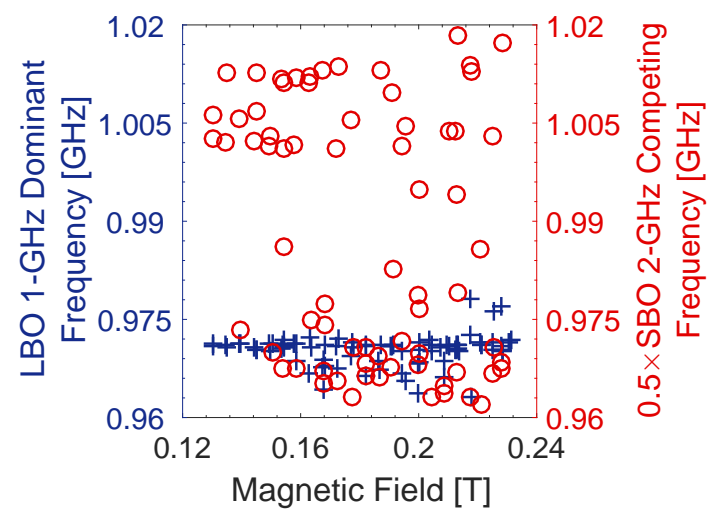

(d) LBO dominant $1 \mathrm{GHz}$ and $\mathrm{SBO}$ competing $0.5 \times 2 \mathrm{GHz}$ frequencies vs. magnetic field.

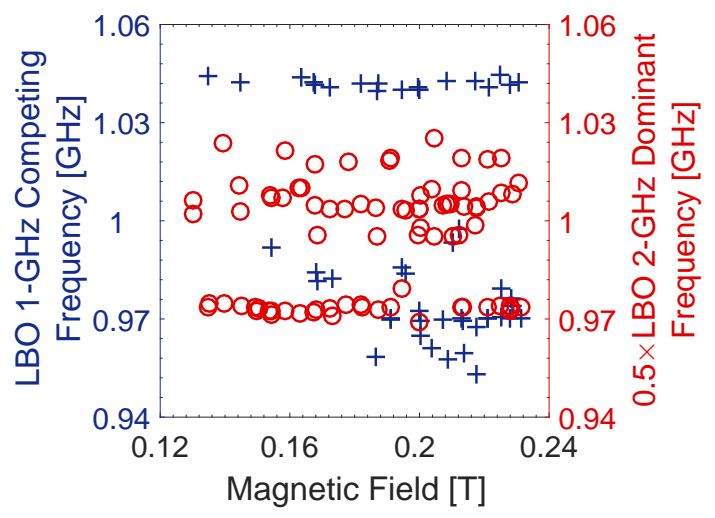

(f) LBO competing $1 \mathrm{GHz}$ and LBO dominant $0.5 \times 2 \mathrm{GHz}$ frequencies vs. magnetic field.

Figure F.1: Taper Load and Nonuniform Magnetic Field: First comparison set of dominant and competing frequencies vs. magnetic field. 


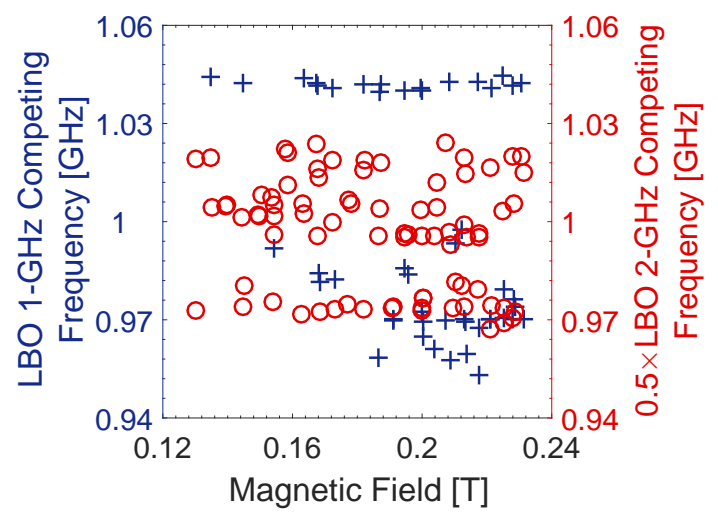

(a) LBO competing $1 \mathrm{GHz}$ and LBO competing $0.5 \times 2 \mathrm{GHz}$ frequencies vs. magnetic field.

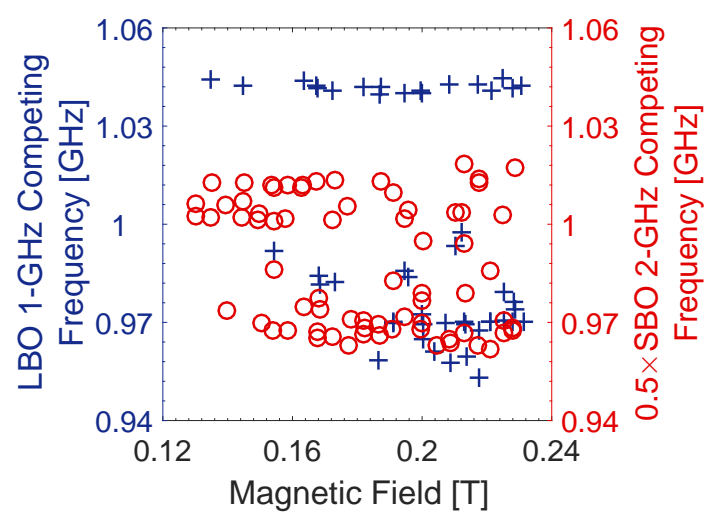

(c) LBO competing $1 \mathrm{GHz}$ and $\mathrm{SBO}$ competing $0.5 \times 2 \mathrm{GHz}$ frequencies vs. magnetic field.

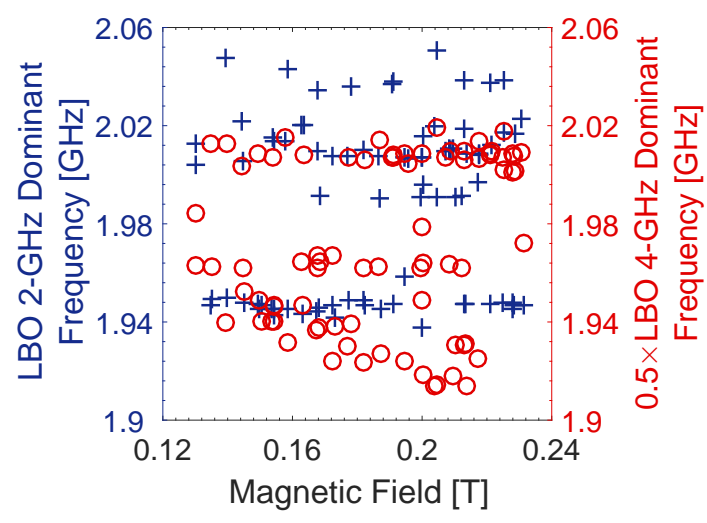

(e) LBO dominant $2 \mathrm{GHz}$ and LBO dominant $0.5 \times 4 \mathrm{GHz}$ frequencies vs. magnetic field.

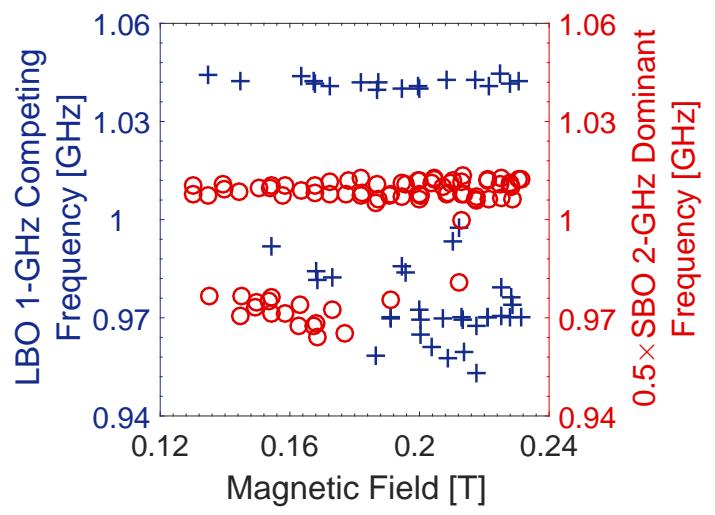

(b) LBO competing $1 \mathrm{GHz}$ and $\mathrm{SBO}$ dominant $0.5 \times 2 \mathrm{GHz}$ frequencies vs. magnetic field.

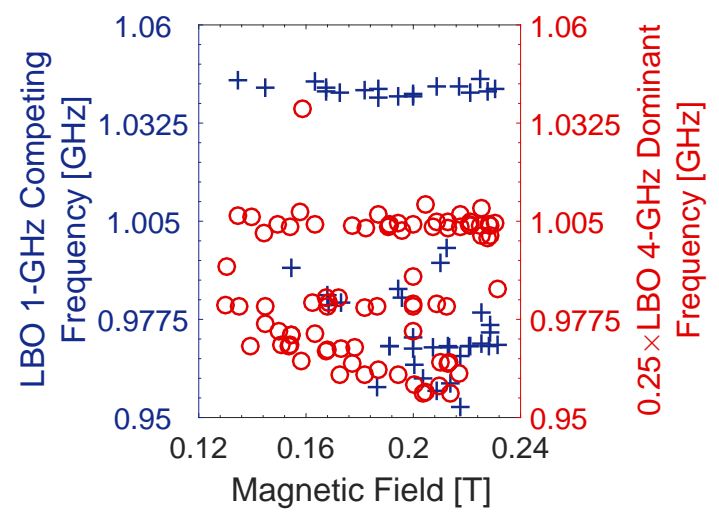

(d) LBO competing $1 \mathrm{GHz}$ and LBO dominant $0.25 \times 4 \mathrm{GHz}$ frequencies vs. magnetic field.

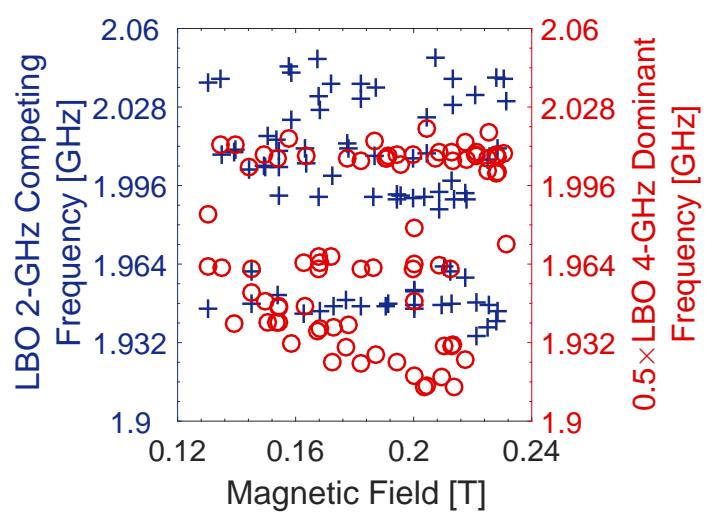

(f) LBO competing $2 \mathrm{GHz}$ and LBO dominant $0.5 \times 4 \mathrm{GHz}$ frequencies vs. magnetic field.

Figure F.2: Taper Load and Nonuniform Magnetic Field: Second comparison set of dominant and competing frequencies vs. magnetic field. 


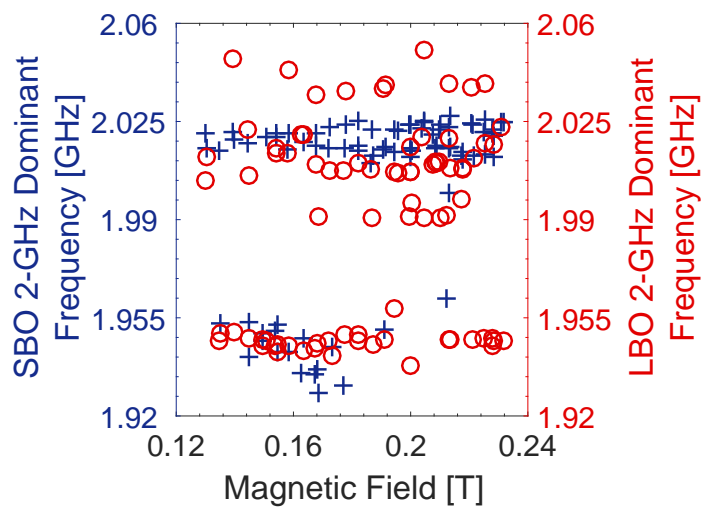

(a) SBO dominant $2 \mathrm{GHz}$ and $\mathrm{LBO}$ dominant $2 \mathrm{GHz}$ frequencies vs. magnetic field.

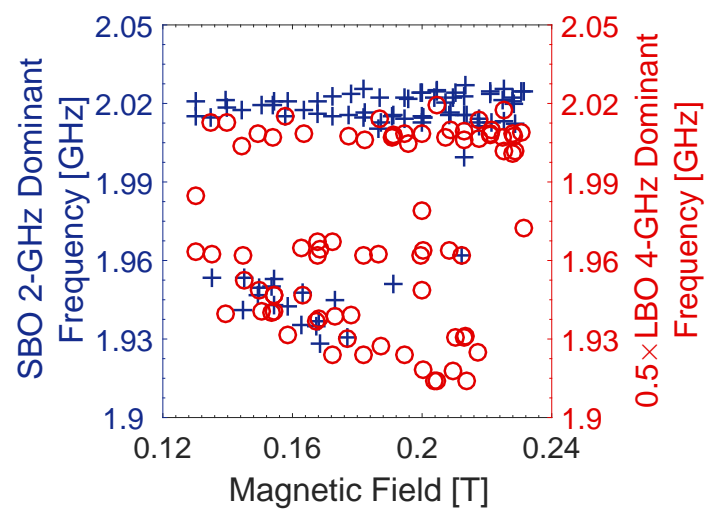

(c) $\mathrm{SBO}$ dominant $2 \mathrm{GHz}$ and $\mathrm{LBO}$ dominant $0.5 \times 4 \mathrm{GHz}$ frequencies vs. magnetic field.

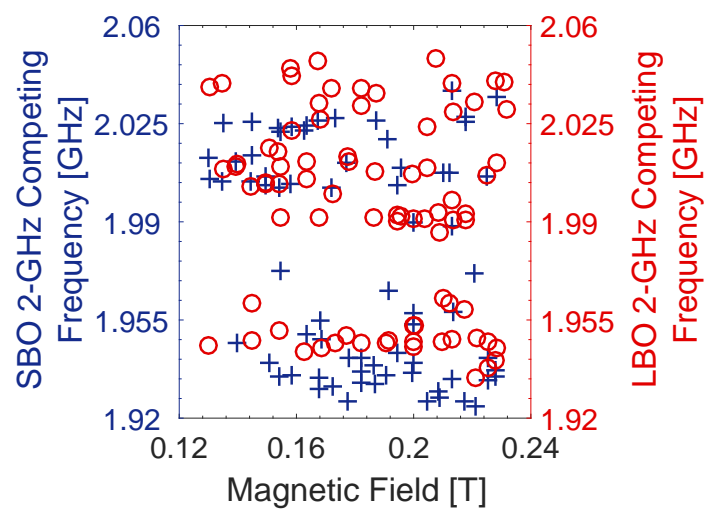

(e) SBO competing $2 \mathrm{GHz}$ and $\mathrm{LBO}$ competing $2 \mathrm{GHz}$ frequencies vs. magnetic field.

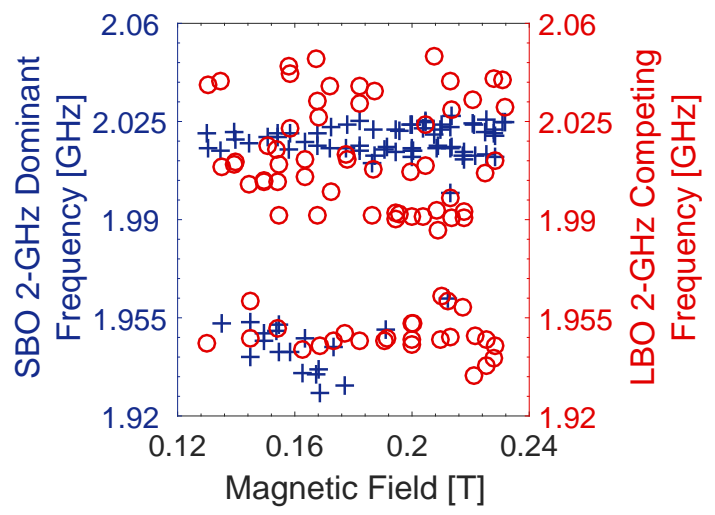

(b) SBO dominant $2 \mathrm{GHz}$ and $\mathrm{LBO}$ competing $2 \mathrm{GHz}$ frequencies vs. magnetic field.

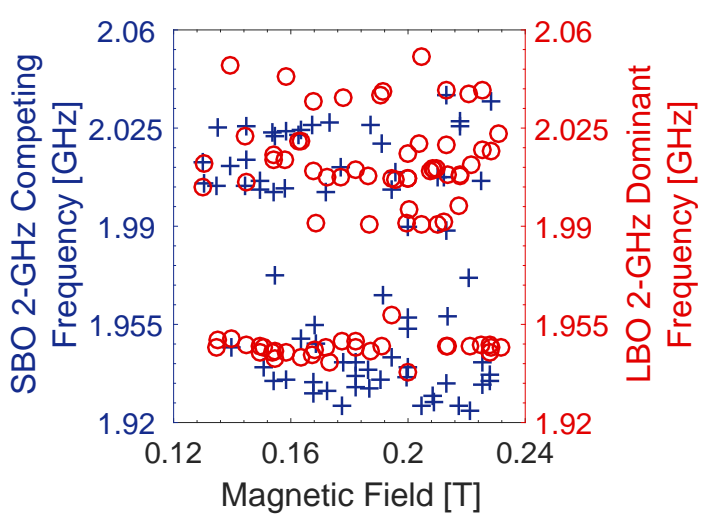

(d) SBO competing $2 \mathrm{GHz}$ and LBO dominant $2 \mathrm{GHz}$ frequencies vs. magnetic field.

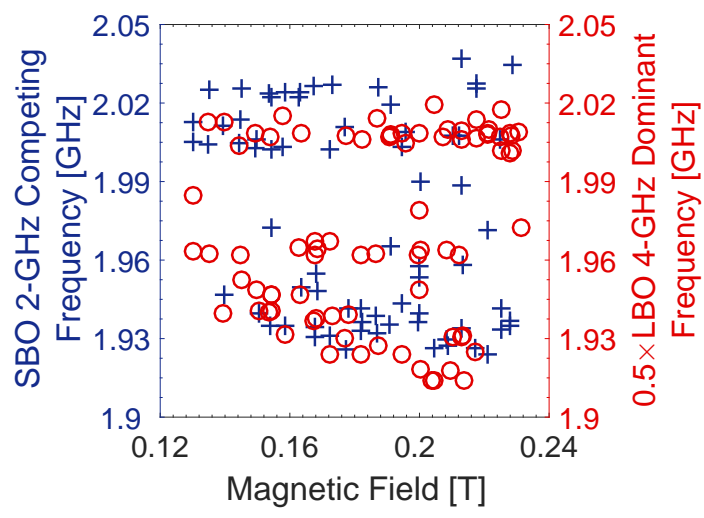

(f) SBO competing $2 \mathrm{GHz}$ and $\mathrm{LBO}$ dominant $0.5 \times 4 \mathrm{GHz}$ frequencies vs. magnetic field.

Figure F.3: Taper Load and Nonuniform Magnetic Field: Third comparison set of dominant and competing frequencies vs. magnetic field. 


\section{APPENDIX G}

\section{MFRPM with Taper Load and Optimized Magnetic Field: Plots of Frequencies vs. Magnetic Field}

This appendix section contains all the plots comparing the fundamental and harmonic frequencies of the LBO and SBO vs. magnetic field. They are included for completeness. Most plots were used to confirm the absence of any meaningful harmonic correlation between different frequencies. Note that the plots omit SBO harmonics because they did not appear to have been generated at detectable power levels. 


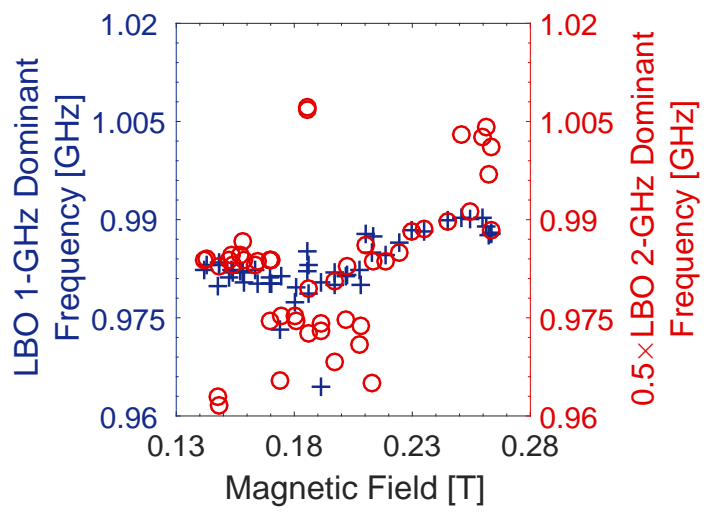

(a) LBO dominant $1 \mathrm{GHz}$ and LBO dominant $0.5 \times 2 \mathrm{GHz}$ frequencies vs. magnetic field.

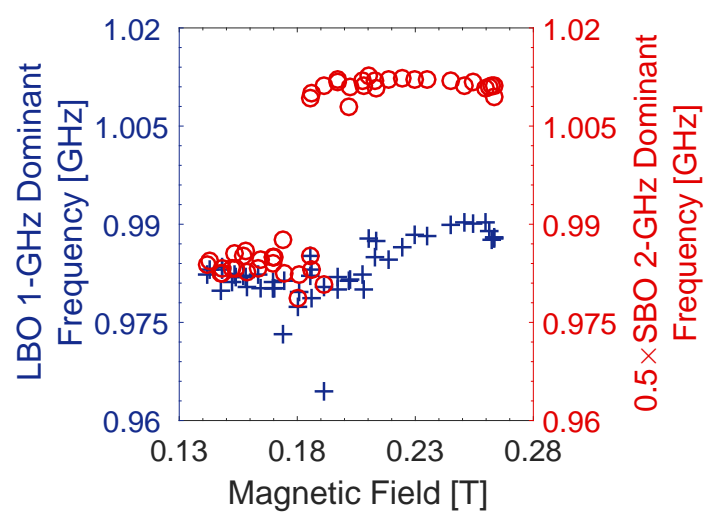

(c) LBO dominant $1 \mathrm{GHz}$ and $\mathrm{SBO}$ dominant $0.5 \times 2 \mathrm{GHz}$ frequencies vs. magnetic field.

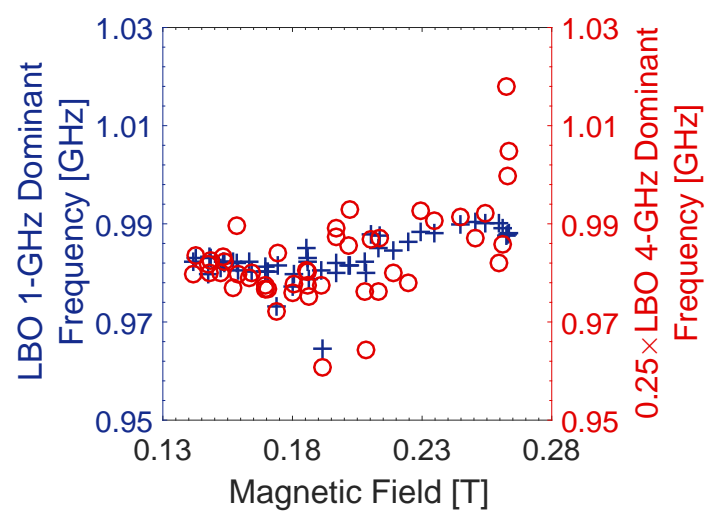

(e) LBO dominant $1 \mathrm{GHz}$ and LBO dominant $0.25 \times 4 \mathrm{GHz}$ frequencies vs. magnetic field.

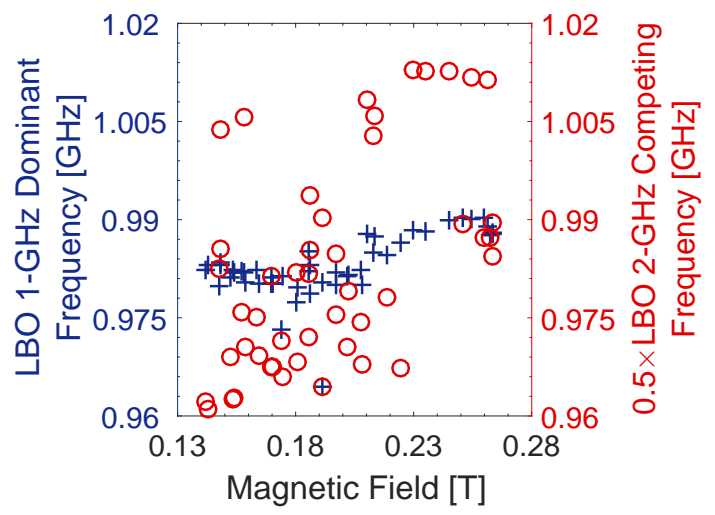

(b) LBO dominant $1 \mathrm{GHz}$ and LBO competing $0.5 \times 2 \mathrm{GHz}$ frequencies vs. magnetic field.

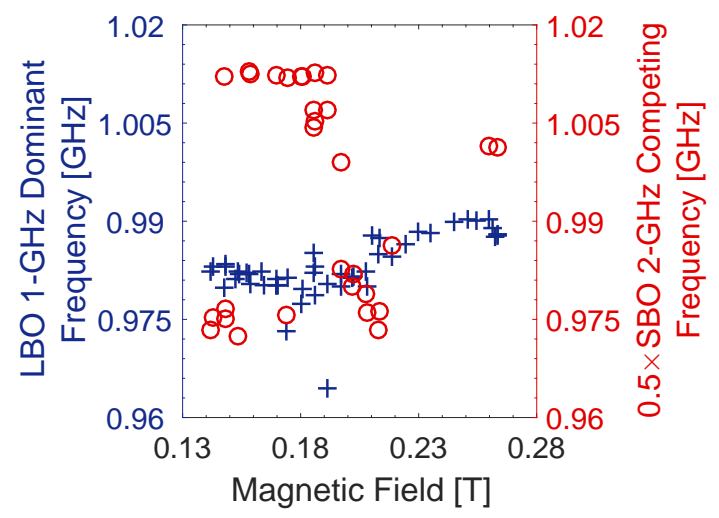

(d) LBO dominant $1 \mathrm{GHz}$ and $\mathrm{SBO}$ competing $0.5 \times 2 \mathrm{GHz}$ frequencies vs. magnetic field.

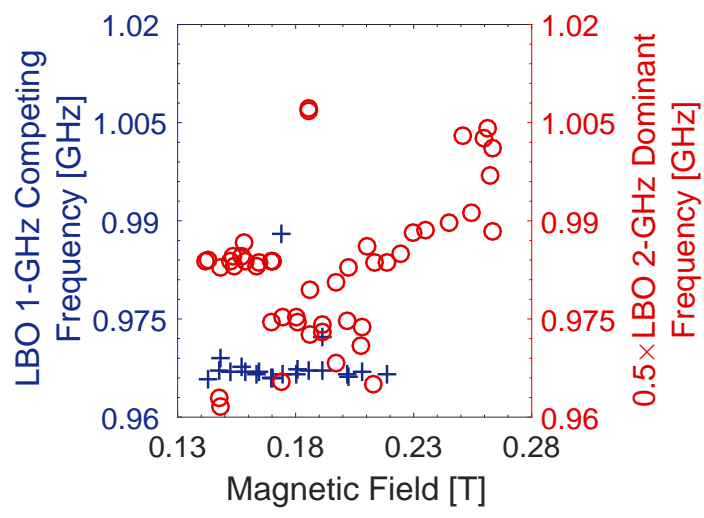

(f) LBO competing $1 \mathrm{GHz}$ and LBO dominant $0.5 \times 2 \mathrm{GHz}$ frequencies vs. magnetic field.

Figure G.1: Taper Load and Uniform Magnetic Field: First comparison set of dominant and competing frequencies vs. magnetic field. 


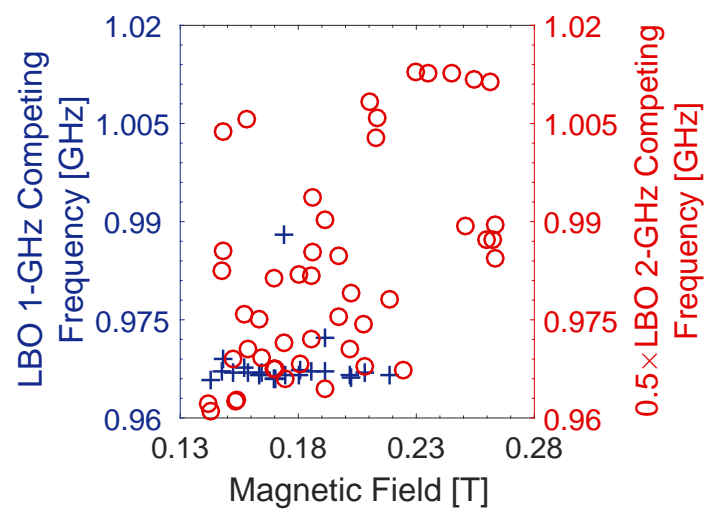

(a) LBO competing $1 \mathrm{GHz}$ and $\mathrm{LBO}$ competing $0.5 \times 2 \mathrm{GHz}$ frequencies vs. magnetic field.

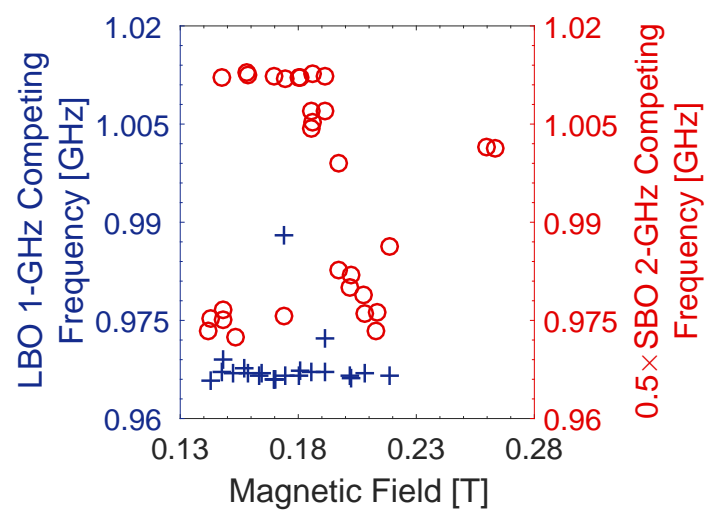

(c) LBO competing $1 \mathrm{GHz}$ and $\mathrm{SBO}$ competing $0.5 \times 2 \mathrm{GHz}$ frequencies vs. magnetic field.

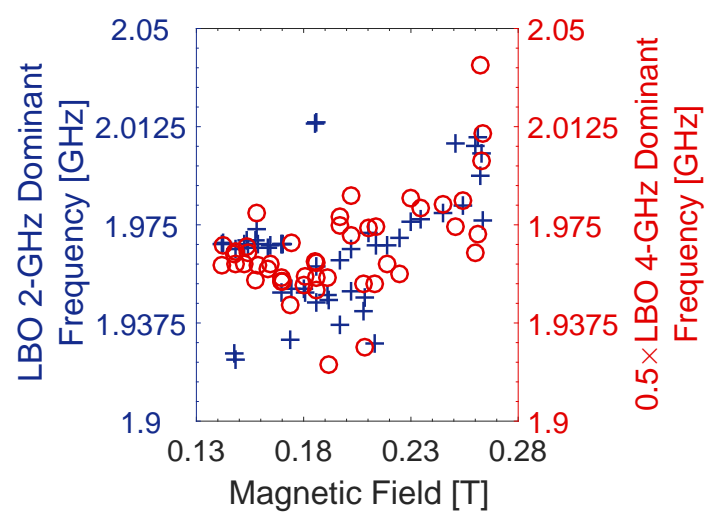

(e) LBO dominant $2 \mathrm{GHz}$ and LBO dominant $0.5 \times 4 \mathrm{GHz}$ frequencies vs. magnetic field.

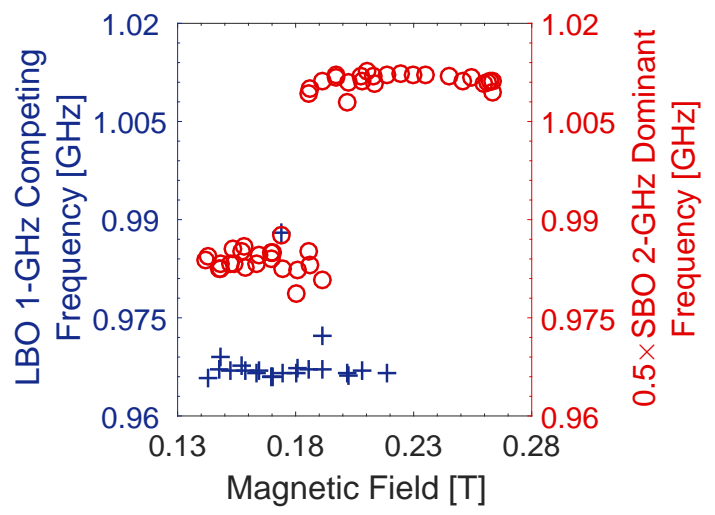

(b) LBO competing $1 \mathrm{GHz}$ and $\mathrm{SBO}$ dominant $0.5 \times 2 \mathrm{GHz}$ frequencies vs. magnetic field.

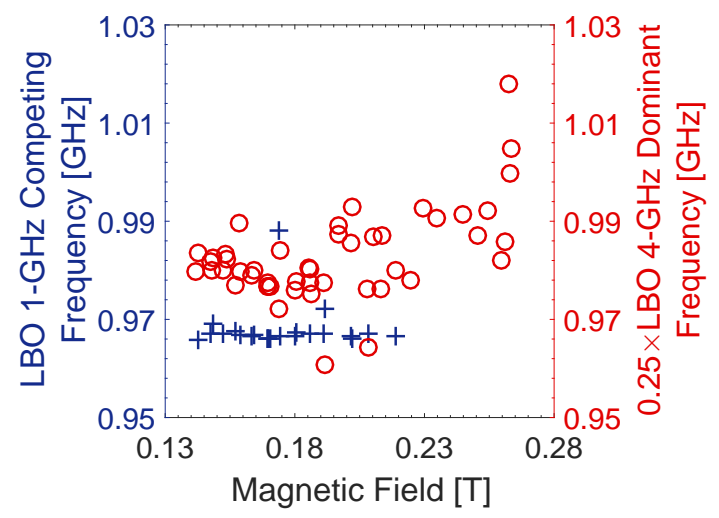

(d) LBO competing $1 \mathrm{GHz}$ and $\mathrm{LBO}$ dominant $0.25 \times 4 \mathrm{GHz}$ frequencies vs. magnetic field.

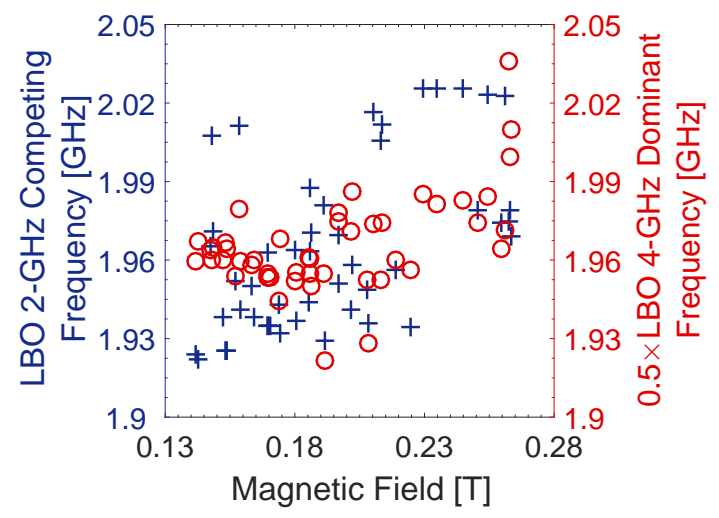

(f) LBO competing $2 \mathrm{GHz}$ and LBO dominant $0.5 \times 4 \mathrm{GHz}$ frequencies vs. magnetic field.

Figure G.2: Taper Load and Uniform Magnetic Field: Second comparison set of dominant and competing frequencies vs. magnetic field. 


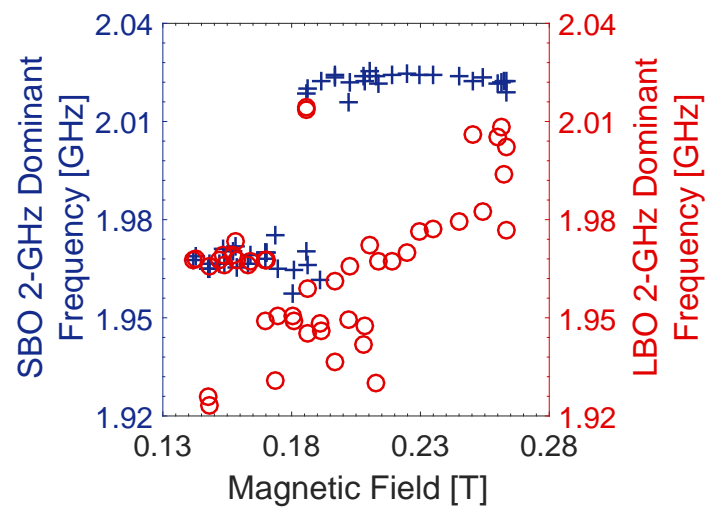

(a) SBO dominant $2 \mathrm{GHz}$ and LBO dominant $2 \mathrm{GHz}$ frequencies vs. magnetic field.

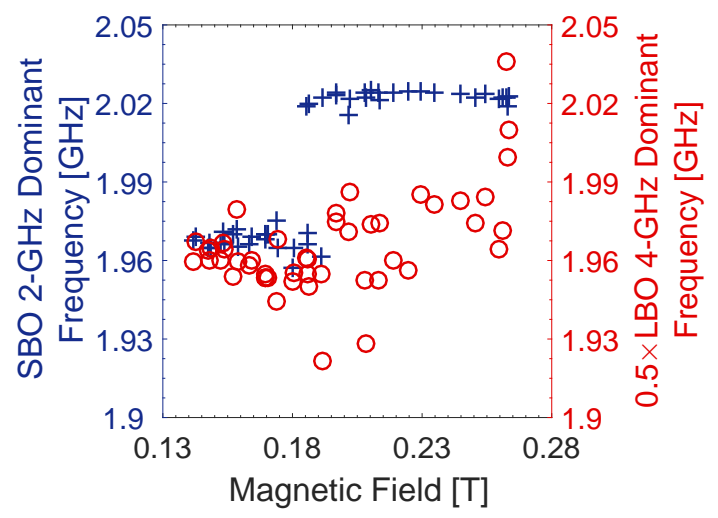

(c) SBO dominant $2 \mathrm{GHz}$ and $\mathrm{LBO}$ dominant $0.5 \times 4 \mathrm{GHz}$ frequencies vs. magnetic field.

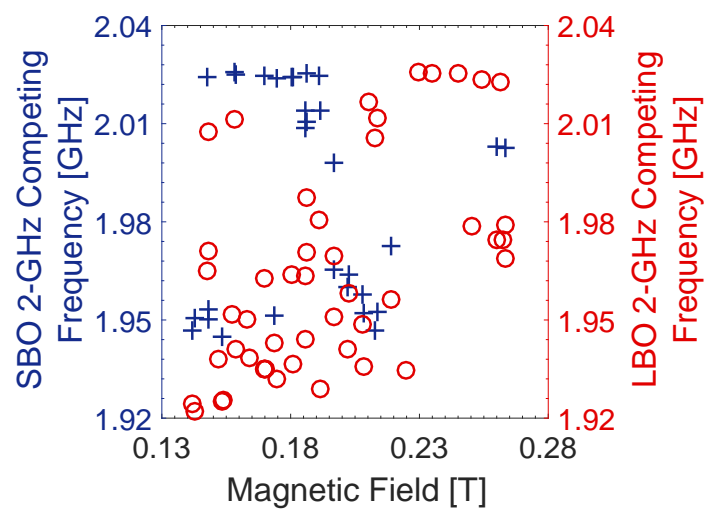

(e) SBO competing $2 \mathrm{GHz}$ and $\mathrm{LBO}$ competing $2 \mathrm{GHz}$ frequencies vs. magnetic field.

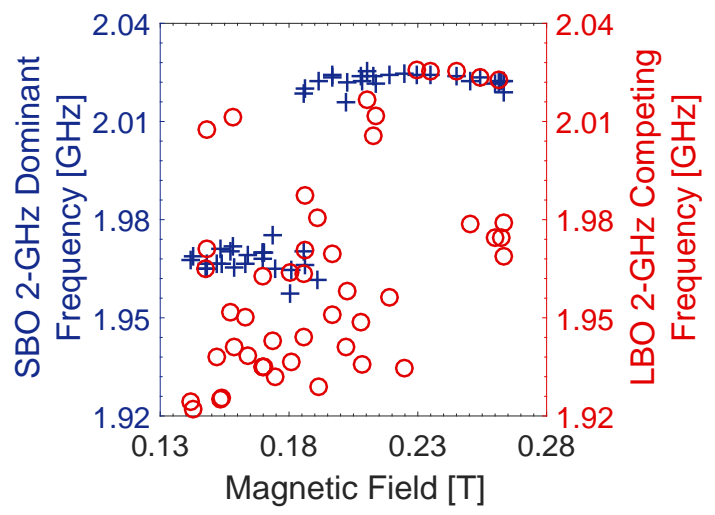

(b) SBO dominant $2 \mathrm{GHz}$ and LBO competing $2 \mathrm{GHz}$ frequencies vs. magnetic field.

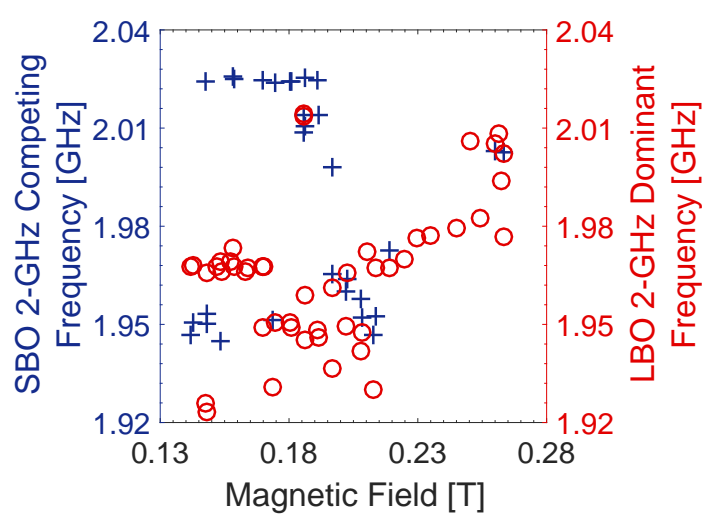

(d) SBO competing $2 \mathrm{GHz}$ and LBO dominant $2 \mathrm{GHz}$ frequencies vs. magnetic field.

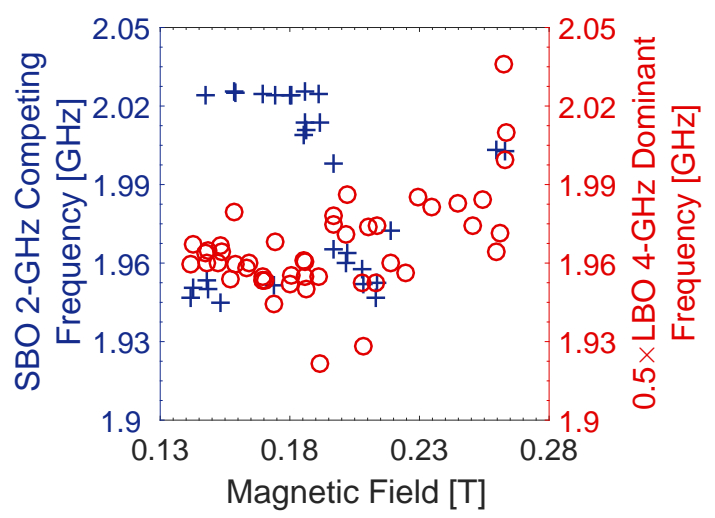

(f) SBO competing $2 \mathrm{GHz}$ and LBO dominant $0.5 \times 4 \mathrm{GHz}$ frequencies vs. magnetic field.

Figure G.3: Taper Load and Uniform Magnetic Field: Third comparison set of dominant and competing frequencies vs. magnetic field. 


\section{APPENDIX H \\ Crowbar Switch Reconfiguration}

\section{H.1 Overview}

The significant jitter present in the erection time of the MELBA-C Marx generator creates the potential for magnetron damage due to crowbar failure and subsequent AK gap closure. Given the rapid start-up of the magnetron and significant pulse-shortening, long voltage pulselengths are unnecessary. This fact, coupled with the jitter, makes operation especially difficult at low magnetic fields. The reason is that magnetron impedances are unusually low at low magnetic fields, meaning the MELBA-C output voltage is relatively low and the current drawn by the magnetron is relatively high, which also exacerbates the process of impedance collapse. Since the trigger time for the crowbar switch is fixed, the actual voltage pulse duration can vary by $200 \mathrm{~ns}$, depending on the MELBA-C erection time. If MELBA-C starts relatively late, the microwave pulse can be cut off by the crowbar. However, if MELBA-C starts relatively early, the longer pulselength can lead to excessive voltage droop, which reduces the voltage across the crowbar switch below the threshold required to crowbar on command, leading to crowbar failure and subsequent magnetron damage. An investigation into previous MELBA-C configurations revealed the fact that the crowbar switch configuration was not optimized for operation at $300 \mathrm{kV}$, which motivated the crowbar switch reconfiguration.

\section{H.2 Switch Description}

The two main components of the crowbar switch are shown in Fig. H.1. The trigger electrode, seen protruding in Fig. H.1b and close up in Fig. H.1c, is an isolated, floating conductor that is ungrounded. The bottom of this electrode, seen in Fig. H.1d, forms one electrode of a spark gap termed the Trigger Isolation Gap (TIG). The purpose of the floating trigger electrode is to introduce a conductor between the ground electrode and 


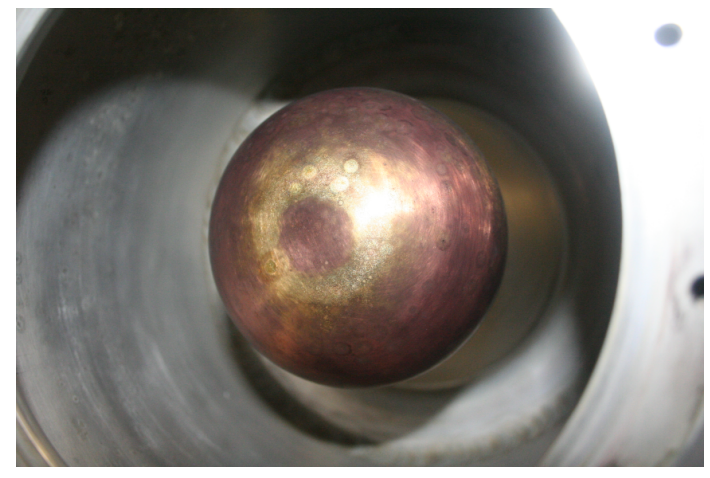

(a) High-potential (MELBA-C side) electrode of the crowbar switch.

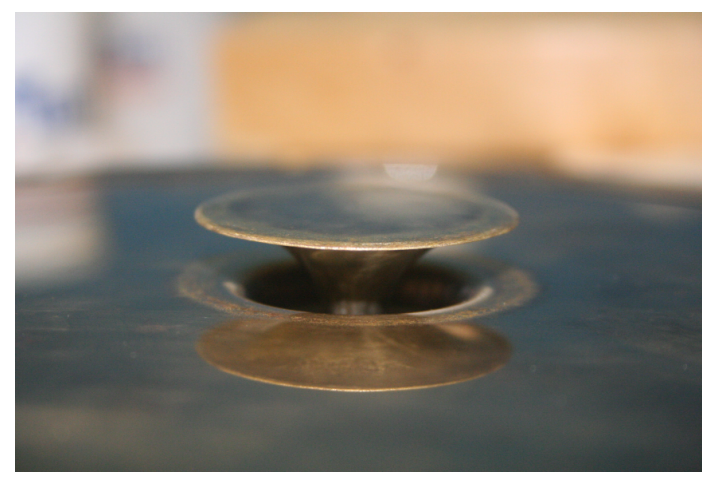

(c) Zoomed photograph of the trigger electrode.

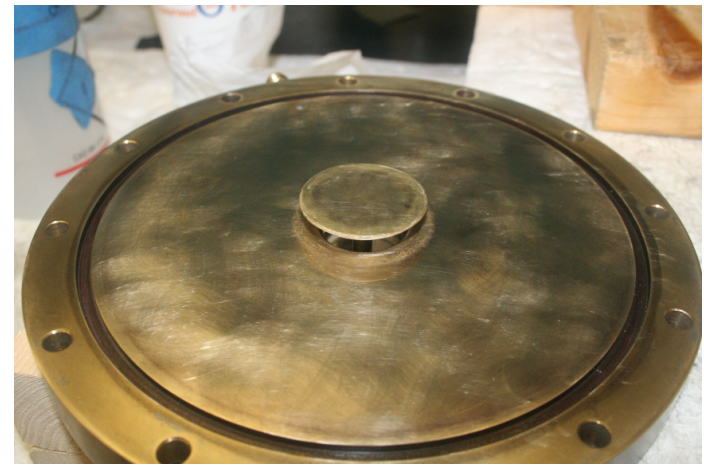

(b) Ground-potential electrode with protruding trigger electrode for the crowbar switch.

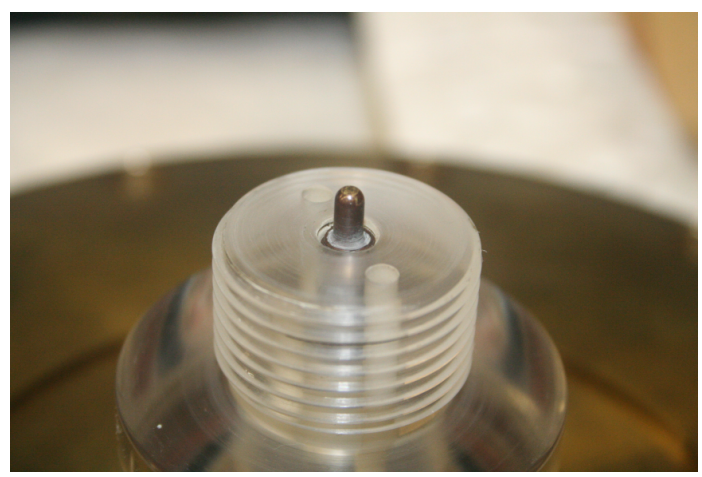

(d) Bottom of the trigger electrode.

Figure H.1: Crowbar switch geometry.

high-potential MELBA-C electrode that 1) does not perturb the natural equipotential lines in the switch to avoid breakdown during the pulse, and 2) introduces, at a preset time, a high-voltage, positive-polarity pulse across the TIG to the trigger electrode to substantially perturb the equipotential lines and induce switch breakdown. At breakdown, an arc forms between the MELBA-C electrode in Fig. H.1a, the trigger electrode in Fig. H.1c, and the ground electrode in Fig. H.1b, thereby shunting the MELBA-C Marx generator energy safely and rapidly to ground. The gas pressure and composition $\left(\mathrm{SF}_{6}\right)$ of the crowbar switch and TIG are the same.

\section{H.3 Modification}

The key to the success of this general switch concept, termed the Trigatron [127], lies in the shape of the trigger electrode. In the presence of an external electric field, a floating conductor's surface describes an equipotential. If the shape of the electrode naturally follows the equipotential lines, it is functionally invisible to the switch. The trigger electrode, 
which protrudes into the switch gap between the high-potential and ground electrodes, therefore has a surface that is at an equivalent potential to the potential in the switch at which the trigger electrode surface is located (which, again, follows the natural contour of an equipotential). The electric field between the ball electrode and the ground plane can be roughly approximated as a constant such that $V_{0}=E \times d$, where $V_{0}$ is the total potential across the gap, $E$ is the electric field, and $d$ is the distance between the ball and ground electrodes. At some position $x$ describing the location of the flat surface of the trigger electrode, $V(x)=V_{0} x / d$. The potential of the floating trigger electrode is therefore approximately known.

The question then becomes: how far should the trigger electrode protrude into the gap? The answer is contingent on the following: 1) the TIG must hold off the potential of the floating electrode, 2) the trigger electrode protrusion should not be so large that it may arc to ground during crowbar in the coaxial stem (a constant distance) rather than under the mushroom-shaped head (a distance that increases as the electrode protrudes further into the gap), 3) the TIG must break down when a positive $85 \mathrm{kV}$ pulse is introduced, and 4) the disparity between the TIG and crowbar switch parameters falling on the Paschen curve for $\mathrm{SF}_{6}$ (resulting from limitations in the TIG spacing) means the TIG will always be much closer to breakdown than the crowbar switch, so the trigger electrode should be as far in the gap as possible (subject to constrains 1,2, and 3).

Calculation of the final parameters revealed the floating potential of the trigger electrode approximately doubles when the new protrusion distance is set, which falls slightly short of what might be desired with a reconfigured MELBA-C output voltage that is approximately one third of its original $1 \mathrm{MV}$ specification. Ultimately, the electrode protrusion distance was changed from $0.394 \mathrm{~cm}$ to $0.777 \mathrm{~cm}$ (which is safely smaller than the constant $0.937 \mathrm{~cm}$ spacing in the coax section), with an estimated change in floating potential from approximately $-23 \mathrm{kV}$ to $-52 \mathrm{kV}$. Thus, when the crowbar switch is triggered, this introduces a voltage swing at an equipotential surface during command crowbar in the crowbar switch from $-52 \mathrm{kV}$ to $85 \mathrm{kV}$, a nearly $30 \mathrm{kV}$ improvement over the old spacing. The TIG was changed from $0.274 \mathrm{~cm}$ to $0.762 \mathrm{~cm}$ in to reliably hold off the $-52 \mathrm{kV}$ potential, but still break down when $85 \mathrm{kV}$ is introduced.

\section{H.4 Outcome}

Tests using the rod cathode and stainless steel current collecting plate demonstrated the ability to regularly crowbar on command, though shots must start with a conservative crowbar pressure that initially produces self-crowbar events. During shots with the 
magnetron load, the crowbar pressure must be carefully adjusted so that MELBA-C selfcrowbars at a time slightly less than the command crowbar time. This provides sufficient defense against shots at low magnetic field where the Marx generator erects unusually quickly. In those cases, where the unexpectedly large voltage droop occurs as previously described, the newly configured crowbar switch provides the voltage swing necessary to force breakdown of the crowbar switch. However, if one relies entirely on the command crowbar, shots at low impedance with rapid Marx erection may fail to crowbar.

It should be noted that this conservative approach is only necessary at low impedances or with loads susceptible to rapid AK gap closure and impedance collapse. If a load exhibits relatively constant impedance falling within MELBA-C specifications, the crowbar switch pressure can be adjusted to eliminate self-crowbar events and only rely on command crowbar. Using this approach, two MELBA-C rebuilds successfully produced data collections of over 250 and 500 shots, respectively, each without a single failure to crowbar. 


\section{APPENDIX I}

\section{Hardware Production Drawings}

The following components were designed and fabricated for the MFRPM project:

1. SBO Anode

2. SBO Microwave Extraction Antenna

3. MFRPM Vacuum Flange

4. L-Band Microwave Window (Flange and Lexan Air-Vacuum Interface)

5. Lexan Vacuum Flange Insulator

6. WR-650 to WR-340 Waveguide Taper

7. WR-340 to WR-187 Waveguide Taper

Previously fabricated components used for this project, such as the LBO anode, recirculation bends, coax-to-waveguide couplers, and cathode are detailed in [10]. O-ring grooves were designed in accordance with the Parker O-Ring Handbook $\$ 4.3$ (Face Type Seals) and Design Chart 4-3 (Face Seal Glands with External Pressure) [128]. 


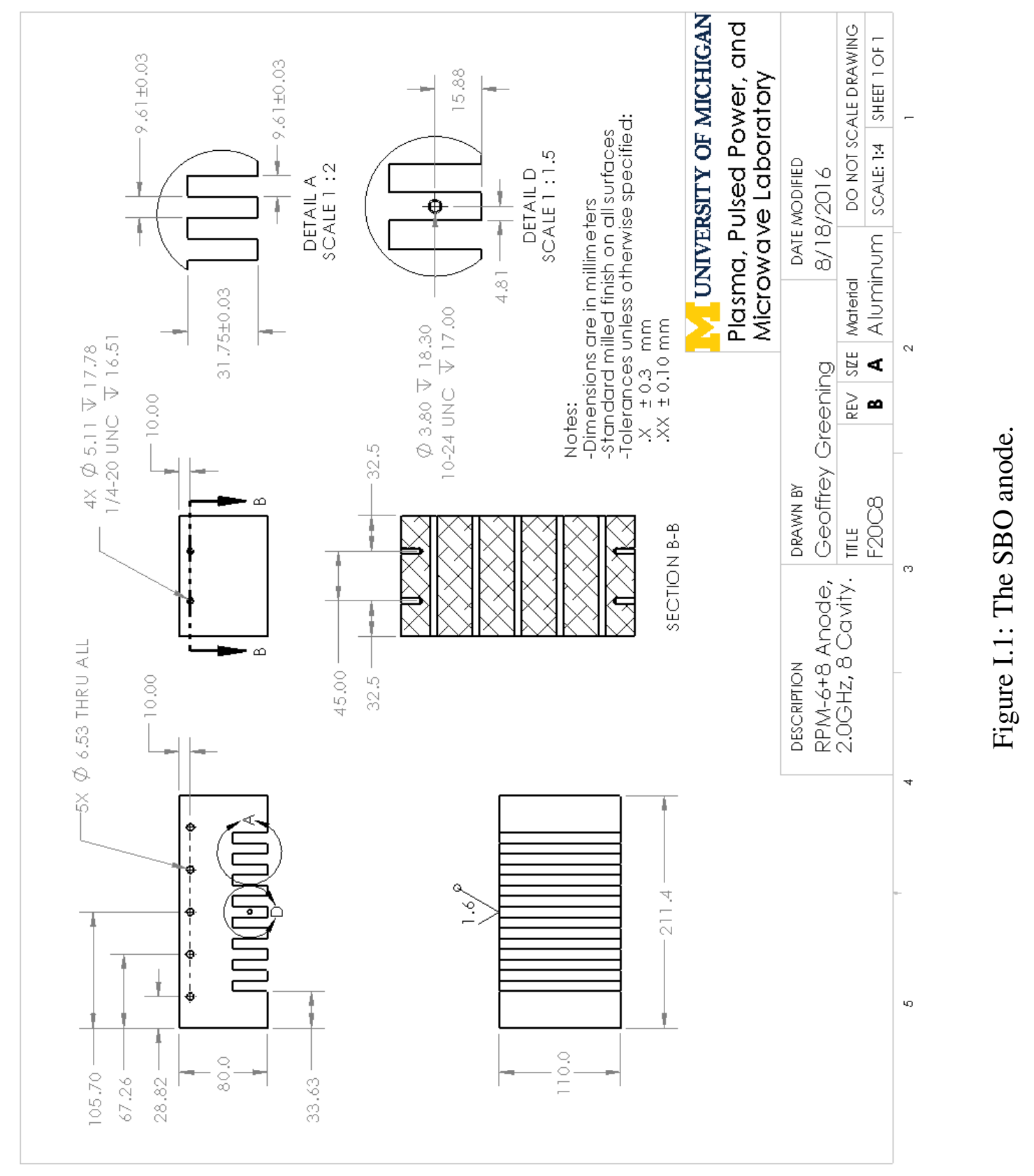




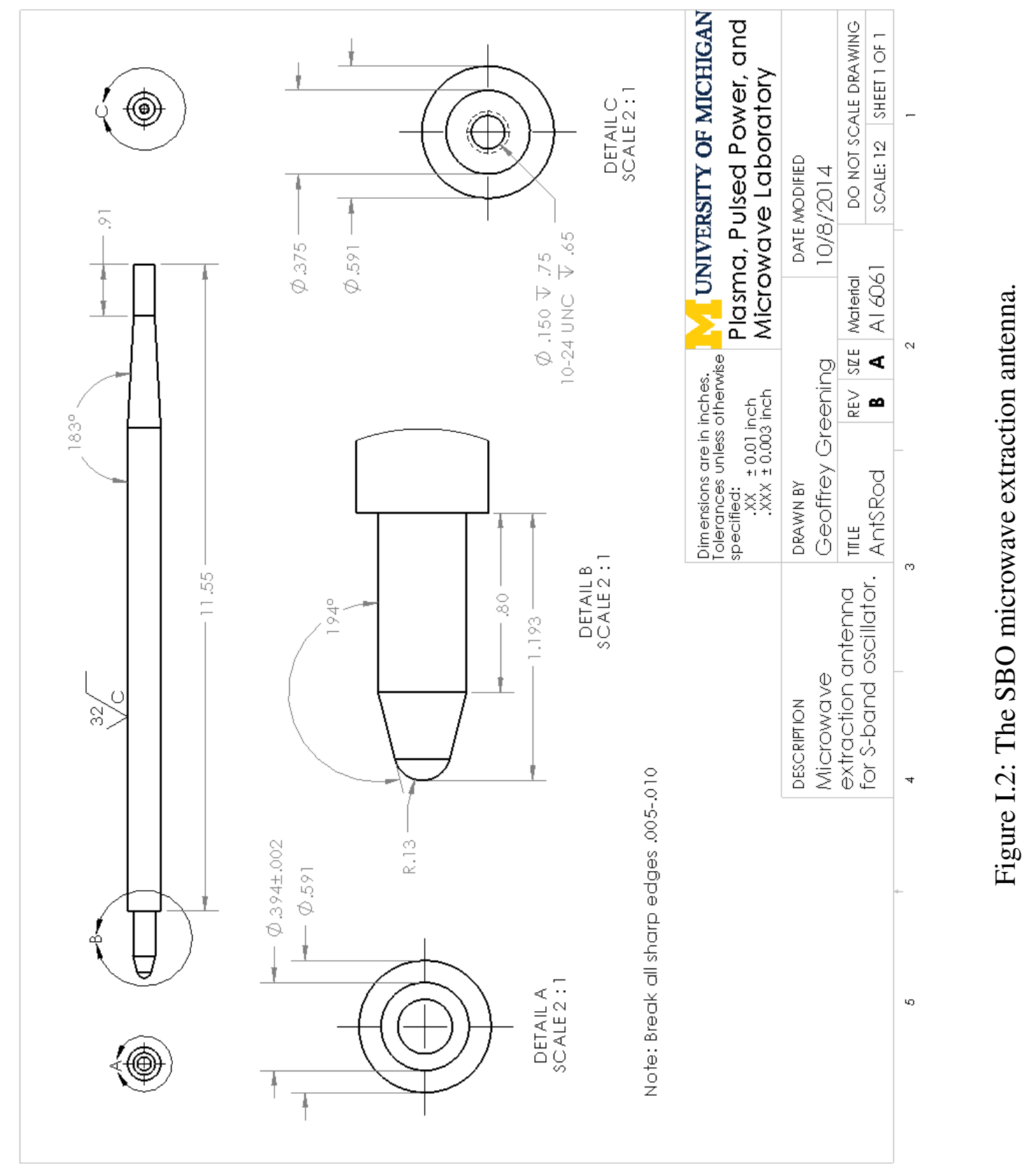




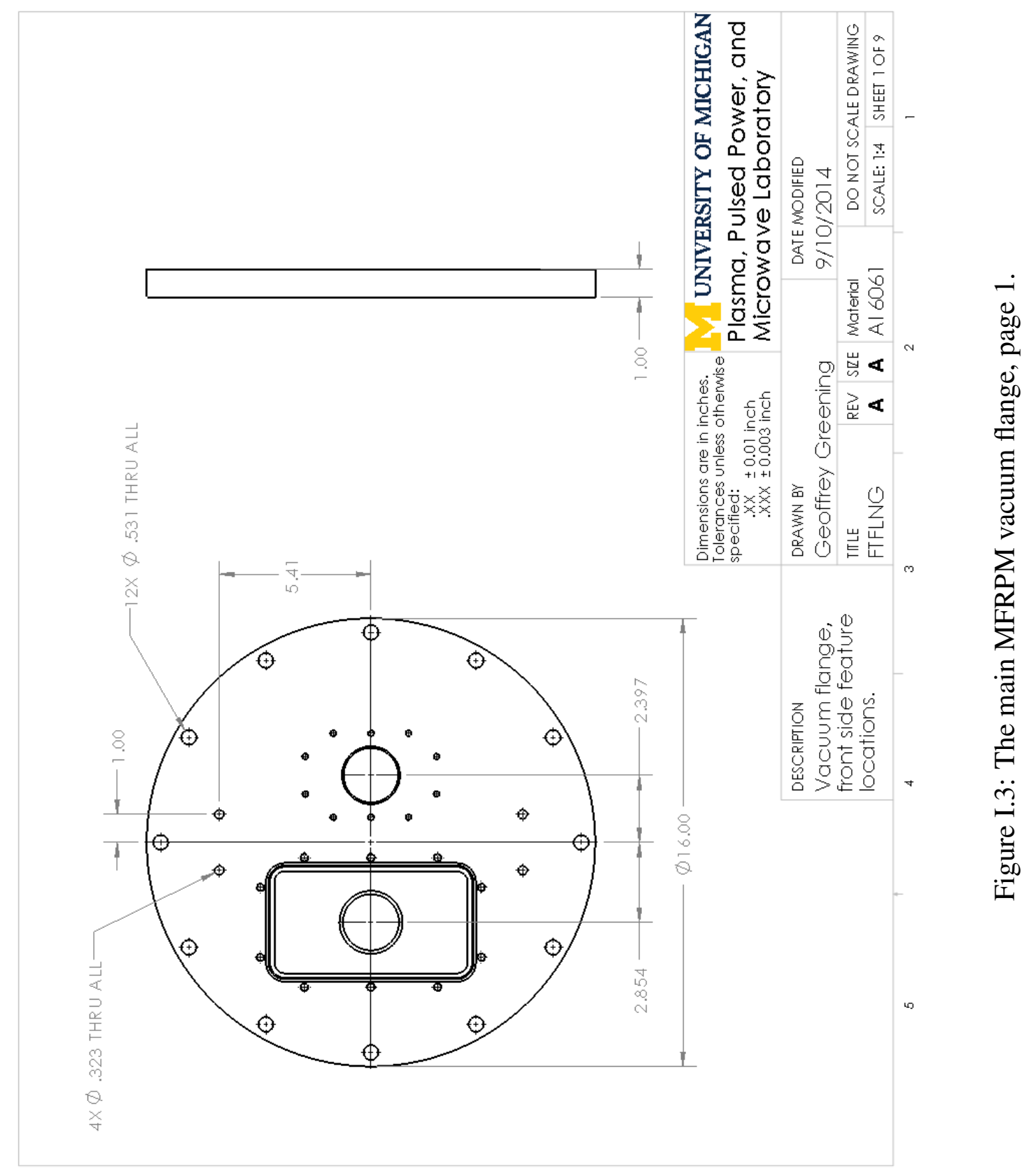




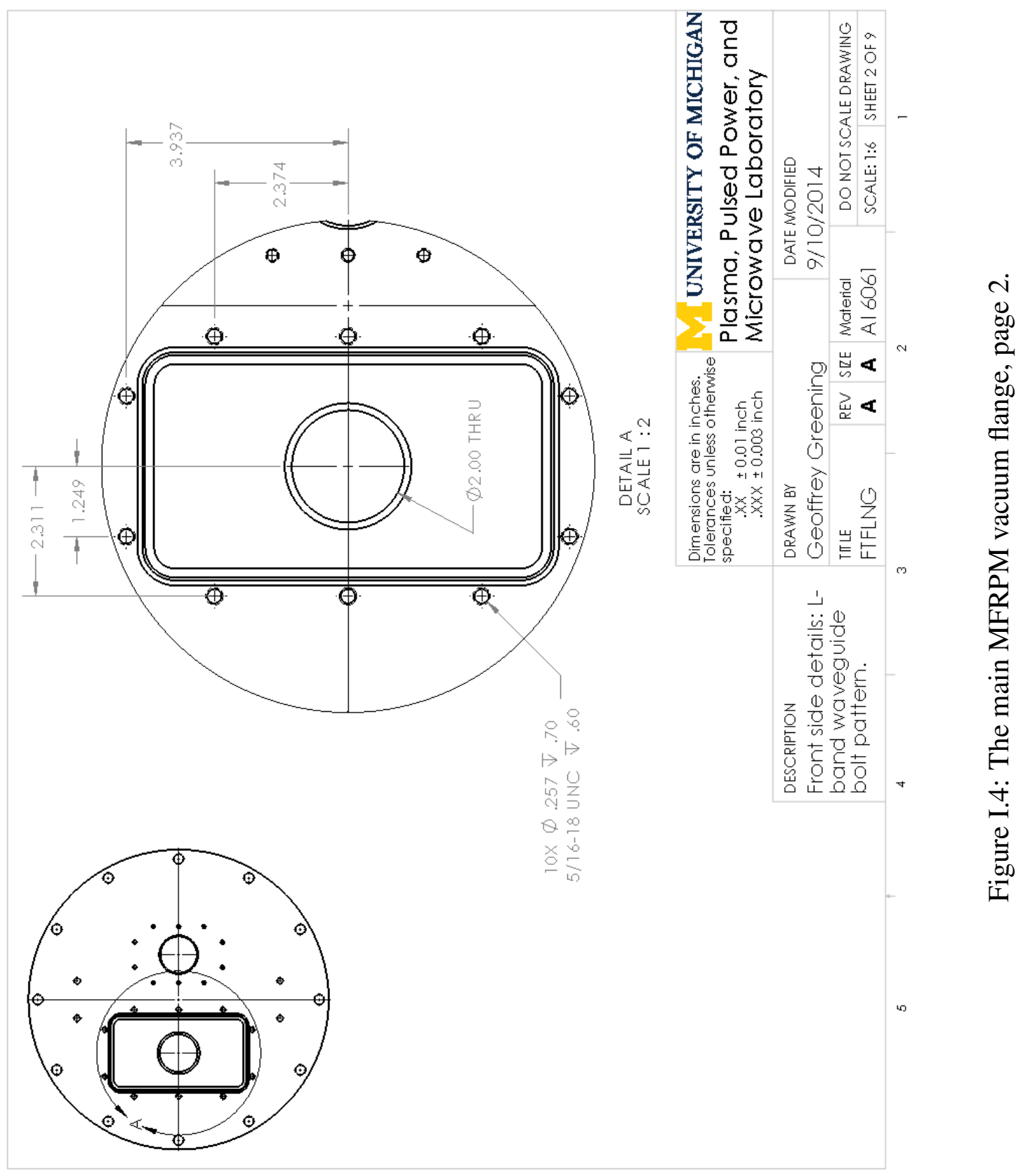




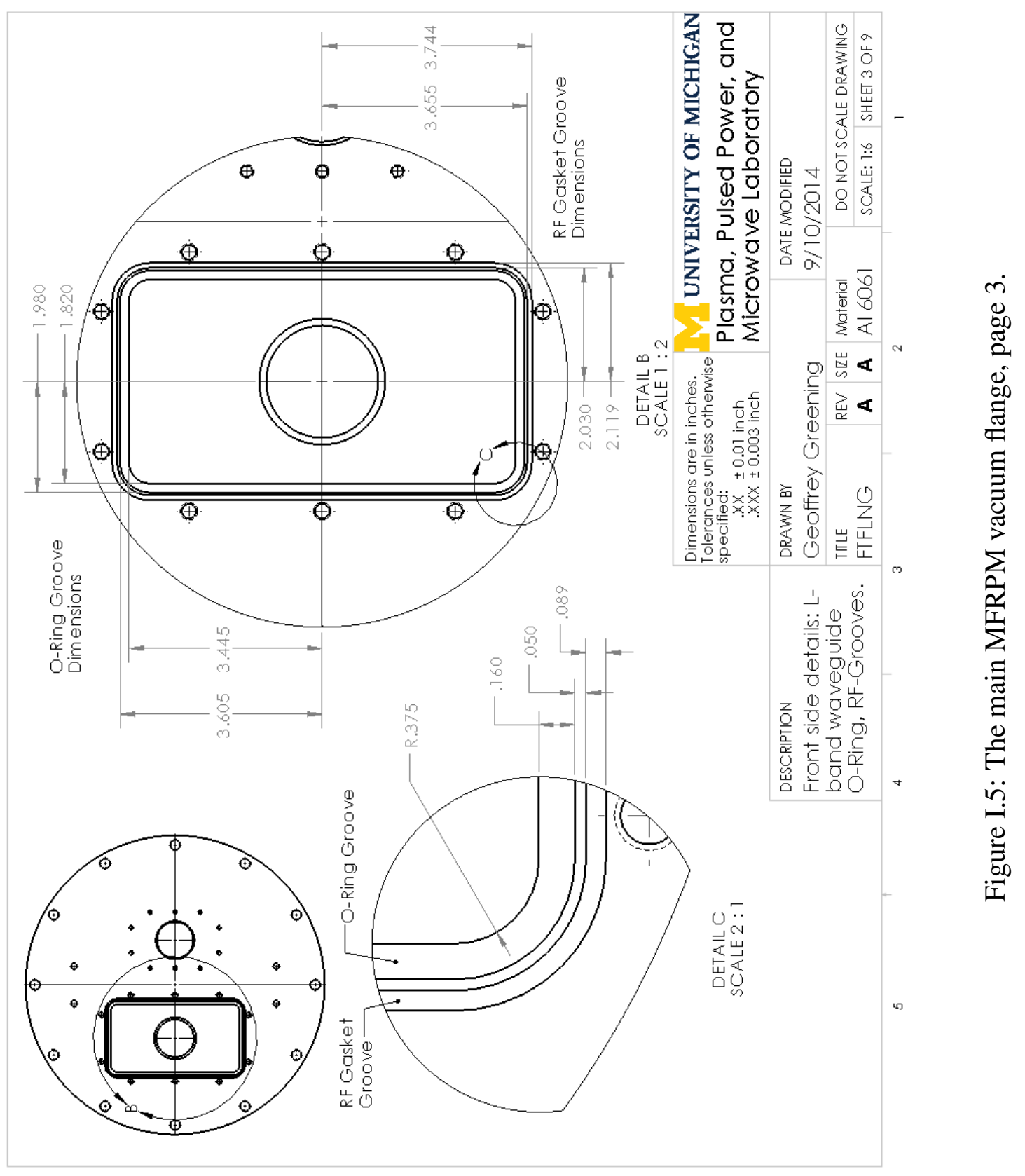




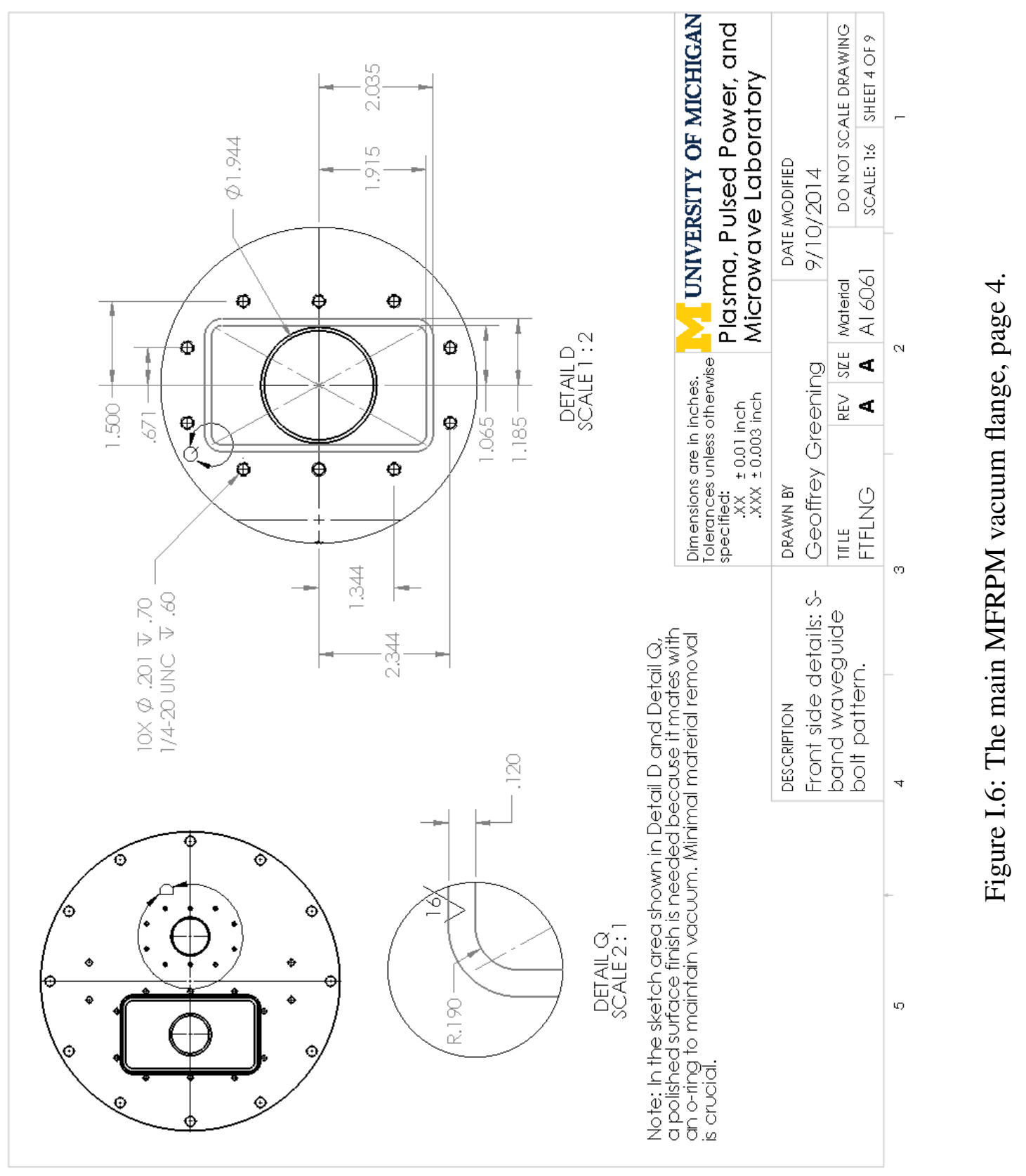




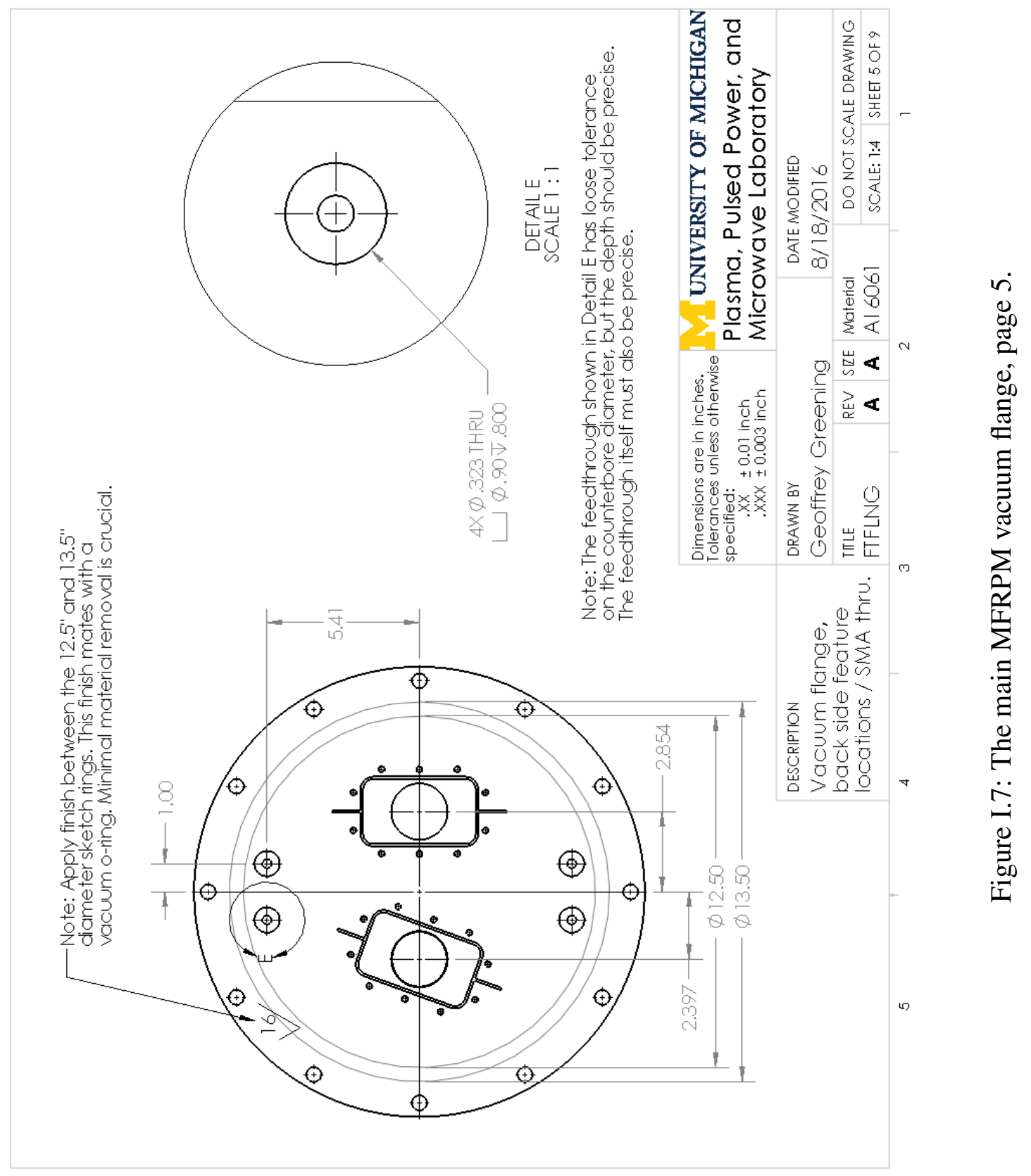




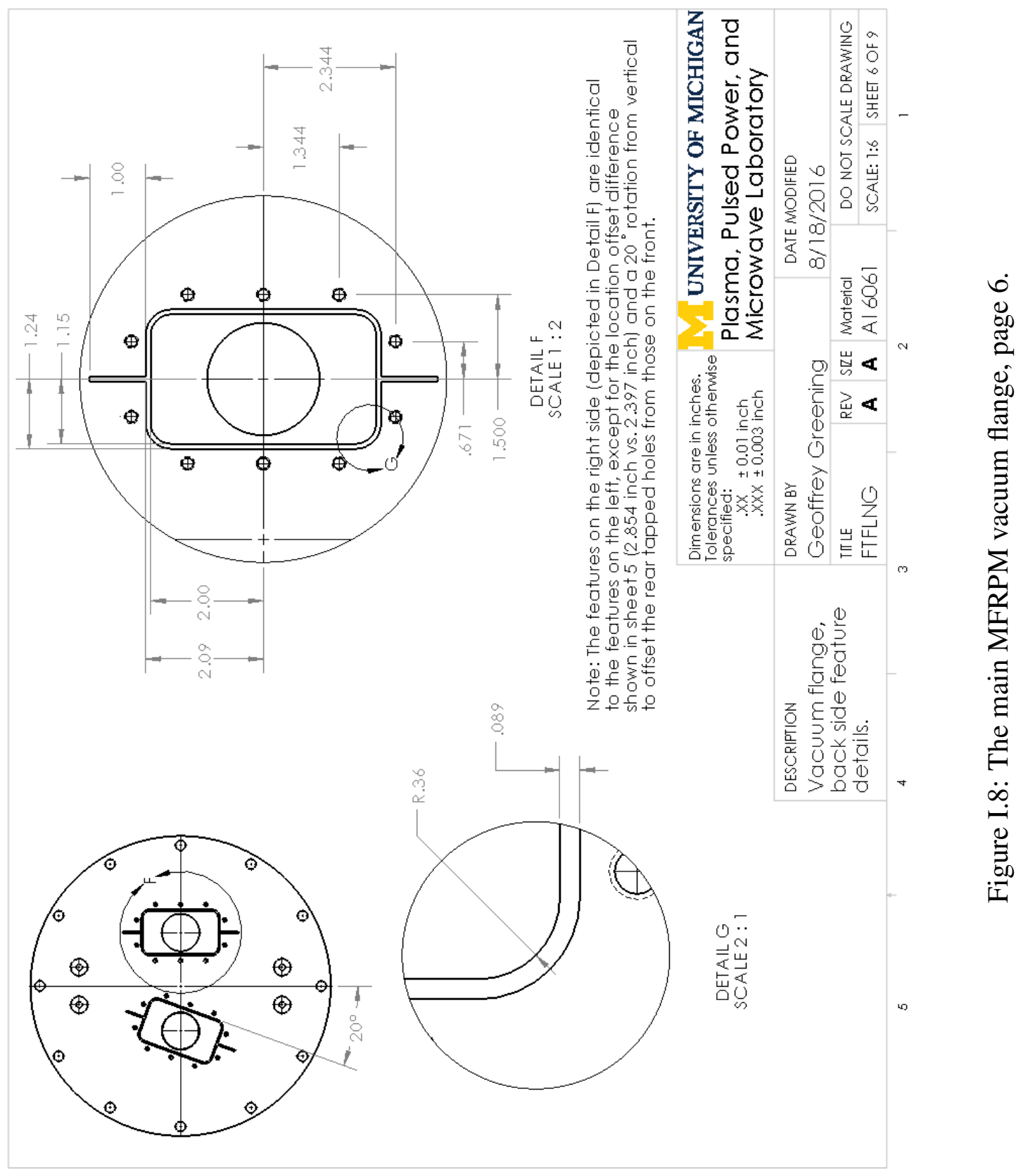




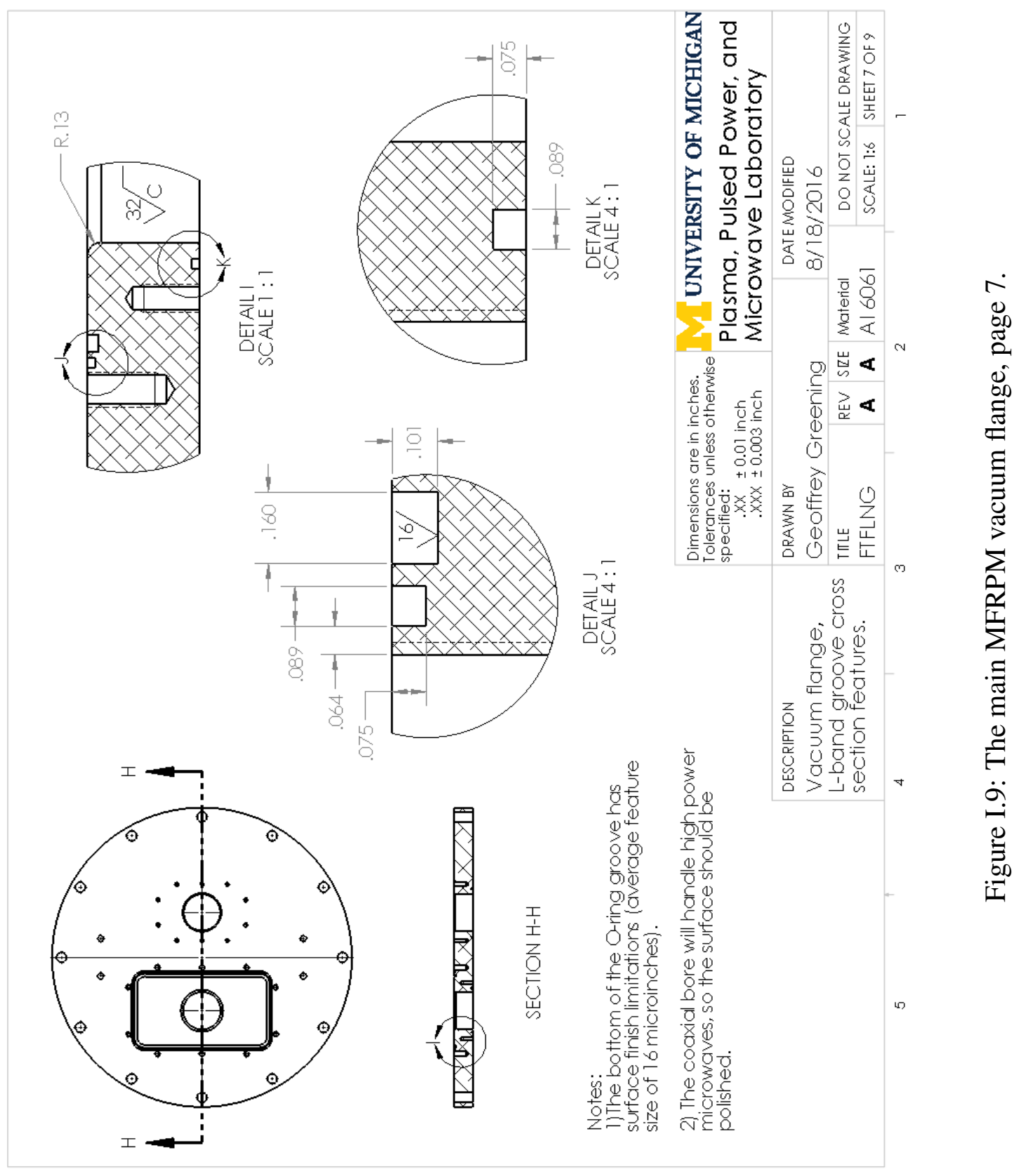




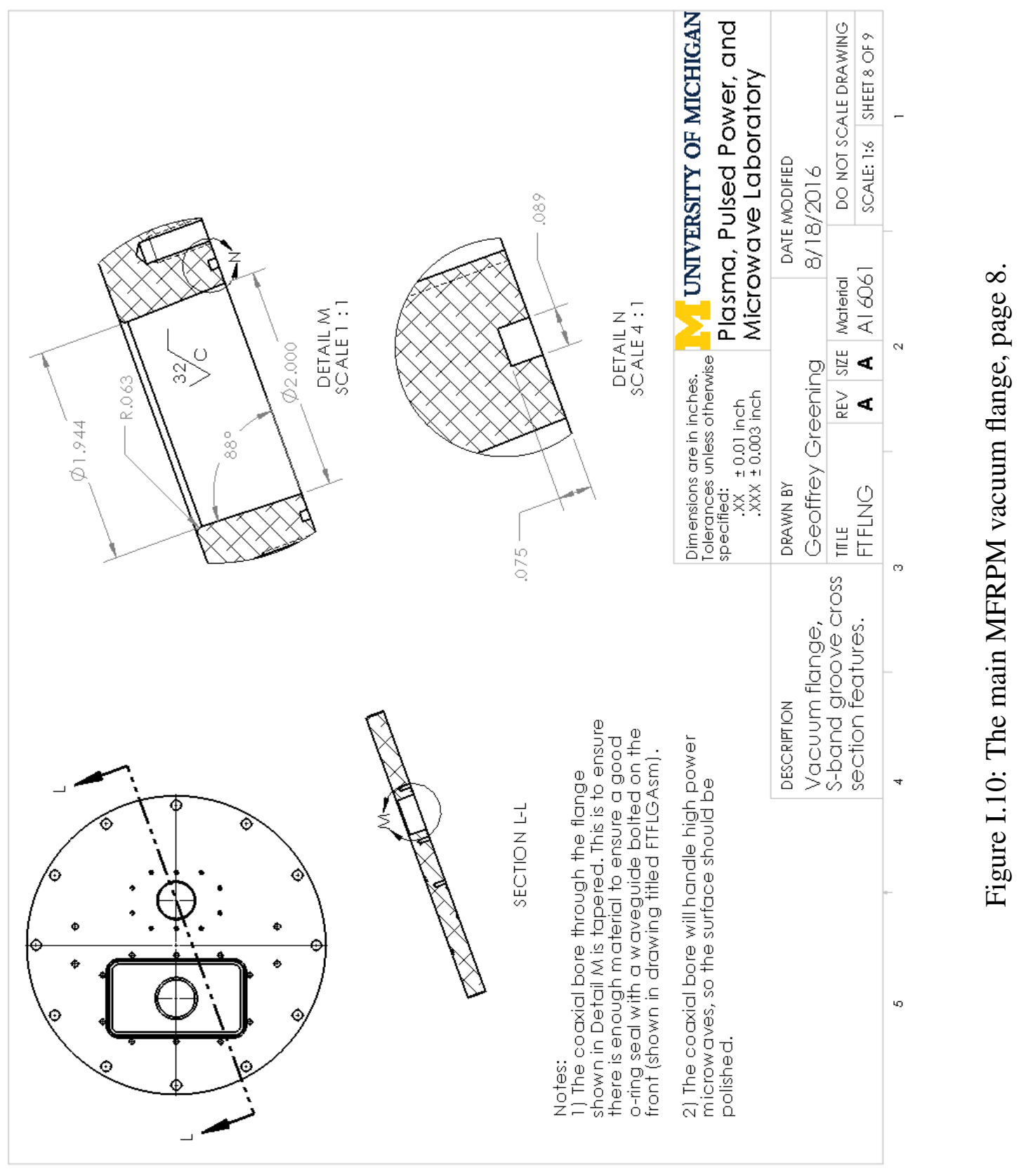




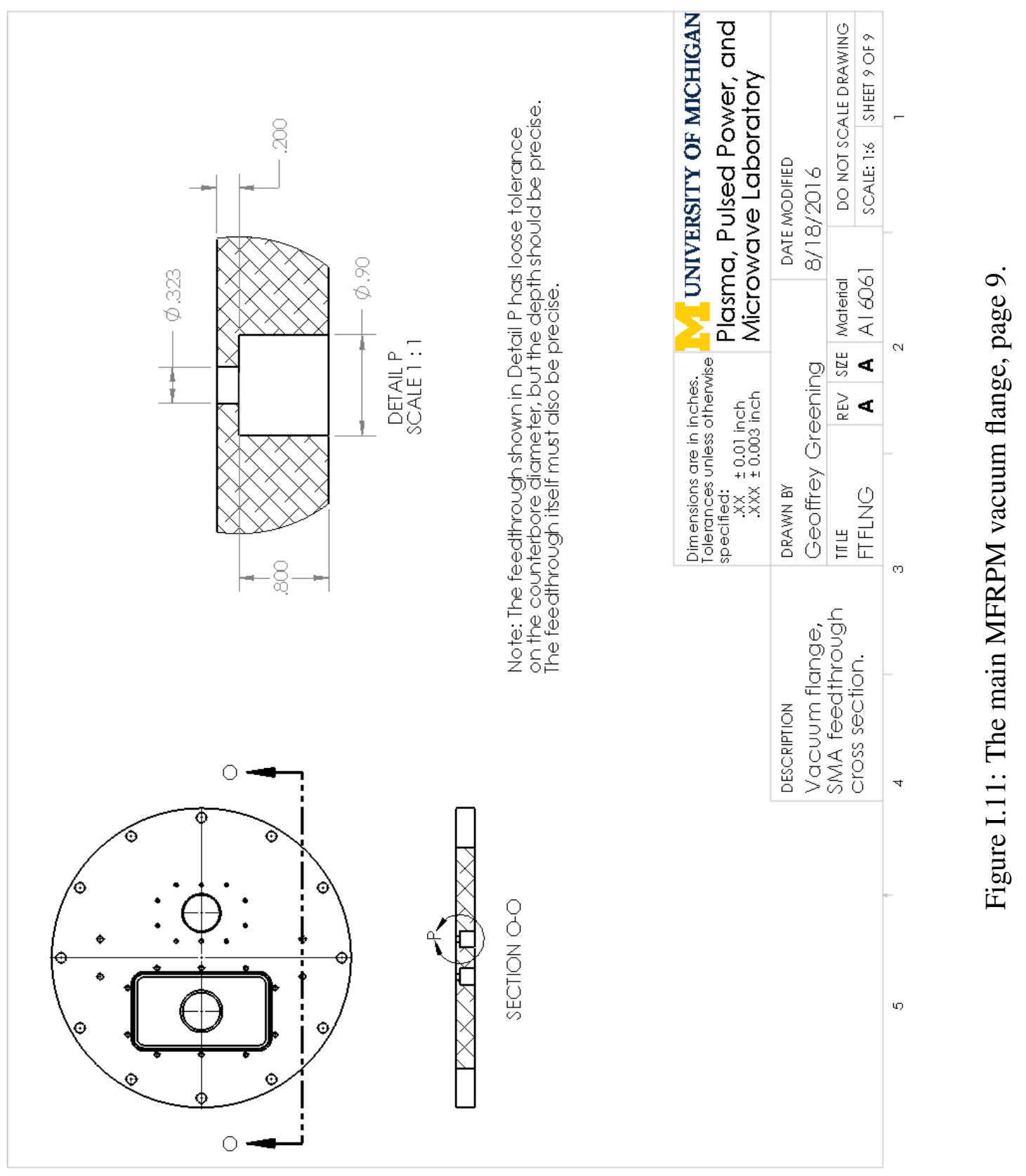




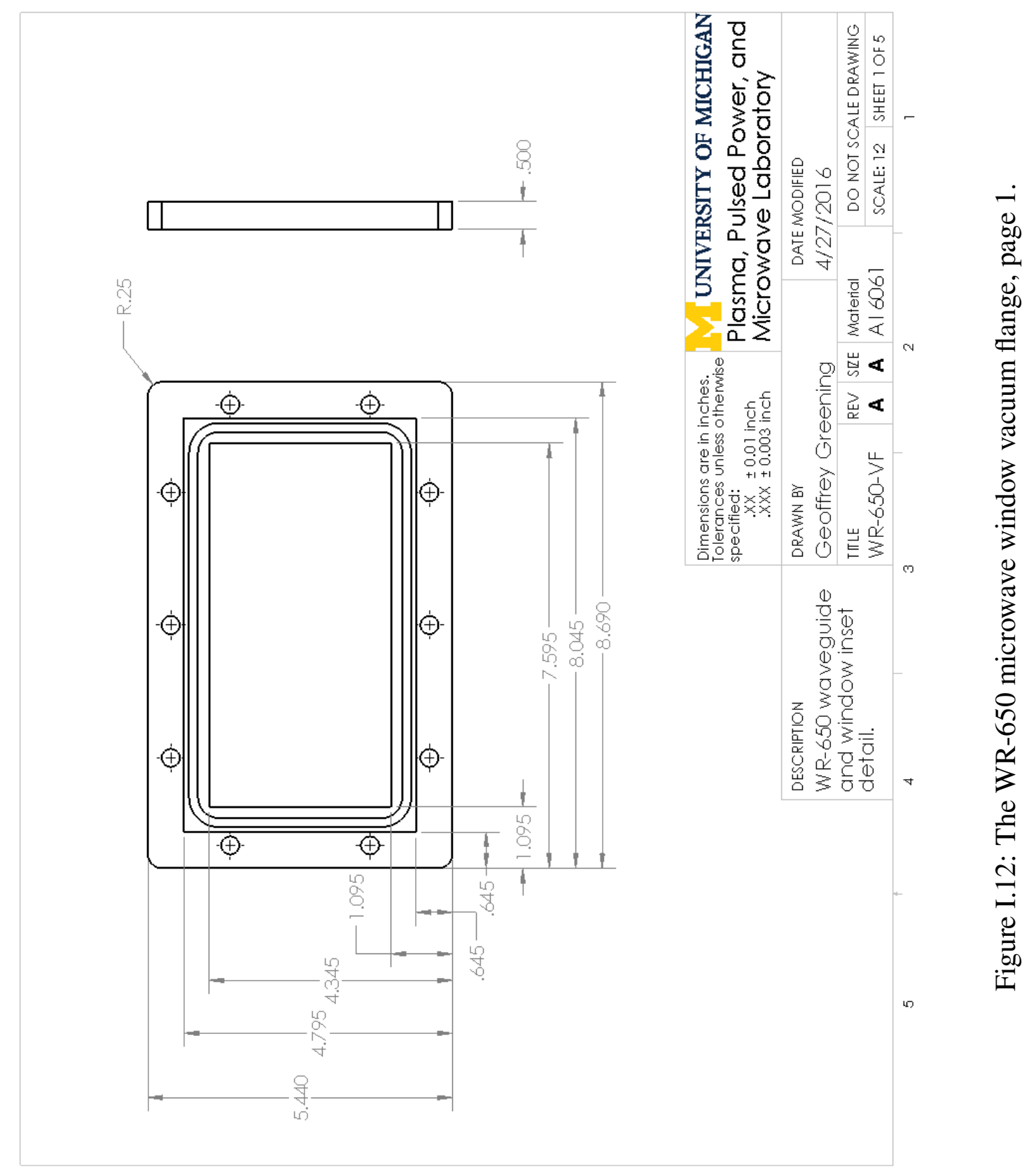



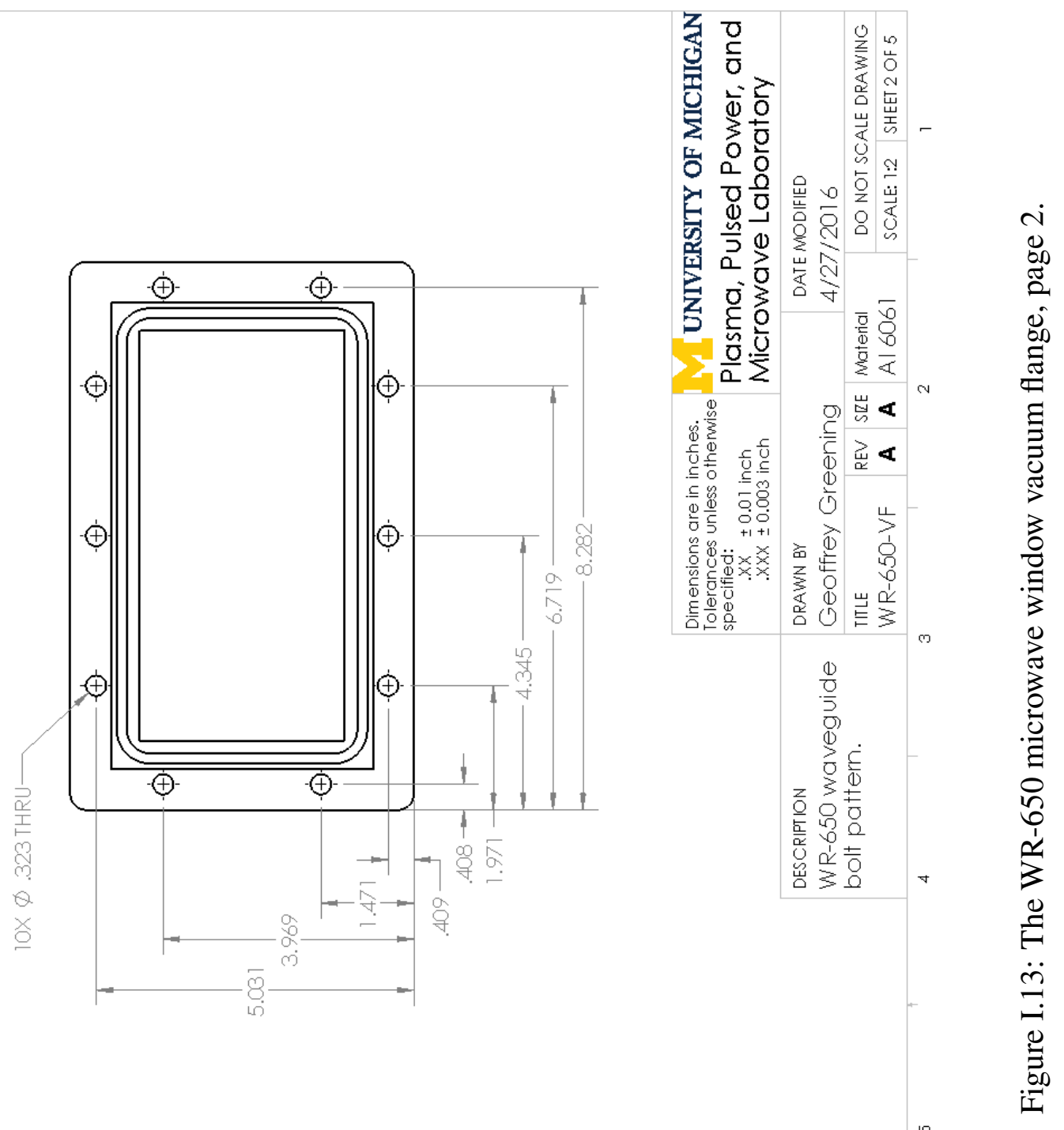


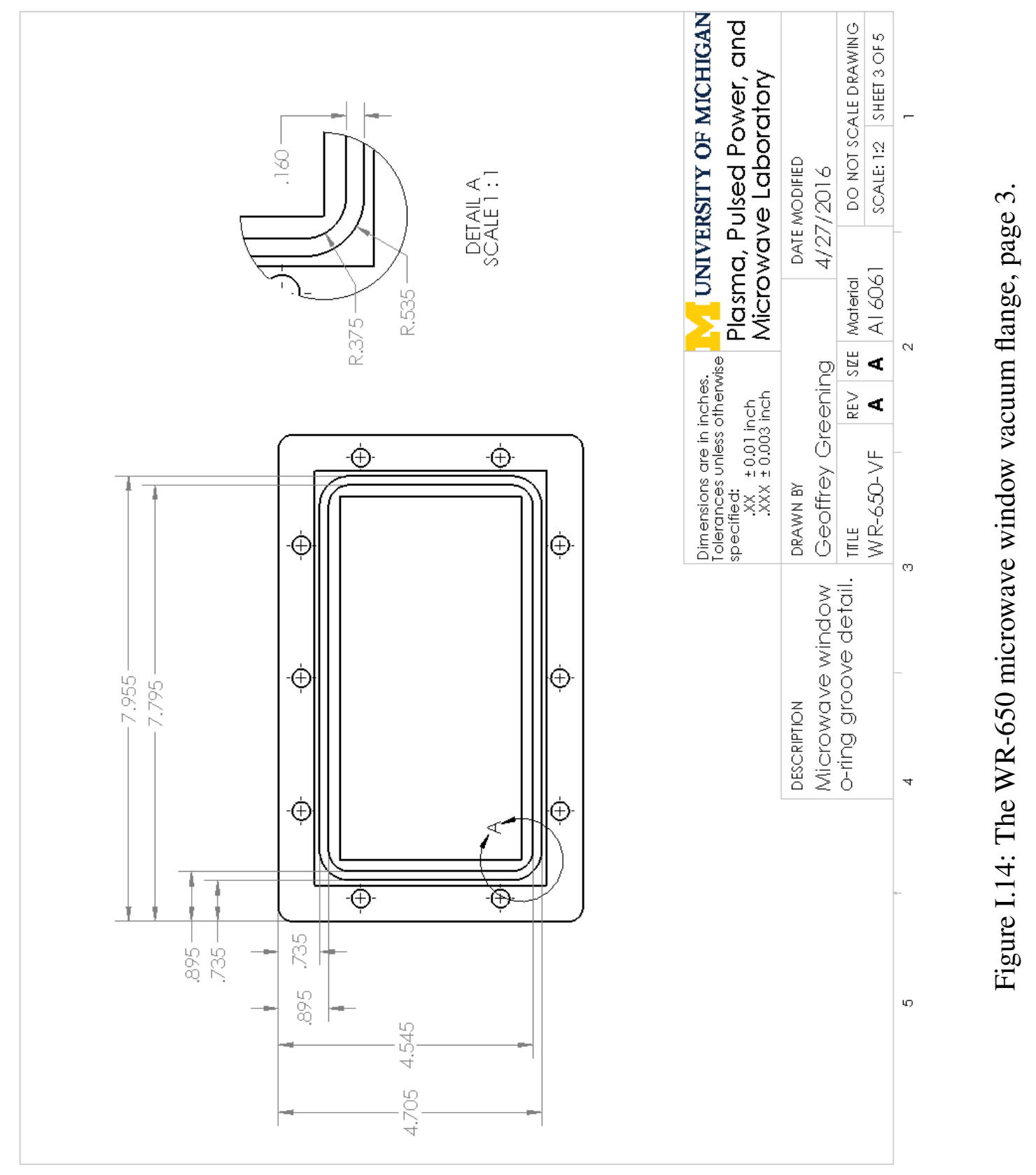




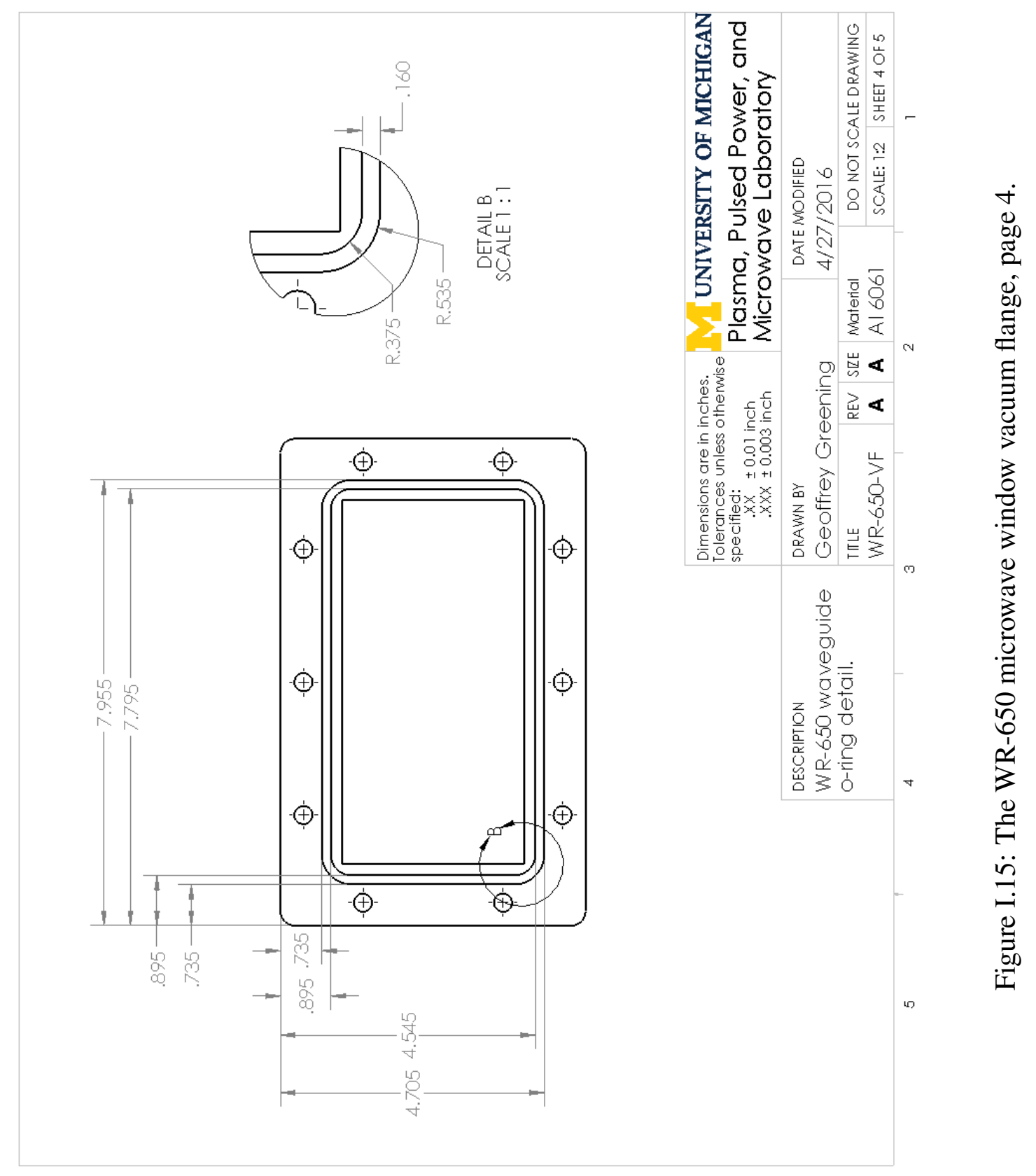




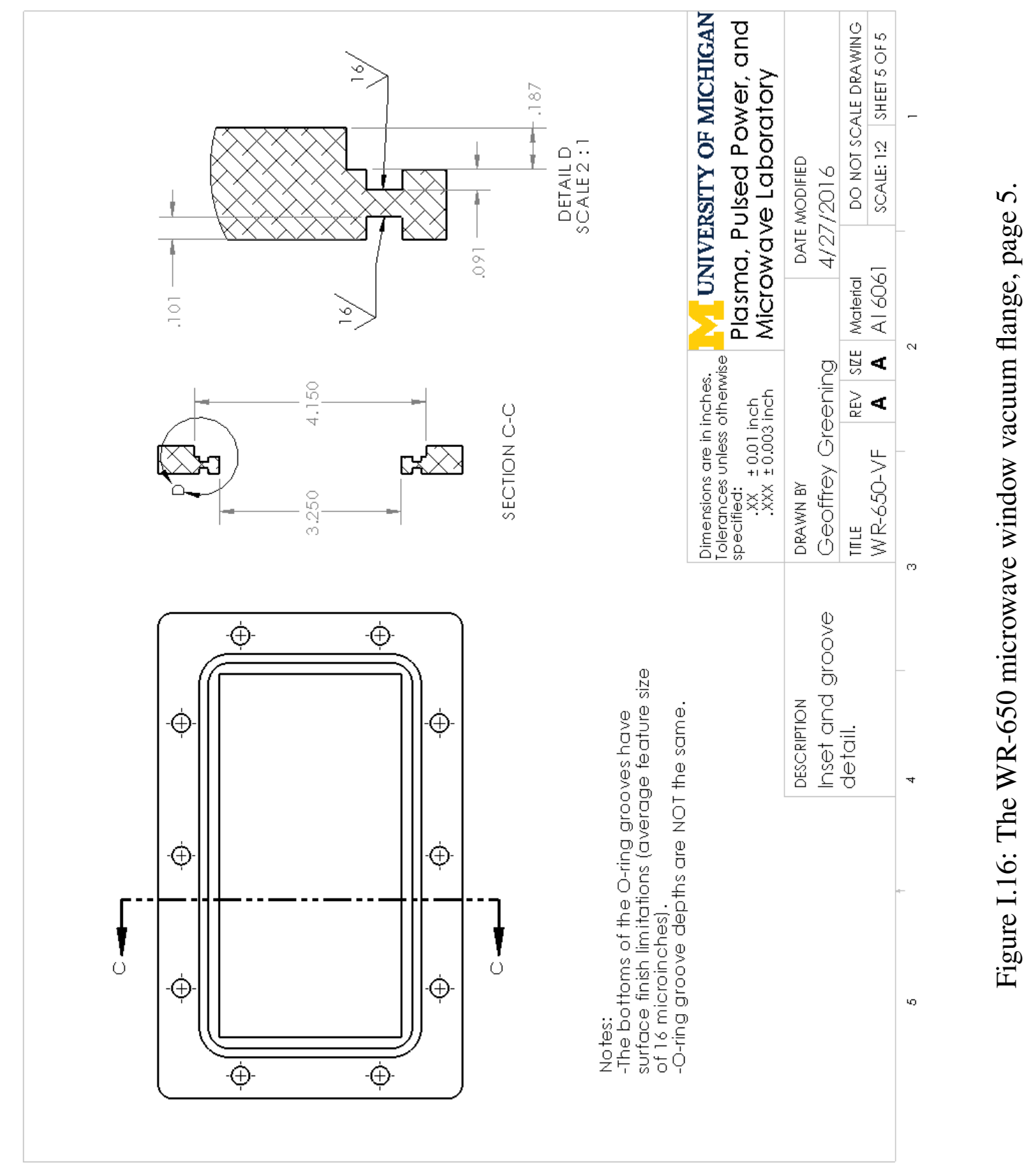




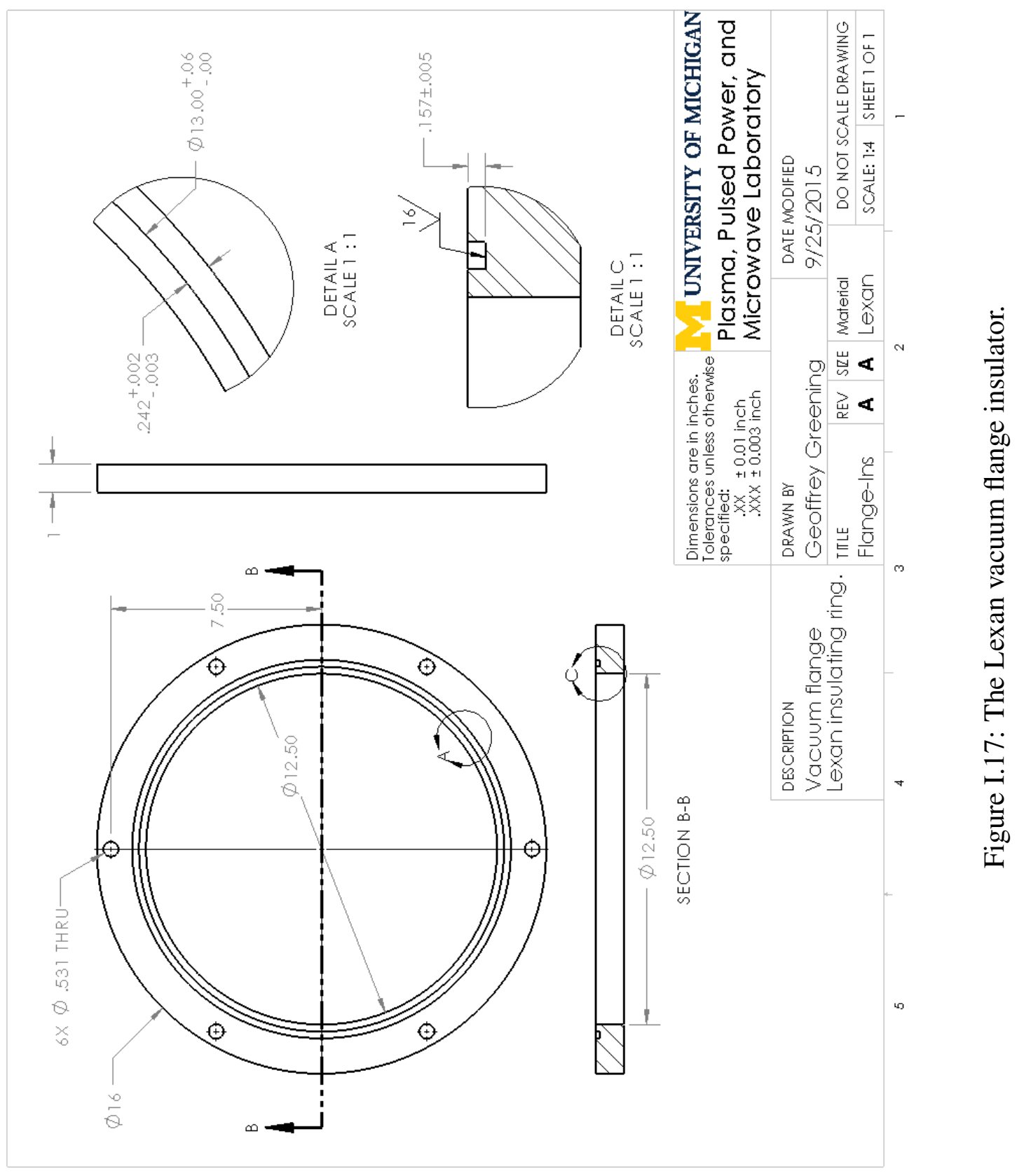




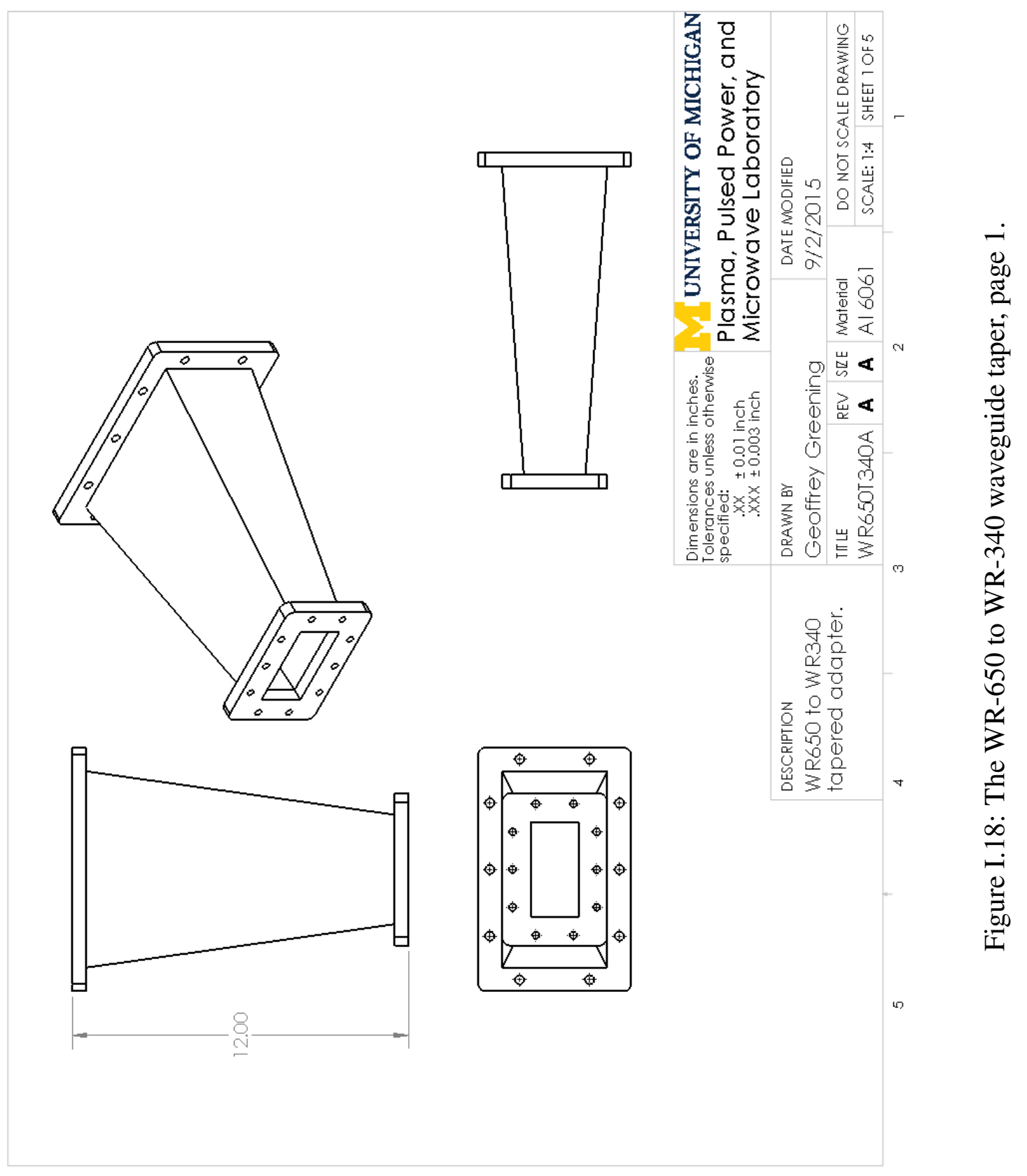




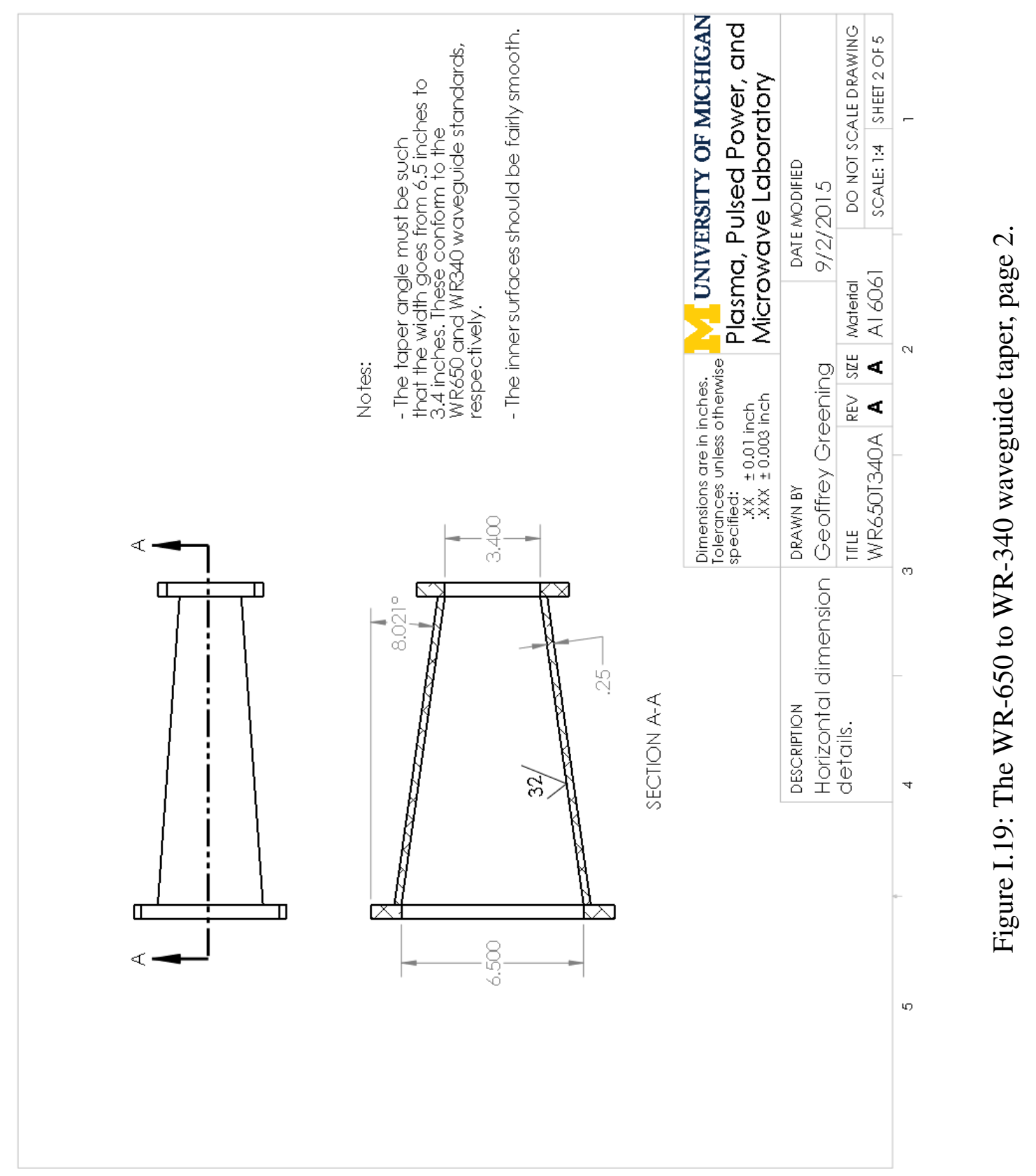




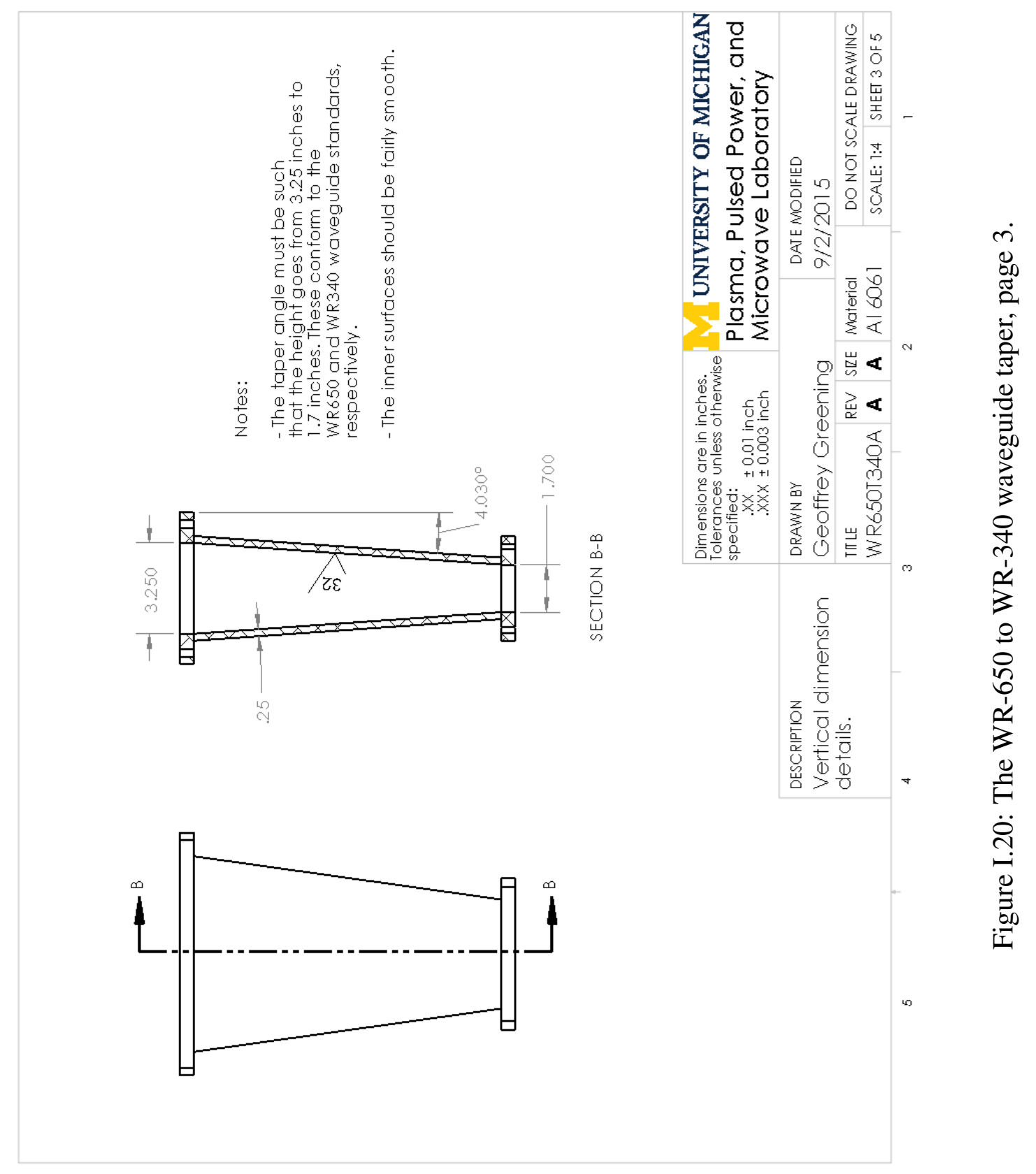




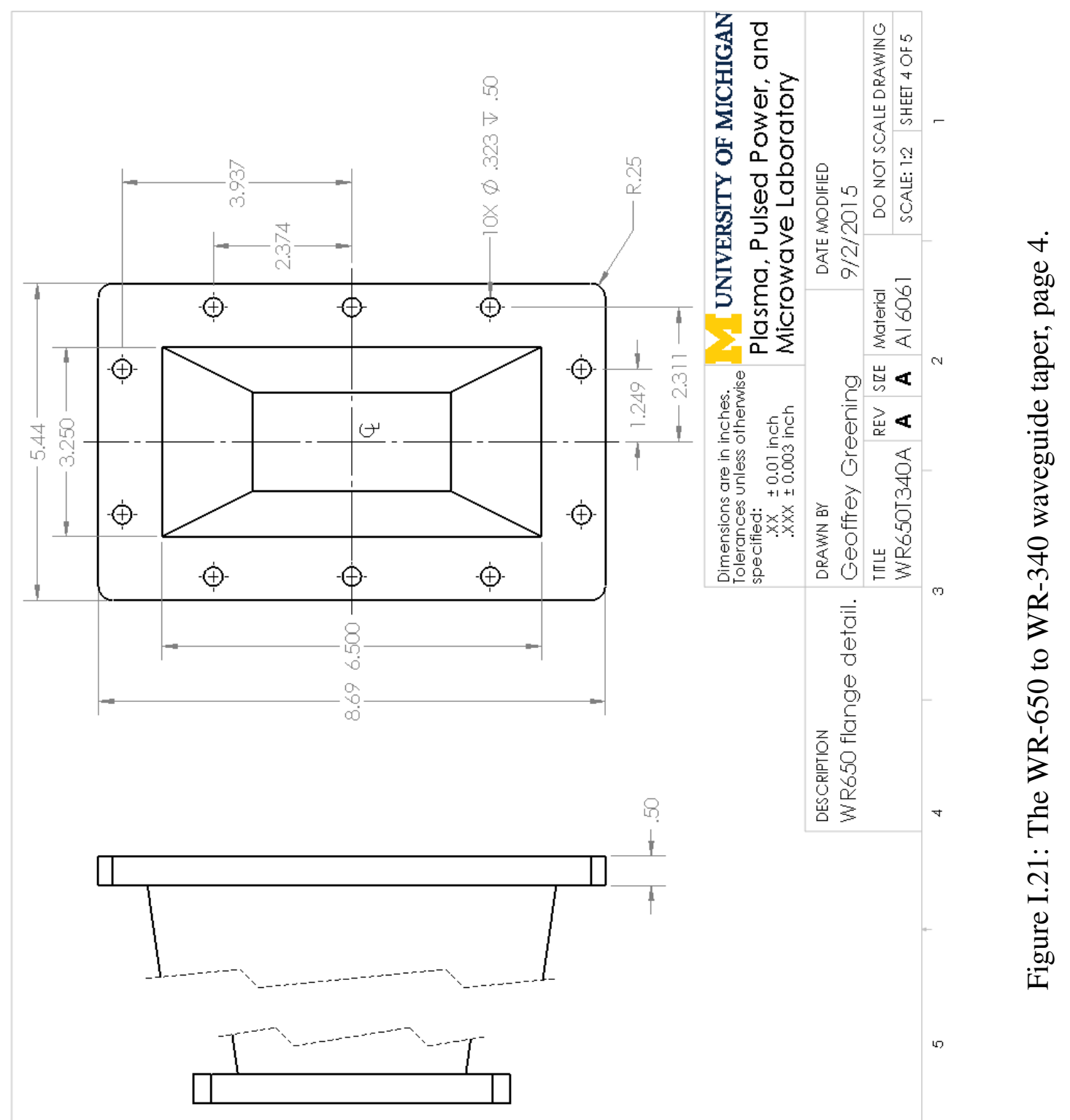




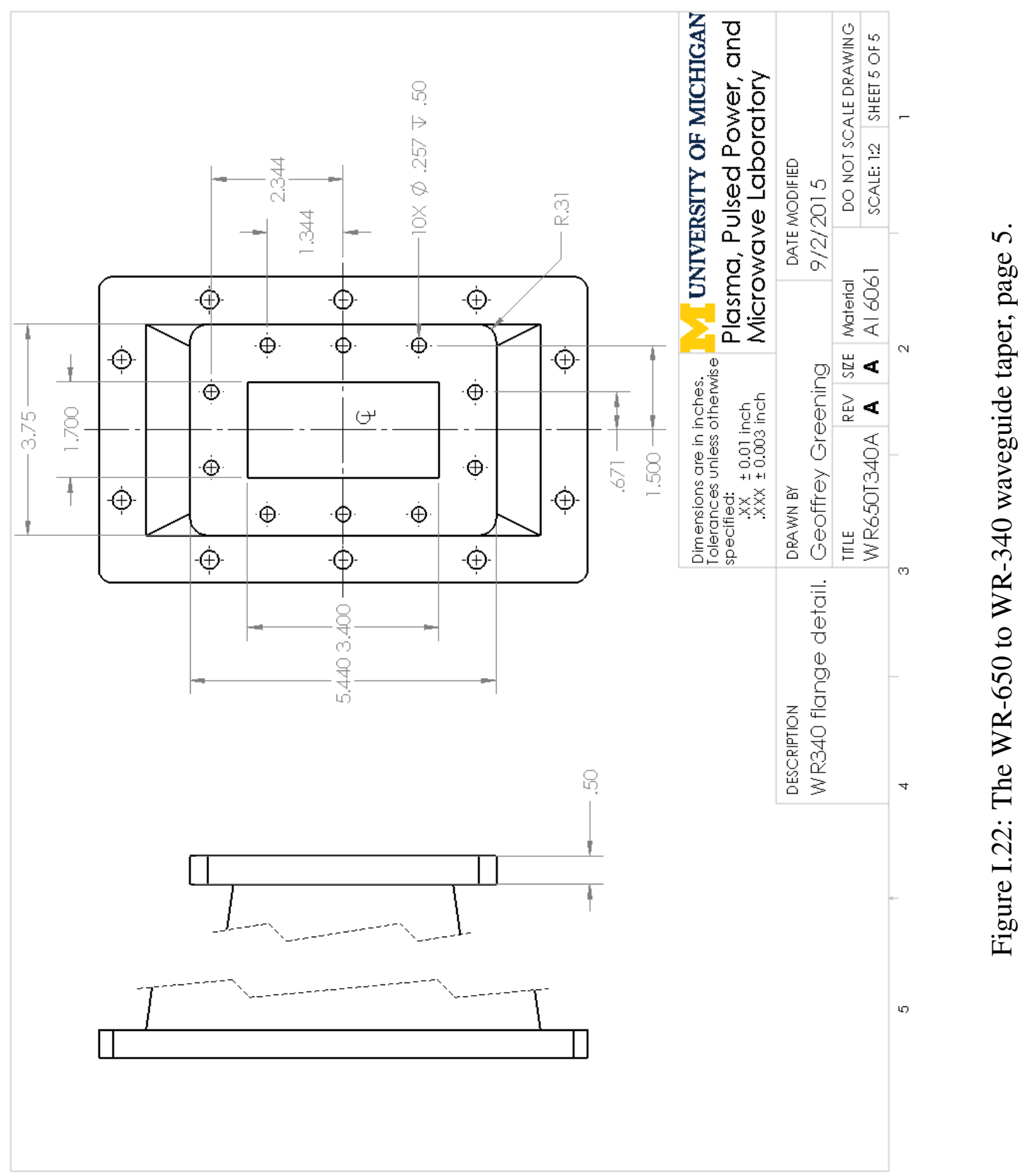




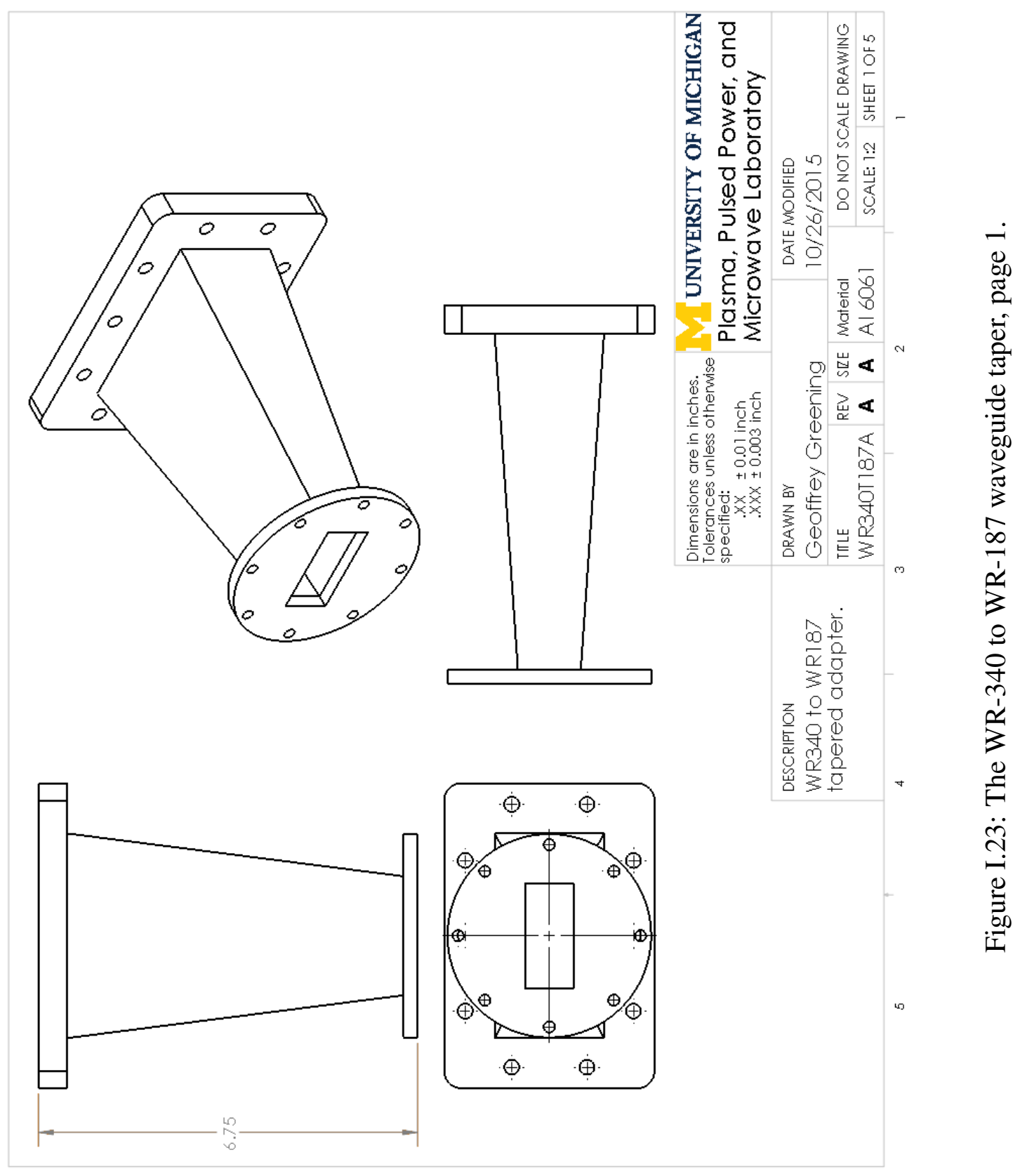




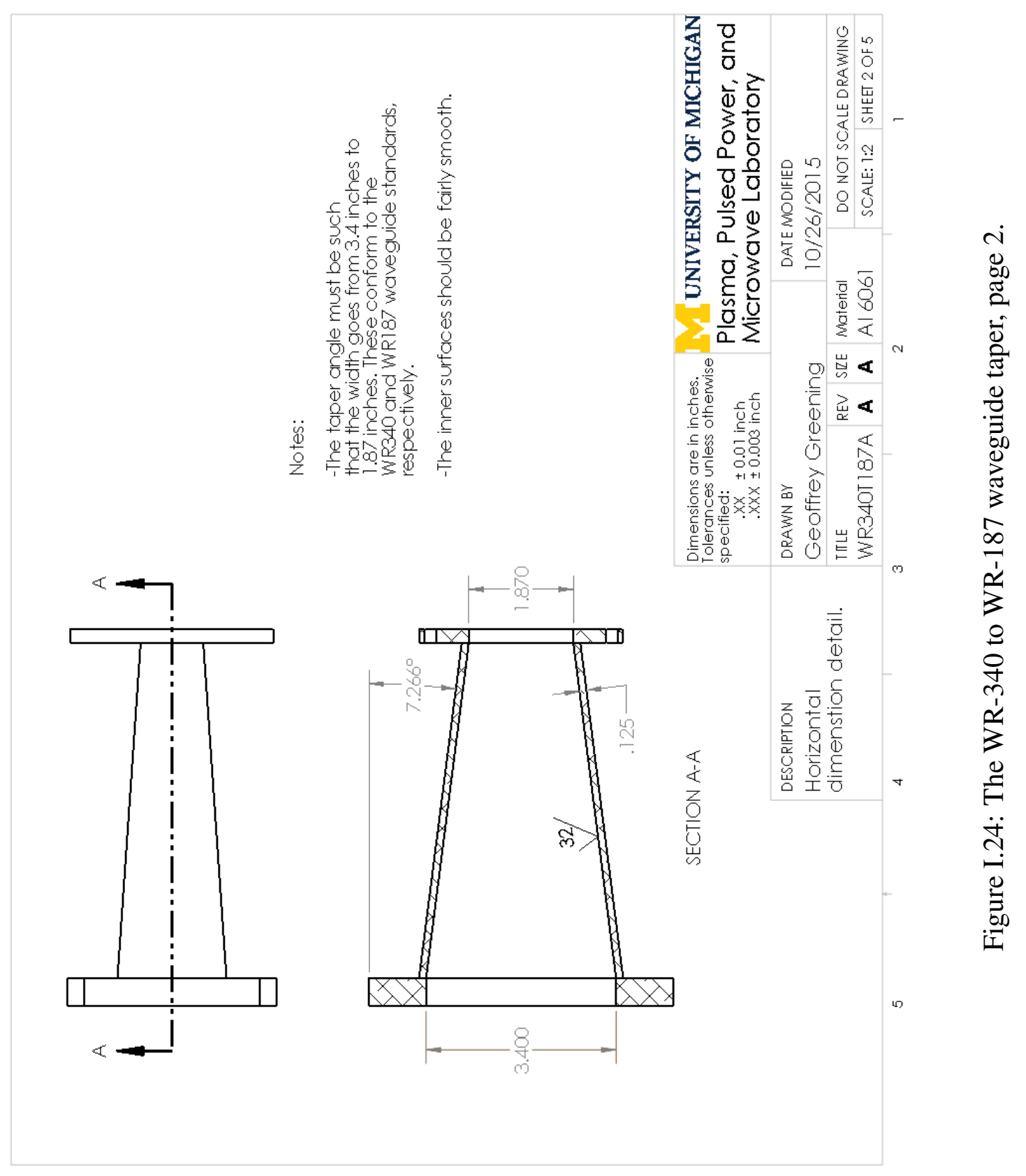




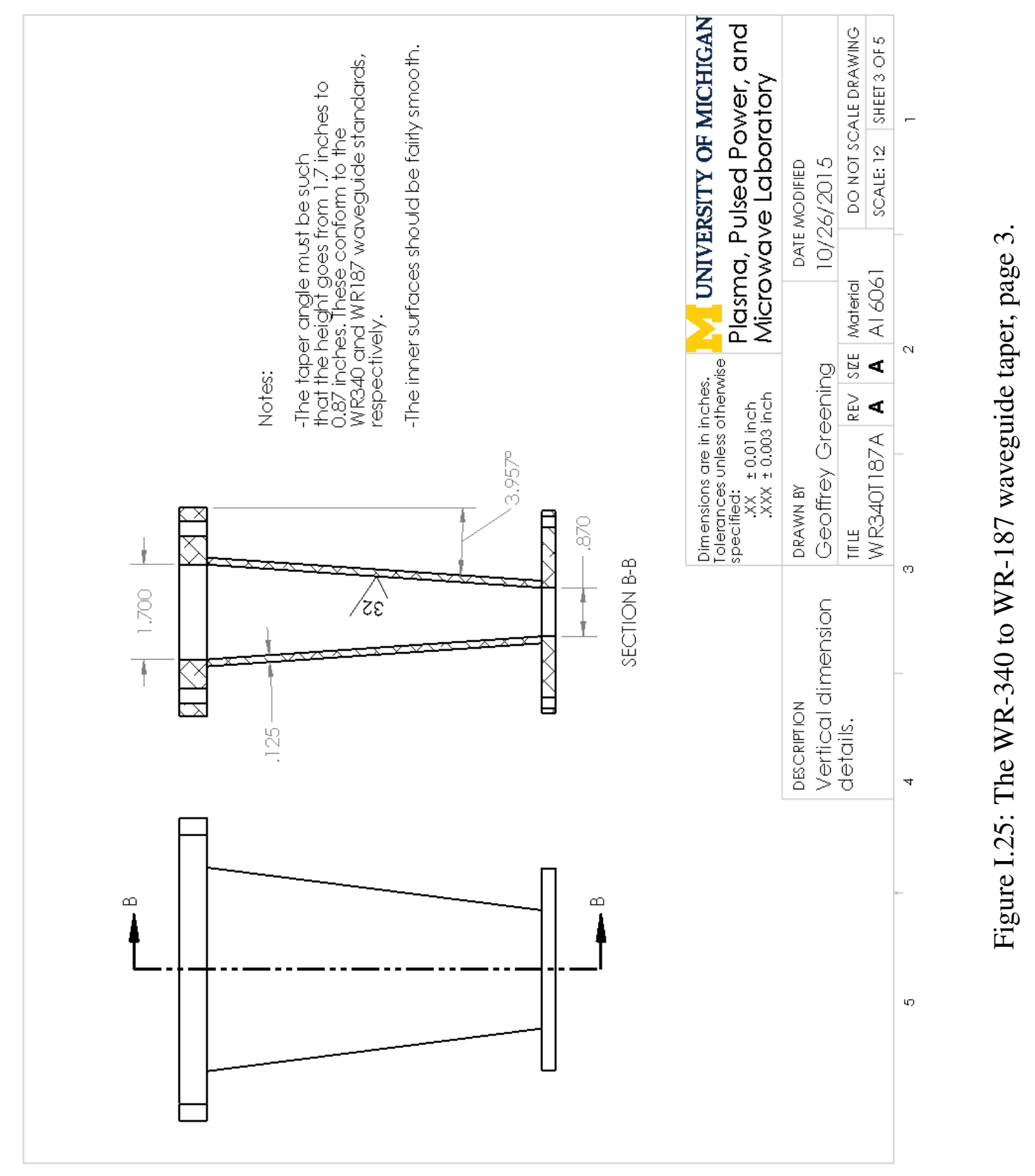



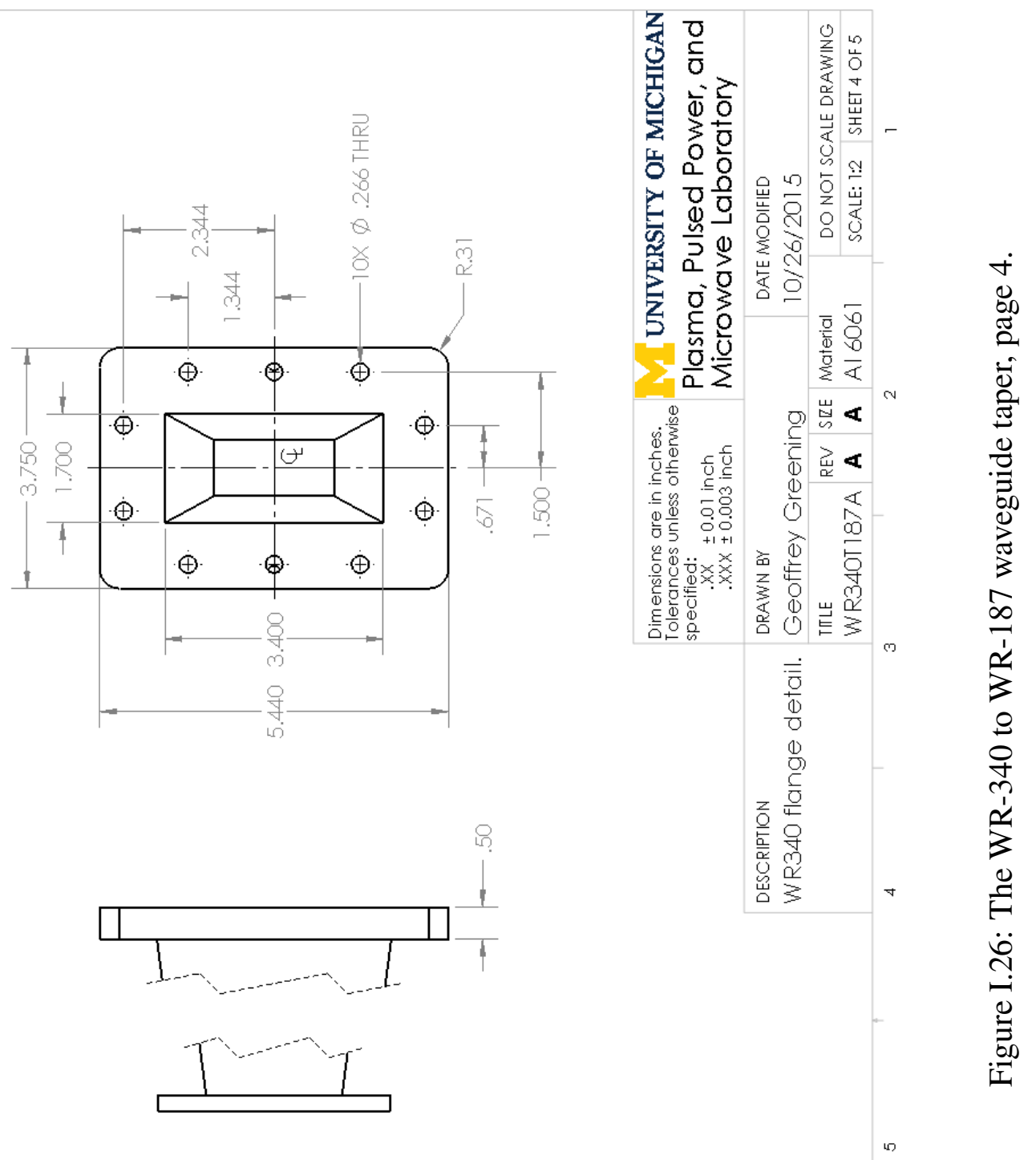


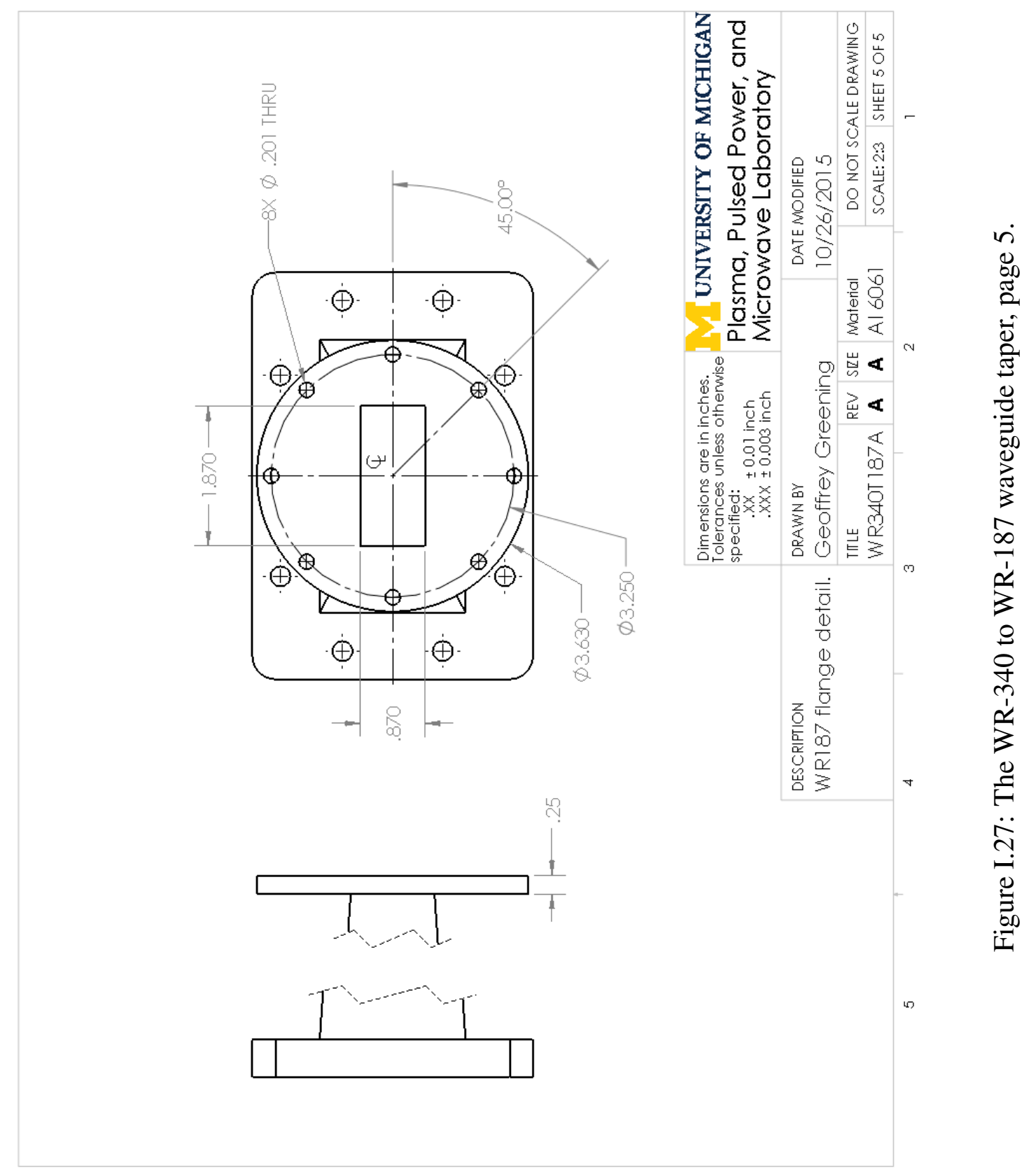




\section{BIBLIOGRAPHY}

[1] R. J. Barker and E. Schamiloglu, Eds., High-Power Microwave Sources and Tech-

nologies, 1st ed. New York: Wiley-IEEE Press, 2001.

[2] R. J. Barker, J. H. Booske, N. C. L. Jr, and G. S. Nusinovich, Eds., Modern Microwave and Millimeter-Wave Power Electronics, 1st ed. Piscataway, NJ: WileyIEEE Press, 2005.

[3] J. Benford, J. A. Swegle, and E. Schamiloglu, High Power Microwaves, 3rd ed. New York: CRC Press, 2016.

[4] R. M. Gilgenbach, M. E. Read, K. E. Hackett, R. Lucey, B. Hui, V. L. Granatstein, K. R. Chu, A. C. England, C. M. Loring, O. C. Eldridge, H. C. Howe, A. G. Kulchar, E. Lazarus, M. Murakami, and J. B. Wilgen, "Heating at the Electron Cyclotron Frequency in the ISX-B Tokamak," Phys. Rev. Lett., vol. 44, no. 10, pp. 647-650, Mar. 1980. [Online]. Available: http://dx.doi.org/10.1103/PhysRevLett.44.647

[5] N. Friedman, The Naval Institute Guide to World Naval Weapon Systems, 5th ed. Naval Institute Press, 2006.

[6] A. S. Gilmour, Klystrons, Traveling Wave Tubes, Magnetrons, Cross-Field Amplifiers, and Gyrotrons. Boston, MA: Artech House, 2011.

[7] G. B. Collins, Ed., Microwave Magnetrons. New York: McGraw-Hill, 1948.

[8] R. M. Gilgenbach, Y. Y. Lau, H. McDowell, K. L. Cartwright, and T. A. Spencer, "Crossed-Field Devices," in Modern Microwave and Millimeter-Wave Power Electronics, 1st ed., R. J. Barker, J. H. Booske, N. C. L. Jr, and G. S. Nusinovich, Eds. Piscataway, NJ: Wiley-IEEE Press, 2005, pp. 289-342.

[9] M. I. Fuks and E. Schamiloglu, "70\% Efficient Relativistic Magnetron With Axial Extraction of Radiation Through a Horn Antenna," IEEE Transactions on Plasma Science, vol. 38, no. 6, pp. 1302-1312, Jun. 2010. [Online]. Available: http://doi.org/10.1109/TPS.2010.2042823

[10] M. A. Franzi, "Relativistic Recirculating Planar Magnetrons," Ph.D. dissertation, University of Michigan, Ann Arbor, MI, USA, 2014.

[11] C. Leach, S. Prasad, M. I. Fuks, J. Buchenauer, J. W. McConaha, and E. Schamiloglu, "Experimental Demonstration of a High-Efficiency Relativistic 
Magnetron With Diffraction Output With Spherical Cathode Endcap," IEEE Transactions on Plasma Science, vol. 45, no. 2, pp. 282-288, Feb. 2017. [Online]. Available: http://doi.org/10.1109/TPS.2016.2644625

[12] J. Benford, H. Sze, T. Young, D. Bromley, and G. Proulx, "Variations on the Relativistic Magnetron," IEEE Transactions on Plasma Science, vol. 13, no. 6, pp. 538-544, Dec. 1985. [Online]. Available: http://dx.doi.org/10.1109/TPS.1985. 4316470

[13] J. Benford, H. Sze, W. Woo, R. R. Smith, and B. Harteneck, "Phase Locking of Relativistic Magnetrons," Phys. Rev. Lett., vol. 62, no. 8, pp. 969-971, Feb. 1989. [Online]. Available: http://dx.doi.org/10.1103/PhysRevLett.62.969

[14] G. B. Greening, N. M. Jordan, S. C. Exelby, D. H. Simon, Y. Y. Lau, and R. M. Gilgenbach, "Multi-frequency recirculating planar magnetrons," Appl. Phys. Lett., vol. 109, no. 7, p. 074101, Aug. 2016. [Online]. Available: http://dx.doi.org/10.1063/1.4961070

[15] T. A. Treado, W. O. Doggett, G. E. Thomas, R. S. Smith, J. Jackson-Ford, and D. J. Jenkins, "Operating modes of relativistic rising-sun and A6 magnetrons," IEEE Trans. Plasma Sci., vol. 16, no. 2, pp. 237-248, Apr. 1988. [Online]. Available: http://dx.doi.org/10.1109/27.3820

[16] A. Palevsky and G. Bekefi, "Microwave emission from pulsed, relativistic e-beam diodes. II. The multiresonator magnetron," The Physics of Fluids, vol. 22, no. 5, pp. 986-996, May 1979. [Online]. Available: http://dx.doi.org/10.1063/1.862663

[17] D. Price, J. S. Levine, and J. N. Benford, "Diode plasma effects on the microwave pulse length from relativistic magnetrons," IEEE Trans. Plasma Sci., vol. 26, no. 3, pp. 348-353, 1998. [Online]. Available: https://doi.org/10.1109/27.700765

[18] J. S. Levine, B. D. Harteneck, and H. D. Price, "Frequency-agile relativistic magnetrons," in SPIE's 1995 International Symposium on Optical Science, Engineering, and Instrumentation. International Society for Optics and Photonics, 1995, pp. 74-79. [Online]. Available: http://dx.doi.org/10.1117/12.218536

[19] Jin-Chuan Ju, Yu-Wei Fan, Hui-Huang Zhong, and Ting Shu, "A Novel Dual-Frequency Magnetically Insulated Transmission Line Oscillator," IEEE Trans. Plasma Sci., vol. 37, no. 10, pp. 2041-2047, Oct. 2009. [Online]. Available: https://doi.org/10.1109/TPS.2009.2027603

[20] X. Zhang, Y. Li, Z. Li, H. Zhong, and B. Qian, "Preliminary experimental investigation of a complex dual-band high power microwave source," Rev. Sci. Instrum., vol. 86, no. 10, p. 104703, Oct. 2015. [Online]. Available: http://dx.doi.org/10.1063/1.4934246

[21] J. He, Y. Cao, J. Zhang, T. Wang, and J. Ling, "Design of a dual-frequency high-power microwave generator," Laser Part. Beams, vol. 29, no. 04, pp. 479-485, Dec. 2011. [Online]. Available: https://doi.org/10.1017/S0263034611000590 
[22] W. Ting, Q. Bao-liang, Z. Jian-de, Z. Xiao-ping, C. Yi-bing, and Z. Qiang, "Preliminary experimental investigation of a dual-band relativistic backward wave oscillator with dual beams," Phys. Plasmas, vol. 18, no. 1, p. 013107, Jan. 2011. [Online]. Available: http://dx.doi.org/10.1063/1.3537818

[23] X. Meng, Y. Gong, T. Tang, H. Gong, and G. Travish, "The research of $140 \mathrm{ghz}$ high harmonic traveling wave tube," in 2016 IEEE International Vacuum Electronics Conference (IVEC), Apr. 2016, pp. 1-2. [Online]. Available: http://doi.org/10.1109/IVEC.2016.7561832

[24] R. M. Gilgenbach, Y. Y. Lau, D. M. French, B. W. Hoff, J. Luginsland, and M. Franzi, "US Patent 8841867b2: Crossed Field Device," U.S. Patent US 8841867 B2, Sep., 2014.

[25] R. M. Gilgenbach, Y.-Y. Lau, D. M. French, B. W. Hoff, M. Franzi, and J. Luginsland, "Recirculating Planar Magnetrons for High-Power High-Frequency Radiation Generation," IEEE Trans. Plasma Sci., vol. 39, no. 4, pp. 980-987, Apr. 2011. [Online]. Available: https://doi.org/10.1109/TPS.2010.2099670

[26] J. W. Luginsland, Y. Y. Lau, and R. M. Gilgenbach, "Two-Dimensional ChildLangmuir Law," Phys. Rev. Lett., vol. 77, no. 22, pp. 4668-4670, Nov. 1996. [Online]. Available: https://doi.org/10.1103/PhysRevLett.77.4668

[27] —- "Two-Dimensional Child-Langmuir Law [Phys. Rev. Lett. 77, 4668 (1996)]," Phys. Rev. Lett., vol. 78, no. 13, pp. 2680-2680, Mar. 1997. [Online]. Available: https://doi.org/10.1103/PhysRevLett.78.2680

[28] Y. Y. Lau, "Simple Theory for the Two-Dimensional Child-Langmuir Law," Phys. Rev. Lett., vol. 87, no. 27, p. 278301, Dec. 2001. [Online]. Available: https://doi.org/10.1103/PhysRevLett.87.278301

[29] R. J. Umstattd, C. G. Carr, C. L. Frenzen, J. W. Luginsland, and Y. Y. Lau, "A simple physical derivation of Child-Langmuir space-charge-limited emission using vacuum capacitance," American Journal of Physics, vol. 73, no. 2, pp. 160-163, Feb. 2005. [Online]. Available: http://dx.doi.org/10.1119/1.1781664

[30] D. Chernin and Y. Y. Lau, "Stability of laminar electron layers," Physics of Fluids (1958-1988), vol. 27, no. 9, pp. 2319-2331, Sep. 1984. [Online]. Available: http://dx.doi.org/10.1063/1.864888

[31] D. M. French, B. W. Hoff, Y. Y. Lau, and R. M. Gilgenbach, "Negative, positive, and infinite mass properties of a rotating electron beam," Applied Physics Letters, vol. 97, no. 11, p. 111501, 2010. [Online]. Available: http://dx.doi.org/10.1063/1.3488833

[32] D. H. Simon, Y. Y. Lau, G. Greening, P. Wong, B. W. Hoff, and R. M. Gilgenbach, "Stability of Brillouin flow in planar, conventional, and inverted magnetrons," Physics of Plasmas, vol. 22, no. 8, p. 082104, Aug. 2015. [Online]. Available: http://dx.doi.org/10.1063/1.4927798 
[33] J. F. Hull, "Inverted magnetron," Proceedings of the IRE, vol. 40, no. 9, pp. 10381041, 1952. [Online]. Available: https://doi.org/10.1109/JRPROC.1952.273869

[34] R. A. Close, A. Palevsky, and G. Bekefi, "Radiation measurements from an inverted relativistic magnetron," Journal of Applied Physics, vol. 54, no. 7, pp. 4147-4151, Jul. 1983. [Online]. Available: http://dx.doi.org/10.1063/1.332549

[35] T. P. Fleming, M. R. Lambrecht, and K. L. Cartwright, "Numerical Simulations of a Relativistic Inverted Magnetron," IEEE Transactions on Plasma Science, vol. 38, no. 7, pp. 1563-1573, Jul. 2010. [Online]. Available: https: //doi.org/10.1109/TPS.2010.2048209

[36] B. W. Hoff, M. Franzi, D. M. French, G. Greening, and R. M. Gilgenbach, "A compact, pi-mode extraction scheme for the axial B-field recirculating planar magnetron," DTIC Document, Tech. Rep., 2012. [Online]. Available: http://oai.dtic. mil/oai/oai?verb=getRecord\&metadataPrefix=html\&identifier=ADA565729

[37] D. H. Simon, Y. Y. Lau, G. Greening, P. Wong, B. Hoff, and R. M. Gilgenbach, "Stability of Brillouin flow in the presence of slow-wave structure," Physics of Plasmas, vol. 23, no. 9, p. 092101, Sep. 2016. [Online]. Available: http://dx.doi.org/10.1063/1.4961917

[38] D. H. Simon, "Equilibrium and Stability of Brillouin Flow in Planar, Conventional, and Inverted Magnetrons," Ph.D. dissertation, University of Michigan, Ann Arbor, MI, USA, 2016.

[39] M. A. Franzi, R. M. Gilgenbach, B. W. Hoff, D. A. Chalenski, D. Simon, Y. Y. Lau, and J. Luginsland, "Recirculating-Planar-Magnetron Simulations and Experiment," IEEE Trans. Plasma Sci., vol. 41, no. 4, pp. 639-645, Apr. 2013. [Online]. Available: https://doi.org/10.1109/TPS.2013.2242493

[40] M. A. Franzi, G. B. Greening, N. M. Jordan, R. M. Gilgenbach, D. H. Simon, Y. Y. Lau, B. W. Hoff, and J. Luginsland, "Microwave Power and Phase Measurements on a Recirculating Planar Magnetron," IEEE Trans. Plasma Sci., vol. 43, no. 5, pp. 1675-1682, May 2015. [Online]. Available: https://doi.org/10.1109/TPS.2015.2417774

[41] N. M. Jordan, G. B. Greening, B. W. Hoff, S. S. Maestas, S. C. Exelby, and R. M. Gilgenbach, "Additively Manufactured High Power Microwave Anodes," IEEE Trans. Plasma Sci., vol. 44, no. 8, pp. 1258-1264, Aug. 2016. [Online]. Available: http://dx.doi.org/10.1109/TPS.2016.2565261

[42] M. Franzi, R. Gilgenbach, Y. Y. Lau, B. Hoff, G. Greening, and P. Zhang, "Passive mode control in the recirculating planar magnetron," Phys. Plasmas, vol. 20, no. 3, p. 033108, 2013. [Online]. Available: http://dx.doi.org/10.1063/1.4794967

[43] Y. Y. Lau, "Private Communication,” Jan. 2017. 
[44] Z. Cendes, "The development of HFSS," in 2016 USNC-URSI Radio Science Meeting, Jun. 2016, pp. 39-40. [Online]. Available: http://doi.org/10.1109/ USNC-URSI.2016.7588501

[45] J. W. Gewartowski and H. A. Watson, Principles of Electron Tubes. Princeton, NJ: D. Van Nostrand, 1965.

[46] Y. Y. Lau and D. Chernin, "A review of the ac space-charge effect in electron-circuit interactions," Phys. Fluids B, vol. 4, no. 11, p. 3473, 1992. [Online]. Available: http://dx.doi.org/10.1063/1.860356

[47] M. Chodorow and C. Susskind, Fundamentals of Microwave Electronics. New York, NY: McGraw-Hill, 1964.

[48] J. C. Slater, Microwave Electronics. Princeton, NJ: D. Van Nostrand, 1950.

[49] E. Okress, G. Mourier, J. Feinstein, E. Kettlewell, and G. R. Feaster, Eds., CrossedField Microwave Devices. $\quad$ New York, NY: Academic Press, Inc., 1961, vol. 1.

[50] L. Schächter, Beam-Wave Interaction in Periodic and Quasi-Periodic Structures, 2nd ed. Berlin, Germany: Springer-Verlag, 2011.

[51] E. Okress, G. Mourier, J. Feinstein, E. Kettlewell, and G. R. Feaster, Eds., CrossedField Microwave Devices. $\quad$ New York, NY: Academic Press, Inc., 1961, vol. 2.

[52] D. M. H. Hung, I. M. Rittersdorf, P. Zhang, D. Chernin, Y. Y. Lau, T. M. Antonsen, J. W. Luginsland, D. H. Simon, and R. M. Gilgenbach, "Absolute Instability near the Band Edge of Traveling-Wave Amplifiers," Phys. Rev. Lett., vol. 115, no. 12, p. 124801, Sep. 2015. [Online]. Available: https://doi.org/10.1103/PhysRevLett.115.124801

[53] A. S. Gilmour, Principles of Traveling Wave Tubes. Boston, MA: Artech House, 2014.

[54] Y. Y. Lau, "Theory of Crossed-Field Devices," in High-Power Microwave Sources, V. L. Granatstein and I. Alexeff, Eds. Norwood, MA: Artech House, 1987, pp. 309-349.

[55] M. R. Lopez, "Experiments on a Relativistic Magnetron Driven by a Microsecond Electron Beam Accelerator with a Ceramic Insulating Stack," Ph.D. dissertation, University of Michigan, Ann Arbor, MI, USA, 2003.

[56] M. C. Jones, "Cathode Priming of a Relativistic Magnetron Using Multi-Emission Zones On Projection Ablation Lithography Cathodes,” Ph.D. dissertation, University of Michigan, Ann Arbor, MI, USA, 2005.

[57] Y. Y. Lau, J. W. Luginsland, K. L. Cartwright, D. H. Simon, W. Tang, B. W. Hoff, and R. M. Gilgenbach, "A re-examination of the Buneman-Hartree condition in a cylindrical smooth-bore relativistic magnetron," Phys. Plasmas, vol. 17, no. 3, p. 033102, 2010. [Online]. Available: http://dx.doi.org/10.1063/1.3328804 
[58] F. F. Chen, Introduction to Plasma Physics and Controlled Fusion, 2nd ed. Springer-Verlag, 2006, vol. 1.

[59] A. Palevsky, G. Bekefi, and A. T. Drobot, "Numerical simulation of oscillating magnetrons," Journal of Applied Physics, vol. 52, no. 8, pp. 4938-4941, Aug. 1981. [Online]. Available: http://dx.doi.org/10.1063/1.329381

[60] P. J. Christenson, D. P. Chernin, A. L. Garner, and Y. Y. Lau, "Resistive destabilization of cycloidal electron flow and universality of (near-) Brillouin flow in a crossed-field gap," Physics of Plasmas, vol. 3, no. 12, pp. 4455-4462, Dec. 1996. [Online]. Available: http://dx.doi.org/10.1063/1.872064

[61] P. J. Christenson, "Equilibrium, Stability, and Turbulence in Cycloidal Electron Flows in Crossed Electric and Magnetic Fields," Ph.D. dissertation, University of Michigan, Ann Arbor, MI, USA, 1996.

[62] S. E. Tsimring, Electron Beams and Microwave Vacuum Electronics. Hoboken, NJ: John Wiley \& Sons, Inc., 2007.

[63] M. Friedman, J. Krall, Y. Y. Lau, and V. Serlin, "Relativistic Klystron Amplifier," in SPIE's 1988 Microwave and Particle Beam Sources and Propagation, vol. 0873, 1988, pp. 92-103. [Online]. Available: http://dx.doi.org/10.1117/12.965085

[64] N. J. Dionne, "Harmonic generation in octave bandwidth traveling-wave tubes," IEEE Transactions on Electron Devices, vol. 17, no. 4, pp. 365-372, Apr. 1970. [Online]. Available: http://doi.org/10.1109/T-ED.1970.16981

[65] C. F. Dong, P. Zhang, D. Chernin, Y. Y. Lau, B. W. Hoff, D. H. Simon, P. Wong, G. B. Greening, and R. M. Gilgenbach, "Harmonic Content in the Beam Current in a Traveling-Wave Tube," IEEE Trans. Electron Dev., vol. 62, no. 12, pp. 4285-4292, Dec. 2015. [Online]. Available: http://dx.doi.org/10.1109/TED.2015.2490584

[66] R. W. Lemke, T. C. Genoni, and T. A. Spencer, "Effects that limit efficiency in relativistic magnetrons," Plasma Science, IEEE Transactions on, vol. 28, no. 3, pp. 887-897, 2000. [Online]. Available: https://doi.org/10.1109/27.887745

[67] P. Pengvanich, V. B. Neculaes, Y. Y. Lau, R. M. Gilgenbach, M. C. Jones, W. M. White, and R. D. Kowalczyk, "Modeling and experimental studies of magnetron injection locking," Journal of Applied Physics, vol. 98, no. 11, p. 114903, 2005. [Online]. Available: http://dx.doi.org/10.1063/1.2132513

[68] P. Pengvanich, "Theory of Injection Locking and Rapid Start-Up of Magnetrons, and Effects of Manufacturing Errors in Terahertz Traveling Wave Tubes," Ph.D. dissertation, University of Michigan, Ann Arbor, MI, USA, 2007.

[69] P. Pengvanich, Y. Y. Lau, E. Cruz, R. M. Gilgenbach, B. Hoff, and J. W. Luginsland, "Analysis of peer-to-peer locking of magnetrons," Physics of Plasmas, vol. 15, no. 10, p. 103104, 2008. [Online]. Available: http://dx.doi.org/10.1063/1.2992526 
[70] E. J. Cruz, B. W. Hoff, P. Pengvanich, Y. Y. Lau, R. M. Gilgenbach, and J. W. Luginsland, "Experiments on peer-to-peer locking of magnetrons," Appl. Phys. Lett., vol. 95, no. 19, p. 191503, 2009. [Online]. Available: http://dx.doi.org/10.1063/1.3262970

[71] E. J. Cruz, "Peer to Peer Magnetron Locking," Ph.D. dissertation, University of Michigan, Ann Arbor, MI, USA, 2011.

[72] R. Adler, "A study of locking phenomena in oscillators," Proceedings of the IEEE, vol. 61, no. 10, pp. 1380-1385, Oct. 1973. [Online]. Available: http://dx.doi.org/10.1109/PROC.1973.9292

[73] D. M. Pozar, Microwave Engineering, 4th ed. Hoboken, NJ: John Wiley \& Sons, Inc., 2012.

[74] E. E. David, "Some Aspects Of R-F Phase Control In Microwave Oscillators," Research Laboratory of Electronics, Massachusetts Institute of Technology, Technical 100, Jun. 1949.

[75] S. C. Chen, "Growth and frequency pushing effects in relativistic magnetron phase-locking," IEEE Transactions on Plasma Science, vol. 18, no. 3, pp. 570-576, Jun. 1990. [Online]. Available: http://dx.doi.org/10.1109/27.55928

[76] T. A. Treado, P. D. Brown, and D. Aiguier, "New experimental results at long pulse and high repetition rate, from Varian's phase-locked magnetron array program," vol. 1872, 1993, pp. 241-251. [Online]. Available: http://dx.doi.org/10.1117/12.147464

[77] A. D. Greenwood, "All Cavity Magnetron Axial Extractor," U.S. Patent US 7106 004B1, Sep., 2006.

[78] B. W. Hoff, A. D. Greenwood, P. J. Mardahl, and M. D. Haworth, "All Cavity-Magnetron Axial Extraction Technique," IEEE Transactions on Plasma Science, vol. 40, no. 11, pp. 3046-3051, Nov. 2012. [Online]. Available: http://doi.org/10.1109/TPS.2012.2217758

[79] I. J. Myers and W. C. Brown, "Magnetron coaxial adaptor having a cap which fits over the magnetron output antenna," U.S. Patent US 5216327 A, Jun., 1993.

[80] B. Goplen, L. Ludeking, D. Smith, and G. Warren, "User-configurable MAGIC for electromagnetic PIC calculations," Comput. Phys. Commun., vol. 87, no. 1, pp. 5486, May 1995. [Online]. Available: https://doi.org/10.1016/0010-4655(95)00010-D

[81] M. R. Gomez, D. M. French, W. Tang, P. Zhang, Y. Y. Lau, and R. M. Gilgenbach, "Experimental validation of a higher dimensional theory of electrical contact resistance," Appl. Phys. Lett., vol. 95, no. 7, p. 072103, Aug. 2009. [Online]. Available: http://dx.doi.org/10.1063/1.3205116 
[82] P. Zhang, Y. Y. Lau, and R. M. Gilgenbach, "Thin film contact resistance with dissimilar materials," Journal of Applied Physics, vol. 109, no. 12, p. 124910, Jun. 2011. [Online]. Available: http://dx.doi.org/10.1063/1.3596759

[83] M. D. Haworth, G. Baca, J. Benford, T. Englert, K. Hackett, K. J. Hendricks, D. Henley, M. LaCour, R. W. Lemke, D. Price, D. Ralph, M. Sena, D. Shiffler, and T. A. Spencer, "Significant pulse-lengthening in a multigigawatt magnetically insulated transmission line oscillator," IEEE Transactions on Plasma Science, vol. 26, no. 3, pp. 312-319, Jun. 1998. [Online]. Available: http://dx.doi.org/10.1109/27.700759

[84] M. Fuks and E. Schamiloglu, "Rapid Start of Oscillations in a Magnetron with a "Transparent" Cathode," Phys. Rev. Lett., vol. 95, no. 20, Nov. 2005. [Online]. Available: https://doi.org/10.1103/PhysRevLett.95.205101

[85] M. C. Jones, V. B. Neculaes, R. M. Gilgenbach, W. M. White, M. R. Lopez, Y. Y. Lau, T. A. Spencer, and D. Price, "Projection ablation lithography cathode for highcurrent, relativistic magnetron," Review of Scientific Instruments, vol. 75, no. 9, pp. 2976-2980, Sep. 2004. [Online]. Available: http://dx.doi.org/10.1063/1.1784561

[86] M. C. Jones, V. B. Neculaes, Y. Y. Lau, R. M. Gilgenbach, and W. M. White, "Cathode priming of a relativistic magnetron," Appl. Phys. Lett., vol. 85, no. 26, p. 6332, 2004. [Online]. Available: http://dx.doi.org/10.1063/1.1841454

[87] T. Fleming and P. Mardahl, "Performance Improvements in the Relativistic Magnetron: The Effect of DC Field Perturbations," IEEE Transactions on Plasma Science, vol. 37, no. 11, pp. 2128-2138, Nov. 2009. [Online]. Available: https://doi.org/10.1109/TPS.2009.2030579

[88] Y. M. Saveliev, S. N. Spark, B. A. Kerr, M. I. Harbour, S. C. Douglas, and W. Sibbet, "Effect of cathode end caps and a cathode emissive surface on relativistic magnetron operation," Plasma Science, IEEE Transactions on, vol. 28, no. 3, pp. 478-484, 2000. [Online]. Available: https://doi.org/10.1109/27.887651

[89] M. Lopez, R. Gilgenbach, D. Jordan, S. Anderson, M. Johnston, M. Keyser, H. Miyake, C. Peters, M. Jones, V. Neculaes, Yue Ying Lau, T. Spencer, J. Luginsland, M. Haworth, R. Lemke, and D. Price, "Cathode effects on a relativistic magnetron driven by a microsecond e-beam accelerator," IEEE Transactions on Plasma Science, vol. 30, no. 3, pp. 947-955, Jun. 2002. [Online]. Available: https://doi.org/10.1109/TPS.2002.801543

[90] C. Leach, S. Prasad, M. I. Fuks, and E. Schamiloglu, "Suppression of Leakage Current in a Relativistic Magnetron Using a Novel Design Cathode Endcap," IEEE Transactions on Plasma Science, vol. 40, no. 8, pp. 2089-2093, Aug. 2012. [Online]. Available: http://doi.org/10.1109/TPS.2012.2199136 
[91] R. B. Miller, "Mechanism of explosive electron emission for dielectric fiber (velvet) cathodes," Journal of Applied Physics, vol. 84, no. 7, p. 3880, 1998. [Online]. Available: http://dx.doi.org/10.1063/1.368567

[92] D. Shiffler, M. Ruebush, M. LaCour, K. Golby, R. Umstattd, M. C. Clark, J. Luginsland, D. Zagar, and M. Sena, "Emission uniformity and emission area of explosive field emission cathodes," Appl. Phys. Lett., vol. 79, no. 18, p. 2871, 2001. [Online]. Available: http://dx.doi.org/10.1063/1.1415408

[93] D. Shiffler, M. LaCour, K. Golby, M. Sena, M. Mithcell, M. Haworth, K. Hendricks, and T. Spencer, "Comparison of velvet-and cesium iodide-coated carbon fiber cathodes," Plasma Science, IEEE Transactions on, vol. 29, no. 3, pp. 445-451, 2001. [Online]. Available: https://doi.org/10.1109/27.928942

[94] D. Shiffler, J. Heggemeier, M. LaCour, K. Golby, and M. Ruebush, "Low level plasma formation in a carbon velvet cesium iodide coated cathode," Physics of Plasmas, vol. 11, no. 4, p. 1680, 2004. [Online]. Available: http://dx.doi.org/10.1063/1.1666571

[95] B. A. Kerr, D. Shiffler, T. Knight, M. LaCour, K. Golby, and T. Spencer, "Carbon velvet cathode implementation on the Orion relativistic magnetron," in Pulsed Power symposium, 2005. The IEE (Reg. No. 2005/11070). IET, 2005, pp. 6-1. [Online]. Available: https://doi.org/10.1049/ic:20050035

[96] L. Courtois, J. Gardelle, and E. Pasini, "Emitted electron beams from velvet cathodes," in 2016 IEEE International Conference on Plasma Science (ICOPS), Jun. 2016, pp. 1-1. [Online]. Available: http://doi.org/10.1109/PLASMA.2016.7534092

[97] E. A. Abramyan, E. N. Efimov, and G. D. Kuleshov, "Energy recovery and power stabilization of pulsed electron beams in Marx generator circuits," in Proceedings of the 2nd International Topical Conference on High Power Electron and Ion Beam Research and Technology, vol. 2, Oct. 1977, pp. 755-760.

[98] Electron Beam Accelerator. Pulse Sciences Inc., Aug. 1983.

[99] R. M. Gilgenbach, L. D. Horton, R. F. Lucey Jr, S. Bidwell, M. Cuneo, J. Miller, and L. Smutek, "Microsecond Electron Beam Diode Closure Experiments," in Proc. IEEE Pulsed Power Conf., 1985, pp. 126-132.

[100] C. Waters, "Current Transformers Provide Accurate, Isolated Measurements," Dec. 1986.

[101] C. L. Enloe, "Ultraviolet-Induced Flashover Of Highly-Angled Polymeric Insulators In Vacuum,” Ph.D. dissertation, University of Michigan, Ann Arbor, MI, USA, 1988.

[102] R. F. Lucey, Jr., "Long-Pulse Relativistic Electron Beam Generation And Propagation In Gases And In Ultraviolet Laser Ionized Channels," Ph.D. dissertation, University of Michigan, Ann Arbor, MI, USA, 1988. 
[103] J. G. Leopold, A. S. Shlapakovski, A. Sayapin, and Y. E. Krasik, "Revisiting Power Flow and Pulse Shortening in a Relativistic Magnetron," IEEE Transactions on Plasma Science, vol. 43, no. 9, pp. 3168-3175, Sep. 2015. [Online]. Available: http://dx.doi.org/10.1109/TPS.2015.2463717

[104] F. J. Agee, "Evolution of pulse shortening research in narrow band, high power microwave sources," Plasma Science, IEEE Transactions on, vol. 26, no. 3, pp. 235-245, 1998. [Online]. Available: http://doi.org/10.1109/27.700749

[105] D. Price and J. N. Benford, "General scaling of pulse shortening in explosiveemission-driven microwave sources," Plasma Science, IEEE Transactions on, vol. 26, no. 3, pp. 256-262, 1998. [Online]. Available: https://doi.org/10.1109/27. 700752

[106] C. W. Peters, W. J. Williams, R. M. Gilgenbach, Y. Y. Lau, R. L. Jaynes, W. E. Cohen, M. R. Lopez, and T. A. Spencer, "Application of time-frequency analysis to high-power microwave devices," in Advanced Signal Processing Algorithms, Architectures, and Implementations X, vol. 4116, 2000, pp. 1-8. [Online]. Available: http://dx.doi.org/10.1117/12.406494

[107] A. Reilly, G. Frazer, and B. Boashash, "Analytic signal generation-tips and traps," IEEE Transactions on Signal Processing, vol. 42, no. 11, pp. 3241-3245, Nov. 1994. [Online]. Available: https://doi.org/10.1109/78.330385

[108] W. J. Williams, "Cross Hilbert time-frequency distributions," in Proc. SPIE, vol. 3461, 1998, pp. 120-129. [Online]. Available: http://dx.doi.org/10.1117/12.325673

[109] C. W. Peters, “Time-Frequency Analysis of High Power Microwave Sources,” Ph.D. dissertation, University of Michigan, Ann Arbor, MI, USA, 2001.

[110] M. Unser, A. Aldroubi, and M. Eden, "Polynomial spline signal approximations: filter design and asymptotic equivalence with Shannon's sampling theorem," IEEE Transactions on Information Theory, vol. 38, no. 1, pp. 95-103, Jan. 1992. [Online]. Available: https://doi.org/10.1109/18.108253

[111] M. E. Cuneo, "The effect of electrode contamination, cleaning and conditioning on high-energy pulsed-power device performance," IEEE Transactions on Dielectrics and Electrical Insulation, vol. 6, no. 4, pp. 469-485, Aug. 1999. [Online]. Available: http://dx.doi.org/10.1109/94.788747

[112] T. A. Treado, R. S. Smith, C. S. Shaughnessy, and G. E. Thomas, "Temporal study of long-pulse relativistic magnetron operation," IEEE Transactions on Plasma Science, vol. 18, no. 3, pp. 594-602, Jun. 1990. [Online]. Available: http://dx.doi.org/10.1109/27.55932

[113] D. A. Shiffler, J. W. Luginsland, R. J. Umstattd, A. LaCour, K. Golby, M. D. Haworth, M. Ruebush, D. Zagar, A. Gibbs, and T. A. Spencer, "Effects of anode materials on the performance of explosive field emission diodes," IEEE 
Transactions on Plasma Science, vol. 30, no. 3, pp. 1232-1237, Jun. 2002. [Online]. Available: http://dx.doi.org/10.1109/TPS.2002.802146

[114] A. S. Gilmour, Microwave Tubes. Dedham, MA: Artech House Publishers, 1986.

[115] A. Sayapin, Y. Hadas, and Y. E. Krasik, "Drastic improvement in the S-band relativistic magnetron operation," Appl. Phys. Lett., vol. 95, no. 7, p. 074101, 2009. [Online]. Available: http://dx.doi.org/10.1063/1.3206939

[116] S. Riyopoulos, "Efficiency reduction caused intense rf-induced $E \times B$ drift during relativistic magnetron operation," Physics of Plasmas, vol. 6, no. 4, p. 1344, 1999. [Online]. Available: http://dx.doi.org/10.1063/1.873714

[117] P. Phillips, Classical Electricity and Magnetism. Reading, MA: Addison-Wesley Publishing Company, Inc., 1955.

[118] “Ansys HFSS 15.0 Online Help,” 2012.

[119] L. Ludeking, A. Woods, and L. Cavey, "MAGIC User Manual," Apr. 2014.

[120] M. Haworth, K. Cartwright, J. Luginsland, D. Shiffler, and R. Umstattd, "Improved electrostatic design for milo cathodes," IEEE Transactions on Plasma Science, vol. 30, no. 3, pp. 992-997, Jun. 2002. [Online]. Available: https://doi.org/10.1109/TPS.2002.801550

[121] A. Iserles, A First Course in the Numerical Analysis of Differential Equations, 2nd ed. Cambridge, UK: Cambridge University Press, 2009.

[122] M. Engelhardt, "LTspice IV User Manual,” 2015.

[123] J. D. Miller, "Transport Of Long-Pulse Relativistic Electron Beams In Preformed Plasma Channels In The Ion Focus Regime," Ph.D. dissertation, University of Michigan, 1989.

[124] R. M. Gilgenbach, J. M. Hochman, R. L. Jaynes, W. E. Cohen, J. I. Rintamaki, C. W. Peters, D. E. Vollers, Y. Y. Lau, and T. A. Spencer, "Optical spectroscopy of plasma in high power microwave pulse shortening experiments driven by a $\mu \mathrm{s}$ e-beam," IEEE Transactions on Plasma Science, vol. 26, no. 3, pp. 282-289, Jun. 1998. [Online]. Available: http://dx.doi.org/10.1109/27.700755

[125] V. Vekselman, J. Gleizer, D. Yarmolich, J. Felsteiner, Y. Krasik, L. Liu, and V. Bernshtam, "Plasma characterization in a diode with a carbon-fiber cathode," Applied Physics Letters, vol. 93, no. 8, p. 081503, 2008. [Online]. Available: http://dx.doi.org/10.1063/1.2976136

[126] T. L. Smith, "Vector Network Analyzer Techniques to Measure WR340 Waveguide Windows," Jun. 2002.

[127] G. A. Mesyats, Pulsed Power. New York, NY: Kluwer Academic / Plenum Publishers, 2005. 
[128] “Parker O-Ring Handbook (ORD 5700),” 2007. 